Florida International University

FIU Digital Commons

FIU Electronic Theses and Dissertations

University Graduate School

$10-28-2010$

\title{
Comparison of Medical and Forensic Profiling Potential of Volatile Biomarkers from Different Biological Specimens from Individuals and Across Populations
}

Maiko Kusano

Florida International University, mkusa001@fiu.edu

DOI: $10.25148 /$ etd.FI10120102

Follow this and additional works at: https://digitalcommons.fiu.edu/etd

\section{Recommended Citation}

Kusano, Maiko, "Comparison of Medical and Forensic Profiling Potential of Volatile Biomarkers from Different Biological Specimens from Individuals and Across Populations" (2010). FIU Electronic Theses and Dissertations. 307.

https://digitalcommons.fiu.edu/etd/307

This work is brought to you for free and open access by the University Graduate School at FIU Digital Commons. It has been accepted for inclusion in FIU Electronic Theses and Dissertations by an authorized administrator of FIU Digital Commons. For more information, please contact dcc@fiu.edu. 


\section{FLORIDA INTERNATIONAL UNIVERSITY}

Miami, Florida

COMPARISON OF MEDICAL AND FORENSIC PROFILING POTENTIAL OF VOLATILE BIOMARKERS FROM DIFFERENT BIOLOGICAL SPECIMENS FROM INDIVIDUALS AND ACROSS POPULATIONS

A dissertation submitted in partial fulfillment of the requirements for the degree of DOCTOR OF PHILOSOPHY

in CHEMISTRY

by

Maiko Kusano 
To: Dean Kenneth Furton

College of Arts and Sciences

This dissertation, written by Maiko Kusano, and entitled Comparison of Medical and Forensic Profiling Potential of Volatile Biomarkers from Different Biological Specimens from Individuals and Across Populations, having been approved in respect to style and intellectual content, is referred to you for judgment.

We have read this dissertation and recommend that it be approved.

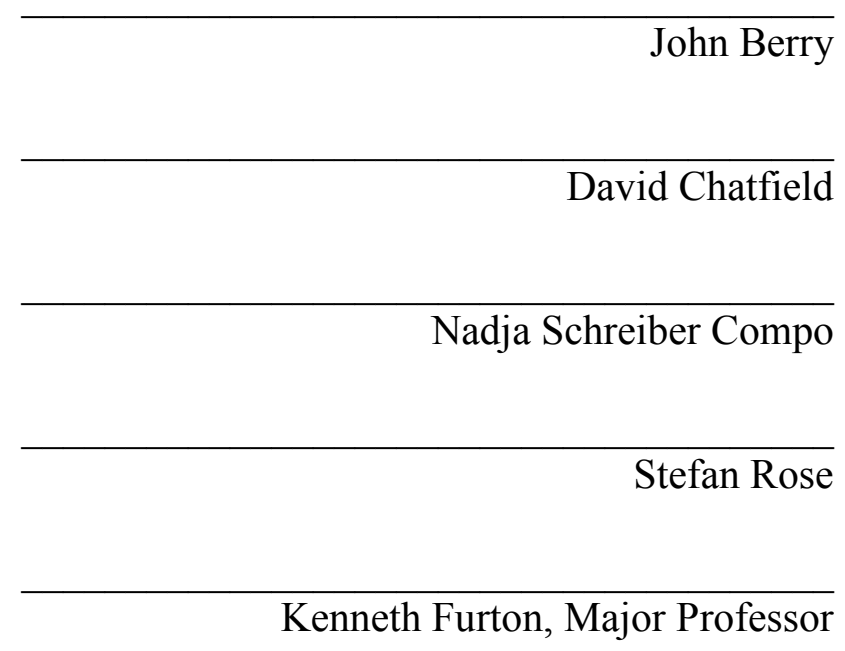

Date of Defense: October 28, 2010

The dissertation of Maiko Kusano is approved.

Dean Kenneth Furton

College of Arts and Sciences

Interim Dean Kevin O'Shea

University Graduate School

Florida International University, 2010 
C Copyright 2010 by Maiko Kusano All rights reserved. 


\section{DEDICATION}

This dissertation is dedicated to my parents, Yoichi and Keiko Kusano.

Without their unconditional love and silent yet continuous support from the other side of the world, the completion of this work would not have been possible. 


\section{ACKNOWLEDGMENTS}

I would like to thank my committee members Dr. John Berry, Dr. David Chatfield, Dr. Stefan Rose, and Dr. Nadja Schreiber Compo for serving on my committee and helping me throughout the course of this dissertation work. I would also like to thank Dr. Albert Sabucedo while he served on my committee for his time and patience with my endless questions. I would like to acknowledge Jackson North Medical Center for allowing me to recruit and sample patients on-site during my study. I would like to thank Dr. Daniel Castellanos for helping me with subject recruitment and acting as my site supervisor at Jackson North Medical Center. I would like to thank my undergraduate research assistant, Eladio Mendez, for helping me out immensely through portions of this research. I would also like to thank Howard Holness who has been an incredible lab manager, friend, and mentor. His generosity in sharing his time, knowledge, and kindness has helped me tremendously in accomplishing my work. Finally, I would like to especially thank my major professor, Dr. Kenneth Furton, for taking me into his group when I became lost, and for his guidance and patience with me throughout this entire process.

I would also like to thank my family and friends for their understanding and continuous support throughout this process. I wish to also thank the members of my Japanese taiko drumming group, Fushu Daiko, for providing me with endless entertainment and a place for me to maintain my sanity during graduate school. Special thanks to Yukako Beatty who has been my closest place to home. Thank you to my graduate school friends for the support and friendship. I would like to say a special thanks to Michael Macias, my former roommate and lab mate, for putting up with me from the beginning until the end. Without 
his support, love, encouragement, faith in me, and slightly more than occasional shoulder to cry on, I doubt that I would have been able to succeed in completing this process. Lastly, I would like to thank my friends who are scattered around the world for their endless support, love and friendship, and places for me to internationally escape to when I needed a break. 


\author{
ABSTRACT OF THE DISSERTATION \\ COMPARISON OF MEDICAL AND FORENSIC PROFILING POTENTIAL OF \\ VOLATILE BIOMARKERS FROM DIFFERENT BIOLOGICAL SPECIMENS FROM \\ INDIVIDUALS AND ACROSS POPULATIONS \\ by \\ Maiko Kusano \\ Florida International University, 2010 \\ Miami, Florida \\ Professor Kenneth Furton, Major Professor
}

There is limited scientific knowledge on the composition of human odor from different biological specimens and the effect that physiological and psychological health conditions could have on them. There is currently no direct comparison of the volatile organic compounds (VOCs) emanating from different biological specimens collected from healthy individuals as well as individuals with certain diagnosed medical conditions. Therefore the question of matching VOCs present in human odor across various biological samples and across health statuses remains unanswered.

The main purpose of this study was to use analytical instrumental methods to compare the VOCs from different biological specimens from the same individual and to compare the populations evaluated in this project. The goals of this study were to utilize headspace solid-phase microextraction gas chromatography mass spectrometry (HS-SPME-GC/MS) to evaluate its potential for profiling VOCs from specimens collected using standard forensic and medical methods over three different populations: healthy group with no 
diagnosed medical or psychological condition, one group with diagnosed type 2 diabetes, and one group with diagnosed major depressive disorder.

The pre-treatment methods of collection materials developed for the study allowed for the removal of targeted VOCs from the sampling kits prior to sampling, extraction and analysis. Optimized SPME-GC/MS conditions has been demonstrated to be capable of sampling, identifying and differentiating the VOCs present in the five biological specimens collected from different subjects and yielded excellent detection limits for the VOCs from buccal swab, breath, blood, and urine with average limits of detection of 8.3 ng.

Visual, Spearman rank correlation, and PCA comparisons of the most abundant and frequent VOCs from each specimen demonstrated that each specimen has characteristic VOCs that allow them to be differentiated for both healthy and diseased individuals. Preliminary comparisons of VOC profiles of healthy individuals, patients with type 2 diabetes, and patients with major depressive disorder revealed compounds that could be used as potential biomarkers to differentiate between healthy and diseased individuals. Finally, a human biological specimen compound database has been created compiling the volatile compounds present in the emanations of human hand odor, oral fluids, breath, blood, and urine. 


\section{TABLE OF CONTENTS}

CHAPTER

PAGE

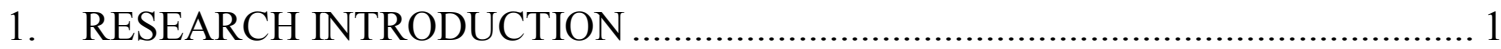

1.1. Basis for research 1

1.2. Research Objectives 2

1.3. Forensic Science and Human Scent 3

1.3.1. Admissibility of Scientific Evidence 3

1.3.2. Human Scent as Scientific Evidence

1.3.3. Canines and Human Scent 7

1.4. Alternative Biological Specimens as Scent Sources 7

1.5. Theory of Instrumental Techniques 9

1.5.1. Solid Phase Microextraction (SPME) 9

1.5.2. Gas-Chromatography/Mass Spectrometry (GC/MS) 13

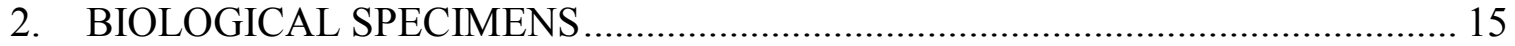

2.1. Human Scent 15

2.1.1. Production of Human Scent 15

2.1.2. Distinctiveness of Human Scent 15

2.1.3. Persistence and Stability of Human Scent 17

2.1.4. Hand odor sampling 18

2.1.4.1. Materials \& Methods 18

2.2. Oral Fluids (Saliva) 19

2.2.1. Materials \& Methods 23

2.2.2. Pre-treatment of buccal swab collection material 24

2.2.2.1. Methods 24

2.2.2.2. Results 24

2.2.3. Buccal swab sampling procedure 26

2.2.4. Determination of optimal extraction conditions for buccal swab 26

2.2.4.1. Methods 26

2.2.4.2. Results 27

2.3. Breath 28

2.3.1. Materials \& Methods 31

2.3.2. Pre-treatment of Bio-VOC $®$ Breath Sampler $\quad 32$

2.3.2.1. Methods $\quad 32$

2.3.2.2. Results $\quad 32$

2.3.3. Breath sampling procedure $\quad 34$

2.3.4. Determination of optimal extraction conditions for breath 34

2.3.4.1. Methods $\quad 34$

2.3.4.2. Results $\quad 35$

2.3.4.2.1. Optimal Extraction Time 35

2.3.4.2.2. Optimal Equilibration Time 38

2.4. Blood 40

2.4.1. Materials \& Methods 43 
2.4.2. Pre-treatment of FTA card 44

$\begin{array}{lll}2.4 .2 .1 . & \text { Methods } & 44\end{array}$

2.4.2.2. Results 44

2.4.3. Blood sampling procedure $\quad 46$

2.4.4. Determination of optimal extraction conditions for blood 46

2.4.4.1. Methods 46

2.4.4.2. Results 47

2.4.4.2.1. Optimal Extraction Time 47

2.4.4.2.2. Optimal Equilibration Time 48

2.4.4.2.3. Effect of Sample Heating 50

2.4.4.2.4. Blood Sample Stability 52

2.5. Urine 53

2.5.1. Materials \& Methods $\quad 56$

2.5.2. Urine sampling procedure 57

2.5.3. Determination of optimal extraction conditions for urine 57

$\begin{array}{lll}\text { 2.5.3.1. Methods } & 57\end{array}$

2.5.3.2. Results 58

2.5.3.2.1. Optimal Extraction Time 58

2.5.3.2.2. Optimal Equilibration Time 59

2.5.3.2.3. Optimal Extraction Temperature 62

2.5.3.2.4. Effect of Salting Out 66

2.6. Comparison of Five Specimens from Individuals 69

$\begin{array}{lll}\text { 2.6.1. Methods } & 69\end{array}$

$\begin{array}{lll}\text { 2.6.2. Results } & 70\end{array}$

2.6.3. Spearman Correlation Ranking $\quad 77$

2.7. Quantitation of VOCs Collected from Specimens 80

2.8. Summary of Biological Specimen Optimization 87

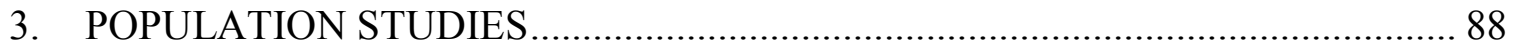

3.1. Scent Biomarkers for Diseases $\quad 88$

3.1.1. Medicine and Human Scent $\quad 91$

3.1.1.1. Disease-Specific Scent 91

3.1.1.2. Canines and Cancer $\quad 92$

3.1.1.3. Rats and Schizophrenia 93

3.2. Diabetes Mellitus 93

3.2.1. Type 1 Diabetes $\quad 94$

3.2.2. Type 2 Diabetes 95

3.3. Mood Disorders 97

3.3.1. Major Depressive Disorder 97

3.4. Comorbidity between Diabetes and Depression 102

$\begin{array}{ll}\text { 3.5. Methods } & 102\end{array}$

3.5.1. Evaluation of Odor Profiles of Individuals over Time 103

3.5.2. Evaluation of the Effect of Fasting Prior to Sampling 103

3.5.3. Population Analysis of the Volatile Organic Compounds Present
Above Collected Odor Samples 
3.5.3.1. Healthy Individuals 103

3.5.3.2. Patients with Type 2 Diabetes 104

3.5.3.3. Patients with Major Depressive Disorder 104

3.6. Results 105

3.6.1. Evaluation of Odor Profiles of Individuals over Time 105

3.6.2. Principal Component Analysis 117

3.6.3. Evaluation of the Effect of Fasting Prior to Sampling 125

3.6.3.1. Cluster Analysis 138

3.6.4. Population Analysis of the Volatile Organic Compounds Present

3.6.4.1. Evaluation of Odor Profiles of Healthy Individuals 144

3.6.4.1.1. Inter-Specimen Matching 172

3.6.4.1.2. Intra-Specimen Matching 175

3.6.4.1.2.1. Type I Error 179

3.6.4.1.2.2. Type II Error 181

3.6.4.2. Evaluation of Odor Profiles of Individuals with Type 2 Diabetes 183

3.6.4.3. Evaluation of Odor Profiles of Individuals with Major Depressive Disorder 195

3.6.4.4. Comparison of the Volatile Organic Compounds Present Above Collected Odor Samples from Five Biological Specimens across Populations 207

3.6.4.4.1. Chi-Square Significance Test 211

3.7. Human Biological Specimen Compound Database 233

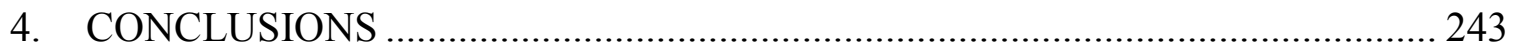

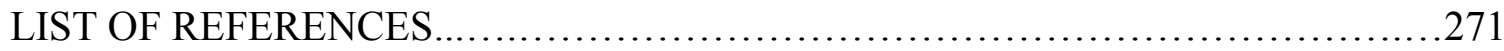

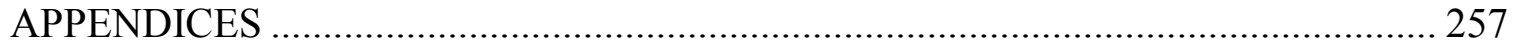

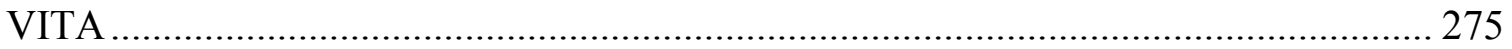




\section{LIST OF TABLES}

TABLE

PAGE

Table 1. U.S. Court Cases with Human Scent Evidence

Table 2. Advantages and disadvantages of hand odor, buccal swabs, breath, blood,

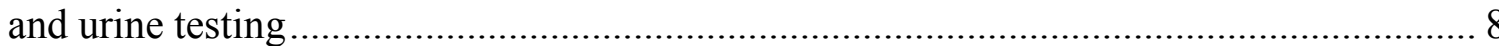

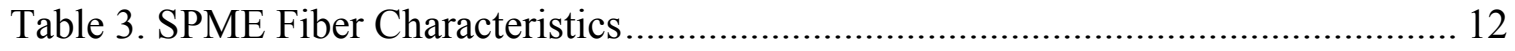

Table 4. Plasma, initial ultrafiltrate and final urine concentrations of major solutes ....... 55

Table 5. Compounds detected under different extraction times for urine SPME

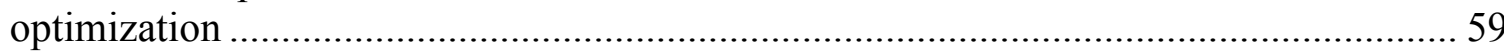

Table 6. Summary of optimized SPME conditions for biological specimens ................. 70

Table 7. Limits of detection and quantitation of VOCs found in the headspace of hand odor

Table 8. Limits of detection and quantitation of VOCs found in the headspace of

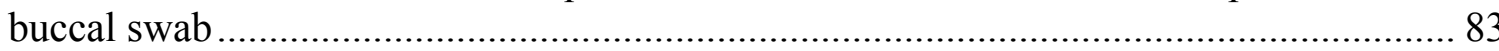

Table 9. Limits of detection and quantitation of VOCs found in the headspace of breath

Table 10. Limits of detection and quantitation of VOCs found in the headspace of blood

Table 11. Limits of detection and quantitation of VOCs found in the headspace of urine

Table 12. Published studies on VOCs in biological specimens identified as potential diagnostic markers of various diseases

Table 13. Metabolic disorders with characteristic odors

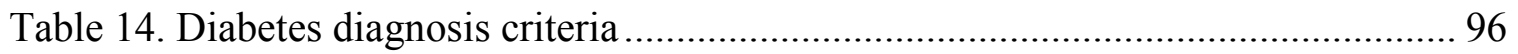

Table 15. Current oral therapeutic agents for type 2 diabetes ....................................... 96

Table 16. VOCs extracted over 6 months for Female 4 ............................................ 108

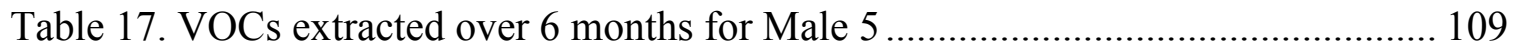


Table 18. VOCs extracted from hand odor from F4 and M5 over 6 months 110

Table 19. VOCs extracted from buccal swabs from F4 and M5 over 6 months. 111

Table 20. VOCs extracted from breath from F4 and M5 over 6 months...................... 112

Table 21. VOCs extracted from blood from F4 and M5 over 6 months........................ 113

Table 22. VOCs extracted from urine from F4 and M5 over 6 months......................... 114

Table 23. Spearman rank correlation coefficients for the peak areas of hand odor VOCs across 6 monthly samplings of F4 and M5

Table 24. Spearman rank correlation coefficients for the peak areas of buccal swab VOCs across 6 monthly samplings of F4 and M5

Table 25. Spearman rank correlation coefficients for the peak areas of breath VOCs across 6 monthly samplings of F4 and M5 115

Table 26. Spearman rank correlation coefficients for the peak areas of blood VOCs across 6 monthly samplings of F4 and M5

Table 27. Spearman rank correlation coefficients for the peak areas of urine VOCs

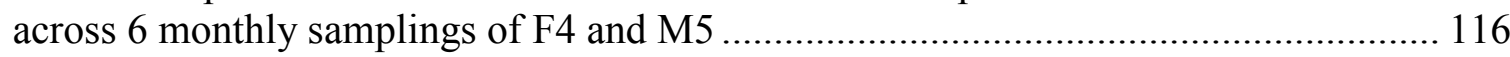

Table 28. PCA results summary for hand odor.................................................... 120

Table 29. PCA results summary for buccal swab ................................................... 121

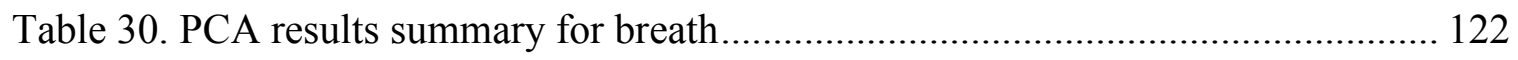

Table 31. PCA results summary for blood............................................................ 123

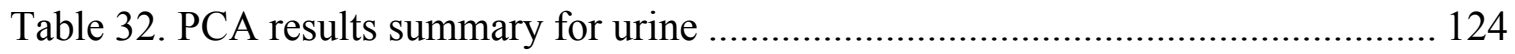

Table 33. Summary of volatile compounds extracted fasting (AM) vs. postprandial (PM) for Female 4.

Table 34. Summary of compounds extracted fasting (AM) vs. postprandial (PM) for Female 15

Table 35. Summary of compounds extracted fasting (AM) vs. postprandial (PM) for Male 2 .

Table 36. Summary of compounds extracted fasting (AM) vs. postprandial (PM) for Male 5. 
Table 37. Identified VOCs in hand odor of thirty-one healthy individuals ranked by frequency of occurrence.

Table 38. Identified VOCs in buccal swab of thirty-one healthy individuals ranked by frequency of occurrence.

Table 39. Identified VOCs in breath odor of thirty-one healthy individuals ranked by frequency of occurrence

Table 40. Identified VOCs in blood odor of thirty-one healthy individuals ranked by frequency of occurrence.

Table 41. Identified VOCs in urine odor of thirty-one healthy individuals ranked by frequency of occurrence. 168

Table 42. Spearman rank correlation coefficients for the peak areas of the VOCs extracted from biological specimens from Male 16

Table 43. Example of Type I error occurrences 180

Table 44. Type I error occurrences for three correlation thresholds across biological specimens

Table 45. Example of Type II error occurrences

Table 46. Type II error occurrences for three correlation thresholds across biological specimens

Table 47. Type I and Type II errors for five biological specimens amongst thirty-one healthy individuals

Table 48. Identified VOCs in hand odor of nineteen individuals with diagnosed type 2 diabetes ranked by frequency of occurrence.

Table 49. Identified VOCs in buccal swabs of nineteen individuals with diagnosed type 2 diabetes ranked by frequency of occurrence. 188

Table 50. Identified VOCs in breath odor of nineteen individuals with diagnosed type 2 diabetes ranked by frequency of occurrence

Table 51. Identified VOCs in blood odor of nineteen individuals with diagnosed type 2 diabetes ranked by frequency of occurrence.

Table 52. Identified VOCs in urine odor of nineteen individuals with diagnosed type 2 diabetes ranked by frequency of occurrence. 
Table 53. Identified VOCs in hand odor of twenty individuals with diagnosed Major

Depressive Disorder ranked by frequency of occurrence 198

Table 54. Identified VOCs in buccal swab of twenty individuals with diagnosed Major Depressive Disorder ranked by frequency of occurrence

Table 55. Identified VOCs in breath of twenty individuals with diagnosed Major Depressive Disorder ranked by frequency of occurrence.

Table 56. Identified VOCs in blood odor of twenty individuals with diagnosed Major Depressive Disorder ranked by frequency of occurrence. 203

Table 57. Identified VOCs in urine odor of twenty individuals with diagnosed Major Depressive Disorder ranked by frequency of occurrence..... 205

Table 58. Type I and Type II errors for five biological specimens amongst healthy individuals.

Table 59. Type I and Type II errors for five biological specimens amongst type 2 diabetic patients 210

Table 60. Type I and Type II errors for five biological specimens amongst individuals diagnosed with Major Depressive Disorder....

Table 61. Comparison of mismatch occurrences for the five biological specimens across populations at 0.9 correlation cutoff threshold

Table 62. Frequency of occurrence of hand odor VOCs in total population and within subgroups....

Table 63. Frequency of occurrence of buccal swab odor VOCs in total population and within subgroups

Table 64. Frequency of occurrence of breath odor VOCs in total population and within subgroups....

Table 65. Frequency of occurrence of breath odor VOCs in total population and within subgroups....

Table 66. Frequency of occurrence of breath odor VOCs in total population and within subgroups.

Table 67. Occurrences of 15 VOCs with significant difference between population subgroups for hand odor through the chi-square test. 228

Table 68. Occurrences of 47 VOCs with significant difference between population subgroups for buccal swab through the chi-square test 
Table 69. Occurrences of 29 VOCs with significant difference between population subgroups for breath through the chi-square test .................................................. 230

Table 70. Occurrences of 29 VOCs with significant difference between population subgroups for blood through the chi-square test.................................................. 231

Table 71. Occurrences of 27 VOCs with significant difference between population subgroups for urine through the chi-square test..................................................... 232

Table 72 Biological specimen compound database ................................................ 235

Table 73. Target ion and qualifying ions for frequently occurring hand odor VOCs

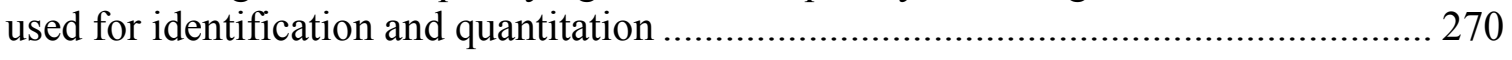

Table 74. Target ion and qualifying ions for frequently occurring buccal swab

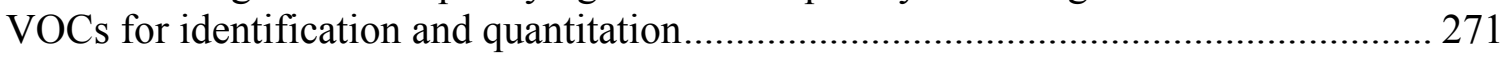

Table 75. Target ion and qualifying ions for frequently occurring breath VOCs for identification and quantitation

Table 76. Target ion and qualifying ions for frequently occurring blood VOCs for

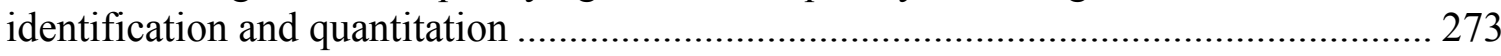

Table 77. Target ion and qualifying ions for frequently occurring urine VOCs for identification and quantitation 


\section{LIST OF FIGURES}

FIGURE

PAGE

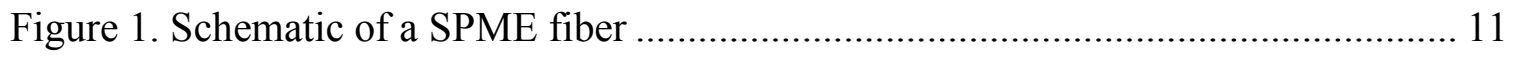

Figure 2. Block diagram of a gas chromatograph coupled to a mass spectrometer.......... 14

Figure 3. Schematic of human salivary glands ................................................... 21

Figure 4. Comparison of untreated and pre-treated cotton swab ................................ 25

Figure 5. Comparison of cotton swab pretreatment for the removal of undesired

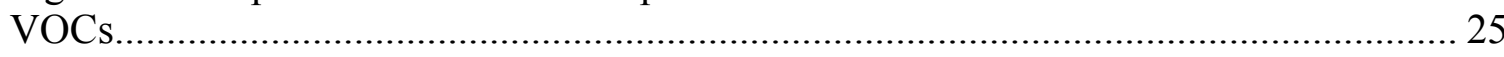

Figure 6. Number of human scent compounds extracted from buccal swab samples vs.

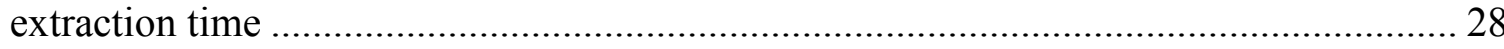

Figure 7. Comparison of untreated and pre-treated Bio-VOC $®$ breath sampler ............. 33

Figure 8. Comparison of breath sampler pretreatment for the removal of undesired

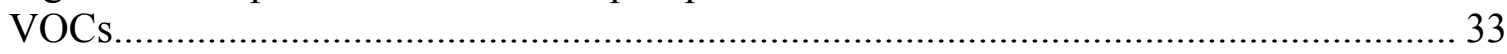

Figure 9. Number of common human scent compounds extracted vs. extraction time for breath samples for subjects M1 and F4 ........................................................ 36

Figure 10. Number of common human scent compounds extracted vs. extraction time for breath samples for subjects M5 and F2 …................................................... 37

Figure 11. Abundances of selected common breath VOCs extracted vs. extraction

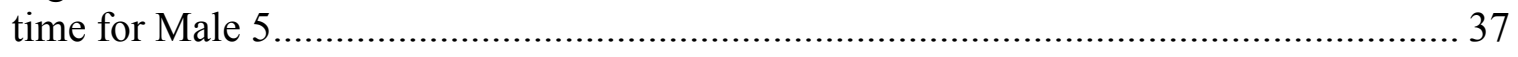

Figure 12. Number of compounds extracted vs. equilibration time for breath................ 39

Figure 13. Abundances of selected common breath VOCs extracted vs. equilibration

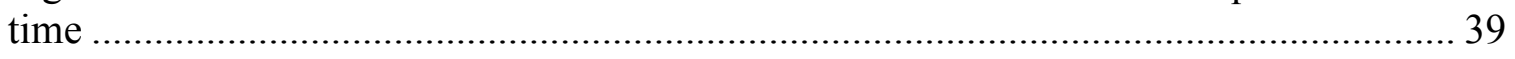

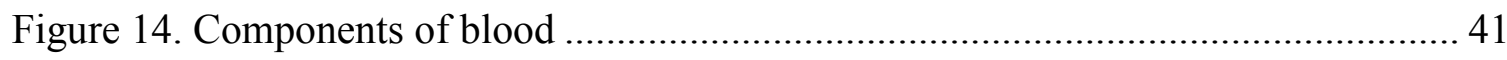

Figure 15. Comparison of untreated and pre-treated FTA card .................................. 45

Figure 16. Comparison of FTA card pretreatment for the removal of undesired VOCs .. 45

Figure 17. Number of compounds extracted vs. extraction time for whole blood ........... 47 
Figure 18. Abundance of selected common compounds extracted vs. extraction time

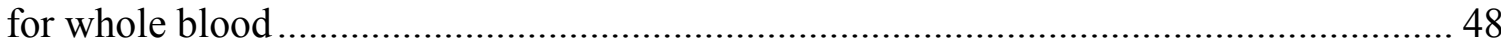

Figure 19. Number of compounds extracted vs. equilibration time for whole blood....... 49

Figure 20. Abundance of selected common blood VOCs extracted vs. equilibration time

Figure 21. Number of compounds extracted at room temperature and $37^{\circ} \mathrm{C}$ for whole blood

Figure 22. Abundance vs. extraction time for undecane at room temperature and $37^{\circ} \mathrm{C} . .51$

Figure 23. VOC profiles of M5 blood fresh, 1 day, and 1 month after storage................ 52

Figure 24. Schematic of urine production........................................................... 53

Figure 25. Abundance vs. equilibration times of aldehydes for urine optimization......... 60

Figure 26. Abundance vs. equilibration times for acids for urine optimization .............. 60

Figure 27. Abundance vs. equilibration times for alcohols for urine optimization .......... 61

Figure 28. Abundance vs. equilibration times for ketones for urine optimization ........... 61

Figure 29. Abundance vs. equilibration time for other functional group compounds

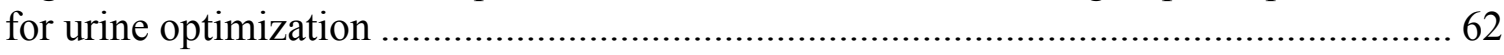

Figure 30. Abundance vs. extraction temperatures of aldehydes for urine optimization.. 63

Figure 31. Abundance vs. extraction temperatures of acids for urine optimization ......... 64

Figure 32. Abundance vs. extraction temperatures of alcohols for urine optimization .... 64

Figure 33. Abundance vs. extraction temperatures of ketones for urine optimization ..... 65

Figure 34. Abundance vs. extraction temperatures of other functional group

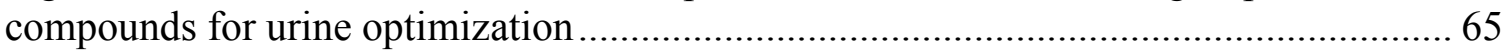

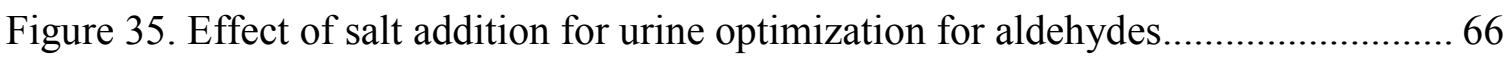

Figure 36. Effect of salt addition for urine optimization for acids .............................. 67

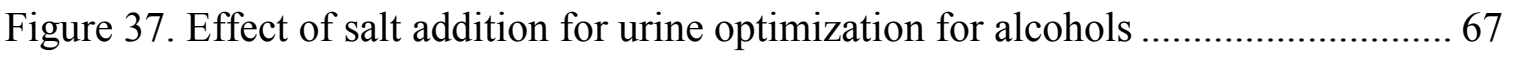

Figure 38. Effect of salt addition for urine optimization for ketones ............................. 68 
Figure 39. Effect of salt addition for urine optimization for other functional group compounds

Figure 40. Typical odor profile chromatogram for a) hand odor, b) buccal swab,

c) breath, d) blood, and e) urine..... 71

Figure 41. Comparison of buccal swab odor profiles from subjects F4, F2, M1, and M5 72

Figure 42. Comparison of breath odor profiles from subjects F4, F2, M1, and M5....... 72

Figure 43. Comparison of blood odor profiles from subjects F4, F2, M1, and M5 ........ 73

Figure 44. Comparison of urine odor profiles from subjects F4, F2, M1, and M5 ......... 73

Figure 45. Buccal swab VOC profile comparison between 4 subjects........................... 74

Figure 46. Subject comparison of breath VOC profiles from subjects F4, F2, M1,

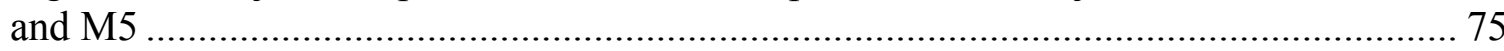

Figure 47. Subject comparison of blood VOC profiles from subjects F4, WB, M1, and M5 76

Figure 48. Subject comparison of urine VOC profiles from subjects F4, F2, M1, and M5....

Figure 49. Spearman rank correlation with respect to Female 4, sample 1 for buccal swab ... 78

Figure 50. Spearman rank correlation with respect to Female 2, sample 1 for breath ..... 78

Figure 51. Spearman rank correlation with respect to Male 1, sample 1 for blood.......... 79

Figure 52. Spearman rank correlation with respect to Male 5, sample 1 for urine.......... 79

Figure 53. Diagram of cell glucose regulation for normal, type 1 and type 2 diabetes .... 95

Figure 54. Difference in the chemical message transduction in the brain cells of normal individual and individual with MDD (adapted from Pfizer Japan Inc.) 100

Figure 55. Common compounds extracted among monthly samplings of hand odor from subjects F4 and M5

Figure 56. Common compounds extracted among monthly samplings of buccal swabs from subjects F4 and M5..... 
Figure 57. Common compounds extracted among monthly samplings of breath from subjects F4 and M5

Figure 58. Common compounds extracted among monthly samplings of blood from subjects F4 and M5

Figure 59. Common compounds extracted among monthly samplings of urine from subjects F4 and M5

Figure 60. Three-dimensional PCA scatter plot of PCs 1, 2, and 3 of Female 4 118

Figure 61. Three-dimensional PCA scatter plot of PCs 1, 2, and 3 of Male 5

Figure 62. Three-dimensional PCA scatter plot of PCs 1, 2, and 3 of hand odor from F4 and M5 over 6 months

Figure 63. Three-dimensional PCA scatter plot of PCs 1, 2, and 3 of buccal swabs from F4 and M5 over 6 months

Figure 64. Three-dimensional PCA scatter plot of PCs 1, 2, and 3 of breath from F4 and M5 over 6 months

Figure 65. Three-dimensional PCA scatter plot of PCs 1, 2, and 3 of blood from F4 and M5 over 6 months

Figure 66. Three-dimensional PCA scatter plot of PCs 1, 2, and 3 of urine from F4 and M5 over 6 months

Figure 67. Average number of compounds extracted from specimens collected after fasting and postprandial

Figure 68. Color chart and dendogram for hand odor for M2 and F15

Figure 69. Color chart and dendogram for buccal swabs for M2 and F15 140

Figure 70. Color chart and dendogram for breath for M2 and F15

Figure 71. Color chart and dendogram for blood for M2 and F15 142

Figure 72. Color chart and dendogram for urine for M2 and F15 143

Figure 73. Frequency distribution of functional groups of extracted volatile organic compounds for hand odor 146

Figure 74. Histogram of VOCs in hand odor across thirty-one healthy individuals 148 
Figure 75. Frequency distribution of functional groups of extracted volatile organic

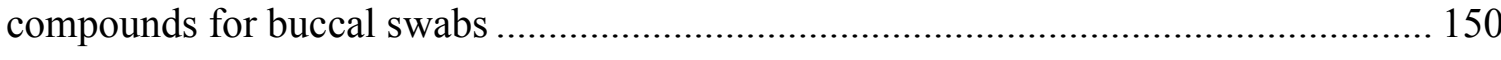

Figure 76. Histogram of VOCs in buccal swab odor from healthy individuals ............. 154

Figure 77. Frequency distribution of functional groups of extracted volatile organic

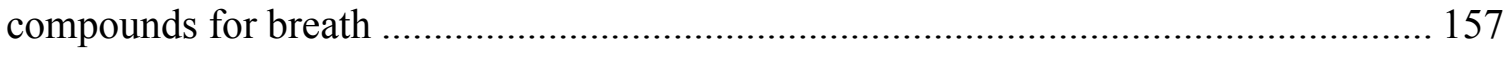

Figure 78. Histogram of VOCs in breath odor across thirty-one healthy individuals .... 160

Figure 79. Frequency distribution of functional groups of extracted volatile organic compounds for blood

Figure 80. Histogram of VOCs in blood odor across thirty-one healthy individuals.... 165

Figure 81. Frequency distribution of functional groups of extracted volatile organic compounds for urine 168

Figure 82. Histogram of VOCs in urine odor across thirty-one healthy individuals...... 171

Figure 83. Relative peak area ratios of VOCs extracted from M16 specimens.............. 173

Figure 84. Three-dimensional PCA scatter plot of PCs 1, 2, and 3 of hand odor from

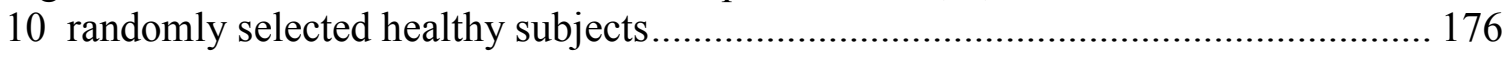

Figure 85. Three-dimensional PCA scatter plot of PCs 1, 2, and 3 of buccal swabs from 10 randomly selected healthy subjects 176

Figure 86. Three-dimensional PCA scatter plot of PCs 1, 2, and 3 of breath from 10 randomly selected healthy subjects

Figure 87. Three-dimensional PCA scatter plot of PCs 1, 2, and 3 of blood from 10 randomly selected healthy subjects.

Figure 88. Three-dimensional PCA scatter plot of PCs 1, 2, and 3 of urine from 10 randomly selected healthy subjects 178

Figure 89. Frequency distribution of functional groups of extracted VOCs in hand odor, buccal swab, breath, blood, and urine across twenty individuals diagnosed with type 2 diabetes 186

Figure 90. Frequency distribution of functional groups of extracted VOCs in hand odor, buccal swab, breath, blood, and urine across twenty individuals diagnosed with Major Depressive Disorder 197

Figure 91. Calibration curve for furfural 257 


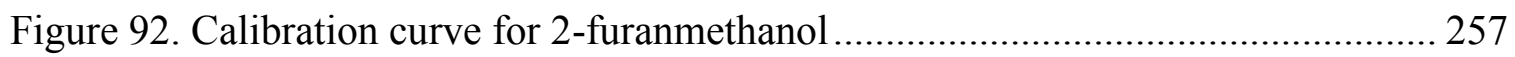

Figure 93. Calibration curve for dimethyl malonate............................................ 258

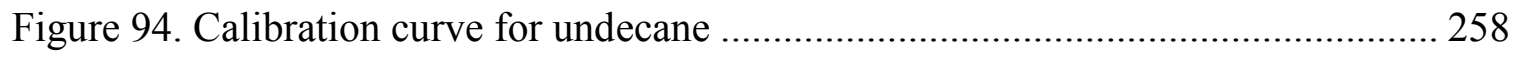

Figure 95. Calibration curve for (E)-6,10-dimethyl-5,9-undecadien-2-one .................. 258

Figure 96. Calibration curve for dodecanoic acid................................................. 259

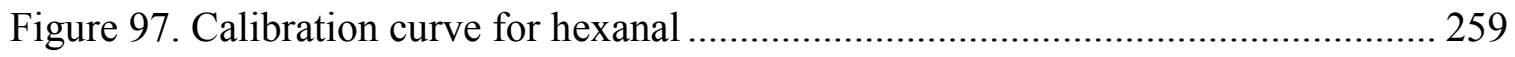

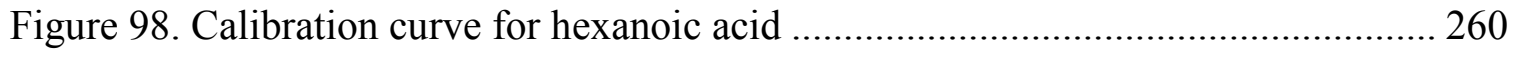

Figure 99. Calibration curve for acetophenone..................................................... 260

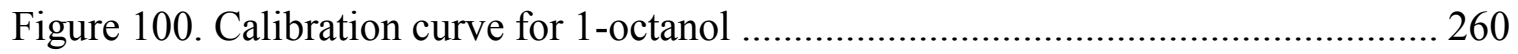

Figure 101. Calibration curve for octanoic acid, ethyl ester..................................... 261

Figure 102. Calibration curve for 1 -methylnaphthalene.......................................... 261

Figure 103. Calibration curve for tetradecane .................................................... 261

Figure 104. Calibration curve for 3 -heptanone..................................................... 262

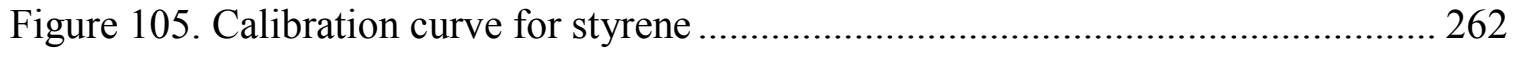

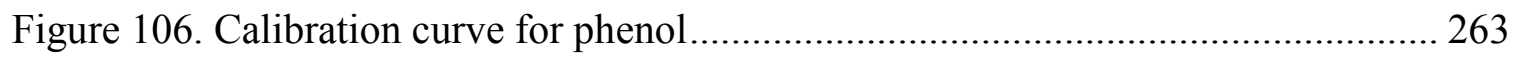

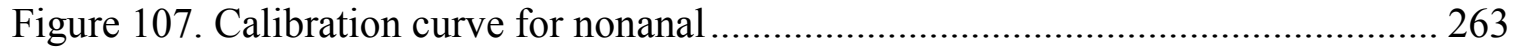

Figure 108. Calibration curve for 1-methylnaphthalene .......................................... 263

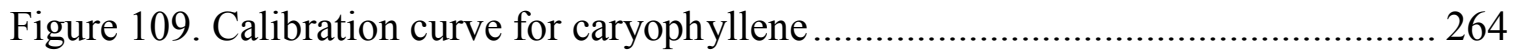

Figure 110. Calibration curve for cyclohexanone.............................................. 264

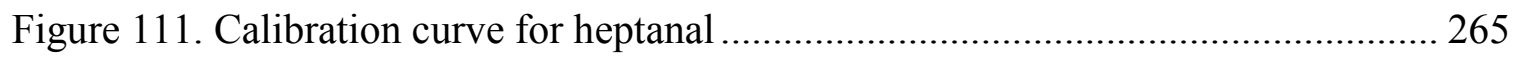

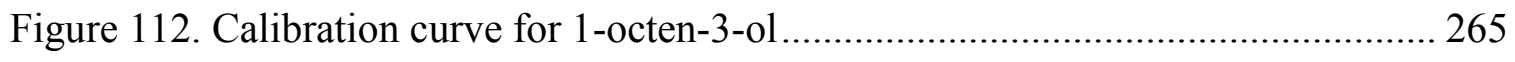

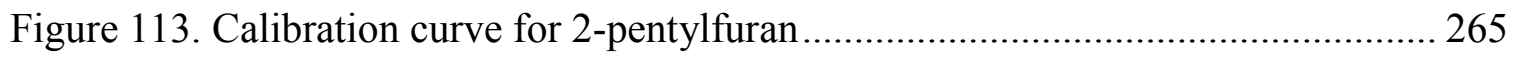

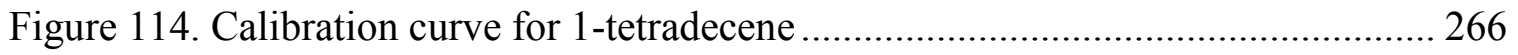


Figure 115. Calibration curve for 2,6-diisopropylnaphthalene.

Figure 116. Calibration curve for 4-heptanone.......................................................... 267

Figure 117. Calibration curve for dimethyl trisulfide ................................................ 267

Figure 118. Calibration curve for 1,2-dichlorobenzene............................................. 268

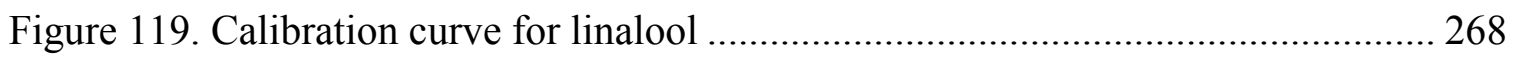

Figure 120. Calibration curve for (E)-2-nonenal .................................................... 268

Figure 121. Calibration curve for diphenylamine....................................................... 269 


\section{RESEARCH INTRODUCTION}

\subsection{Basis for research}

Cases have been reported where pet dogs have detected cancers on their owners ${ }^{1,2}$. In each case, the patient's dog paid excessive attention to a particular part of the owner's body and, upon seeking medical advice, the owners were diagnosed with cancer. Cases like these have triggered interest in the scientific community to investigate the odors emitted by the human body. In the forensic science field, this is especially true in terms of understanding how canines differentiate people by their scents.

Volatile organic compounds (VOCs) are naturally occurring chemical compounds that are characterized by having low boiling points. The human body produces odors made up of a variety of VOCs. The individual odor hypothesis theorizes that each individual possesses a unique scent, referred to as "human scent", which allows for scent identification using canines in law enforcement and forensic fields. However, if persons with certain diseases are known to emit disease-specific odors, it is possible that problems may arise in scent identification line-ups as a result of diseases or disorders which could result in false matching of persons of interest. Therefore, the analysis of the scent biomarkers among different populations can provide valuable information as to what impact it could have on law enforcement in terms of matching people. Identification of such biomarkers is also useful for the medical field for diagnostic purposes. To date, no studies have been published that compare the volatile organic compounds obtained from different biological specimens collected from healthy volunteers, those with type 2 diabetes, and clinically depressed patients to establish a profile that can be used to 
differentiate between healthy and diseased persons. Results obtained from this research will provide valuable information not only for the medical perspectives but also for the law enforcement/person detection perspectives helping to determine the uniqueness of odor profiles from different individuals for diagnostic and/or identification purposes.

\subsection{Research Objectives}

This research was conducted to test two main hypotheses:

Hypothesis I: Individuals can be differentiated using any biological fluids.

Hypothesis II: Subjects can be grouped based on being healthy or having a medical or psychiatric condition.

The main objectives of this research were to employ analytical instrumental methods to compare the VOCs from different samples taken from the same individual and to compare among the populations evaluated in this project. The content of this report focuses on data obtained via instrumental evaluation of human scent samples from different biological specimens, as well as samples taken from different populations. The different tasks that were addressed are listed below.

a. Optimization of collection and analysis methods for human scent samples from different biological specimens using SPME to extract the volatile compounds and GC/MS as separation and identification technique

b. Evaluation of odor profiles of different specimens of individuals over time

c. Evaluation of the similarities/differences in sampling from different biological specimens using SPME-GC/MS 
d. Population analyses of the VOCs present above collected odor samples using SPME-GC/MS

i. Healthy individuals

ii. Patients with type 2 diabetes

iii. Patients with Major Depressive Disorder (MDD)

e. Creation of human biological specimen compound database

\subsection{Forensic Science and Human Scent}

\subsubsection{Admissibility of Scientific Evidence}

For scientific evidence to be admissible in a United States court of law, it must satisfy the requirements of either the Frye standard, the Daubert standard, or the Federal Rules of Evidence. The appropriate standard is dependent on the case and in which jurisdiction the case is being handled. The Federal Rules of Evidence govern the admission of evidence in civil and criminal proceedings in federal courts. These rules are applied through the cases of Frye v. United States (1923) and Daubert v. Merrell Dow Pharmaceuticals $(1993)^{3}$.

The Frye standard, or the general acceptance test, is applied to determine the admissibility of scientific evidence. Under the Frye standard, scientific evidence is admissible if the technology is "generally accepted" in that field. Frye defines general acceptance as follows:

Just when a scientific principle or discovery crosses the line between the experimental and demonstrable stages is difficult to define. Somewhere in this twilight zone the evidential force of the principle must be recognized, and while courts will go a long way in admitting expert testimony deduced from a well recognized scientific principle or discovery, the thing from which the deduction is 
made must be sufficiently established to have gained general acceptance in the particular field in which it belongs ${ }^{3}$.

The issue with the Frye standard is that it does not specify in which field the technology is accepted. It also does not distinguish between science and pseudo-science. In 1975, the Frye test was superseded by the Federal Rules of Evidence (Rule 702) governing expert testimony:

If scientific, technical, or other specialized knowledge will assist the trier of fact to understand the evidence or to determine a fact in issue, a witness qualified as an expert by knowledge, skill, experience, training, or education, may testify thereto in the form of an opinion or otherwise, if (1) the testimony is based upon sufficient facts or data, (2) the testimony is the product of reliable principles and methods, and (3) the witness has applied the principles and methods reliably to the facts of the case $e^{4}$.

While many states (including Florida, New York, Alaska, California, and Washington) still follow the Frye standard, the U.S. federal court and half of the states now use the Daubert standard in their proceedings governing the admissibility of expert testimony. With the Daubert standard, scientific expert testimony's admissibility is regulated by several guidelines. The judge is given the task of "gatekeeping" in which s/he must ensure the relevance and reliability of the expert's testimony to the case. Beyond "general acceptance" as is the case with Frye, Daubert requires that the scientific knowledge is a product of a scientific methodology which has been tested for its validity and reliability, subjected to peer review and publication, tested for its error rate, have standards and controls of operation, and has gained widespread acceptance in the relevant scientific community ${ }^{3}$. 


\subsubsection{Human Scent as Scientific Evidence}

Human scent as scientific evidence is still very controversial and often challenged in court ${ }^{5}$. While research on human scent has been a growing interest in the scientific and law enforcement communities, there are still many unanswered questions concerning the use of human scent and there is not a common method of evidence collection, storage, and analysis that is implemented across different law enforcement agencies and research groups. For this reason, introduction of human scent evidence in a United States court is not an easy task; however, there are instances wherein human scent evidence has been accepted as scientific evidence in court cases.

The most recent U.S. court ruling pertaining to human scent evidence was a court case in 2009 of U.S. v. Wade where the defendant requested a Daubert hearing regarding the reliability of human scent trailing canines ${ }^{6}$. The prosecution introduced evidence that the scent of the victim was trailed to the defendant's residence by a trained trailing dog. Scent trailing was performed using scent collected with the scent transfer unit from the victim's clothing. The introduction of this human scent (dog trailing) evidence was challenged by the defendant on the durability of human scent, the reliability of the scent transfer unit, and the dog trailing methods in general. The court ruled that there was no prejudice from the admission of the scent evidence because of other sufficient corroborating pieces of evidence that justified the issuance of the search warrant. In this case, the dog trail evidence met the threshold for reliability under Evidence Rule 702 and Daubert and was ruled admissible. Table 1 lists several court rulings pertaining to the use of human scent evidence. 
Table 1. U.S. Court Cases with Human Scent Evidence

\begin{tabular}{|l|l|l|l|}
\hline Court Case & Year & Standard & \\
\hline $\begin{array}{l}\text { People v. Gonzalez } \\
\text { 218 Cal.App.3d 403 }\end{array}$ & 1990 & Frye & $\begin{array}{l}\text { Corroborating evidence is required when } \\
\text { using canine human scent evidence. }\end{array}$ \\
\hline $\begin{array}{l}\text { People of the State of } \\
\text { California v. Jeffrey } \\
\text { Dewyane Mitchell" }\end{array}$ & 2003 & Frye & $\begin{array}{l}\text { Evidence was scent collected from scent } \\
\text { transfer unit. Issues on the novelty of the } \\
\text { device as well as the degradation of } \\
\text { human scent after collection were } \\
\text { challenged. }\end{array}$ \\
\hline People v. Ryan Willis ${ }^{8}$ & 2004 & Frye & $\begin{array}{l}\text { Dog scent identification was challenged } \\
\text { on the persistence and uniqueness of } \\
\text { human scent, as well as the adequacy of } \\
\text { the certification procedures for scent } \\
\text { identification. }\end{array}$ \\
\hline $\begin{array}{l}\text { People of the State of } \\
\text { California v. Benigo } \\
\text { Salcido }\end{array}$ & 2005 & Frye & $\begin{array}{l}\text { Scent identification line-up by canines } \\
\text { was challenged on the basis of the } \\
\text { uniqueness and survivability of human } \\
\text { scent. Court ruled that human scent } \\
\text { evidence can be admissible if "the person } \\
\text { performing the technique used the correct } \\
\text { scientific procedures, the training and } \\
\text { experience of the dog and dog handler } \\
\text { prove them to be proficient, and the } \\
\text { methods used by the dog handler in the } \\
\text { case are reliable.", }\end{array}$ \\
\hline U.S. v. Wade & 2008 & $\begin{array}{l}\text { Dog trailing evidence was accepted as } \\
\text { meeting the standard for admissibility } \\
\text { under Rule 702 and Daubert because dog } \\
\text { trail evidence is "based on scientifically } \\
\text { valid principles". }\end{array}$ \\
\hline
\end{tabular}




\subsubsection{Canines and Human Scent}

Specialized use of human scent in criminal investigations is well-established by detector canines. Scent-discriminating canines have been used in criminal investigations for over 100 years. Many European countries have been using trained dogs for scent identification line-ups, where the dogs match the scent sample from a crime scene to scent collected from the hands of a suspect. Human scent identification line-ups have gained widespread acceptance in Europe, and have been admitted as evidence in the court. However, it is still very controversial and debated upon in the United States. Studies have indicated that with sufficient training, detector canines are capable of matching scents emanating from

different areas of the body ${ }^{10,11}$. In addition to criminal investigations, canines are being trained on human scent for search and rescue to search for missing persons, disaster site survivors, and decomposing human remains ${ }^{12}$.

\subsection{Alternative Biological Specimens as Scent Sources}

There is currently no direct comparison made between different biological specimens as scent sources. In the forensic field, skin odor and sweat (on clothing articles) are the main sources used for human scent identification. Forensic research on human scent have utilized scent collected on gauze, stainless steel bars, and glass beads from skin contact $^{10,12-14}$. In the medical field, blood and urine are the specimens of choice for testing, diagnostics, and metabolic profiling. The advantages and disadvantages of the collection and testing for the five biological specimens of interest to this study are outlined in Table 2 . 
Table 2. Advantages and disadvantages of hand odor, buccal swabs, breath, blood, and urine testing

\begin{tabular}{|l|c|c|c|c|c|}
\hline & Hand Odor & Buccal Swab & Breath & Blood & Urine \\
\hline Sample Collection & Noninvasive & Noninvasive & Noninvasive & Invasive & $\begin{array}{c}\text { Privacy } \\
\text { concerns }\end{array}$ \\
\hline $\begin{array}{l}\text { Sample } \\
\text { Availability }\end{array}$ & $\begin{array}{c}\text { Microliters of } \\
\text { insensible } \\
\text { sweat }\end{array}$ & $1-5 \mathrm{~mL}$ & $>50 \mathrm{~mL}$ & $>5 \mathrm{~mL}$ & $>50 \mathrm{~mL}$ \\
\hline $\begin{array}{l}\text { Speed of } \\
\text { Collection }\end{array}$ & $\begin{array}{c}20 \text { minutes } \\
\text { with hand- } \\
\text { washing } \\
\text { procedure }\end{array}$ & $<1$ minute & $<1$ minute & Minutes & Minutes \\
\hline $\begin{array}{l}\text { Currently Routine } \\
\text { Method? }\end{array}$ & No & For DNA & $\begin{array}{c}\text { For breath- } \\
\text { alcohol only }\end{array}$ & Yes & Yes \\
\hline
\end{tabular}

Published research on VOC detection and identification on different specimens generally focuses on only one specimen. The VOC research on the biological specimens of interest to this study will be discussed in detail in subsequent sections. Research on VOC detection across multiple specimens is usually only limited to two, at most three specimens ${ }^{15-17}$. Statheropoulos et al. reported the use of VOCs from human expired air, blood, and urine for locating entrapped people in earthquakes to determine whether a relatively small target group of VOCs common to all three matrices can be determined ${ }^{18}$. The VOCs from blood and urine were analyzed by headspace SPME-GC/MS and breath samples were analyzed by thermal desorption GC/MS. Acetone was found in all three matrices, and isoprene was found in both breath and blood samples.

Identification of target odor compounds can provide valuable information to both the medical and forensic communities. From the medical perspective, analysis of VOCs in biological fluids can reveal interesting diagnostic properties of different biomarkers. In addition to the disease diagnostic potential, analysis of VOCs in biological samples may 
be useful in differentiating populations (i.e. healthy vs. illness). The differences found among the different populations can lead to potential for early diagnosis of certain medical diseases.

From the forensic perspective, biological evidence collected may be useful for human identification in terms of matching individuals to odor from a crime scene. Canines have the ability to discriminate human scent because people vary in their odors. However, if persons with certain diseases are known to emit disease-specific VOCs, it is possible that problems may arise in scent identification line-ups which could result in false matching between two people with the same disease. Therefore, the analysis of the volatile biomarkers among different populations can provide valuable information as to the impact of matching people for law enforcement purposes.

\subsection{Theory of Instrumental Techniques}

\subsubsection{Solid Phase Microextraction (SPME)}

Solid phase microextraction was invented in 1989 by Pawliszyn as a new preconcentration technology ${ }^{19}$. Since then, SPME methods have been developed and widely adopted for various applications. Solid phase microextraction gained its popularity over the recent years for numerous advantageous characteristics. The extraction process does not require a solvent, making SPME economically and environmentally friendly. Minimal sample volume is required. SPME is known for its high sensitivity when coupled to analytical instruments. It is also field portable, providing a simple and convenient mean for sampling on-site. SPME can be used for the extraction 
of volatile and semi-volatile components in a sample matrix from both liquid and gaseous phases.

A SPME device has a very simple yet sophisticated configuration similar to a syringe (Figure 1). The SPME fiber, a fused silica fiber coated with an absorbent polymer about $1.0 \mathrm{~cm}$ in length, is attached to a stainless steel needle and encased by a metal sheath for the purpose of fiber protection in between extractions. The body of the SPME holder consists of a spring loaded plunger, barrel, and an adjustable depth gauge. By pushing the plunger down the fiber is exposed out of the needle and can perform extraction of the sample. Extraction can be done by immersing the SPME fiber directly into a sample, by exposing the fiber to the headspace of a sample, or by membrane protection where the fiber is separated from the sample with a selective membrane ${ }^{20}$. In this study, extraction mode of interest was headspace sampling. 


\section{Figure 1. Schematic of a SPME fiber}

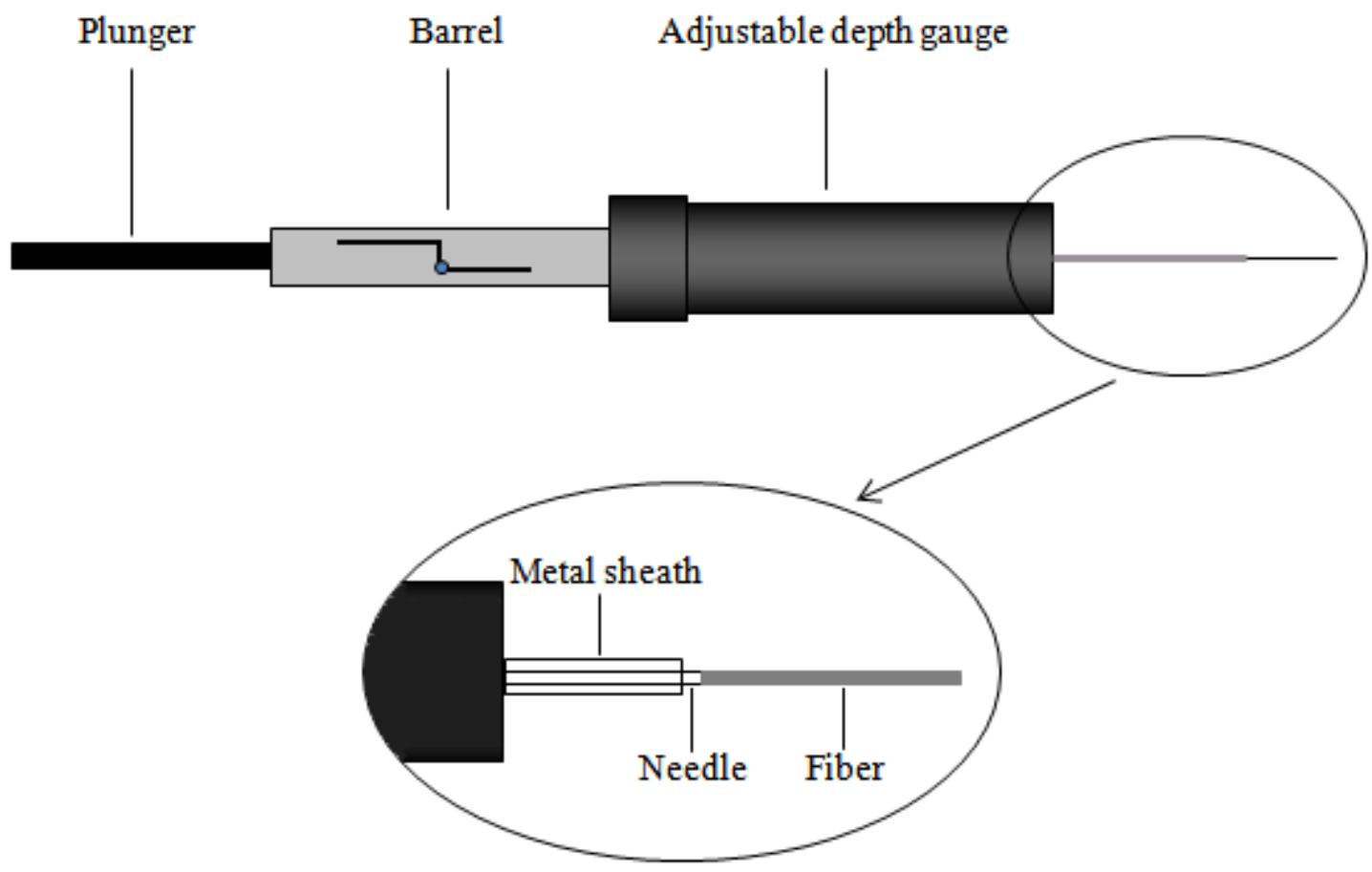

Fiber coating varies in terms of polarity and thickness. Choosing the appropriate fiber type depends on the properties of the analytes of interest. Commercially available fibers come in various polymeric phases, some of which are shown in Table 3. Polar compounds have a higher affinity to polar coatings than non-polar coatings, where nonpolar compounds will be retained more effectively with non-polar coatings. Therefore, fiber optimization and choosing the correct fiber type is essential in analysis using the SPME sampling method. 
Table 3. SPME Fiber Characteristics

\begin{tabular}{|l|l|l|l|}
\hline Fiber Coating & Polarity & Extraction & Applications \\
\hline Polydimethylsiloxane (PDMS) & Non-polar & Absorbent & $\begin{array}{l}\text { Volatiles, mid- to } \\
\text { non-polar semi- } \\
\text { volatiles }\end{array}$ \\
\hline $\begin{array}{l}\text { Polydimethylsiloxane/divinylbenzene } \\
\text { (PDMS/DVB) }\end{array}$ & Bi-polar & Adsorbent & Polar volatiles \\
\hline Polyacrylate (PA) & Polar & Absorbent & $\begin{array}{l}\text { Polar semi- } \\
\text { volatiles }\end{array}$ \\
\hline Carbowax/Polyethylene Glycol (PEG) & Polar & Adsorbent & Polar analytes \\
\hline $\begin{array}{l}\text { Polydimethylsiloxane/carboxen } \\
\text { (PDMS/CAR) }\end{array}$ & Bi-polar & Adsorbent & $\begin{array}{l}\text { Gases and } \\
\text { volatiles }\end{array}$ \\
\hline $\begin{array}{l}\text { Divinylbenzene/carboxen/polydimethyl } \\
\text { siloxane (DVB/CAR/PDMS) }\end{array}$ & Bi-polar & Adsorbent & Odors and flavors \\
\hline
\end{tabular}

SPME is a multiphase equilibration process involving two main steps. The first step involves the partitioning of the analytes between the fiber coating and the sample matrix via adsorption or absorption. Extraction is considered complete when equilibrium is reached between the coating and the matrix. The second step is desorption of the extracted analytes from the fiber into an analytical instrument. In headspace sampling, the phases in the SPME equilibrium system are the solid or liquid sample, the headspace above the sample, and the fiber coating. The equilibrium condition can be described with the following equation:

$$
n_{f}=\frac{K_{f s} V_{f} V_{s} C_{0}}{K_{f s} V_{f}+K_{h s} V_{h}+V_{s}} \quad \text { Equation } 1
$$

where $n_{f}$ is the amount of analyte extracted by the fiber coating, $K_{f s}$ is the fiber coatingsample matrix distribution constant, $V_{f}$ is the fiber-coating volume, $K_{h s} V_{h}$ is the analyte capacity of the headspace, $V_{s}$ is the sample volume, and $C_{0}$ is the initial concentration of a 
given analyte in the sample matrix ${ }^{21}$. When equilibrium is reached, the sample concentration is directly proportional to the amount of analyte extracted.

\subsubsection{Gas-Chromatography/Mass Spectrometry (GC/MS)}

Gas chromatography/mass spectrometry is the synergistic combination of two wellestablished and powerful analytical instruments. Separation of analytes in complex mixture samples is performed by the gas chromatograph; structural identification of these analytes is then provided by the mass spectrometer through the measurement of mass and abundance of ions. The complimentary characteristics of GC and MS allow for the separation, quantification, and identification of substances at low concentrations and have led to the general acceptance of GC/MS as the "gold standard" in forensic and clinical laboratories.

Gas chromatography was first introduced in 1952 by James and Martin ${ }^{22}$. The basic operation sequence of the gas chromatograph is as follows: the volatilization of the sample introduced at the heated injection port, the separation of the analytes in the sample through selective distribution between the mobile phase (inert carrier gas, typically helium or hydrogen) and the stationary phase (coating of the column), and the detection of the separated analytes by the detector. The amount of time the analyte resides in the column, the retention time, is based on the interaction of the analyte with the stationary phase. Because the samples require volatilization at the time of sample introduction, separation by GC requires that the sample be volatile and thermally stable. Optimization of the column parameters such as polarity, phase thickness, internal diameter, and column length determine the selectivity and efficiency of the separation of 
the analytes of interest ${ }^{22-24}$. Separated analytes are then introduced into the mass spectrometer downstream for further identification.

The first mass spectrometer was constructed by J.J. Thomson in $1912^{25}$. In the mass spectrometer, neutral sample molecules are ionized to generate a charged molecule or fragments of a molecule and the mass-to-charge $(\mathrm{m} / \mathrm{z})$ ratio of gaseous ions is measured. The sample is introduced into the ion source where the vaporized analyte is converted into ions via electron, chemical, or field ionization. The ions are then accelerated into and sorted by the mass analyzer according to their $\mathrm{m} / \mathrm{z}$ ratio in either time or space. The sorted ions are detected by the detector in proportion to their abundance. The resulting mass spectrum of a molecule is thus a plot of $\mathrm{m} / \mathrm{z}$ ratio against ion abundance.

Figure 2. Block diagram of a gas chromatograph coupled to a mass spectrometer

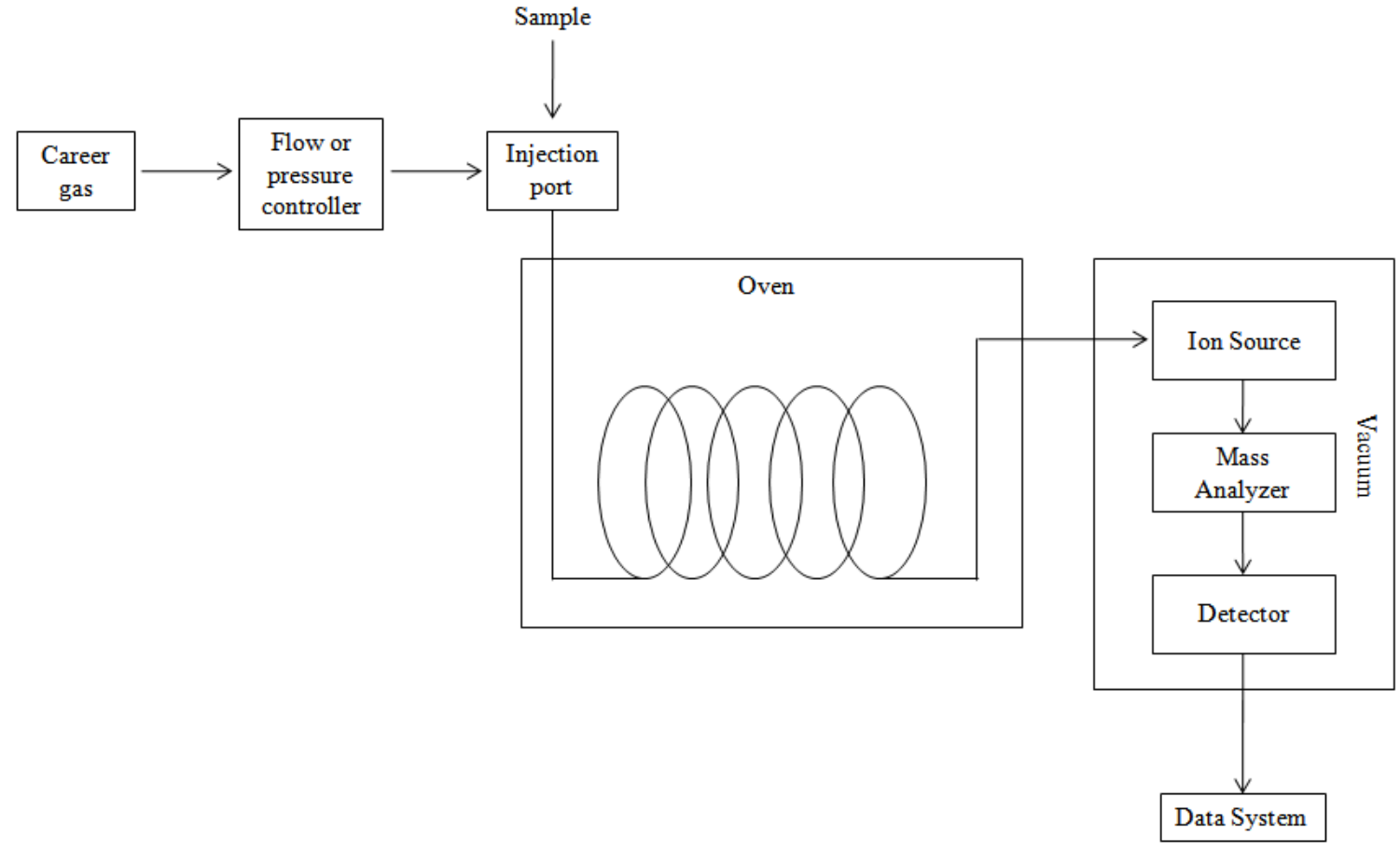




\section{BIOLOGICAL SPECIMENS}

\subsection{Human Scent}

\subsubsection{Production of Human Scent}

Human odor production is a complex process that is yet to be fully understood. The most common interpretation of the human odor production rests on the idea that glandular secretions, volatile organic compounds synthesized by metabolism, and bacterial action on dead skin cells play key roles in the generation of human scent. There is a current of warm air that surrounds the human body carrying germs that come from the bacteria that are shed with dead skin cells (called "rafts") ${ }^{26}$. This warm current of air, or the human thermal plume, allows for the deposition of human scent to the environment.

\subsubsection{Distinctiveness of Human Scent}

The concept that individuals have their own distinctive scent that is retained by a variety of factors such as diet, exercise, circadian and seasonal changes, menstrual cycle, emotional and physical health status has been around for over a century ${ }^{27}$. This individual odor hypothesis has triggered many researchers to investigate the fingerprint characteristics in and discriminatory power of compounds found in human scent. A large-scale study involving 197 subjects whose scent samples were collected five times each over a ten-week period revealed strong evidence of individual fingerprints, particularly from axillary sweat samples ${ }^{28}$. Statistical evaluations of data demonstrated repeat samples of individuals clustering closely. In another study, subjects were sampled during different seasons and the results showed that although emission behavior of the 
scents were different between seasons, the ratios of the significant fingerprint peaks did not fluctuate as much ${ }^{29}$.

A very recently published study by a research group at Florida International University also demonstrated that distinguishable human scent profiles can be produced by utilizing the relative ratio patterns of a combination of compounds in hand scent profiles which vary in degree of frequency of detection ${ }^{13}$. This research group has categorized human scent components into three distinguishing categories: primary, secondary, and tertiary ${ }^{5}$. The primary odor of a person contains constituents that are stable over time regardless of diet or environmental factors. Secondary odor constituents are present as a result of diet and environmental factors. Tertiary odor constituents are those which are present because of outside source influences (i.e., lotions, shampoos, and perfumes). Individual scent is likely to be a combination of various primary odor compounds differing in ratio from person to person as well as other compounds that vary among individuals. To date, no specific marker has been found that is unique or exclusive to one gender. There is still much work to be done to classify what specific compounds are exclusively "human scent", or to determine how and from where these human scent compounds originate. While fingerprint characteristics of human scent are being researched extensively, current knowledge on human scent indicates that human odor may be analogous to facial features in that there is no single measurement on the face that characterizes an individual, and characterization requires the combination of features and inclusion of other traits. The same can be said for human odor. Hence, human odor can be considered an "extended 
phenotype" where the variations may be genetically or environmentally induced, or by combination of both ${ }^{28}$.

\subsubsection{Persistence and Stability of Human Scent}

The persistence of human scent has also been investigated by examining the effects of aging on scent samples. In a scent-weight dissipation study, results revealed that measurable amounts of human scent compounds were still present in a controlled environment three months after the scent compounds were deposited onto sterile gauze and sealed in a glass vial ${ }^{30}$. However, aging of samples seems to affect the scent profiles to some extent, especially depending on the storage conditions under which they were kept. In an aging study on crime scene objects performed by the Netherlands National Police, trials were conducted where canine performance was tested to determine how well dogs could match fresh and stored (aged) scent samples ${ }^{31}$. Dogs were able to faultlessly match fresh scent samples to the originating subject, but when tested with stored scent samples, their performances decreased. A storage and scent stability study conducted in a laboratory setting demonstrated that scent profiles changed over time, with the greatest profile variations seen between week 0 and week $3^{12}$. Excessive exposure of samples to UVA/UVB also altered the human scent profile, indicating that choosing the appropriate storage conditions is vital in human scent sample storage ${ }^{12}$. 


\subsubsection{Hand odor sampling}

Scent collected from hand odor sampling using the method previously established served as a base human scent sample to which odor profiles of the remaining biological specimens were compared.

\subsubsection{Materials \& Methods}

DUKAL brand, $100 \%$ cotton, sterile, 2 X 2, 8 ply, gauze pads used to collect hand odor were purchased from DUKAL Corporation (Syosset, NY, USA). The soap used for hand washing was Natural, Clear Olive Oil Soap from Life of the Party (North Brunswick, NJ, USA). Ten ml glass, clear, screw top headspace vials with PTFE/Silicone septa were used to hold the samples (SUPELCO, Bellefonte, PA, USA). The methanol used for the pretreatment of gauze pads was HPLC grade (Fisher Scientific, Pittsburgh, PA, USA). Divinylbenzene/Carboxen/Polydimethylsiloxane (DVB/CAR/PDMS) $\quad(50 / 30 \mu \mathrm{m}$ film thickness) SPME fibers and SPME fiber holders were obtained from SUPELCO (Bellefonte, PA, USA).

Prior to sample collection, DUKAL gauze pads were pretreated by spiking $1000 \mu \mathrm{L}$ of HPLC-grade methanol and baking in a $105^{\circ} \mathrm{C}$ oven for 45 minutes in an Isotemp Oven, Model 655G (Fisher Scientific, Pittsburgh, PA, USA). Pretreated gauze pads were analyzed to ensure analytical cleanliness by SPME-GC/MS.

The hand odor sampling protocol is as follows: 30 seconds washing of the hands and forearms with olive oil based soap, two minutes rinsing of the washed areas with water, two minutes air drying, and five minutes of rubbing the palms of the hands over the forearms. Subjects were given a pre-treated sterile gauze pad to hold between the palms 
of their hands for ten minutes. Samples were collected in triplicates with ten minutebreaks in between each sampling. The gauze pad was re-sealed back into the $10 \mathrm{ml}$ glass headspace vial. All samples were stored at room temperature and were allowed to sit for approximately 24 hours prior to extraction. No attempt was made to control microbial interactions with the samples as that may make contributions to the overall odor profile.

The GC/MS analysis was carried out using an Agilent Technologies $6890 \mathrm{~N}$ gas chromatograph coupled to an Agilent Technologies 5973N mass selective detector (Palo Alto, CA, USA). The column used to separate the analytes was a HP5-MS, $30 \mathrm{~m}, 0.25$ um, $0.25 \mathrm{~mm}$ with helium as the carrier gas with a flow rate of $1.0 \mathrm{~mL} / \mathrm{min}$. The extracted VOCs were desorbed in the injection port of the $\mathrm{GC}$ with a temperature of $250^{\circ} \mathrm{C}$ for five minutes in splitless mode. The GC oven temperature program was as follows: an initial oven temperature of $40^{\circ} \mathrm{C}$ for five minutes, $10^{\circ} \mathrm{C} / \mathrm{min}$ ramp to a final temperature of $250^{\circ} \mathrm{C}$, followed by a final hold for two minutes for a total run time of 28 minutes. The mass spectrometer used was an HP 5973 MSD with a quadrupole analyzer operated in electron ionization mode. The mass spectrometer transfer line was maintained at $280^{\circ} \mathrm{C}$ and the source temperature was $230^{\circ} \mathrm{C}$. The analytes were acquired in full-scan mode in $41-550 \mathrm{~m} / \mathrm{z}$ range.

\subsection{Oral Fluids (Saliva)}

Saliva is an exocrine secretion composed of water, salts, mucus and the digestive enzyme $\alpha$-amylase ${ }^{32-35}$. Human saliva has several functions pertaining to oral health and homeostasis. Protective functions of saliva include lubrication, antimicrobial, mucosal integrity, cleansing, buffering, and remineralization of the teeth. Digestive functions 
include food preparation, digestion, and tasting. When food is introduced into the mouth, as chewing starts the process of digestion, saliva moistens the food and provides lubrication so that the food can be swallowed. Preliminary digestion of food starts by the polysaccharide-digesting enzyme $\alpha$-amylase.

Saliva is secreted by three main salivary glands, the parotid, sublingual, and submandibular glands which are located in the mouth (Figure $3^{36}$ ). The parotid gland is found wrapped around the ramus of the mandible, and the sublingual and submandibular glands are found below the tongue. There are also numerous minor salivary glands including Von Ebner glands and Blandin-Nühm mucous glands. Submandibular glands contribute the greatest to the unstimulated saliva secretion at $65 \%-70 \%$, followed by $20 \%$ by the parotid glands, $7 \%-8 \%$ by the sublingual glands, and less than $10 \%$ by the minor salivary glands ${ }^{32,33,37}$. The contributions of these salivary glands change with stimulated saliva secretion, where the parotid glands contribute the greatest at over $50 \%$ of the total saliva secretion. Saliva production from a healthy adult ranges from $500 \mathrm{~mL}$ to $1500 \mathrm{~mL}$ per day. Salivary $\mathrm{pH}$ is close to neutral (about six or seven), and changes with the salivary flow where the $\mathrm{pH}$ becomes more acidic $(\mathrm{pH}$ 5.3) at low salivary flow and more basic $(\mathrm{pH} 7.8)$ at high salivary flow ${ }^{33}$. Quantitative and qualitative saliva production can be affected by different conditions, pathological and physiological, including chewing, health status, stimulation by smell or taste, age, and oral hygiene ${ }^{32}$. 
Figure 3. Schematic of human salivary glands (source: Causes of Dry Mouth by Morefocus Group, Inc.)

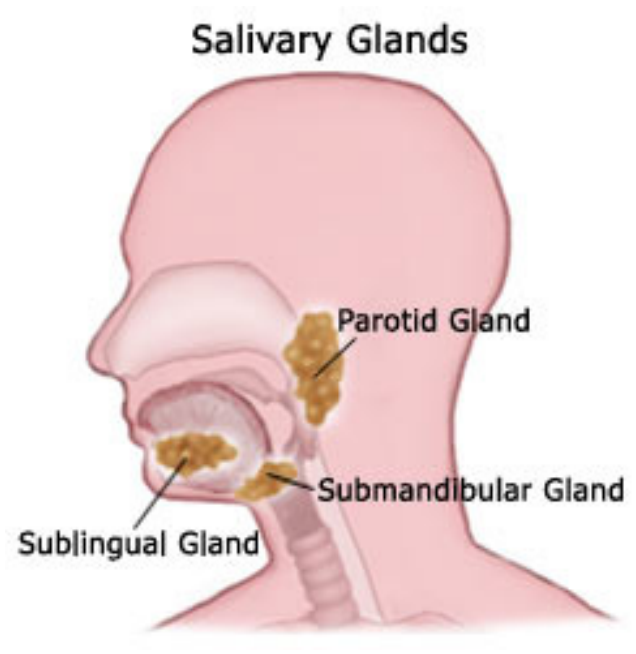

In addition to the fluids secreted from the salivary glands, components of whole saliva also include serum and blood derivatives, oral bacterial and viral products, fluids from bronchial and nasal secretions, epithelial lining cells, and exogenous substances like food debris. There are both inorganic and organic components in saliva. Inorganic components of saliva are ions and electrolytes $\left(\mathrm{Na}^{+}, \mathrm{K}^{+}, \mathrm{Cl}^{-}, \mathrm{Ca}^{2+}, \mathrm{H}^{+}, \mathrm{HCO}_{3}^{-}\right)$that help with the buffering capacity (bicarbonate and phosphate ions), remineralization of teeth enamels (calcium and phosphate ions), and maintenance of mucosal integrity. Organic components of saliva include a wide range of proteins and glycoproteins that contribute to the protective and food- and speech-related functions of saliva. Proline-rich proteins (PRPs) are the proteins that contain high levels of proline that account for about $70 \%$ of the parotid saliva protein content ${ }^{38}$. Acidic PRPs contribute to the protection of oral tissues and surfaces by forming pellicle, and by maintaining saliva calcium in equilibrium with the enamel. Other acidic and basic PRPs also play roles in food processing as well 
as microbial management in the mouth. Statherin, histatins, and mucins are other proteins that are major contributors to the protective, food processing, and microbial management functions of saliva. Some of the other proteins that are present in lower concentrations but also play anti-microbial roles include lysozyme, lactoferrins, cystatins, peroxidases, and secretory immunoglobulin $\mathrm{A}^{33}$. Most of the aforementioned proteins are multifunctional.

There are some constituents of saliva that are not normally a part of salivary secretion. Drugs and hormones are examples of such non-ordinary saliva constituents. These constituents are serum constituents that are transported from the blood to saliva via different transport mechanisms. The transport mechanisms can either be intracellular or extracellular ${ }^{34}$. Intracellular transport mechanisms include passive diffusion across a concentration gradient and active transport through protein channels, of which passive diffusion is the more common of the two. The most common route of extracellular transport is ultrafiltration through tight gap junctions between the cells. Constituents transported to saliva must have some water solubility to be retained in saliva, since saliva is $99 \%$ water. The saliva/plasma concentrations of the non-salivary constituents depend on the $\mathrm{pH}$ of the saliva ${ }^{37}$.

Analysis of odiferous volatile saliva compounds have generally been in relation to disease of the mouth, such as periodontitis ${ }^{39-41}$. Volatile sulfur compounds and aromatic nitrogen containing compounds have been found at elevated levels in the mouth air and saliva samples of patients with moderate to severe preriodontitis ${ }^{40,42}$. More recently, saliva analysis has gained interest for its diagnostic tool potential for cancer, infections 
from virus and bacteria, hormonal abnormality, and drug testing ${ }^{32,37,43}$. Saliva sampling is noninvasive and simple. In the clinical and forensic fields, buccal swab sampling is a routine sampling method for the collection of DNA. Analysis of VOCs from such swab samples has not yet been investigated.

\subsubsection{Materials \& Methods}

Sterile cotton-tipped applicators to collect cheek cells and saliva were purchased from Solon Manufacturing Co (Skowhegan, ME, USA). Reagent quality (200 proof) ethyl alcohol used for the pre-treatment of cotton swabs was purchased from Florida Distillers Co (Lake Alfred, FL, USA). Ten ml glass, clear, screw top headspace vials with PTFE/Silicone septa were used to hold the samples (SUPELCO, Bellefonte, PA, USA). Divinylbenzene/Carboxen/Polydimethylsiloxane (DVB/CAR/PDMS) $\quad(50 / 30 \mu \mathrm{m}$ film thickness) SPME fibers and SPME fiber holders were obtained from SUPELCO (Bellefonte, PA, USA).

The GC/MS analysis was carried out using an Agilent Technologies 6890N gas chromatograph coupled to an Agilent Technologies 5973N mass selective detector (Palo Alto, CA, USA). The column used to separate the analytes was a HP5-MS, $30 \mathrm{~m}, 0.25$ um, $0.25 \mathrm{~mm}$ with helium as the carrier gas with a flow rate of $1.0 \mathrm{~mL} / \mathrm{min}$. The extracted VOCs were desorbed in the injection port of the $\mathrm{GC}$ with a temperature of $250^{\circ} \mathrm{C}$ for five minutes in splitless mode. The GC method begins with an initial oven temperature of $40^{\circ} \mathrm{C}$ for five minutes, then ramped at $10^{\circ} \mathrm{C} / \mathrm{min}$ to a final temperature of $270^{\circ} \mathrm{C}$, and held at $270^{\circ} \mathrm{C}$ for two minutes for a total run time of 30 minutes. The mass spectrometer used was an HP 5973 MSD with a quadrupole analyzer operated in electron ionization mode. 
The mass spectrometer transfer line was maintained at $280^{\circ} \mathrm{C}$ and the source temperature was $230^{\circ} \mathrm{C}$. The analytes were acquired in full-scan mode in $41-550 \mathrm{~m} / \mathrm{z}$ range.

\subsubsection{Pre-treatment of buccal swab collection material}

\subsubsection{Methods}

Sterile cotton-tipped swabs were pre-treated prior to sample collection to eliminate any background compounds. Swabs were spiked with $200 \mu \mathrm{L}$ of ethanol, then baked in a $105^{\circ} \mathrm{C}$ oven for one hour, and the procedure was repeated again. Pre-treated swabs were analyzed using SPME-GC/MS with a 21-hour extraction time to ensure they were free of undesired compounds and analytically clean.

\subsubsection{Results}

Pretreated cotton swabs were analyzed using SPME-GC/MS to ensure they were free of undesired human scent compounds. Example of a chromatogram of pre-and post-cleaning treatment of the sterile cotton swabs prior to sample collection are shown in Figure 4. As

seen in Figure 5, the pre-treatment procedure effectively removes the numerous compounds that were originally present in the scent collection materials. 
Figure 4. Comparison of untreated and pre-treated cotton swab

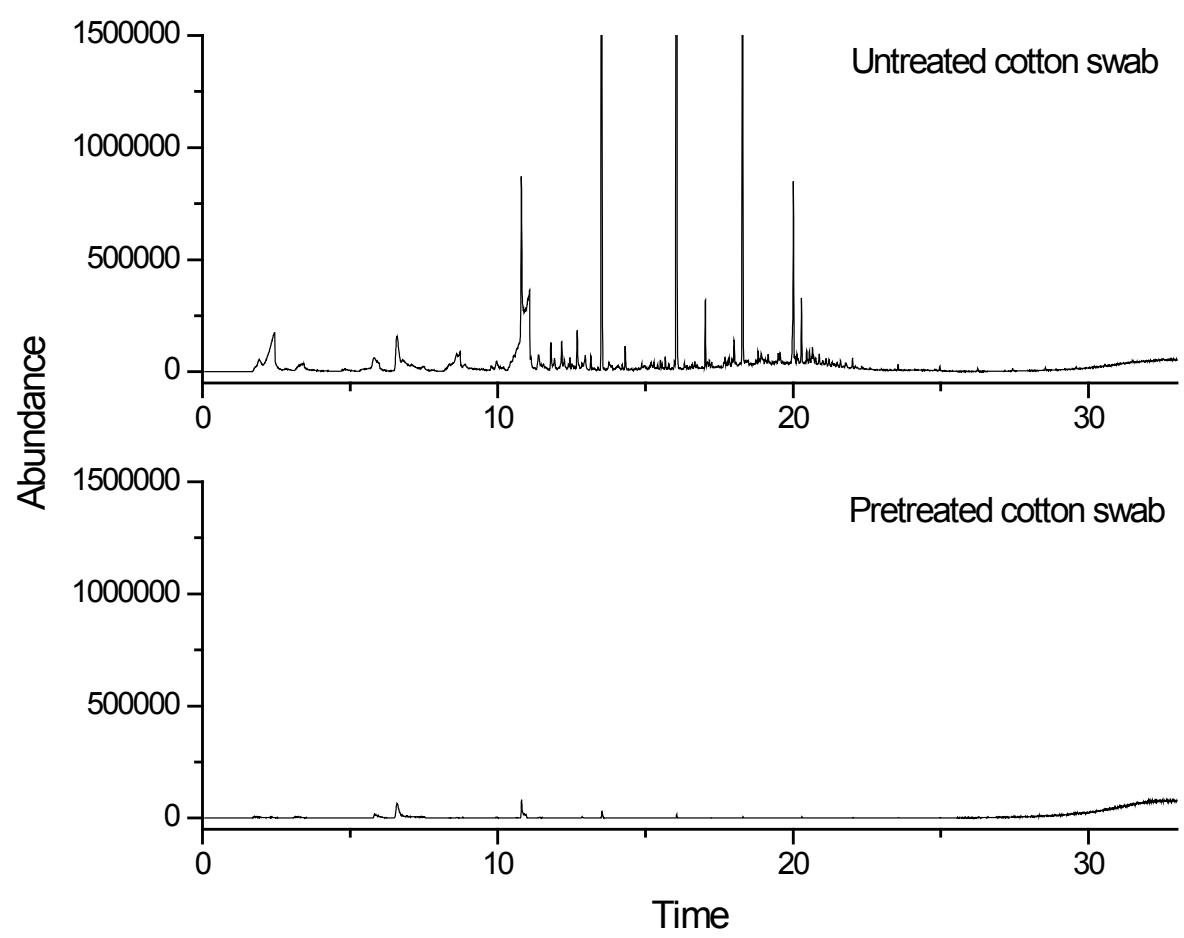

Figure 5 Comparison of cotton swab pretreatment for the removal of undesired VOCs

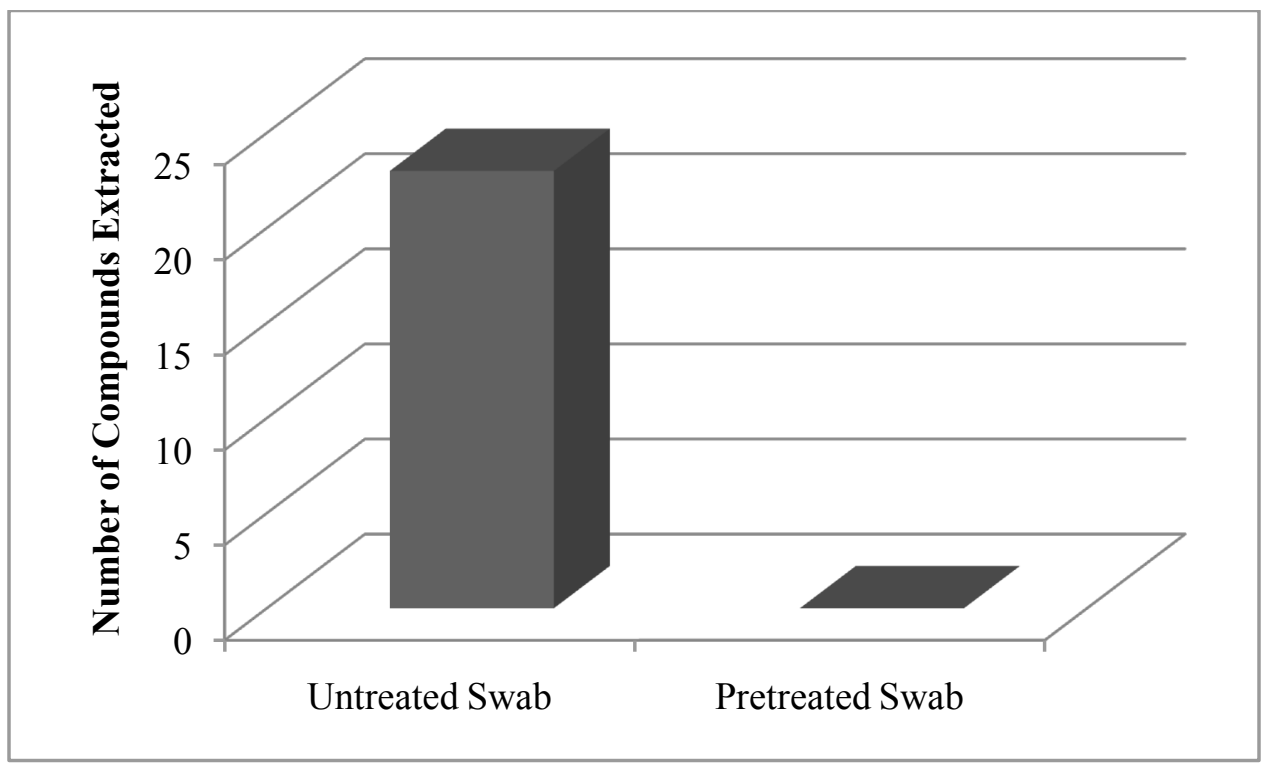




\subsubsection{Buccal swab sampling procedure}

Each subject was asked not to eat or drink anything other than water at least two hours prior to buccal swab and breath samplings. Subjects sampled themselves with a pretreated cotton-tipped sterile swab. Subjects followed a set sampling procedure of rinsing their mouths with water for 30 seconds followed by rubbing the swabs on the inside of both cheek sides (rubbing 20 times up and down per side of cheek). Samples were collected in triplicates with ten minute breaks in between each sampling. Swabs were resealed back into the ten $\mathrm{ml}$ glass headspace vial. All samples were stored at room temperature and were allowed to sit for approximately 24 hours prior to extraction. No attempt was made to control microbial interactions with the samples as that may make contributions to the overall odor profile.

\subsubsection{Determination of optimal extraction conditions for buccal swab}

\subsubsection{Methods}

Buccal swab samples were collected from subjects Male 1, Male 5, and Female 4 following a set sampling procedure as described in section 2.2.3. Each subject sampled themselves using a pre-treated sterile cotton-tipped swab. Samples were taken in triplicates with 10 minutes break in between each sampling. Swabs were re-sealed back into the $10 \mathrm{ml}$ glass vial. All samples were stored in the ten $\mathrm{ml}$ glass vials at room temperature, and were allowed to equilibrate for approximately 24 hours prior to extraction. During optimization, the odor exposures were done at room temperature on multiple samples for $3,6,12,15,21$, and 24 hours. The 50/30 $\mu \mathrm{m}$ DVB/CAR/PDMS SPME fibers (SUPELCO, Bellefonte, PA, USA) were used to extract the VOCs from the 
headspace of the buccal swab samples in the vials. All samples were run using the GC/MS method for buccal swab samples previously mentioned in section 2.2.1.

\subsubsection{Results}

Figure 6 shows the number of compounds that have been previously reported as "human scent compounds" extracted from buccal swab samples against extraction times. "Common compounds" denote the number of VOCs present in all triplicate samples. The optimal extraction time for buccal swab samples was evaluated on a combination of the number of human scent compounds extracted as well as the abundances of six selected common buccal swab compounds (1-octen-3-ol, acetophenone, benzaldehyde, nonanal, decanal, and nonanoic acid methyl ester) present in the collected samples. Twenty-one (21) hours was determined to be the optimal extraction time for collected buccal swab odor compounds. 
Figure 6. Number of human scent compounds extracted from buccal swab samples vs. extraction time

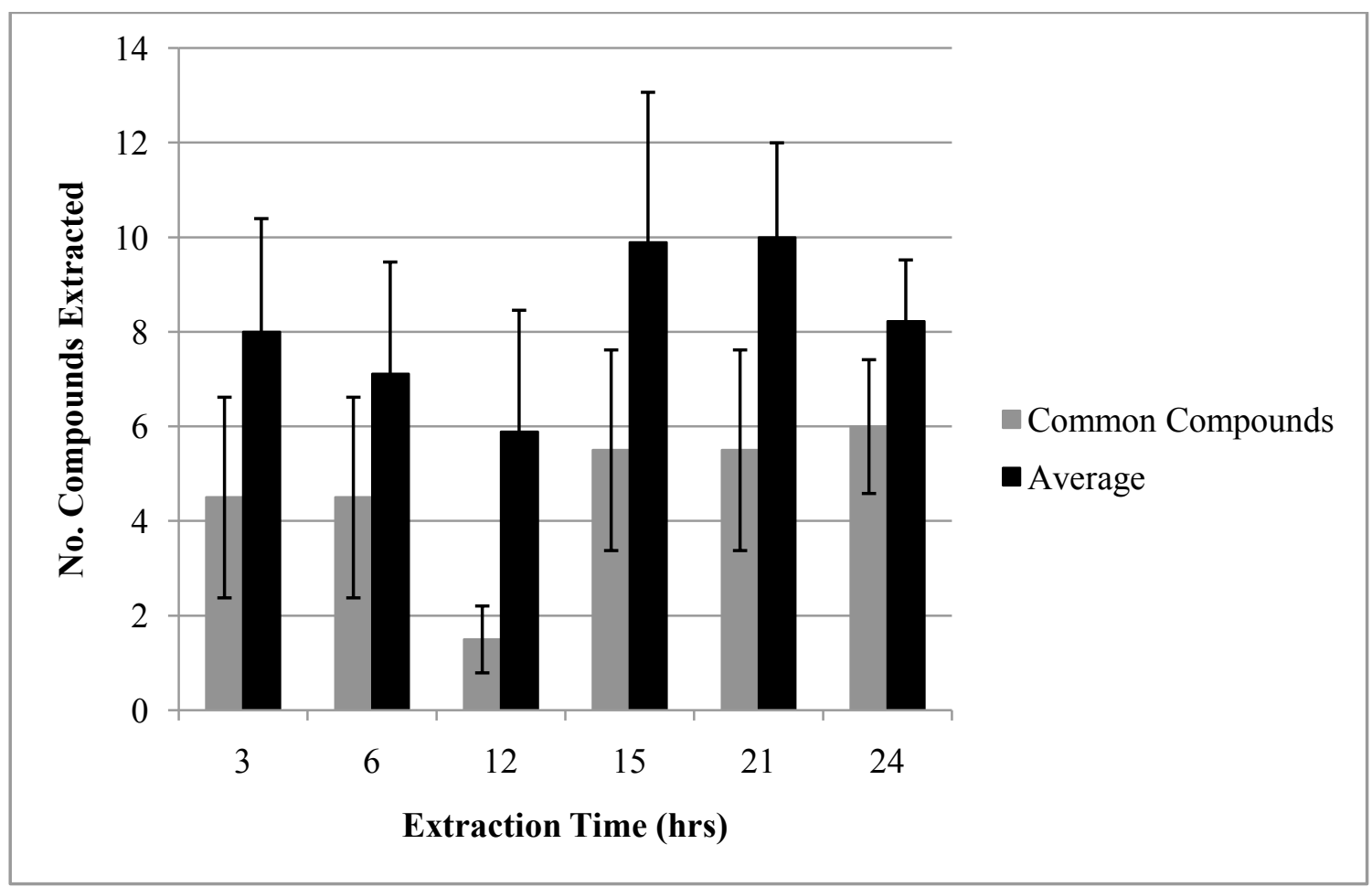

\subsection{Breath}

Human breath is "a bulk matrix consisting of nitrogen, oxygen, carbon dioxide, water vapor, inert gases and some trace components; volatile organic compounds" that is produced in the concentrations of $\mathrm{nmol} / 1$ to $\mathrm{pmol} / 1$ range $\mathrm{e}^{44,45}$. For humans, breathing is the process that exchanges oxygen and carbon dioxide between the body and the external environment and is governed by the respiratory system. Some of the major functions of the respiratory system are to provide oxygen, eliminate carbon dioxide, regulate the blood $\mathrm{pH}$, and to defend the body against microbes ${ }^{46}$. The anatomical features of the human respiratory system include the lungs, the airways, and the respiratory muscles. 
The trachea branches into two bronchi, one entering the right lung and the other entering the left lung. The bronchi branch further into smaller, narrower bronchioles. At the end of the bronchioles are the round air sacs called alveoli where the gas exchange occurs between the capillaries and alveoli. The mechanics of breathing follows Boyle's law, which states that at in a closed system at constant temperature, pressure and volume of a gas are inversely proportional to each other ${ }^{46}$. The closed system in the respiratory system is the thoracic cavity, and the mechanics of breathing depends on the changes in volume and pressure of the thoracic cavity. Breathing occurs in two phases, inspiration (inhaling) and expiration (exhaling). The diaphragm contracts during inspiration, increasing the thoracic cavity volume and thereby decreasing the pressure within. The lungs expand; the air pressure inside the alveoli becomes subatmospheric resulting in the air rushing in to fill the lungs until equilibrium is reached. Conversely, during expiration, the diaphragm relaxes, decreasing the thoracic volume and increasing the pressure within (alveolar pressure becomes larger than atmospheric pressure), and, as a result, the lungs contract and air is expelled out into the atmosphere. The VOCs are also transported in the bloodstream and expired through the lungs along with the other gases that compose the "bulk matrix" of the human breath.

The VOCs in breath may be of endogenous or exogenous origins. Volatile substances of exogenous origins are contaminants from the environment that are breathed in and absorbed by the body. Inorganic gases such as nitric oxide and carbon monoxide are endogenous compounds that are generated in the airways. Other endogenous VOCs that are exhaled can be produced virtually anywhere in the body as a result of metabolic and 
biochemical processes. These VOCs are transported through the bloodstream and eventually exhaled through the lungs. The VOCs can diffuse across the pulmonary alveolar membrane which separates the alveolar air from the blood in the capillaries, thereby crossing from blood to air and air to blood ${ }^{44}$. The exhalation rates and route of transport of individual blood-borne VOCs depends on whether the VOC has a high or low Henry's constant, as having a low Henry's constant means that the VOC is not readily soluble in water ${ }^{44}$. In other words, the exhalation rates of the VOCs in human breath depend on their molecular weight and hydrophobicity.

Modern breath analysis started in 1971 when Pauling et al. determined more than 200 components in human breath using gas chromatography ${ }^{15}$. Since then, variations in the VOCs as well as the effect of age and gender on the profile of volatile components in normal human breath have been studied widely resulting in the detection of over 3000 compounds $^{47,48}$. Some known endogenous VOCs present in human breath include ethane, pentane, isoprene, and acetone ${ }^{48-53}$. Acetone and isoprene are two of the most common VOCs in human breath, and the biochemical pathways of these compounds are well known. Acetone production is a result of decarboxylation of excess acetyl-CoA which generates ketone bodies ${ }^{45,49}$. Isoprene generation is a result of mevalonate metabolism of cholesterol synthesis ${ }^{49}$. However, the source and the biochemical pathways of most VOCs that have been detected in human expired breath are still not known.

There has been an increasing interest in human breath analysis for its investigation potential for biomarkers for diseases such as certain types of cancer (lung and breast), oxidative stress, pulmonary tuberculosis, diabetes mellitus, and kidney impairment ${ }^{52,54-57}$. 
The interest in human exhaled breath is based on the non-invasiveness and safeness in the sampling process as compared to blood testing. Breath VOC measurements can be used to estimate the body burden and different processes in the body because there exists a dynamic equilibrium between the blood VOCs and the expired breath VOC concentrations. However, a standard breath sampling and analysis protocol for clinical settings has yet to be settled. Studies on the analysis of endogenous compounds found in humane exhaled breath are still challenged because of the low concentrations of the VOCs. Currently, breath-alcohol testing is the only typical routine breath analysis application implemented in the medical and forensic fields.

\subsubsection{Materials \& Methods}

Breath samples were collected using a Teflon Bio-VOC® breath sampler (Markes International Ltd., Rhondda Cynon Taff, UK). Disposable cardboard pediatric mouthpieces attached to the Bio-VOC® apparatus were purchased from Alliance Tech Medical, Inc. ${ }^{\mathrm{TM}}$ (Grandbury, TX, USA). To seal the apparatus, $11 \mathrm{~mm}$ crimp seals with PTFE/Silicone septa (Sun Sri, Rockwood, TN, USA) were used. The acetone used for the pre-treatment of Bio-VOC $®$ breath sampler apparatus was ACS grade acetone purchased from Fisher Scientific, Pittsburgh, PA, USA).

The GC/MS analysis was carried out using an Agilent Technologies 6890N gas chromatograph coupled to an Agilent Technologies 5973N mass selective detector (Palo Alto, CA, USA). The column used to separate the analytes was a HP5-MS, $30 \mathrm{~m}, 0.25$ um, $0.25 \mathrm{~mm}$ with helium as the carrier gas with a flow rate of $1.0 \mathrm{~mL} / \mathrm{min}$. The extracted VOCs were desorbed in the injection port of the GC for five minutes in splitless mode. 
The injection port temperature was set at $280^{\circ} \mathrm{C}$ for breath samples. The GC method begins with an initial oven temperature of $40^{\circ} \mathrm{C}$ for five minutes, then ramped at $10^{\circ} \mathrm{C} / \mathrm{min}$ to a final temperature of $270^{\circ} \mathrm{C}$, and held at $270^{\circ} \mathrm{C}$ for two minutes for a total run time of 30 minutes. The mass spectrometer used was an HP 5973 MSD with a quadrupole analyzer operated in electron ionization mode. The mass spectrometer transfer line was maintained at $280^{\circ} \mathrm{C}$ and the source temperature was $230^{\circ} \mathrm{C}$. The analytes were acquired in full-scan mode in $41-550 \mathrm{~m} / \mathrm{z}$ range.

\subsubsection{Pre-treatment of Bio-VOC® Breath Sampler}

\subsubsection{Methods}

Sample collection materials were pre-treated prior to sample collection to eliminate any background compounds. The Bio-VOC® apparatus was rinsed with acetone and placed in an oven of $40^{\circ} \mathrm{C}$ for at least one hour followed by 30 minutes in a $105^{\circ} \mathrm{C}$ oven, then pure nitrogen gas was passed through the bulb for two minutes. The breath sampler was crimp sealed until time of breath sampling.

\subsubsection{Results}

Pretreated Bio-VOC® breath samplers were analyzed using SPME-GC/MS to ensure they were free of undesired human scent compounds. Example of a chromatogram of preand post-cleaning treatment of the breath samplers prior to sample collection are shown in Figure 7. As seen in Figure 8, the pre-treatment procedure effectively removes the numerous compounds that were originally present in the scent collection materials. 
Figure 7. Comparison of untreated and pre-treated Bio-VOC® breath sampler

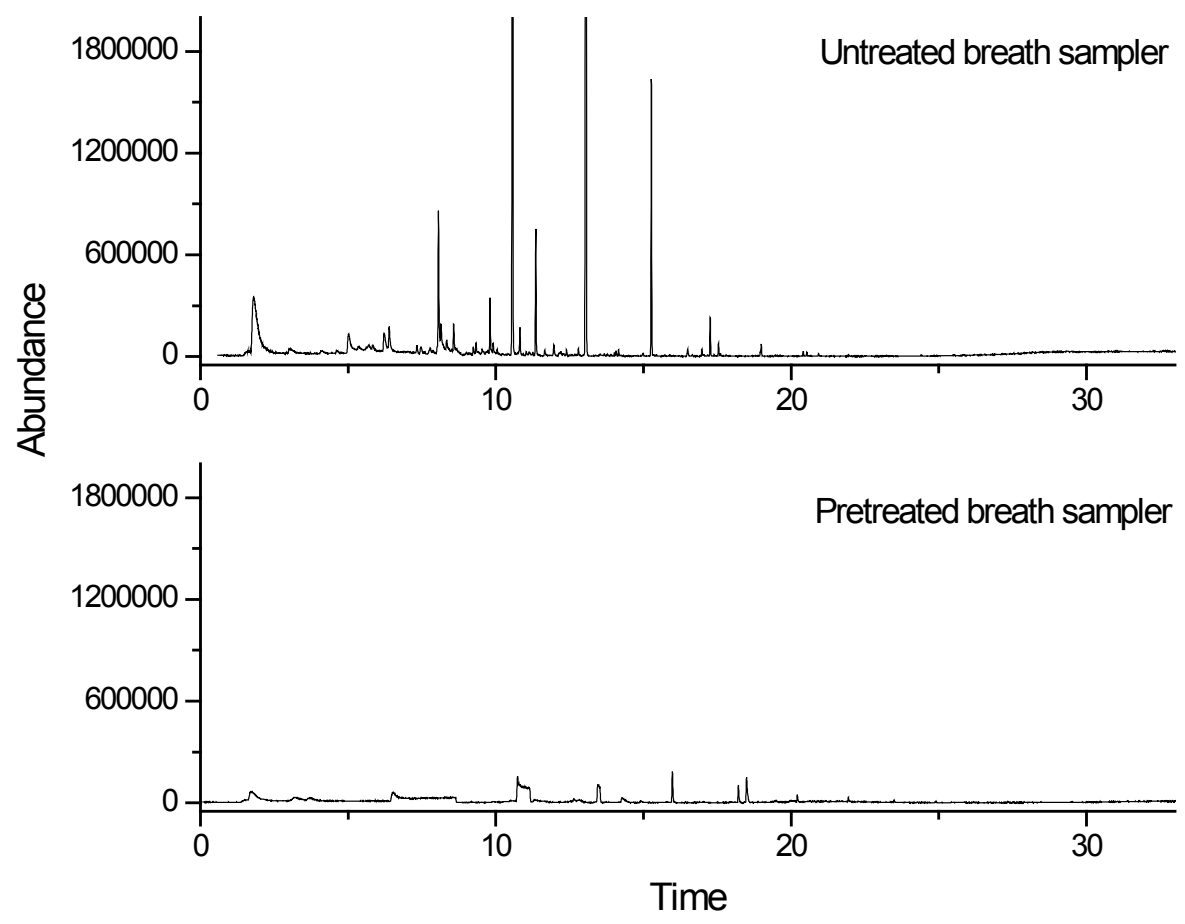

Figure 8. Comparison of breath sampler pretreatment for the removal of undesired VOCs

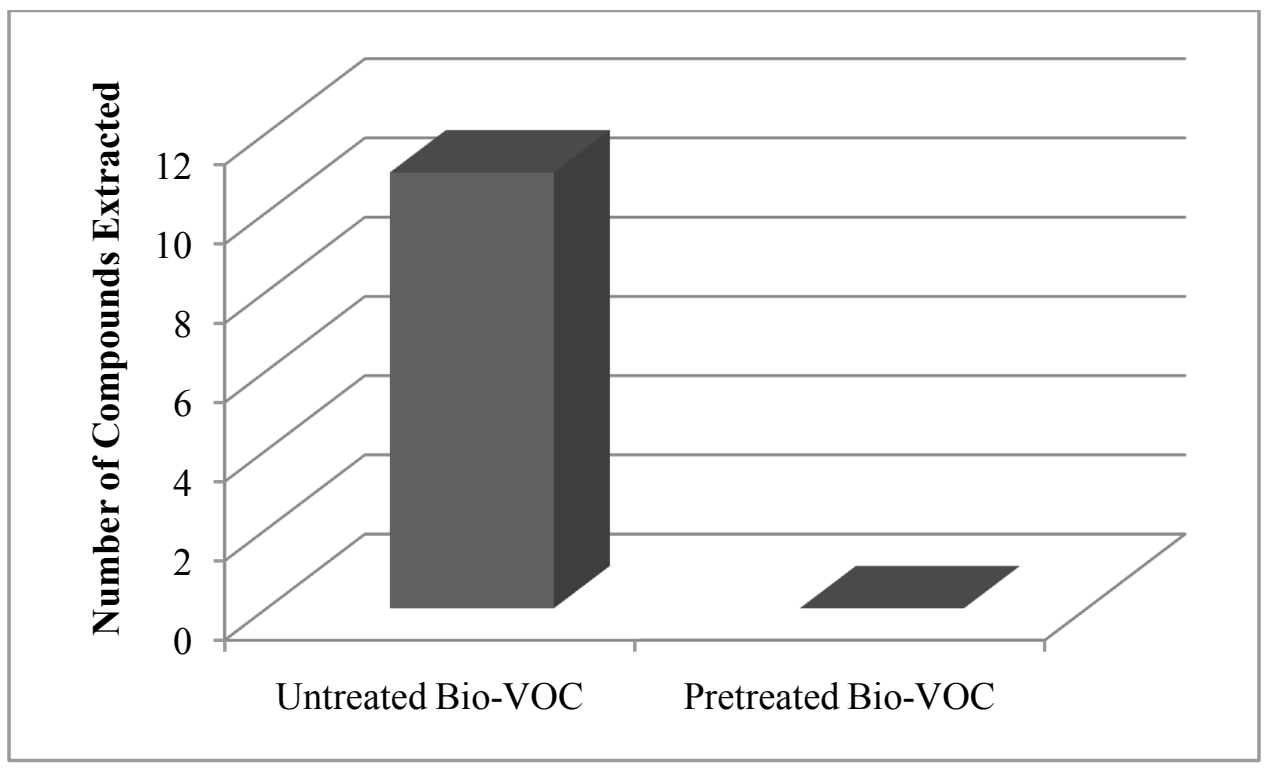




\subsubsection{Breath sampling procedure}

Breath samples were collected following a set sampling procedure. Subjects were asked to perform a single slow vital capacity breath into a disposable cardboard mouthpiece connected to a $150 \mathrm{ml}$ Teflon ${ }^{\circledR}$-bulb, which traps the end-expired air. The Teflon ${ }^{\circledR}$-bulb was immediately sealed after sampling.

\subsubsection{Determination of optimal extraction conditions for breath}

\subsubsection{Methods}

Breath samples were collected from subjects Male 1 and Female 4 following a set sampling procedure as described in section 2.3.3. Each subject sampled themselves by performing a single slow vital capacity breath into a disposable cardboard mouthpiece connected to a $150 \mathrm{ml}$ Teflon ${ }^{\circledR}$ Bio-VOC $®$ breath sampler (Markes International Ltd., Rhondda Cynon Taff, UK), which traps the end-expired air. The Teflon®-bulb was immediately sealed using an $11 \mathrm{~mm}$ crimp seal with PTFE/Silicone septa (Sun Sri, Rockwood, TN, USA) after sampling. The exhaled breath VOCs were extracted by 50/30 $\mu \mathrm{m}$ DVB/CAR/PDMS SPME fibers (SUPELCO, Bellefonte, PA, USA) directly inserted into the Teflon ${ }^{\circledR}$-bulb. During extraction optimization, the breath exposures were done at room temperature on multiple samples for $10 \mathrm{~min}, 15 \mathrm{~min}, 30 \mathrm{~min}, 45 \mathrm{~min}, 1 \mathrm{hr}, 3 \mathrm{hrs}, 6$ hrs, $12 \mathrm{hrs}, 15 \mathrm{hrs}, 18 \mathrm{hrs}, 21 \mathrm{hrs}$, and $24 \mathrm{hrs}$. Optimization of sample equilibration time was also performed at $15 \mathrm{~min}, 1 \mathrm{hr}$, and $24 \mathrm{hrs}$. All samples were run using the GC/MS method for breath samples previously mentioned in section 2.3.1. 


\subsubsection{Results}

\subsection{Optimal Extraction Time}

The exhaled breath VOCs from four subjects (Male 1, Male 5, Female 2, and Female 4) were extracted by SPME fibers inserted directly into the Teflon ${ }^{\circledR}-$ bulb portion of the BioVOC® Breath Sampler. During optimization, the breath exposures were done at room temperature on duplicate samples taken from subjects Male 1 and Female 4 for 10 min, $15 \mathrm{~min}, 30 \mathrm{~min}, 45 \mathrm{~min}, 1 \mathrm{hr}, 3 \mathrm{hrs}, 6 \mathrm{hrs}, 12 \mathrm{hrs}, 15 \mathrm{hrs}, 18 \mathrm{hrs}, 21 \mathrm{hrs}$, and $24 \mathrm{hrs}$ (Figure 9). Extraction time optimization was only investigated for the 15, 18, 21, and 24 hours exposures for subjects Male 5 and Female 2, since fewer compounds were extracted at extraction times below 12 hours (Figure 10). The optimal extraction time for breath samples was evaluated on a combination of the number of human scent compounds extracted as well as the abundances of selected common breath VOCs present in the subject breath samples: phenol, styrene, nonanal, decanal, and acetophenone. Figure 11 shows an example of the abundances of common breath VOCs extracted for the 15, 18, 21, and 24-hour extraction times for subject M5. Similar results were seen for the other three subjects. Twenty-one (21) hours was determined to be the optimal extraction time for collected breath odor compounds using the Bio-VOC® breath sampler through the evaluation parameters stated. 
Figure 9. Number of common human scent compounds extracted vs. extraction time for breath samples for subjects M1 and F4

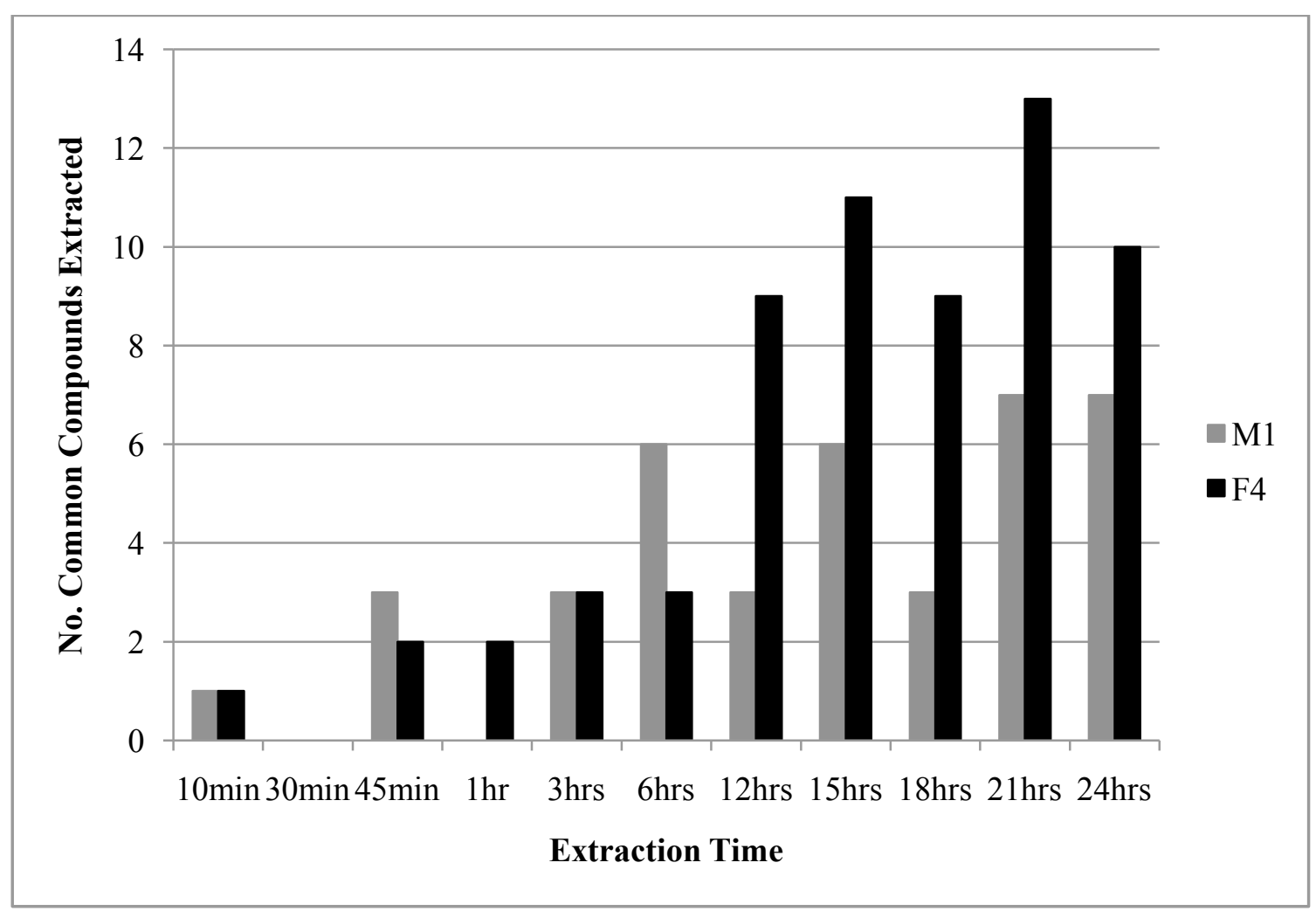


Figure 10. Number of common human scent compounds extracted vs. extraction time for breath samples for subjects M5 and F2

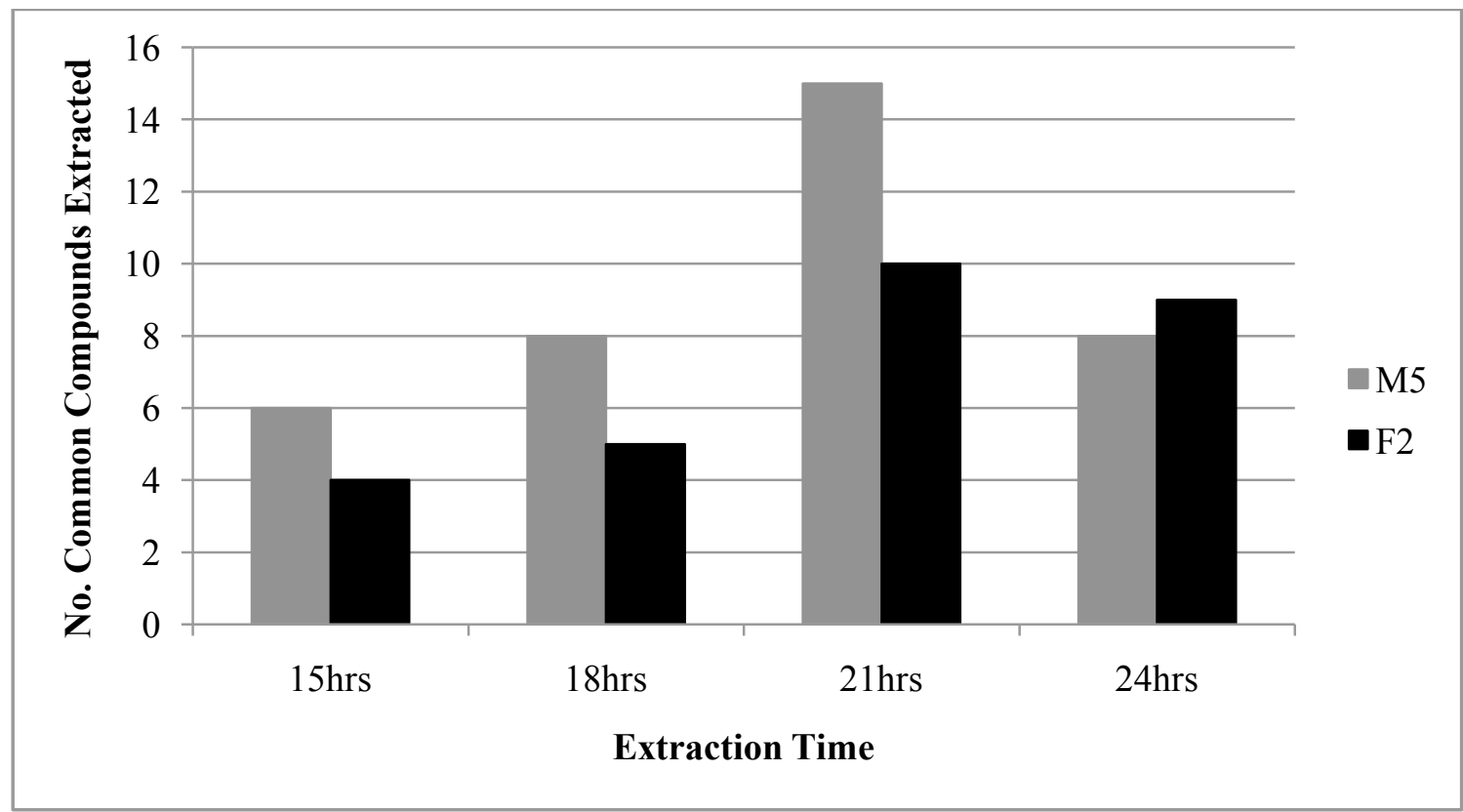

Figure 11 Abundances of selected common breath VOCs extracted vs. extraction time for Male 5

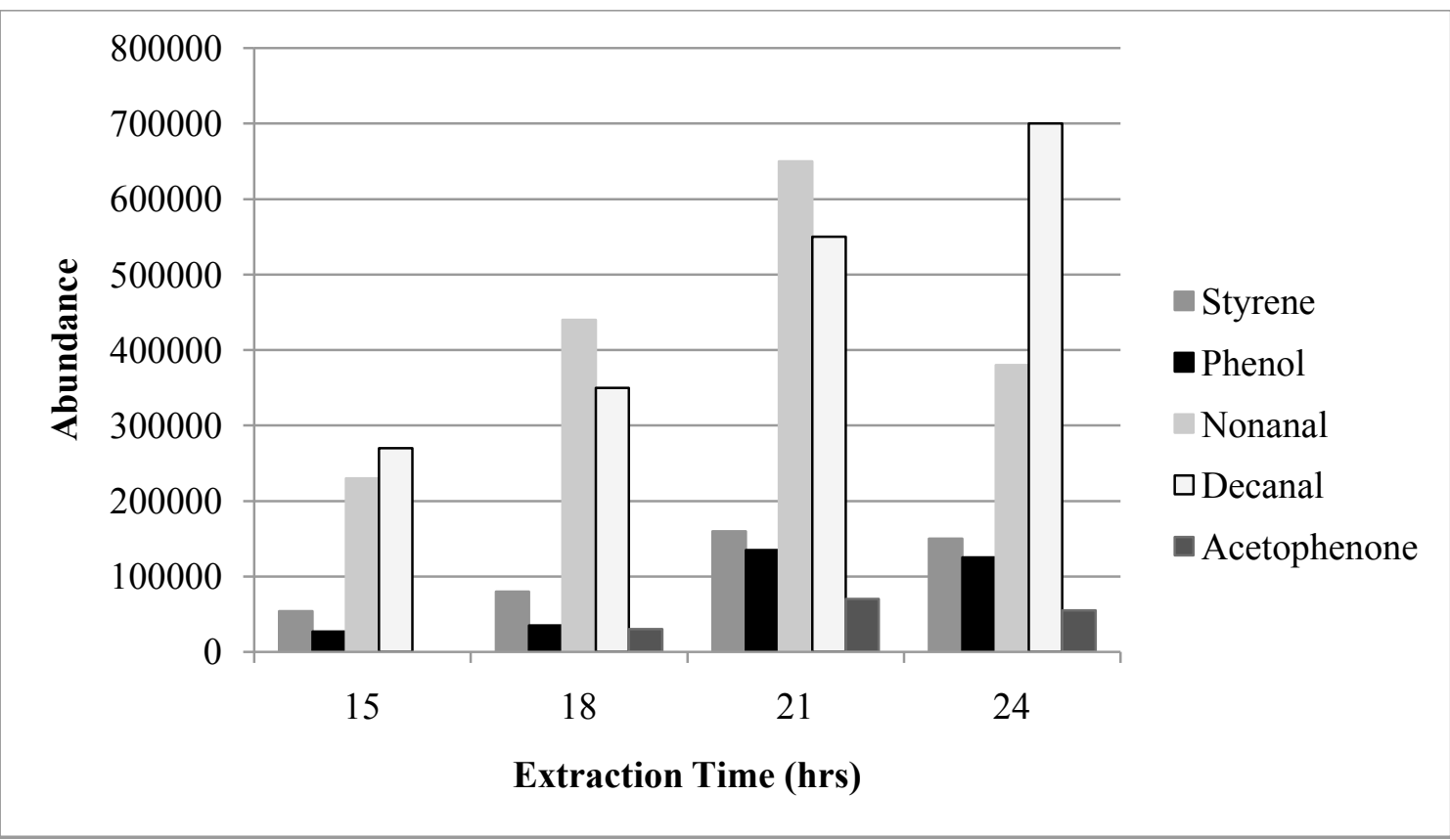




\subsection{Optimal Equilibration Time}

Equilibration time is the time which the collected sample is allowed to sit for in order for the VOCs in the sample to reach a steady state between the headspace and the sample collection matrix/material. The optimal equilibration time for breath samples collected in Bio-VOC ${ }^{\circledR}$ breath sampler was evaluated with the previously-determined 21-hr extraction time. Optimal equilibration time was evaluated on a combination of the number of human scent compounds extracted and the abundances of selected common human scent compounds, phenol, styrene, nonanal, decanal, and acetophenone, present in the breath samples. Fifteen (15) minutes was determined to be the most optimal equilibration time as shown in Figure 12 and Figure 13. At longer equilibration times, fewer compounds were extracted. There is a potential loss of compounds at longer equilibration times; with longer equilibration times it is possible that some of the compounds dissipate through the breath sampler and, therefore, the compounds are no longer present in the breath sampler at the time of extraction by SPME. 
Figure 12. Number of compounds extracted vs. equilibration time for breath

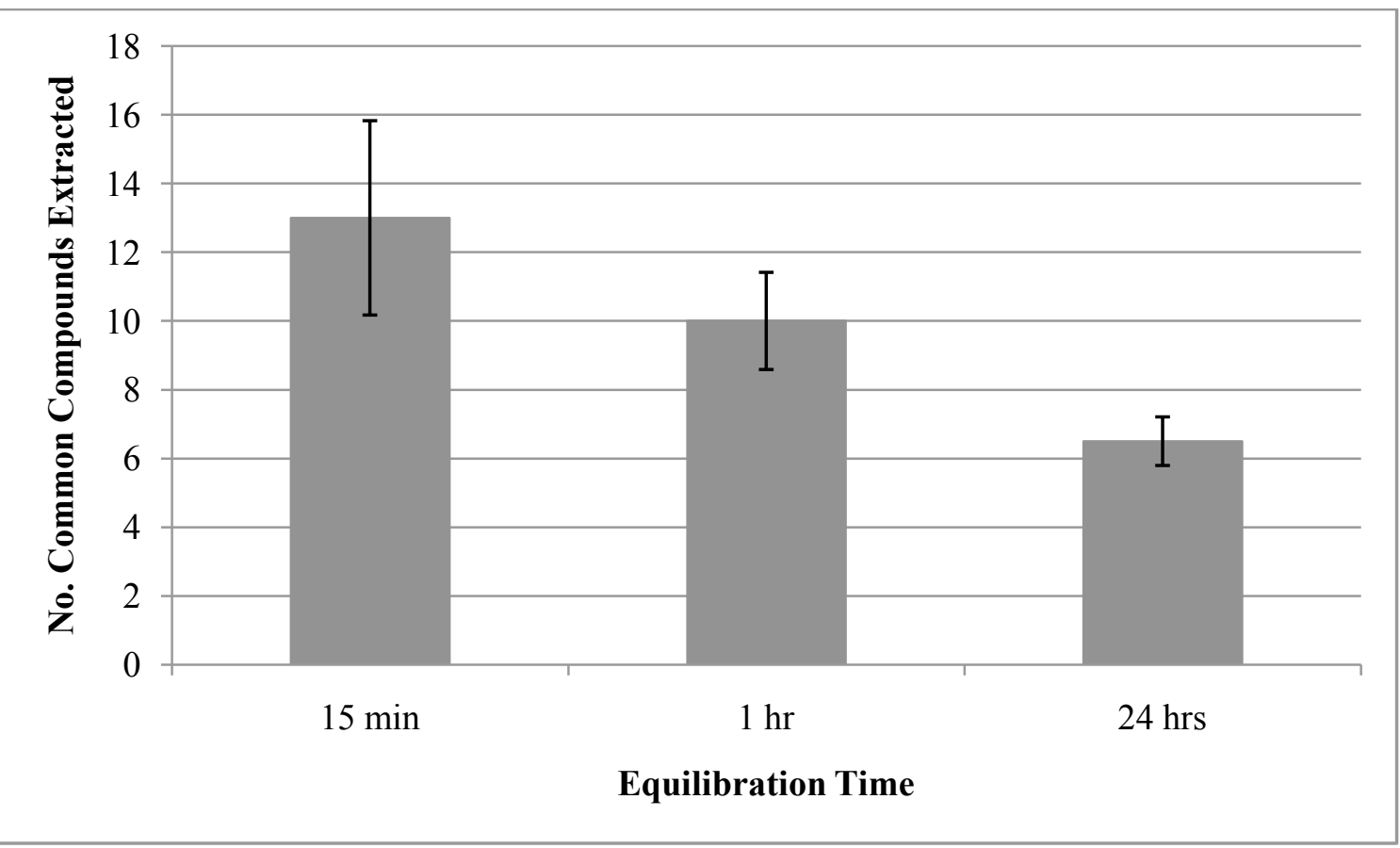

Figure 13. Abundances of selected common breath VOCs extracted vs. equilibration time

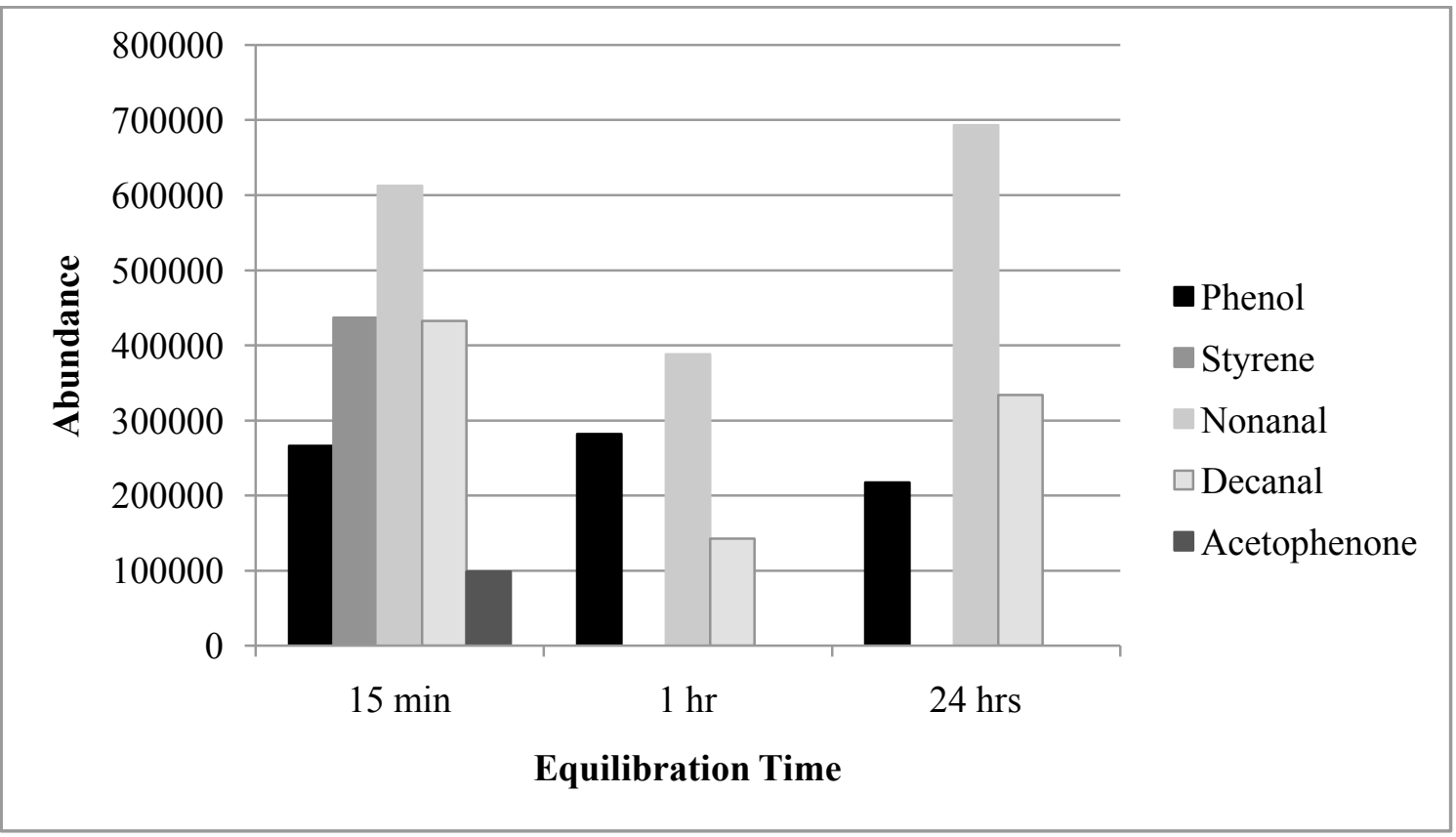




\subsection{Blood}

Blood is the essential body fluid which transports oxygen and nutrients to the body cells and carries carbon dioxide and waste materials away from the cells. Blood accounts for about $7 \%$ to $9 \%$ of a person's total body weight, and the volume is about 4-6 L of blood for an average adult ${ }^{58}$. Blood is composed of cells and plasma; plasma constitutes about $55 \%$ of blood volume and is the liquid portion of the blood in which the cells are suspended. The blood cells, called formed elements, are erythrocytes (red blood cells), leukocytes (white blood cells), and platelets (fragment cells). Erythrocytes carry oxygen and carbon dioxide and account for majority of the blood cells (>99\%). Erythrocytes contain the protein hemoglobin which binds the oxygen and carbon dioxide molecules, resulting in the characteristic red color of blood. The functions of leucocytes and platelets are infection/cancer protection and blood clotting, respectively. Plasma is mostly water (91\% of plasma by weight), and a variety of inorganic electrolytes, proteins, gases,

nutrients, hormones, and waste products ${ }^{46}$ (Figure 14). The other functions of blood include immunological functions, self-repair mechanism (blood clotting and coagulation), messenger functions, homeostatic functions such as $\mathrm{pH}$ and body temperature regulations. Blood $\mathrm{pH}$ is maintained via homeostasis between 7.35 and $7.45^{59}$. 
Figure 14. Components of blood

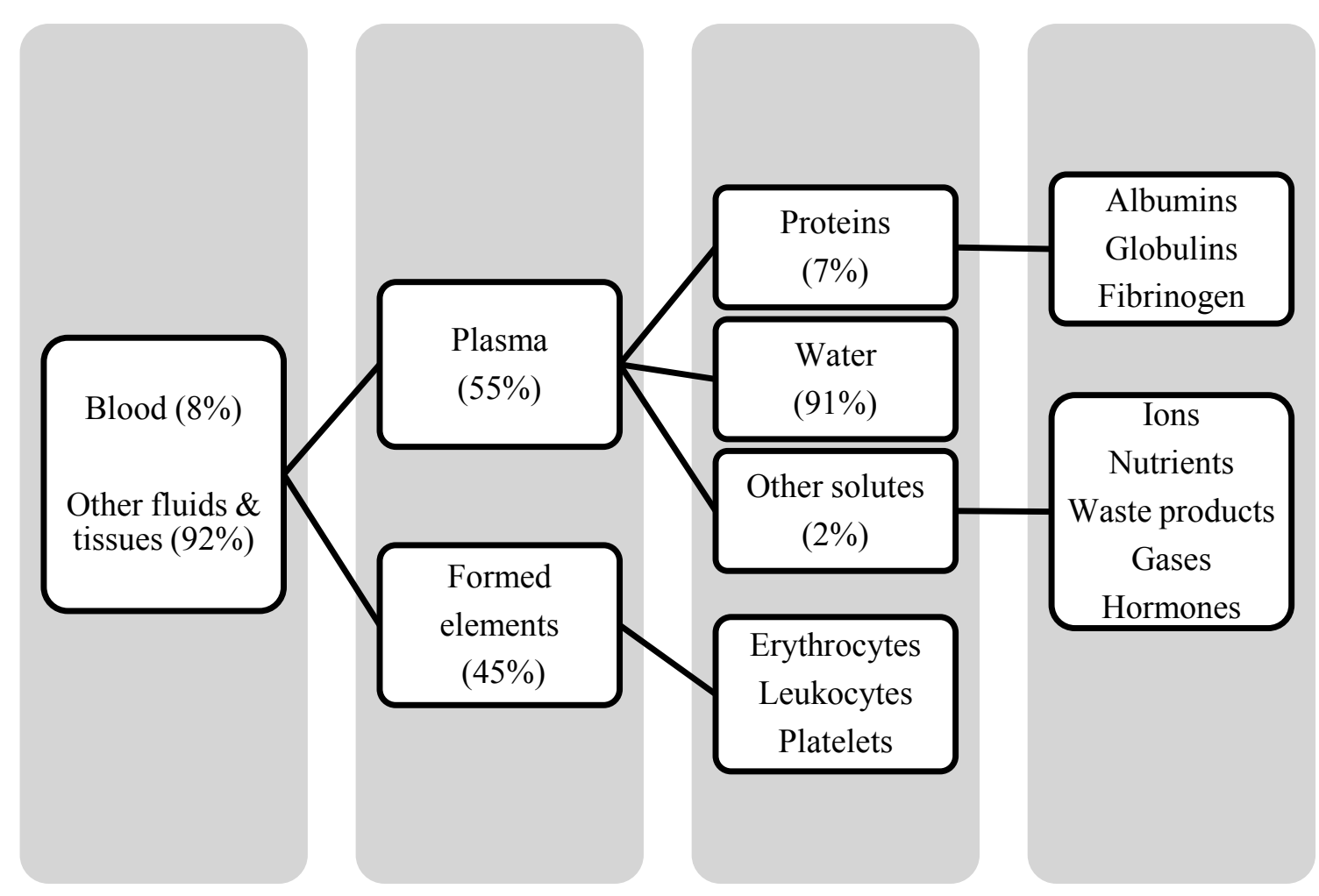

Blood, nutrients, gases, ions, and hormones are transported among the cells in the body via the circulatory system. The circulatory system is made up of the cardiovascular system and the lymphatic system; blood flow is through the former. The cardiovascular system is further composed of two pumping systems: pulmonary circulation and systemic circulation $^{58,60}$. The pulmonary circuit involves the right side of the heart where oxygendepleted blood is pumped to the lungs. Carbon dioxide and waste products are eliminated from the blood via the pulmonary circuit. Deoxygenated blood is carried from the right heart chamber to the lungs via the pulmonary arteries; oxygenated blood is transported from the lungs back to the heart via the pulmonary veins. Returned oxygenated blood is then pumped from the left side of the heart and is circulated to the rest of the body by the 
systemic circuit. The blood moves from the heart into aorta which feeds into the arteries, to arterioles, and finally to capillaries where the two-way exchange of gases, nutrients, and waste materials between blood and cells occurs. The blood-cell exchange of water (via osmosis) and dissolved solutes mainly occurs by process of diffusion, and to a lesser extent, by the blood pressure exerted against the capillary walls as blood flows through the capillaries.

Blood VOCs have been studied for the health effects of occupational and environmental exposures $^{61-63}$. When increased levels of volatile aromatic compounds such as benzene, toluene, and xylene isomers are present in most human blood, they are indicative of occupational or environmental VOC exposures and/or smoking ${ }^{16,61-64}$. Benzene and toluene concentrations in the blood of smokers (median concentration of $493 \mathrm{ng} / 1$ and $2001 \mathrm{ng} / 1$ for benzene and toluene, respectively) have been reported to be significantly different ( $p<0.0001$ for benzene, $p<0.05$ for toluene)from those of non-smokers (median concentrations of $190 \mathrm{ng} / 1$ and $1141 \mathrm{ng} / 1$ for benzene and toluene, respectively $)^{61}$. Blood sampling is the most invasive technique compared to collection of other biological specimens, as it requires needles to withdraw blood or breaking of the skin with a lancet, causing physical pain and psychological discomfort to the subject. Despite the sampling method being more invasive compared to breath or urine, blood VOC analysis is more representative of the internal environment of human biological activities. For this reason, analyses of volatile biomarkers for diseases have been gaining interest in the recent years $^{65-68}$. Hexanal and heptanal were found in higher levels (greater than $1.8 \mu \mathrm{M}$ ) in lung cancer blood compared to normal control blood (lower than $0.20 \mu \mathrm{M})^{66,67}$. In a study 
comparing blood VOCs of healthy participants and liver cancer patients, hexanal, 1octen-3-ol, and octane were found with positive rates greater than $84 \%$ in lung cancer patient blood $(\mathrm{p} \leq 0.0001)^{68}$.

Blood testing is still the most definitive testing method for DNA testing and disease diagnostics and therefore is widely performed in medical, toxicological, and forensic fields. Blood testing normally involves venipuncture to obtain venous blood, and requires several milliliters of blood in quantity. FTA cards have been used as a blood DNA storage method, and only require several drops of blood. To date, blood VOC detection using FTA cards as the collection medium has not been performed.

\subsubsection{Materials \& Methods}

Blood samples were collected on Whatman FTA® MiniCard (Whatman International Ltd, Maidstone, UK). Unistik2 Super $(21 \mathrm{G}, 0.81 \mathrm{~mm})$ single-use capillary sampling devices used for obtaining blood were purchased from Fisher HealthCare (Houston, TX, USA). Human whole blood containing anticoagulant sodium EDTA was obtained from $\begin{array}{llll}\text { Bioreclamation } & \text { Inc. } & \text { (Hicksville, } & \text { NY, }\end{array}$ Divinylbenzene/Carboxen/Polydimethylsiloxane (DVB/CAR/PDMS) $\quad(50 / 30 \mu \mathrm{m}$ film thickness) SPME fibers and SPME fiber holders were obtained from SUPELCO (Bellefonte, PA, USA).

The GC/MS analysis was carried out using an Agilent Technologies 6890N gas chromatograph coupled to an Agilent Technologies 5973N mass selective detector (Palo Alto, CA, USA). The column used to separate the analytes was a HP5-MS, $30 \mathrm{~m}, 0.25$ um, $0.25 \mathrm{~mm}$ with helium as the carrier gas with a flow rate of $1.0 \mathrm{~mL} / \mathrm{min}$. The extracted 
VOCs were desorbed in the injection port of the $\mathrm{GC}$ with a temperature of $250^{\circ} \mathrm{C}$ for five minutes in splitless mode. For blood, the GC method begins with an initial oven temperature of $30^{\circ} \mathrm{C}$ ramped at $10^{\circ} \mathrm{C} / \mathrm{min}$ to a final temperature of $200^{\circ} \mathrm{C}$ where it is held for two minutes, for a total run time of 19 minutes. The mass spectrometer used was an HP 5973 MSD with a quadrupole analyzer operated in electron ionization mode. The mass spectrometer transfer line was maintained at $280^{\circ} \mathrm{C}$ and the source temperature was $230^{\circ} \mathrm{C}$. The analytes were acquired in full-scan mode in $41-550 \mathrm{~m} / \mathrm{z}$ range.

\subsubsection{Pre-treatment of FTA card}

\subsubsection{Methods}

Sample collection materials were pre-treated prior to sample collection to eliminate any background compounds. One half of the Whatman FTA ${ }^{\circledR}$ MiniCard (one circle) was inserted in a sterile ten $\mathrm{ml}$ glass headspace vial and baked in a $105^{\circ} \mathrm{C}$ oven for 45

minutes. Pre-treated materials were analyzed using SPME-GC/MS to make sure they were free of undesired compounds and analytically clean.

\subsubsection{Results}

Pretreated FTA cards were analyzed using SPME-GC/MS to ensure they were free of undesired human scent compounds. An example of a chromatogram of pre-and postcleaning treatment of the FTA cards prior to sample collection is shown in Figure 15. As seen in Figure 16, the pre-treatment procedure effectively removes the numerous compounds that were originally present in the scent collection materials. 
Figure 15. Comparison of untreated and pre-treated FTA card

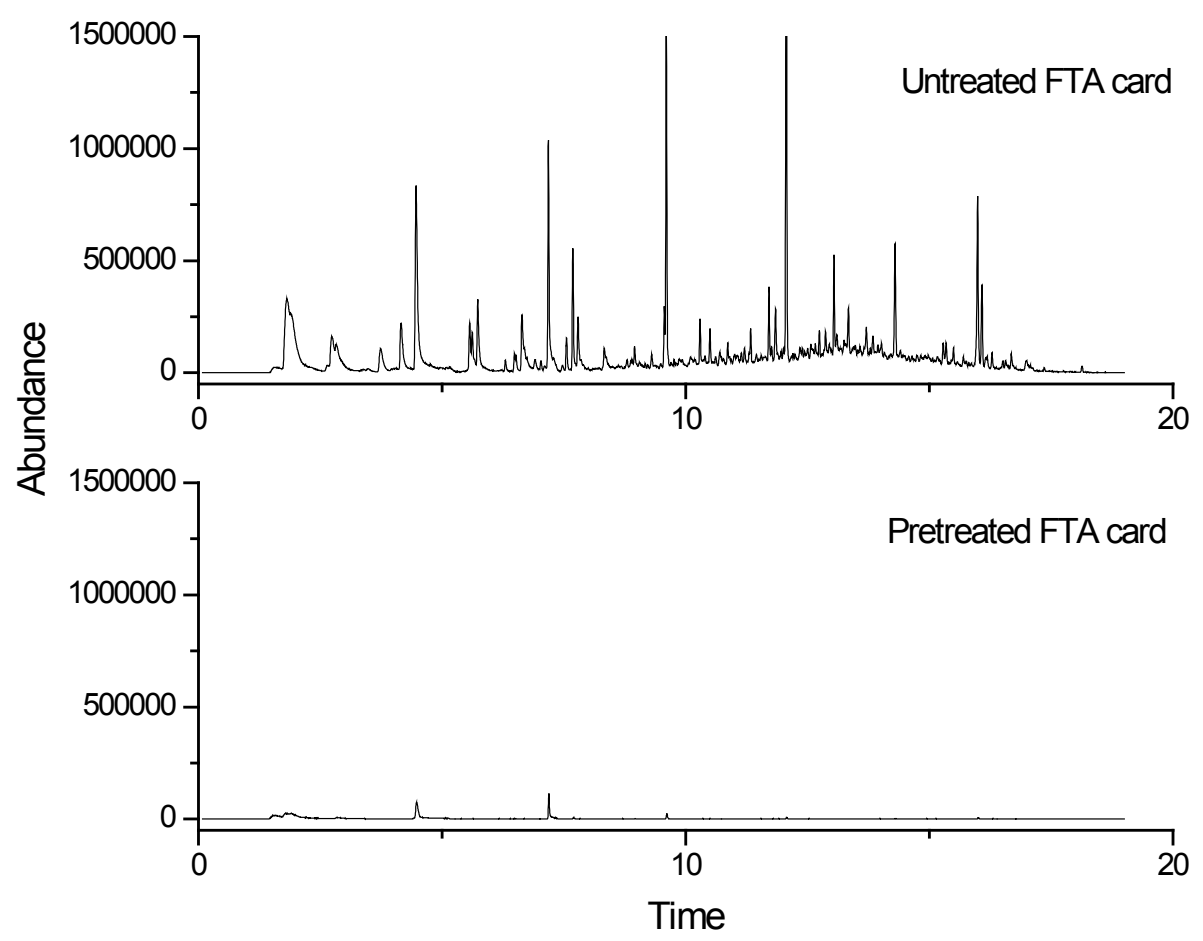

Figure 16. Comparison of FTA card pretreatment for the removal of undesired VOCs

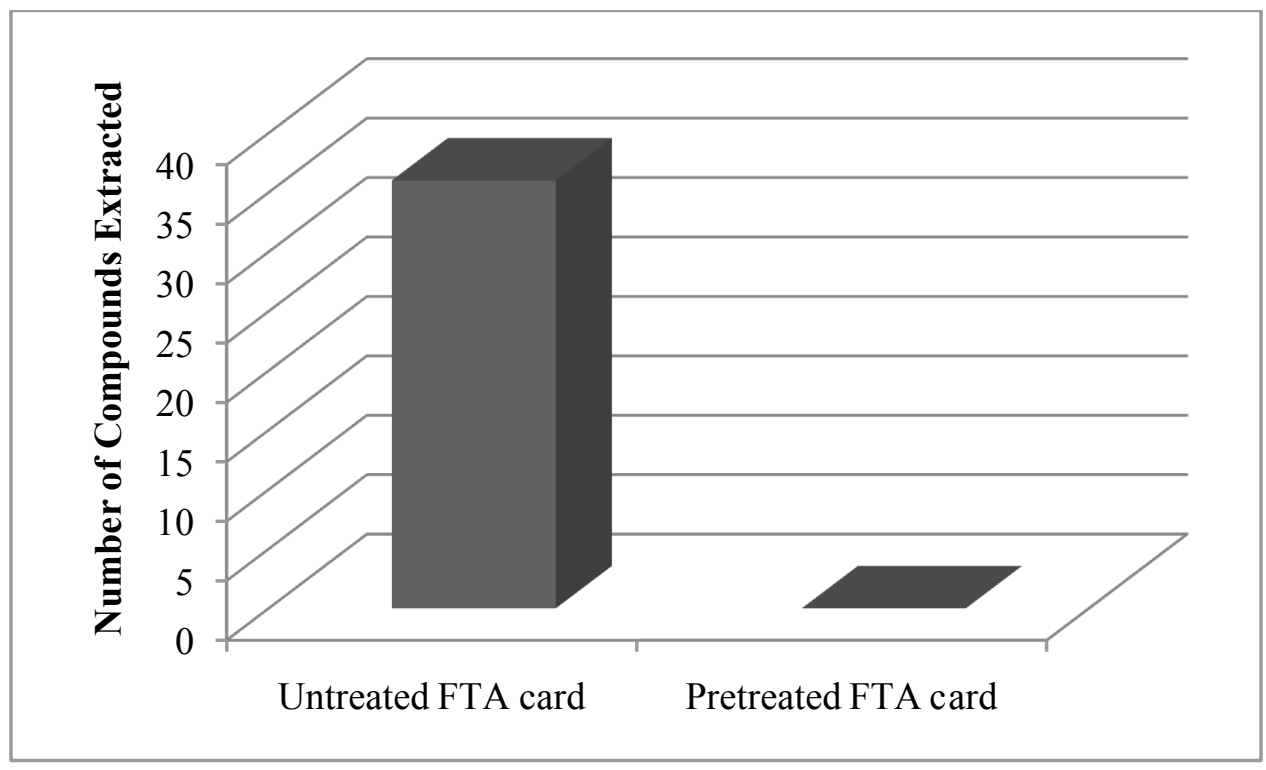




\subsubsection{Blood sampling procedure}

Blood samples were collected from two fingers (right and left ring fingers) using a fingerstick apparatus. Subjects sampled themselves by filling the circle of the FTA card with their blood. All samples were stored in the ten $\mathrm{ml}$ vials at room temperature.

\subsubsection{Determination of optimal extraction conditions for blood}

\subsubsection{Methods}

Human whole blood containing the anticoagulant sodium EDTA (Bioreclamation Inc., Hicksville, NY, USA) was used for the optimization study for blood. Whole blood samples were collected on pre-treated Whatman FTA® MiniCard (Whatman International Ltd, Maidstone, UK). To each individual FTA card, $200 \mu \mathrm{L}$ of whole blood was added, where blood was evenly distributed within the FTA circle. FTA cards were then resealed in their respective vials. During optimization, the blood exposures were done in a $37^{\circ} \mathrm{C}$ sand bath in triplicate samples for $1 \mathrm{~min}, 5 \mathrm{~min}, 10 \mathrm{~min}, 30 \mathrm{~min}, 1 \mathrm{hr}, 3$ hrs, $12 \mathrm{hrs}, 18 \mathrm{hrs}, 21 \mathrm{hrs}$, and $24 \mathrm{hrs}$. Optimization studies were also performed for sample equilibration time at $5 \mathrm{~min}, 1 \mathrm{hr}, 8 \mathrm{hrs}, 18 \mathrm{hrs}$, and $24 \mathrm{hrs}$ as well as the effect of sample heating. The 50/30 $\mu \mathrm{m}$ DVB/CAR/PDMS SPME fibers (SUPELCO, Bellefonte, PA, USA) were used to extract the VOCs from the headspace of the blood samples in the vials. All samples were run using the GC/MS method for blood samples previously mentioned in section 2.4.1. 


\subsubsection{Results}

\subsection{Optimal Extraction Time}

During optimization, the blood sample exposures were done in a $37^{\circ} \mathrm{C}$ sand bath in triplicate for one $\min , 5 \mathrm{~min}, 10 \mathrm{~min}, 30 \mathrm{~min}, 1 \mathrm{hr}, 3 \mathrm{hrs}, 12 \mathrm{hrs}, 18 \mathrm{hrs}, 21 \mathrm{hrs}$, and 24 hrs. The optimal extraction time for blood samples was evaluated on a combination of the number of human scent compounds extracted as well as the abundance of selected human scent compounds of various functional groups (decane, toluene, heptanal, and 1-octen-3ol). Eighteen (18) hours was determined to be the optimal extraction time for collected blood VOCs as shown in Figure 17 and Figure 18. No compounds were detected below the one hour extraction time. Beyond 18 hours, the abundance of majority of the extracted compounds decreased. The 24-hour extraction resulted in half the abundance of what was extracted under the 18-hour extraction time.

Figure 17. Number of compounds extracted vs. extraction time for whole blood

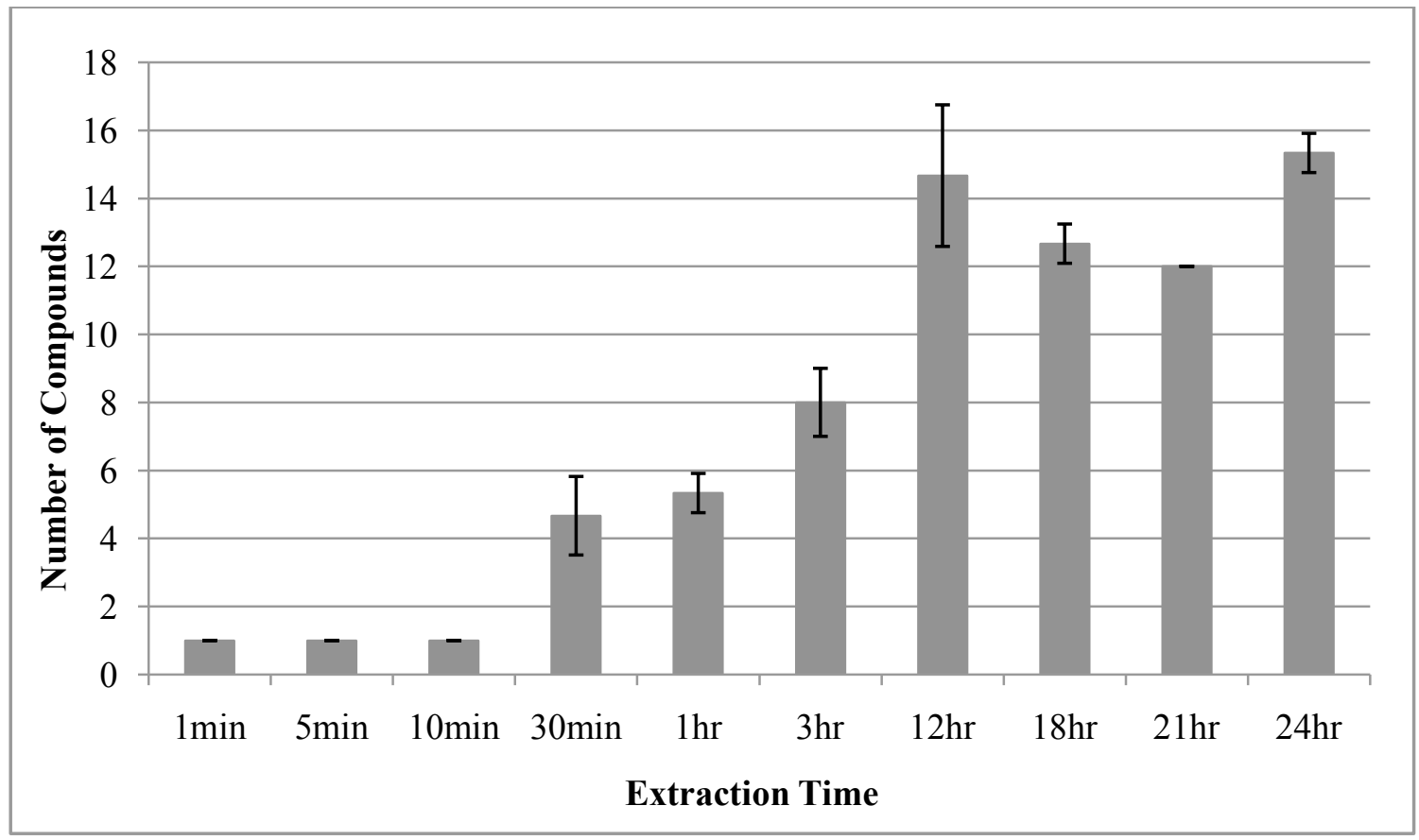


Figure 18. Abundance of selected common compounds extracted vs. extraction time for whole blood

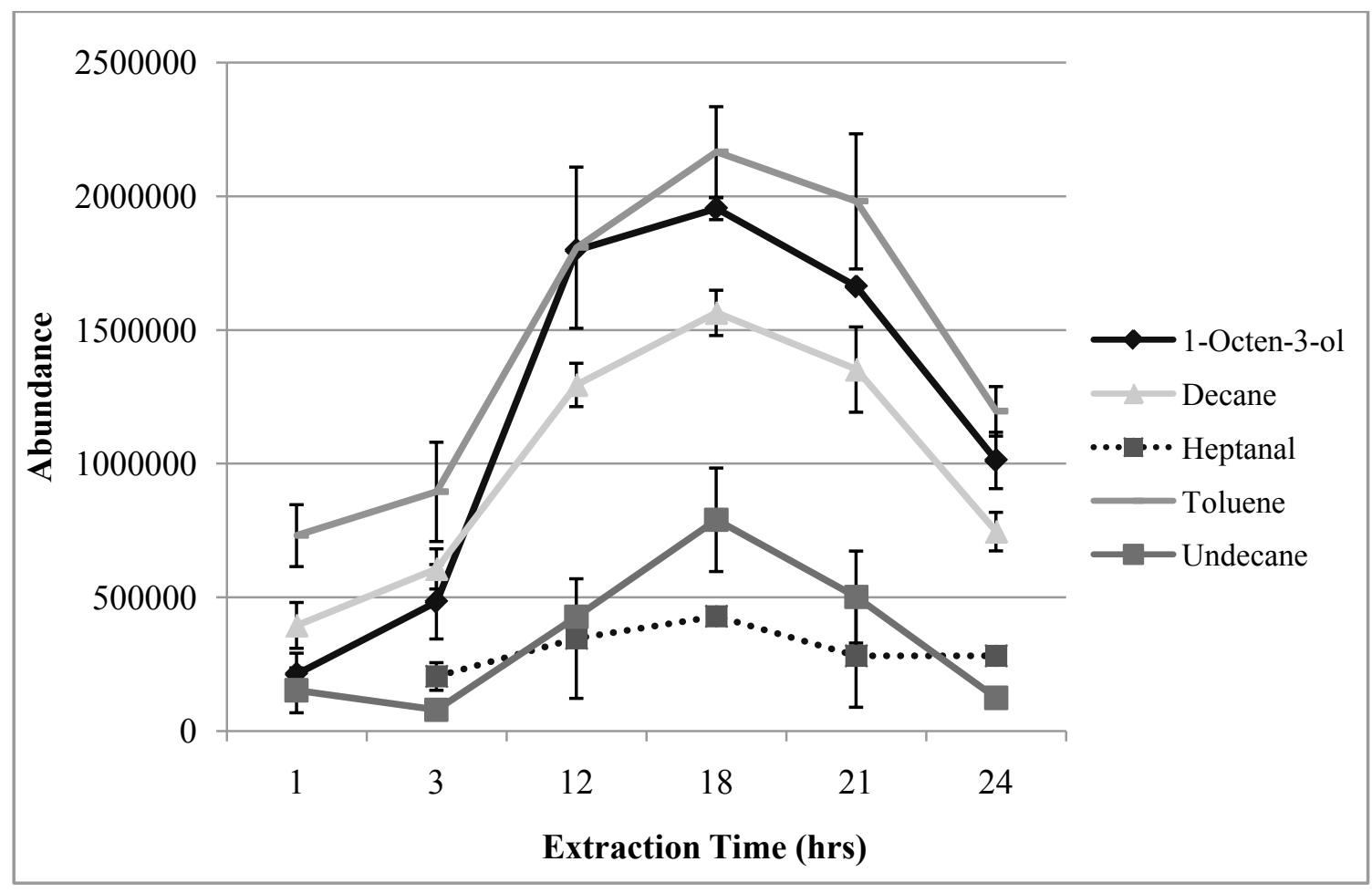

\subsection{Optimal Equilibration Time}

Once the optimal extraction time was determined, the optimal equilibration time was investigated. Various equilibration times ( $5 \mathrm{~min}, 1 \mathrm{hr}, 8 \mathrm{hrs}, 18 \mathrm{hrs}$, and $24 \mathrm{hrs}$ ) were investigated with the optimized 18 hour extraction time in a $37^{\circ} \mathrm{C}$ sand bath. One hour equilibration resulted in the greatest number of compounds extracted with SPME (Figure 19). The optimal equilibration time for blood samples were evaluated on a combination of the number of human scent compounds extracted as well as the abundance of selected human scent compounds of various functional groups (decane, undecane, toluene, heptanal, and 1-octen-3-ol). The abundance of the compounds detected was not greatly affected by the different equilibration times (Figure 20). No single obvious trend was 
seen in the abundances of compounds with varying equilibration times; however, with the exception of 1-octen-3-ol, a general decrease in the abundance was seen with increasing equilibration times. While abundances of extracted compounds were slightly higher for the 5-minute equilibration time than for the 1-hour equilibration time, the number of compounds extracted was greater for the latter. Therefore, the combination of a 1-hour equilibration time and 18-hour extraction time was chosen for the blood samples.

Figure 19. Number of compounds extracted vs. equilibration time for whole blood

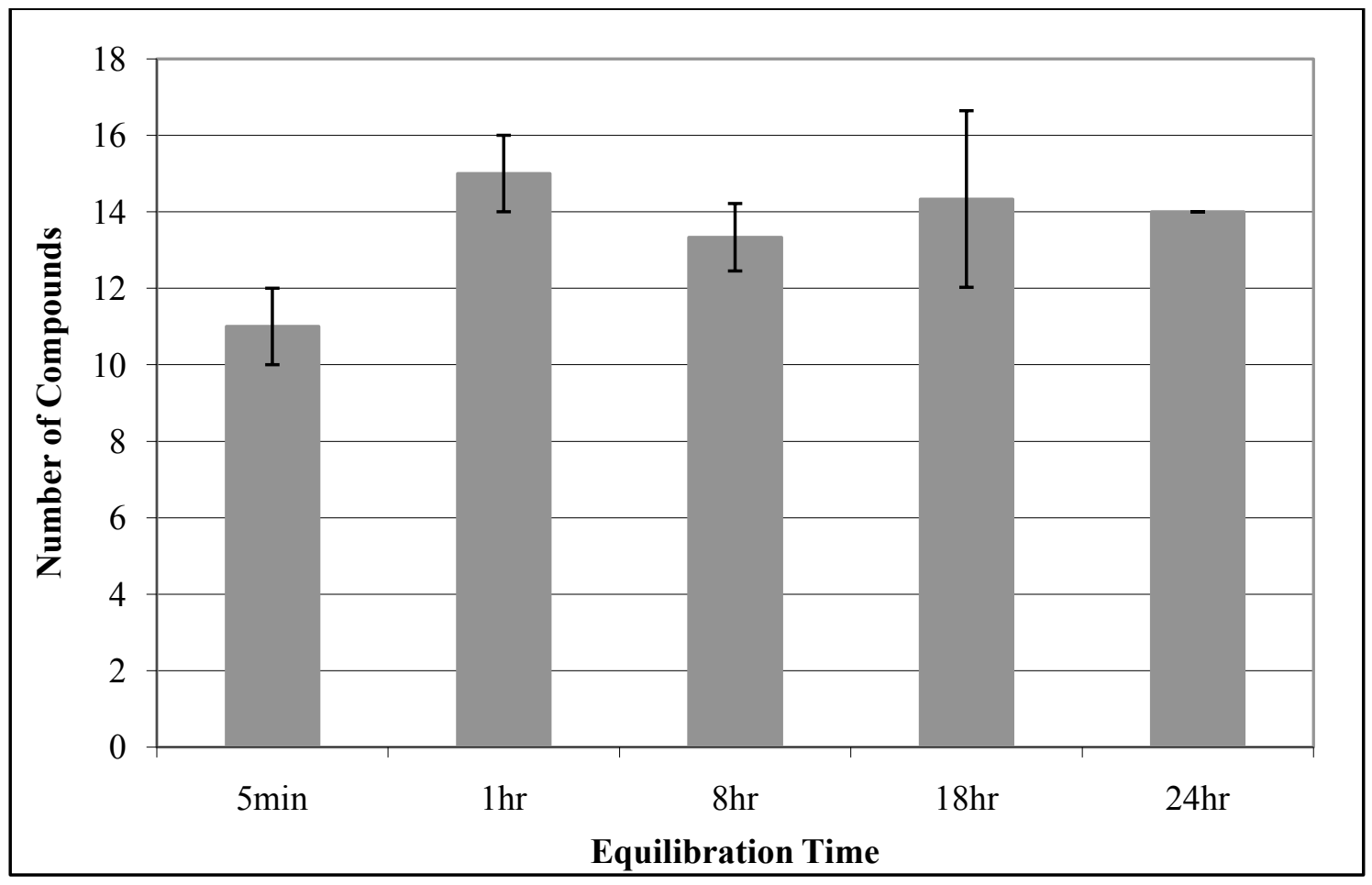


Figure 20. Abundance of selected common blood VOCs extracted vs. equilibration time

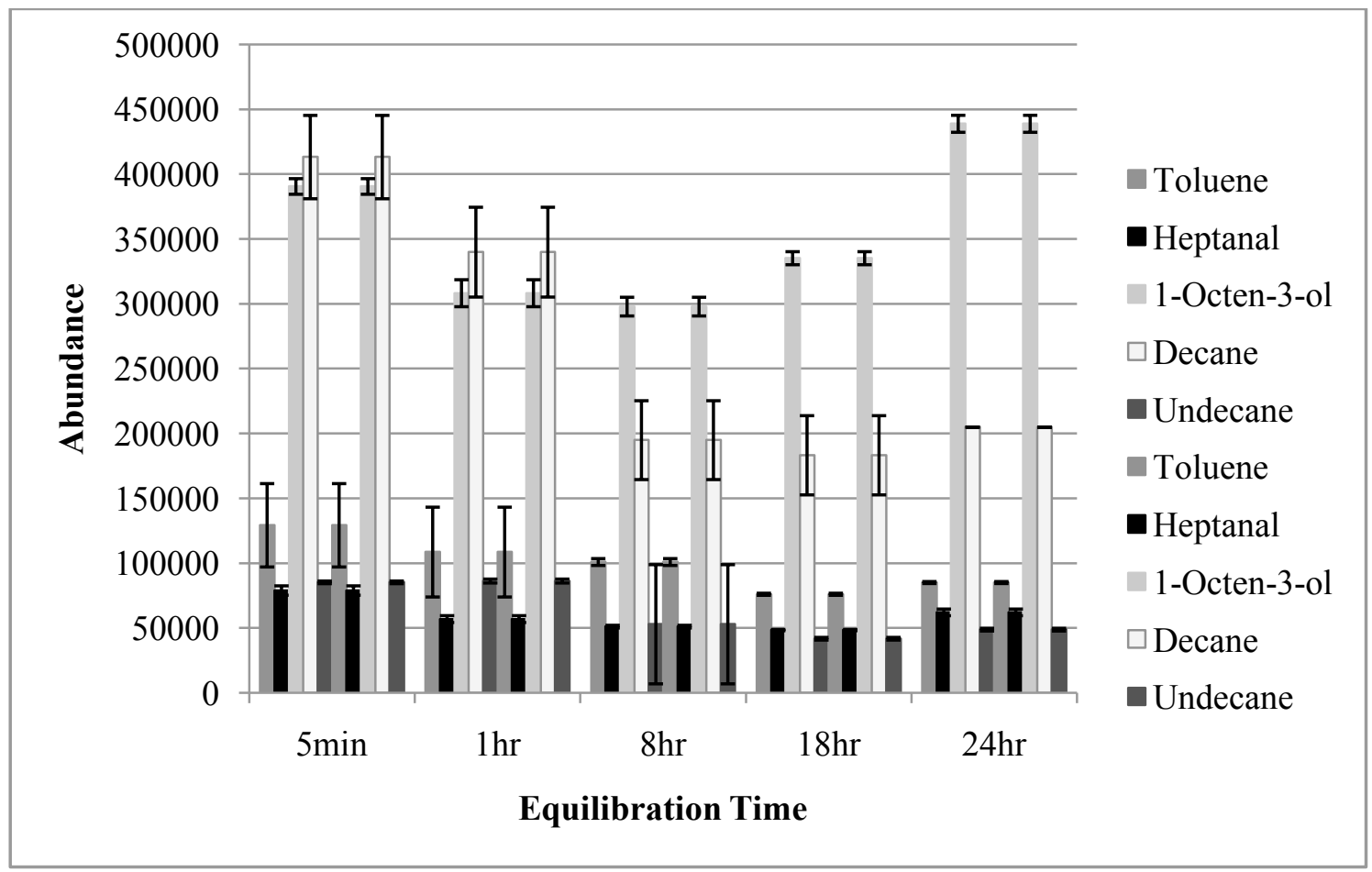

\subsection{Effect of Sample Heating}

The extraction efficiency of heating the blood samples was investigated. High temperatures degrade the blood samples so a comparison was made between unheated samples (room temperature equilibration and extraction) and samples heated to $37^{\circ} \mathrm{C}$ (human body temperature). More VOCs were extracted from the samples heated in the $37^{\circ} \mathrm{C}$ sand bath regardless of the extraction time, although the difference decreased as extraction time increased (Figure 21). Abundance of compounds significantly increased for the heated samples also. An example is shown for a common VOC extracted from blood samples, undecane, in Figure 22. 
Figure 21. Number of compounds extracted at room temperature and $37^{\circ} \mathrm{C}$ for whole blood

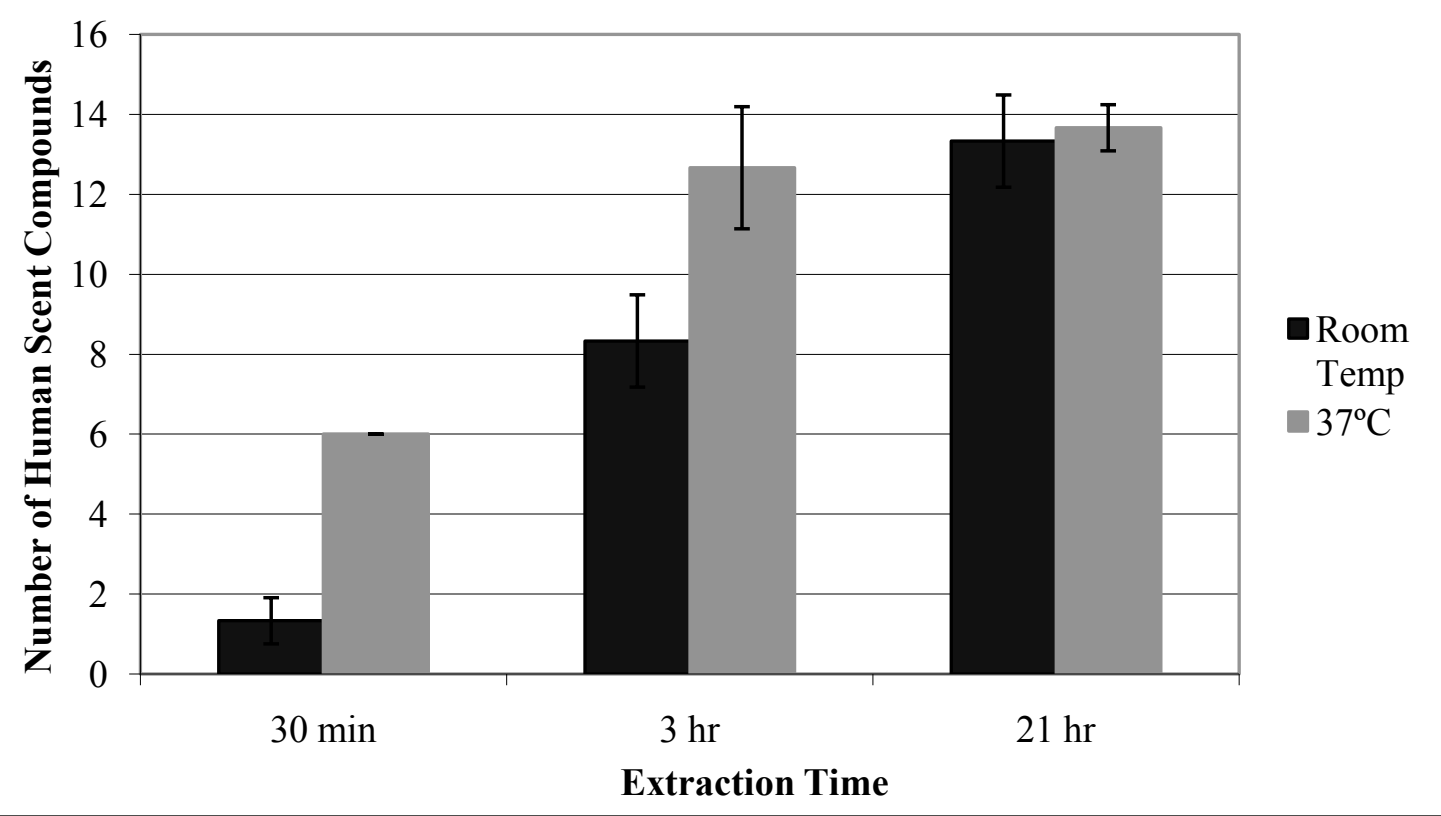

Figure 22. Abundance vs. extraction time for undecane at room temperature and $37^{\circ} \mathrm{C}$

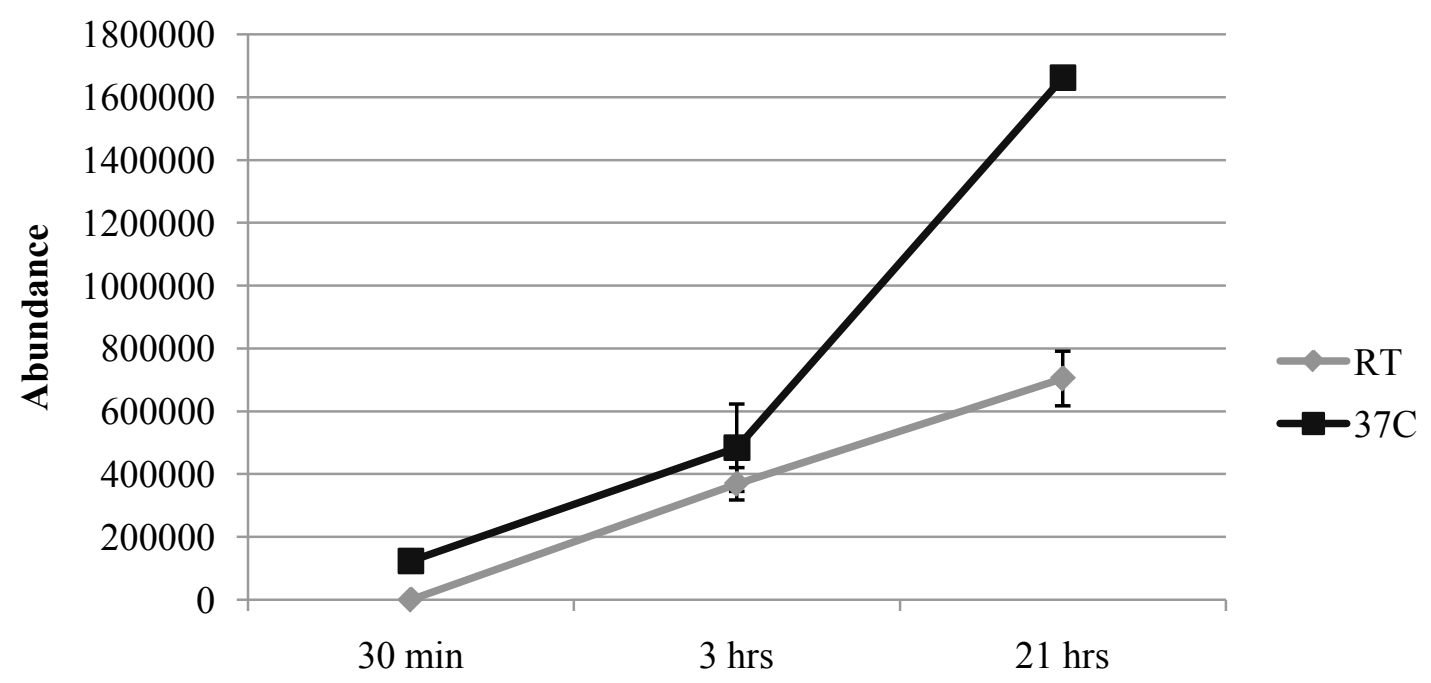

Extraction Time 


\subsection{Blood Sample Stability}

The stability of blood stored over time below $3^{\circ} \mathrm{C}$ was investigated. Blood collection tubes used to collect and store the blood only contained the anticoagulant $\mathrm{K}_{2}$ EDTA, and no preservatives. The VOC profiles of subject Male 5 were shown to be relatively stable even after one month of storage below $3^{\circ} \mathrm{C}$ as shown in Figure 23. Decanal, hexadecane, heptadecane, and octadecane were not present after one month of storage.

Figure 23. VOC profiles of M5 blood fresh, 1 day, and 1 month after storage

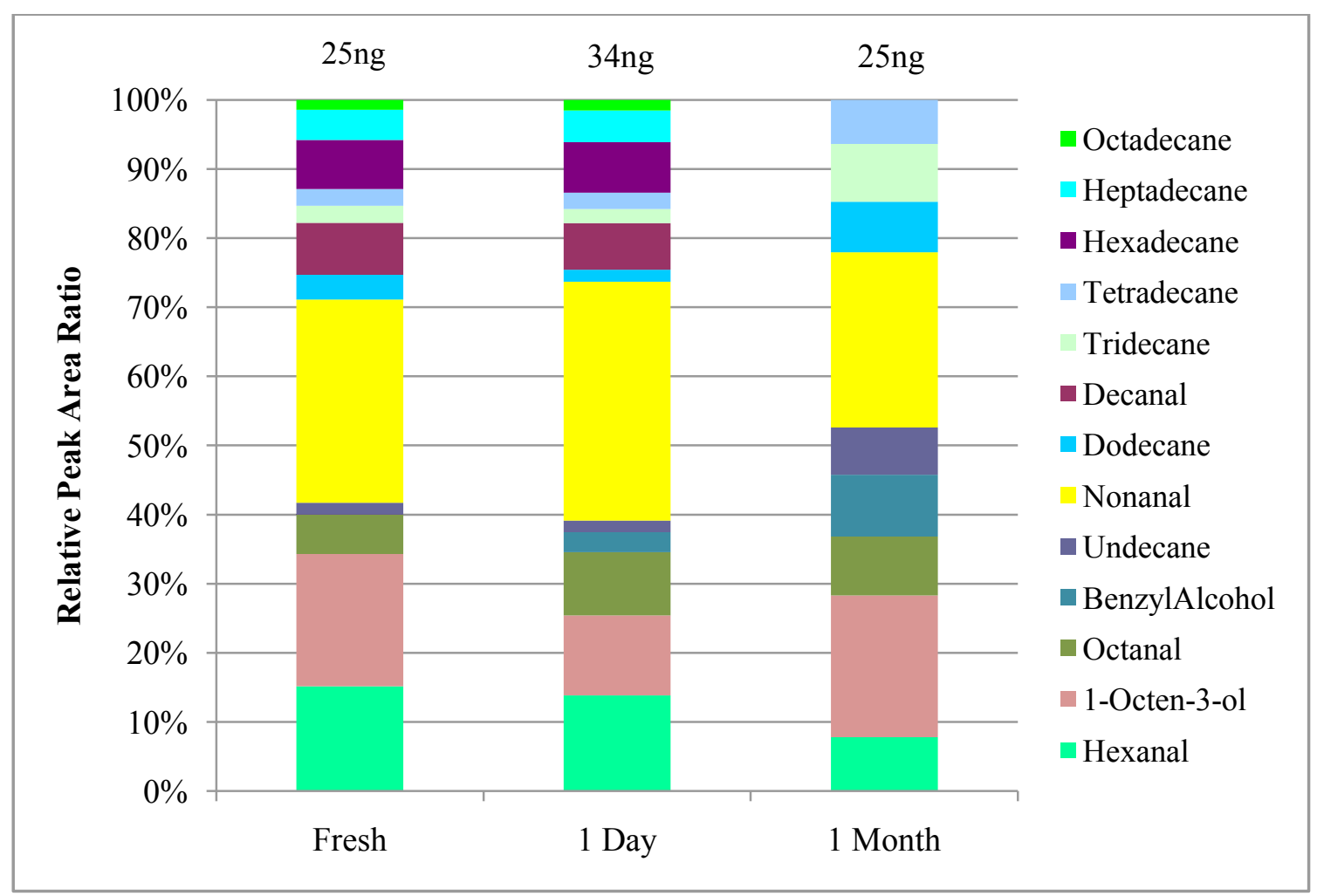




\subsection{Urine}

Urine is a transparent, aqueous liquid of mostly metabolic waste products that is secreted by the kidneys. Urine is composed of mostly water (95\%), and the rest are dissolved organic waste products, inorganic salts, and gases ${ }^{60}$. Organic waste materials include urea, creatinine, ammonia, and uric acid ${ }^{46,69}$. Inorganic constituents of urine include sodium, potassium, calcium, magnesium, phosphates, and sulfates. It is usually paleyellow in color; however, the color may range from yellow to amber, depending on the solute concentration in the urine and ingestion of certain foods. Urine secreted by the kidneys flows to the bladder through the ureters and is stored there until it is eliminated by the urethra during micturition.

\section{Figure 24. Schematic of urine production}

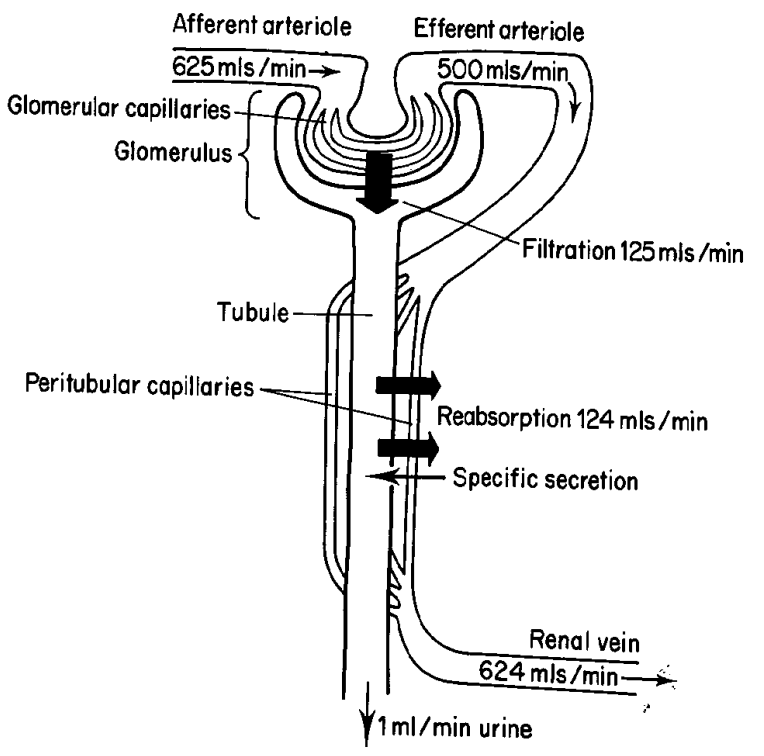

The urinary excretion mechanism begins by filtration by the nephrons of the kidneys (Figure 24). Initially, urine is an ultrafiltrate of the plasma from the glomerular capillaries into the glomerular capsule ${ }^{58,59}$. The glomerular filtration process is a bulk flow 
movement of the solvent (water) and the solutes within. Molecular weight determines what molecules are allowed to pass through the filter. The ultrafiltrate that results from the process is essentially all of the plasma constituents in almost exactly the same concentration, with the exception of proteins. The ultrafiltrate does not contain any cells. Following filtration, kidney tubular transport mechanisms of reabsorption and secretion occur. Reabsorption of tubular fluid, either by active transport or passive diffusion, moves water and solutes and transports back into the bloodstream ${ }^{59}$. There are two potential routes of reabsorption, the paracellular pathway where solutes are reabsorbed between tight junctions, and the transcellular pathway where solutes are reabsorbed through epithelial cells. Passive reabsorption via diffusion depends on the polarity and therefore the lipid solubility of the solute. Secretion is the opposite of reabsorption, where substances are moved from the blood into the tubular fluid to be excreted. The few mLs of urine containing water and solutes that were not reabsorbed are excreted. Table 4 shows the solute concentrations in plasma, ultrafiltrate in the glomerular capsule, and final urine. On average, humans produce approximately one to two liters per day. The average $\mathrm{pH}$ of freshly collected urine is 6.0 , but it can range between 4.5 and $8.0^{60}$. 
Table 4. Plasma, initial ultrafiltrate and final urine concentrations of major solutes ${ }^{54}$

\begin{tabular}{|l|c|c|c|}
\hline & $\begin{array}{c}\text { Plasma } \\
(\mathrm{mmol} / \mathrm{L})\end{array}$ & $\begin{array}{c}\text { Bowman's capsule } \\
(\mathrm{mmol} / \mathrm{L})\end{array}$ & $\begin{array}{c}\text { Urine } \\
(\mathrm{mmol} / \mathrm{L})\end{array}$ \\
\hline Sodium & 142 & 142 & $50-150$ \\
\hline Potassium & 4.0 & 4.0 & $20-100$ \\
\hline Chloride & 103 & 113 & $50-150$ \\
\hline Bicarbonate & $24-27$ & $27-30$ & $0-25$ \\
\hline Glucose & 5.5 & 5.9 & 0 \\
\hline Protein & $6 \mathrm{~g} / 100 \mathrm{~mL}$ & $0.020 \mathrm{~g} / 100 \mathrm{~mL}$ & $<0.010 \mathrm{~g} / 100 \mathrm{~mL}$ \\
\hline
\end{tabular}

Urine as collected in the bladder is sterile until it reaches the urethra ${ }^{59}$. However, bacterial colonies reside in the epithelial cells of the urethra, and when urine passes through the urethra and comes in contact with the bacteria, the interaction between the urine and the bacterial community can result in strong odor. Urinary odor depends on the volume and concentration of the solutes excreted by the kidneys. Dilute urine is nearly odorless; concentrated urine may result in strong odor of ammonia, resulting from the breakdown of urea. Diet and physiological conditions may also affect the odor of urine. Unusual urinary odor may be related to pathological conditions, infections, or renal failure.

Volatile organic compounds in urine result from food, drinks, air pollutants, drugs, bacterial interaction within the body, and metabolic processes ${ }^{70,71}$. Studies on the profiles of volatile metabolites in urine have demonstrated the presence of compounds of wide range of functional groups including alcohols, aldehydes, ketones, oxygen- and nitrogencontaining compounds, sulfur-containing compounds, and other heterocyclic compounds $^{70-73}$. The VOCs in the headspace of urine have been studied in a variety of fields including toxicology (drug detection and recovery) ${ }^{74-76}$, occupational and 
environmental exposures $^{77-80}$, and metabolic investigations ${ }^{70,71,81}$. Early studies on the urinary VOC profiles showed that there was a significant variance between different individuals, but intra-person urinary VOC profiles remained constant over time even with variation factors such as diet, exercise, circadian and seasonal changes ${ }^{73}$. Disturbances of volatile compounds found in urine have been associated with physiological disorders including diabetes mellitus, liver and kidney disease, and trimethylaminuria ${ }^{71,82,83}$.

\subsubsection{Materials \& Methods}

Sterile, disposable specimen collection cups were purchased from Dynarex Corporation (Orangeburg, NY, USA). Laboratory grade artificial urine was obtained from WARD'S Natural Science (Rochester, NY, USA). $\mathrm{NaCl}, \mathrm{KCl}, \mathrm{MgSO}_{4}, \mathrm{~K}_{2} \mathrm{CO}_{3}$, and $\mathrm{Na}_{2} \mathrm{CO}_{3}$ used for the urine optimization studies were purchased from Fisher Scientific (Fair Lawn, NJ, USA). Ten ml glass, clear, screw top headspace vials with PTFE/Silicone septa were used to hold the samples (SUPELCO, Bellefonte, PA, USA). Divinylbenzene/Carboxen/Polydimethylsiloxane (DVB/CAR/PDMS) $(50 / 30 \mu \mathrm{m}$ film thickness) SPME fibers and SPME fiber holders were obtained from SUPELCO (Bellefonte, PA, USA).

The GC/MS analysis was carried out using an Agilent Technologies $6890 \mathrm{~N}$ gas chromatograph coupled to an Agilent Technologies 5973N mass selective detector (Palo Alto, CA, USA). The column used to separate the analytes was a HP5-MS, $30 \mathrm{~m}, 0.25$ um, $0.25 \mathrm{~mm}$ with helium as the carrier gas with a flow rate of $1.0 \mathrm{~mL} / \mathrm{min}$. The extracted VOCs were desorbed in the injection port of the $\mathrm{GC}$ with a temperature of $250^{\circ} \mathrm{C}$ for five minutes in splitless mode. The GC oven temperature programming was as follows: the 
initial oven temperature of $40^{\circ} \mathrm{C}$ held for five minutes, and then ramped at $15^{\circ} \mathrm{C} / \mathrm{min}$ to a final temperature of $280^{\circ} \mathrm{C}$ where it was held for two minutes, for a total run time of 23 minutes. The mass spectrometer used was an HP 5973 MSD with a quadrupole analyzer operated in electron ionization mode. The mass spectrometer transfer line was maintained at $280^{\circ} \mathrm{C}$ and the source temperature was $230^{\circ} \mathrm{C}$. The analytes were acquired in full-scan mode in $41-550 \mathrm{~m} / \mathrm{z}$ range.

\subsubsection{Urine sampling procedure}

Subjects sampled themselves by collecting urine in a disposable sterile specimen collection cup. Urine samples were stored in a $4^{\circ} \mathrm{C}$ refrigerator until ready for use.

\subsubsection{Determination of optimal extraction conditions for urine}

\subsubsection{Methods}

Laboratory grade artificial urine (WARD'S Natural Science, Rochester, NY, USA) was spiked with acetone, 2-pentanone, dimethyl disulfide, 1H-pyrrole, toluene, hexanal, 4heptanone, 3-heptanone, 2-heptanone, benzaldehyde, phenol, 1-octanol, octanoic acid, and nonanoic acid for the optimization study. Urine samples were prepared in sterile 10 $\mathrm{ml}$ glass headspace vials with $2 \mathrm{ml}$ urine, $0.5 \mathrm{ml}$ deionized water, $1.5 \mathrm{~g} \mathrm{NaCl}$ (except during salting-out study), and spiked with $25 \mu \mathrm{L}$ of 200 ppm standard mix comprised of the 14 volatile compounds mentioned above. Samples were made in triplicates and vortexed at $2800 \mathrm{rpm}$ using lab dancer vortex (IKA ${ }^{\circledR}$ Works, Inc., Wilmington, NC, USA) for 30 seconds. Optimization studies were performed for SPME fiber exposure time $(1,5,15,30 \mathrm{~min}$, and $1 \mathrm{hr})$, sample equilibration time $(5,15$, and $30 \mathrm{~min})$, extraction temperature (room temperature, $40^{\circ} \mathrm{C}, 50^{\circ} \mathrm{C}, 60^{\circ} \mathrm{C}$, and $70^{\circ} \mathrm{C}$ ), and salting-out effects of 
5 different inorganic salts $\left(\mathrm{NaCl}, \mathrm{KCl}, \mathrm{MgSO}_{4}, \mathrm{~K}_{2} \mathrm{CO}_{3}\right.$, and $\left.\mathrm{Na}_{2} \mathrm{CO}_{3}\right)$. The $50 / 30 \mu \mathrm{m}$ DVB/CAR/PDMS SPME fibers (SUPELCO, Bellefonte, PA, USA) were used to extract the VOCs from the headspace of the urine samples in the vials. All samples were run using the GC/MS method for urine samples previously mentioned in section 2.5.1.

\subsubsection{Results}

\subsection{Optimal Extraction Time}

Laboratory grade artificial urine was spiked with $25 \mu l$ of $200 \mathrm{ppm}$ standard mix comprised of 14 volatile compounds listed in section 2.5.3.1. Urine samples were exposed for $1 \mathrm{~min}, 5 \mathrm{~min}, 15 \mathrm{~min}, 30 \mathrm{~min}$, and 1 hour to determine the optimal extraction time in a $50^{\circ} \mathrm{C}$ water bath with a 30 minute equilibration time. Signal increased with increasing extraction time until 30 minutes. The signal remained the same for the 30 minute- and one hour-extraction times. As shown in Table 5, 13 out of the 14 spiked compounds were recovered for both 30 minute- and one hour-extraction times. No acetone was detected in any of the samples, as it was masked by the acetonitrile solvent peak. From the results, 30 minutes was determined to be the optimal extraction time. 
Table 5. Compounds detected under different extraction times for urine SPME optimization

\begin{tabular}{|c|c|c|c|c|c|c|}
\hline \multirow[t]{2}{*}{$\mathrm{RT}$} & \multirow[t]{2}{*}{ Compound } & \multicolumn{5}{|c|}{ Extraction Time } \\
\hline & & $1 \mathrm{~min}$ & $5 \mathrm{~min}$ & $15 \mathrm{~min}$ & $30 \mathrm{~min}$ & $1 \mathrm{hr}$ \\
\hline 1.89 & Acetone & & & & & \\
\hline 3.28 & 2-Pentanone & $\mathrm{X}$ & $\mathrm{X}$ & $\mathrm{X}$ & $\mathrm{X}$ & $\mathrm{X}$ \\
\hline 4.44 & Dimethyl Disulfide & $\mathrm{x}$ & $\mathrm{x}$ & $\mathrm{x}$ & $\mathrm{x}$ & $\mathrm{x}$ \\
\hline 4.76 & Pyrrole & & $\mathrm{x}$ & $\mathrm{x}$ & $\mathrm{x}$ & $\mathrm{x}$ \\
\hline 5.07 & Toluene & $\mathrm{X}$ & $\mathrm{x}$ & $\mathrm{x}$ & $\mathrm{x}$ & $\mathrm{x}$ \\
\hline 6.02 & Hexanal & $\mathrm{x}$ & $\mathrm{x}$ & $\mathrm{x}$ & $\mathrm{x}$ & $\mathrm{x}$ \\
\hline 7.73 & 4-Heptanone & $\mathrm{x}$ & $\mathrm{x}$ & $\mathrm{x}$ & $\mathrm{x}$ & $\mathrm{x}$ \\
\hline 8.05 & 3-Heptanone & $\mathrm{x}$ & $\mathrm{x}$ & $\mathrm{x}$ & $\mathrm{x}$ & $\mathrm{x}$ \\
\hline 8.13 & 2-Heptanone & $\mathrm{x}$ & $\mathrm{x}$ & $\mathrm{x}$ & $\mathrm{x}$ & $\mathrm{x}$ \\
\hline 9.25 & Benzaldehyde & $\mathrm{x}$ & $\mathrm{X}$ & $\mathrm{x}$ & $\mathrm{x}$ & $\mathrm{x}$ \\
\hline 9.58 & Phenol & $\mathrm{x}$ & $\mathrm{x}$ & $\mathrm{x}$ & $\mathrm{x}$ & $\mathrm{x}$ \\
\hline 10.86 & 1-Octanol & & $\mathrm{x}$ & $\mathrm{x}$ & $\mathrm{x}$ & $\mathrm{x}$ \\
\hline 12.15 & Octanoic acid & & & & $\mathrm{x}$ & $\mathrm{x}$ \\
\hline 12.94 & Nonanoic acid & & & $\mathrm{X}$ & $\mathrm{x}$ & $\mathrm{x}$ \\
\hline
\end{tabular}

\subsection{Optimal Equilibration Time}

Equilibration times were varied $(5,15$, and 30 minutes) with a 30 minute extraction time at $50^{\circ} \mathrm{C}$. Results demonstrated that peak areas for the three equilibration times were comparable with one another (Figure 25 through Figure 29). Longer equilibration time did not affect the signal strength or peak area; therefore, the shortest equilibration time of five minutes was chosen. 
Figure 25. Abundance vs. equilibration times of aldehydes for urine optimization

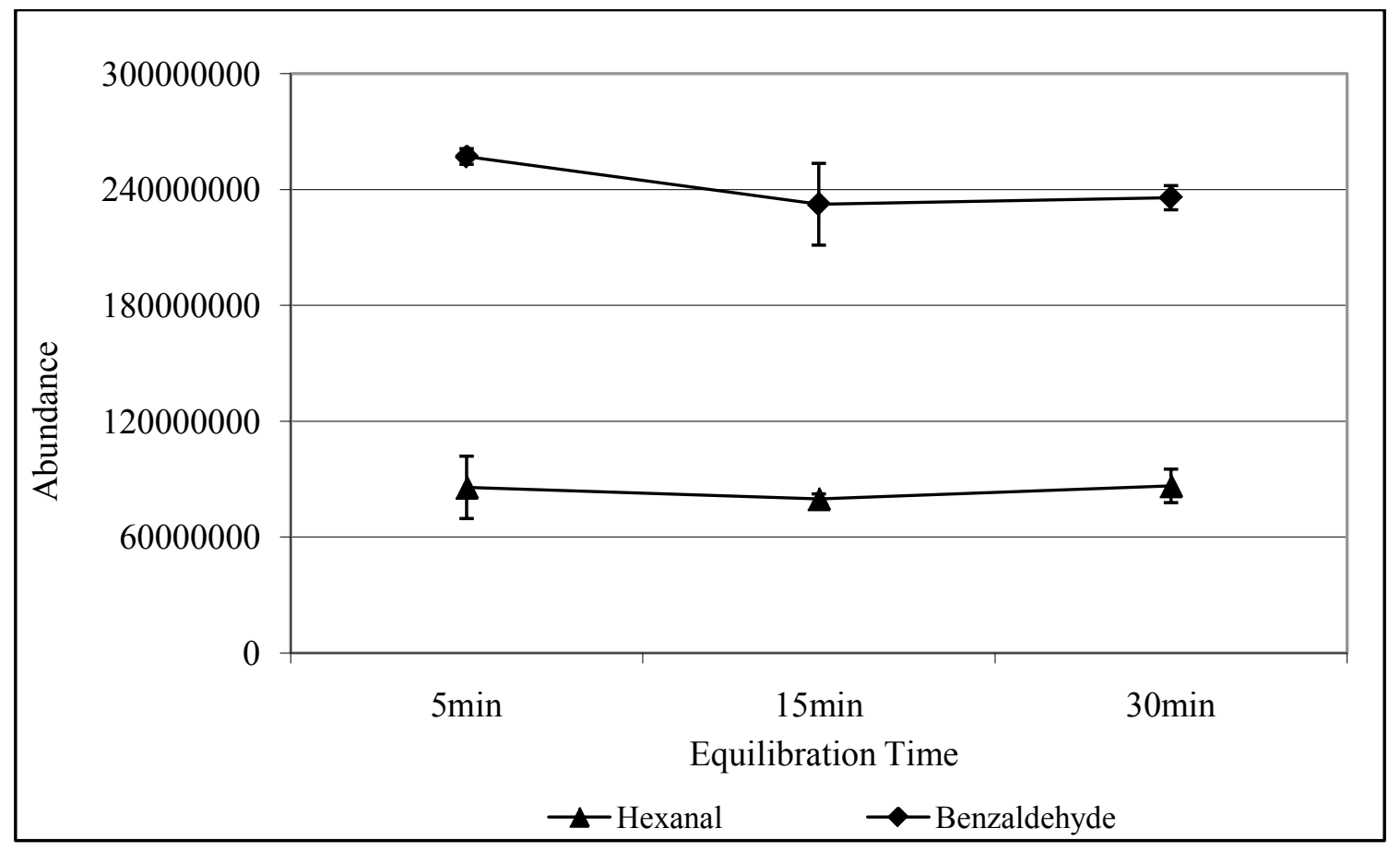

Figure 26. Abundance vs. equilibration times for acids for urine optimization

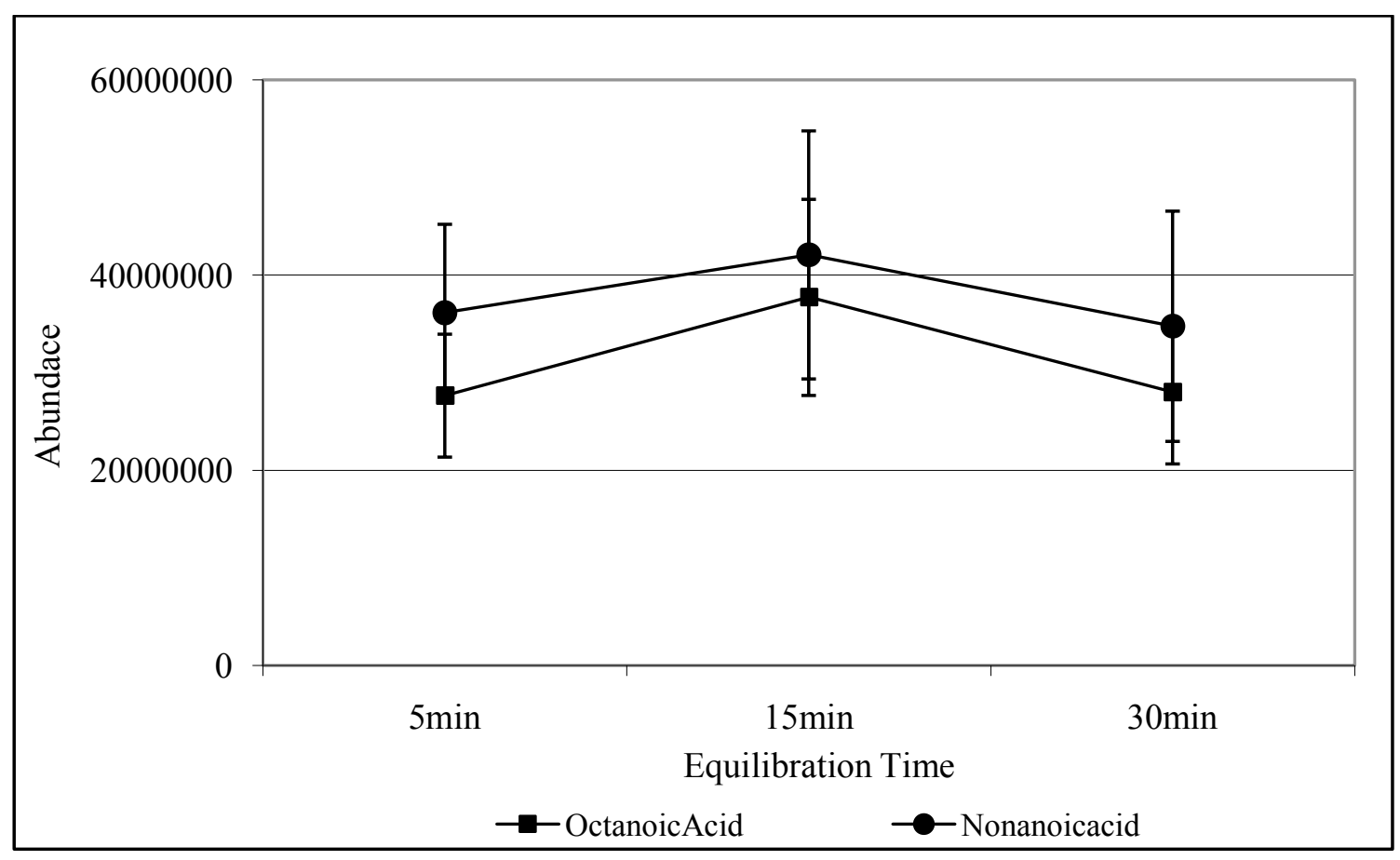


Figure 27. Abundance vs. equilibration times for alcohols for urine optimization

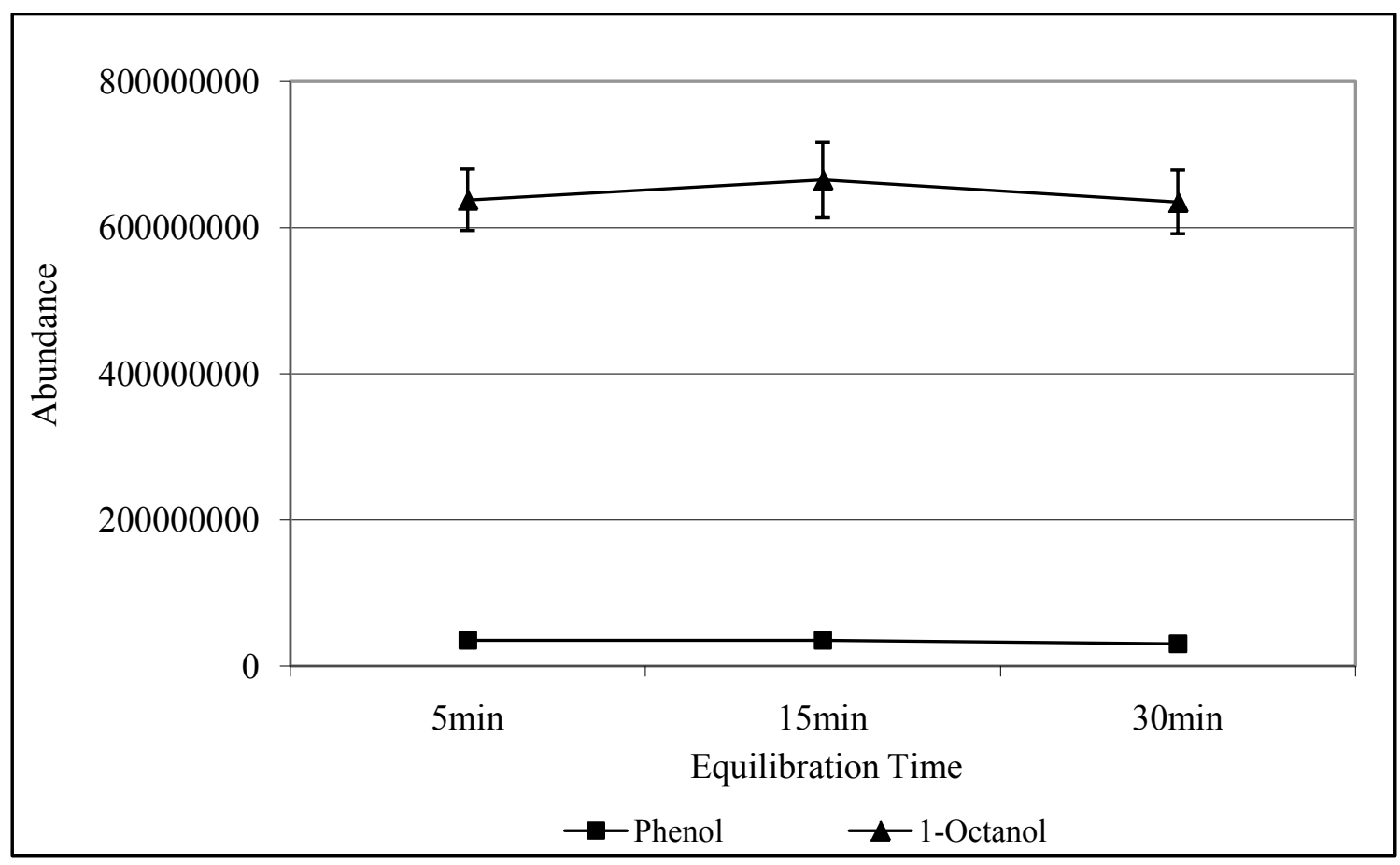

Figure 28. Abundance vs. equilibration times for ketones for urine optimization

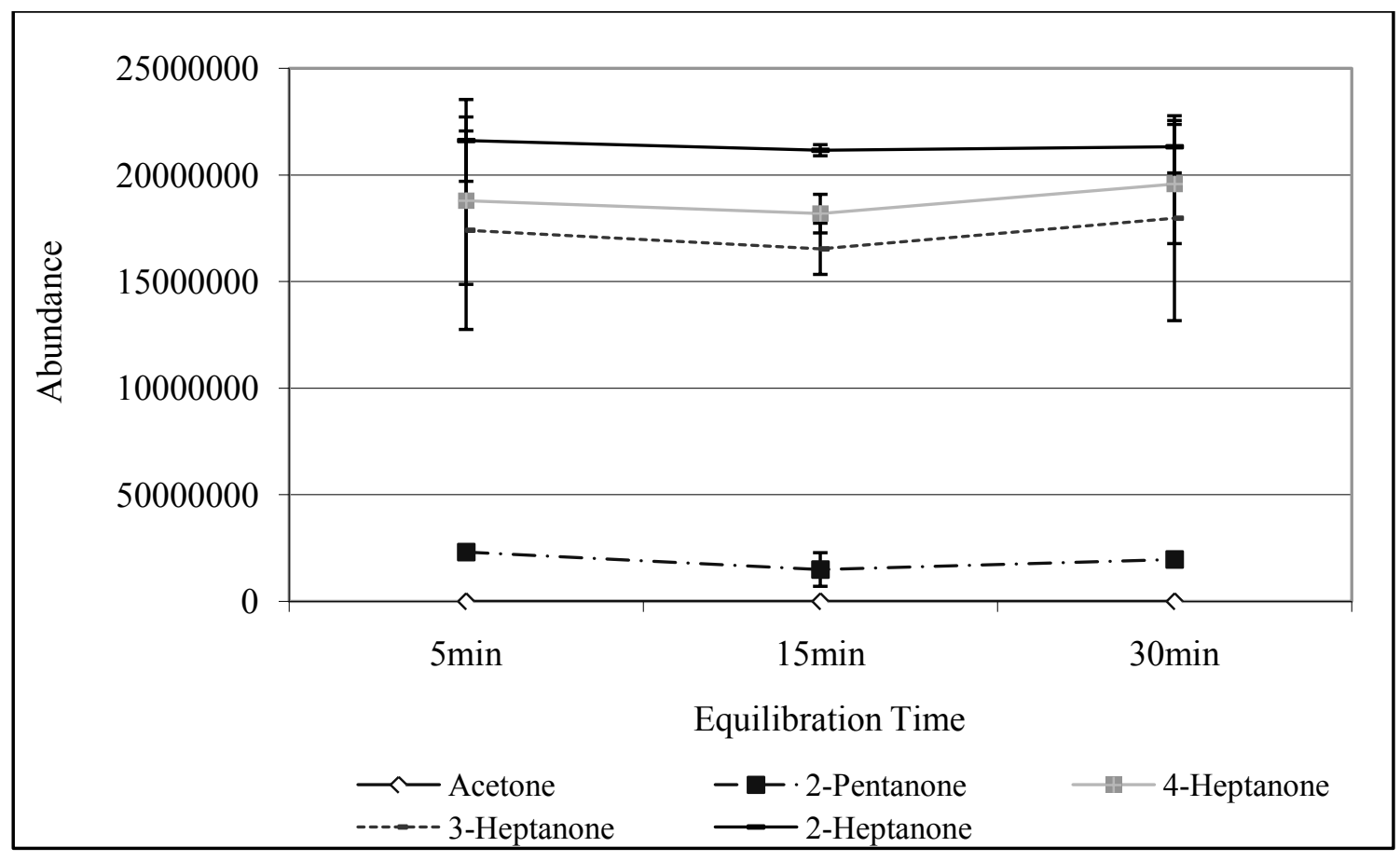


Figure 29. Abundance vs. equilibration time for other functional group compounds for urine optimization

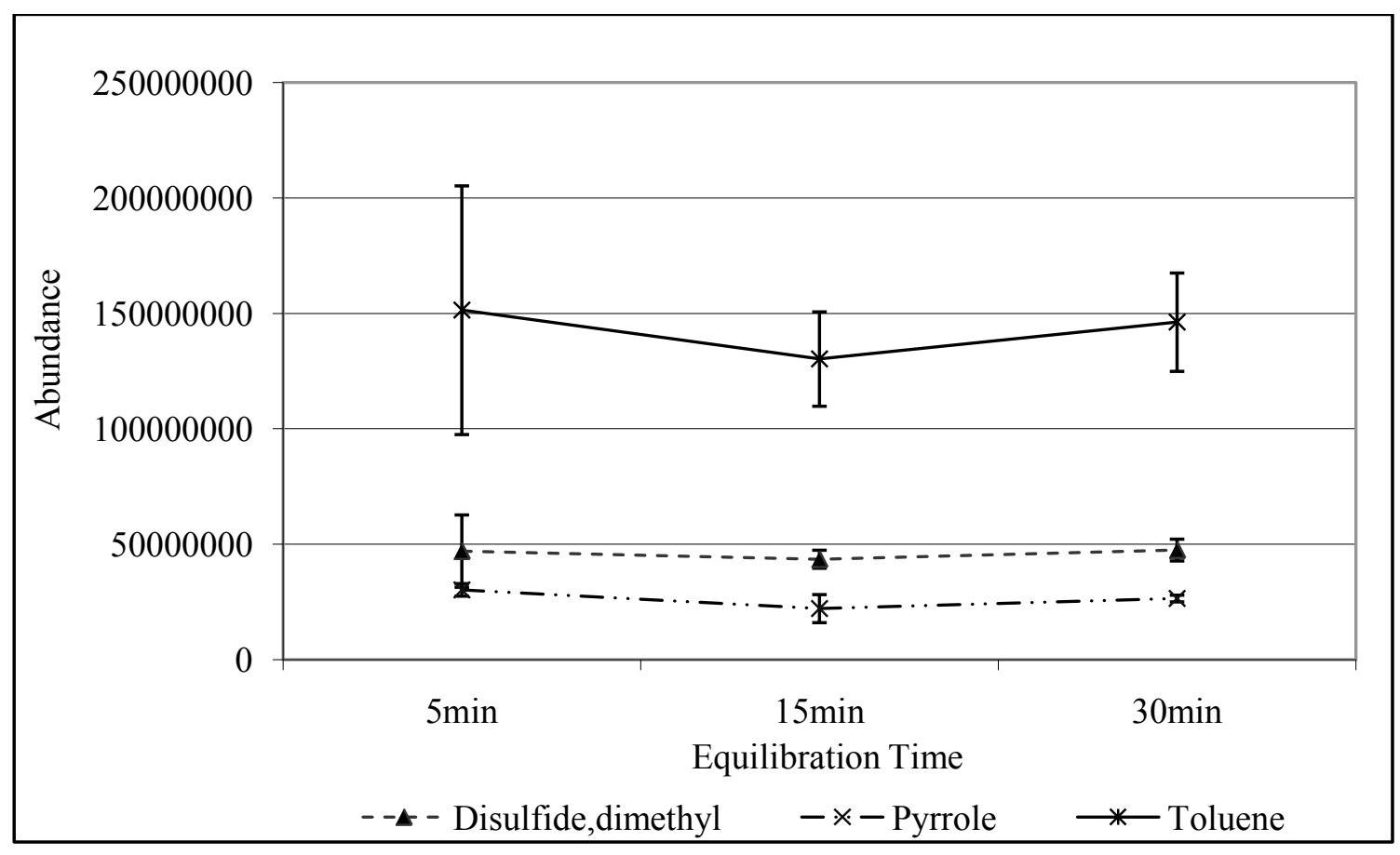

\subsection{Optimal Extraction Temperature}

Temperature of the hot water bath was varied (room temperature $\left(\sim 25^{\circ} \mathrm{C}, 40^{\circ} \mathrm{C}, 50^{\circ} \mathrm{C}\right.$, $60^{\circ} \mathrm{C}$, and $70^{\circ} \mathrm{C}$ ) using the five minute equilibration and 30 minute extraction times. Results demonstrated that $60^{\circ} \mathrm{C}$ gave the best results in terms of the abundance of the compounds extracted for the majority of the compounds. As shown in Figure 31, for the carboxylic acids nonanoic acid and octanoic acid, the abundance of extracted compounds was highest at $70^{\circ} \mathrm{C}$. However, for aldehydes, alcohols, ketones, and pyrrole, the abundance of the extracted compounds decreased at $70^{\circ} \mathrm{C}$. For dimethyl disulfide and toluene, there was a slight increase in the abundance of the extracted compounds. Raising the temperature beyond $60^{\circ} \mathrm{C}$ introduces the additional concern of fiber coating swelling and powdering which could risk damage to the fiber through fragmentation 
when it is withdrawn back into the needle. Therefore, $60^{\circ} \mathrm{C}$ was chosen as the optimal extraction temperature.

Figure 30. Abundance vs. extraction temperatures of aldehydes for urine optimization

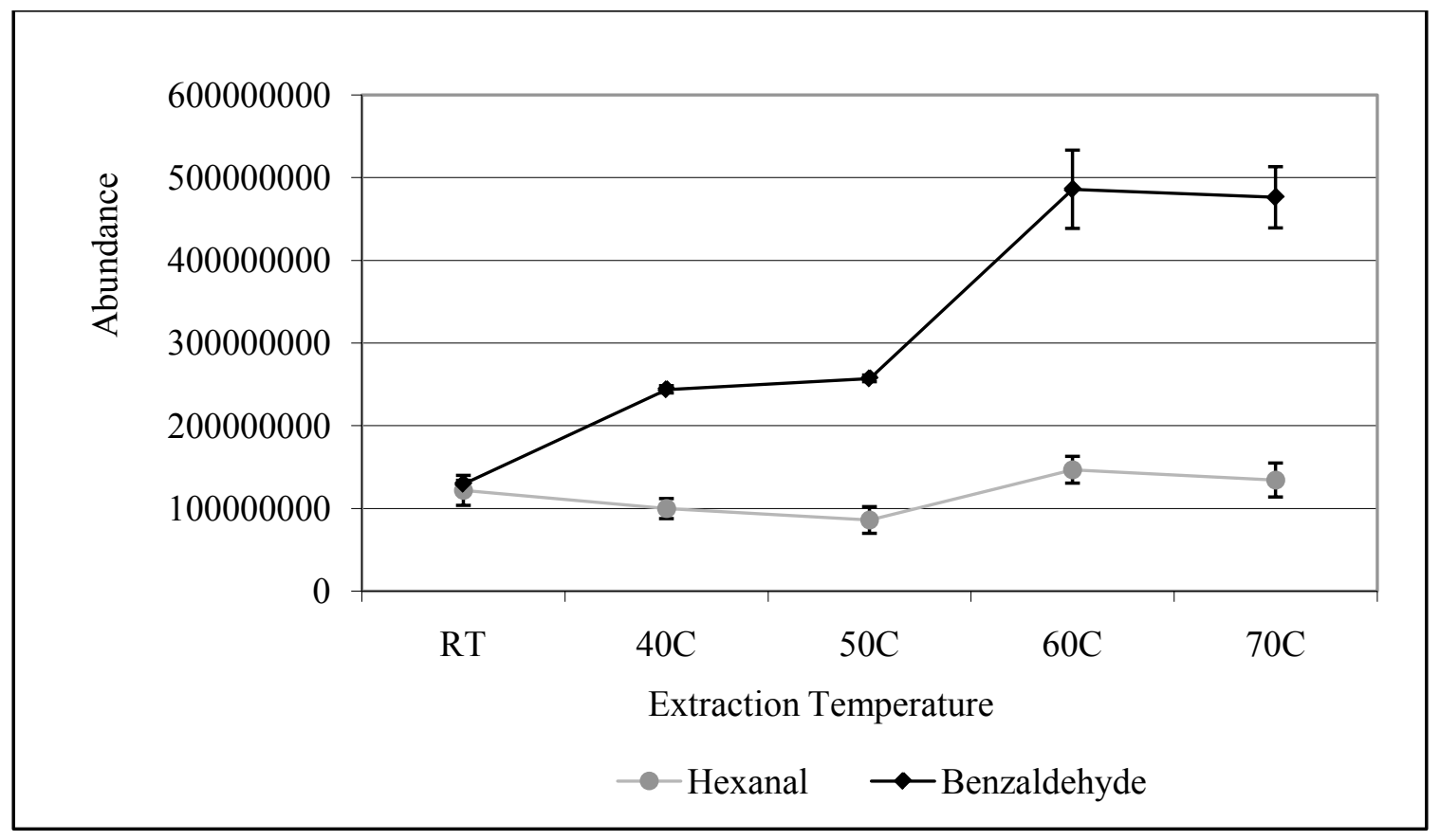


Figure 31. Abundance vs. extraction temperatures of acids for urine optimization

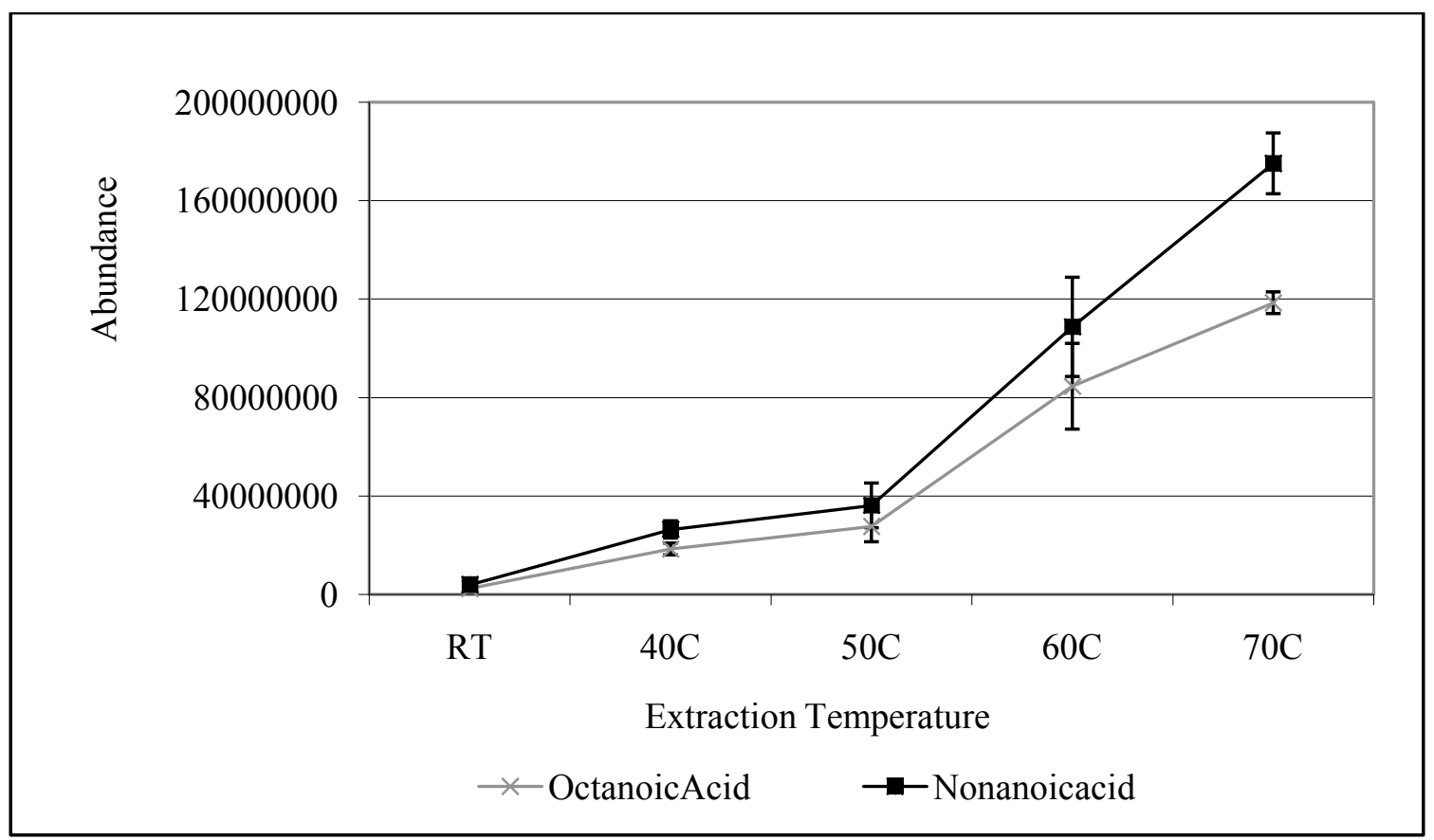

Figure 32. Abundance vs. extraction temperatures of alcohols for urine optimization

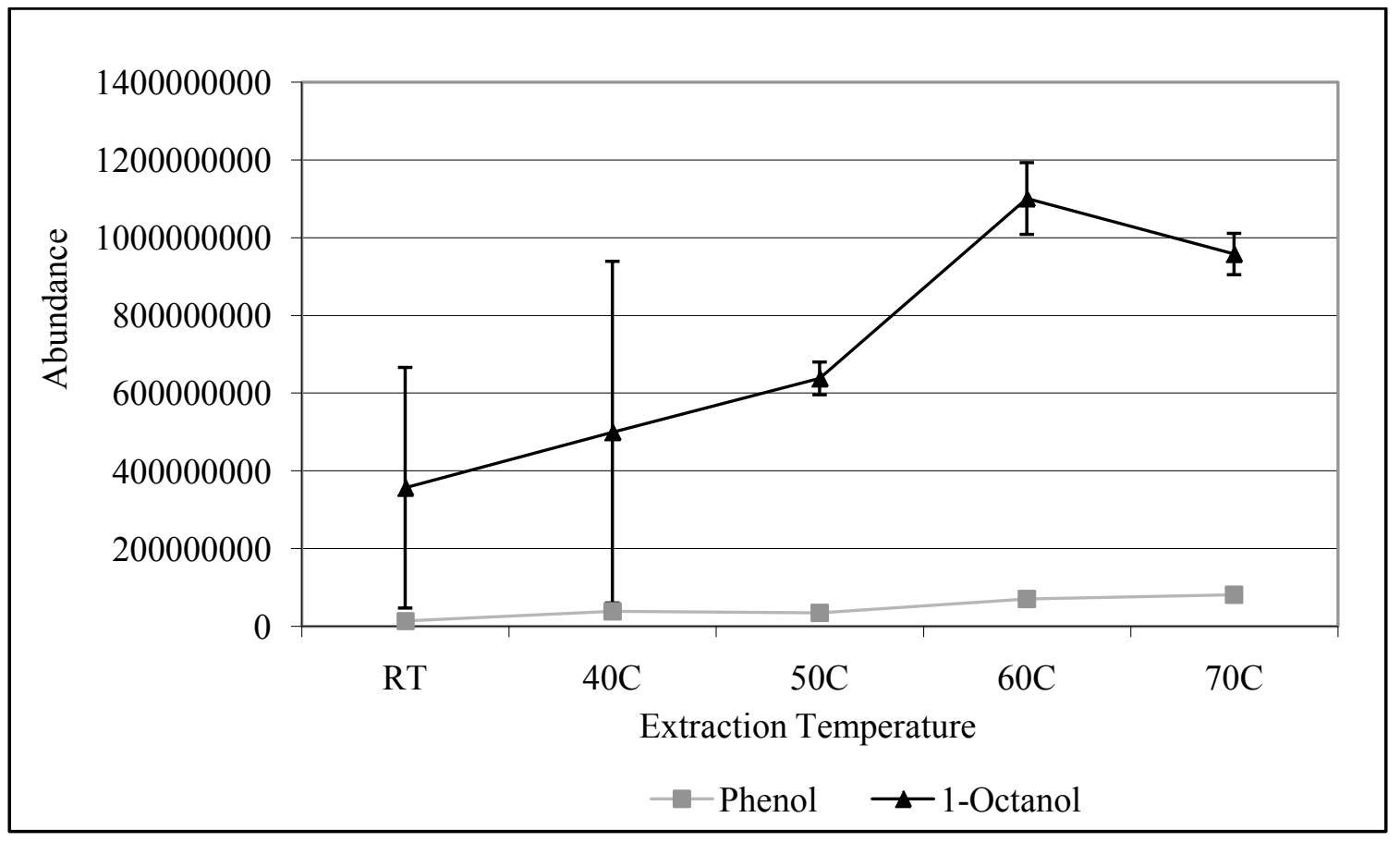


Figure 33. Abundance vs. extraction temperatures of ketones for urine optimization

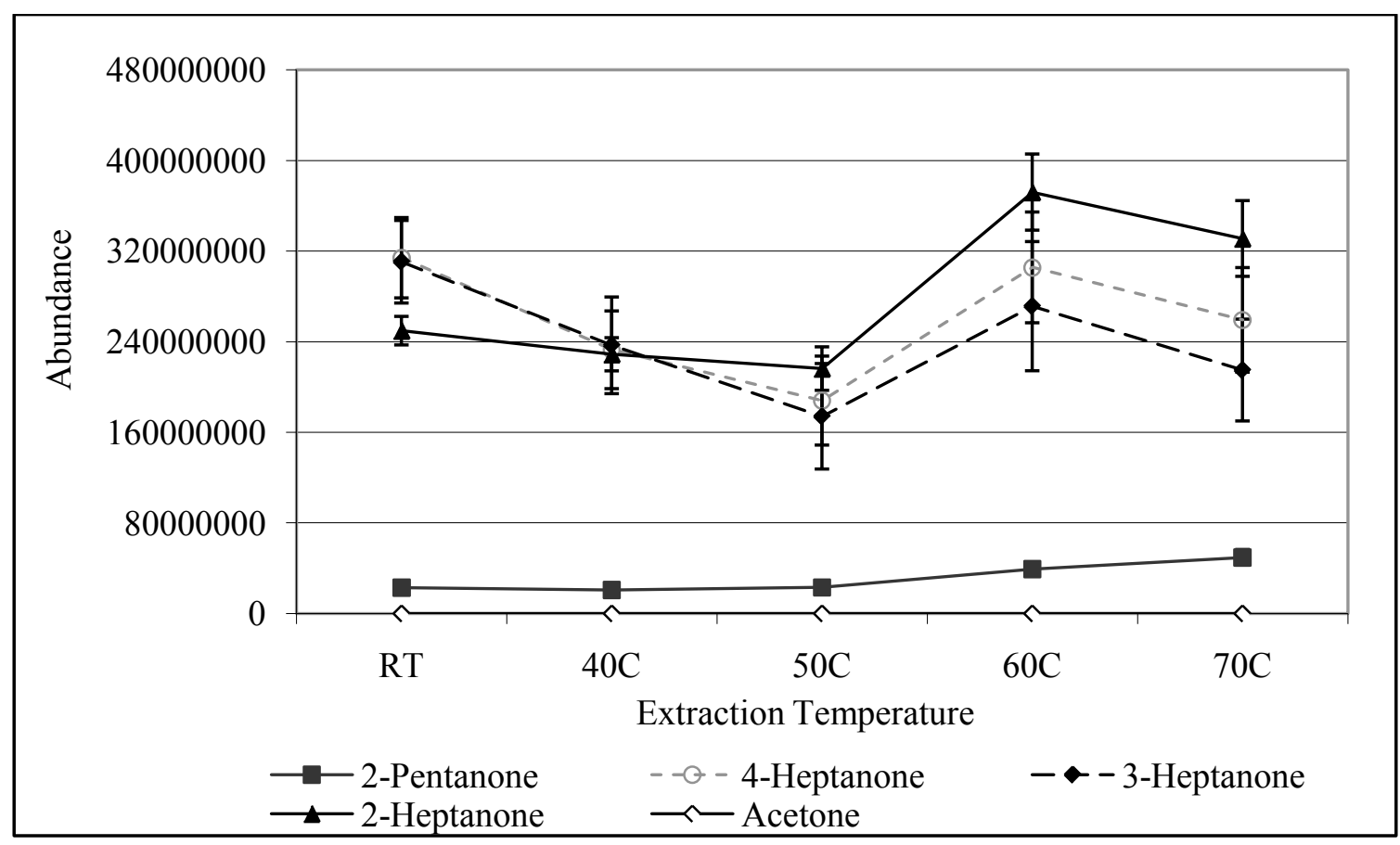

Figure 34. Abundance vs. extraction temperatures of other functional group compounds for urine optimization

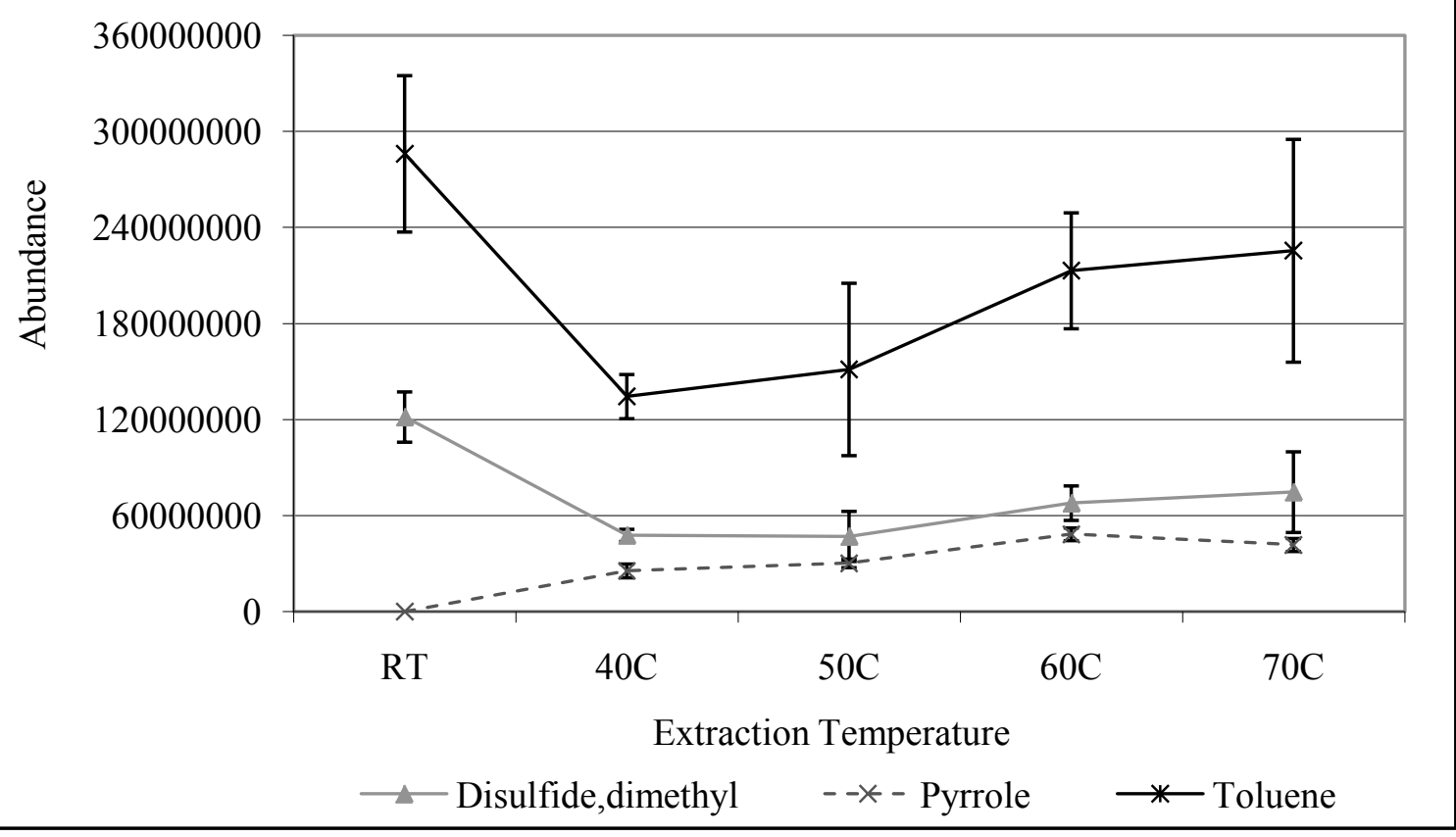




\subsection{Effect of Salting Out}

Five inorganic salts $\left(\mathrm{NaCl}, \mathrm{KCl}, \mathrm{MgSO}_{4}, \mathrm{~K}_{2} \mathrm{CO}_{3}\right.$, and $\left.\mathrm{Na}_{2} \mathrm{CO}_{3}\right)$ were tested for their salting-out abilities. Adding an inorganic salt to a sample solution improves extraction efficiency for volatile compounds in biological fluids. Salting out can be used not only to lower the detection limits, but also to buffer random salt concentration in body fluids. Salt (1.5g) was added to completely saturate the sample solution. Samples were equilibrated for five minutes and SPME fibers were exposed for 30 minutes in a $60^{\circ} \mathrm{C}$ hot water bath. As shown in Figure 35 through Figure 39, $\mathrm{NaCl}$ improved the extraction efficiency of the VOCs best out of the five inorganic salts investigated. No acids were detected with the addition of $\mathrm{K}_{2} \mathrm{CO}_{3}$ or $\mathrm{Na}_{2} \mathrm{CO}_{3}$ (Figure 36).

Figure 35. Effect of salt addition for urine optimization for aldehydes

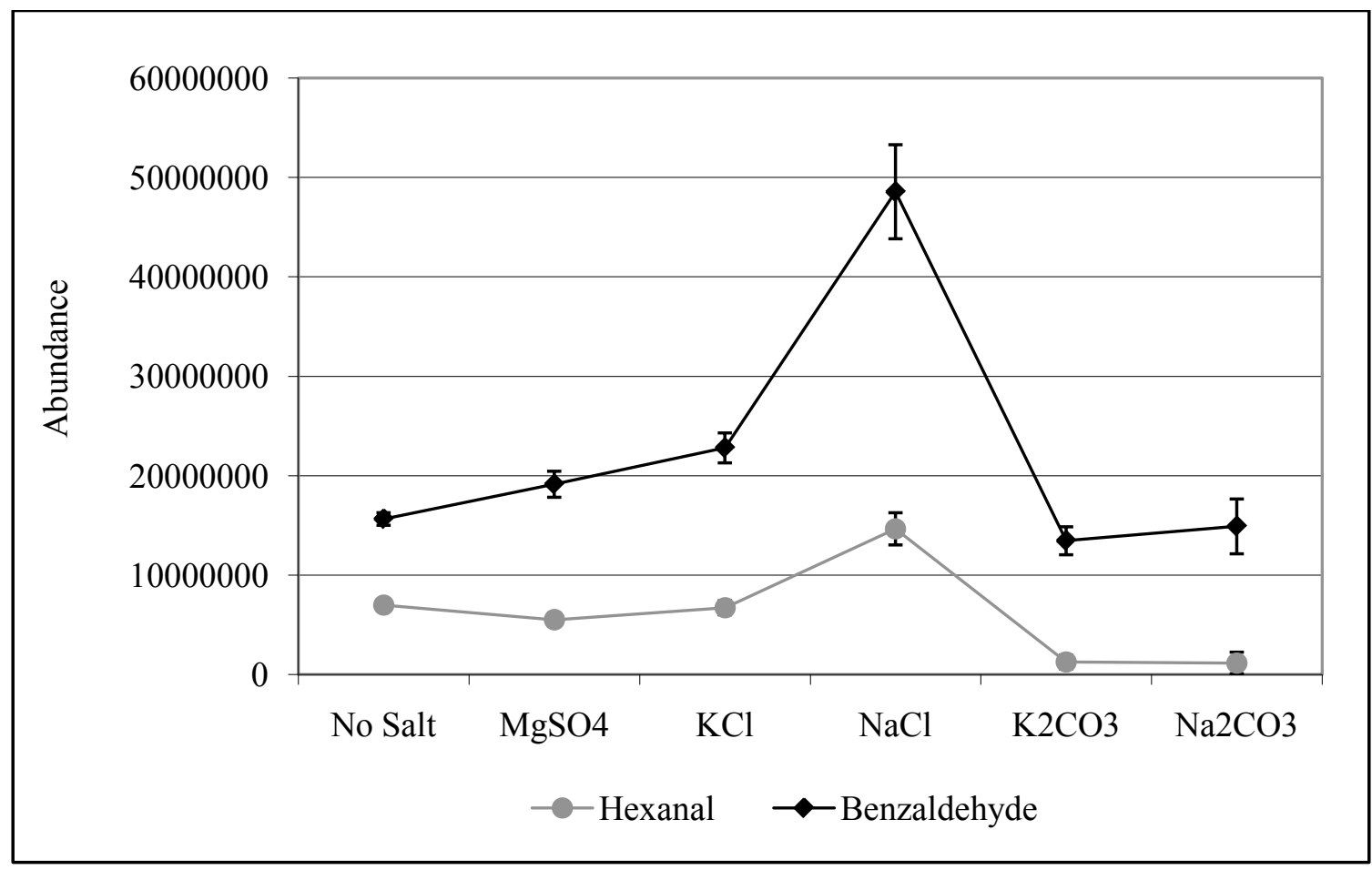


Figure 36. Effect of salt addition for urine optimization for acids

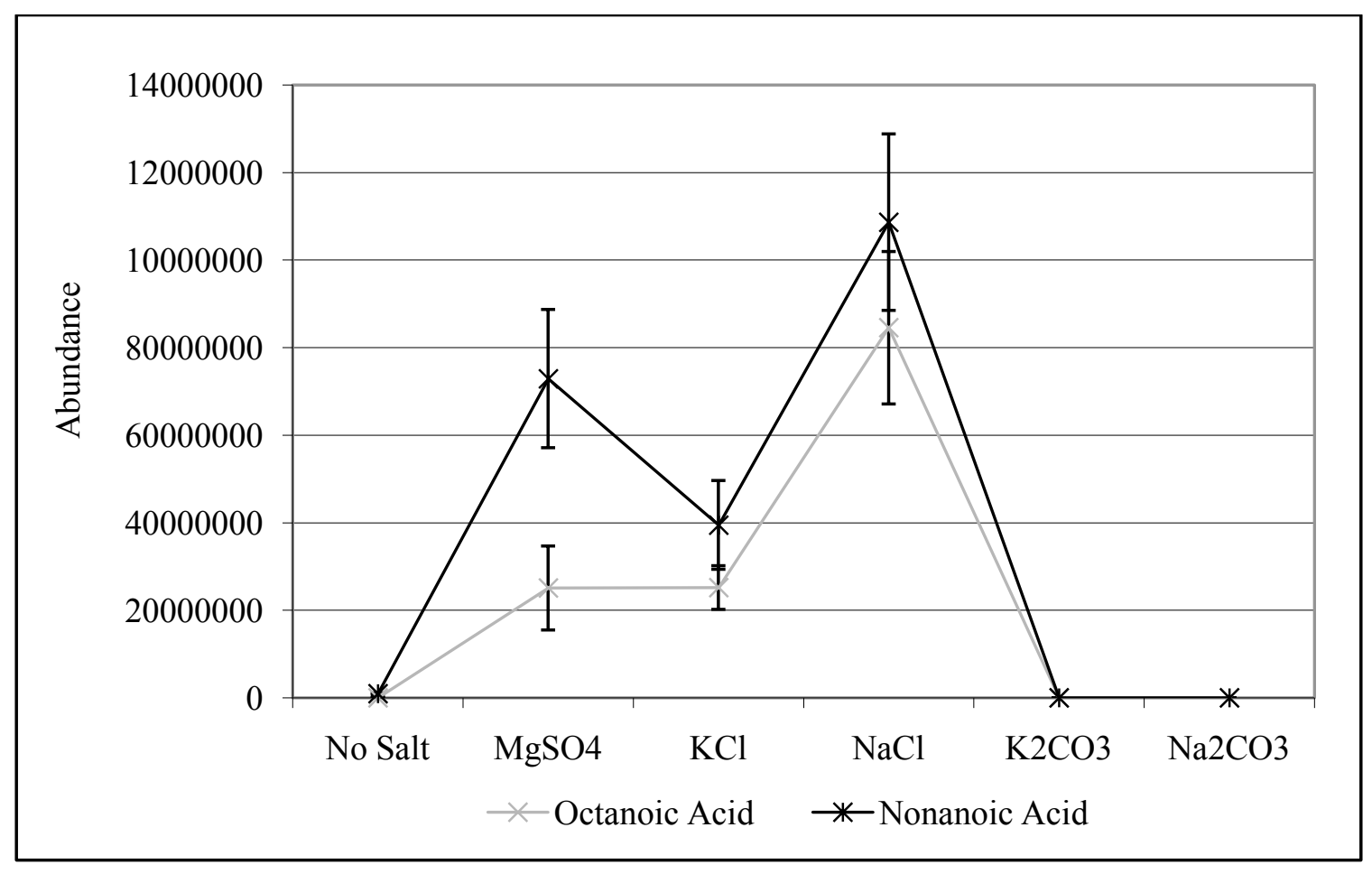

Figure 37. Effect of salt addition for urine optimization for alcohols

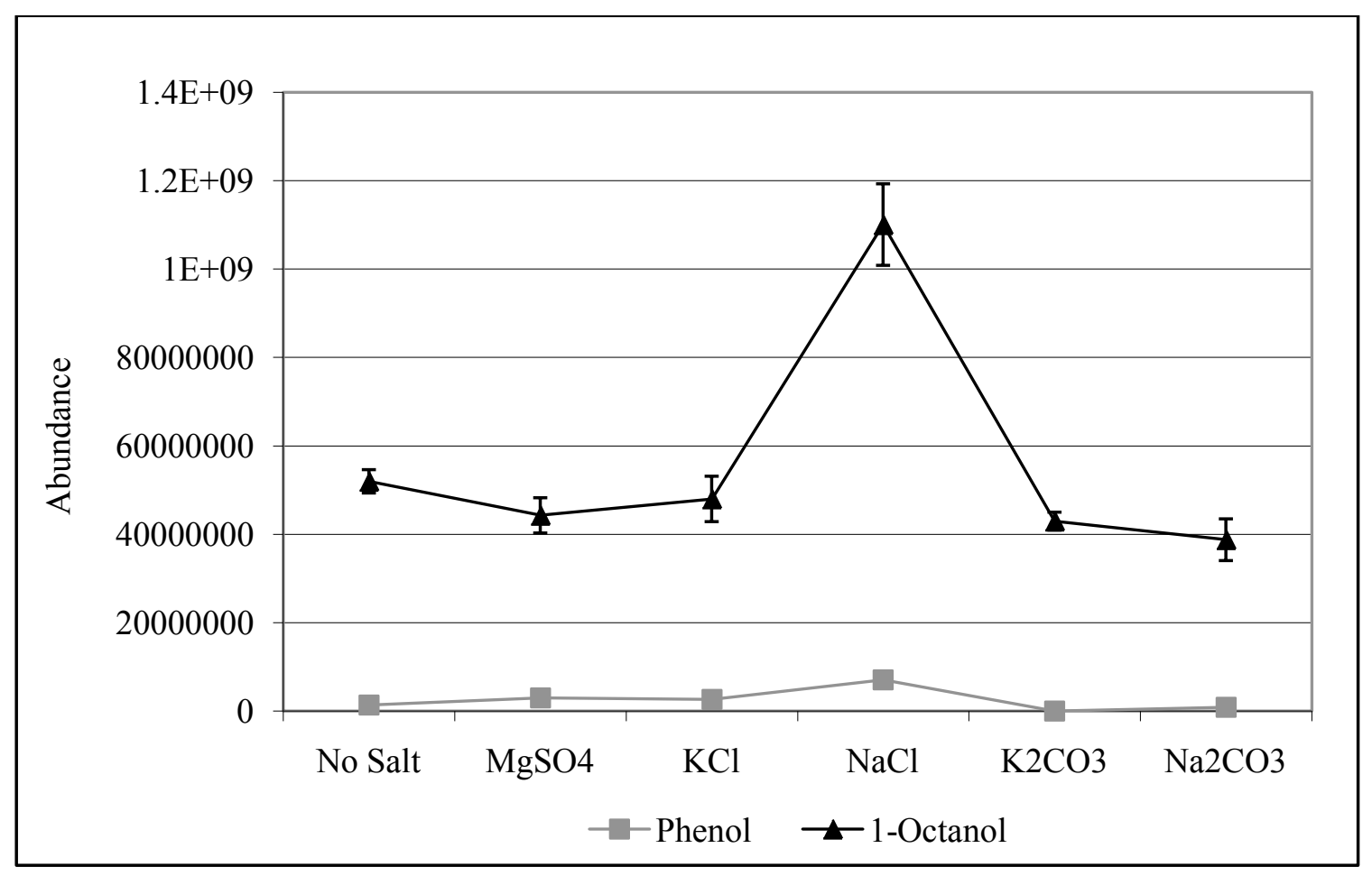


Figure 38. Effect of salt addition for urine optimization for ketones

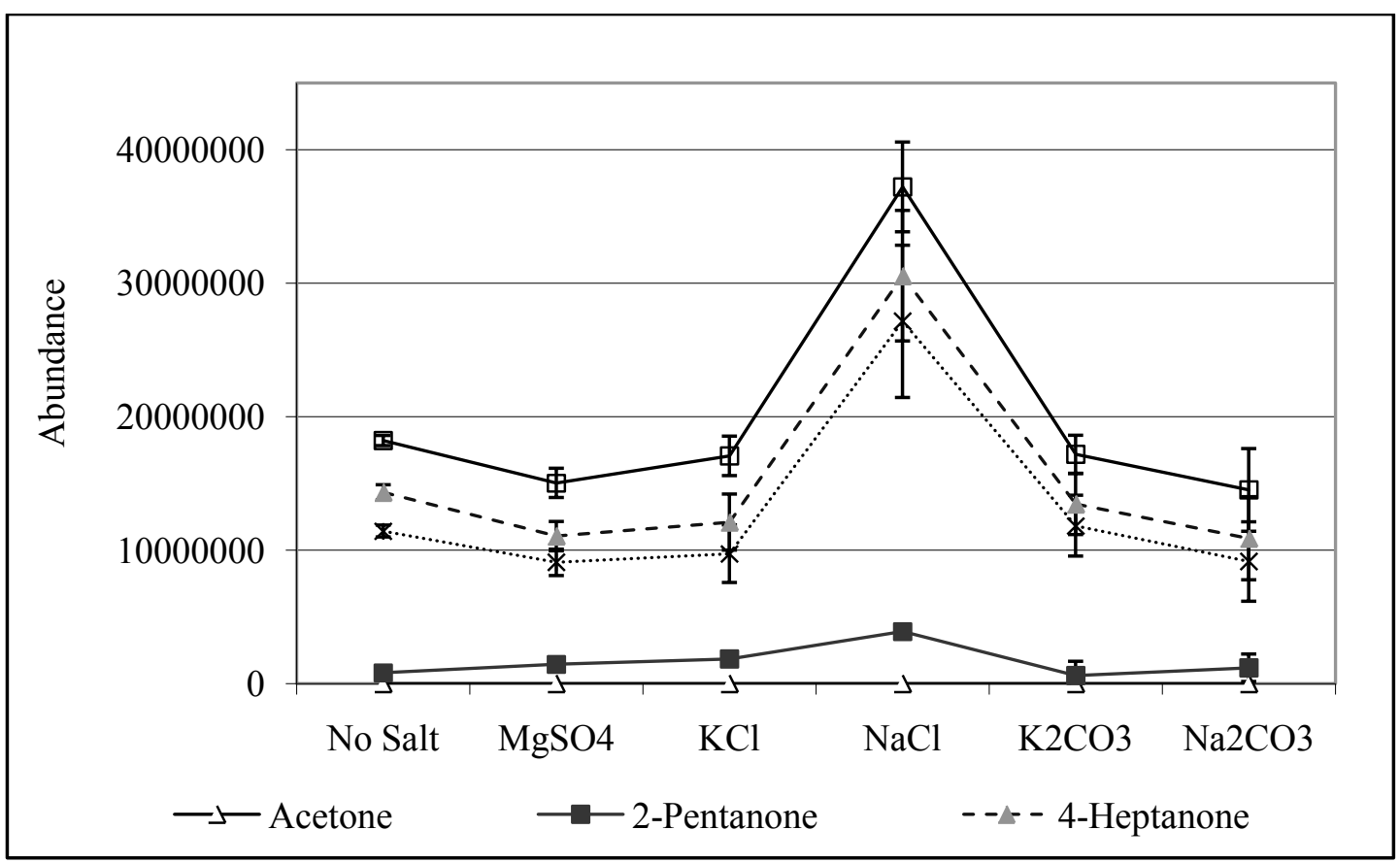

Figure 39. Effect of salt addition for urine optimization for other functional group compounds

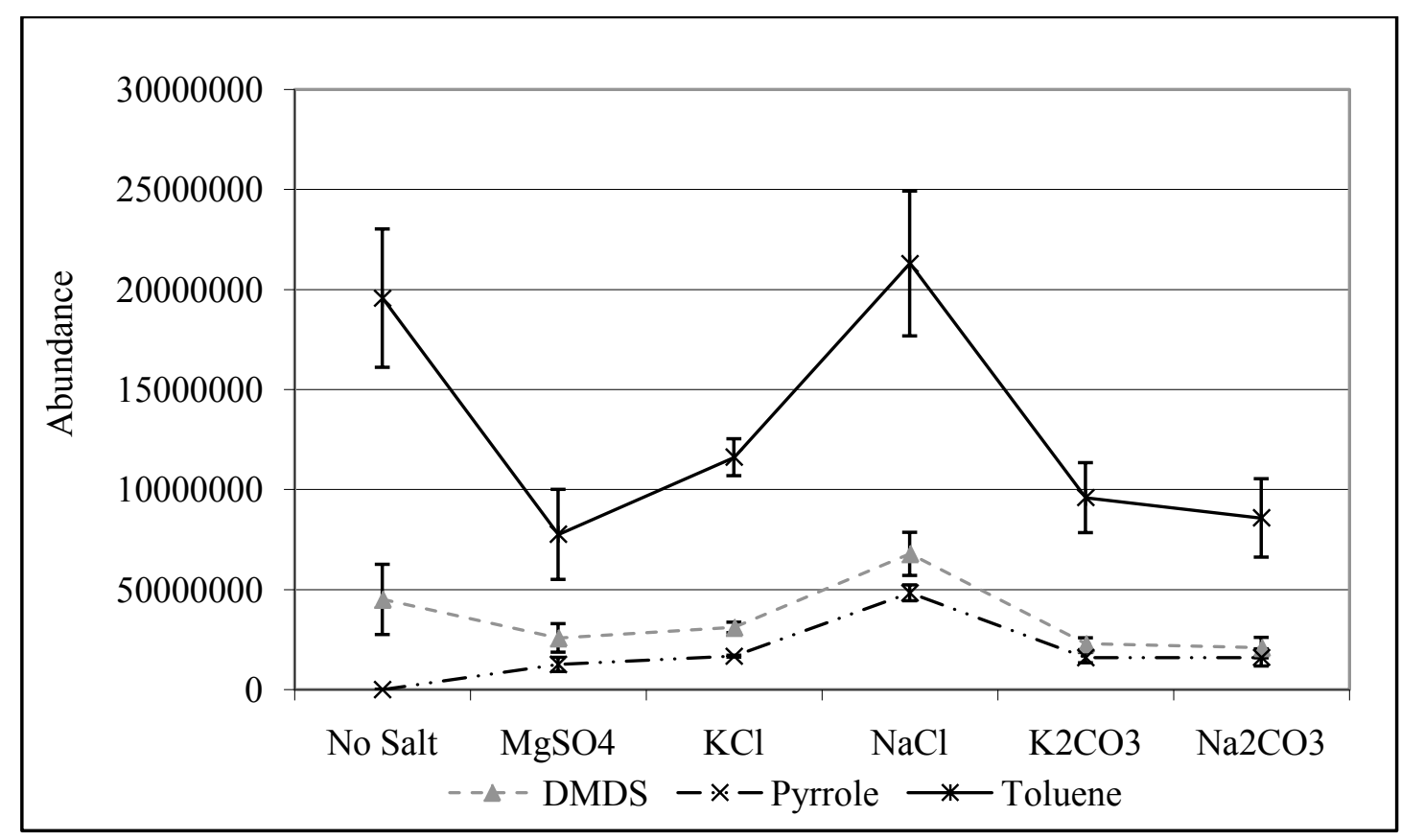




\subsection{Comparison of Five Specimens from Individuals}

\subsubsection{Methods}

Five biological specimen samples were collected from four subjects: two females (F2 and F4) and two males (M1 and M5) ranging in age from 27 to 31 years old. Each specimen sample was collected in duplicate (hand odor and breath) or triplicate (buccal swab, blood, and urine) and analyzed by the SPME-GC/MS methods mentioned in the preceding sections of this chapter and subjected to chromatographic and statistical analyses as described below to determine the similarities and differences in the VOCs extracted from the five samples. Hand odor, buccal swab, breath, and urine samples were collected using the specimen sampling procedures as described previously. Blood was obtained using venipuncture method from subjects F4, M1, and M5 in a BD Vacutainer

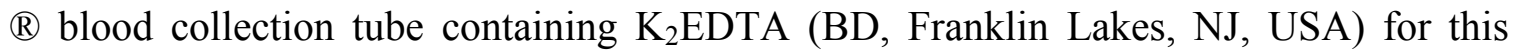
portion of study. Subject F2 was not able to give the blood sample, therefore human whole blood containing anticoagulant sodium EDTA (Bioreclamation Inc., Hicksville, NY, USA) was used in place of F2 blood sample for the VOC profile comparison of blood. Hand odor, buccal swab, and breath samples were stored at room temperature. Blood and urine samples were stored at $4^{\circ} \mathrm{C}$ until ready for use. The optimized conditions are summarized in Table 6. Extraction conditions for hand odor were determined previously by Curran ${ }^{84}$. All subsequent studies involving human subject samplings utilized the optimized SPME conditions established here. Methods for GC/MS analyses of samples from each specimen are described in the previous sections also. 
Table 6. Summary of optimized SPME conditions for biological specimens

\begin{tabular}{|l|l|l|l|l|l|}
\hline & $\begin{array}{l}\text { Hand } \\
\text { Odor }\end{array}$ & $\begin{array}{l}\text { Buccal } \\
\text { Swab }\end{array}$ & Breath & Blood & Urine \\
\hline Equilibration Time & 24 hours & 24 hours & 15 minutes & 1 hour & 5 minutes \\
\hline Extraction Time & 21 hours & 21 hours & 21 hours & 18 hours & 30 minutes \\
\hline Extraction Temperature & RT & RT & RT & $37^{\circ} \mathrm{C}$ & $60^{\circ} \mathrm{C}$ \\
\hline Other & -- & -- & -- & -- & $\mathrm{NaCl}$ \\
\hline
\end{tabular}

\subsubsection{Results}

Using the optimized sample collection and SMPE conditions, preliminary human subject odor profiles were compared for the five biological specimens prior to larger population sampling. Examples of a typical chromatogram for each specimen are shown in Figure 40. Specimen samples were compared among four subjects (two female subjects F2 and F4, two male subjects M1 and M5). Figure 41 through Figure 44 show the chromatogram expanded and siloxane peaks removed to compare the odor profiles produced from the four subjects (F4, F2, M1, and M5) for the four specimens (buccal swab, breath, blood, and urine). As seen from these figures, qualitatively there are commonalities in the compounds present in the headspace of the collected samples from different individuals. Figure 45 shows the comparison of the relative peak area ratios for the common VOCs present in triplicate samples of buccal swabs collected from the four subjects. Figure 45 also demonstrates the reproducibility between samples collected from the same individual. For buccal swab samples, hexanoic acid and decanal were common to all subjects; however, the ratios of all compounds demonstrate unique overall VOC profiles per individual. 
Figure 40. Typical odor profile chromatogram for a) hand odor, b) buccal swab, c) breath, d) blood, and e) urine

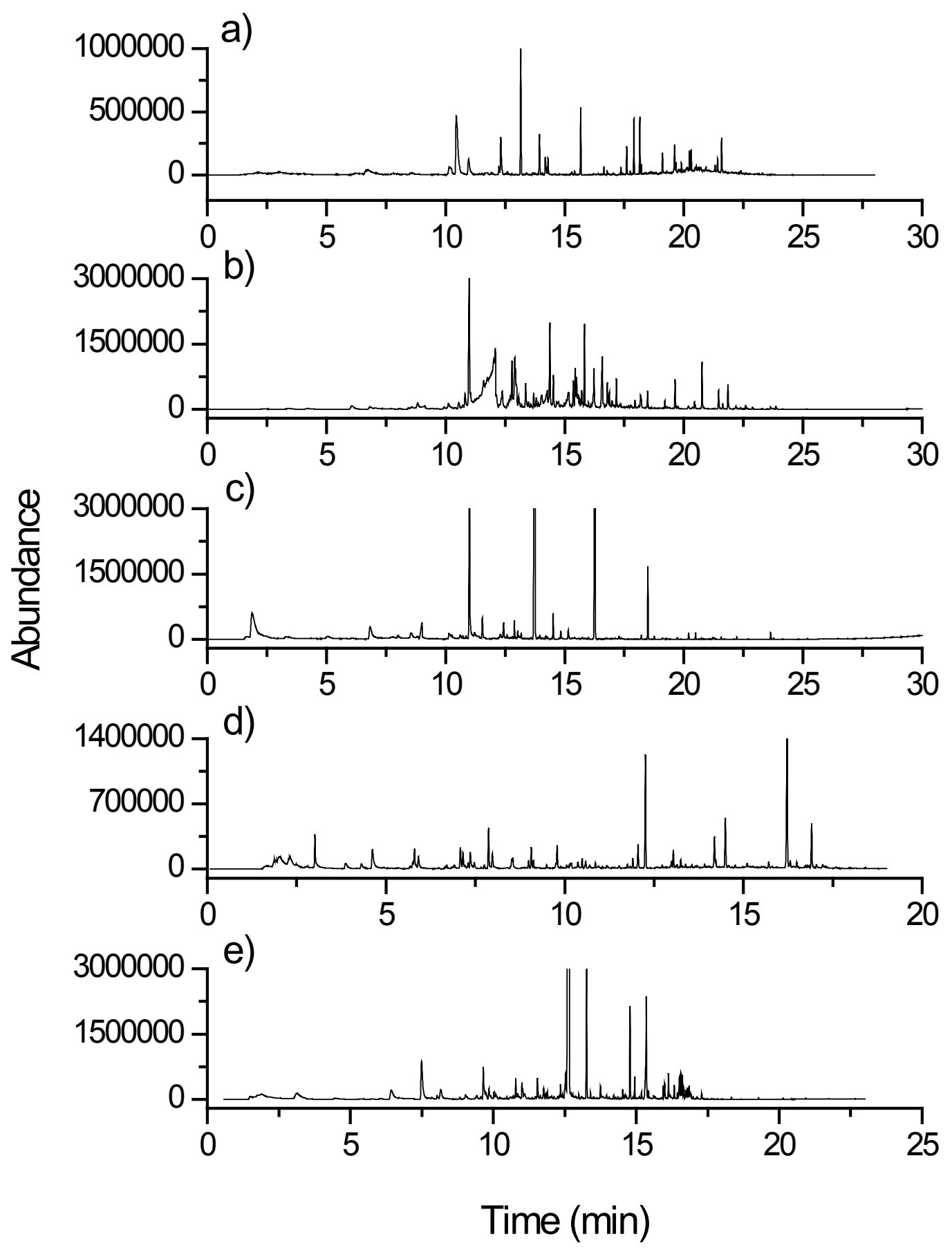


Figure 41. Comparison of buccal swab odor profiles from subjects F4, F2, M1, and M5

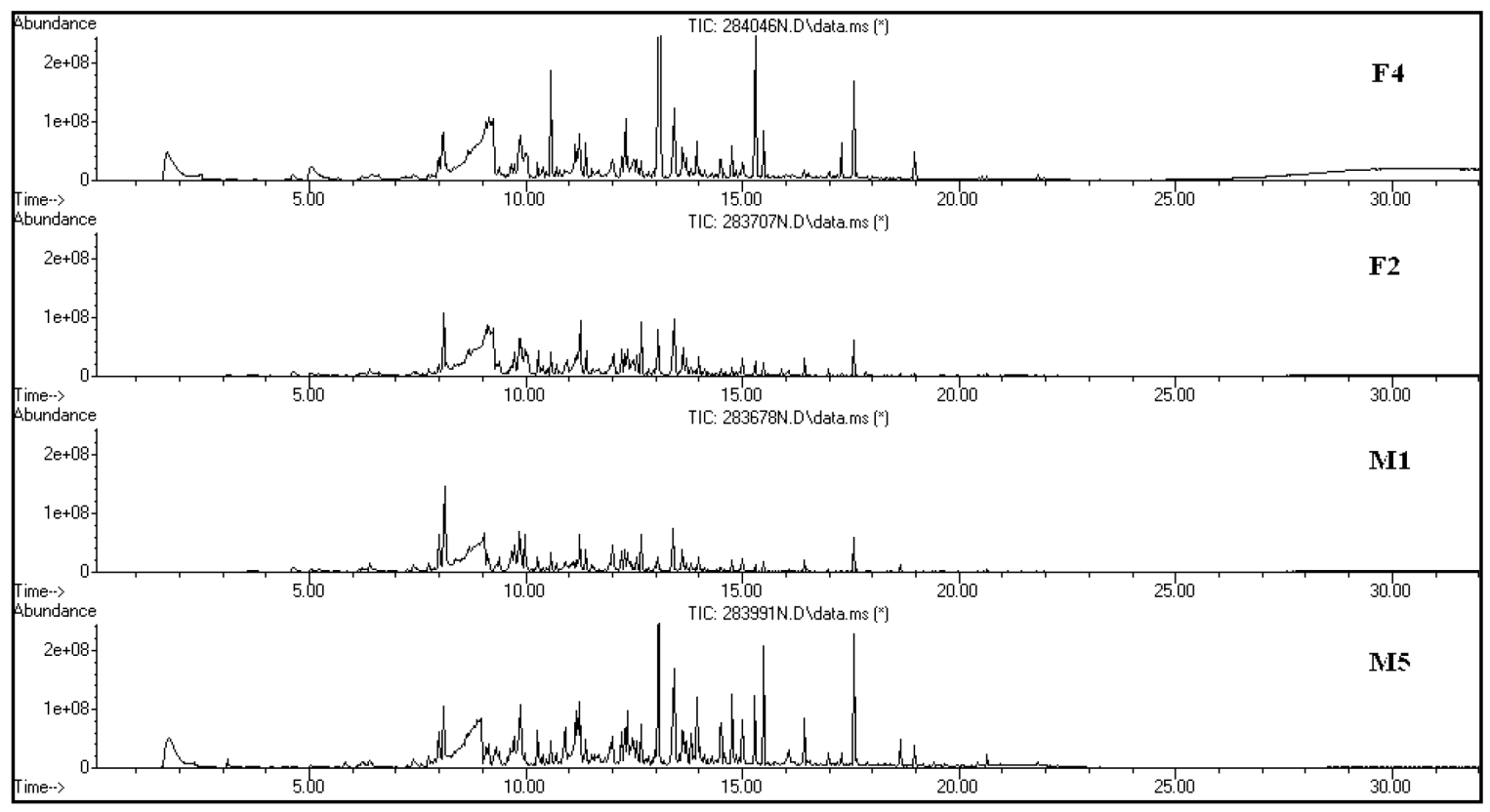

Figure 42. Comparison of breath odor profiles from subjects F4, F2, M1, and M5

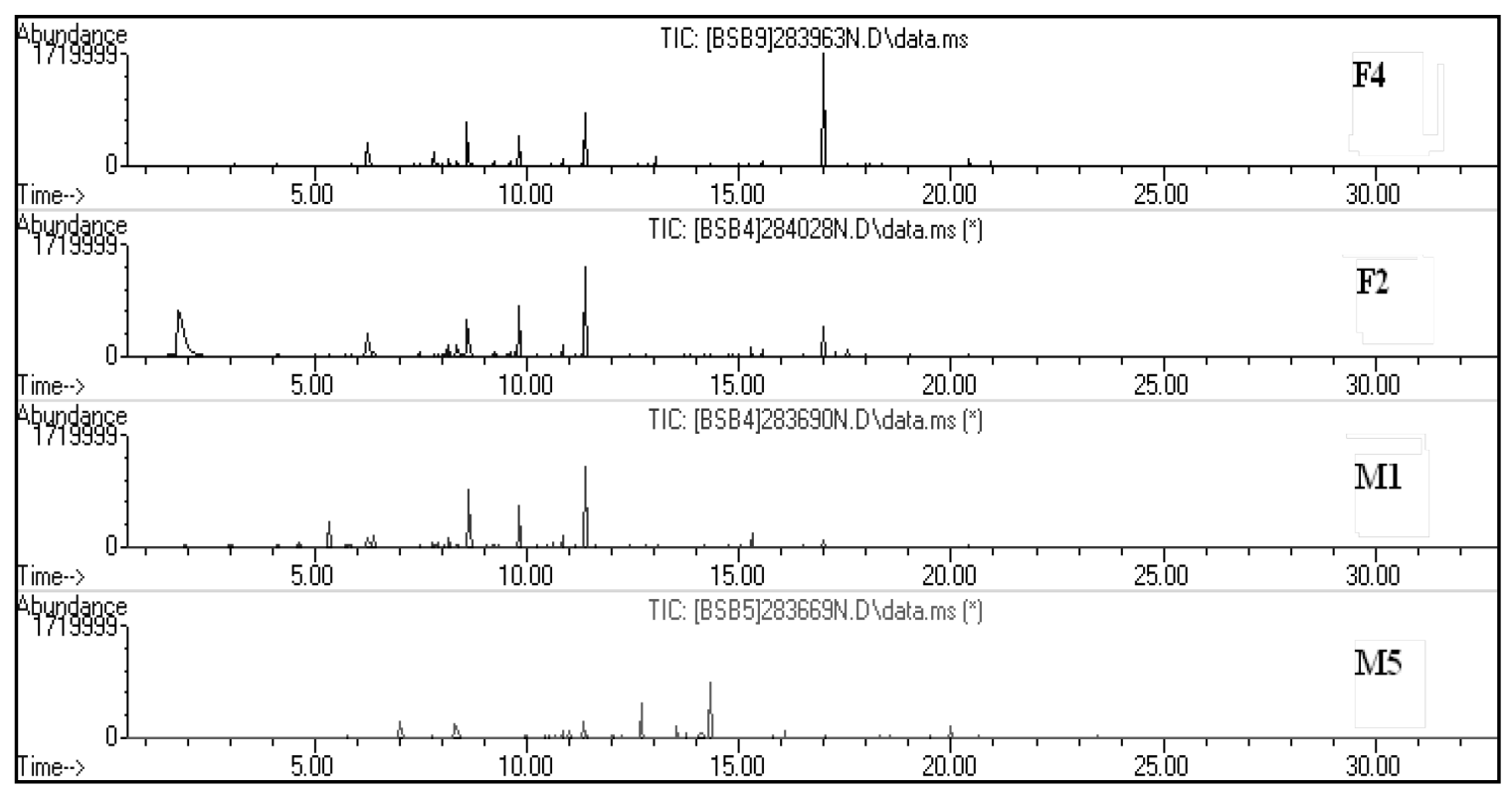


Figure 43. Comparison of blood odor profiles from subjects F4, F2, M1, and M5

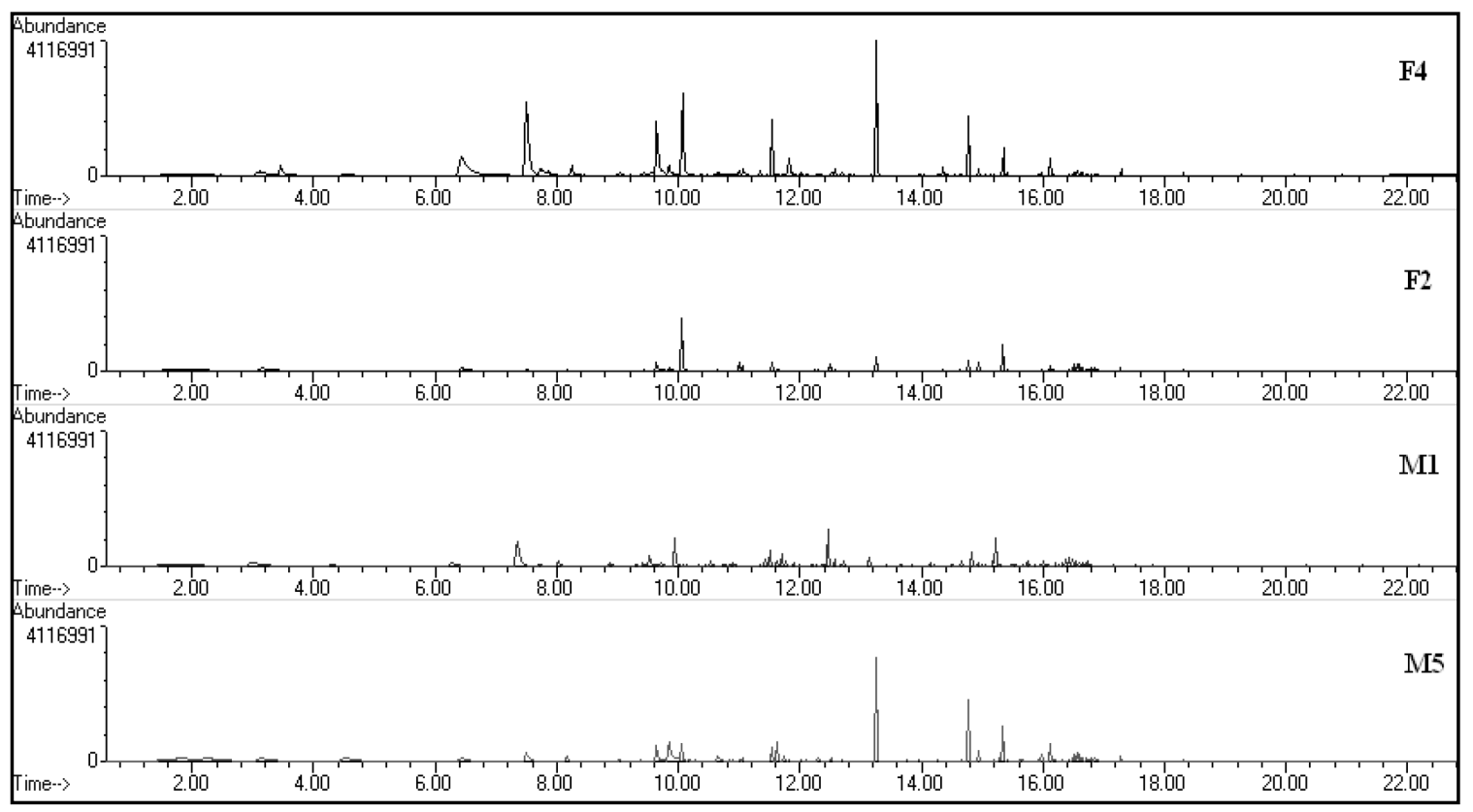

Figure 44. Comparison of urine odor profiles from subjects F4, F2, M1, and M5

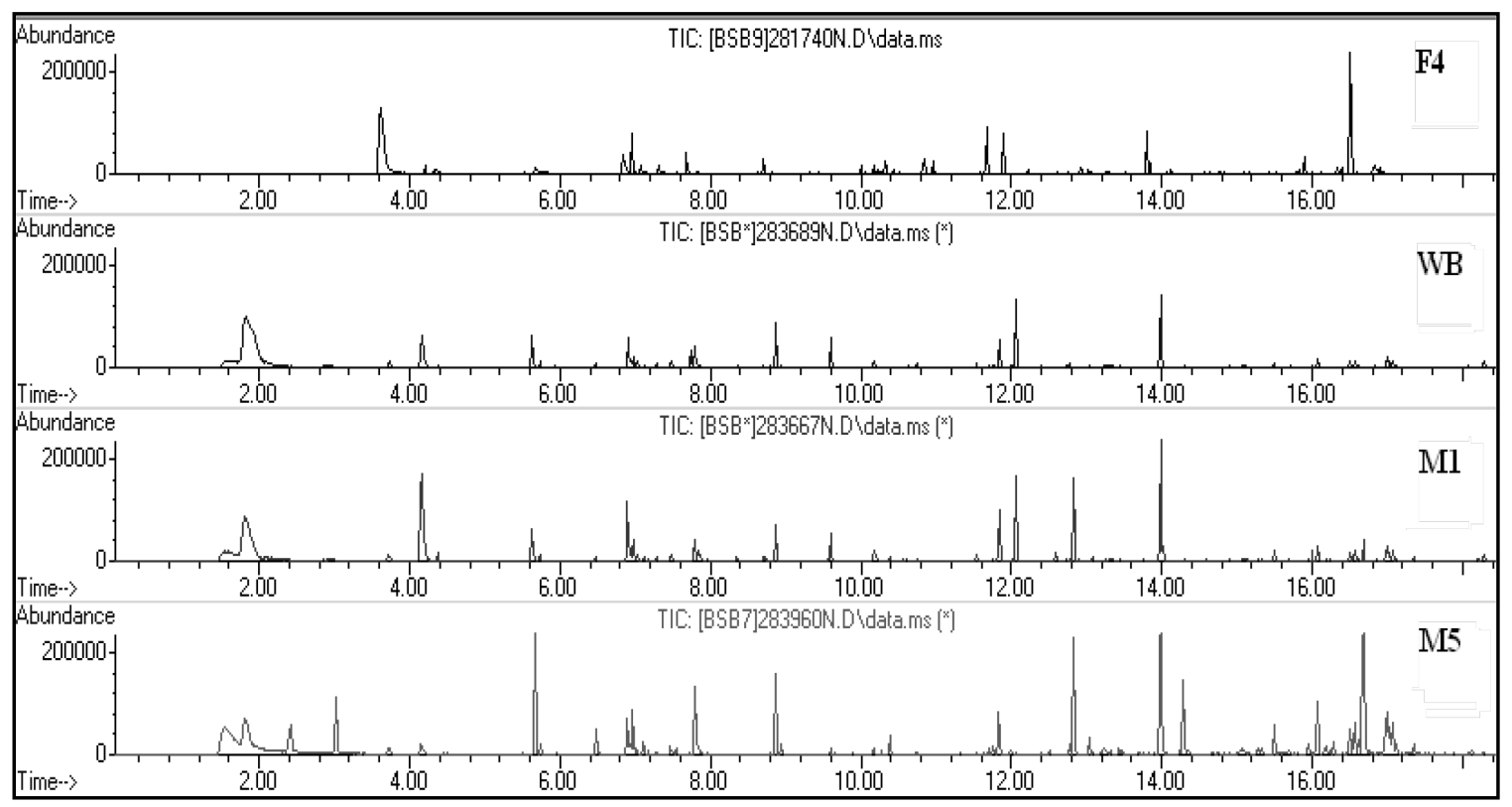


Figure 45. Buccal swab VOC profile comparison between 4 subjects

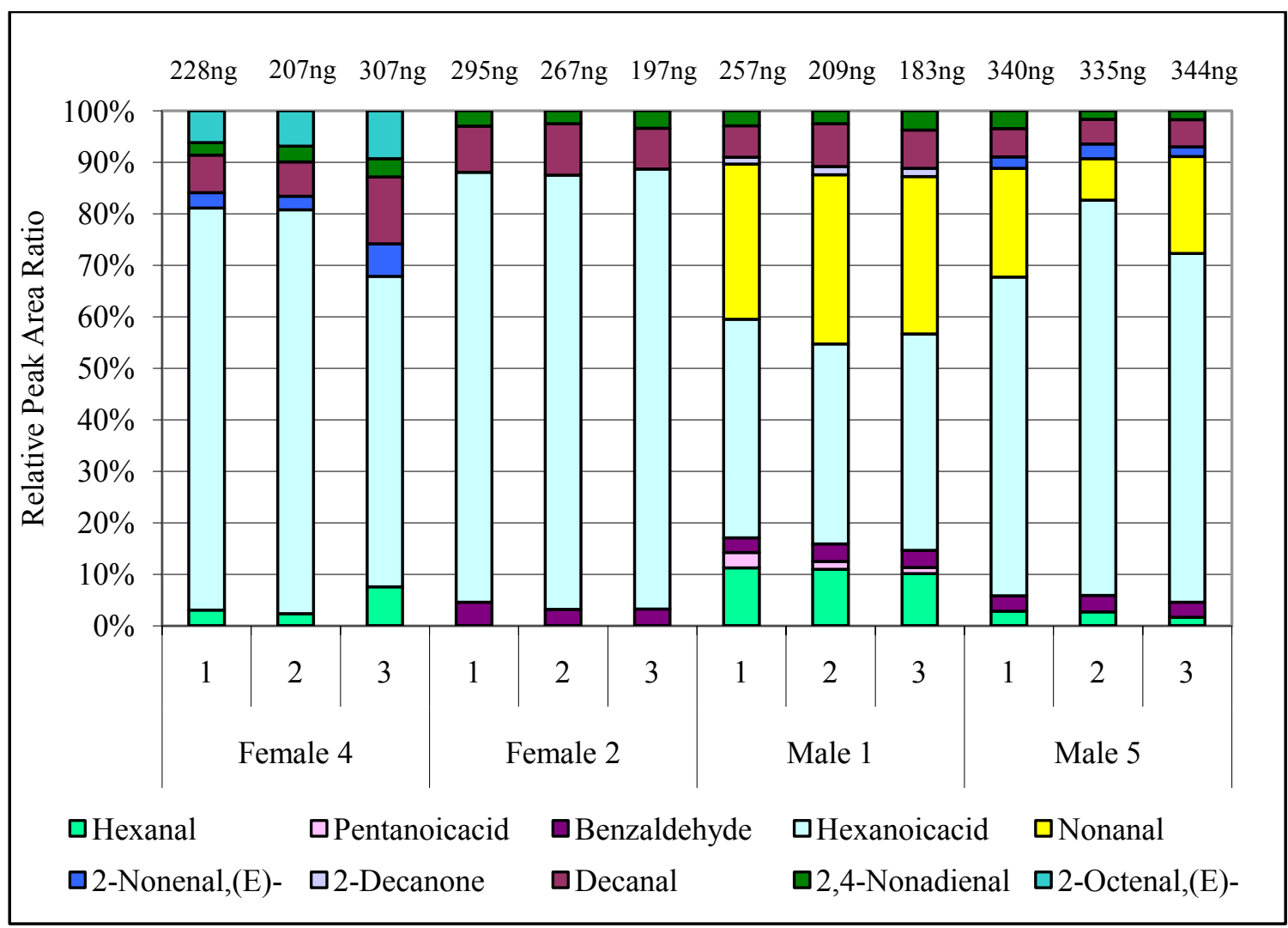

Figure 46 through Figure 48 show the comparisons of the VOC profiles for breath, blood, and urine for the four subjects compared side by side. For breath, acetone, nonanal, decanal, and 2-ethyl-1-hexanol were common to all subjects, but in varying ratios (Figure 46). Blood was collected from subjects Female 4, Male 1, and Male 5 in a BD Vacutainer ${ }^{\circledR}$ blood collection tube containing $\mathrm{K}_{2}$ EDTA. The blood VOC profiles were compared along with the whole blood (WB) used during the optimization studies. Compounds common to all blood samples were undecane, nonanal, dodecane, tridecane, and tetradecane (Figure 47). For urine, only 4-heptanone was present in all subject samples, and was also the predominant VOC in urine (Figure 48). For each specimen, reproducibility of VOC profiles was demonstrated between the multiple samples 
collected from the same individual. Compounds that were present in all four subjects varied in the ratios of the compounds present in the profile, demonstrating the individuality of the overall VOC profiles per individual.

Figure 46. Subject comparison of breath VOC profiles from subjects F4, F2, M1, and M5

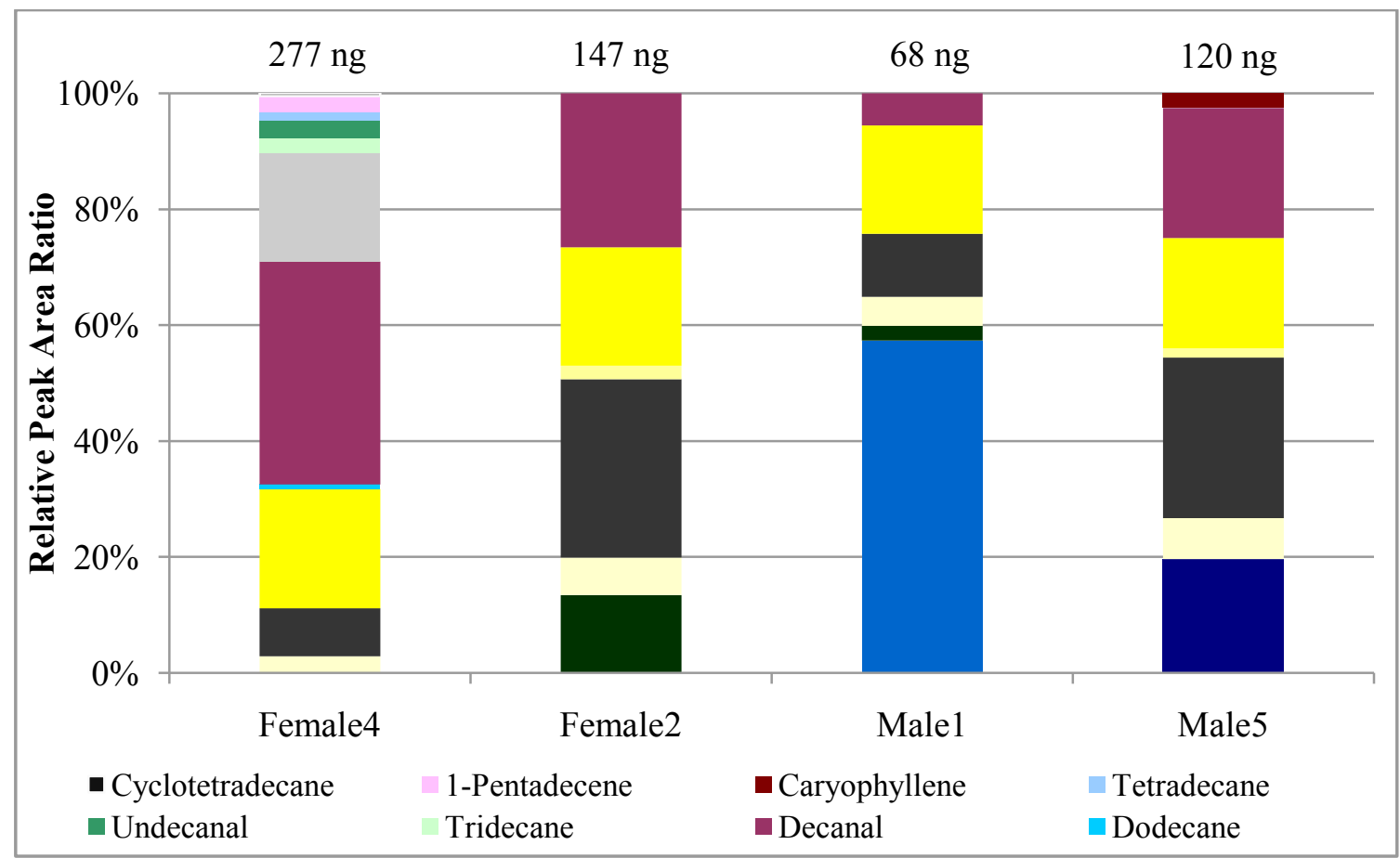


Figure 47. Subject comparison of blood VOC profiles from subjects F4, WB, M1, and M5

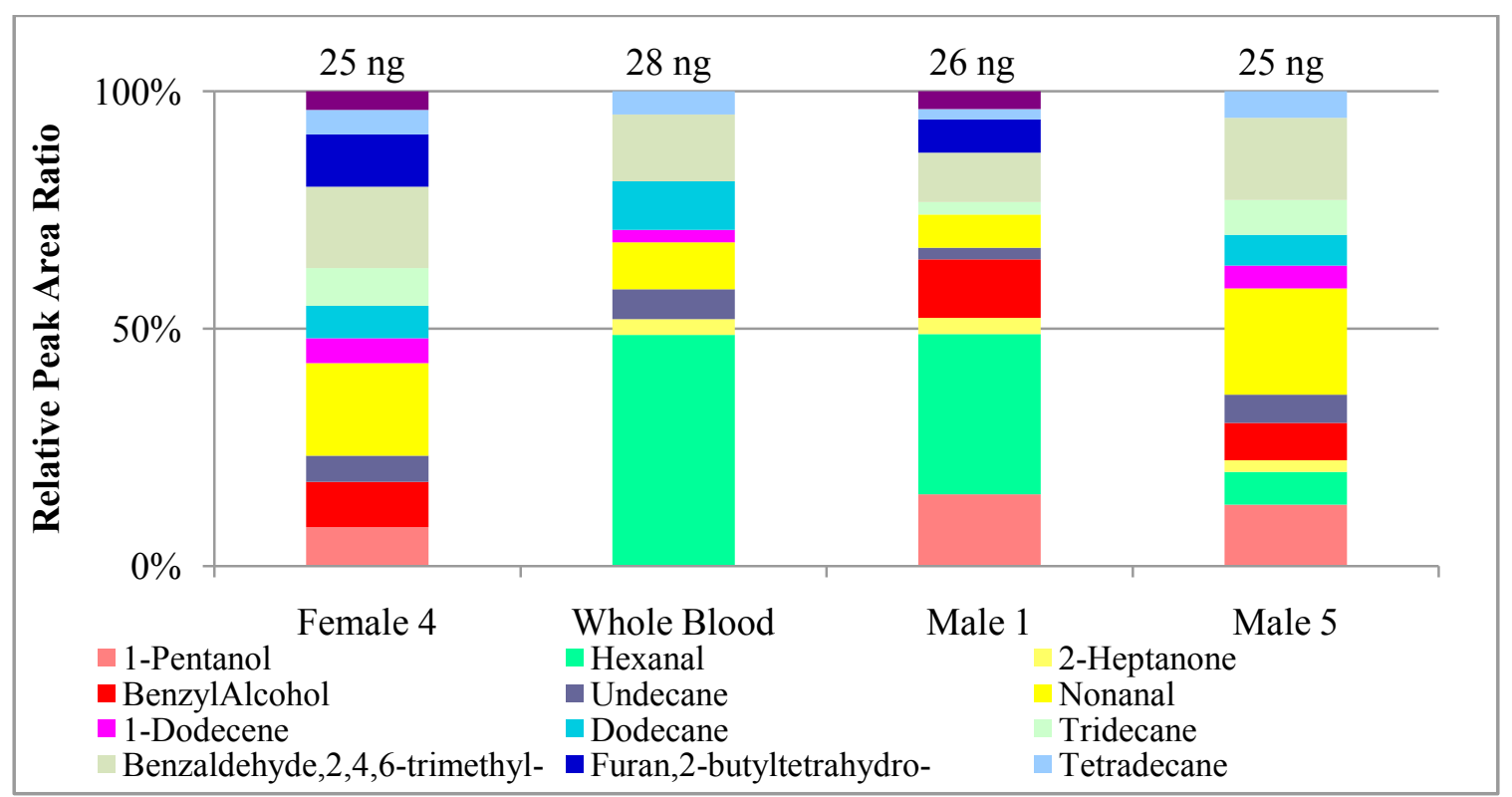

Figure 48. Subject comparison of urine VOC profiles from subjects F4, F2, M1, and M5

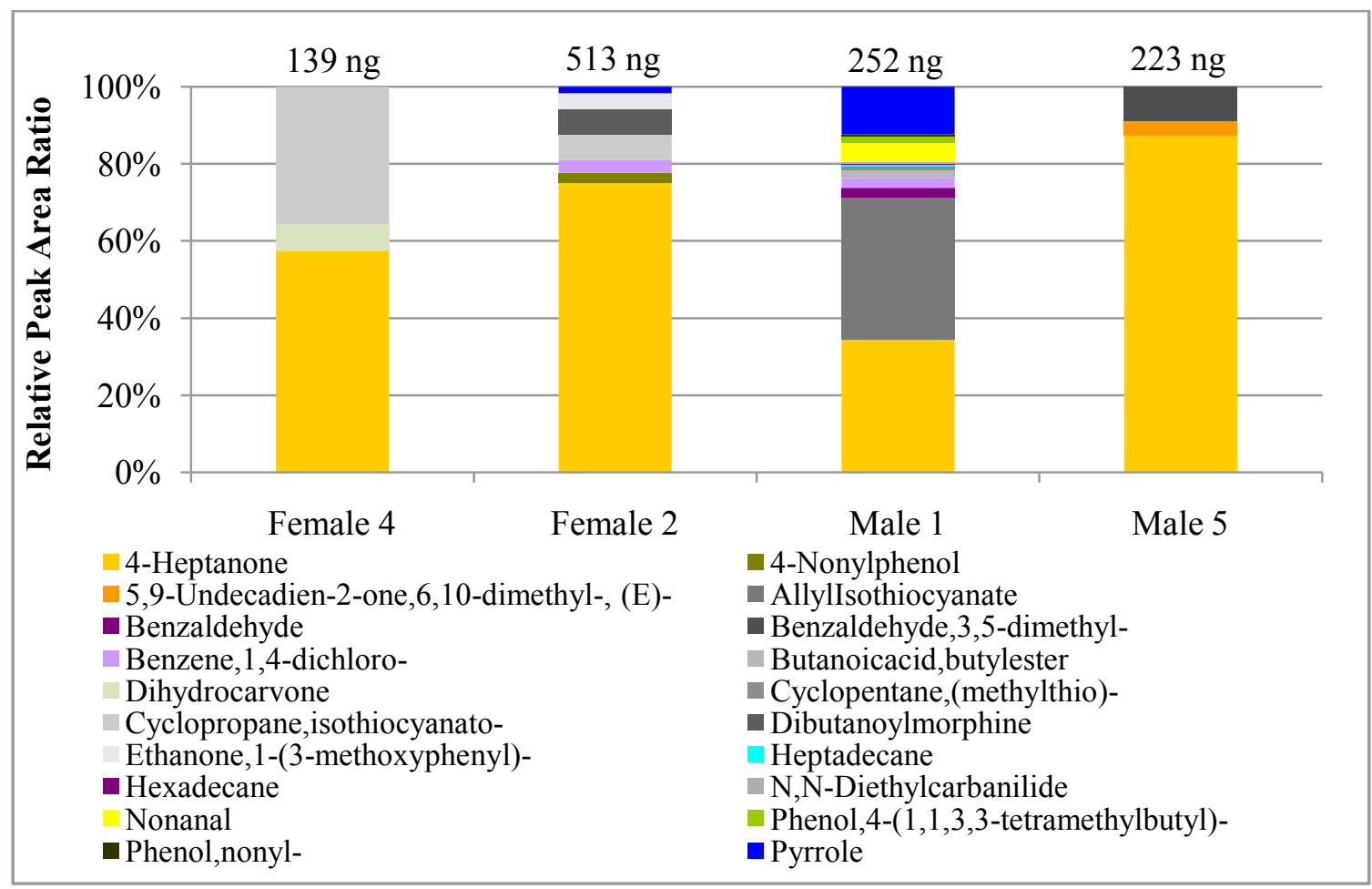




\subsubsection{Spearman Correlation Ranking}

Spearman's rank correlation is a statistical technique that is used to test the statistical dependence, in terms of direction and strength of the relationships, between two variables $^{85}$. The Spearman rank correlation coefficient method is a nonparametric, or distribution-free technique, that allows for ranked data to be compared to determine the correlation between the variables. The null hypothesis is that there is no relationship between the variables being compared. The Spearman correlation coefficient ranges from -1 to +1 . The Spearman rank correlation coefficient is defined by the following equation:

$$
r_{s}=1-\frac{6 \sum d^{2}}{n\left(n^{2}-1\right)} \quad \text { Equation } 2
$$

The correlation coefficient, $r_{s}$, is determined in terms of the difference between the ranked compounds, $d$, and the number of compounds being compared, $n$. The Spearman rank correlation determined the possible correlation between the chemical odor profile (set of VOCs) of a single individual or a single specimen with a population or other specimens $^{13}$.

Spearman correlation coefficient comparisons were conducted utilizing the VOCs detected in the headspace of the four specimens investigated in this study. Using a Spearman correlation macro developed in-house ${ }^{13}$, analysis of specimen profiles from subjects demonstrated that intra-subject samples have a high correlation (correlation coefficient $>0.9$ ) while inter-subject samples comparison shows a low correlation (correlation coefficient $<0.6$ ), reiterating the individuality of odor profiles characteristic for each subject (Figure 49). Similarly, Spearman rank correlation comparisons for breath 
(Figure 50), blood (Figure 51), and urine (Figure 52) were performed and showed similar results as that for buccal swab samples shown above. High correlation (correlation coefficient $>0.9$ ) was seen between the samples from the same subject, while low correlation (correlation coefficient $\leq 0.6$ ) was seen between different sub jects, reiterating the individuality of odor profiles for each subject.

Figure 49. Spearman rank correlation with respect to Female 4, sample 1 for buccal swab

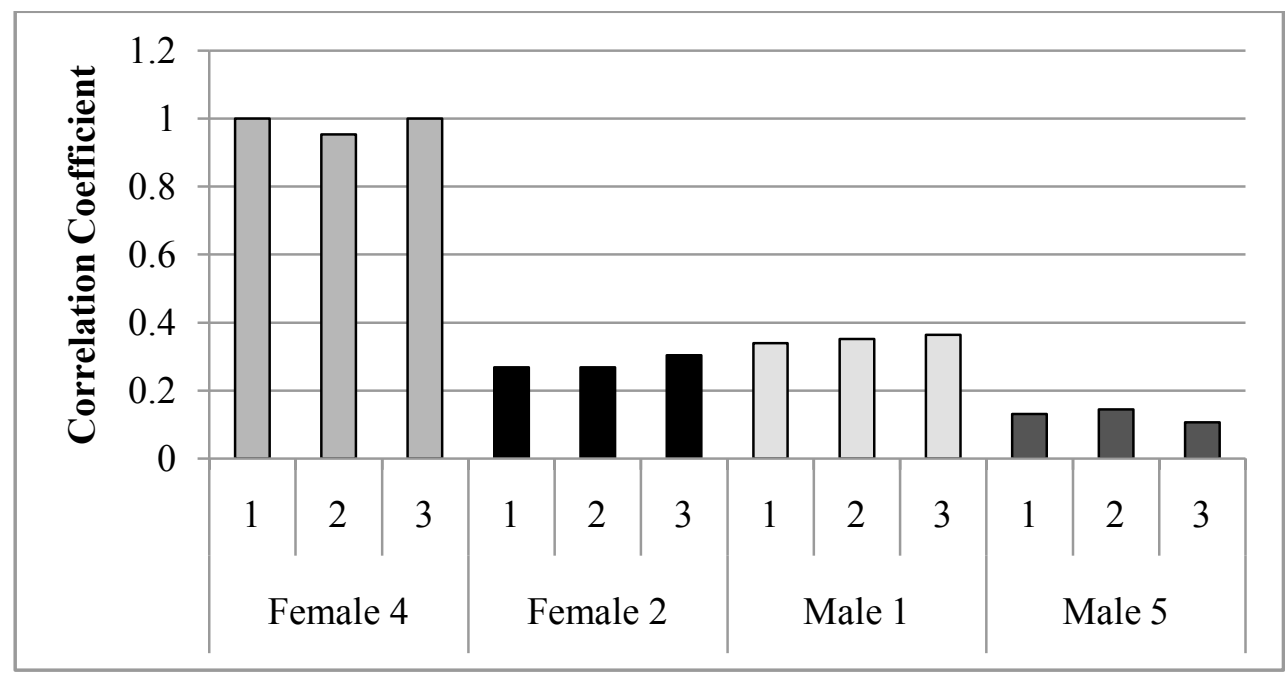

Figure 50. Spearman rank correlation with respect to Female 2, sample 1 for breath

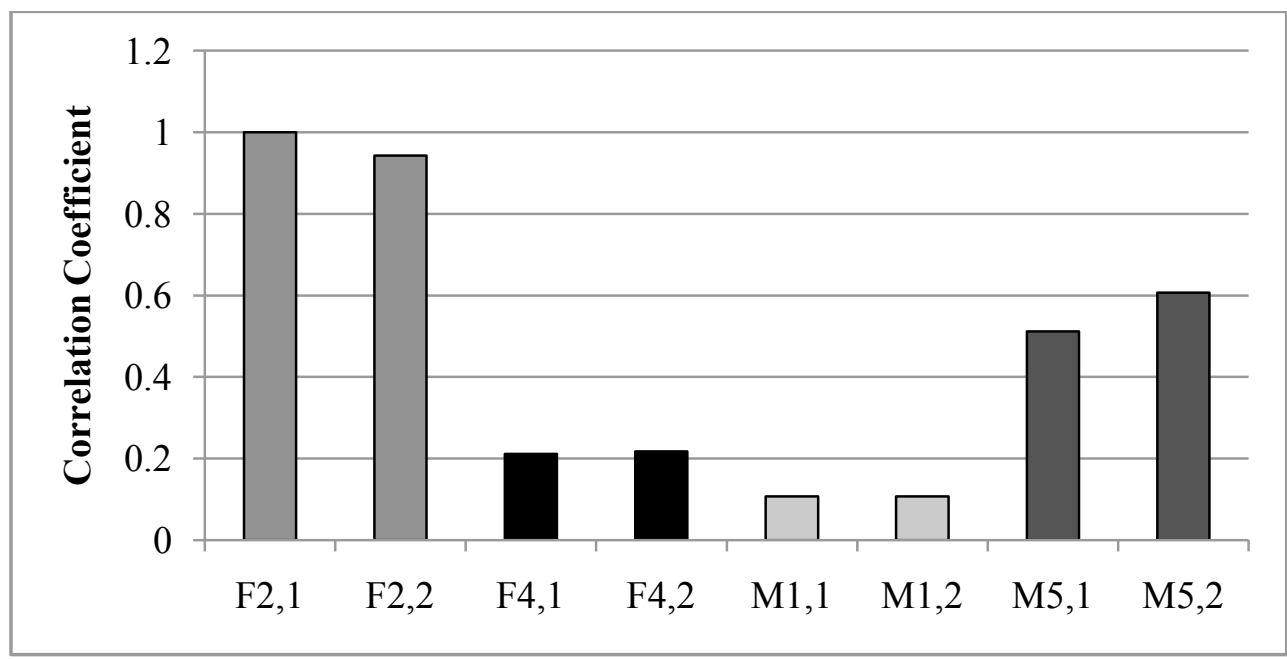


Figure 51. Spearman rank correlation with respect to Male 1, sample 1 for blood

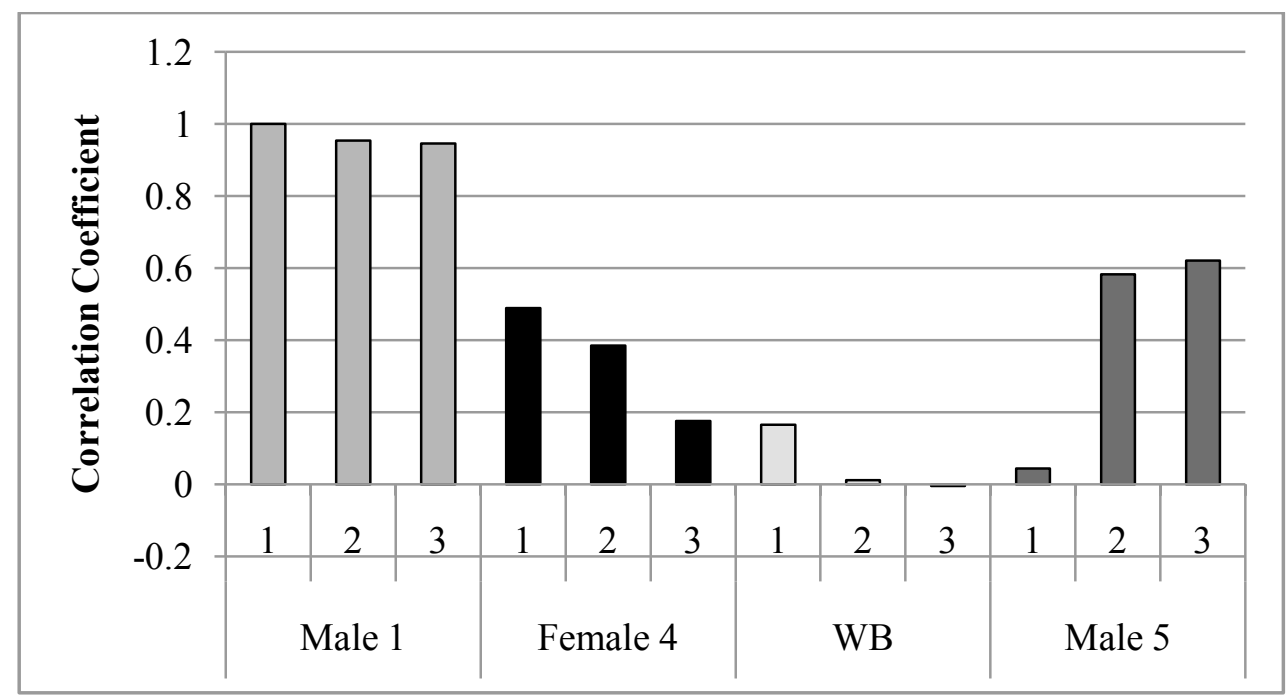

Figure 52. Spearman rank correlation with respect to Male 5, sample 1 for urine

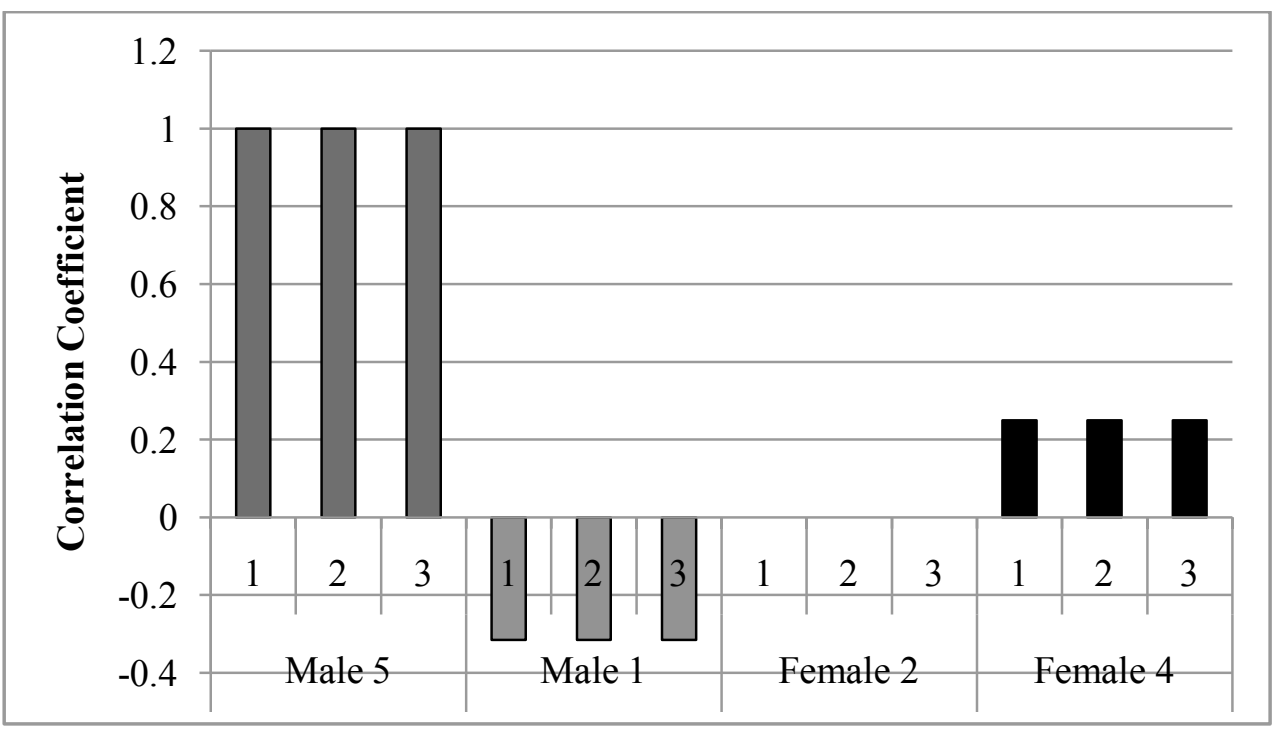




\subsection{Quantitation of VOCs Collected from Specimens}

It is important to determine the level of the signal of the analyte where it could be distinguished from the background noise of the instrument. With human scent where the amounts of volatile organic compounds emitted from the specimens are very low. The limit of detection (LOD) and limit of quantitation (LOQ) must be determined for each analyte using the instrument of analyses. In analytical chemistry, the limit of detection is defined as the concentration of the analyte which gives an instrument a signal three times greater than the background noise. The limit of quantitation is defined as the lower limit of the analyte concentration for precise quantitative measurements. LOD and LOQ can be determined by Equation 3 and Equation 4, respectively:

$$
\begin{array}{ll}
y_{\text {lod }}=a+3 s_{x / y} & \text { Equation 3 } \\
y_{\text {loq }}=a+10 s_{x / y} & \text { Equation 4 }
\end{array}
$$

where $y_{l o d}$ is the y-coordinate of the limit of detection for the calibration curve, $a$ is the yintercept, and $3 \mathrm{~s}_{x / y}$ is the standard error for the predicted $y$ value for all $x$ values in the regression.

Limits of detection and quantitation were investigated for selected compounds most commonly found in the five biological specimens. To determine the LODs and LOQs of the analytes found in each specimen, a calibration graph was prepared for each analyte using $5,10,20,40,60$, and $80 \mathrm{ng} / \mathrm{uL}$ concentrations of standard solutions prepared in dichloromethane and analyzed by GC/MS. The LODs and LOQs were calculated using the slopes from the calibration curves of each analyte in conjunction with a regression 
equation as shown in Equation 3. The LODs and LOQs for all analytes tested are presented in Table 7 through Table 11. The average LOD values of the VOCs commonly found in hand odor, buccal swab, breath, blood, and urine were $3.65 \mathrm{ng}, 7.98 \mathrm{ng}, 6.88 \mathrm{ng}$, 5.07ng, and 8.46ng, respectively. The average LOQ values were $12.15 \mathrm{ng}$ (hand odor), 29.60ng (buccal swab), 22.92ng (breath), 16.90ng (blood), and 28.20ng (urine). Sample calibration curves of selected compounds of various functional groups for each specimen can be found in the Appendices section. 
Table 7. Limits of detection and quantitation of VOCs found in the headspace of hand odor

\begin{tabular}{|l|l|c|c|}
\hline RT (min) & Compound Name & LOD (ng) & LO (ng) \\
\hline 6.25 & Furfural & 4.86 & 16.19 \\
\hline 6.93 & 2-Furanmethanol & 4.08 & 13.60 \\
\hline 8.10 & Heptanal & 4.57 & 15.23 \\
\hline 8.85 & Propanedioicacid,dimethylester & 3.78 & 12.59 \\
\hline 9.44 & Benzaldehyde & 2.24 & 7.48 \\
\hline 10.01 & Phenol & 1.58 & 5.27 \\
\hline 10.06 & 5-Hepten-2-one,6-methyl- & 2.44 & 8.13 \\
\hline 10.25 & Hexanoicacid & $\mathrm{ND}$ & $\mathrm{ND}$ \\
\hline 10.36 & Octanal & 6.88 & 22.92 \\
\hline 10.97 & BenzylAlcohol & 2.32 & 7.74 \\
\hline 11.56 & Acetophenone & 1.82 & 6.06 \\
\hline 12.13 & Undecane & 3.83 & 12.78 \\
\hline 12.16 & 1,6-Octadien-3-ol,3,7-dimethyl- & 5.77 & 19.22 \\
\hline 12.22 & Nonanal & 3.97 & 13.24 \\
\hline 12.56 & Octanoicacid,methylester & 1.68 & 5.60 \\
\hline 13.14 & 2-Nonenal,(E)- & 2.99 & 9.95 \\
\hline 13.31 & Nonanol & 3.05 & 10.17 \\
\hline 13.65 & 2-Decanone & 1.59 & 5.30 \\
\hline 13.75 & Dodecane & 2.20 & 7.32 \\
\hline 13.85 & Decanal & 1.85 & 6.16 \\
\hline 15.21 & Tridecane & 2.15 & 7.18 \\
\hline 15.33 & Undecanal & 2.25 & 7.51 \\
\hline 16.57 & Tetradecane & 2.40 & 8.01 \\
\hline 16.70 & Dodecanal & 2.22 & 7.41 \\
\hline 17.04 & $5,9-$ Undecadien-2-one,6,10-dimethyl-, (E)- & 2.18 & 7.27 \\
\hline 17.74 & 1-Pentadecene & 2.61 & 8.69 \\
\hline 17.83 & Pentadecane & 3.11 & 10.35 \\
\hline 18.65 & Dodecanoic acid & 4.33 & 14.42 \\
\hline 19.02 & Hexadecane & 3.11 & 10.36 \\
\hline 20.16 & Heptadecane & 5.38 & 17.92 \\
\hline 23.23 & Eicosane & 3.22 & 10.73 \\
\hline 24.17 & Heneicosane & 4.11 & 13.71 \\
\hline 25.06 & Docosane & 18.09 & 60.30 \\
\hline & Average & $\mathbf{3 . 6 5}$ & $\mathbf{1 2 . 1 5}$ \\
\hline
\end{tabular}


Table 8. Limits of detection and quantitation of VOCs found in the headspace of buccal swab

\begin{tabular}{|c|c|c|c|}
\hline RT (min) & Compound Name & LOD (ng) & LOQ (ng) \\
\hline 5.24 & Hexanal & 10.20 & 34.00 \\
\hline 6.25 & Furfural & 6.60 & 22.01 \\
\hline 7.28 & 1-Hexanol & 7.54 & 25.14 \\
\hline 8.16 & Pentanoicacid & 12.42 & 41.41 \\
\hline 9.44 & Benzaldehyde & 5.78 & 19.27 \\
\hline 9.95 & Phenol & 9.13 & 30.44 \\
\hline 10.12 & Furan,2-pentyl- & 9.23 & 30.77 \\
\hline 10.13 & 1-Decene & ND & ND \\
\hline 10.40 & Hexanoicacid & 5.19 & 17.29 \\
\hline 11.41 & 2-Octenal,(E)- & 5.61 & 18.69 \\
\hline 11.56 & Acetophenone & 9.96 & 33.21 \\
\hline 11.64 & 1-Octanol & 6.78 & 22.60 \\
\hline 11.83 & Heptanoicacid & 4.88 & 16.27 \\
\hline 12.12 & Heptanoicacid,ethylester & 8.47 & 28.23 \\
\hline 13.14 & 2-Nonenal,(E)- & 5.06 & 16.87 \\
\hline 13.37 & Menthol & 6.51 & 21.69 \\
\hline 13.48 & OctanoicAcid & 4.79 & 15.98 \\
\hline 13.63 & 1-Dodecene & 6.89 & 22.96 \\
\hline 13.71 & Octanoicacid,ethylester & 16.98 & 56.60 \\
\hline 13.85 & Decanal & 5.84 & 19.47 \\
\hline 13.99 & 2,4-Nonadienal,(E,E)- & 5.43 & 18.10 \\
\hline 15.16 & Nonanoicacid,ethylester & 12.11 & 40.36 \\
\hline 15.21 & Tridecane & 5.73 & 19.10 \\
\hline 15.46 & Naphthalene,1-methyl- & 7.19 & 23.98 \\
\hline 16.46 & 1-Tetradecene & 7.55 & 25.17 \\
\hline 16.51 & Decanoicacid,ethylester & 10.54 & 35.14 \\
\hline 16.57 & Tetradecane & 6.49 & 21.62 \\
\hline 16.70 & Dodecanal & 5.82 & 19.41 \\
\hline 16.97 & Caryophyllene & 7.56 & 25.20 \\
\hline 17.03 & 5,9-Undecadien-2-one,6,10-dimethyl-, (E)- & 9.92 & 33.05 \\
\hline 17.74 & 1-Pentadecene & 6.82 & 22.72 \\
\hline 18.13 & Dodecanoicacid,methylester & 9.36 & 31.19 \\
\hline 18.59 & Dodecanoicacid & 9.47 & 31.58 \\
\hline 19.02 & Hexadecane & 7.05 & 23.51 \\
\hline 19.48 & Benzophenone & 10.09 & 33.65 \\
\hline 20.16 & Heptadecane & 5.87 & 19.56 \\
\hline 23.23 & Eicosane & 6.99 & 23.29 \\
\hline 24.17 & Heneicosane & 7.20 & 24.02 \\
\hline \multirow[t]{2}{*}{25.06} & Docosane & 14.16 & 47.19 \\
\hline & Average & 7.98 & 26.60 \\
\hline
\end{tabular}


Table 9. Limits of detection and quantitation of VOCs found in the headspace of breath

\begin{tabular}{|c|c|c|c|}
\hline RT (min) & Compound Name & LOD (ng) & LOQ (ng) \\
\hline 4.16 & Octane & 6.93 & 23.09 \\
\hline 4.28 & Hexanal & $\mathrm{ND}$ & ND \\
\hline 5.28 & Ethylbenzene & 5.73 & 19.11 \\
\hline 5.42 & Xylenes & 11.00 & 36.67 \\
\hline 5.75 & 3-Heptanone & 5.72 & 19.06 \\
\hline 5.79 & Styrene & 6.14 & 20.46 \\
\hline 6.52 & 1R-.alpha.-Pinene & 6.18 & 20.60 \\
\hline 6.89 & Benzene,propyl- & 5.27 & 17.55 \\
\hline 7.03 & Benzaldehyde & 6.03 & 20.09 \\
\hline 7.37 & Phenol & 4.38 & 14.61 \\
\hline 7.47 & 5-Hepten-2-one,6-methyl- & 4.89 & 16.29 \\
\hline 8.16 & Limonene & 6.12 & 20.40 \\
\hline 8.26 & Benzene,1,2-dichloro- & 5.26 & 17.54 \\
\hline 8.80 & Acetophenone & 4.96 & 16.53 \\
\hline 9.29 & Undecane & 6.14 & 20.46 \\
\hline 9.37 & Nonanal & 4.47 & 14.90 \\
\hline 9.58 & Benzene,1,2,4,5-tetramethyl- & 5.10 & 16.99 \\
\hline 10.46 & Menthol & 6.54 & 21.79 \\
\hline 10.65 & Naphthalene & 22.41 & 74.70 \\
\hline 10.69 & 1-Dodecene & 3.73 & 12.42 \\
\hline 10.81 & Methyl Salicylate & 5.16 & 17.19 \\
\hline 10.81 & Dodecane & 4.69 & 15.62 \\
\hline 10.91 & Decanal & 5.30 & 17.67 \\
\hline 12.23 & Tridecane & 5.65 & 18.83 \\
\hline 12.35 & Undecanal & 4.90 & 16.34 \\
\hline 12.49 & Naphthalene,1-methyl- & 5.79 & 19.29 \\
\hline 13.12 & n-Decanoicacid & 9.04 & 30.13 \\
\hline 13.57 & Tetradecane & 6.00 & 20.00 \\
\hline 13.71 & Dodecanal & 5.11 & 17.02 \\
\hline 13.98 & Caryophyllene & 6.22 & 20.74 \\
\hline 14.04 & 5,9-Undecadien-2-one,6,10-dimethyl- & 5.54 & 18.45 \\
\hline 14.28 & 5,9-Undecadien-2-one,6,10-dimethyl-, (E)- & 11.39 & 37.98 \\
\hline 15.07 & ButylatedHydroxytoluene & 5.63 & 18.76 \\
\hline 15.57 & Dodecanoicacid & 11.07 & 36.89 \\
\hline 16.02 & Hexadecane & 6.59 & 21.95 \\
\hline 16.48 & Benzophenone & 6.16 & 20.55 \\
\hline 17.16 & Heptadecane & 6.71 & 22.37 \\
\hline 17.57 & 2,6-Diisopropylnaphthalene & 6.17 & 20.57 \\
\hline 19.70 & n-Hexadecanoicacid & 17.13 & 57.11 \\
\hline \multirow[t]{2}{*}{20.02} & Eicosane & 6.98 & 23.26 \\
\hline & Average & 6.88 & 22.92 \\
\hline
\end{tabular}


Table 10. Limits of detection and quantitation of VOCs found in the headspace of blood

\begin{tabular}{|l|l|c|c|}
\hline RT $(\mathbf{m i n}$ & Compound Name & LOD (ng) & LOQ (ng) \\
\hline 4.80 & 1-Hexanol & 2.73 & 9.11 \\
\hline 4.88 & 4-Heptanone & 3.69 & 12.29 \\
\hline 5.08 & 3-Heptanone & 2.81 & 9.38 \\
\hline 5.14 & 2-Heptanone & 4.19 & 13.95 \\
\hline 5.20 & Cyclohexanone & 4.34 & 14.47 \\
\hline 5.23 & Nonane & 5.88 & 19.61 \\
\hline 5.30 & Heptanal & 3.21 & 10.68 \\
\hline 6.23 & Benzaldehyde & 3.74 & 12.45 \\
\hline 6.33 & 1-Heptanol & 3.31 & 11.02 \\
\hline 6.49 & 1-Octen-3-ol & 18.66 & 62.18 \\
\hline 6.53 & Phenol & 17.14 & 57.14 \\
\hline 6.62 & 5-Hepten-2-one,6-methyl- & 5.04 & 16.81 \\
\hline 6.67 & 1-Decene & 10.84 & 36.14 \\
\hline 6.68 & Furan,2-pentyl- & 16.89 & 56.31 \\
\hline 7.28 & Limonene & 2.81 & 9.38 \\
\hline 7.38 & Benzene,1,2-dichloro- & 1.58 & 5.28 \\
\hline 7.39 & Benzyl Alcohol & 4.63 & 15.42 \\
\hline 7.89 & Acetophenone & 4.66 & 15.52 \\
\hline 7.92 & 1-Octanol & 3.34 & 11.15 \\
\hline 8.35 & Undecane & 2.68 & 8.95 \\
\hline 8.43 & Nonanal & 2.57 & 8.58 \\
\hline 8.64 & Benzene,1,2,4,5-tetramethyl- & 4.39 & 14.64 \\
\hline 9.49 & Menthol & 4.68 & 15.61 \\
\hline 9.69 & Naphthalene & 10.50 & 35.01 \\
\hline 9.71 & 1-Dodecene & 5.71 & 19.03 \\
\hline 9.74 & 2-Decanone & 5.39 & 17.97 \\
\hline 9.84 & Dodecane & 2.76 & 9.21 \\
\hline 9.94 & Decanal & 3.22 & 10.73 \\
\hline 11.25 & Tridecane & 2.47 & 8.22 \\
\hline 11.51 & Naphthalene,1-methyl- & 3.71 & 12.37 \\
\hline 12.48 & 1-Tetradecene & 2.36 & 7.85 \\
\hline 12.58 & Tetradecane & 2.41 & 8.04 \\
\hline 12.71 & Dodecanal & 2.49 & 8.31 \\
\hline 13.05 & $5,9-$-Undecadien-2-one,6,10-dimethyl-,(E)- & 5.01 & 16.69 \\
\hline 13.52 & 1-Dodecanol & 4.78 & 15.92 \\
\hline 15.02 & Hexadecane & 2.45 & 8.16 \\
\hline 16.16 & Heptadecane & 1.63 & 5.42 \\
\hline 16.57 & 2,6-Diisopropylnaphthalene & 3.92 & 13.08 \\
\hline & Average & $\mathbf{5 . 0 7}$ & $\mathbf{1 6 . 9 0}$ \\
\hline & & & \\
\hline
\end{tabular}


Table 11. Limits of detection and quantitation of VOCs found in the headspace of urine

\begin{tabular}{|c|c|c|c|}
\hline RT (min) & Compound Name & LOD (ng) & LOQ (ng) \\
\hline & Toluene & ND & ND \\
\hline & 2-Pentanone & ND & ND \\
\hline & Pyrrole & ND & ND \\
\hline & 4-Nonylphenol & ND & ND \\
\hline 7.08 & 4-Heptanone & 7.91 & 26.36 \\
\hline 7.38 & 3-Heptanone & 9.52 & 31.73 \\
\hline 7.46 & 2-Heptanone & 7.54 & 25.15 \\
\hline 8.69 & Benzaldehyde & 10.07 & 33.56 \\
\hline 8.80 & Dimethyltrisulfide & 7.15 & 23.83 \\
\hline 9.15 & 1-Decene & 7.59 & 25.30 \\
\hline 9.77 & Benzene,1,2-dichloro- & 8.23 & 27.42 \\
\hline 10.24 & 1-Octanol & 7.89 & 26.28 \\
\hline 10.30 & Phenol,4-methyl- & 9.39 & 31.30 \\
\hline 10.60 & 1,6-Octadien-3-ol,3,7-dimethyl- & 7.98 & 26.59 \\
\hline 10.65 & Nonanal & 5.21 & 17.36 \\
\hline 11.29 & 2-Nonenal,(E)- & 8.25 & 27.51 \\
\hline 11.46 & Menthol & 7.41 & 24.71 \\
\hline 11.71 & MethylSalicylate & 8.65 & 28.85 \\
\hline 11.77 & Decanal & 6.50 & 21.66 \\
\hline 12.67 & 1,3-Benzodioxole,5-(2-propenyl)- & 8.06 & 26.85 \\
\hline 14.10 & 5,9-Undecadien-2-one,6,10-dimethyl-, (E)- & 7.44 & 24.79 \\
\hline 14.64 & ButylatedHydroxytoluene & 7.00 & 23.34 \\
\hline 14.95 & Dodecanoicacid & 16.52 & 55.08 \\
\hline 15.26 & Hexadecane & 8.09 & 26.98 \\
\hline 15.38 & Phenol,4-(1,1,3,3-tetramethylbutyl)- & 7.92 & 26.40 \\
\hline 15.55 & Diphenylamine & 9.78 & 32.58 \\
\hline 15.63 & Benzophenone & 10.34 & 34.47 \\
\hline \multirow[t]{2}{*}{16.02} & Heptadecane & 8.59 & 28.62 \\
\hline & Average & 8.46 & 28.20 \\
\hline
\end{tabular}




\subsection{Summary of Biological Specimen Optimization}

Thus far, SPME-GC/MS conditions of VOCs above the headspace of collected buccal swab, breath, blood, and urine specimens have been optimized. Data obtained using these optimized conditions yielded promising results as VOC profiles of different subjects are distinct from one another and reproducible within themselves. The specimen sampling methods described through the present chapter were utilized for the simultaneous

collection of hand odor, buccal swab, breath, blood, and urine for the subsequent population studies on healthy, diabetic, and depressed individuals. The optimized SPMEGC/MS conditions were used for the extraction, separation, and analysis of the VOCs extracted above the headspace of the collected specimen samples. 


\section{POPULATION STUDIES}

\subsection{Scent Biomarkers for Diseases}

"By the sense of smell we can recognize the peculiar perspiration of many diseases, which has an important bearing on their identification. ${ }^{86 "}$ - Susruta Samhita

The above quote was taken from an ancient ayurvedic medicine transcript over 2000 years ago. Thus it is clear that people have long used the smell of individuals to diagnose diseases, and the above statement holds true today. The difference is that today, instead of physicians simply using the sense of smell or taste, there are analytical methods and instruments available to detect and identify such potential biomarkers. The VOC analysis in biological specimens is of research interest because of its potential to identify diagnostic biomarkers for various diseases. The appearance of specific biomarkers can indicate physiological malfunction. Odor can be a diagnostic marker that can lead to early diagnosis of diseases. Identification of target VOCs for a particular disease would be a useful screening tool. Over the last couple decades, research describing the detection and identification of VOCs that are specific to certain diseases has been growing in published literature (Table 12).

Since the initial demonstration of a peculiar odor emanating from schizophrenic patients, there have been several studies attempting to identify the odoriferous substance which could perhaps provide an approach to an etiologic diagnosis of schizophrenia ${ }^{87,88}$. It has been reported that the mean alveolar gradients of carbon disulfide and pentane were significantly higher in schizophrenic patients compared to patients with other psychiatric disorders and subjects with no psychiatric disorder. 
There are numerous studies on the investigation of volatile biomarkers for cancer. Deng et al. have developed a procedure for headspace SPME-GC/MS with on-fiber derivatization to determine the levels of hexanal and heptanal in the blood of cancer patients, as aldehydes with low molecular weight have been proposed to be cancer biomarkers ${ }^{66}$. It was found that high levels of hexanal and heptanal were found only in blood taken from patients with lung cancer. Their results corresponded with other studies that also found that aldehyde compounds were only present in breath and urine of cancer patients ${ }^{67}$. These findings suggest that early screening of lung cancer may be carried out by analysis of these aldehydes in biological fluids. Other studies on volatile biomarkers in breath samples of lung cancer patients have also identified potential VOCs that could discriminate between individuals with and without lung cancer, although there is no single compound that marks the disease $\mathrm{e}^{54,57,89,90}$.

There are many published studies on the profiling potential of VOCs in biological specimens in attempt to associate disturbances of volatile compounds with many other metabolic illnesses (Table 12). While many of the reported VOCs are also present in healthy individuals, studies have reported an elevation in the levels of these compounds in patients with asthma ${ }^{91-93}$, diabetes ${ }^{56,94}$, liver and kidney disease/impairment ${ }^{70,71,83}$, gastrointestinal diseases $^{95}$, and oxidative stress ${ }^{56,96}$. 
Table 12. Published studies on VOCs in biological specimens identified as potential diagnostic markers of various diseases

\begin{tabular}{|l|l|l|l|}
\hline Diseases & VOCs Detected & Specimen & References \\
\hline $\begin{array}{l}\text { Lung or breast } \\
\text { cancer }\end{array}$ & $\begin{array}{l}\text { Alkanes, monomethylated alkanes, } \\
\text { benzene and benzene derivatives }\end{array}$ & Breath, blood & $\begin{array}{l}54,57,66- \\
68,89,90,97\end{array}$ \\
\hline Oxidative stress & $\begin{array}{l}\text { Ethane, pentane, other alkanes and } \\
\text { methylated alkanes }\end{array}$ & Breath & $56,87,96$ \\
\hline Asthma & NO, pentane, ethane, 8-isoprostane & Breath & $91-93$ \\
\hline Diabetes mellitus & $\begin{array}{l}\text { Acetone, ethanol, methyl nitrate, 4- } \\
\text { heptanone }\end{array}$ & Breath, urine & 55,94 \\
\hline Schizophrenia & Carbon disulfide, pentane, ethane & Breath & 87,88 \\
\hline Cystic fibrosis & Carbonyl sulfide, alkanes & Breath & 98 \\
\hline Kidney impairment & $\begin{array}{l}\text { Nitrogen-containing compounds } \\
\text { (ammonia, dimethylamine, } \\
\text { trimethylamine) }\end{array}$ & Urine & 71 \\
\hline $\begin{array}{l}\text { Liver } \\
\text { impairment/failure }\end{array}$ & $\begin{array}{l}\text { Benzaldehyde and phenolics } \\
\text { (phenylketonuria), } \\
\text { dimethyl disulfide and sulfur- } \\
\text { containing compounds (hepatitis), }\end{array}$ & Urine & $70,71,83$ \\
\hline $\begin{array}{l}\text { Gastrointestinal } \\
\text { disease }\end{array}$ & $\begin{array}{l}\text { Sulfur-containing compounds, } \\
\text { p-menth-1-en-8-ol }\end{array}$ & Feces & 95 \\
\hline
\end{tabular}




\subsubsection{Medicine and Human Scent}

\subsubsection{Disease-Specific Scent}

Certain medical conditions are known to cause odor symptoms. Patients of acidosis have acetone smelling breath ${ }^{86}$. Maple syrup urine disease is an inherited disease of amino acid metabolism, which causes urine to have a characteristic sweet smell like maple syrup ${ }^{99}$. This disease is an autosomal recessively inherited disorder in which branched-chain amino acids and their $\alpha$-keto and $\alpha$-hydroxy acids accumulate in the body fluids, leading to mental retardation and neurological damage. The odor of this disease is a result of the presence of a ketone called sotolone, which is a component of maple syrup. Sotolone is only present in the urine of patients with maple syrup urine disease, and not in urine of healthy individuals. Trimethylaminuria, or fish odor syndrome (or fish malodor syndrome), is a genetically inherited enzyme deficiency where the patient fails to metabolize trimethylaminuria, causing the body to emit a persistent fish odor ${ }^{100}$. Unusual amounts of unoxidized trimethylamine are excreted in the sweat, urine, breath, and other bodily secretions. Patients with diabetes mellitus may have a breath odor resembling the smell of fruity acetone. The smell of fresh baked brown bread is associated with typhoid fever $^{86}$. Other diseases known to exhibit characteristic (mostly unpleasant, putrid) odors include foetor hepaticus of the liver, smallpox, scurvy, scrofula (tuberculosis of the neck), yellow fever, gout, and diseases of the respiratory tract such as bronchiectasis, lubabscessea and ozaena ${ }^{86}$. Examples of metabolic disorders characterized by unusual odors are shown in Table 13. 
Table 13. Metabolic disorders with characteristic odors

\begin{tabular}{|l|l|}
\hline Disease & Characteristic Odor \\
\hline Phenylketonuria & Musty or mousey odor \\
Maple syrup urine disease & Maple syrup \\
Isovaleric academia & Sweaty feet \\
Trimethylaminuria & Fish odor \\
Diabetes Mellitus & Sweet fruity acetone \\
Acidosis & Acetone smelling breath \\
\hline
\end{tabular}

\subsubsection{Canines and Cancer}

In 1989, Williams and Pembroke described a case of malignant melanoma detected by the patient's pet $\operatorname{dog}^{2}$. The dog constantly sniffed and even tried to bite a specific mole on her leg. Upon seeking medical advice, the patient was diagnosed with basal cell carcinoma. More instances of this kind have been reported in the recent years. In 2001, a case was reported by Church where a pet Labrador constantly pushing and sniffing at his owner's lesion on his left thigh led to the owner to have the lesion examined ${ }^{101}$. The lesion was found to be a basal cell carcinoma. After the lesion was surgically removed, the dog showed no more interest in the area. In 2005, Welsh et al. reported yet another similar case where a patient's dog sniffed and poked at her left axilla which was later found to be infiltrating ductal carcinoma: breast cancer ${ }^{1}$. Following these reports, researchers at Cambridge Universality Veterinary School in England in collaboration with the Cambridge Institute of Dog Behavior \& Training are testing the viability of dogs to be trained to detect cancer - what is now referred to as "dognoseis". 


\subsubsection{Rats and Schizophrenia}

Patients with schizophrenia have been reported to emit a characteristic unpleasant odor that is unrelated to hygiene. In 1959, Smith and Sines' study of the peculiar odor in the sweat of schizophrenic patients demonstrated the differences to that of non-schizophrenic individuals' sweat through the use of trained rats ${ }^{102}$. A human panel testing the odor was also able to discriminate between the sweat of the patients and controls.

\subsection{Diabetes Mellitus}

Diabetes mellitus (commonly just called diabetes) is a metabolic disorder characterized by an insulin deficiency or to a hyporesponsiveness to insulin ${ }^{46,103}$. These conditions result in high blood glucose levels to the point where glucose spills over into the urine hence the name diabetes ("syphon" or "running through") and mellitus ("sweet") in Greek. Despite the high blood glucose levels, the body cells do not absorb the glucose and therefore "starve" while the glucose accumulates in the blood, a condition known as hyperglycemia. High blood glucose results in the acceleration of other metabolic pathways like triacylglycerol hydrolysis, gluconeogenesis, fatty acid oxidation, and ketone body formation. When plasma ketone body level becomes abnormally high, blood $\mathrm{pH}$-buffering capacity and renal functions are impaired. Ketosis leads to increased plasma $\mathrm{H}^{+}$concentration, where excess $\mathrm{H}^{+}$together with other important ions $\left(\mathrm{Na}^{+}, \mathrm{K}^{+}\right)$is excreted into the urine. Severe dehydration may occur from loss of these ions and water

excretion. In addition, $\mathrm{H}^{+}$accumulation causes brain dysfunction. Complications of diabetes include heart disease, high blood pressure, blindness, kidney disease, nervous system disease, circulatory problems, dental disease, and complication of pregnancy ${ }^{104}$. 
Uncontrolled diabetes often results in acute life-threatening emergencies such as diabetic ketoacidosis, hypotention, coma, and death ${ }^{105}$. Diabetes is the third leading cause of death in the U.S., following heart disease and cancer. The 2007 National Diabetes Fact Sheet estimated 23.6 million children and adults in the U.S. ( $7.8 \%$ of the population) having diabetes, of which 17.9 million are diagnosed diabetes patients and the remaining 5.7 million are undiagnosed ${ }^{106}$.

\subsubsection{Type 1 Diabetes}

Type 1 diabetes or insulin-dependent diabetes mellitus (IDDM) is caused by a deficiency of pancreatic $\beta$ cells resulting in insulin deficiency ${ }^{46}$. Type 1 diabetes was previously also known as juvenile-onset diabetes mellitus, for its onset is most often during childhood. Pancreatic $\beta$ cells are selectively destroyed as an autoimmune response, and individuals with type 1 diabetes require insulin therapy with daily injections and a specific diet. The cause of this autoimmune destruction is not yet fully understood. Type 1 diabetes is the less common type of diabetes, affecting about 5-10 percent of the diagnosed diabetic patients ${ }^{107}$. Diabetic ketoacidosis occurs predominantly in type 1 diabetes patients, because it results from a total inability of insulin secretion. The body responds to this insulin shortage by converting to burning fatty acids and producing acidic ketone bodies. As noted before, management of type 1 diabetes involves daily insulin replacement therapy. Pancreas transplantation and a lesser invasive islet cell transplantation are treatments of extreme cases, although these still do not fully cure the disease. The lifespan of type 1 diabetic are generally reduced by up to one-third as a result of degenerative complications mentioned before. 


\section{Figure 53. Diagram of cell glucose regulation for normal, type 1 and type 2 diabetes}

NORMAL

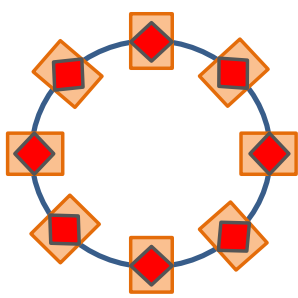

Insulin lets cells take up glucose from the blood. Glucose in the cell is transformed into energy for the body.
TYPE 1 DIABETES

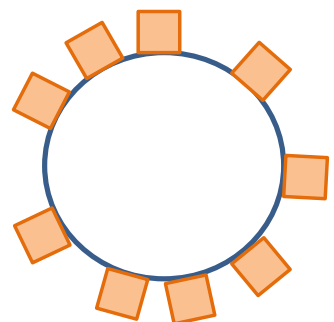

In Type 1 diabetes the pancreas is not producing insulin; therefore the glucose cannot enter the cell.
TYPE 2 DIABETES

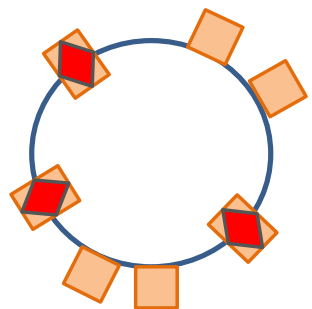

In Type 2 diabetes the pancreas still produces insulin, but not enough to help adequate amounts of glucose into the cells.

\section{glucose insulin $\bigcirc$ cell}

\subsubsection{Type 2 Diabetes}

Type 2 diabetes or non-insulin-dependent diabetes mellitus accounts for over $90 \%$ of the diagnosed diabetes cases ${ }^{106}$. Unlike type 1 diabetes, type 2 diabetics have normal or even elevated insulin levels. The issue with type 2 diabetes lies in that there is a lack of insulin receptors on normally insulin-responsive cells (Figure 53). The cells do not respond appropriately to insulin, hence these cells are said to be insulin-resistant. Consequently, the blood glucose level becomes elevated, particularly after a meal. Table 14 shows the criteria for diagnosing diabetes.

Type 2 diabetes is also commonly known as adult-onset diabetes and usually occurs in obese individuals with a predisposition for this condition. High-cholesterol, hypertension, high-fat diet, and low exercise lifestyle are all factors that increase the risk of type 2 diabetes. In addition to insulin resistance, type 2 diabetes is characterized by impaired regulation of hepatic glucose production and declining $\beta$-cell function. In extreme cases, 
the conditions could lead up to $\beta$-cell failure where the patient will require insulin therapy for survival. Major complications from improperly managed type 2 diabetes include retinopathy leading to blindness, nephropathy, neuropathy, and cardiovascular diseases such as coronary artery disease ${ }^{46}$. Treatment (and prevention for onset) for type 2 diabetes mainly involves diet therapy for weight reduction, and exercise. There are also oral therapeutic agents that are used in the management of type 2 diabetes (Table $15)^{105,108}$. Since type 2 diabetes accounts for the majority of diabetic patients in the U.S., the type 2 will be the diabetic population of interest in this study.

\section{Table 14. Diabetes diagnosis criteria}

\begin{tabular}{|c|c|c|c|}
\hline \multirow{2}{*}{ Stage } & \multicolumn{3}{|c|}{ Test } \\
\cline { 2 - 4 } & $\begin{array}{c}\text { Fasting Plasma } \\
\text { Glucose }\end{array}$ & $\begin{array}{c}\text { Random Plasma } \\
\text { Glucose }\end{array}$ & $\begin{array}{c}\text { Oral Glucose } \\
\text { Tolerance Test }(75-\mathrm{g})\end{array}$ \\
\hline Normal & $<100 \mathrm{mg} / \mathrm{dL}$ & $\mathrm{hPG}^{\mathrm{a}}<140 \mathrm{mg} / \mathrm{dL}$ \\
\hline Diabetes & $\geq 126 \mathrm{mg} / \mathrm{dL}$ & $\begin{array}{c}\geq 200 \mathrm{mg} / \mathrm{dL} \text { plus } \\
\text { typical diabetes } \\
\text { symptoms }\end{array}$ & $2 \mathrm{hPG} \geq 200 \mathrm{mg} / \mathrm{dL}$ \\
\hline
\end{tabular}

a 2-hour plasma glucose

Table 15. Current oral therapeutic agents for type 2 diabetes

\begin{tabular}{|l|l|l|}
\hline \multicolumn{1}{|c|}{ Drug Class } & \multicolumn{1}{|c|}{ Mechanism of Action } & \multicolumn{1}{c|}{ Indication(s) } \\
\hline $\begin{array}{l}\text { Sulphonylureas and } \\
\text { repaglinide }\end{array}$ & Increase insulin secretion & $\begin{array}{l}\text { Deficient pancreatic } \\
\text { secretion of insulin } \\
\text { resulting in hyperglycemia }\end{array}$ \\
\hline $\begin{array}{l}\text { Biguanides } \\
\text { (Metformin) }\end{array}$ & $\begin{array}{l}\text { Decrease peripheral insulin } \\
\text { resistance } \\
\text { Decrease hepatic gluconeogenesis }\end{array}$ & $\begin{array}{l}\text { Obesity } \\
\text { Insulin resistance }\end{array}$ \\
\hline Thiazolidenediones & $\begin{array}{l}\text { Decrease peripheral insulin } \\
\text { resistance } \\
\text { Reduce fatty acids }\end{array}$ & Insulin resistance \\
\hline $\begin{array}{l}\text { a-glucosidase } \\
\text { inhibitors }\end{array}$ & Slow absorption of carbohydrates & Hyperglycemia after meal \\
\hline
\end{tabular}




\subsection{Mood Disorders}

Mood disorders are mental disorders in which the predominant feature is having a disturbance in mood. The National Institute of Mental Health estimates 26.2 percent of Americans ages 18 and older suffer from some form of diagnosable mental disorder in a given year ${ }^{109}$. Of these mental disorders, the prevalence of mood disorders (major depressive disorder (MDD), dysthymic disorder, and bipolar disorder) is approximately 9.5 percent of the U.S. population age 18 and older, or about 20.9 million American adults. In particular, in a given year, approximately 14.8 million American adults or about 6.7 percent of the U.S. population (age 18 or older) suffer from MDD. Worldwide, MDD affects about 121 million people and is among the leading causes of disability ${ }^{110}$.

\subsubsection{Major Depressive Disorder}

Depression is common. It is an episodic medical disorder in which patients are overwhelmed by feeling sad, hopeless, helpless, and worthless ${ }^{111-113}$. Depression commonly affects sleep, appetite, and general interest in life. The latest edition of the Diagnostic and Statistical Manual of Mental Disorders (DSM-IV-TR) defines MDD to be characterized by one or more Major Depressive Episodes where the depressed mood must be present for most of the day, almost every day, for at least a 2-week period ${ }^{111}$. Major Depressive Disorder can be a single episode where there is only a single presence of a Major Depressive Episode, or recurrent if two or more Major Depressive Episodes are present. To be classified as having MDD, there could not have been a Manic Episode, a Mixed Episode, or a Hypomanic Episode, as the diagnosis would be changed to Bipolar Disorder if this were the case. It is also different from a depressive mood disorder as a 
result of a medical condition, side effect of medications or drugs of abuse. Lastly, Dysthymic Disorder is very similar to MDD in terms of symptoms and, therefore, is often difficult to distinguish from MDD. The difference between the two mood disorders lies in severity, chronicity, and persistence. Usually Dysthymic Disorder consists of chronic (depressed mood present for most days over at least two years), less severe depressive symptoms compared to MDD.

Other mental disorders not under the Mood Disorders category may have depressive symptoms also; however, they are different from MDD. Such disorders include Schizophrenia, Delusional Disorder, and Psychotic Disorder Not Otherwise Specified. Schizoaffective Disorder is also similar to MDD with Psychotic Features, but the difference is that to be diagnosed with Schizoaffective Disorder, "there must be at least 2 weeks of delusions or hallucinations occurring in the absence of prominent mood symptoms. $^{111,}$

The average onset age of MDD is in the early 30s and the peak age of risk is between 25 and 44 , although it can develop at any age, even prepubescent ${ }^{113}$. Major Depressive Disorder is approximately twice as common in women as men, with prevalence of lifetime risk for MDD varying around 10-25\% for women and 5-12\% for men

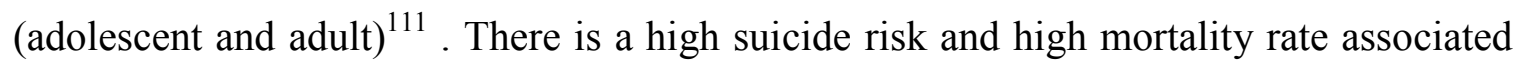
with MDD, as completed suicide occurs in up to $15 \%$ of individuals with severe MDD ${ }^{114}$. Death rates increase even more for elder individuals suffering $\mathrm{MDD}^{115}$.

While depression is so common and treatment of the disorder has been very successful, the exact cause of depression is yet to be understood. Many hypotheses have been 
presented to suggest the cause of depression to be the result of genetic, biological, psychological, and environmental factors; however, depression most likely results from a combination of these factors and not from one single cause ${ }^{12,113}$. Family, twin, and adoption studies support the idea that there are genetic influences that affect development of depression. Prevalence of depression is greatly increased among blood relatives of individuals with diagnosed MDD when compared to the general population ${ }^{112}$. Biochemically, unusual levels of monoamine neurotransmitters, especially norepinephrine, serotonin, and dopamine, have been accounted as causal factors of depression. In the brain, chemical messages and information are communicated between neurons. Information from one neuron is transmitted to another neuron at the synaptic cleft by neurotransmitters that are released by the presynaptic neuron. Altered neurotransmitter activities such as depletion or complete absence of one or all of these monoamine transmitters, or increases in them are thought to result in depression (Figure $54)$. 
Figure 54. Difference in the chemical message transduction in the brain cells of normal individual and individual with MDD (adapted from Pfizer Japan Inc.)

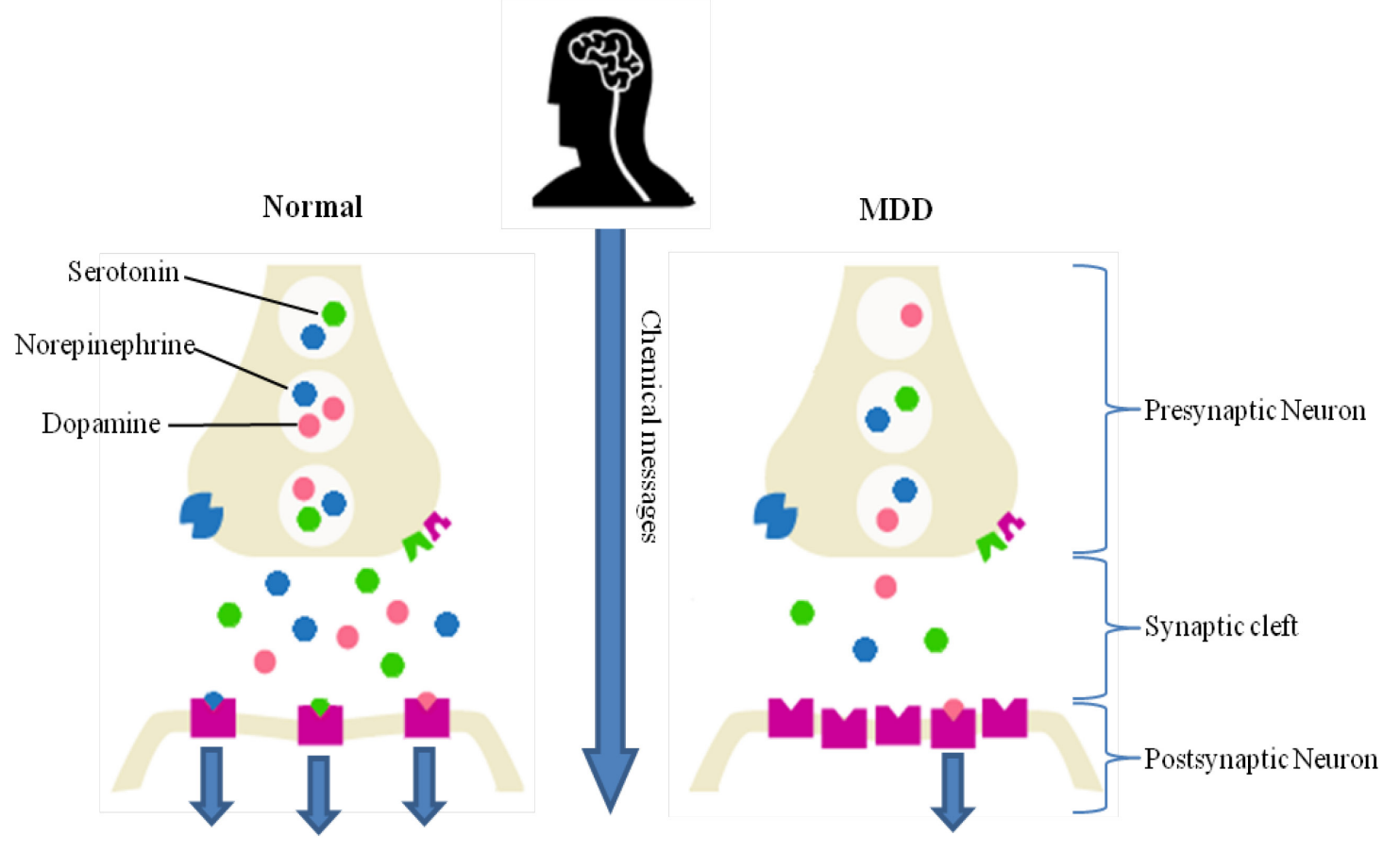

Treatment of MDD usually involves psychotherapy and/or pharmacotherapy (antidepressant medications). Psychological treatments include cognitive-behavioral therapy, interpersonal psychotherapy, and behavioral activation which all focus on the relationship between thoughts, perceptions, and behaviors and depressed mood. Other forms of treatment include electroconvulsive therapy (ECT) and light therapy. Antidepressant drugs have proven to be very effective in the treatment of depression. There are over 20 antidepressant drugs available today, and most of them work by normalizing the chemical imbalance in the brain. Major classes of antidepressant drugs are: 
- Tricyclic antidepressants (TCAs)

- Monoamine oxidase inhibitors (MAOIs)

- Selective serotonin reuptake inhibitors (SSRIs)

- Serotonin-norepinephrine reuptake inhibitors (SNRIs)

- Others

- Lithium

- Dopamine-norepinephrine reuptake inhibitors

- Serotonin modulators

- Norepinephrine-serotonin modulator

Tricyclic antidepressants and MAOIs are first generation antidepressants. Tricyclic antidepressants work by slowing down the reabsorption of norepinephrine and serotonin by brain cells. Monoamine oxidase inhibitors are one of the oldest classes of antidepressant drugs and are recommended to be restricted to patients who have not responded well to other antidepressant medications due to their potential adverse side effects and requirement for strict dietary restrictions ${ }^{116}$. Monoamine oxidase inhibitors work by inhibiting the enzyme monoamine oxidase from breaking down the monoamine neurotransmitters, thereby increasing the level of active neurotransmitters in the brain. Selective serotonin reuptake inhibitors, as the name suggests, selectively inhibits the reabsorption of serotonin by the nerve cells, thereby providing higher levels of serotonin at the brain receptor site. 


\subsection{Comorbidity between Diabetes and Depression}

Since 1684, it has been recognized that there is an association between diabetes and depression ${ }^{117}$. Since then, the persistence and prevalence of MDD in diabetic adults have been studied through a number of epidemiological surveys, longitudinal, follow-up, and prospective studies ${ }^{118-122}$. The exact factors that link the two illnesses still remain uncertain; however, there are hypotheses on the interrelationship between MDD and diabetes. One hypothesis suggests that MDD in diabetics results from biochemical factors related to the illness and the treatments. Another hypothesis suggests that the psychosocial or psychological burden from having the chronic illness (diabetes) accountable for MDD in diabetes. Intensive review of the current literature published on the covariance of MDD and diabetes found that the latter hypothesis is not supported ${ }^{122}$. Studies have reported that MDD doubles the risk for onset of type 2 diabetes in otherwise healthy individuals and over the general population ${ }^{117,122}$.

\subsection{Methods}

For all subjects sampled in the population studies, hand odor, buccal swabs, breath, blood, and urine were collected using the specimen sampling methods as described in the previous chapter. All collected specimens except for urine were stored at room temperature under normal laboratory conditions until ready for SPME analysis. Urine samples were stored at $4^{\circ} \mathrm{C}$ until ready for SPME analysis. The volatile organic compounds from the headspace of collected specimen samples were extracted using 50/30 $\mu \mathrm{m} \mathrm{DVB/CAR/PDMS} \mathrm{SPME} \mathrm{fibers.} \mathrm{Single} \mathrm{headspace} \mathrm{extractions} \mathrm{were} \mathrm{performed}$ 
for each sample. VOC extraction conditions using SPME are described in Table 6 under section 2.6.1.

\subsubsection{Evaluation of Odor Profiles of Individuals over Time}

Unrelated subjects Male 5 and Female 4 were sampled over six months. No attempt was made to control the diet of the subjects during the course of sampling. Samples were collected on a monthly interval (month 0 to month 6 ). The average temperature and the average humidity of the sampling environment during the samplings in the laboratory were $22.5^{\circ} \mathrm{C}$ and $75 \%$, respectively.

\subsubsection{Evaluation of the Effect of Fasting Prior to Sampling}

Four subjects were evaluated: two females (F4 and F15) and two males (M2 and M5) ranging in age from 24 to 29. Each subject was sampled twice during the course of a day. The first sample was collected at least two hours after the subject had consumed any food or beverage (except water) and the second sample was collected immediately after consuming a meal. No attempt was made to control the meal consumed by the subjects. The average temperature and the average humidity of the sampling environment during the samplings in the laboratory were $23.6^{\circ} \mathrm{C}$ and $56 \%$, respectively.

\subsubsection{Population Analysis of the Volatile Organic Compounds Present Above Collected Odor Samples}

\subsubsection{Healthy Individuals}

All sampling protocols were approved by the Florida International University Institutional Review Board (IRB) for research involving human subjects. Subjects were asked to read and sign an IRB-approved informed consent form prior to starting the 
sampling protocol. Thirty-one healthy subjects (15 males, 16 females), ages 19 to 36, were sampled. Subjects were also asked to answer a confidential medical questionnaire form prior to sampling to ensure that they were not diagnosed with any major physiological or psychological illnesses (particularly diabetes and depression) and to be aware of any over-the-counter or prescription medication they may be taking at the time of sampling that could account for any unusual metabolites in the extracted VOCs. No attempt was made to control the diet of the subjects being sampled.

\subsubsection{Patients with Type 2 Diabetes}

All sampling protocols were approved by the Florida International University Institutional Review Board (IRB) for research involving human subjects as well by the Jackson Health System Clinical Trials Office. Subjects were asked to read and sign an IRB-approved informed consent form prior to starting the sampling protocol. Nineteen diabetic subjects ( 11 males, 8 females), ages 25 to 60 were sampled. Nine of these subjects were inpatients at Jackson North Medical Center (North Miami Beach, FL) and were sampled at the hospital. Subjects were also asked to answer a confidential medical questionnaire form. No attempt was made to control the diet of the subjects being sampled. Blood glucose level was tested and noted at the time of sampling for all subjects.

\subsubsection{Patients with Major Depressive Disorder}

All sampling protocols were approved by the Florida International University Institutional Review Board for research involving human subjects as well by the Jackson Health System Clinical Trials Office. Subjects were asked to read and sign an IRB- 
approved informed consent form prior to starting the sampling protocol. Twenty healthy subjects ( 10 males, 10 females), ages 18 to 49 were sampled. Nine of these subjects were inpatients at Jackson North Medical Center (North Miami Beach, FL) and were sampled at the hospital. Subjects were also asked to answer a confidential medical questionnaire form. No attempt was made to control the diet of the subjects being sampled.

\subsection{Results}

\subsubsection{Evaluation of Odor Profiles of Individuals over Time}

The VOC profiles of subjects Female 4 and Male 5 were followed over a six-month period (month 0 to month 6). Fifty-five (55) compounds were extracted across the five biological specimens over six monthly samplings. Compounds that did not appear at least five times over the monthly samplings were disregarded. Table 16 and Table 17 show the common compounds that were extracted over the six-month period for Female 4 and Male 5, respectively. The VOCs from the five biological specimens included a variety of compounds including aldehydes, alcohols, alkanes, acids, esters, ketones, and aromatics. Specimen-specific compounds were detected, such as 4-heptanone and 4-nonylphenol for urine, which did not appear in the other four specimens. No single compound was found to be present across all five specimens in all six months. The variability of compounds present between subjects Female 4 and Male 5 for each specimen can be seen in Table 18 through Table 22, which lists the common compounds that were extracted among the monthly samplings of these subjects. Ten human compounds were extracted from hand odor samples, of which seven were common to both subjects Female 4 and Male 5. These common compounds were: nonanal, decanal, undecanal, tetradecane, hexadecane, 
heptadecane, and octadecane. Twenty-five compounds were extracted from buccal swab samples, of which fourteen were common to both subjects Female 4 and Male 5. These common compounds were: 2-pentylfuran, hexanoicacid, (E)-2-nonenal, (E,E)-2,4nonadienal, decanal, nonanoic acid, nonanoic acid ethyl ester, 6-dodecanone, 1tetradecene, (E)-6,10-dimethyl-5,9-undecadien-2-one, dodecanoic acid ethyl ester, 2,2'diethyl-1,1'-biphenyl, tetradecanoic acid ethyl ester, and hexadecanoic acid ethyl ester. Ten compounds were extracted from breath samples, of which eight were common to both subjects: styrene, phenol, 1,2-dichlorobenzene, 2-ethyl-1-hexanol, nonanal, decanal, butylated hydroxytoluene, and benzophenone. Sixteen compounds were extracted from blood samples, of which ten were common to both subjects. The ten common compounds were: hexanal, 2-pentylfuran, 1,2-dichlorobenzene, undecane, nonanal, decanal, tridecane, 2,4,6-trimethylbenzaldehyde, hexadecane, and 2,6-diisopropylnaphthalene. Finally, nine compounds were extracted from urine samples, of which two were common to both subjects: 4-heptanone and 4-nonylphenol. Figure 55 through Figure 59 show the variability of the relative peak area ratio patterns for the common compounds present in the monthly samplings of subjects Female 4 and Male 5.

Curran determined that multiple sampling of one individual's odor profile over time does not contain as much variation as that seen amongst members of a population using hand odor $^{84}$. Similar results have been obtained with different biological specimens, showing that peak area arrays for the common human compounds extracted among six monthly samples for an individual have greater correlation than when compared between subjects. Table 23 through Table 27 show the correlation coefficients calculated between six 
monthly samplings for subjects Female 4 and Male 5. Correlation coefficients are much higher when comparing samples of the same subject over the six-month period, as compared to the low coefficients when comparing samples between the subjects. 
Table 16. VOCs extracted over 6 months for Female 4

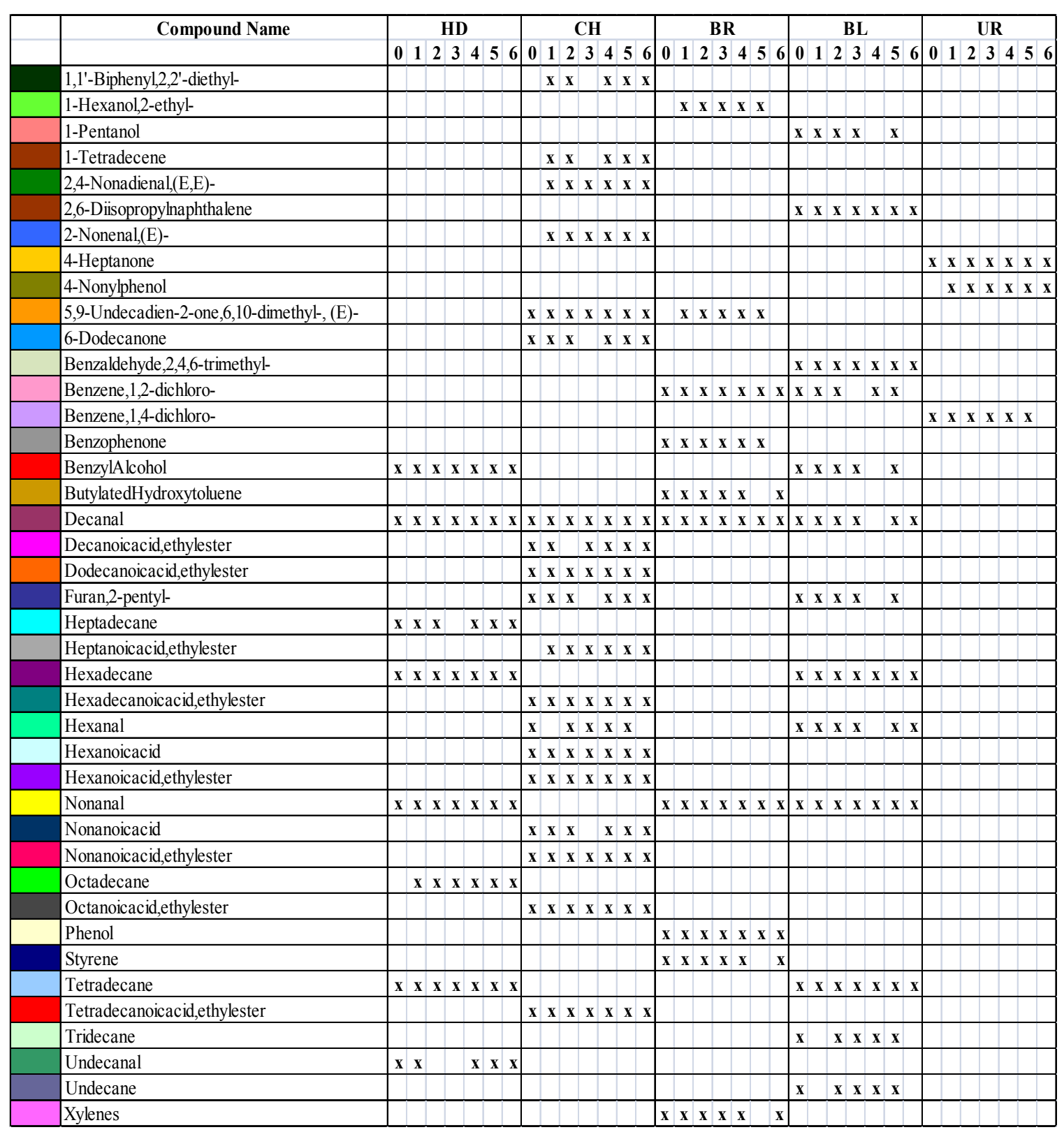


Table 17. VOCs extracted over 6 months for Male 5

\begin{tabular}{|c|c|c|c|c|c|c|c|c|c|c|c|c|c|c|c|c|c|c|c|c|c|c|c|c|c|c|c|c|c|c|c|c|c|c|c|}
\hline \multirow[t]{2}{*}{ Compound Name } & \multicolumn{7}{|c|}{ HD } & \multicolumn{7}{|c|}{$\mathrm{CH}$} & \multicolumn{7}{|c|}{ BR } & \multicolumn{7}{|c|}{ BL } & \multicolumn{7}{|c|}{ UR } \\
\hline & 0 & 1 & 2 & 3 & 4 & 5 & 6 & 0 & 1 & 2 & 3 & 4 & 5 & 6 & 0 & 1 & 2 & 3 & & 5 & 6 & & & 2 & 3 & & & 6 & 0 & & & & & & 6 \\
\hline 1,1'-Biphenyl,2,2'-diethyl- & & & & & & & & & $\mathbf{x}$ & $\mathbf{x}$ & $\mathbf{x}$ & $\mathbf{x}$ & $\mathbf{x}$ & $\mathbf{x}$ & & & & & & & & & & & & & & & & & & & & & \\
\hline 1-Dodecene & & & & & & & & $\mathbf{x}$ & $\mathbf{x}$ & $\mathbf{x}$ & & $\mathbf{x}$ & $\mathbf{x}$ & $\mathbf{x}$ & & & & & & & & & & & & & & & & & & & & & \\
\hline 1-Hexanol,2-ethyl- & & & & & & & & & & & & & & & $\mathbf{x}$ & $\mathbf{x}$ & & $\mathbf{x}$ & $\mathbf{x}$ & $\mathbf{x}$ & $\mathbf{x}$ & & & & & & & & & & & & & & \\
\hline 1-Tetradecene & & & & & & & & $\mathbf{x}$ & $\mathbf{x}$ & $\mathbf{x}$ & $\mathbf{x}$ & $\mathbf{x}$ & $\mathbf{x}$ & $\mathbf{x}$ & & & & & & & & & & & & & & & & & & & & & \\
\hline 2,4-Nonadienal,(E,E)- & & & & & & & & $\mathbf{x}$ & $\mathbf{x}$ & $\mathbf{x}$ & $\mathbf{x}$ & $\mathbf{x}$ & $\mathbf{x}$ & $\mathbf{x}$ & & & & & & & & & & & & & & & & & & & & & \\
\hline 2,6-Diisopropylnaphthalene & & & & & & & & & & & & & & & & & & & & & & $\mathbf{x}$ & $\mathbf{x}$ & & $\mathbf{x}$ & $\mathbf{x}$ & $\mathbf{x}$ & & & & & & & & \\
\hline 2-Heptanone & & & & & & & & & & & & & & & & & & & & & & $\mathbf{x}$ & $\mathbf{x}$ & $\mathbf{x}$ & & $\mathbf{x}$ & $\mathbf{x}$ & & & & & & & & \\
\hline 2-Nonenal,(E)- & & & & & & & & $\mathbf{x}$ & $\mathbf{x}$ & $\mathbf{x}$ & & $\mathbf{x}$ & $\mathbf{x}$ & $\mathbf{x}$ & & & & & & & & & & & & & & & & & & & & & \\
\hline 2-Undecanone & & & & & & & & & & & & & & & & & & & & & & $\mathbf{x}$ & & & $\mathbf{x}$ & $\mathbf{x}$ & $\mathbf{x}$ & $\mathbf{x}$ & & & & & & & \\
\hline 4-Heptanone & & & & & & & & & & & & & & & & & & & & & & & & & & & & & $\mathbf{x}$ & $\mathbf{x}$ & $\mathbf{x}$ & $\mathbf{x}$ & $\mathbf{x}$ & $\mathbf{x}$ & $\mathbf{x}$ \\
\hline 4-Nonylphenol & & & & & & & & & & & & & & & & & & & & & & & & & & & & 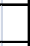 & $\mathbf{x}$ & $\mathbf{x}$ & $\mathbf{x}$ & $\mathbf{x}$ & $\mathbf{x}$ & $\mathbf{x}$ & $\mathbf{x}$ \\
\hline 4-Terpineol & & & & & & & & & & & & & & & & & & & & & & & & & & & & & & $\mathbf{x}$ & $\mathbf{x}$ & $\mathbf{x}$ & & $\mathbf{x}$ & $\mathbf{x}$ \\
\hline 5,9-Undecadien-2-one,6,10-dimethyl-, (E)- & $\mathbf{x}$ & & $\mathbf{x}$ & $\mathbf{x}$ & $\mathbf{x}$ & $\mathbf{x}$ & $\mathbf{x}$ & $\mathbf{x}$ & $\mathbf{x}$ & $\mathbf{x}$ & $\mathbf{x}$ & $\mathbf{x}$ & $\mathbf{x}$ & $\mathbf{x}$ & & & & & & & & & & & & & & & & & & & & & \\
\hline 6-Dodecanone & & & & & & & & $\mathbf{x}$ & & & $\mathbf{x}$ & $\mathbf{x}$ & $\mathbf{x}$ & $\mathbf{x}$ & & & & & & & & & & & & & & & & & & & & & \\
\hline 6-Methyl-3,5-heptadiene-2-one & & & & & & & & $\mathbf{x}$ & $\mathbf{x}$ & $\mathbf{x}$ & & $\mathbf{x}$ & $\mathbf{x}$ & & & & & & & & & & & & & & & & & & & & & & \\
\hline Benzaldehyde,2,4,6-trimethyl- & & & & & & & & & & & & & & & & & & & & & & $\mathbf{x}$ & $\mathbf{x}$ & & $\mathbf{x}$ & $\mathbf{x}$ & $\mathbf{x}$ & $\mathbf{x}$ & & & & & & & \\
\hline Benzene,1,2-dichloro- & & & & & & & & & & & & & & & $\mathbf{x}$ & $\mathbf{x}$ & $\mathbf{x}$ & & & $\mathbf{x}$ & $\mathbf{x}$ & $\mathbf{x}$ & $\mathbf{x}$ & & $\mathbf{x}$ & $\mathbf{x}$ & $\mathbf{x}$ & $\mathbf{x}$ & & & & & & & \\
\hline Benzophenone & & & & & & & & & & & & & & & $\mathbf{x}$ & & & $\mathbf{x}$ & $\mathbf{x}$ & $\mathbf{x}$ & $\mathbf{x}$ & & & & & & & & & & & & & & \\
\hline ButylatedHydroxytoluene & & & & & & & & & & & & & & & $\mathbf{x}$ & $\mathbf{x}$ & $\mathbf{x}$ & $\mathbf{x}$ & $\mathbf{x}$ & $\mathbf{x}$ & $\mathbf{x}$ & & & & & & & & & & & & & 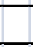 & \\
\hline Carvone & & & & & & & & & & & & & 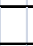 & & & & & & & & & & & & & & & . & $\mathbf{x}$ & $\mathbf{x}$ & $\mathbf{x}$ & $\mathbf{x}$ & $\mathbf{x}$ & $\mathbf{x}$ & $\mathbf{x}$ \\
\hline Caryophyllene & & & & & & & & & & $\mathbf{x}$ & $\mathbf{x}$ & $\mathbf{x}$ & $\mathbf{x}$ & $\mathbf{x}$ & & & & & - & 7 & & & & & & & & & & & & & & & \\
\hline Decanal & $\mathbf{x}$ & $\mathbf{x}$ & $\mathbf{x}$ & $\mathbf{x}$ & $\mathbf{x}$ & $\mathbf{x}$ & $\mathbf{x}$ & $\mathbf{x}$ & $\mathbf{x}$ & $\mathbf{x}$ & $\mathbf{x}$ & $\mathbf{x}$ & $\mathbf{x}$ & $\mathbf{x}$ & $\mathbf{x}$ & $\mathbf{x}$ & $\mathbf{x}$ & $\mathbf{x}$ & $\mathbf{x}$ & $\mathbf{x}$ & $\mathbf{x}$ & & & $\mathbf{x}$ & $\mathbf{x}$ & $\mathbf{x}$ & $\mathbf{x}$ & $\mathbf{x}$ & & & & & & & \\
\hline Dodecane & & & & & & & & & & & & & & & & & & & & & & $\mathbf{x}$ & & & $\mathbf{x}$ & $\mathbf{x}$ & $\mathbf{x}$ & $\mathbf{x}$ & & & & & & 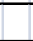 & \\
\hline Dodecanoicacid & & & & & & & & & & & & & & & & & & & & & & & & & & & & & $\mathbf{x}$ & $\mathbf{x}$ & $\mathbf{x}$ & $\mathbf{x}$ & & $\mathbf{x}$ & \\
\hline Dodecanoicacid,ethylester & & & & & & & & $\mathbf{x}$ & $\mathbf{x}$ & $\mathbf{x}$ & $\mathbf{x}$ & $\mathbf{x}$ & $\mathbf{x}$ & $\mathbf{x}$ & & & & & & & & & & & - & & & & & & & & & & \\
\hline Furan,2-pentyl- & & & & & 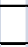 & 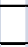 & 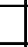 & $\mathbf{x}$ & $\mathbf{x}$ & $\mathbf{x}$ & $\mathbf{x}$ & $\mathbf{x}$ & $\mathbf{x}$ & $\mathbf{x}$ & & & & & 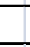 & & & $\mathbf{x}$ & $\mathbf{x}$ & $\mathbf{x}$ & $\mathbf{x}$ & $\mathbf{x}$ & $\mathbf{x}$ & $\mathbf{x}$ & & & & & & & \\
\hline Heptadecane & $\mathbf{x}$ & $\mathbf{x}$ & $\mathbf{x}$ & $\mathbf{x}$ & $\mathbf{x}$ & $\mathbf{x}$ & $\mathbf{x}$ & & & & & & & & & & & & & & & & & & & & & & & & & & & & \\
\hline Hexadecane & $\mathbf{x}$ & $\mathbf{x}$ & $\mathbf{x}$ & $\mathbf{x}$ & $\mathbf{x}$ & $\mathbf{x}$ & $\mathbf{x}$ & & & & & & & & & & & & & & & $\mathbf{x}$ & $\mathbf{x}$ & $\mathbf{x}$ & $\mathbf{x}$ & $\mathbf{x}$ & $\mathbf{x}$ & $\mathbf{x}$ & & & & & & & \\
\hline Hexadecanoicacid,ethylester & & & & & & & & $\mathbf{x}$ & $\mathbf{x}$ & $\mathbf{x}$ & $\mathbf{x}$ & $\mathbf{x}$ & $\mathbf{x}$ & & & & & & & & & & & & & & & & & & & & & & \\
\hline Hexanal & & & & & & & & & & & & & & & & & & & & & & $\mathbf{x}$ & $\mathbf{x}$ & $\mathbf{x}$ & $\mathbf{x}$ & $\mathbf{x}$ & $\mathbf{x}$ & & & & & & & & \\
\hline Hexanoicacid & & & & & & & & $\mathbf{x}$ & $\mathbf{x}$ & $\mathbf{x}$ & $\mathbf{x}$ & $\mathbf{x}$ & $\mathbf{x}$ & $\mathbf{x}$ & & & & & & & & & & & & & & & & & & & & & \\
\hline Linalool Oxide & & & & & & & & $\mathbf{x}$ & $\mathbf{x}$ & $\mathbf{x}$ & $\mathbf{x}$ & & $\mathbf{x}$ & $\mathbf{x}$ & & & & & & & & & & & & & & & & & & & & & \\
\hline Menthol & & & & & & & & & & & & & & & & & & & & & & & & & & & & & $\mathbf{x}$ & $\mathbf{x}$ & $\mathbf{x}$ & $\mathbf{x}$ & $\mathbf{x}$ & $\mathbf{x}$ & \\
\hline Naphthalene,2-methyl- & & & & & & & & $\mathbf{x}$ & $\mathbf{x}$ & $\mathbf{x}$ & $\mathbf{x}$ & $\mathbf{x}$ & $\mathbf{x}$ & $\mathbf{x}$ & & & & & & & & & & & & & & & & & & & & & \\
\hline Nonanal & $\mathbf{x}$ & $\mathbf{x}$ & $\mathbf{x}$ & $\mathbf{x}$ & $\mathbf{x}$ & $\mathbf{x}$ & $\mathbf{x}$ & & & & & & & & $\mathbf{x}$ & $\mathbf{x}$ & $\mathbf{x}$ & $\mathbf{x}$ & $\mathbf{x}$ & $\mathbf{x}$ & $\mathbf{x}$ & $\mathbf{x}$ & $\mathbf{x}$ & $\mathbf{x}$ & $\mathbf{x}$ & $\mathbf{x}$ & $\mathbf{x}$ & $\mathbf{x}$ & & & & & & & \\
\hline Nonanoicacid & & & & & & & & $\mathbf{x}$ & $\mathbf{x}$ & $\mathbf{x}$ & $\mathbf{x}$ & $\mathbf{x}$ & $\mathbf{x}$ & $\mathbf{x}$ & & & & & & & & & & & & & & & & & & & & & \\
\hline Nonanoicacid,ethylester & & & & & & & & $\mathbf{x}$ & $\overline{\mathbf{x}}$ & $\overline{\mathbf{x}}$ & $\overline{\mathbf{x}}$ & $\mathbf{x}$ & $\overline{\mathbf{x}}$ & $\mathbf{x}$ & & & & & & & & & & & & & & & & & & & & & \\
\hline Octadecane & $\mathbf{x}$ & $\mathbf{x}$ & $\mathbf{x}$ & $\mathbf{x}$ & $\mathbf{x}$ & $\mathbf{x}$ & $\mathbf{x}$ & $\mathbf{x}$ & & $\mathbf{x}$ & $\mathbf{x}$ & $\mathbf{x}$ & $\mathbf{x}$ & & & & & & & & & & & & & & & & & & & & & & \\
\hline Phenol & & & & & & & & & & & & & & & $\mathbf{x}$ & & $\mathbf{x}$ & $\mathbf{x}$ & $\mathbf{x}$ & $\mathbf{x}$ & $\mathbf{x}$ & & & & & & & & & & & & & & \\
\hline Phenol,4-(1,1,3,3-tetramethylbutyl)- & & & & & & & & & & & & & & & & & & & & & & & & & & & & & $\mathbf{x}$ & $\mathbf{x}$ & & $\mathbf{x}$ & $\mathbf{x}$ & $\mathbf{x}$ & \\
\hline Pyrrole & & & & & & & & & & & & & & & & & & & & & & & & & & & & & $\mathbf{x}$ & $\mathbf{x}$ & & $\mathbf{x}$ & $\mathbf{x}$ & $\mathbf{x}$ & $\mathbf{x}$ \\
\hline Styrene & & & & & & & & & & & & & & & $\mathbf{x}$ & $\mathbf{x}$ & $\mathbf{x}$ & $\mathbf{x}$ & $\mathbf{x}$ & $\mathbf{x}$ & $\mathbf{x}$ & & & & & & & & & & & & & & \\
\hline Tetradecane & $\mathbf{x}$ & $\mathbf{x}$ & $\mathbf{x}$ & $\mathbf{x}$ & $\mathbf{x}$ & $\mathbf{x}$ & $\mathbf{x}$ & & & & & & & & & & & & & & & & & & & & & & & & & & & & \\
\hline Tetradecanoicacid,ethylester & & & & & & & & $\mathbf{x}$ & $\mathbf{x}$ & $\overline{\mathbf{x}}$ & $\overline{\mathbf{x}}$ & & $\mathbf{x}$ & & & & & & & & & & & & & & & & & & & & & & \\
\hline Tridecane & $\mathbf{x}$ & & & $\mathbf{x}$ & $\mathbf{x}$ & $\mathbf{x}$ & $\mathbf{x}$ & & & & & & & & & & & & & & & $\mathbf{x}$ & & & $\mathbf{x}$ & $\mathbf{x}$ & $\mathbf{x}$ & $\mathbf{x}$ & & & & & & & \\
\hline Undecanal & $\mathbf{x}$ & $\mathbf{x}$ & $\mathbf{x}$ & $\mathbf{x}$ & $\mathbf{x}$ & $\mathbf{x}$ & $\mathbf{x}$ & & & & & & & & & & & & & & & & & & & & & & & & & & & & \\
\hline Undecane & & & & & & & & & & & & & & & & & & & & & & $\mathbf{x}$ & & & $\mathbf{x}$ & $\mathbf{x}$ & $\mathbf{x}$ & $\mathbf{x}$ & & & & & & & \\
\hline
\end{tabular}


Table 18. VOCs extracted from hand odor from F4 and M5 over 6 months

\begin{tabular}{|c|c|c|c|c|c|c|c|c|c|c|c|c|c|c|}
\hline \multirow{2}{*}{$\begin{array}{l}\text { RT } \\
(\mathrm{min})\end{array}$} & \multirow[t]{2}{*}{ Compound Name } & \multicolumn{7}{|c|}{ F4 } & \multicolumn{6}{|c|}{ M5 } \\
\hline & & $\mathbf{0}$ & 1 & 2 & & & 5 & & 0 & & & 3 & & 56 \\
\hline 11.39 & BenzylAlcohol & $\mathbf{x}$ & $\mathbf{x}$ & $\mathbf{x}$ & $\mathbf{x}$ & $\mathbf{x}$ & $\mathbf{x}$ & $\mathbf{x}$ & & & & & & \\
\hline 12.64 & Nonanal & $\mathbf{x}$ & $\mathbf{x}$ & $\mathbf{x}$ & $\mathbf{x}$ & $\overline{\mathbf{x}}$ & $\mathbf{x}$ & & $\mathbf{X}$ & $\mathbf{x}$ & $\mathbf{x}$ & $\mathbf{x}$ & & \begin{tabular}{l|l}
$\mathbf{X}$ & $\mathbf{x}$
\end{tabular} \\
\hline 14.27 & Decanal & $\mathbf{x}$ & $\mathbf{x}$ & $\underline{\mathbf{x}}$ & $\underline{\mathbf{x}}$ & $\mathbf{x}$ & $\mathbf{x}$ & & $\underline{\mathbf{x}}$ & $\mathbf{x}$ & $\mathbf{x}$ & $\mathbf{x}$ & & \begin{tabular}{l|l}
$\mathbf{X}$ & $\mathbf{x}$ \\
\end{tabular} \\
\hline 15.62 & Tridecane & & & & & & & & $\underline{\mathbf{x}}$ & & & $\mathbf{x}$ & & $\mathbf{X} \mathbf{x}$ \\
\hline 15.74 & Undecanal & $\mathbf{x}$ & $\mathbf{x}$ & & & $\mathbf{x}$ & $\underline{\mathbf{x}}$ & $\underline{x}$ & $\underline{\mathbf{x}}$ & $\mathbf{X}$ & $\mathbf{x}$ & $\mathbf{x}$ & & \begin{tabular}{l|l}
$\mathbf{x}$ & $\mathbf{x}$ \\
\end{tabular} \\
\hline 16.49 & Hexadecane & $\mathbf{x}$ & $\overline{\mathbf{x}}$ & $\mathbf{x}$ & $\mathbf{X}$ & $\mathbf{x}$ & $\mathbf{x}$ & $\mathbf{x}$ & $\mathbf{x}$ & $\mathbf{X}$ & $\mathbf{x}$ & $\mathbf{x}$ & & \begin{tabular}{l|l}
$\mathbf{X}$ & $\mathbf{x}$ \\
\end{tabular} \\
\hline 17.63 & Heptadecane & $\mathbf{x}$ & $\mathbf{x}$ & $\mathbf{x}$ & & $\mathbf{x}$ & $\mathbf{x}$ & $\mathbf{x}$ & $\mathbf{x}$ & $\mathbf{x}$ & $\mathbf{x}$ & $\mathbf{x}$ & & \begin{tabular}{l|l}
$\mathbf{x}$ \\
\end{tabular} \\
\hline 17.69 & 5,9-Undecadien-2-one,6,10-dimethyl-, (E)- & & & & & & & & $\mathbf{x}$ & & $\mathbf{x}$ & $\mathbf{x}$ & & $\mathbf{x} \mathbf{x}$ \\
\hline 20.17 & Tetradecane & $\mathbf{x}$ & $\mathbf{x}$ & $\underline{\mathbf{x}}$ & $\underline{\mathbf{x}}$ & $\mathbf{x}$ & $\underline{\mathbf{x}}$ & $\mathrm{x}$ & $\underline{\mathbf{x}}$ & $\underline{\boldsymbol{x}}$ & $\underline{\mathbf{x}}$ & $\mathbf{x}$ & & $\mathbf{x} \mathbf{x}$ \\
\hline 21.65 & Octadecane & & $\mathbf{x}$ & $\mathbf{x}$ & $\mathbf{x}$ & $\mathbf{x}$ & $\mathbf{x}$ & & $\mathbf{x}$ & & $\mathbf{x}$ & $\mathbf{x}$ & & $\overline{\mathbf{x}} \mathbf{x}$ \\
\hline
\end{tabular}

Figure 55. Common compounds extracted among monthly samplings of hand odor from subjects F4 and M5

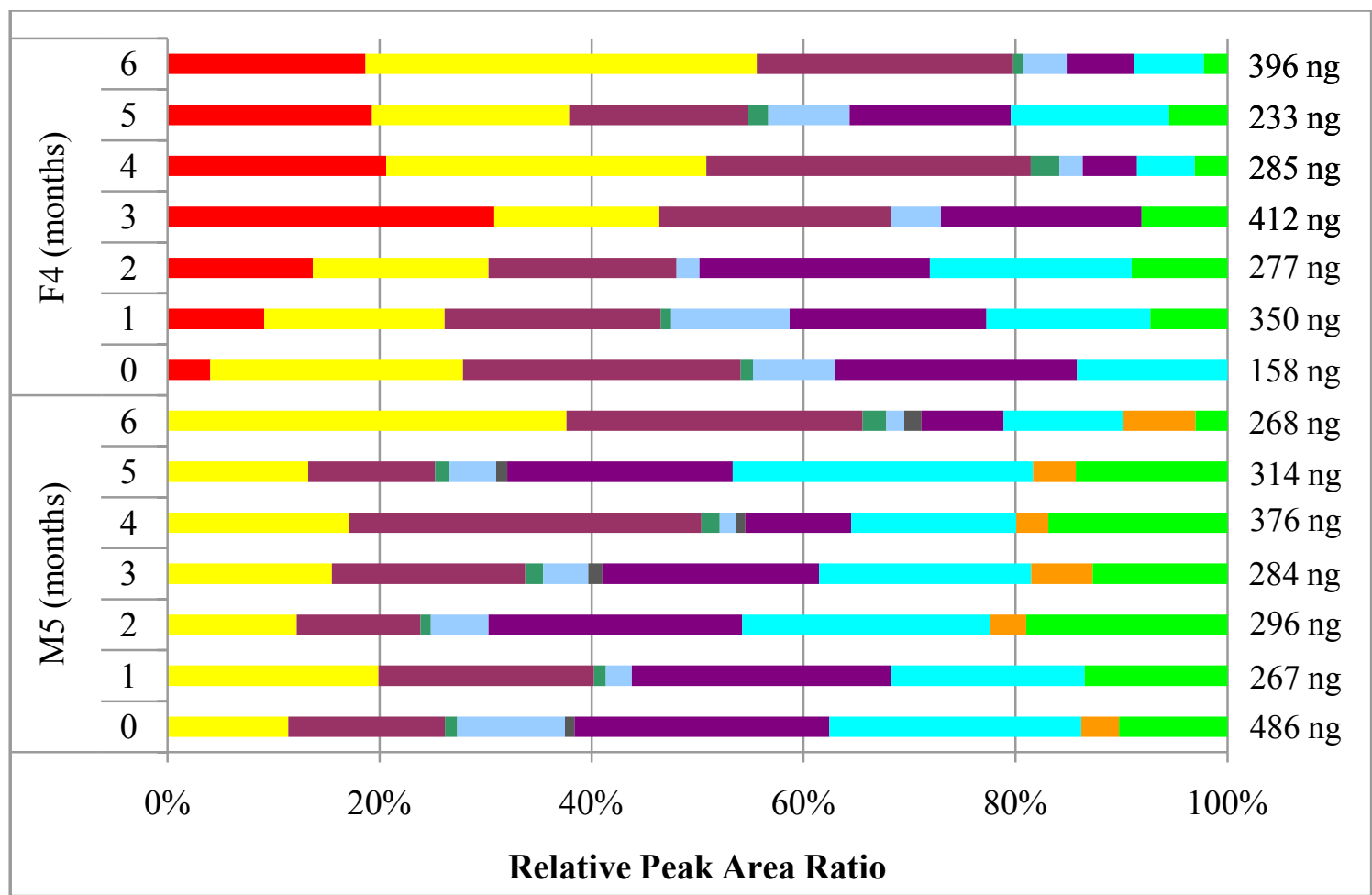


Table 19. VOCs extracted from buccal swabs from F4 and M5 over 6 months

\begin{tabular}{|c|c|c|c|c|c|c|c|c|c|c|c|c|c|c|c|}
\hline \multirow{2}{*}{\begin{tabular}{|c|} 
RT \\
(min)
\end{tabular}} & \multirow[t]{2}{*}{ Compound Name } & \multicolumn{7}{|c|}{ F4 } & \multicolumn{7}{|c|}{ M5 } \\
\hline & & $\mathbf{0}$ & $\mathbf{1}$ & 2 & 3 & 4 & 5 & 6 & $\mathbf{0}$ & $\mathbf{1}$ & 2 & 3 & 4 & 5 & 6 \\
\hline 4.63 & Hexanal & $\mathbf{x}$ & & $\mathbf{x}$ & $\mathbf{x}$ & $\mathbf{x}$ & $\mathbf{x}$ & & & & & & & & \\
\hline 7.98 & Furan,2-pentyl- & $\overline{\mathbf{x}}$ & $\mathbf{x}$ & $\mathbf{x}$ & & $\mathbf{x}$ & $\mathbf{x}$ & $\mathbf{x}$ & $\mathbf{x}$ & $\mathbf{x}$ & $\mathbf{x}$ & $\mathbf{x}$ & $\mathbf{x}$ & $\mathbf{x}$ & $\mathbf{x}$ \\
\hline 8.12 & Hexanoicacid,ethylester & $\mathbf{x}$ & $\mathbf{x}$ & $\mathbf{x}$ & $\mathbf{x}$ & $\mathbf{x}$ & $\mathbf{x}$ & $\mathbf{x}$ & & & & & & & \\
\hline 9.25 & Hexanoicacid & $\mathbf{x}$ & $\mathbf{x}$ & $\mathbf{x}$ & $\mathbf{x}$ & $\mathbf{x}$ & $\mathbf{x}$ & $\mathbf{x}$ & $\mathbf{x}$ & $\mathbf{x}$ & $\mathbf{x}$ & $\mathbf{x}$ & $\mathbf{x}$ & $\mathbf{x}$ & $\mathbf{x}$ \\
\hline 9.37 & Linalool Oxide & & & & & & & & $\mathbf{x}$ & $\mathbf{x}$ & $\mathbf{x}$ & $\mathbf{x}$ & & $\overline{\mathbf{x}}$ & $\mathbf{x}$ \\
\hline 9.73 & Heptanoicacid,ethylester & & $\mathbf{x}$ & $\mathbf{x}$ & $\mathbf{x}$ & $\mathbf{x}$ & $\mathbf{x}$ & $\mathbf{x}$ & & & & & & & \\
\hline 9.87 & 6-Methyl-3,5-heptadiene-2-one & & & & & & & & $\mathbf{x}$ & $\mathbf{x}$ & $\mathbf{x}$ & & $\mathbf{x}$ & $\mathbf{x}$ & \\
\hline 10.70 & 2-Nonenal,(E)- & & $\mathbf{x}$ & $\mathbf{x}$ & $\mathbf{x}$ & $\mathbf{x}$ & $\mathbf{x}$ & $\mathbf{x}$ & $\mathbf{x}$ & $\mathbf{x}$ & $\mathbf{x}$ & & $\overline{\mathbf{x}}$ & $\mathbf{x}$ & $\mathbf{x}$ \\
\hline 11.25 & Octanoicacid,ethylester & $\mathbf{x}$ & $\mathbf{x}$ & $\mathbf{x}$ & $\mathbf{x}$ & $\mathbf{x}$ & $\mathbf{x}$ & $\mathbf{x}$ & & & & & & & \\
\hline 11.36 & 2,4-Nonadienal,(E,E)- & & $\mathbf{x}$ & $\mathbf{x}$ & $\mathbf{x}$ & $\mathbf{x}$ & $\mathbf{x}$ & $\mathbf{x}$ & $\mathbf{x}$ & $\mathbf{x}$ & $\mathbf{x}$ & $\mathbf{x}$ & $\mathbf{x}$ & $\mathbf{x}$ & $\mathbf{x}$ \\
\hline 11.39 & Decanal & $\mathbf{x}$ & $\mathbf{x}$ & $\mathbf{x}$ & $\mathbf{x}$ & $\mathbf{x}$ & $\mathbf{x}$ & $\mathbf{x}$ & $\mathbf{x}$ & $\mathbf{x}$ & $\mathbf{x}$ & $\mathbf{x}$ & $\mathbf{x}$ & $\mathbf{x}$ & $\mathbf{x}$ \\
\hline 12.48 & Nonanoicacid & $\mathbf{x}$ & $\mathbf{x}$ & $\mathbf{x}$ & & $\mathbf{x}$ & $\mathbf{x}$ & $\mathbf{x}$ & $\mathbf{x}$ & $\mathbf{x}$ & $\mathbf{x}$ & $\mathbf{x}$ & $\mathbf{x}$ & $\overline{\mathbf{x}}$ & $\mathbf{x}$ \\
\hline 12.67 & Nonanoicacid,ethylester & $\mathbf{x}$ & $\mathbf{x}$ & $\mathbf{x}$ & $\mathbf{x}$ & $\mathbf{x}$ & $\mathbf{x}$ & $\mathbf{x}$ & $\mathbf{x}$ & $\mathbf{x}$ & $\mathbf{x}$ & $\mathbf{x}$ & $\mathbf{x}$ & $\mathbf{x}$ & $\mathbf{x}$ \\
\hline 12.76 & Naphthalene,2-methyl- & & & & & & & & $\mathbf{x}$ & $\mathbf{x}$ & $\mathbf{x}$ & $\mathbf{x}$ & $\mathbf{x}$ & $\mathbf{x}$ & $\mathbf{x}$ \\
\hline 13.71 & 6-Dodecanone & $\mathbf{x}$ & $\mathbf{x}$ & $\mathbf{x}$ & & $\mathbf{x}$ & $\mathbf{x}$ & $\mathbf{x}$ & $\mathbf{x}$ & & & $\mathbf{x}$ & $\mathbf{x}$ & $\mathbf{x}$ & $\mathbf{x}$ \\
\hline 13.91 & Decanoicacid,ethylester & $\mathbf{x}$ & $\mathbf{x}$ & & $\mathbf{x}$ & $\mathbf{x}$ & $\mathbf{x}$ & $\mathbf{x}$ & & & & & & & \\
\hline 13.95 & 1-Tetradecene & & $\mathbf{x}$ & $\mathbf{x}$ & & $\mathbf{x}$ & $\mathbf{x}$ & $\mathbf{x}$ & $\mathbf{x}$ & $\mathbf{x}$ & $\mathbf{x}$ & $\mathbf{x}$ & $\mathbf{x}$ & $\mathbf{x}$ & $\mathbf{x}$ \\
\hline 14.49 & Caryophyllene & & & & & & & & & & $\mathbf{x}$ & $\mathbf{x}$ & $\mathbf{x}$ & $\mathbf{x}$ & $\mathbf{x}$ \\
\hline 14.76 & 5,9-Undecadien-2-one,6,10-dimethyl-, (E)- & $\mathbf{x}$ & $\mathbf{x}$ & $\mathbf{x}$ & $\mathbf{x}$ & $\mathbf{x}$ & $\mathbf{x}$ & $\mathbf{x}$ & $\mathbf{x}$ & $\mathbf{x}$ & $\mathbf{x}$ & $\mathbf{x}$ & $\mathbf{x}$ & $\mathbf{x}$ & $\mathbf{x}$ \\
\hline 15.00 & 1-Dodecene & & & & & & & & $\mathbf{x}$ & $\overline{\mathbf{x}}$ & $\mathbf{x}$ & & $\mathbf{x}$ & $\mathbf{x}$ & $\mathbf{x}$ \\
\hline 16.44 & Dodecanoicacid,ethylester & $\mathbf{x}$ & $\mathbf{x}$ & $\mathbf{x}$ & $\mathbf{x}$ & $\mathbf{x}$ & $\mathbf{x}$ & $\mathbf{x}$ & $\mathbf{x}$ & $\mathbf{x}$ & $\mathbf{x}$ & $\mathbf{x}$ & $\mathbf{x}$ & $\mathbf{x}$ & $\mathbf{x}$ \\
\hline 17.70 & 1,1'-Biphenyl,2,2'-diethyl- & & $\mathbf{x}$ & $\mathbf{x}$ & & $\mathbf{x}$ & $\mathbf{x}$ & $\mathbf{x}$ & & $\mathbf{x}$ & $\mathbf{x}$ & $\mathbf{x}$ & $\mathbf{x}$ & $\mathbf{x}$ & $\mathbf{x}$ \\
\hline 18.57 & Tetradecanoicacid,ethylester & $\mathbf{x}$ & $\mathbf{x}$ & $\mathbf{x}$ & $\mathbf{x}$ & $\mathbf{x}$ & $\mathbf{x}$ & $\mathbf{x}$ & $\mathbf{x}$ & $\mathbf{x}$ & $\mathbf{x}$ & $\mathbf{x}$ & & $\mathbf{x}$ & \\
\hline 18.70 & Octadecane & & & & & & & & $\mathbf{x}$ & & $\mathbf{x}$ & $\mathbf{x}$ & $\mathbf{x}$ & $\mathbf{x}$ & \\
\hline 20.66 & Hexadecanoicacid,ethylester & $\mathbf{x}$ & $\mathbf{x}$ & $\mathbf{x}$ & $\mathbf{x}$ & $\mathbf{x}$ & $\mathbf{x}$ & $\mathbf{x}$ & $\mathbf{x}$ & $\mathbf{x}$ & $\mathbf{x}$ & $\mathbf{x}$ & $\mathbf{x}$ & $\mathbf{x}$ & \\
\hline
\end{tabular}

Figure 56. Common compounds extracted among monthly samplings of buccal swabs from subjects F4 and M5

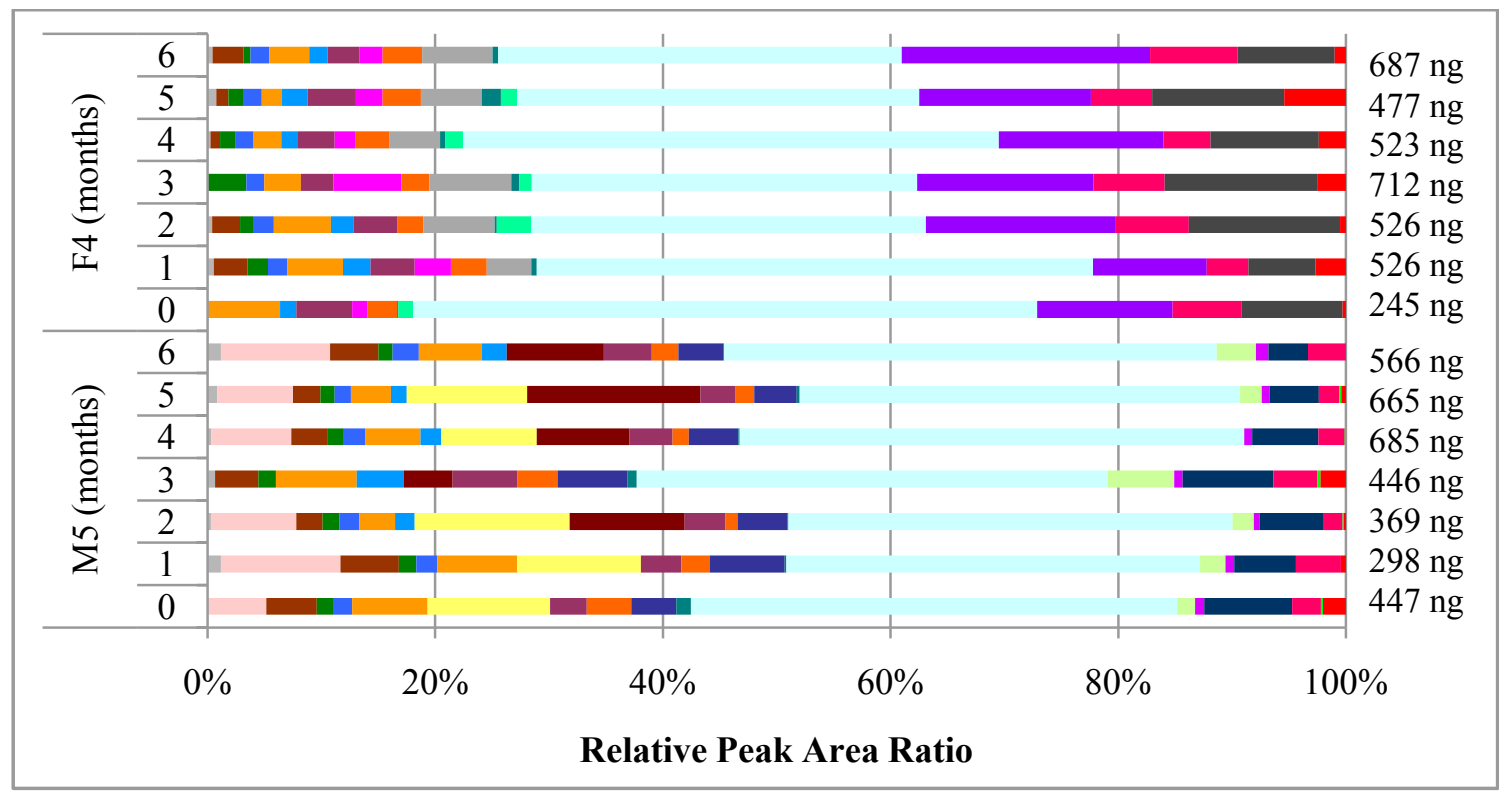


Table 20. VOCs extracted from breath from F4 and M5 over 6 months

\begin{tabular}{|c|c|c|c|c|c|c|c|c|c|c|c|c|c|c|c|}
\hline \multirow{2}{*}{$\begin{array}{c}\text { RT } \\
(\mathbf{m i n}) \\
\end{array}$} & \multirow[t]{2}{*}{ Compound Name } & \multicolumn{7}{|c|}{ F4 } & \multicolumn{7}{|c|}{ M5 } \\
\hline & & $\mathbf{0}$ & 1 & 2 & 3 & 4 & 5 & 6 & 0 & 1 & 2 & 3 & 4 & & 6 \\
\hline 5.85 & Xylenes & $\mathbf{x}$ & $\mathbf{x}$ & $\underline{x}$ & $\underline{x}$ & $\underline{x}$ & & $\underline{\mathbf{x}}$ & & & & & & & \\
\hline 6.23 & Styrene & $\mathbf{x}$ & $\mathbf{x}$ & $\underline{x}$ & $\underline{x}$ & $\underline{x}$ & & $\mathbf{x}$ & $\mathbf{x}$ & $\mathbf{x}$ & $\mathbf{x}$ & $\mathbf{x}$ & $\underline{\mathbf{x}}$ & $\mathbf{x}$ & $\mathbf{x}$ \\
\hline 7.79 & Phenol & $\mathbf{x}$ & $\mathbf{x}$ & $\mathbf{x}$ & $\mathbf{x}$ & $\mathbf{x}$ & $\mathbf{x}$ & $\mathbf{x}$ & $\mathbf{x}$ & & $\mathbf{x}$ & $\mathbf{x}$ & $\mathbf{x}$ & $\mathbf{x}$ & $\mathbf{x}$ \\
\hline 8.35 & Benzene,1,2-dichloro- & $\mathbf{x}$ & $\mathbf{x}$ & $\mathbf{x}$ & $\mathbf{x}$ & $\mathbf{x}$ & $\mathbf{x}$ & $\mathbf{x}$ & $\mathbf{x}$ & $\mathbf{x}$ & $\mathbf{x}$ & & & & $\mathbf{x}$ \\
\hline 8.60 & 1-Hexanol,2-ethyl- & & $\mathbf{x}$ & $\mathbf{x}$ & $\mathbf{x}$ & $\mathbf{x}$ & $\underline{x}$ & & $\mathbf{x}$ & $\mathbf{x}$ & & $\mathbf{x}$ & $\mathbf{x}$ & & $\mathbf{x}$ \\
\hline 9.83 & Nonanal & $\mathbf{x}$ & $\mathbf{x}$ & $\mathbf{x}$ & $\mathbf{x}$ & $\underline{\mathbf{x}}$ & $\underline{x}$ & $\mathbf{x}$ & $\mathbf{x}$ & $\mathbf{x}$ & $\mathbf{x}$ & $\mathbf{x}$ & $\mathbf{x}$ & $\mathbf{x}$ & $\mathbf{x}$ \\
\hline 11.38 & Decanal & $\mathbf{x}$ & $\mathbf{x}$ & $\mathbf{x}$ & $\mathbf{x}$ & $\mathbf{x}$ & $\mathbf{x}$ & $\mathbf{x}$ & $\mathbf{x}$ & $\mathbf{x}$ & $\mathbf{x}$ & $\mathbf{x}$ & $\underline{\mathbf{x}}$ & $\mathbf{x}$ & $\mathbf{x}$ \\
\hline 14.75 & 5,9-Undecadien-2-one,6,10-dimethyl-, (E)- & & $\mathbf{x}$ & $\mathbf{x}$ & $\mathbf{x}$ & $\mathbf{x}$ & $\mathbf{x}$ & & & & & & & & \\
\hline 15.56 & ButylatedHydroxytoluene & $\mathbf{x}$ & $\mathbf{x}$ & $\mathbf{x}$ & $\mathbf{x}$ & $\mathbf{x}$ & & $\mathbf{x}$ & $\mathbf{x}$ & $\mathbf{x}$ & $\mathbf{x}$ & $\mathbf{x}$ & $\underline{\mathbf{x}}$ & & $\underline{\mathbf{x}}$ \\
\hline 16.99 & Benzophenone & $\mathbf{x}$ & $\mathbf{x}$ & $\mathbf{x}$ & $\mathbf{x}$ & $\underline{x}$ & $\underline{\mathbf{x}}$ & & $\mathbf{x}$ & & & $\mathbf{x}$ & $\mathbf{x}$ & $\mathbf{x}$ & $\mathbf{X}$ \\
\hline
\end{tabular}

Figure 57. Common compounds extracted among monthly samplings of breath from subjects F4 and M5

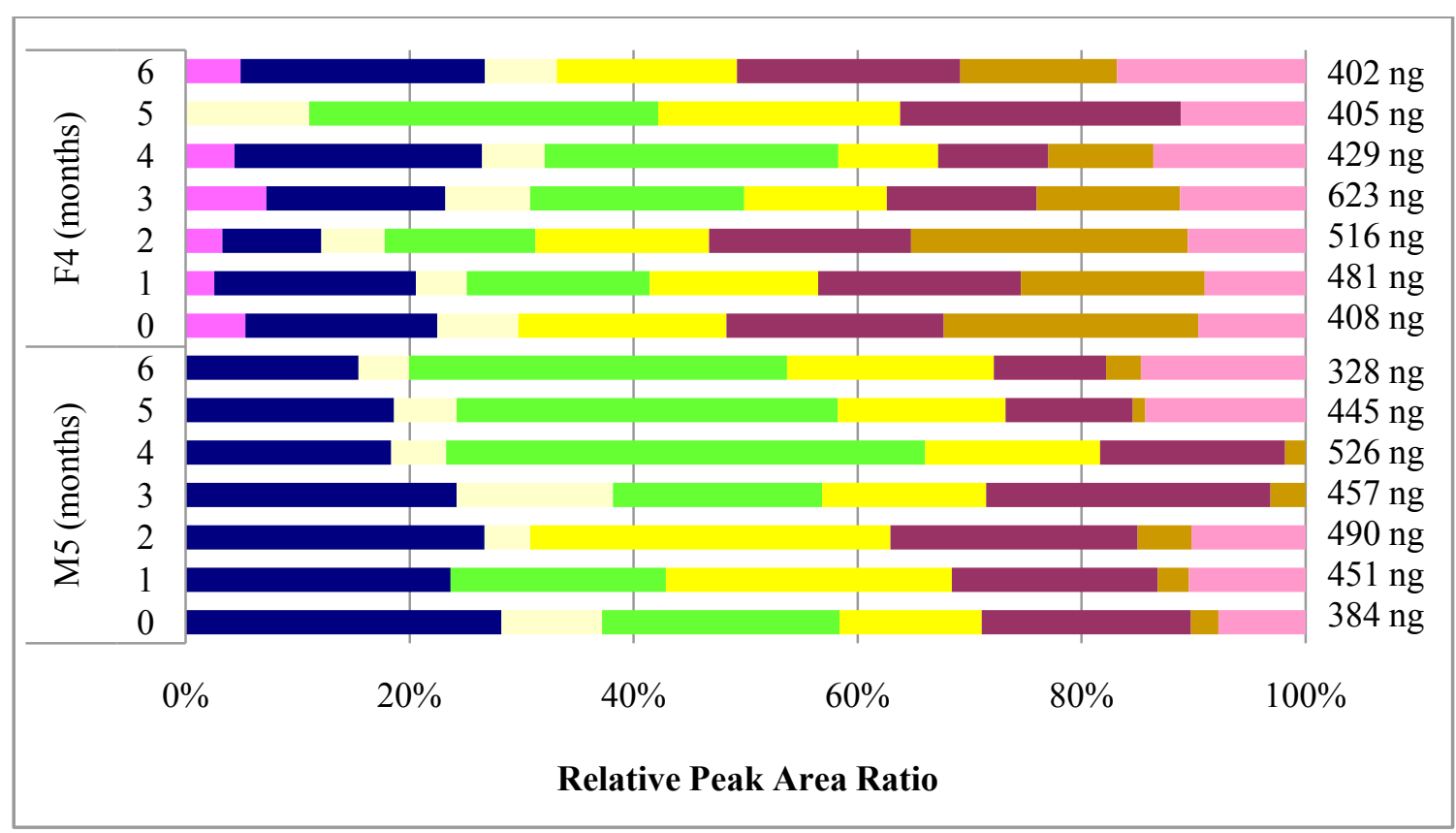


Table 21. VOCs extracted from blood from F4 and M5 over 6 months

\begin{tabular}{|c|c|c|c|c|c|c|c|c|c|c|c|c|c|c|c|}
\hline \multirow{2}{*}{$\begin{array}{c}\mathrm{RT} \\
(\mathrm{min})\end{array}$} & \multirow[t]{2}{*}{ Compound Name } & \multicolumn{7}{|c|}{ F4 } & \multicolumn{7}{|c|}{ M5 } \\
\hline & & $\mathbf{0}$ & 1 & 2 & 3 & 4 & 5 & 6 & $\mathbf{0}$ & 1 & 2 & 3 & 4 & 5 & 6 \\
\hline 3.72 & 1-Pentanol & $\mathbf{x}$ & $\mathbf{x}$ & $\mathbf{x}$ & $\mathbf{x}$ & & $\mathbf{x}$ & & & & & & & & \\
\hline 4.14 & Hexanal & $\mathbf{x}$ & $\mathbf{x}$ & $\mathbf{x}$ & $\mathbf{x}$ & & $\mathbf{x}$ & $\mathbf{x}$ & $\mathbf{x}$ & $\mathbf{x}$ & $\mathbf{x}$ & $\mathbf{x}$ & $\mathbf{x}$ & $\mathbf{x}$ & \\
\hline 5.50 & 2-Heptanone & & & & & & & & $\mathbf{x}$ & $\mathbf{x}$ & $\mathbf{x}$ & & $\mathbf{x}$ & $\mathbf{x}$ & \\
\hline 7.1 & Furan,2-pentyl- & $\mathbf{x}$ & $\mathbf{x}$ & $\mathbf{x}$ & $\mathbf{x}$ & & $\mathbf{x}$ & & $\mathbf{x}$ & $\mathbf{x}$ & $\mathbf{x}$ & $\mathbf{x}$ & $\mathbf{x}$ & $\mathbf{x}$ & $\mathbf{x}$ \\
\hline 7.47 & Benzene,1,2-dichloro- & $\mathbf{x}$ & $\mathbf{x}$ & $\mathbf{x}$ & & $\mathbf{x}$ & $\mathbf{x}$ & & $\mathbf{x}$ & $\mathbf{x}$ & & $\mathbf{x}$ & $\mathbf{x}$ & $\mathbf{x}$ & $\mathbf{x}$ \\
\hline 7.79 & BenzylAlcohol & $\mathbf{x}$ & $\mathbf{x}$ & $\mathbf{x}$ & $\mathbf{x}$ & & $\mathbf{x}$ & & & & & & & & \\
\hline 8.77 & Undecane & $\mathbf{x}$ & & $\mathbf{x}$ & $\mathbf{x}$ & $\mathbf{x}$ & $\mathbf{x}$ & & $\mathbf{x}$ & & & $\mathbf{x}$ & $\mathbf{x}$ & $\mathbf{x}$ & $\mathbf{x}$ \\
\hline 8.8 & Nonanal & $\mathbf{x}$ & $\mathbf{x}$ & $\mathbf{x}$ & $\mathbf{x}$ & $\mathbf{x}$ & $\mathbf{x}$ & $\mathbf{x}$ & $\mathbf{x}$ & $\mathbf{x}$ & $\mathbf{x}$ & $\mathbf{x}$ & $\mathbf{x}$ & $\mathbf{x}$ & $\mathbf{x}$ \\
\hline 10.29 & Dodecane & & & & & & & & $\mathbf{x}$ & & & $\mathbf{x}$ & $\mathbf{x}$ & $\mathbf{x}$ & $\mathbf{x}$ \\
\hline 10.39 & Decanal & $\mathbf{x}$ & $\mathbf{x}$ & $\mathbf{x}$ & $\mathbf{x}$ & & $\mathbf{x}$ & $\mathbf{x}$ & & & $\mathbf{x}$ & $\mathbf{x}$ & $\mathbf{x}$ & $\mathbf{x}$ & $\mathbf{x}$ \\
\hline 11.63 & 2-Undecanone & & & & & & & & $\mathbf{x}$ & & & $\mathbf{x}$ & $\mathbf{x}$ & $\mathbf{x}$ & $\mathbf{x}$ \\
\hline 11.63 & Tridecane & $\mathbf{x}$ & & $\mathbf{x}$ & $\mathbf{x}$ & $\mathbf{x}$ & $\mathbf{x}$ & & $\mathbf{x}$ & & & $\mathbf{x}$ & $\mathbf{x}$ & $\mathbf{x}$ & $\mathbf{x}$ \\
\hline 11.84 & Benzaldehyde,2,4,6-trimethyl- & $\mathbf{x}$ & $\mathbf{x}$ & $\mathbf{x}$ & $\mathbf{x}$ & $\mathbf{x}$ & $\mathbf{x}$ & $\mathbf{x}$ & $\mathbf{x}$ & $\mathbf{x}$ & & $\mathbf{x}$ & $\mathbf{x}$ & $\mathbf{x}$ & $\mathbf{x}$ \\
\hline 13.04 & Tetradecane & $\mathbf{x}$ & $\mathbf{x}$ & $\mathbf{x}$ & $\mathbf{x}$ & $\mathbf{x}$ & $\mathbf{x}$ & $\mathbf{x}$ & & & & & & & \\
\hline 15.49 & Hexadecane & $\mathbf{x}$ & $\mathbf{x}$ & $\mathbf{x}$ & $\underline{\mathbf{x}}$ & $\mathbf{x}$ & $\mathbf{x}$ & $\mathbf{x}$ & $\mathbf{x}$ & $\mathbf{x}$ & $\mathbf{x}$ & $\mathbf{x}$ & $\underline{\mathbf{x}}$ & $\mathbf{x}$ & $\mathbf{x}$ \\
\hline 16.99 & 2,6-Diisopropylnaphthalene & $\mathbf{x}$ & $\mathbf{x}$ & $\mathbf{x}$ & $\mathbf{x}$ & $\mathbf{x}$ & $\mathbf{X}$ & $\mathbf{x}$ & $\mathbf{x}$ & $\mathbf{x}$ & & $\mathbf{x}$ & $\mathbf{x}$ & $\mathbf{x}$ & \\
\hline
\end{tabular}

Figure 58. Common compounds extracted among monthly samplings of blood from subjects F4 and M5

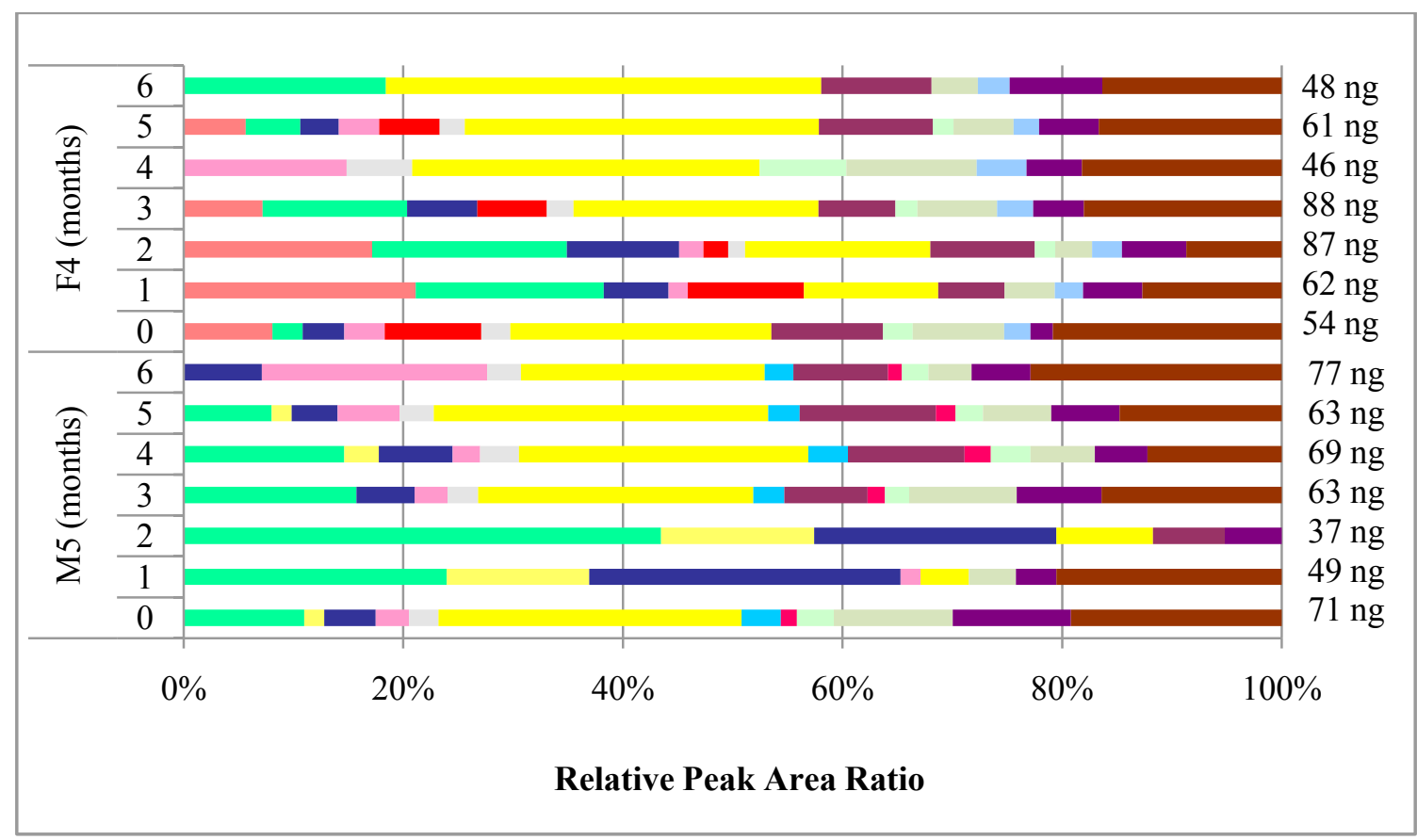


Table 22. VOCs extracted from urine from F4 and M5 over 6 months

\begin{tabular}{|c|c|c|c|c|c|c|c|c|c|c|c|c|}
\hline \multirow{2}{*}{$\begin{array}{c}\text { RT } \\
\text { (min) }\end{array}$} & \multirow[t]{2}{*}{ Compound Name } & \multicolumn{6}{|c|}{ F4 } & \multicolumn{5}{|c|}{ M5 } \\
\hline & & $\mathbf{0}$ & 12 & & & 5 & 6 & $\mathbf{0}$ & 1 & 23 & & 56 \\
\hline 4.50 & Pyrrole & & & & & & & $\mathbf{x}$ & $\mathbf{x}$ & $\mathbf{x}$ & $\mathbf{x}$ & $\mathbf{X} \mathbf{x}$ \\
\hline 7.51 & 4-Heptanone & $\mathbf{x}$ & $\begin{array}{ll}\mathrm{X} \\
\end{array}$ & $x$ & $x$ & $\mathbf{x}$ & $\mathbf{x}$ & $\mathbf{x}$ & $\mathbf{x}$ & \begin{tabular}{l|l}
$\mathbf{X}$ & $\mathbf{x}$ \\
\end{tabular} & $\mathbf{x}$ & \begin{tabular}{l|l}
$\mathbf{X}$ & $\mathbf{X}$ \\
\end{tabular} \\
\hline 9.85 & Benzene,1,4-dichloro- & $\overline{\mathbf{x}}$ & $\bar{x}$ & $x$ & $\mathbf{X}$ & $\mathbf{x}$ & & & & & & \\
\hline 11.82 & Menthol & & & & & & & $\mathbf{x}$ & $\mathbf{x}$ & \begin{tabular}{l|l}
$\mathbf{x}$ \\
\end{tabular} & $\mathbf{x}$ & $\mathbf{x}$ \\
\hline 11.88 & 4-Terpineol & & & & & & & & $\mathbf{x}$ & \begin{tabular}{l|l}
$\mathbf{x}$ & $\mathbf{x}$ \\
\end{tabular} & & \begin{tabular}{l|l}
$\mathbf{x}$ & $\mathbf{x}$ \\
\end{tabular} \\
\hline 12.58 & Carvone & & & & & & & $\mathbf{x}$ & $\mathbf{x}$ & \begin{tabular}{l|l}
$\mathbf{x}$ & $\mathbf{x}$ \\
\end{tabular} & $\mathbf{x}$ & \begin{tabular}{l|l}
$\mathbf{x}$ & $\mathbf{X}$ \\
\end{tabular} \\
\hline 15.30 & Dodecanoicacid & & & & & & & $\mathbf{x}$ & $\mathbf{x}$ & $\overline{\mathbf{X}} \mathbf{x}$ & & $\mathbf{x}$ \\
\hline 16.51 & Phenol,4-(1,1,3,3-tetramethylbutyl)- & & & & & & & $\mathbf{x}$ & $\mathbf{x}$ & $\mathbf{x}$ & $\mathbf{x}$ & $\mathbf{x}$ \\
\hline 16.62 & 4-Nonylphenol & $I$ & $\mathrm{x} x$ & 1 & $x$ & $\mathbf{x}$ & $\mathbf{x}$ & $\mathbf{x}$ & $\mathbf{x}$ & & $\mathbf{x}$ & \begin{tabular}{|l|l|}
$\mathbf{X}$ & $\mathbf{X}$ \\
\end{tabular} \\
\hline
\end{tabular}

Figure 59. Common compounds extracted among monthly samplings of urine from subjects F4 and M5

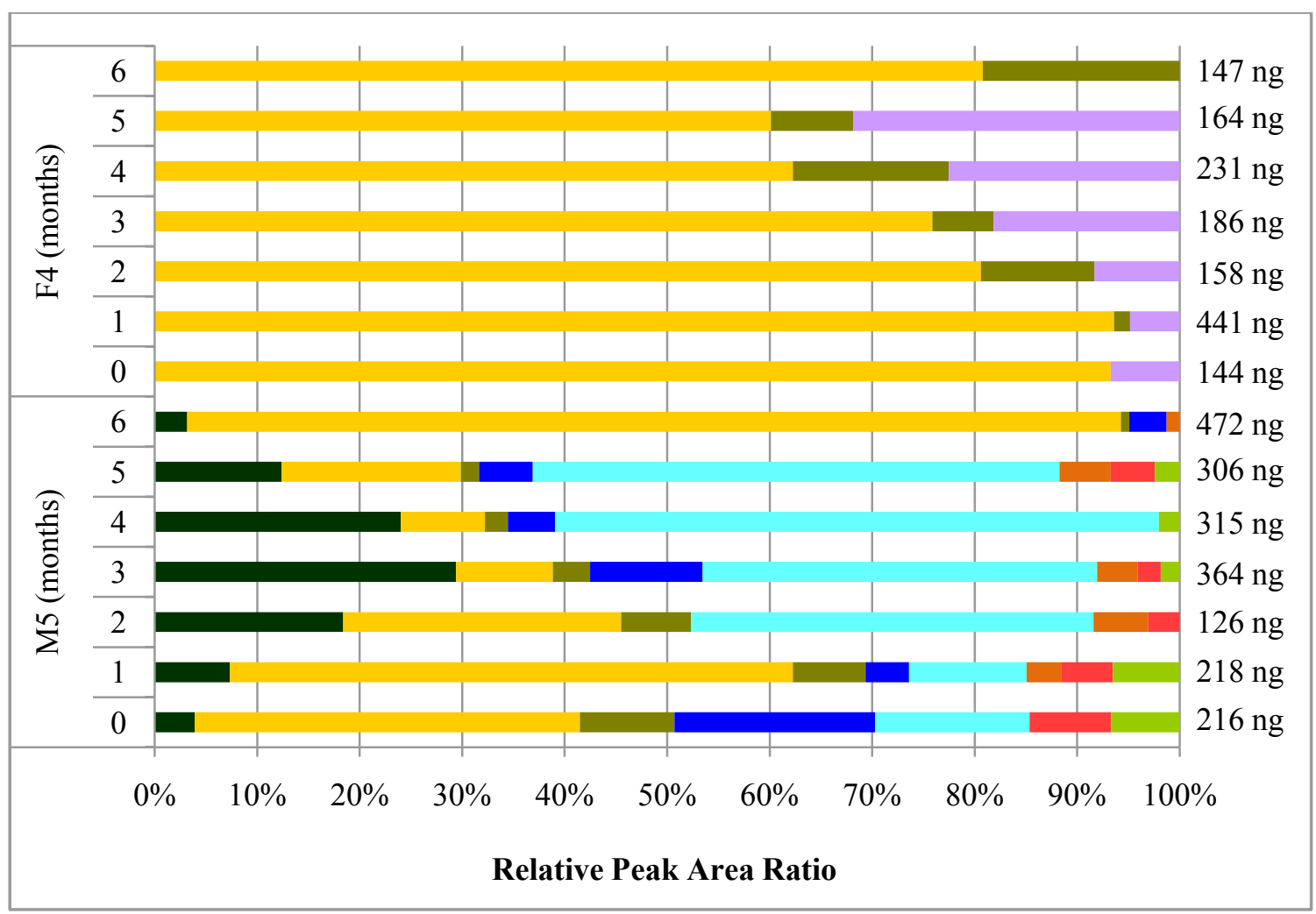


Table 23. Spearman rank correlation coefficients for the peak areas of hand odor VOCs across 6 monthly samplings of F4 and M5

\begin{tabular}{|c|c|c|c|c|c|c|c|c|c|c|c|c|c|c|}
\hline & \multicolumn{10}{|c|}{ Spearman Rank Correlation Coeffient Matrix (Hand Odor) } \\
\hline & F4 (m0) & F4 (m1) & F4 (m2) & F4 (m3) & F4 (m4) & F4 (m5) & F4 (m6) & M5 (m0) & M5 (m1) & M5 (m2) & M5 (m3) & M5 (m4) & M5 (m5) & M5 (m6) \\
\hline F4 (m0) & 1 & 0.740 & 0.721 & 0.000 & 0.572 & -0.139 & 0.572 & 0.214 & 0.401 & 0.000 & 0.313 & 0.324 & -0.077 & 0.686 \\
\hline F4 (m1) & 0.740 & 1 & 0.910 & 0.271 & 0.265 & -0.364 & 0.265 & 0.407 & 0.557 & 0.233 & 0.459 & 0.175 & 0.100 & 0.592 \\
\hline F4 (m2) & 0.721 & 0.910 & 1 & 0.323 & 0.560 & 0.000 & 0.560 & 0.280 & 0.436 & 0.126 & 0.341 & 0.132 & 0.000 & 0.560 \\
\hline F4 (m3) & 0.000 & 0.271 & 0.323 & 1 & 0.407 & 0.447 & 0.407 & 0.103 & 0.188 & -0.077 & 0.113 & -0.211 & -0.201 & 0.172 \\
\hline F4 (m4) & 0.572 & 0.265 & 0.560 & 0.407 & 1 & 0.728 & 1 & 0.000 & 0.173 & -0.166 & 0.099 & 0.175 & -0.233 & 0.518 \\
\hline F4 (m5) & -0.139 & -0.364 & 0.000 & 0.447 & 0.728 & 1 & 0.728 & -0.232 & -0.129 & -0.342 & -0.181 & -0.141 & -0.402 & 0.165 \\
\hline F4 (m6) & 0.572 & 0.265 & 0.560 & 0.407 & 1 & 0.728 & 1 & 0.000 & 0.173 & -0.166 & 0.099 & 0.175 & -0.233 & 0.518 \\
\hline M5 (m) & 0.214 & 0.407 & 0.280 & 0.103 & 0.000 & -0.232 & 0.000 & 1 & 0.925 & 0.815 & 0.963 & 0.000 & 0.815 & 0.410 \\
\hline M5 (m1) & 0.401 & 0.557 & 0.436 & 0.188 & 0.173 & -0.129 & 0.173 & 0.925 & 1 & 0.815 & 0.983 & 0.278 & 0.772 & 0.552 \\
\hline M5 (m2) & 0.000 & 0.233 & 0.126 & -0.077 & -0.166 & -0.342 & -0.166 & 0.815 & 0.815 & 1 & 0.830 & 0.154 & 0.964 & 0.078 \\
\hline M5 (m3) & 0.313 & 0.459 & 0.341 & 0.113 & 0.099 & -0.181 & 0.099 & 0.963 & 0.983 & 0.830 & 1 & 0.256 & 0.830 & 0.534 \\
\hline M5 (m4) & 0.324 & 0.175 & 0.132 & -0.211 & 0.175 & -0.141 & 0.175 & 0.000 & 0.278 & 0.154 & 0.256 & 1 & 0.231 & 0.503 \\
\hline M5 (m5) & -0.077 & 0.100 & 0.000 & -0.201 & -0.233 & -0.402 & -0.233 & 0.815 & 0.772 & 0.964 & 0.830 & 0.231 & 1 & 0.116 \\
\hline M5 (m6) & 0.686 & 0.592 & 0.560 & 0.172 & 0.518 & 0.165 & 0.518 & 0.410 & 0.552 & 0.078 & 0.534 & 0.503 & 0.116 & 1 \\
\hline
\end{tabular}

Table 24. Spearman rank correlation coefficients for the peak areas of buccal swab

\section{VOCs across 6 monthly samplings of F4 and M5}

\begin{tabular}{|c|c|c|c|c|c|c|c|c|c|c|c|c|c|c|c|}
\hline & \multicolumn{10}{|c|}{ Spearman Rank Correlation Coeffient Matrix (Buccal Swab) } \\
\hline & F4 (m0) & F4 (m1) & F4 (m2) & F4 (m3) & F4 (m4) & F4 (m5) & F4 (m6) & M5 (m0) & M5 (m1) & M5 (m2) & M5 (m3) & M5 (m4) & M5 (m5) & M5 (m) \\
\hline F4 (m0) & 1 & 0.933 & 0.917 & 0.783 & 0.967 & 0.967 & 0.933 & -0.184 & -0.128 & -0.145 & -0.145 & -0.145 & -0.139 & -0.100 \\
\hline F4 (m1) & 0.933 & 1 & 0.983 & 0.867 & 0.933 & 0.933 & 1.000 & -0.161 & -0.100 & -0.156 & -0.117 & -0.156 & -0.128 & -0.067 \\
\hline F4 (m2) & 0.917 & 0.983 & 1 & 0.833 & 0.950 & 0.950 & 0.983 & -0.172 & -0.083 & -0.133 & -0.106 & -0.133 & -0.111 & -0.044 \\
\hline F4 (m3) & 0.783 & 0.867 & 0.833 & 1 & 0.700 & 0.700 & 0.867 & -0.083 & -0.011 & -0.106 & -0.033 & -0.106 & -0.061 & 0.028 \\
\hline F4 (m4) & 0.967 & 0.933 & 0.950 & 0.700 & 1 & 1 & 0.933 & -0.206 & -0.133 & -0.145 & -0.150 & -0.145 & -0.139 & -0.100 \\
\hline F4 (m5) & 0.967 & 0.933 & 0.950 & 0.700 & 1 & 1 & 0.933 & -0.206 & -0.133 & -0.145 & -0.150 & -0.145 & -0.139 & -0.100 \\
\hline F4 (m6) & 0.933 & 1.000 & 0.983 & 0.867 & 0.933 & 0.933 & 1 & -0.161 & -0.100 & -0.156 & -0.117 & -0.156 & -0.128 & -0.067 \\
\hline M5 (m0) & -0.184 & -0.161 & -0.172 & -0.083 & -0.206 & -0.206 & -0.161 & 1 & 0.855 & 0.764 & 0.891 & 0.800 & 0.882 & 0.736 \\
\hline M5 (m1) & -0.128 & -0.100 & -0.083 & -0.011 & -0.133 & -0.133 & -0.100 & 0.855 & 1 & 0.936 & 0.945 & 0.945 & 0.955 & 0.827 \\
\hline M5 (m2) & -0.145 & -0.156 & -0.133 & -0.106 & -0.145 & -0.145 & -0.156 & 0.764 & 0.936 & 1 & 0.882 & 0.991 & 0.909 & 0.727 \\
\hline M5 (m3) & -0.145 & -0.156 & -0.133 & -0.106 & -0.145 & -0.145 & -0.156 & 0.800 & 0.945 & 0.991 & 1 & 0.909 & 0.973 & 0.882 \\
\hline M5 (m4) & -0.145 & -0.156 & -0.133 & -0.106 & -0.145 & -0.145 & -0.156 & 0.800 & 0.945 & 0.991 & 0.909 & 1 & 0.945 & 0.755 \\
\hline M5(m5) & -0.139 & -0.128 & -0.111 & -0.061 & -0.139 & -0.139 & -0.128 & 0.882 & 0.955 & 0.909 & 0.973 & 0.945 & 1 & 0.855 \\
\hline M5 (m6) & -0.100 & -0.067 & -0.044 & 0.028 & -0.100 & -0.100 & -0.067 & 0.736 & 0.827 & 0.727 & 0.882 & 0.755 & 0.855 & 1 \\
\hline
\end{tabular}

Table 25. Spearman rank correlation coefficients for the peak areas of breath VOCs across 6 monthly samplings of F4 and M5

\begin{tabular}{|c|c|c|c|c|c|c|c|c|c|c|c|c|c|c|}
\hline & \multicolumn{14}{|c|}{ Spearman Rank Correlation Coefficient Matrix (Breath) } \\
\hline & $\mathrm{F} 4(\mathrm{~m} 0)$ & $\mathrm{F} 4(\mathrm{~m} 1)$ & $\mathrm{F} 4(\mathrm{~m} 2)$ & $\mathrm{F} 4(\mathrm{~m} 3)$ & F4 (m4) & F4 (m5) & F4 (m6) & M5 (m0) & M5 (m1) & M5 (m2) & M5 (m3) & M5 (m4) & M5 (m5) & M5 (m6) \\
\hline $\mathrm{F} 4(\mathrm{~m} 0)$ & 1 & 1 & \begin{tabular}{|l|}
1 \\
\end{tabular} & 0.800 & 0.400 & 1 & 0.400 & -0.143 & 0.143 & -0.191 & 0.238 & -0.143 & -0.191 & -0.048 \\
\hline $\mathrm{F} 4(\mathrm{~m} 1)$ & 1 & 1 & 1 & 0.800 & 0.400 & 1 & 0.400 & -0.143 & 0.143 & -0.191 & 0.238 & -0.143 & -0.191 & -0.048 \\
\hline $\mathrm{F} 4(\mathrm{~m} 2)$ & 1 & 1 & 1 & 0.800 & 0.400 & 1 & 0.400 & -0.143 & 0.143 & -0.191 & 0.238 & -0.143 & -0.191 & -0.048 \\
\hline $\mathrm{F} 4(\mathrm{~m} 3)$ & 0.800 & 0.800 & 0.800 & 1 & 0.200 & 0.800 & 0.800 & -0.191 & 0.191 & -0.143 & 0.095 & -0.191 & -0.143 & 0.048 \\
\hline F4 (m4) & 0.400 & 0.400 & 0.400 & 0.200 & 1 & 0.400 & 0.400 & & & -0.524 & -0.191 & -0.477 & -0.524 & -0.429 \\
\hline F4 (m5) & 1 & 1 & 1 & 0.800 & 0.400 & 1 & 0.400 & -0.143 & 0.143 & -0.191 & 0.238 & -0.143 & -0.191 & -0.048 \\
\hline F4 (m6) & 0.400 & 0.400 & 0.400 & 0.800 & 0.400 & 0.400 & 1 & -0.524 & -0.238 & -0.477 & -0.334 & -0.524 & -0.477 & -0.334 \\
\hline M5 (m0) & -0.143 & -0.143 & -0.143 & -0.191 & -0.477 & -0.143 & -0.524 & 1 & 0.771 & 0.943 & 0.829 & 0.943 & 0.886 & 0.714 \\
\hline M5 (m1) & 0.143 & 0.143 & 0.143 & 0.191 & -0.286 & 0.143 & -0.238 & 0.771 & 1 & 0.829 & 0.714 & 0.600 & 0.657 & 0.600 \\
\hline M5 (m2) & -0.191 & -0.191 & -0.191 & -0.143 & -0.524 & -0.191 & -0.477 & 0.943 & 0.829 & 1 & 0.657 & 0.886 & 0.943 & 0.829 \\
\hline M5 (m3) & 0.238 & 0.238 & 0.238 & 0.095 & -0.191 & 0.238 & -0.334 & 0.829 & 0.714 & 0.657 & 1 & 0.771 & 0.600 & 0.486 \\
\hline M5 (m4) & -0.143 & -0.143 & -0.143 & -0.191 & -0.477 & -0.143 & -0.524 & 0.943 & 0.600 & 0.886 & 0.771 & 1 & 0.943 & 0.829 \\
\hline M5 (m5) & -0.191 & -0.191 & -0.191 & -0.143 & -0.524 & -0.191 & -0.477 & 0.886 & 0.657 & 0.943 & 0.600 & 0.943 & 1 & 0.943 \\
\hline M5 (m6) & -0.048 & -0.048 & -0.048 & 0.048 & -0.429 & -0.048 & -0.334 & 0.714 & 0.600 & 0.829 & 0.486 & 0.829 & 0.943 & 1 \\
\hline
\end{tabular}


Table 26. Spearman rank correlation coefficients for the peak areas of blood VOCs across 6 monthly samplings of F4 and M5

\begin{tabular}{|c|c|c|c|c|c|c|c|c|c|c|c|c|c|c|}
\hline & \multicolumn{10}{|c|}{ Spearman Rank Correlation Coefficient Matrix (Blood) } \\
\hline & F4 (m0) & F4 (m1) & F4 (m2) & F4 (m3) & F4 (m4) & F4 (m5) & F4 (m6) & M5 (m0) & M5 (m1) & M5 (m2) & M5 (m3) & M5 (m4) & M5 (m5) & M5 (m6) \\
\hline F4 (m0) & 1 & 0.771 & 0.829 & 0.943 & 0.943 & 0.943 & 0.829 & 0.524 & 0.238 & 0.238 & 0.524 & 0.477 & 0.524 & 0.477 \\
\hline F4 (m1) & 0.771 & 1 & 0.943 & 0.886 & 0.886 & 0.886 & 0.943 & 0.572 & 0.238 & 0.238 & 0.572 & 0.429 & 0.572 & 0.429 \\
\hline F4 (m2) & 0.829 & 0.943 & 1 & 0.943 & 0.943 & 0.943 & 1.000 & 0.715 & 0.334 & 0.334 & 0.715 & 0.572 & 0.715 & 0.572 \\
\hline F4 (m3) & 0.943 & 0.886 & 0.943 & 1 & 1 & 1 & 0.943 & 0.620 & 0.286 & 0.286 & 0.620 & 0.524 & 0.620 & 0.524 \\
\hline F4 (m4) & 0.943 & 0.886 & 0.943 & 1 & 1 & 1 & 0.943 & 0.620 & 0.286 & 0.286 & 0.620 & 0.524 & 0.620 & 0.524 \\
\hline F4 (m5) & 0.943 & 0.886 & 0.943 & 1 & 1 & 1 & 0.943 & 0.620 & 0.286 & 0.286 & 0.620 & 0.524 & 0.620 & 0.524 \\
\hline F4 (m6) & 0.829 & 0.943 & 1.000 & 0.943 & 0.943 & 0.943 & 1 & 0.715 & 0.334 & 0.334 & 0.715 & 0.572 & 0.715 & 0.572 \\
\hline M5 (m0) & 0.524 & 0.572 & 0.715 & 0.620 & 0.620 & 0.620 & 0.715 & 1 & 0.400 & 0.400 & 1.000 & 0.800 & 1.000 & 0.800 \\
\hline M5 (m1) & 0.238 & 0.238 & 0.334 & 0.286 & 0.286 & 0.286 & 0.334 & 0.400 & 1 & 1 & 0.400 & 0.800 & 0.400 & 0.800 \\
\hline M5 (m2) & 0.238 & 0.238 & 0.334 & 0.286 & 0.286 & 0.286 & 0.334 & 0.400 & 1 & 1 & 0.400 & 0.800 & 0.400 & 0.800 \\
\hline M5 (m3) & 0.524 & 0.572 & 0.715 & 0.620 & 0.620 & 0.620 & 0.715 & 1.000 & 0.400 & 0.400 & 1 & 0.800 & 1 & 0.800 \\
\hline M5 (m4) & 0.477 & 0.429 & 0.572 & 0.524 & 0.524 & 0.524 & 0.572 & 0.800 & 0.800 & 0.800 & 0.800 & 1 & 0.800 & 1 \\
\hline M5 (m5) & 0.524 & 0.572 & 0.715 & 0.620 & 0.620 & 0.620 & 0.715 & 1.000 & 0.400 & 0.400 & 1.000 & 0.800 & 1 & 0.800 \\
\hline M5 (m6) & 0.477 & 0.429 & 0.572 & 0.524 & 0.524 & 0.524 & 0.572 & 0.800 & 0.800 & 0.800 & 0.800 & 1 & 0.800 & 1 \\
\hline
\end{tabular}

Table 27. Spearman rank correlation coefficients for the peak areas of urine VOCs across 6 monthly samplings of F4 and M5

\begin{tabular}{|l|c|c|c|c|c|c|c|c|c|c|c|c|c|c|c|c|c|}
\hline & \multicolumn{10}{|c|}{ Spearman Rank Correlation Coefficient Matrix (Urine) } \\
\hline & F4 (m0) & F4 (m1) & F4 (m2) & F4 (m3) & F4 (m4) & F4 (m5) & F4 (m6) & M5 (m0) & M5 (m1) & M5 (m2) & M5 (m3) & M5 (m4) & M5 (m5) & M5 (m6) \\
\hline F4 (m0) & 1 & 0.980 & 0.750 & 0.540 & 0.960 & 0.750 & 0.829 & 0.350 & 0.110 & -0.010 & -0.180 & -0.150 & -0.080 & 0.260 \\
\hline F4 (m1) & 0.980 & 1 & 0.400 & 0.320 & 0.790 & 0.676 & 0.310 & 0.190 & 0.140 & -0.090 & -0.290 & -0.220 & -0.130 & -0.010 \\
\hline F4 (m) & 0.750 & 0.400 & 1 & 0.490 & 0.908 & 0.680 & 0.940 & 0.470 & 0.160 & 0.110 & 0.020 & -0.060 & 0.100 & 0.440 \\
\hline F4 (m3) & 0.540 & 0.320 & 0.490 & 1 & 0.460 & 0.930 & 0.260 & 0.390 & 0.120 & -0.120 & -0.130 & -0.220 & 0.040 & 0.120 \\
\hline F4 (m4) & 0.960 & 0.790 & 0.908 & 0.460 & 1 & 0.640 & 0.938 & 0.430 & 0.140 & 0.050 & -0.130 & -0.130 & -0.040 & 0.310 \\
\hline F4 (m5) & 0.750 & 0.680 & 0.680 & 0.930 & 0.640 & 1 & 0.510 & 0.290 & 0.080 & -0.140 & -0.280 & -0.290 & 0.120 & 0.120 \\
\hline F4 (m6) & 0.860 & 0.310 & 0.940 & 0.260 & 0.938 & 0.510 & 1 & 0.420 & 0.180 & 0.210 & 0.080 & -0.010 & 0.120 & 0.480 \\
\hline M5 (m0) & 0.350 & 0.190 & 0.470 & 0.390 & 0.430 & 0.290 & 0.420 & 1 & 0.270 & 0.000 & 0.260 & 0.060 & 0.200 & 0.780 \\
\hline M5 (m1) & 0.110 & 0.140 & 0.160 & 0.120 & 0.140 & 0.080 & 0.180 & 0.270 & 1 & 0.510 & 0.420 & 0.685 & 0.520 & 0.300 \\
\hline M5 (m2) & -0.010 & -0.090 & 0.110 & -0.120 & 0.050 & -0.140 & 0.210 & 0.000 & 0.510 & 1 & 0.886 & 0.755 & 0.920 & 0.337 \\
\hline M5 (m3) & -0.180 & -0.290 & 0.020 & -0.130 & -0.130 & -0.280 & 0.080 & 0.260 & 0.420 & 0.886 & 1 & 0.810 & 0.808 & 0.320 \\
\hline M5 (m4) & -0.150 & -0.220 & -0.060 & -0.220 & -0.130 & -0.290 & -0.010 & 0.060 & 0.685 & 0.755 & 0.810 & 1 & 0.730 & 0.090 \\
\hline M5 (m5) & -0.080 & -0.130 & 0.100 & 0.040 & -0.040 & 0.120 & 0.120 & 0.200 & 0.410 & 0.920 & 0.808 & 0.730 & 1 & 0.350 \\
\hline M5 (m6) & 0.260 & -0.010 & 0.440 & 0.120 & 0.310 & 0.120 & 0.480 & 0.780 & 0.300 & 0.337 & 0.320 & 0.090 & 0.350 & 1 \\
\hline
\end{tabular}




\subsubsection{Principal Component Analysis}

Principal component analysis (PCA) was conducted on the six month time study sampling dataset. Figure 60 and Figure 61 shows a three-dimensional plot of the first three principal components of subjects Female 4 and Male 5, respectively. Groupings can be seen among the different biological specimen samples. As shown in purple in the figure, buccal swab samples group together, as do hand odor (blue), breath (green), blood (red), and urine (orange) samples. Additionally, PCA was individually conducted on the multiple-samplings dataset between Female 4 and Male 5 for each specimen. When the first three principal components of these datasets are plotted, groupings can be noted among the multiple samples collected from the same individual. Female 4's samplings from the time study group together and Male 5's samplings group together, but there is no overlap between subjects. Figure 62 to Figure 66 display PCA data for the hand odor, buccal swab, breath, blood, and urine samples from Female 4 and Male 5 over the six month time period. With the exception of a few outliers, each specimen's samples from Female 4 group together as do the samples from Male 5. These three-dimensional plots provide a convenient visual aid for identifying the groupings according to specimen or individual. 
Figure 60. Three-dimensional PCA scatter plot of PCs 1, 2, and 3 of Female 4

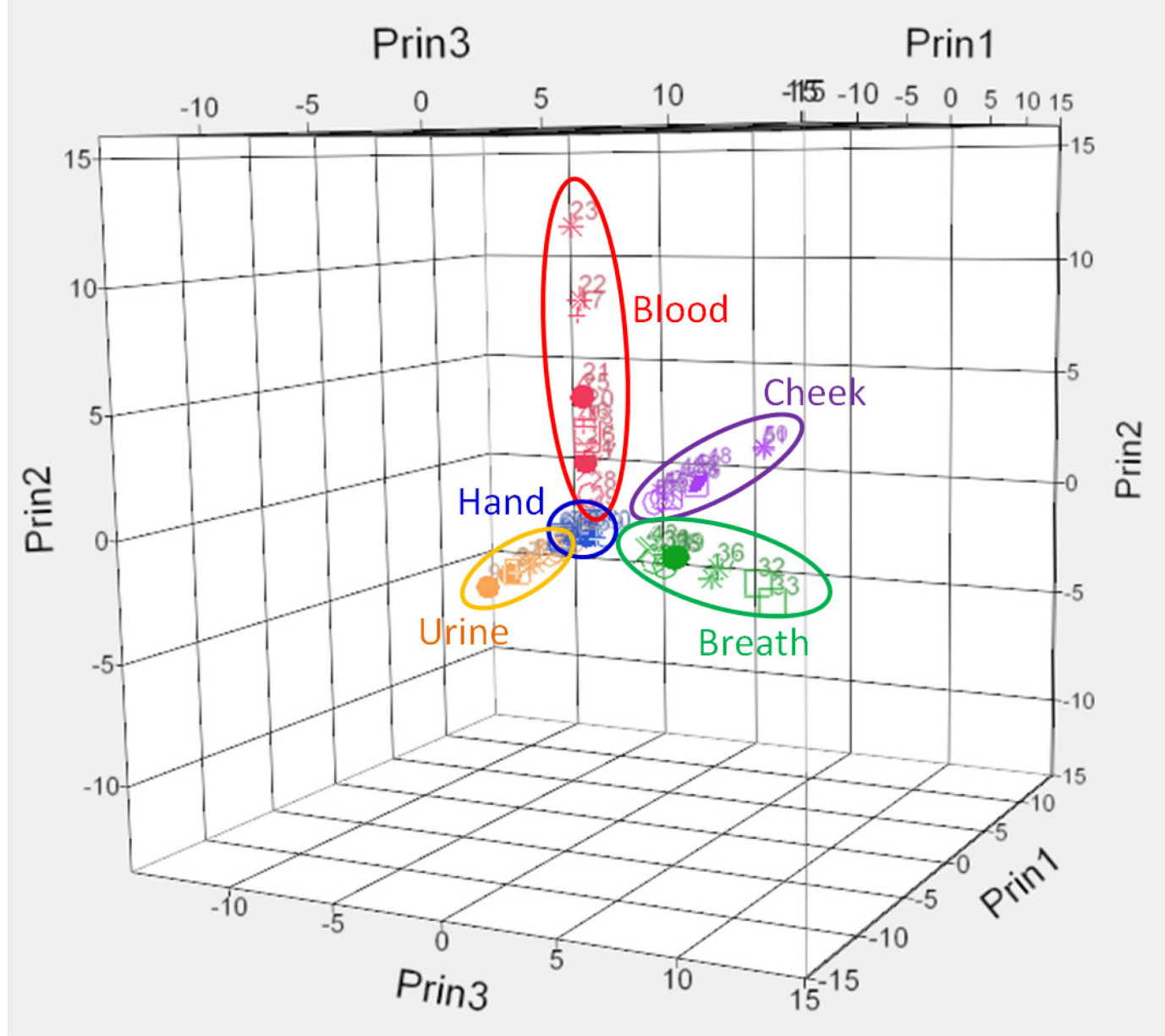


Figure 61. Three-dimensional PCA scatter plot of PCs 1, 2, and 3 of Male 5

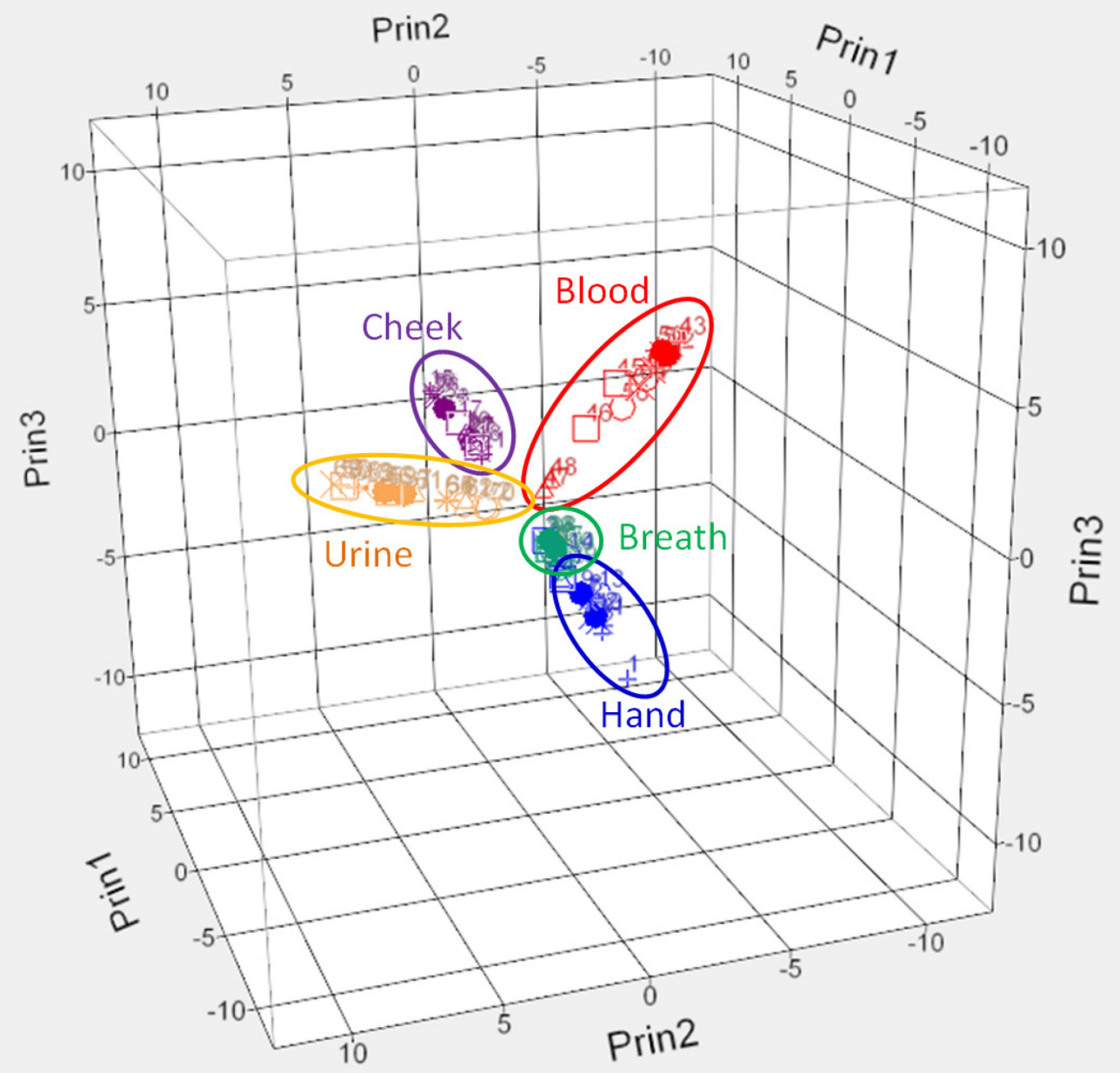


Table 28. PCA results summary for hand odor

\begin{tabular}{|l|l|l|l|l|l|}
\hline Number & 1 & 2 & 3 & 4 & 5 \\
\hline Eigenvalue & 3.643 & 1.864 & 1.458 & 0.549 & 0.275 \\
\hline Percent & 45.531 & 23.306 & 18.230 & 6.862 & 3.438 \\
\hline Cum Percent & 45.531 & 68.838 & 87.068 & 93.929 & 97.367 \\
\hline
\end{tabular}

Figure 62. Three-dimensional PCA scatter plot of PCs 1, 2, and 3 of hand odor from F4 and M5 over 6 months

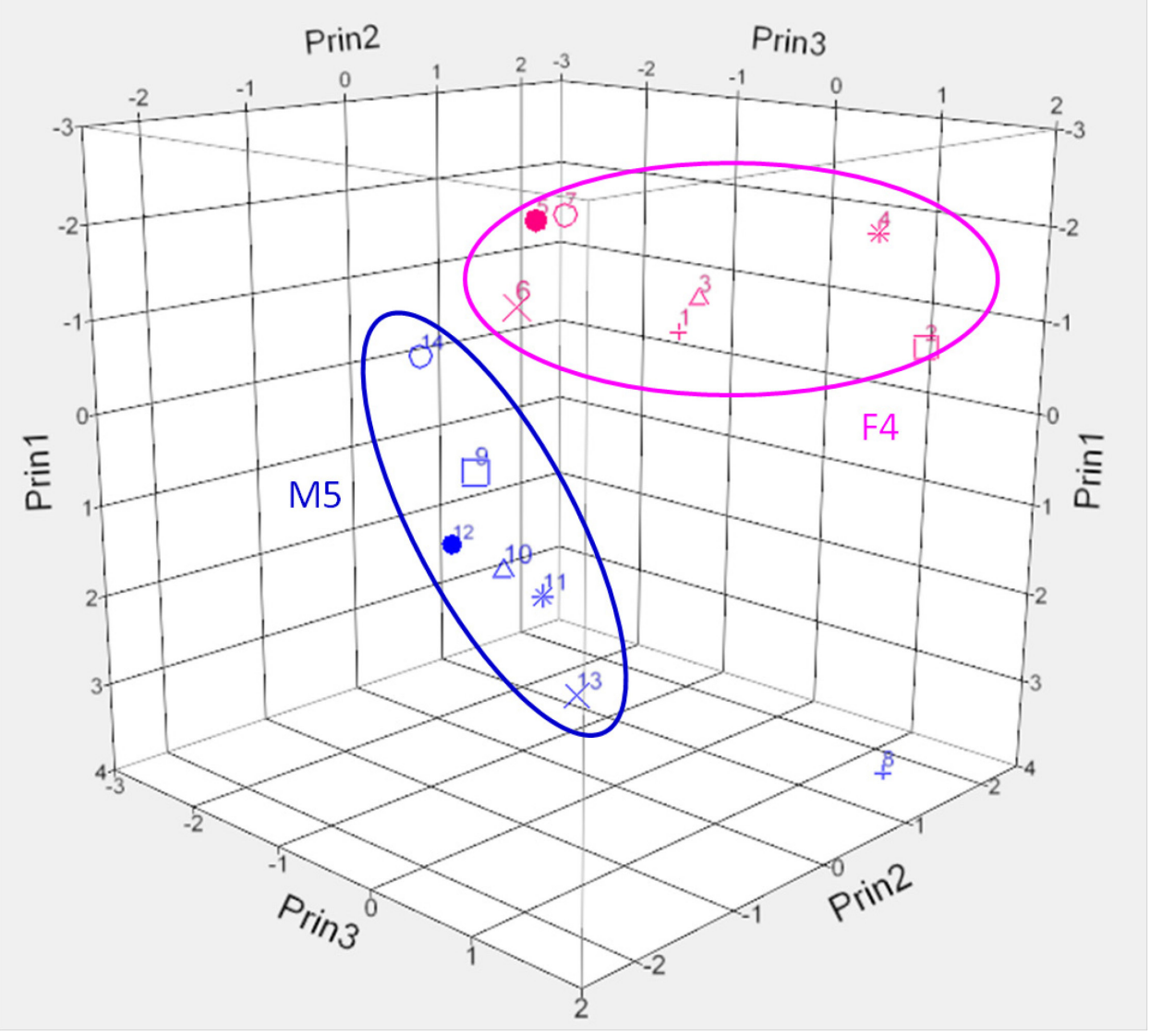


Table 29. PCA results summary for buccal swab

\begin{tabular}{|l|l|l|l|l|l|}
\hline Number & 1 & 2 & 3 & 4 & 5 \\
\hline Eigenvalue & 9.739 & 3.239 & 0.706 & 0.468 & 0.386 \\
\hline Percent & 64.926 & 21.599 & 4.705 & 3.118 & 2.576 \\
\hline Cum Percent & 64.926 & 86.526 & 91.231 & 94.349 & 96.925 \\
\hline
\end{tabular}

Figure 63. Three-dimensional PCA scatter plot of PCs 1, 2, and 3 of buccal swabs from $F 4$ and M5 over 6 months

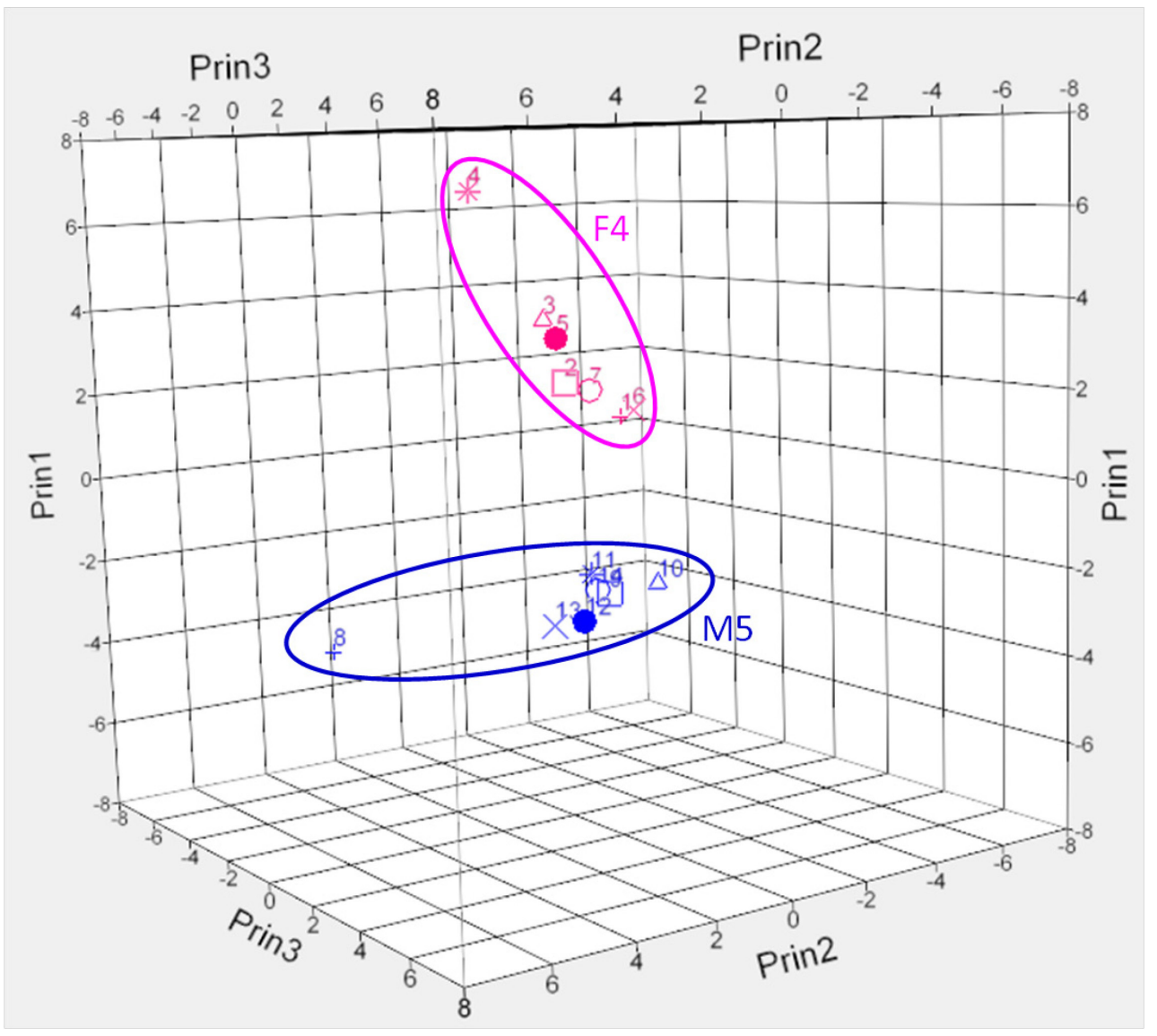


Table 30. PCA results summary for breath

\begin{tabular}{|l|l|l|l|l|l|}
\hline Number & 1 & 2 & 3 & 4 & 5 \\
\hline Eigenvalue & 3.533 & 2.026 & 0.886 & 0.241 & 0.182 \\
\hline Percent & 50.469 & 28.940 & 12.658 & 3.446 & 2.606 \\
\hline Cum Percent & 50.469 & 79.409 & 92.068 & 95.514 & 98.120 \\
\hline
\end{tabular}

Figure 64. Three-dimensional PCA scatter plot of PCs 1, 2, and 3 of breath from F4 and M5 over 6 months

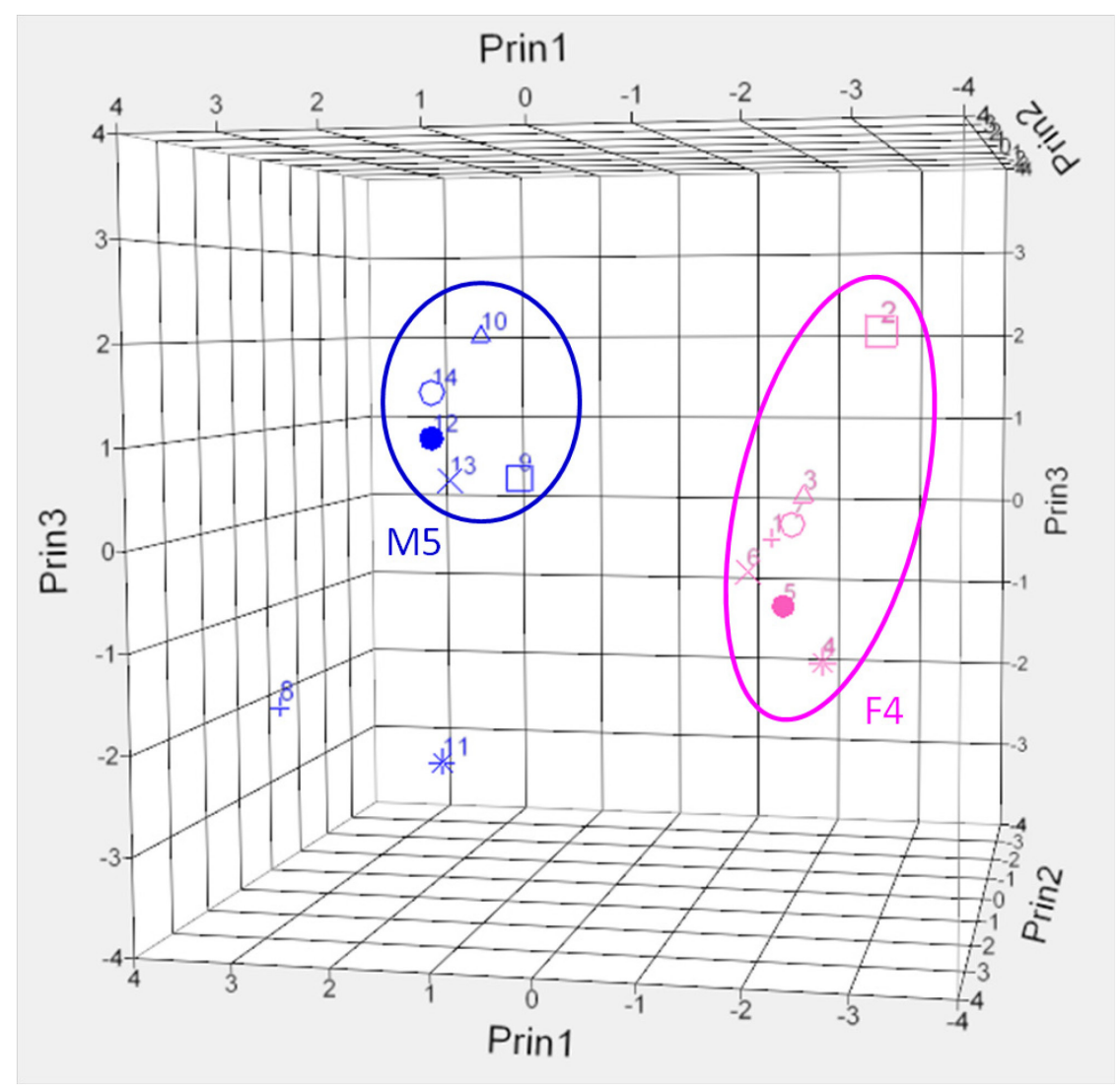


Table 31. PCA results summary for blood

\begin{tabular}{|l|l|l|l|l|l|}
\hline Number & 1 & 2 & 3 & 4 & 5 \\
\hline Eigenvalue & 3.636 & 2.176 & 0.742 & 0.252 & 0.153 \\
\hline Percent & 51.939 & 31.090 & 10.602 & 3.594 & 2.188 \\
\hline Cum Percent & 51.939 & 83.029 & 93.631 & 97.225 & 99.413 \\
\hline
\end{tabular}

Figure 65. Three-dimensional PCA scatter plot of PCs 1, 2, and 3 of blood from F4 and M5 over 6 months

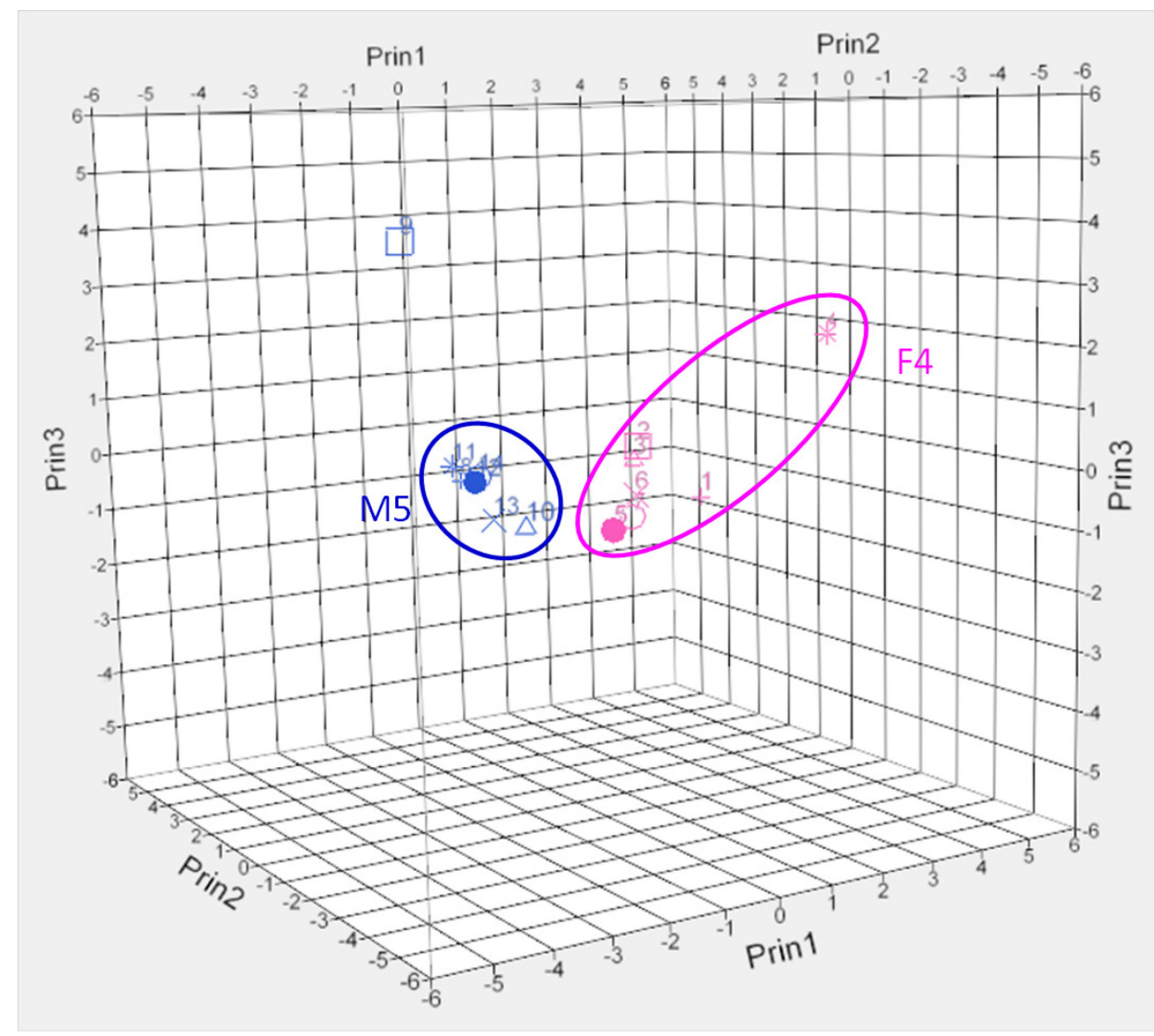


Table 32. PCA results summary for urine

\begin{tabular}{|l|l|l|l|l|l|}
\hline Number & 1 & 2 & 3 & 4 & 5 \\
\hline Eigenvalue & 2.389 & 1.023 & 0.899 & 0.487 & 0.202 \\
\hline Percent & 47.781 & 20.454 & 17.988 & 9.737 & 4.040 \\
\hline Cum Percent & 47.781 & 68.235 & 86.223 & 95.959 & 100 \\
\hline
\end{tabular}

Figure 66. Three-dimensional PCA scatter plot of PCs 1, 2, and 3 of urine from F4 and M5 over 6 months

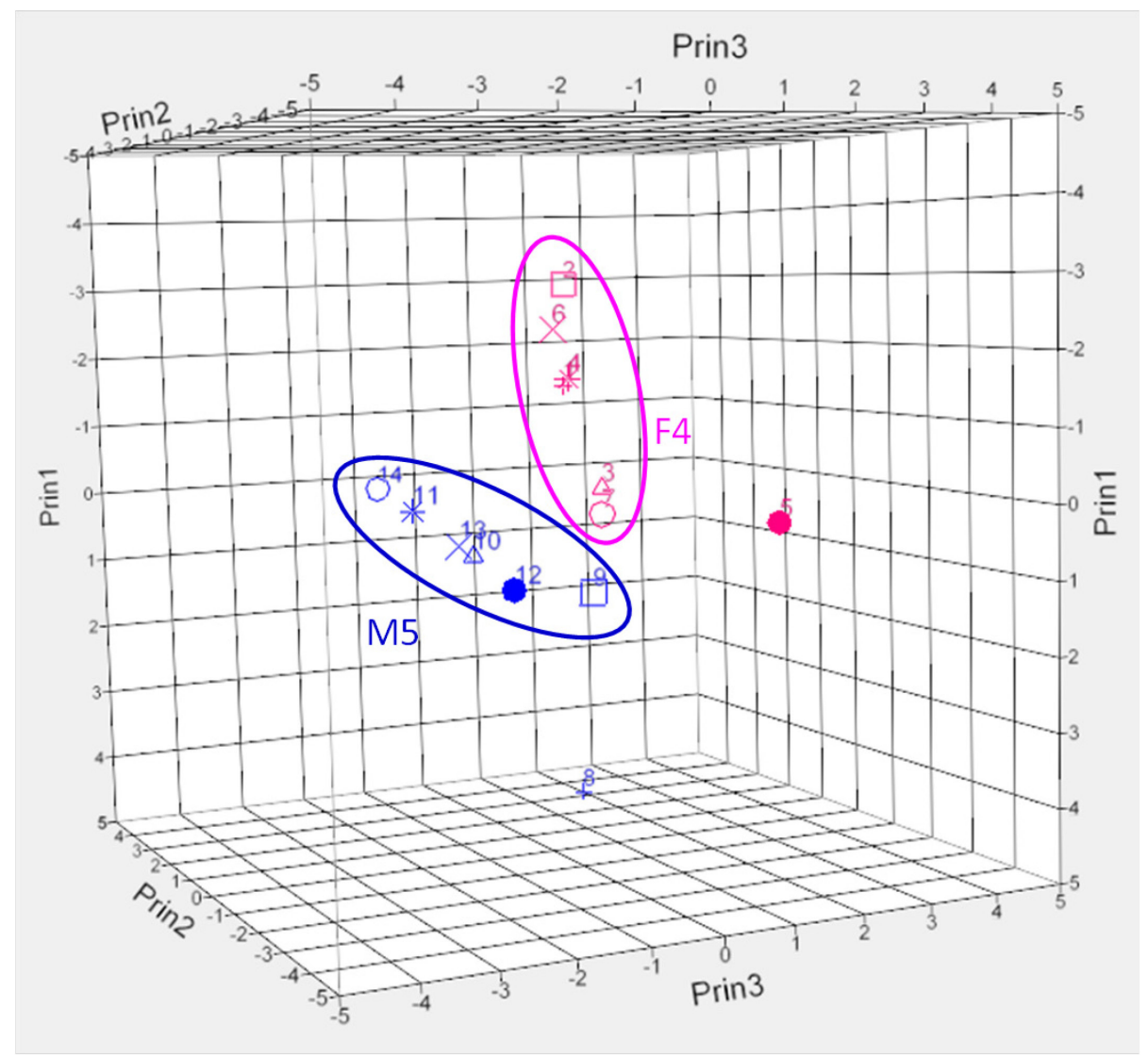


Results from the six-month study of the VOC profiles of two subjects were in agreement with the previously reported findings of the similar study done with hand odor. Relative peak area ratio comparisons, Spearman rank correlation coefficient comparisons, and PCA results all demonstrate that VOC profiles of one individual taken over a period of time do not contain as much variation as that seen between different individuals. The VOCs from the same specimen over time group together, and VOCs from the same individual for different specimens group together. These findings further support the hypothesis that human scent, not only hand odor but also extending to other biological specimens, is stable over time and distinguishable between individuals.

\subsubsection{Evaluation of the Effect of Fasting Prior to Sampling}

Four unrelated subjects (Female 4, Female 15, Male 2, and Male 15) were sampled intraday to evaluate the effect of fasting prior to specimen collections. The "fasting" sampling was done in the morning where the subjects had not consumed any food or drinks, with the exception of water, since the previous night. The "postprandial" sampling was done in the afternoon, immediately after the subject had consumed a meal (within thirty minutes after eating). Figure 67 demonstrates the comparison of the average number of compounds extracted from each specimen. A small increase in the total number of common compounds extracted was observed for each of the five specimens. Buccal swab and breath samples were anticipated to have the largest differences between the fasting and postprandial samples; however, the differences in the number of compounds extracted were not drastic. 
Figure 67. Average number of compounds extracted from specimens collected after fasting and postprandial

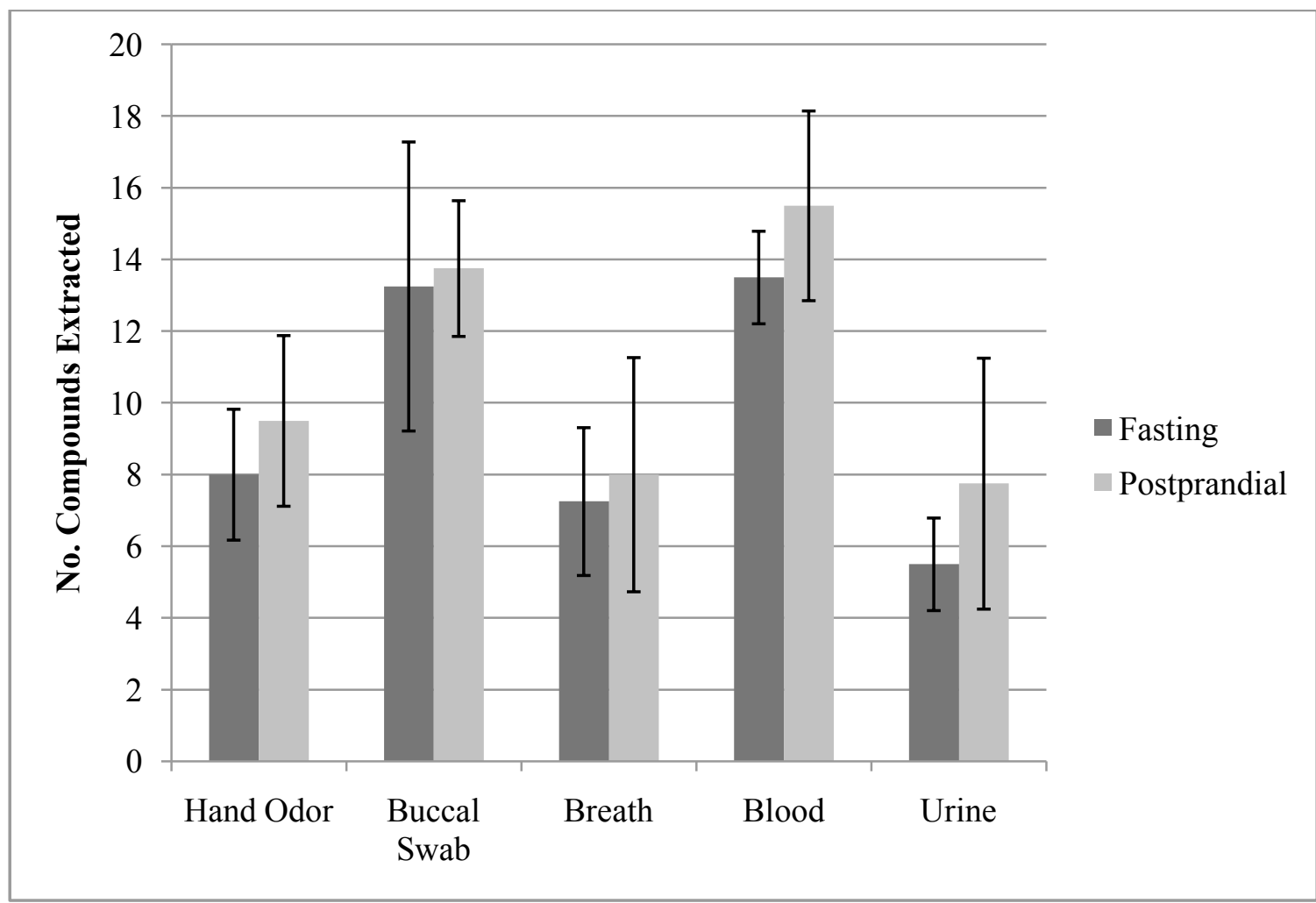

Comparisons of compounds that were extracted at fasting and postprandial sampling times from the five biological specimens for subject Female 4 are summarized in Table 33. For each specimen, the compounds that were extracted from both fasting and postprandial samplings were subjected to paired $t$-test of significance to evaluate whether the variation in the amounts of the compound extracted was statistically significant or not. For hand odor, eight out of the twelve compounds extracted were common across fasting and postprandial samplings: benzyl alcohol, nonanal, decanal, tridecane, tetradecane, (E)-6,10-dimethyl-5,9-undecadien-2-one, hexadecane, and heptadecane. The extracted amounts were statistically significant only for tridecane. For buccal swabs, nine 
out of the fifteen compounds extracted were common across fasting and postprandial samplings: 2-pentylfuran, hexanoic acid, octanoic acid ethyl ester, decanal, nonanoic acid, nonanoic acid ethyl ester, (E)- 6,10-dimethyl-5,9-undecadien-2-one, 1-decene, and dodecanoic acid ethyl ester. When subjected to paired $t$-test of significance, the difference in the amounts of these nine common extracted compounds was not found to be statistically significant. For breath, four out of the six compounds extracted were common across fasting and postprandial samplings: toluene, 2-ethyl-1-hexanol, nonanal, and decanal. The differences in the amounts of these four compounds extracted between samplings were not statistically significant. For blood, nine out of the eighteen compounds extracted were common between samplings: undecane, nonanal, tridecane, 2,4,6-trimethylbenzaldehyde, 2-butyltetrahydrofuran, tetradecane, hexadecane, diisopropylnaphthalene, and 2,6-diisopropylnaphthalene. None of the common compounds were have to have a statistically significant difference in the amount extracted betweens samplings. Finally for urine, two out of the five compounds extracted were common between samplings: 4-heptanone and carvone. Carvone was found to be statistically significantly different in the amount extracted between fasting and postprandial samplings. 
Table 33. Summary of volatile compounds extracted fasting (AM) vs. postprandial (PM) for Female 4

\begin{tabular}{|c|c|c|c|c|c|c|c|c|c|c|c|c|c|}
\hline & \multicolumn{2}{|c|}{ Hand } & \multicolumn{3}{|c|}{ Buccal } & \multicolumn{3}{|c|}{ Breath } & \multicolumn{3}{|c|}{ Blood } & \multicolumn{2}{|c|}{ Urine } \\
\hline & AM & PM & $\mathrm{AN}$ & & & $\mathrm{AM}$ & $\mathrm{P}$ & & AM & $\mathrm{PN}$ & & $\mathrm{AM}$ & PM \\
\hline 1-Decene & & & $\mathrm{x}$ & & & & & & $\mathrm{x}$ & & & & \\
\hline 1-Hexanol,2-ethyl- & & & & & & $\mathrm{x}$ & rata & & & & & & \\
\hline 1-Octanol & & & & & & & & & & & & & $\mathrm{x}$ \\
\hline 2,6-Diisopropylnaphthalene & & & & & & & & & $\mathrm{x}$ & $\mathrm{x}$ & & & \\
\hline 2-Dodecene,(Z)- & & & & & & & & & & $\mathrm{X}$ & & & \\
\hline 4-Heptanone & & & & & & & & & & & & $\mathrm{x}$ & $\mathrm{x}$ \\
\hline 5,9-Undecadien-2-one,6,10-dimethyl-,(E)- & $\mathrm{x}$ & $\mathrm{x}$ & $\mathrm{x}$ & & & & & & & $\mathrm{x}$ & & & \\
\hline 6-Dodecanone & & & & & & & & & & & & & \\
\hline Acetone & & & & & & $\mathrm{x}$ & & & & & & & \\
\hline Benzaldehyde & $\mathrm{x}$ & & & & & & & & & & & & \\
\hline Benzaldehyde,2,4,6-trimethyl- & & & & & & & & & $\mathrm{x}$ & $\mathrm{x}$ & & & \\
\hline BenzylAlcohol & $\mathrm{x}$ & $\mathrm{x}$ & & & & & & & $\mathrm{X}$ & & & & \\
\hline Carvone & & & & & & & & & & & & $\mathrm{x}$ & $\mathrm{x}$ \\
\hline Caryophyllene & & & & & & & & & & & & & \\
\hline Cyclododecane & & & & & & & & & & $\mathrm{x}$ & & & \\
\hline Cyclopropane,1-ethyl-2-heptyl- & & & & & & & & & & & & & $\mathrm{X}$ \\
\hline Decanal & $\mathrm{x}$ & $\mathrm{x}$ & $\mathrm{x}$ & & & $\mathrm{x}$ & & & & $\mathrm{x}$ & & & \\
\hline Diisopropylnaphthalene & & & & & & & & & $\mathrm{x}$ & $\mathrm{x}$ & & & \\
\hline Dodecane & & & & & & & & & $\mathrm{x}$ & $\mathrm{x}$ & & & \\
\hline Dodecanoicacid & & & & & & & & & & & & & \\
\hline Dodecanoicacid,ethylester & & & $\mathrm{x}$ & & & & & & & & & & \\
\hline Furan,2-butyltetrahydro- & & & & & & & & & $\mathrm{x}$ & $\mathrm{x}$ & & & \\
\hline Furan,2-pentyl- & & & $\mathrm{x}$ & & & & & & & $\mathrm{x}$ & & & \\
\hline Heptadecane & $\mathrm{x}$ & $\mathrm{x}$ & & & & & & & & & & & \\
\hline Heptanoicacid,ethylester & & & & & & & & & & & & & \\
\hline Hexadecane & $\mathrm{x}$ & $\mathrm{x}$ & & & & & & & $\mathrm{X}$ & $\mathrm{x}$ & & & \\
\hline Hexanal & & & & & & & & & $\mathrm{x}$ & & & & \\
\hline Hexanoicacid & & & $\mathrm{x}$ & & & & & & & & & & \\
\hline Hexanoicacid,pentylester & & & & & & & & & & & & & \\
\hline Menthol & & & & & & & & & & & & $\mathrm{x}$ & \\
\hline Nonanal & $\mathrm{x}$ & $\mathrm{x}$ & & & & $\mathrm{x}$ & & & $\mathrm{X}$ & $\mathrm{x}$ & & & \\
\hline Nonanoicacid & & & $\mathrm{x}$ & & & & & & & & & & \\
\hline Nonanoicacid,ethylester & & & $\mathrm{x}$ & & & & & & & & & & \\
\hline Octadecane & $\mathrm{x}$ & & & & & & & & & & & & \\
\hline Octanoicacid,ethylester & & & $\mathrm{x}$ & & & & & & & & & & \\
\hline Octanoicacid,methylester & & $\mathrm{x}$ & & & & & & & & & & & \\
\hline Phenol & & & & & & $\mathrm{x}$ & & & & & & & \\
\hline Tetradecane & $\mathrm{x}$ & $\mathrm{x}$ & & & & & & & $\mathrm{x}$ & $\mathrm{x}$ & & & \\
\hline Toluene & & & & & & $\mathrm{x}$ & & & & & & & \\
\hline Tridecane & $\mathrm{x}$ & $\mathrm{x}$ & & & & & & & $\mathrm{x}$ & $\mathrm{x}$ & & & \\
\hline Undecane & & $\mathrm{x}$ & & & & & & & $\mathrm{x}$ & $\mathrm{x}$ & & & \\
\hline
\end{tabular}


Comparisons of compounds that were extracted at fasting and postprandial sampling times from the five biological specimens for subject Female 15 are summarized in Table 34. For each specimen, the compounds that were extracted from both fasting and postprandial samplings were subjected to paired $t$-test of significance to evaluate whether the variation in the amounts of the compound extracted was statistically significant or not. For hand odor, seven out of the eleven compounds extracted were common across fasting and postprandial samplings: benzyl alcohol, undecane, nonanal, decanal, tetradecane, (E)-6,10-dimethyl-5,9-undecadien-2-one, and octadecane. The extracted amounts were not statistically significant for any of the common compounds. For buccal swabs, nine out of the twenty-four compounds extracted were common across fasting and postprandial samplings: benzaldehyde, 2-pentylfuran, hexanoic acid, decanal, nonanoic acid, nonanoic acid ethyl ester, (E)-6,10-dimethyl-5,9-undecadien-2-one, diethyl phthalate, and isopropyl myristate. When subjected to paired $t$-test of significance, the difference in the amounts of these nine common extracted compounds was not found to be statistically significant. For breath, eight out of the nine compounds extracted were common across fasting and postprandial samplings: toluene, p-xylene, 1,2dichlorobenzene, 2-ethyl-1-hexanol, nonanal, decanal, butylated hydroxytoluene, and benzophenone. The differences in the amounts of these eight compounds extracted between samplings were not statistically significant. For blood, eleven out of the sixteen compounds extracted were common between samplings: 2-pentylfuran, nonanal menthol, 1-dodecene, decanal, (Z)-2-dodecene, tridecane, 2-butyltetrahydrofuran, tetradecane, hexadecane, and 2,6-diisopropylnaphthalene. None of the common compounds were have to have a statistically significant difference in the amount extracted betweens samplings. 
Finally for urine, four out of the eight compounds extracted were common between samplings: 4-heptanone, menthol, carvone, and N-N-diethylcarbanilide. Menthol and carvone were found to be statistically significantly different in the amount extracted between fasting and postprandial samplings. 
Table 34. Summary of compounds extracted fasting (AM) vs. postprandial (PM) for Female 15

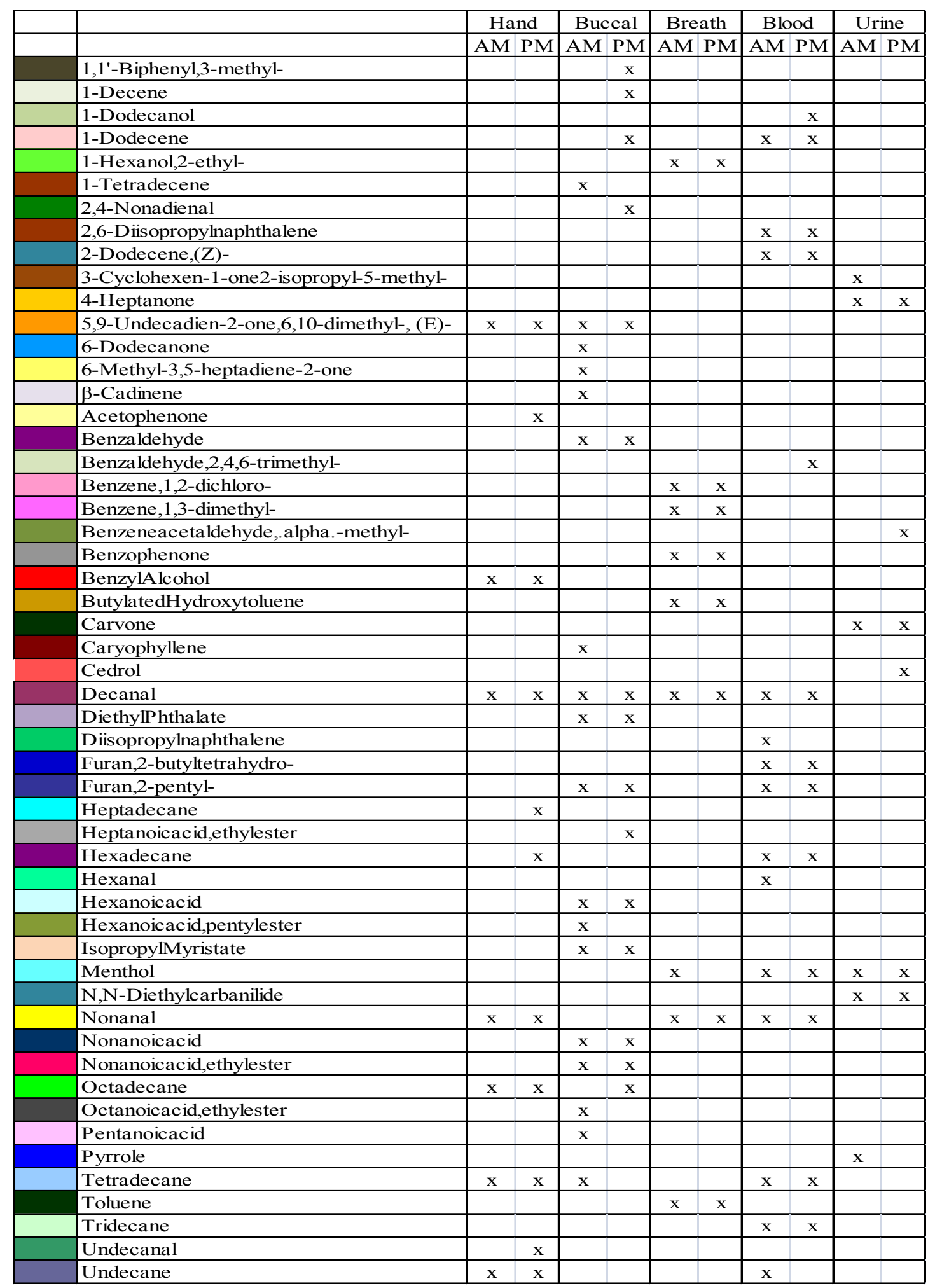


Comparisons of compounds that were extracted at fasting and postprandial sampling times from the five biological specimens for subject Male 2 are summarized in Table 35. For each specimen, the compounds that were extracted from both fasting and postprandial samplings were subjected to paired $t$-test of significance to evaluate whether the variation in the amounts of the compound extracted was statistically significant or not. For hand odor, five out of the seven compounds extracted were common across fasting and postprandial samplings: nonanal, decanal, tetradecane, hexadecane, and heptadecane. The extracted amounts were not statistically significant for any of the common compounds. For buccal swabs, eight out of the fourteen compounds extracted were common across fasting and postprandial samplings: 2-pentylfuran, hexanoic acid, octanoic acid ethyl ester, decanal, nonanoic acid ethyl ester, 6-dodecanone, (E)-6,10dimethyl-5,9-undecadien-2-one, and dodecanoic acid ethyl ester. When subjected to paired $t$-test of significance, the difference in the amounts of dodecanoic acid ethyl ester extracted between fasting and postprandial samplings was found to be statistically significant. For breath, five out of the eight compounds extracted were common across fasting and postprandial samplings: phenol, 2-ethyl-1-hexanol, nonanal, decanal, and butylated hydroxytoluene. The differences in the amounts of these five compounds extracted between samplings were not statistically significant. For blood, ten out of the seventeen compounds extracted were common between samplings: hexanal, nonanal, dodecane, tridecane, 2,4,6-trimethylbenzaldehyde, tetradecane, 1-decene, hexadecane, 2,6-diisopropylnaphthalene, and octadecane. None of the common compounds were have to have a statistically significant difference in the amount extracted betweens samplings. Finally for urine, three out of the eight compounds extracted were common between 
samplings: menthol, 2-isopropylbenzaldehyde, and carvone. 2-Isopropylbenzaldehyde was found to be statistically significantly different in the amount extracted between fasting and postprandial samplings. 
Table 35. Summary of compounds extracted fasting (AM) vs. postprandial (PM) for

Male 2

\begin{tabular}{|c|c|c|c|c|c|c|c|c|c|c|c|c|}
\hline & \multicolumn{2}{|c|}{ Hand } & \multicolumn{2}{|c|}{ Buccal } & \multicolumn{3}{|c|}{ Breath } & \multicolumn{2}{|c|}{ Blood } & \multicolumn{3}{|c|}{ Urine } \\
\hline & $\mathrm{AM}$ & $\mathrm{PM}$ & $\mathrm{AN}$ & $\mathrm{PI}$ & & $\overline{\mathrm{AM}}$ & PM & $\mathrm{AM}$ & $\mathrm{PN}$ & & $\overline{\mathrm{AM}}$ & $\mathrm{PM}$ \\
\hline 1-Decene & & & & & & & & $\mathrm{x}$ & $\mathrm{x}$ & & & \\
\hline 1-Hexanol,2-ethyl- & & & & & & $\mathrm{x}$ & $\mathrm{x}$ & & & & & \\
\hline 1-Tetradecene & & & & $\mathrm{x}$ & & & & & & & & \\
\hline 2,6-Diisopropylnaphthalene & & & & & & & & $\mathrm{x}$ & $\mathrm{x}$ & & & \\
\hline 2-Cyclohexen-1-one,3-methyl-6-(1-methylethyl)- & & & & & & & & & & & & $\mathrm{x}$ \\
\hline 2-Isopropylbenzaldehyde & & & & & & & & & & & $\mathrm{x}$ & $\mathrm{x}$ \\
\hline 4-Heptanone & & & & & & & & & & & $\mathrm{x}$ & \\
\hline 5,9-Undecadien-2-one,6,10-dimethyl-, (E)- & $\mathrm{x}$ & $\mathrm{x}$ & $\mathrm{x}$ & $\mathrm{x}$ & & & & & $\mathrm{x}$ & & & \\
\hline 6-Dodecanone & & & $\mathrm{x}$ & $\mathrm{x}$ & & & & & & & & \\
\hline Allyllsothiocyanate & & & & & & & & & & & & $\mathrm{x}$ \\
\hline Benzaldehyde,2,4,6-trimethyl- & & & & & & & & $\mathrm{x}$ & $\mathrm{x}$ & & & \\
\hline Benzene,1,3-dichloro- & & & & & & & & & & & & $\mathrm{X}$ \\
\hline Benzeneacetaldehyde,.alpha.-methyl- & & & & & & & & & & & $\mathrm{x}$ & \\
\hline Benzophenone & & & & & & & $\mathrm{x}$ & & & & & \\
\hline ButylatedHydroxytoluene & & & & & & $\mathrm{x}$ & $\mathrm{x}$ & & & & & \\
\hline Carvone & & & & & & & $\mathrm{x}$ & & & & $\mathrm{x}$ & $\mathrm{x}$ \\
\hline Cyclopropane,nonyl- & & & $\mathrm{x}$ & & & & & & & & & \\
\hline Decanal & $\mathrm{x}$ & $\mathrm{x}$ & $\mathrm{x}$ & $\mathrm{x}$ & & $\mathrm{x}$ & $\mathrm{x}$ & $\mathrm{x}$ & & & & \\
\hline Diisopropylnaphthalene & & & & & & & & $\mathrm{x}$ & & & & \\
\hline Dodecane & & & & & & & & $\mathrm{x}$ & $\mathrm{x}$ & & & \\
\hline Dodecanoicacid,ethylester & & & $\mathrm{x}$ & $\mathrm{x}$ & & & & & & & & \\
\hline Furan,2-pentyl- & & & $\mathrm{x}$ & $\mathrm{x}$ & & & & & $\mathrm{x}$ & & & \\
\hline Heptadecane & $\mathrm{x}$ & $\mathrm{x}$ & & & & & & $\mathrm{x}$ & & & & \\
\hline Hexadecane & $\mathrm{x}$ & $\mathrm{x}$ & & & & & & $\mathrm{x}$ & $\mathrm{x}$ & & & \\
\hline Hexadecanoicacid,ethylester & & & $\mathrm{x}$ & & & & & & & & & \\
\hline Hexanal & & & & & & & & $\mathrm{x}$ & $\mathrm{x}$ & & & \\
\hline Hexanoicacid & & & $\mathrm{x}$ & $\mathrm{x}$ & & & & & & & & \\
\hline Hexanoicacid,pentylester & & & & $\mathrm{x}$ & & & & & & & & \\
\hline Menthol & & & & & & & $\mathrm{x}$ & & & & $\mathrm{x}$ & $\mathrm{x}$ \\
\hline Naphthalene & & & & & & & & $\mathrm{x}$ & & & & \\
\hline Nonanal & $\mathrm{x}$ & $\mathrm{x}$ & & & & $\mathrm{x}$ & $\mathrm{x}$ & $\mathrm{x}$ & $\mathrm{x}$ & & & \\
\hline Nonanoicacid & & & & $\mathrm{x}$ & & & & & & & & \\
\hline Nonanoicacid,ethylester & & & $\mathrm{x}$ & $\mathrm{x}$ & & & & & & & & \\
\hline Octadecane & & & & & & & & $\mathrm{x}$ & $\mathrm{x}$ & & & \\
\hline Octanoicacid,ethylester & & & $\mathrm{x}$ & $\mathrm{x}$ & & & & & & & & \\
\hline Phenol & & & & & & $\mathrm{x}$ & $\mathrm{x}$ & & & & & \\
\hline Tetradecane & $\mathrm{x}$ & $\mathrm{x}$ & & & & & & $\mathrm{x}$ & $\mathrm{x}$ & & & \\
\hline Tetradecanoicacid,ethylester & & & $\mathrm{x}$ & & & & & & & & & \\
\hline Tridecane & & & & & & & & $\mathrm{x}$ & $\mathrm{x}$ & & & \\
\hline Undecane & $\mathrm{x}$ & & & & & & & & $\mathrm{x}$ & & & \\
\hline
\end{tabular}


Comparisons of compounds that were extracted at fasting and postprandial sampling times from the five biological specimens for subject Male 5 are summarized in Table 36 . For each specimen, the compounds that were extracted from both fasting and postprandial samplings were subjected to paired $t$-test of significance to evaluate whether the variation in the amounts of the compound extracted was statistically significant or not. For hand odor, nine out of the twelve compounds extracted were common across fasting and postprandial samplings: undecane, nonanal, decanal, tridecane, tetradecane, hexadecane, heptadecane, and octadecane. The extracted amounts were not statistically significant for any of the common compounds. For buccal swabs, ten out of the twenty compounds extracted were common across fasting and postprandial samplings: 2pentylfuran, hexanoic acid, 6-methyl-3,5-heptadiene-2-one, 1-dodecene, decanal, nonanoic acid, 6-dodecanone, 1-tetradecene, (E)-6,10-dimethyl-5,9-undecadien-2-one, and 1-decene. When subjected to paired $t$-test of significance, the differences in the amounts of these common compounds extracted were not statistically significant. For breath, eight out of the thirteen compounds extracted were common across fasting and postprandial samplings: toluene, p-xylene, phenol, 1,2-dichlorobenzene, nonanal, decanal, tetradecane, and butylated hydroxytoluene. The amount extracted for phenol was statistically significantly different between the two samplings. For blood, ten out of the twenty-one compounds extracted were common between samplings: hexanal, dimethylsulfone, undecane, nonanal, dodecane, tridecane, tetradecane, 1-decene, hexadecane, diisopropylnaphthalene, and 2,6-diisopropylnaphthalene. None of the common compounds were found to have a statistically significant difference in the amount extracted betweens samplings. Finally for urine, five out of the fourteen 
compounds extracted were common between samplings: pyrrole, 4-heptanone, dimethylsulfone, nonanal, and menthol. Menthol was found to be statistically significantly different in the amount extracted between fasting and postprandial samplings. 
Table 36. Summary of compounds extracted fasting (AM) vs. postprandial (PM) for Male 5

\begin{tabular}{|c|c|c|c|c|c|c|c|c|c|c|}
\hline & \multicolumn{2}{|c|}{ Hand } & \multicolumn{2}{|c|}{ Buccal } & \multicolumn{2}{|c|}{ Breath } & \multicolumn{2}{|c|}{ Blood } & \multicolumn{2}{|c|}{ Urine } \\
\hline & $\mathrm{AM}$ & PM & $\mathrm{AM}$ & PM & $\mathrm{AM}$ & PM & $\mathrm{AM}$ & PM & $\mathrm{AM}$ & PM \\
\hline beta.-Phellandrene & & & & & & & & & & $\mathrm{x}$ \\
\hline 1,3-Cyclohexadiene,1-methyl-4-(1-methylethyl)- & & & & & & & & & & $\mathrm{x}$ \\
\hline 1,4-Cyclohexadiene,1-methyl-4-(1-methylethyl)- & & & & & & & & & & $\mathrm{x}$ \\
\hline 1-Decene & & & $\mathrm{x}$ & $\mathrm{x}$ & & & $\mathrm{x}$ & $\mathrm{x}$ & & \\
\hline 1-Dodecene & & & $\mathrm{x}$ & $\mathrm{x}$ & & & & $\mathrm{x}$ & & \\
\hline 1-Heptanol & & & & & & & & $\mathrm{x}$ & & \\
\hline 1-Hexadecene & & & $\mathrm{x}$ & & & & & & & \\
\hline 1-Hexanol & & & $\mathrm{x}$ & & & & & & & \\
\hline 1-Hexanol,2-ethyl- & & & & & $\mathrm{x}$ & & & & & \\
\hline 1-Pentanol & & & & & & & & $\mathrm{x}$ & & \\
\hline 1-Tetradecene & & & $\mathrm{x}$ & $\mathrm{x}$ & & & & & & \\
\hline 2,4-Nonadienal & & & & $\mathrm{x}$ & & & & . & & \\
\hline 2,5-Octanedione & & & & & & & & $\mathrm{x}$ & & \\
\hline 2,6-Diis opropylnaphthalene & & & & & & & $\mathrm{x}$ & $\mathrm{x}$ & & \\
\hline 4-Heptanone & & & & & & & & & $\mathrm{x}$ & $\mathrm{x}$ \\
\hline 5,9-Undecadien-2-one,6,10-dimethyl-, (E)- & & $\mathrm{x}$ & $\mathrm{x}$ & $\mathrm{x}$ & & & & & & \\
\hline 6-Dodecanone & & & $\mathrm{x}$ & $\mathrm{x}$ & & & & & & \\
\hline 6-Methyl-3,5-heptadiene-2-one & & & $\mathrm{x}$ & $\mathrm{x}$ & & & & & & \\
\hline Benzaldehyde,2,4,6-trimethyl- & & & & & & & $\mathrm{x}$ & & & \\
\hline Benzene,1,2-dichloro- & & & & & $\mathrm{x}$ & $\mathrm{x}$ & & & & \\
\hline Benzene,1,3-dimethyl- & & & & & $\mathrm{x}$ & $\mathrm{x}$ & & & & \\
\hline Benzene,1-methyl-4-(1-methyletheny1)- & & & & & & & & & & $\mathrm{x}$ \\
\hline Benzene,1-methyl-4-(1-methylethyl)- & & & & & & & & & & $\mathrm{x}$ \\
\hline Benzenemethanol,.alpha.,.alpha.,4-trimethyl- & & & & & & & & & & $\mathrm{x}$ \\
\hline Benzophenone & & & & & & $\mathrm{x}$ & & & & \\
\hline BenzylAlcohol & & & & & & & $\mathrm{x}$ & & & \\
\hline ButylatedHydroxy toluene & & & & $\mathrm{x}$ & $\mathrm{x}$ & $\mathrm{x}$ & & & & \\
\hline Carvone & & & & & & & & & $\mathrm{x}$ & \\
\hline Caryophyllene & & & & $\mathrm{x}$ & & & & & & \\
\hline Cedrol & & & & & & & & & & $\mathrm{x}$ \\
\hline Cyclopropane,nonyl- & & & $\mathrm{x}$ & & & & & & & \\
\hline Decanal & $\mathrm{x}$ & $\mathrm{x}$ & $\mathrm{x}$ & $\mathrm{x}$ & $\mathrm{x}$ & $\mathrm{x}$ & & $\mathrm{x}$ & & \\
\hline DiethylPhthalate & & & & $\mathrm{x}$ & & & & & & \\
\hline Diis opropylnaphthalene & & & & & & & $\mathrm{x}$ & $\mathrm{x}$ & & \\
\hline Dimethylsulfone & & & & & & & $\mathrm{x}$ & $\mathrm{x}$ & $\mathrm{x}$ & $\mathrm{x}$ \\
\hline Dimethyltrisulfide & & & & & & 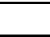 & & & & $\mathrm{x}$ \\
\hline D-Limonene & & & & & & $\mathrm{x}$ & & 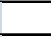 & & \\
\hline Dodecane & & & & & & & $\mathrm{x}$ & $\mathrm{x}$ & & \\
\hline Dodecanoicacid & & $\mathrm{x}$ & & & & & & & & \\
\hline Furan,2-pentyl- & & & $\mathrm{x}$ & $\mathrm{x}$ & & & & $\mathrm{x}$ & & \\
\hline Heptadecane & $\mathrm{x}$ & $\mathrm{x}$ & & & & & & & & \\
\hline Hexadecane & $\mathrm{x}$ & $\mathrm{x}$ & & & & & $\mathrm{x}$ & $\mathrm{x}$ & & \\
\hline Hexanal & & & & & & $\mathrm{x}$ & $\mathrm{x}$ & $\mathrm{x}$ & & \\
\hline Hexanoicacid & & & $\mathrm{x}$ & $\mathrm{x}$ & & & & & & \\
\hline Menthol & & & & & & $\mathrm{x}$ & & $\mathrm{x}$ & $\mathrm{x}$ & $\mathrm{x}$ \\
\hline Naphthalene,2-methyl- & & & $\mathrm{x}$ & & & & & & & \\
\hline Nonanal & $\mathrm{x}$ & $\mathrm{x}$ & & & $\mathrm{x}$ & $\mathrm{x}$ & $\mathrm{x}$ & $\mathrm{x}$ & $\mathrm{x}$ & $\mathrm{x}$ \\
\hline Nonanoicacid & & & $\mathrm{x}$ & $\mathrm{x}$ & & & & & & \\
\hline Nonanoicacid,ethylester & & & & $\mathrm{x}$ & & & & & & \\
\hline Octadecane & $\mathrm{x}$ & $\mathrm{x}$ & & & & & & $\mathrm{x}$ & & \\
\hline Pentadecane & $\mathrm{x}$ & & & & & & & & & \\
\hline Phenol & & & & & $\mathrm{x}$ & $\mathrm{x}$ & & & & \\
\hline Pyrrole & & & & & & & & & $\mathrm{x}$ & $\mathrm{x}$ \\
\hline Tetradecane & $\mathrm{x}$ & $\mathrm{x}$ & & & $\mathrm{x}$ & $\mathrm{x}$ & $\mathrm{x}$ & $\mathrm{x}$ & & \\
\hline Toluene & & & & & $\mathrm{x}$ & $\mathrm{x}$ & & & & \\
\hline Tridecane & $\mathrm{x}$ & $\mathrm{x}$ & & & & & $\mathrm{x}$ & $\mathrm{x}$ & & \\
\hline Undecanal & & $\mathrm{x}$ & & & & & 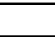 & & & \\
\hline Undecane & $\mathrm{x}$ & $\mathrm{x}$ & & & & & $\mathrm{x}$ & $\mathrm{x}$ & & \\
\hline$\Delta$-Cadinene & & & $\mathrm{x}$ & & & & & & & \\
\hline
\end{tabular}




\subsubsection{Cluster Analysis}

Cluster analysis is a statistical method which classifies a set of observations into subsets, or clusters, based on similarities or dissimilarities (distances). The Bray-Curtis Similarity Index (BSCI) is a normalization method which measures the resemblance, or similarity, between samples. Bray-Curtis, originally a dissimilarity measure that falls between zero and one, is multiplied by 100 to give percentage similarity. The equation for BCSI is as follows:

$$
B S C I=100\left(1-\frac{\sum_{i=1}^{n}\left|X_{i j}-X_{i k}\right|}{\sum_{i=1}^{n}\left(X_{i j}+X_{i k}\right)}\right) \quad \text { Equation } 5
$$

$\mathrm{X} i j$ is the peak area for the $i$ th compound from sample $j$. Data classified using BSCI can be displayed in the form of a two-dimensional diagram known as a dendogram. Hierarchical clusters represented by dendograms were constructed using the Minitab 15 Statistical Software (Minitab Inc., State College, PA, USA).

The following figures (Figure 68 through Figure 72) demonstrate, in semi-quantitative fashion, examples of the relative ratios of the peak areas of the compounds extracted in the headspace above the collected specimen samples along with the corresponding dendograms which show the similarity between the VOC profiles in the intra-day (fasting vs. postprandial) specimen samples from a male subject and a female subject. The greatest variation between the intra-day samples were observed in urine. Urinary volatiles should be most susceptible to variation because urinary components are filtered waste products being secreted from the body. The intra-day variation of urine is affected by the food and beverages consumed by the subject. However, sampling immediately after 
consuming a meal as it was done in this study should not have influenced the VOC profiles, because the metabolites and waste products from the meal consumed prior to sampling will not have been secreted yet. Therefore, it should be noted that the intra-day variation of urine samples in this study are not results from fasting or postprandial prior to sampling.

Figure 68. Color chart and dendogram for hand odor for M2 and F15

M2

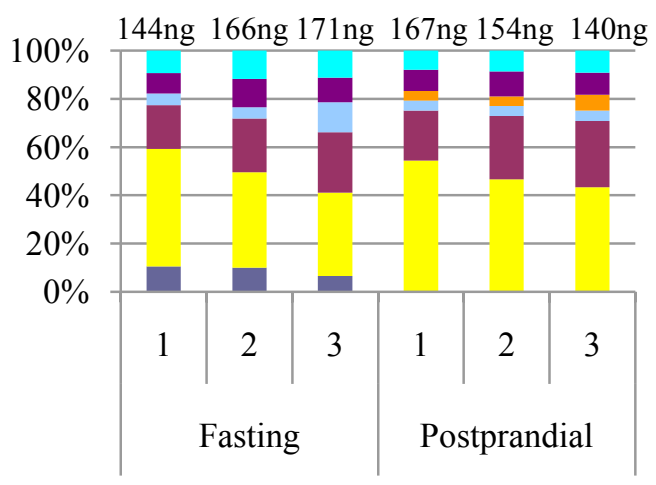

F15

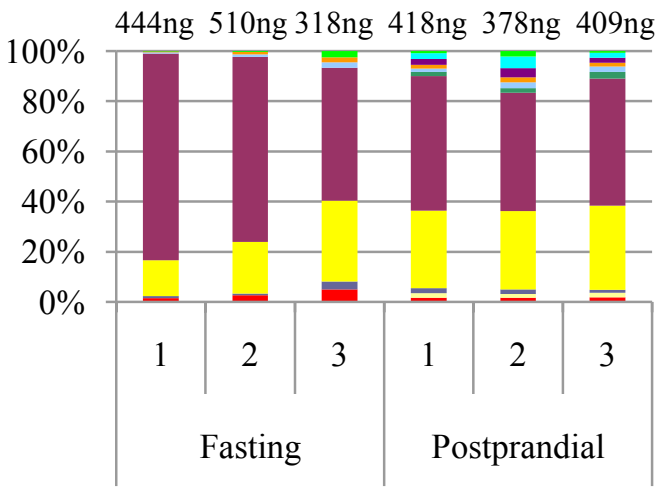

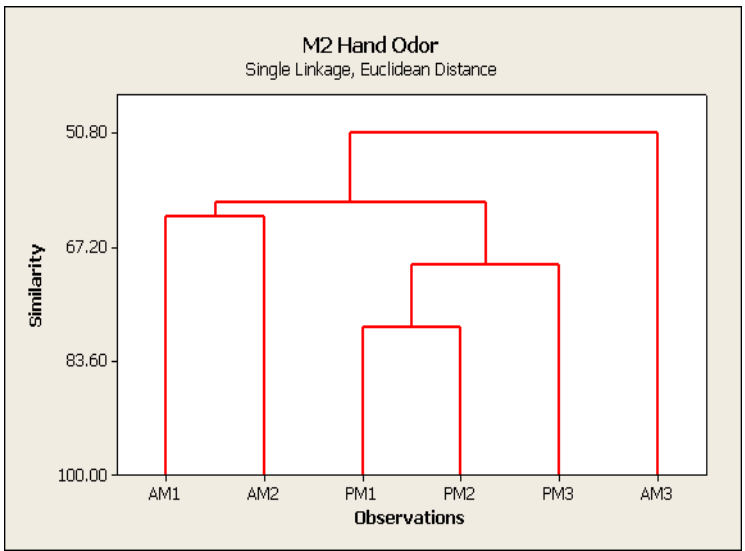

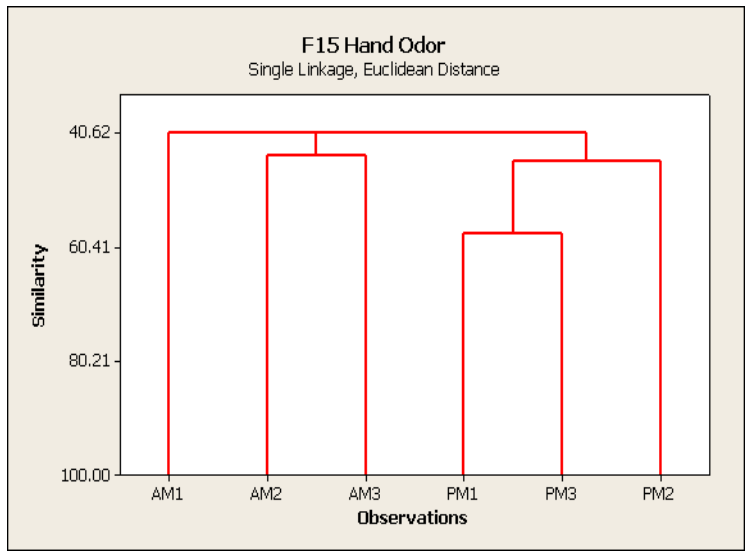


Figure 69. Color chart and dendogram for buccal swabs for M2 and F15

M2

266ng 259ng 292ng 363ng 377ng 387ng

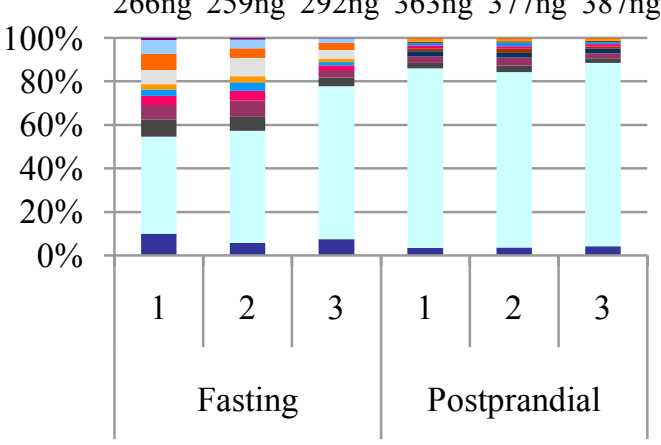

F15

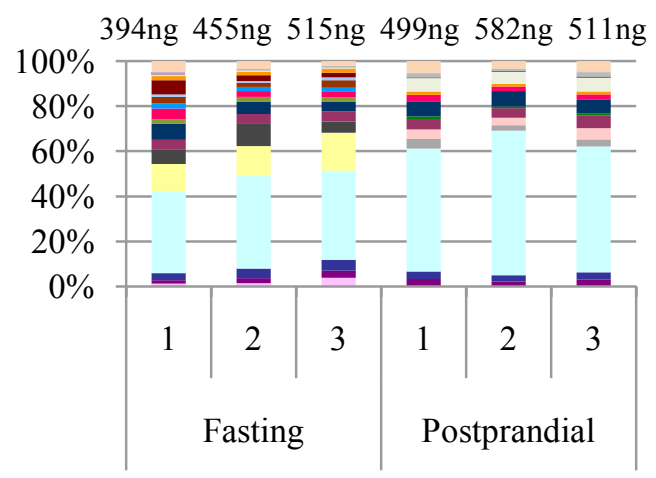

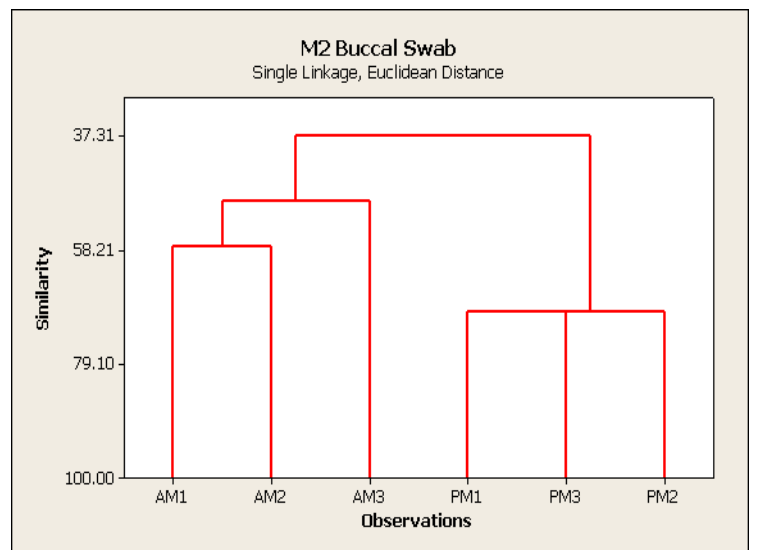

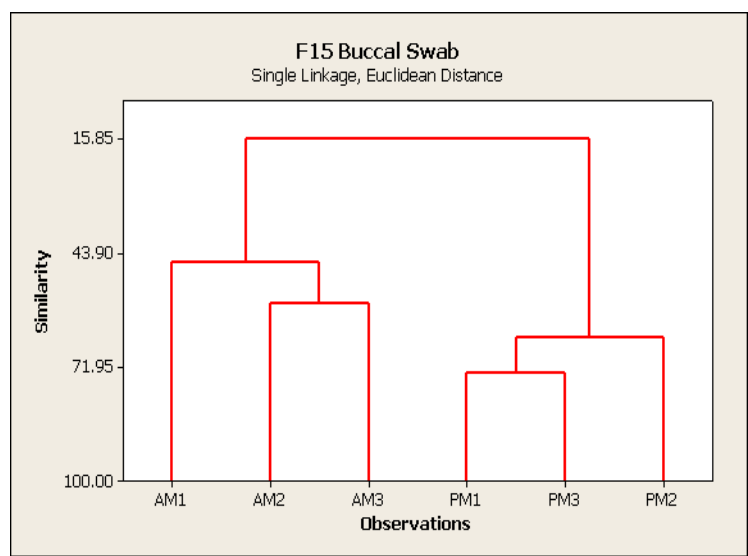


Figure 70. Color chart and dendogram for breath for M2 and F15

M2
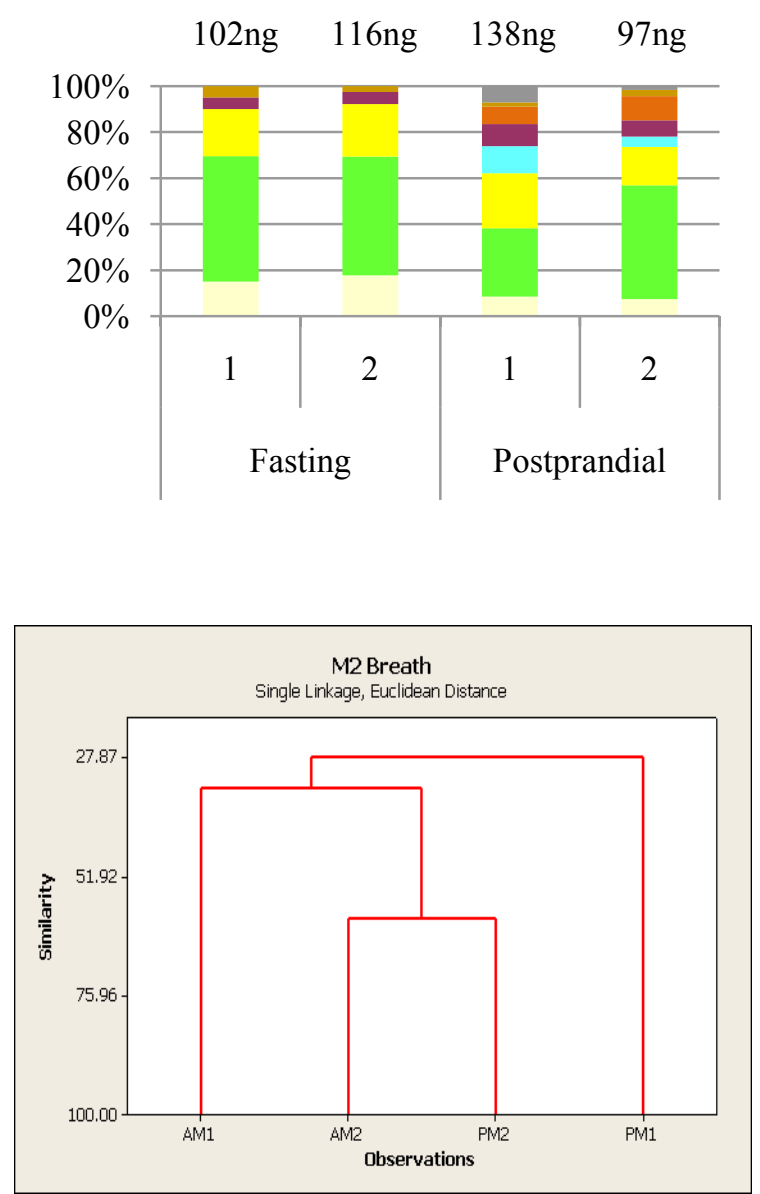

F15
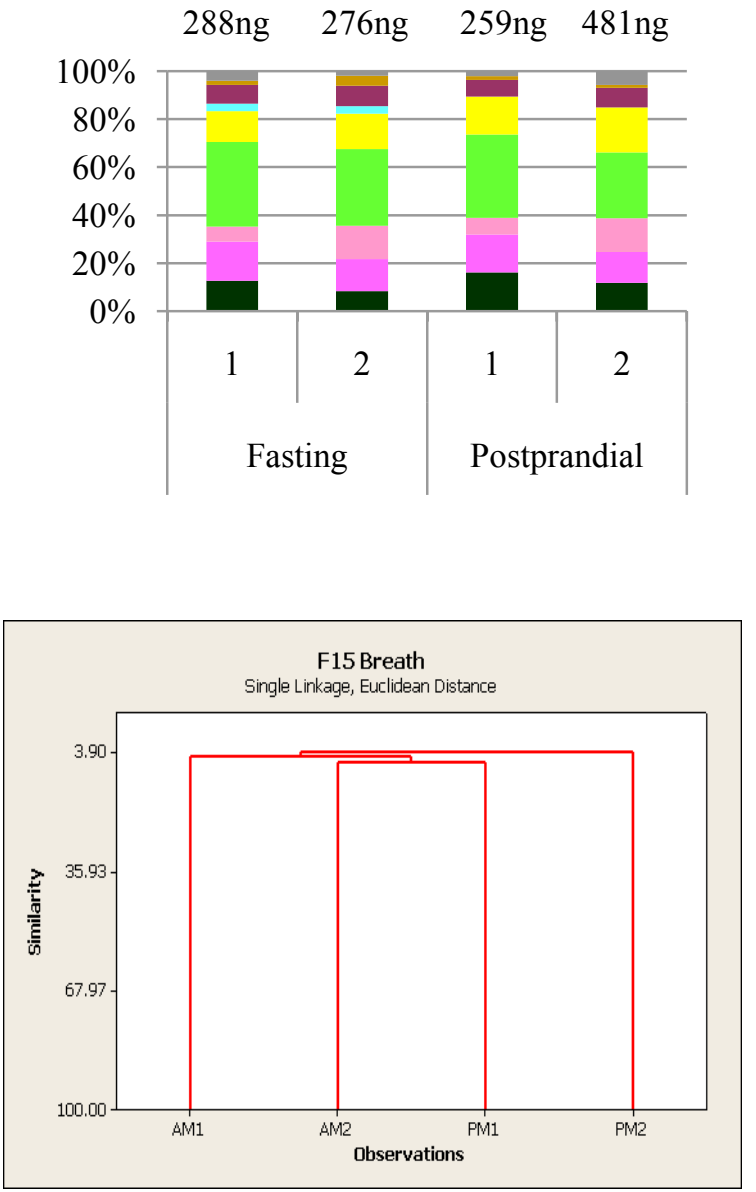
Figure 71. Color chart and dendogram for blood for M2 and F15

M2
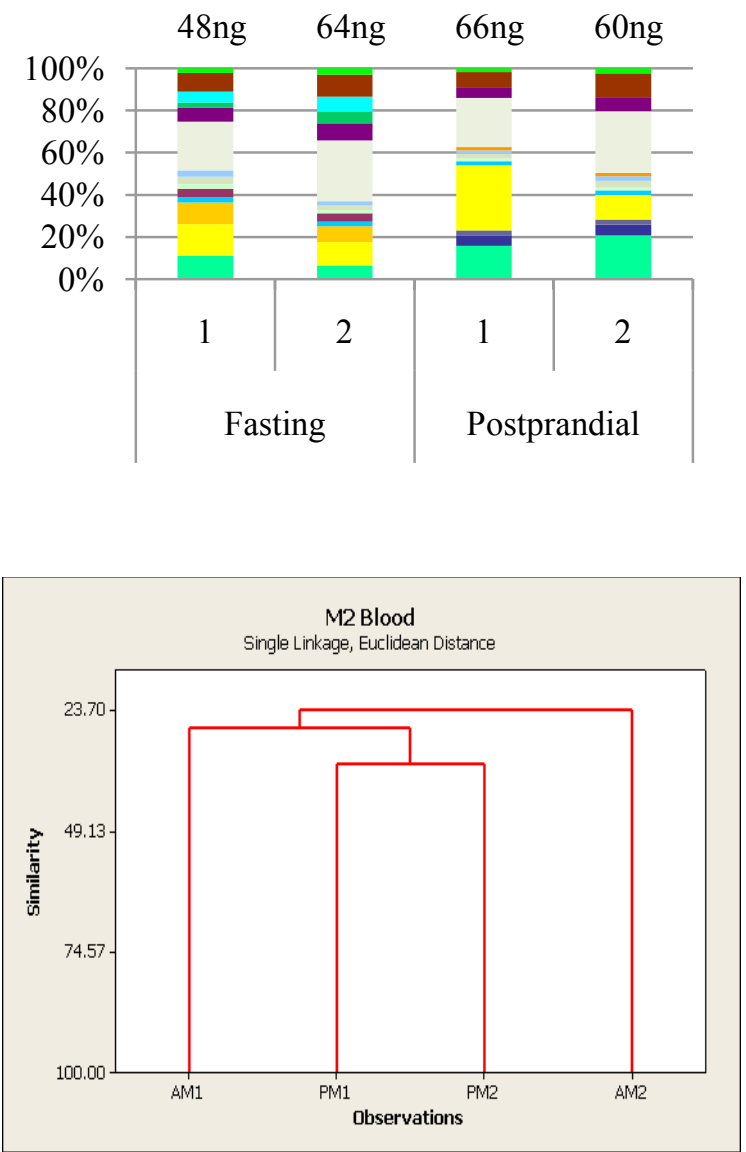

F15
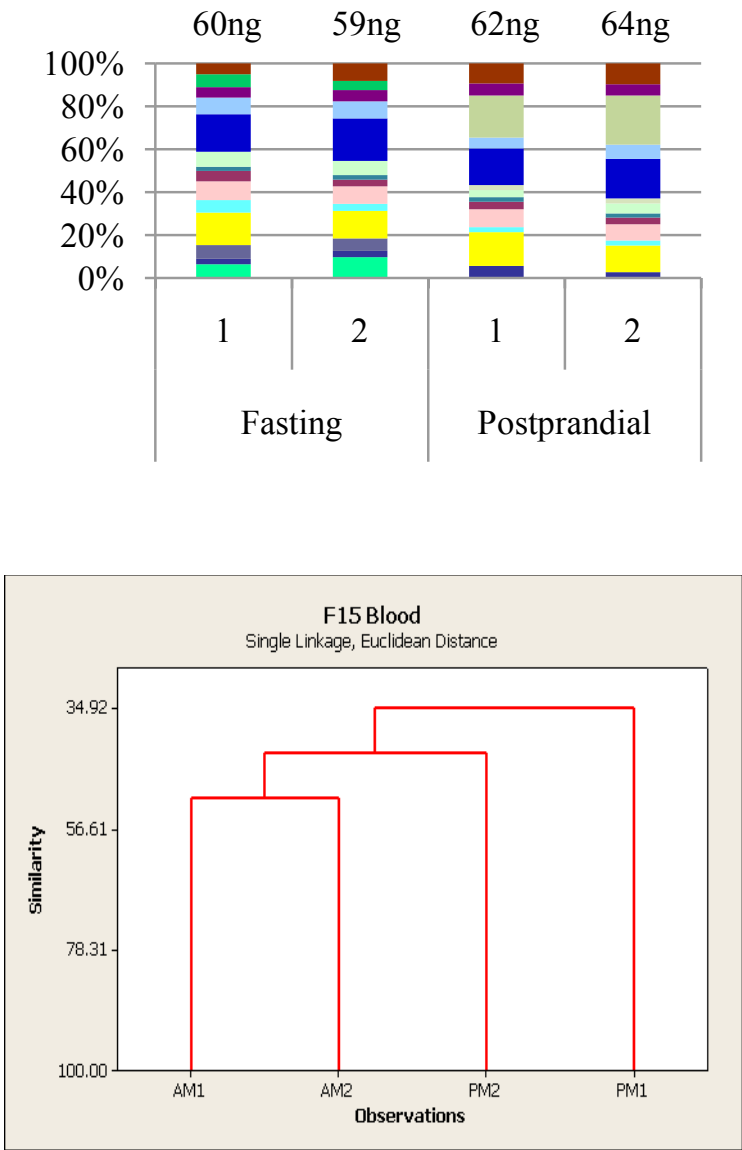
Figure 72. Color chart and dendogram for urine for M2 and F15

M2
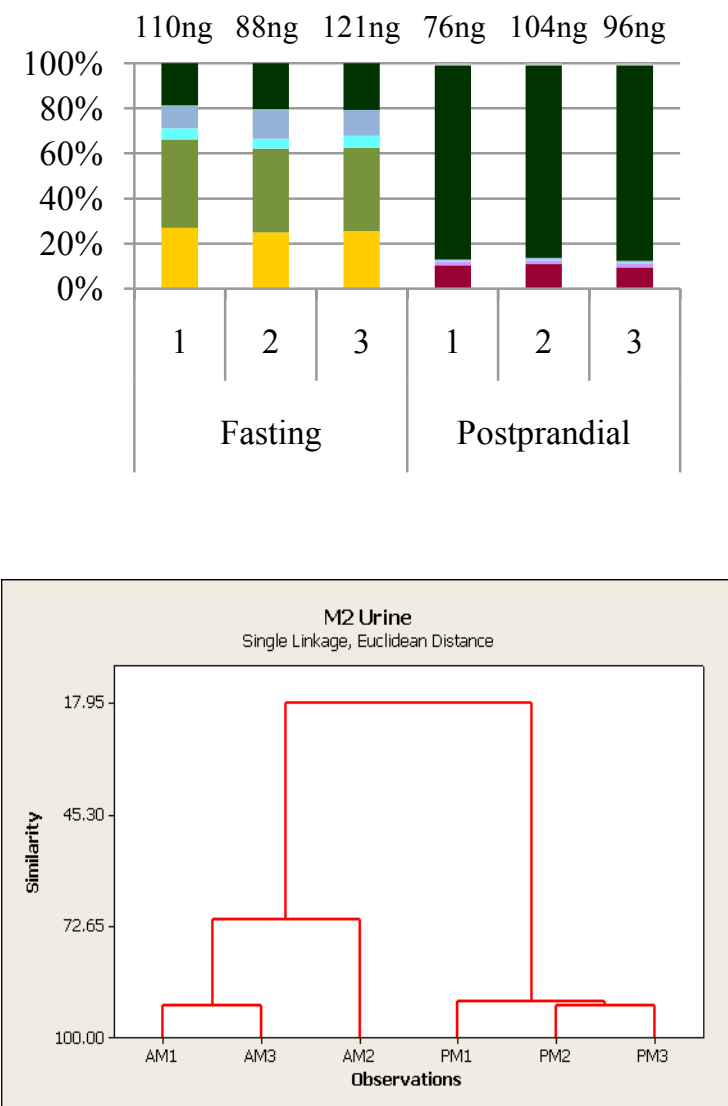

F15
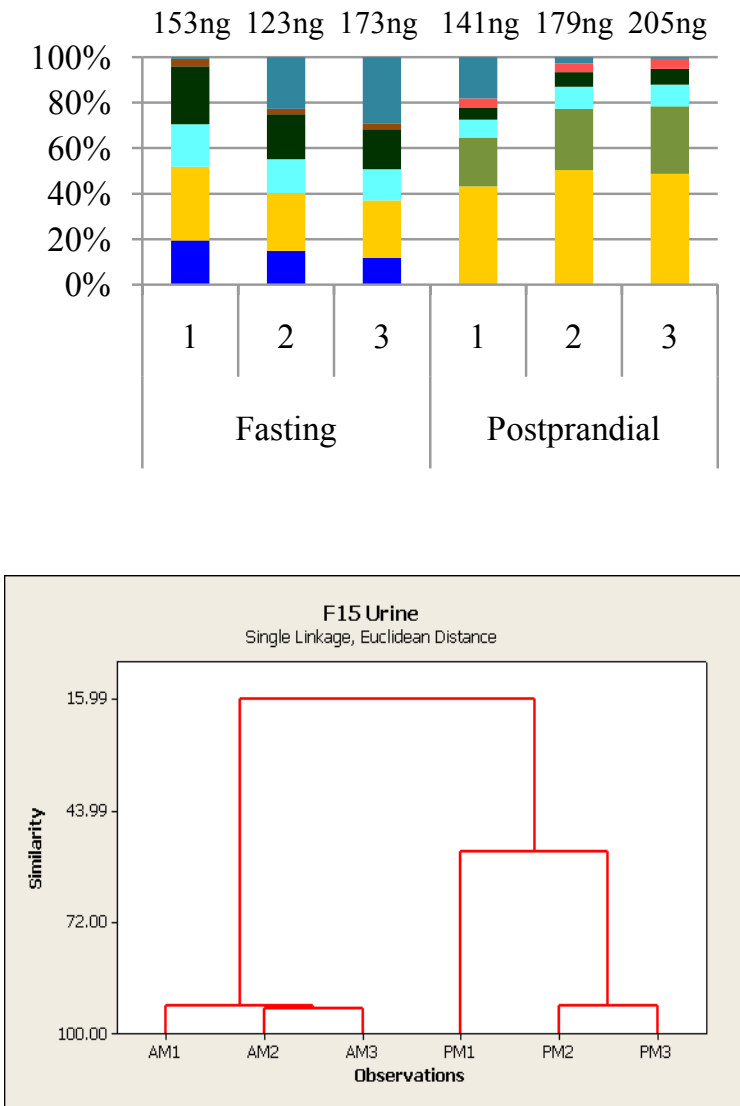

The effect of fasting prior to sampling was evaluated through the number of compounds extracted, paired $t$-test of significance of the amount of compounds extracted both at fasting and postprandial samplings, relative peak area ratios of the compounds extracted, and cluster analysis using BCSI. While the fasting samples and postprandial samples are more similar within the same sampling condition, the difference in the VOC profiles of samples collected at fasting and postprandial were not drastic enough to conclude that fasting is crucial prior to sampling. Fasting prior to sampling is more important for 
medical purposes, particularly for blood testing (i.e., blood glucose or blood cholesterol). This is particularly true for patients with diabetes. Food intake disturbs blood glucose homeostasis and changes metabolic response of the body to ingested materials. Fasting can also affect the presence of ketone bodies in urine, resulting in higher production of ketone bodies (ketonuria). Additionally, for medical purposes, first morning urine is usually a preferred screening sample because it is most concentrated and prevents falsenegative tests.

Forensically, fasting prior to sampling is not as important as it would be for medical profiling purposes. In real life scenarios, samples collected from suspects or subjects will have been sampled at random time of the day and will most likely not be fasting. With only a small increase in the number of compounds extracted and the amount of common compounds extracted between fasting and postprandial sampling being mostly not statistically significant, whether or not the suspect or subject of interest was fasting or had just consumed food should not greatly influence the specimen VOC profile in terms of forensic applications.

\subsubsection{Population Analysis of the Volatile Organic Compounds Present Above Collected} Odor Samples

\subsubsection{Evaluation of Odor Profiles of Healthy Individuals}

Odor profiles of thirty-one healthy individuals were evaluated using SPME-GC/MS. Compounds were identified by spectral library (NIST) and/or by standard reference comparison. Thirty-six VOCs were extracted across the hand odor samples collected from each of the thirty-one subjects. The compounds ranged in functionality, including: 
acids $(2.8 \%)$, alcohols $(11.1 \%)$, aldehydes $(27.8 \%)$, aliphatics $(38.9 \%)$, esters $(11.1 \%)$, and ketones (8.3\%) as shown in Figure 73 . The frequency of the occurrence of the VOCs extracted in healthy subjects' hand odor are listed in Table 37 and displayed in the histogram in Figure 74. Of the short to mid-chain aldehydes $\left(\mathrm{C}_{5}-\mathrm{C}_{14}\right)$ extracted from hand odor, nonanal, decanal, and undecanal were present in more than $90 \%$ of the subjects' odor profiles. Hand odor (skin of palms of hands and forearms) is comprised of secretions from the sebaceous and the eccrine glands. Aldehydes have been reported to be oxidative degradation products of sebaceous secretion components ${ }^{5,14}$. Likewise, alcohols and free fatty acids derive from the interaction of sebaceous secretion components and bacteria residing on $\operatorname{skin}^{14}$. Hydrolysis of triglycerides from cutaneous bacterial activity results in free fatty acids (mostly shorter than $\mathrm{C}_{20}$, as was the case in the present study). Acids however are not as prominent in skin odor as aldehydes or aliphatics, as many of the shorter chain free fatty acids $\left(\mathrm{C}_{6}-\mathrm{C}_{10}\right)$ are reduced to alcohols on skin ${ }^{123}$. Mid- to longchain alkanes $\left(\mathrm{C}_{11}-\mathrm{C}_{20}\right)$ were observed in the present study, of which tetradecane, hexadecane, and heptadecane were present in over $90 \%$ of the subjects. Three ketones were extracted, of which (E)-6,10-dimethyl-5,9-undecadien-2-one (geranyl acetone) was present in over two thirds of the subjects. 
Figure 73. Frequency distribution of functional groups of extracted volatile organic compounds for hand odor

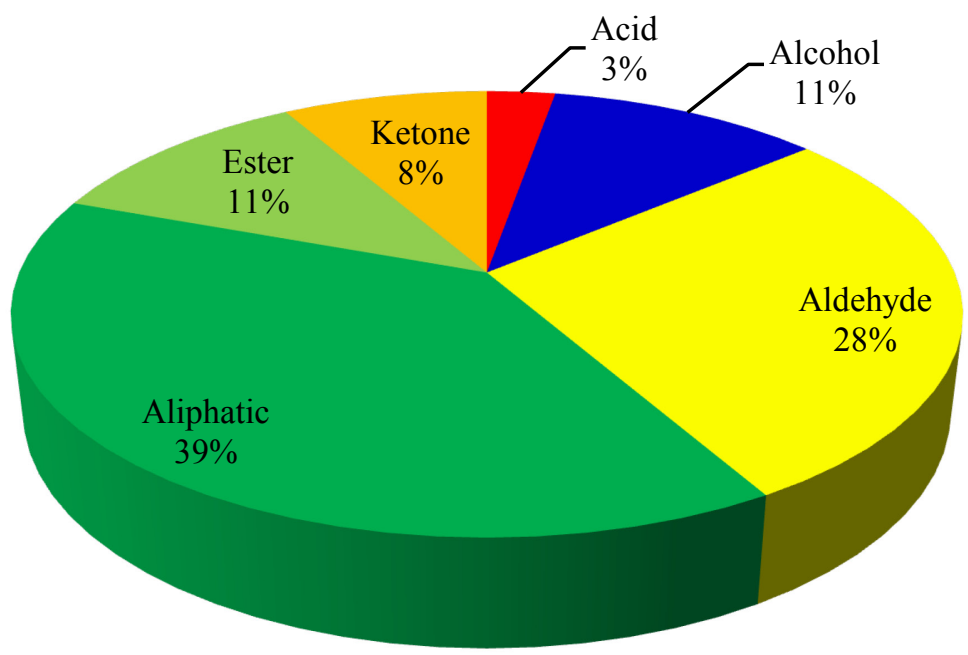


Table 37. Identified VOCs in hand odor of thirty-one healthy individuals ranked by frequency of occurrence (Note: * denotes compound was verified by reference standard)

\begin{tabular}{|l|ccc|c|}
\hline \multicolumn{1}{|c|}{ Compound Name } & \multicolumn{3}{|c|}{ Frequency } & Occurrence \\
& Female & Male & Total & $(\%)$ \\
\hline Decanal* & 16 & 15 & 31 & 100 \\
\hline Nonanal* $^{*}$ (16 & 15 & 31 & 100 \\
\hline Tetradecane* & 16 & 15 & 31 & 100 \\
\hline Hexadecane* & 15 & 15 & 30 & 96.8 \\
\hline Heptadecan** & 14 & 14 & 28 & 90.3 \\
\hline Undecanal* $^{*}$ & 14 & 14 & 28 & 90.3 \\
\hline 5,9-Undecadien-2-one,6,10-dimethyl-, (E)-* & 9 & 14 & 23 & 74.2 \\
\hline Tridecane* & 9 & 13 & 22 & 71.0 \\
\hline Octadecane* & 8 & 10 & 18 & 58.1 \\
\hline Undecane & 8 & 7 & 15 & 48.4 \\
\hline Dodecane* & 9 & 5 & 14 & 45.2 \\
\hline Benzyl Alcohol* & 5 & 8 & 13 & 41.9 \\
\hline Dodecanal* & 5 & 5 & 10 & 32.3 \\
\hline 1,6-Octadien-3-ol,3,7-dimethyl-* & 4 & 3 & 7 & 22.6 \\
\hline 1-Hexanol,2-ethyl-* & 4 & 2 & 6 & 19.4 \\
\hline 2-Decanone* & 3 & 3 & 6 & 19.4 \\
\hline Benzaldehyde* & 3 & 1 & 4 & 12.9 \\
\hline Nonanoicacid,methylester & 2 & 2 & 4 & 12.9 \\
\hline 2-Nonenal,(E)-* & 2 & 1 & 3 & 9.7 \\
\hline Dodecanoicacid,methylester & 2 & 1 & 3 & 9.7 \\
\hline Dodecanoicacid* & 0 & 2 & 2 & 6.5 \\
\hline Eicosane* & 0 & 2 & 2 & 6.5 \\
\hline Furfural* & 1 & 1 & 2 & 6.5 \\
\hline Octanoicacid,methylester* & 0 & 2 & 2 & 6.5 \\
\hline Phenol* & 1 & 1 & 2 & 6.5 \\
\hline 1-Pentadecene* & 1 & 0 & 1 & 3.2 \\
\hline Docosane* & 0 & 1 & 1 & 3.2 \\
\hline Heneicosane* & 0 & 1 & 1 & 3.2 \\
\hline Heptanal* & 0 & 1 & 1 & 3.2 \\
\hline Hexanoicacid & 0 & 1 & 1 & 3.2 \\
\hline Octanal* & 1 & 0 & 1 & 3.2 \\
\hline Propanedioicacid,dimethylester* & 1 & 0 & 1 & 3.2 \\
\hline Tetradecanal & 1 & 0 & 1 & 3.2 \\
\hline Acetophenone* & 2 & 1 & 3 & 0.1 \\
\hline Pentadecane* & 0 & 2 & 2 & 0.1 \\
\hline & & & & \\
\hline & & & 7 \\
\hline
\end{tabular}


Figure 74. Histogram of VOCs in hand odor across thirty-one healthy individuals

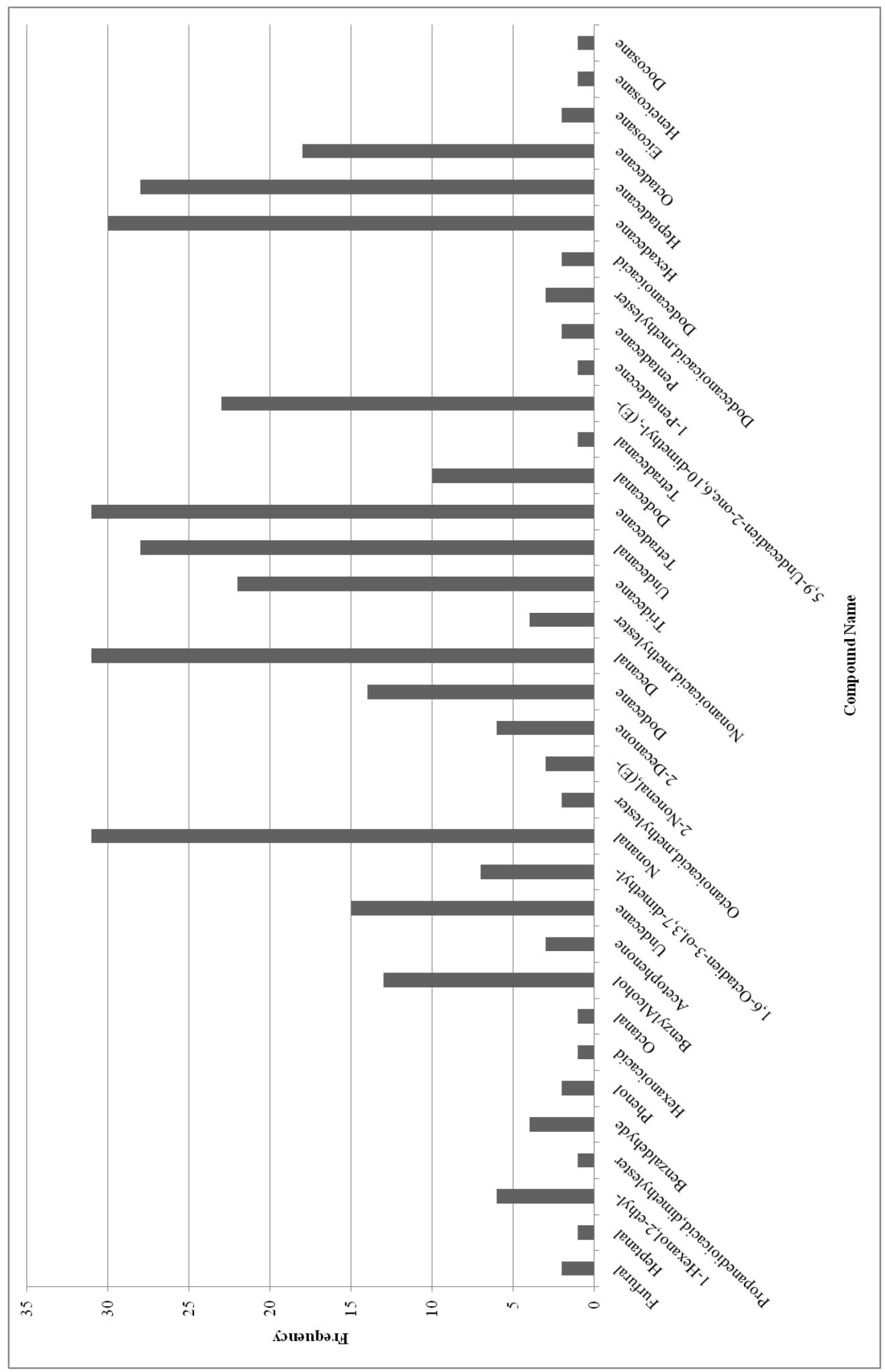


For buccal swab samples, 109 VOCs were extracted across the samples collected from the thirty-one healthy individuals. The functionality distribution of the extracted volatile compounds was as follows: acids (10.1\%), alcohols (5.5\%), aldehydes (11.0\%), aliphatics (26.6\%), aromatics $(12.8 \%)$, esters $(21.1 \%)$, ketones $(11.9 \%)$, and others $(0.9 \%)$. Figure 75 displays a pie chart of the functionality distribution. The frequency of the occurrence of the buccal swab VOCs from the thirty-one healthy individuals are listed in Table 38 and displayed in the histogram in Figure 76. The compounds with the highest frequency of occurrences (>80.0\%) were (E)-6,10-dimethyl-5,9-undecadiene-2-one, decanal, hexanoic acid, 2-pentylfuran, (E,E)-2,4-nonadienal, nonanoic acid ethyl ester, and (E)-2nonenal.

While aliphatic compounds have the highest frequency distribution of functional groups in buccal swab odor, top two-thirds of the compounds detected in the headspace of buccal swab samples were fatty acid ester compounds, and specifically ethyl esters of long chain fatty acids $\left(\mathrm{C}_{8}-\mathrm{C}_{16}\right)$. Carboxylic acids were also widely present in buccal swab samples. Hexanoic acid was the most predominant compound extracted from buccal swabs (and present in $100 \%$ of the subjects); its chromatographic peaks were usually very large and with a wide peak width also. Hexanoic acid peaks in the gas chromatograms of buccal swabs were generally not a chromatographically ideal peak, making quantitation for hexanoic acid difficult.

It is difficult to specify the exact origin of the VOCs found in buccal swab, as there are many possible routes of VOC entry into the salivary flow. Volatile constituents of oral fluids can be attributed to environmental and occupational exposure through inhalation of 
air and/or water vapor through the lungs, ingestion through the mouth, and transdermal absorption through the skin. Putrefactive activities of microorganisms in the mouth also affect the production of volatiles in buccal swab samples. Blood-saliva partition must be studied from the size and solubility (in water or lipids) of the volatile compounds.

Figure 75. Frequency distribution of functional groups of extracted volatile organic compounds for buccal swabs

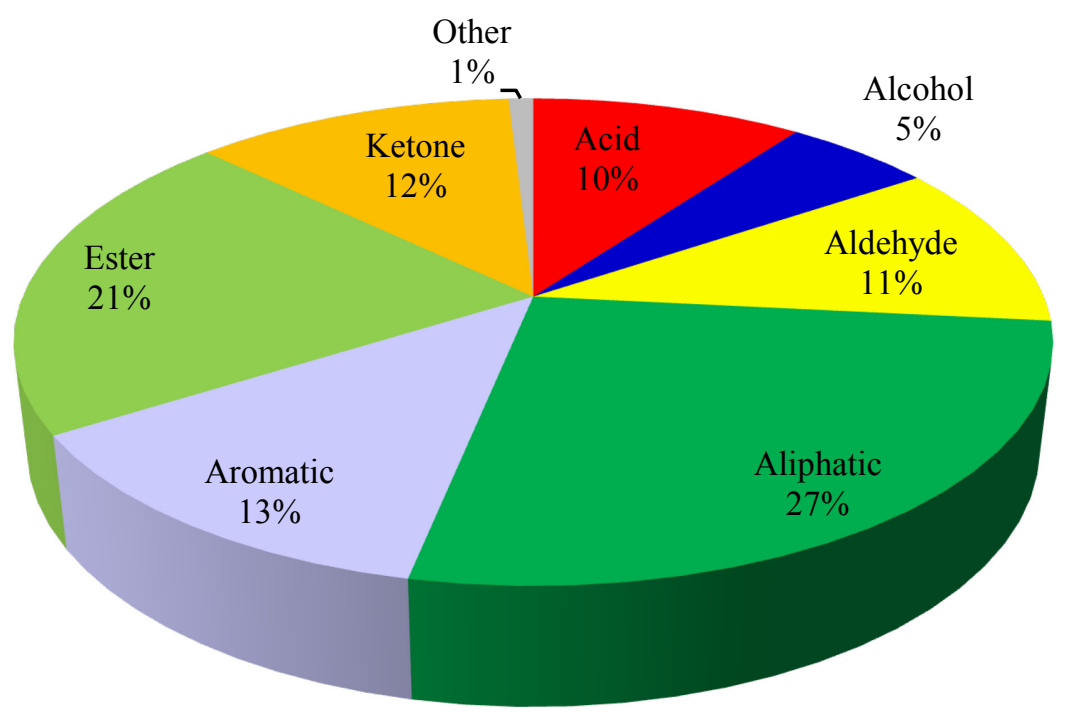


Table 38. Identified VOCs in buccal swab of thirty-one healthy individuals ranked by frequency of occurrence (Note: *denotes compound was verified by reference standard)

\begin{tabular}{|c|c|c|c|c|}
\hline \multirow{2}{*}{ Compound } & \multicolumn{3}{|c|}{ Frequency } & \multirow{2}{*}{$\begin{array}{c}\text { Occurrence } \\
(\%)\end{array}$} \\
\hline & Female & Male & Total & \\
\hline 5,9-Undecadien-2-one,6,10-dimethyl-, (E)-* & 16 & 15 & 31 & 100 \\
\hline Decanal* & 16 & 15 & 31 & 100 \\
\hline Hexanoicacid* & 16 & 15 & 31 & 100 \\
\hline Furan,2-pentyl-* & 15 & 15 & 30 & 96.8 \\
\hline 2,4-Nonadienal,(E,E)-* & 14 & 14 & 28 & 90.3 \\
\hline Nonanoicacid,ethylester* & 13 & 14 & 27 & 87.1 \\
\hline 2-Nonenal,(E)-* & 12 & 13 & 25 & 80.7 \\
\hline 6-Dodecanone* & 14 & 10 & 24 & 77.4 \\
\hline Dodecanoicacid,ethylester* & 11 & 13 & 24 & 77.4 \\
\hline Nonanoicacid & 13 & 11 & 24 & 77.4 \\
\hline Hexadecanoicacid,ethylester & 8 & 12 & 20 & 64.5 \\
\hline Hexanoicacid,ethylester & 7 & 13 & 20 & 64.5 \\
\hline Tetradecanoicacid,ethylester & 8 & 12 & 20 & 64.5 \\
\hline Octanoicacid,ethylester* & 9 & 10 & 19 & 61.3 \\
\hline Hexanal* & 10 & 8 & 18 & 58.1 \\
\hline 1-Tetradecene* & 6 & 11 & 17 & 54.8 \\
\hline Naphthalene,1-methyl-* & 8 & 6 & 14 & 45.2 \\
\hline 1,1'-Biphenyl,2,2'-diethyl- & 6 & 7 & 13 & 41.9 \\
\hline 1-Dodecene* & 4 & 9 & 13 & 41.9 \\
\hline Hexanoicacid,pentylester & 6 & 7 & 13 & 41.9 \\
\hline Benzaldehyde* & 9 & 3 & 12 & 38.7 \\
\hline Naphthalene,2-methyl- & 6 & 6 & 12 & 38.7 \\
\hline Dibutanoylmorphine & 3 & 8 & 11 & 35.5 \\
\hline Linalool oxide & 5 & 6 & 11 & 35.5 \\
\hline 6-Methyl-3,5-heptadiene-2-one & 3 & 7 & 10 & 32.3 \\
\hline Tetradecane* & 5 & 5 & 10 & 32.3 \\
\hline 3-Nonen-2-one & 4 & 6 & 10 & 32.2 \\
\hline 1-Octanol* & 4 & 5 & 9 & 29.0 \\
\hline 2-Octenoicacid & 5 & 4 & 9 & 29.0 \\
\hline DiethylPhthalate & 3 & 6 & 9 & 29.0 \\
\hline 1-Decene* & 3 & 5 & 8 & 25.8 \\
\hline Cyclododecane & 6 & 2 & 8 & 25.8 \\
\hline Furfural* & 1 & 7 & 8 & 25.8 \\
\hline Heptanoicacid,ethylester* & 4 & 4 & 8 & 25.8 \\
\hline 1-Hexanol* & 2 & 5 & 7 & 22.6 \\
\hline 2-Octenal,(E)-* & 1 & 6 & 7 & 22.6 \\
\hline 2-Tetradecene,(E)- & 5 & 2 & 7 & 22.6 \\
\hline Dodecanoicacid* & 1 & 6 & 7 & 22.6 \\
\hline Pentadecanoicacid,ethylester & 0 & 7 & 7 & 22.6 \\
\hline Decanoicacid,ethylester* & 2 & 4 & 6 & 19.4 \\
\hline
\end{tabular}




\begin{tabular}{|c|c|c|c|c|}
\hline Hexadecane* & 2 & 4 & 6 & 19.4 \\
\hline Naphthalene,2,7-dimethyl- & 2 & 4 & 6 & 19.4 \\
\hline Benzoicacid,ethylester & 3 & 2 & 5 & 16.1 \\
\hline Caryophyllene* & 2 & 3 & 5 & 16.1 \\
\hline IsopropylMyristate & 3 & 2 & 5 & 16.1 \\
\hline Vanillin & 0 & 5 & 5 & 16.1 \\
\hline 1-Pentadecene* & 2 & 2 & 4 & 12.9 \\
\hline p-Benzoquinone & 3 & 1 & 4 & 12.9 \\
\hline Benzophenone* & 1 & 3 & 4 & 12.9 \\
\hline Cyclopropane,nonyl- & 1 & 3 & 4 & 12.9 \\
\hline 2(3H)-Furanone,5-ethyldihydro- & 3 & 0 & 3 & 9.7 \\
\hline 2(3H)-Furanone, dihydro-5-pentyl- & 0 & 3 & 3 & 9.7 \\
\hline 3,5,9-Undecatrien-2-one,6,10-dimethyl-, (E,Z)- & 0 & 3 & 3 & 9.7 \\
\hline 3,7-Dimethyl-octa-1,6-diene & 1 & 2 & 3 & 9.7 \\
\hline Acetophenone* & 2 & 1 & 3 & 9.7 \\
\hline Benzene, 1,1'-methylenebis[4-methyl- & 2 & 1 & 3 & 9.7 \\
\hline Benzene,1-methyl-2-[(4-methylphenyl)methyl]- & 0 & 3 & 3 & 9.7 \\
\hline Benzothiazole & 2 & 1 & 3 & 9.7 \\
\hline Calamenene & 1 & 2 & 3 & 9.7 \\
\hline Heptadecane* & 1 & 2 & 3 & 9.7 \\
\hline Hexanoicacid,anhydride & 1 & 2 & 3 & 9.7 \\
\hline Octadecanal & 1 & 2 & 3 & 9.7 \\
\hline OctanoicAcid* & 2 & 1 & 3 & 9.7 \\
\hline$\beta$-Bourbonene & 2 & 1 & 3 & 9.7 \\
\hline 1,1'-Biphenyl,4-methyl- & 0 & 2 & 2 & 6.5 \\
\hline 1-Heptadecene & 0 & 2 & 2 & 6.5 \\
\hline 1-Hexadecene & 0 & 2 & 2 & 6.5 \\
\hline 1-Pentanol & 1 & 1 & 2 & 6.5 \\
\hline Cyclodecane & 0 & 0 & 0 & 6.5 \\
\hline Cyclotetradecane & 1 & 1 & 2 & 6.5 \\
\hline E-11-Hexadecenoicacid,ethylester & 1 & 1 & 2 & 6.5 \\
\hline Ethyl9-hexadecenoate & 0 & 2 & 2 & 6.5 \\
\hline Galaxolide & 1 & 1 & 2 & 6.5 \\
\hline IsopropylPalmitate & 1 & 1 & 2 & 6.5 \\
\hline Octadecane & 1 & 1 & 2 & 6.5 \\
\hline Pentanoicacid* & 1 & 1 & 2 & 6.5 \\
\hline Phenol* & 2 & 0 & 2 & 6.5 \\
\hline$\beta$-Farnesene & 1 & 1 & 2 & 6.5 \\
\hline 1,1'-Biphenyl,2-ethyl- & 1 & 0 & 1 & 3.2 \\
\hline 1,1'-Biphenyl,3-methyl- & 0 & 1 & 1 & 3.2 \\
\hline 2(3H)-Furanone, dihydro-5-propyl- & 0 & 1 & 1 & 3.2 \\
\hline 2-Dodecenal,(E)- & 0 & 1 & 1 & 3.2 \\
\hline 2-Heptenoicacid & 0 & 1 & 1 & 3.2 \\
\hline 3-Eicosene,(E)- & 0 & 1 & 1 & 3.2 \\
\hline 3-Heptadecene,(Z)- & 1 & 0 & 1 & 3.2 \\
\hline 4,8-Dimethyl-nona-3,8-dien-2-one & 1 & 0 & 1 & 3.2 \\
\hline 7-Hexadecene,(Z)- & 1 & 0 & 1 & 3.2 \\
\hline
\end{tabular}




\begin{tabular}{|l|lll|l|}
\hline 9-Octadecenoicacid,(E)- & 0 & 1 & 1 & 3.2 \\
\hline Aceticacid & 0 & 1 & 1 & 3.2 \\
\hline Benzaldehyde,4-(1-methylethyl)- & 1 & 0 & 1 & 3.2 \\
\hline Benzene, 1-methyl-2-[(3-methylphenyl)methyl]- & 1 & 0 & 1 & 3.2 \\
\hline ButylatedHydroxytoluene & 1 & 0 & 1 & 3.2 \\
\hline Docosane* & 1 & 0 & 1 & 3.2 \\
\hline Dodecanal* & 1 & 0 & 1 & 3.2 \\
\hline Dodecanoicacid,methylester* & 0 & 1 & 1 & 3.2 \\
\hline Eicosane* & 1 & 0 & 1 & 3.2 \\
\hline Ethyltridecanoate & 0 & 1 & 1 & 3.2 \\
\hline Heneicosane* & 1 & 0 & 1 & 3.2 \\
\hline Heptanoicacid* & 0 & 1 & 1 & 3.2 \\
\hline Hexadecanoicacid,methylester & 1 & 0 & 1 & 3.2 \\
\hline Nonadecanoicacid,ethylester & 0 & 1 & 1 & 3.2 \\
\hline Pentadecane,7-methyl- & 1 & 0 & 1 & 3.2 \\
\hline Tetradecanoicacid,2-methyl-,methyl ester & 0 & 1 & 1 & 3.2 \\
\hline$\gamma$-Cadinene & 1 & 0 & 1 & 3.2 \\
\hline$\Delta$-Cadinene & 0 & 1 & 1 & 3.2 \\
\hline
\end{tabular}


Figure 76. Histogram of VOCs in buccal swab odor from healthy individuals

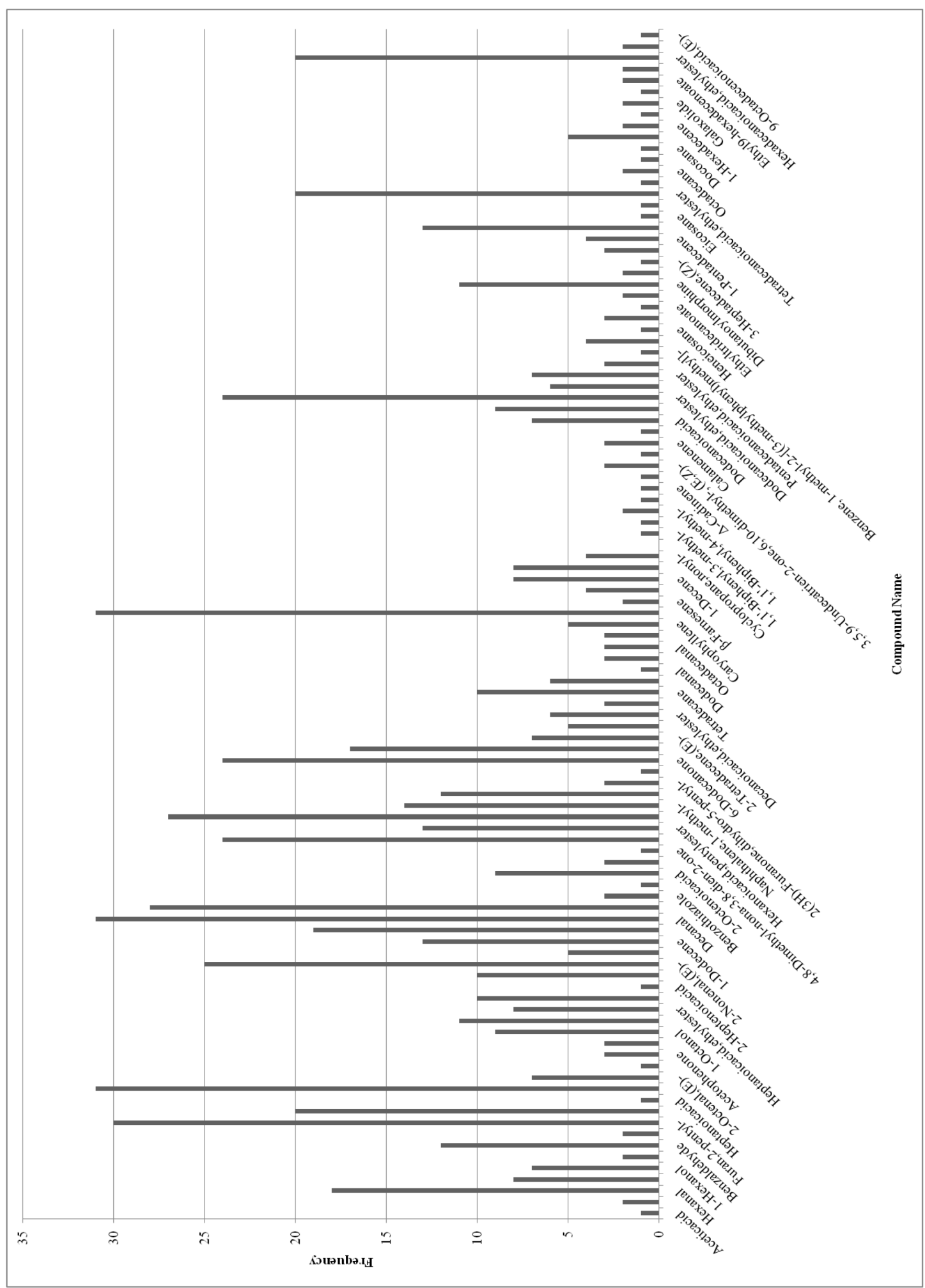


Across the breath samples collected from the healthy subjects, 83 volatile compounds were extracted. The functionality distribution of the extracted volatile compounds was as follows: acids (2.4\%), alcohols (8.4\%), aldehydes (10.8\%), aliphatics (30.1\%), aromatics $(34.9 \%)$, esters $(7.2 \%)$, and ketones $(6.0 \%)$. Figure 77 displays a pie chart of the distribution of the seven functional groups. The frequency of the occurrence of the breath VOCs from the thirty-one healthy individuals are listed in Table 39 and displayed in the histogram in Figure 78. The highest frequency occurring VOCs for breath samples were decanal, nonanal, styrene, benzophenone, 2-ethyl-1-hexanol, and 1,2-dichlorobezene.

Exhaled breath VOCs origin can be attributed to the lungs, nasal cavity, or some systemic pathway from which the VOCs are exhaled following blood-to-alveolar diffusion. Diffusion property depends on the compound's polarity, fat solubility, volatility, and Henry's constant as described in section 0 . Blood-breath partition ratio of a compound depends on the compound's lipid or water solubility. Water soluble compounds such as alcohols and ketones can be seen in breath by diffusion through the blood circulation of the respiratory tree to the water-bearing membranes. Therefore, higher concentrations of water-soluble compounds are expected to be observed in exhaled breath. However, water-insoluble (lipophilic) compounds such as straight-chain hydrocarbons can also be observed in exhaled breath because of their ability to diffuse through the lungs. High lipid soluble compounds are eliminated more slowly than lipophobic compounds because they can be deposited and stored in fat before being eliminated ${ }^{62}$.

Straight-chain hydrocarbons $\left(\mathrm{C}_{10}-\mathrm{C}_{20}\right)$ were present in the breath odor VOCs extracted from the healthy individuals. These compounds are products of lipid peroxidation of the 
polyunsaturated fatty acids ${ }^{49}$. Oxidation by enzymes of the polyunsaturated fatty acids also produce fatty acid hydroperoxides, which are eventually converted to aldehydes, as observed across most subjects (nonanal and decanal are present in $95 \%$ of the subjects). Branched alkanes (i.e., 2,6-dimethylundecane) and highly substituted benzenes (i.e., trimethyl-benzenes) were commonly present across all subjects. These compounds are most likely of environmental contaminant origin as they possess negative polarity alveolar gradient which are consistent with VOCs that are of exogenous origin ${ }^{48}$. In other words, VOCs with negative alveolar gradient are eliminated faster than the rate they are synthesized in the body. Naphthalene and methylnaphthalenes have been suggested to be steroid degradation products but the exact origin is still unknown ${ }^{48}$.

One compound that is a known volatile constituent of human breath that was not detected in the present study is isoprene. Isoprene is present in all human breath resulting from the mevalonic pathway of cholesterol synthesis. It is important to note here that under the current instrumental conditions isoprene was not detected, most likely due to its high vapor pressure $\left(560 \mathrm{mmHg}\right.$ at $\left.20^{\circ} \mathrm{C}\right)$ therefore making isoprene a highly volatile compound that is also extremely unstable and thermally labile to cause degradation at the GC injection port with the current settings. 
Figure 77. Frequency distribution of functional groups of extracted volatile organic compounds for breath

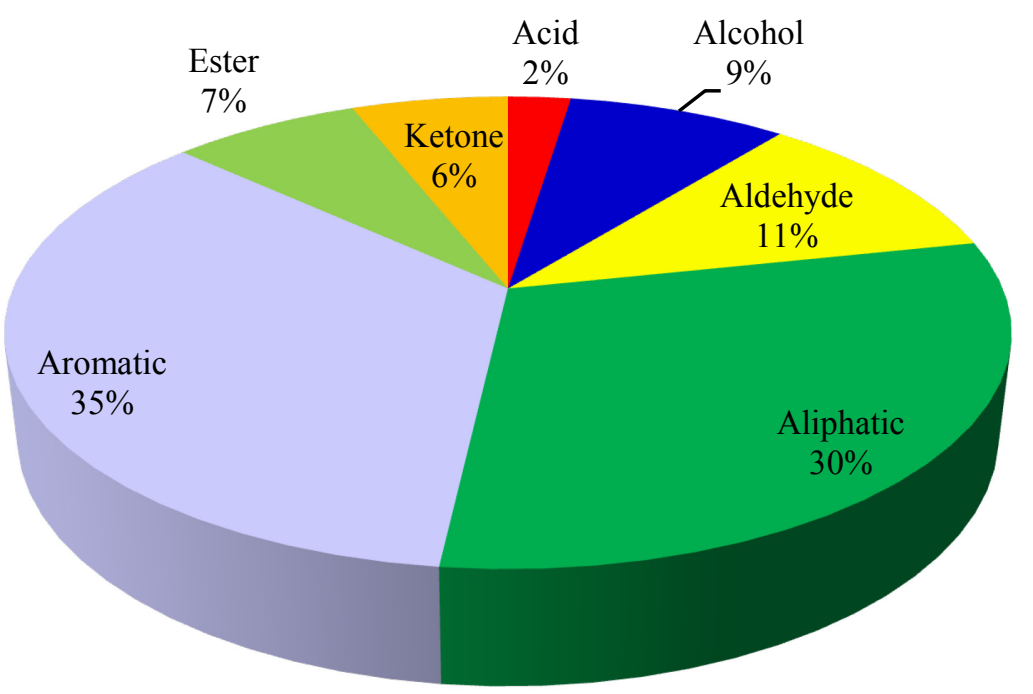


Table 39. Identified VOCs in breath odor of thirty-one healthy individuals ranked by frequency of occurrence (Note: *denotes compound was verified by reference standard)

\begin{tabular}{|l|ccc|c|}
\hline \multicolumn{1}{|c|}{ Compound } & \multicolumn{3}{|c|}{ Frequency } & Occurrence \\
& Female & Male & Total & $(\%)$ \\
\hline Decanal* & 16 & 15 & 31 & 100 \\
\hline Nonanal* $^{*}$ & 15 & 15 & 30 & 96.8 \\
\hline Styrene* & 15 & 14 & 29 & 93.6 \\
\hline Benzophenone* & 13 & 15 & 28 & 90.3 \\
\hline Benzene,1,2-dichloro-* & 13 & 12 & 25 & 80.7 \\
\hline 1-Hexanol,2-ethyl-* & 11 & 12 & 23 & 80.7 \\
\hline ButylatedHydroxytoluene* & 14 & 10 & 24 & 77.4 \\
\hline Xylenes* & 11 & 11 & 22 & 71.0 \\
\hline Phenol* & 9 & 9 & 18 & 58.1 \\
\hline Dibutylphthalate & 10 & 6 & 16 & 51.6 \\
\hline Undecane* & 9 & 4 & 13 & 41.9 \\
\hline Dodecane* & 9 & 4 & 13 & 41.9 \\
\hline 2,6-Diisopropylnaphthalene* & 7 & 6 & 13 & 41.9 \\
\hline Tetradecane* & 6 & 5 & 11 & 35.5 \\
\hline Acetophenone* & 4 & 6 & 10 & 32.3 \\
\hline 1-Dodecene* & 5 & 5 & 10 & 32.3 \\
\hline Tridecane* & 6 & 4 & 10 & 32.3 \\
\hline Longifolene & 6 & 4 & 10 & 32.3 \\
\hline Undecanal* & 5 & 4 & 9 & 29.0 \\
\hline 5,9-Undecadien-2-one,6,10-dimethyl-, (E)-* & 6 & 3 & 9 & 29.0 \\
\hline Dodecanal* & 4 & 4 & 8 & 25.8 \\
\hline Toluene* & 5 & 3 & 8 & 25.8 \\
\hline Phenol,2-(1,1-dimethylethyl)- & 5 & 2 & 7 & 22.6 \\
\hline Indole & 2 & 4 & 6 & 19.4 \\
\hline Naphthalene* & 2 & 3 & 5 & 16.1 \\
\hline Nonanoicacid & 3 & 2 & 5 & 16.1 \\
\hline Benzene,1,3,5-trimethyl- & 2 & 2 & 4 & 12.9 \\
\hline Diisopropylnaphthalene & 3 & 1 & 4 & 12.9 \\
\hline Benzene,1,4-dichloro- & 1 & 2 & 3 & 9.7 \\
\hline Longicyclene & 1 & 2 & 3 & 9.7 \\
\hline Hexadecane* & 1 & 2 & 3 & 9.7 \\
\hline Cyclododecane & 2 & 1 & 3 & 9.7 \\
\hline Limonene* & 1 & 1 & 2 & 6.5 \\
\hline 5-Hepten-2-one,6-methyl-* & 1 & 1 & 2 & 6.1 \\
\hline Benzene,1,2,3-trimethyl- & 1 & 1 & 2 & 6.1 \\
\hline Benzene,4-ethyl-1,2-dimethyl- & 1 & 1 & 2 & 6.1 \\
\hline n-Decanoicacid* & 2 & 0 & 2 & 6.1 \\
\hline$\beta$-Maaliene & 1 & 1 & 2 & 6.1 \\
\hline 1,1'-Biphenyl,4-methyl- & 0 & 2 & 2 & 6.1 \\
\hline Heptadecane** & 1 & 1 & 2 & 6.1 \\
\hline & & & & \\
\hline & & 4 & \\
\hline
\end{tabular}




\begin{tabular}{|c|c|c|c|c|}
\hline Hexanal* & 0 & 1 & 1 & 3.0 \\
\hline 2-Propanol,1-propoxy- & 0 & 1 & 1 & 3.0 \\
\hline Cyclohexane,methyl- & 1 & 0 & 1 & 3.0 \\
\hline 1,3,5,7-Cyclooctatetraene & 1 & 0 & 1 & 3.0 \\
\hline Benzene,propyl- & 1 & 0 & 1 & 3.0 \\
\hline Benzene,1-ethyl-2-methyl- & 1 & 0 & 1 & 3.0 \\
\hline Nonane,3-methyl- & 1 & 0 & 1 & 3.0 \\
\hline Benzaldehyde* & 0 & 1 & 1 & 3.0 \\
\hline Octanal & 1 & 0 & 1 & 3.0 \\
\hline 4-Cyanocyclohexene & 0 & 1 & 1 & 3.0 \\
\hline Benzene,diethyl- & 1 & 0 & 1 & 3.0 \\
\hline 1S-.alpha.-Pinene & 0 & 1 & 1 & 3.0 \\
\hline Eicosane* & 1 & 0 & 1 & 3.0 \\
\hline Decane,3-methyl- & 1 & 0 & 1 & 3.0 \\
\hline Benzene,1-ethyl-3,5-dimethyl- & 1 & 0 & 1 & 3.0 \\
\hline Undecane,3,6-dimethyl- & 1 & 0 & 1 & 3.0 \\
\hline Benzene,1-ethyl-2,4-dimethyl- & 1 & 0 & 1 & 3.0 \\
\hline Benzene,1-methyl-3-(1-methylethyl)- & 1 & 0 & 1 & 3.0 \\
\hline 3-Octanol,3,7-dimethyl-,(.+/-.)- & 0 & 1 & 1 & 3.0 \\
\hline Nonadecane & 1 & 0 & 1 & 3.0 \\
\hline Cyclohexane,pentyl- & 1 & 0 & 1 & 3.0 \\
\hline Benzene,1,3-diethyl-5-methyl- & 1 & 0 & 1 & 3.0 \\
\hline Benzene,1-methyl-4-(1-methylpropyl)- & 1 & 0 & 1 & 3.0 \\
\hline Menthol* & 0 & 1 & 1 & 3.0 \\
\hline MethylSalicylate* & 0 & 1 & 1 & 3.0 \\
\hline Undecane,2,6-dimethyl- & 1 & 0 & 1 & 3.0 \\
\hline Ethanol,2-phenoxy- & 0 & 1 & 1 & 3.0 \\
\hline Benzaldehyde,4-methoxy- & 1 & 0 & 1 & 3.0 \\
\hline Naphthalene,2-methyl- & 1 & 0 & 1 & 3.0 \\
\hline Indolizine & 1 & 0 & 1 & 3.0 \\
\hline Benzene,3-cyclohexen-1-yl- & 0 & 1 & 1 & 3.0 \\
\hline Caryophyllene* & 0 & 1 & 1 & 3.0 \\
\hline$\beta$-Gurjunene & 1 & 0 & 1 & 3.0 \\
\hline DiethylPhthalate & 1 & 0 & 1 & 3.0 \\
\hline Tetradecanal & 1 & 0 & 1 & 3.0 \\
\hline p-Benzoquinone & 1 & 0 & 1 & 3.0 \\
\hline Dodecanoicacid,methylester & 0 & 1 & 1 & 3.0 \\
\hline Galaxolide & 0 & 1 & 1 & 3.0 \\
\hline Homomenthylsalicylate & 0 & 1 & 1 & 3.0 \\
\hline IsopropylPalmitate & 0 & 1 & 1 & 3.0 \\
\hline
\end{tabular}


Figure 78. Histogram of VOCs in breath odor across thirty-one healthy individuals

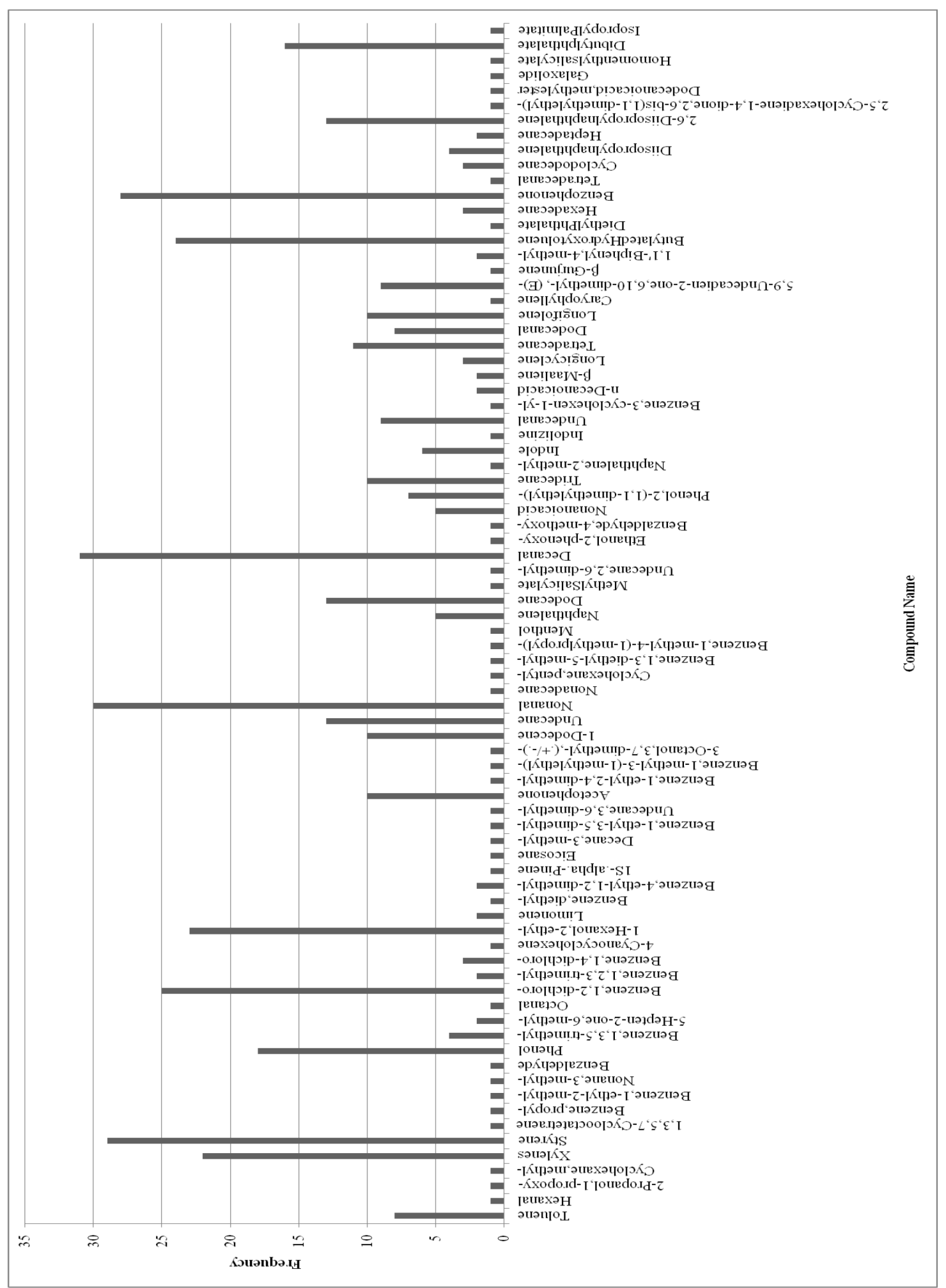


For blood samples, 77 VOCs were extracted across the samples collected from each of the thirty-one individuals. The compounds ranged in functionality, including: alcohols (18.2\%), aldehydes (18.2\%), aliphatics (27.3\%), aromatics (22.1\%), esters (1.3\%), ketones $(14.3 \%)$, and others (3.9\%) as shown in Figure 79. Volatile compounds included in the "others" category included ethers and sulfur compound (dimethylsulfone). The frequency of occurrence and histogram of the VOCs found in blood odor from thirty-one healthy individuals are shown in Table 40 and Figure 80.

Among the alcohols that were present in blood odor samples, benzyl alcohol was the only compound found at high frequency of $80.6 \%$. Benzyl alcohol is commonly found in nature in essential oils and is very widely used in the flavor and fragrance industries and thus the exposure of normal humans to benzyl alcohol is very high. 1-Octen-3-ol is a secondary alcohol that is produced by mushrooms and also accounted for the odor of cows $^{68}$. The alcohol 1-octen-3-ol has been reported to be a constituent of human sweat and breath and has been suggested to be an oxidation product from milk fat. The $\mathrm{C}_{7}-\mathrm{C}_{10}$ aldehydes were commonly observed in the blood odor of healthy individuals. Hexanal and heptanal were both observed across the subjects. These two compounds have been suggested to be lung cancer biomarkers at elevated levels, but are also known to be present in healthy individuals as a breakdown product of lipid peroxidation in the body ${ }^{67,68}$. Aliphatic compounds included $\mathrm{C}_{7}-\mathrm{C}_{25}$ cycloalkanes, $\mathrm{C}_{11}-\mathrm{C}_{25}$ higher alkanes, and $\mathrm{C}_{10}-\mathrm{C}_{15}$ higher alkenes, of which $\mathrm{C}_{11}-\mathrm{C}_{16}$ straight-chain higher alkanes were present in over $90 \%$ of subjects. 
Major sources of aromatic VOCs including substituted and chlorinated benzenes and xylenes that are of exogenous origin include tobacco products, automobile exhaust, and chlorinated water which today are all extremely difficult to avoid exposure from even for nonoccupationally and nonenvironmentally exposed workers. With increasing air pollutant levels especially in developed countries such as the U.S. where the present study was conducted, it is inevitable to see an above-detection level of exogenous VOCs in human biological specimens even for nonoccupational exposure workers. Alkylnaphthalenes (diisopropylnaphthalene, 2,6-diisopropylnaphthalane) have seen a significant increase in the uses as substitutes for polychlorinated biphenyls (PCBs) over the last several decades, resulting in the increase in their concentrations in the environment. Concentration of 2,6-diisopropylnaphthalene in blood has been shown to be directly proportional to the exposure dosage of the compound, as it is readily absorbed and redistributed in blood and tissues of the body and rapidly bioaccumulated ${ }^{124}$.

Figure 79. Frequency distribution of functional groups of extracted volatile organic compounds for blood

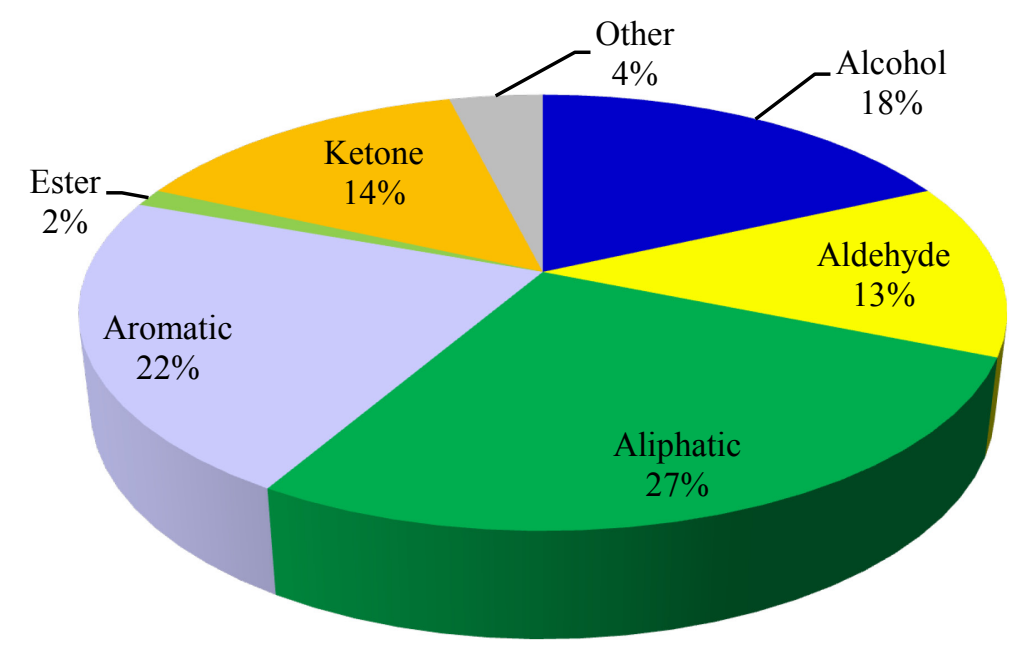


Table 40. Identified VOCs in blood odor of thirty-one healthy individuals ranked by frequency of occurrence (Note: *denotes compound was verified by reference standard)

\begin{tabular}{|l|ccc|c|}
\hline \multicolumn{1}{|c|}{ Compound } & \multicolumn{3}{|c|}{ Frequency } & Occurrence \\
& Female & Male & Total & $(\%)$ \\
\hline Benzaldehyde,2,4,6-trimethyl- & 16 & 15 & 31 & 100 \\
\hline 2,6-Diisopropylnaphthalene* & 15 & 15 & 30 & 96.8 \\
\hline Hexadecane* & 15 & 15 & 30 & 96.8 \\
\hline Tetradecane* & 15 & 15 & 30 & 96.8 \\
\hline Tridecane* & 14 & 15 & 29 & 93.6 \\
\hline Undecane* & 14 & 15 & 29 & 93.6 \\
\hline Dodecane* & 13 & 15 & 28 & 90.3 \\
\hline Nonanal* & 14 & 14 & 28 & 90.3 \\
\hline Hexanal & 15 & 11 & 26 & 83.9 \\
\hline BenzylAlcohol* & 13 & 12 & 25 & 80.7 \\
\hline Furan,2-butyltetrahydro- & 12 & 11 & 23 & 74.2 \\
\hline Heptadecane* & 13 & 9 & 22 & 71.0 \\
\hline Furan,2-pentyl-* & 9 & 11 & 20 & 64.5 \\
\hline Cyclohexanone* & 10 & 9 & 19 & 61.3 \\
\hline Diisopropylnaphthalene & 10 & 9 & 19 & 61.3 \\
\hline 1-Dodecen** & 6 & 12 & 18 & 58.1 \\
\hline Decanal* & 6 & 9 & 15 & 48.4 \\
\hline 5,9-Undecadien-2-one,6,10-dimethyl-, (E)-* & 6 & 8 & 14 & 45.2 \\
\hline 1-Pentanol & 6 & 6 & 12 & 38.7 \\
\hline 2-Dodecene,(Z)- & 6 & 6 & 12 & 38.7 \\
\hline Benzene,1,2-dichloro-* & 6 & 6 & 12 & 38.7 \\
\hline 2-Undecanone & 4 & 7 & 11 & 35.5 \\
\hline Cyclododecane & 5 & 5 & 10 & 32.3 \\
\hline 2-Heptanone* & 5 & 3 & 8 & 25.8 \\
\hline 1-Octen-3-ol* & 4 & 4 & 8 & 25.5 \\
\hline 1-Decene* & 1 & 6 & 7 & 22.6 \\
\hline Cyclopropane,nonyl- & 4 & 3 & 7 & 22.6 \\
\hline Longifolene & 4 & 2 & 6 & 19.4 \\
\hline Octadecane & 2 & 4 & 6 & 19.4 \\
\hline Octanal & 2 & 4 & 6 & 19.4 \\
\hline 1-Dodecanol & 3 & 2 & 5 & 16.1 \\
\hline 1-Octanol* & 1 & 4 & 5 & 16.1 \\
\hline 2-Heptanone,6-methyl- & 4 & 1 & 5 & 16.1 \\
\hline Heptana1* & 3 & 2 & 5 & 16.1 \\
\hline Benzene,1,4-dichloro- & 2 & 2 & 4 & 12.9 \\
\hline Menthol* & 2 & 2 & 4 & 12.9 \\
\hline 1-Phenyl-2-butanone & 1 & 2 & 3 & 9.7 \\
\hline & & & & \\
\hline
\end{tabular}




\begin{tabular}{|c|c|c|c|c|}
\hline 2,4-Diphenyl-4-methyl-1(E)-pentene & 2 & 1 & 3 & 9.7 \\
\hline Benzenemethanol,.alpha.,.alpha.-dimethyl- & 1 & 2 & 3 & 9.7 \\
\hline Ethanol,2-butoxy- & 1 & 2 & 3 & 9.7 \\
\hline 4-Cyanocyclohexene & 0 & 2 & 2 & 6.5 \\
\hline 4-Heptanone* & 2 & 0 & 2 & 6.5 \\
\hline 5-Hepten-2-one,6-methyl-* & 1 & 1 & 2 & 6.5 \\
\hline Benzaldehyde* & 1 & 1 & 2 & 6.5 \\
\hline Benzaldehyde,4-(methylthio)- & 0 & 2 & 2 & 6.5 \\
\hline Heptanol* & 1 & 1 & 2 & 6.5 \\
\hline Phenol* & 2 & 0 & 2 & 6.5 \\
\hline Phenol,2,4,6-trimethyl- & 2 & 0 & 2 & 6.5 \\
\hline Xylenes* & 2 & 0 & 2 & 6.5 \\
\hline 1,1'-Biphenyl,2-methyl- & 1 & 0 & 1 & 3.2 \\
\hline 1,1'-Biphenyl,4-methyl- & 0 & 1 & 1 & 3.2 \\
\hline 1,2-Benzenediol,3,5-bis(1,1-dimethylethyl)- & 0 & 1 & 1 & 3.2 \\
\hline 1-Tetradecene* & 0 & 1 & 1 & 3.2 \\
\hline 2-Decanone* & 1 & 0 & 1 & 3.2 \\
\hline 2-Propanol,1-propoxy- & 1 & 0 & 1 & 3.2 \\
\hline 3,7-Dimethyl-octa-1,6-diene & 1 & 0 & 1 & 3.2 \\
\hline 3-Isopropylbenzaldehyde & 1 & 0 & 1 & 3.2 \\
\hline Acetone & 1 & 0 & 1 & 3.2 \\
\hline Acetophenone* & 0 & 1 & 1 & 3.2 \\
\hline Benzaldehyde,3-hydroxy- & 0 & 1 & 1 & 3.2 \\
\hline Benzene,1,3-dichloro- & 1 & 0 & 1 & 3.2 \\
\hline Benzene,1-methyl-3-propyl- & 1 & 0 & 1 & 3.2 \\
\hline Benzene,1-methyl-4-(1-methylpropyl)- & 1 & 0 & 1 & 3.2 \\
\hline Benzene,4-ethyl-1,2-dimethyl- & 1 & 0 & 1 & 3.2 \\
\hline Benzene,diethyl- & 1 & 0 & 1 & 3.2 \\
\hline Cyclodecane & 1 & 0 & 1 & 3.2 \\
\hline Cyclooctane & 1 & 0 & 1 & 3.2 \\
\hline Dimethylsulfone & 0 & 1 & 1 & 3.2 \\
\hline D-Limonene* & 1 & 0 & 1 & 3.2 \\
\hline Heptadecane,9-octyl- & 0 & 1 & 1 & 3.2 \\
\hline IsopropylMyristate & 0 & 1 & 1 & 3.2 \\
\hline Naphthalene* & 0 & 1 & 1 & 3.2 \\
\hline Naphthalene,2,3,6-trimethyl- & 0 & 1 & 1 & 3.2 \\
\hline Tetradecanal & 1 & 0 & 1 & 3.2 \\
\hline$\beta$-Maaliene & 0 & 1 & 1 & 3.2 \\
\hline
\end{tabular}


Figure 80. Histogram of VOCs in blood odor across thirty-one healthy individuals

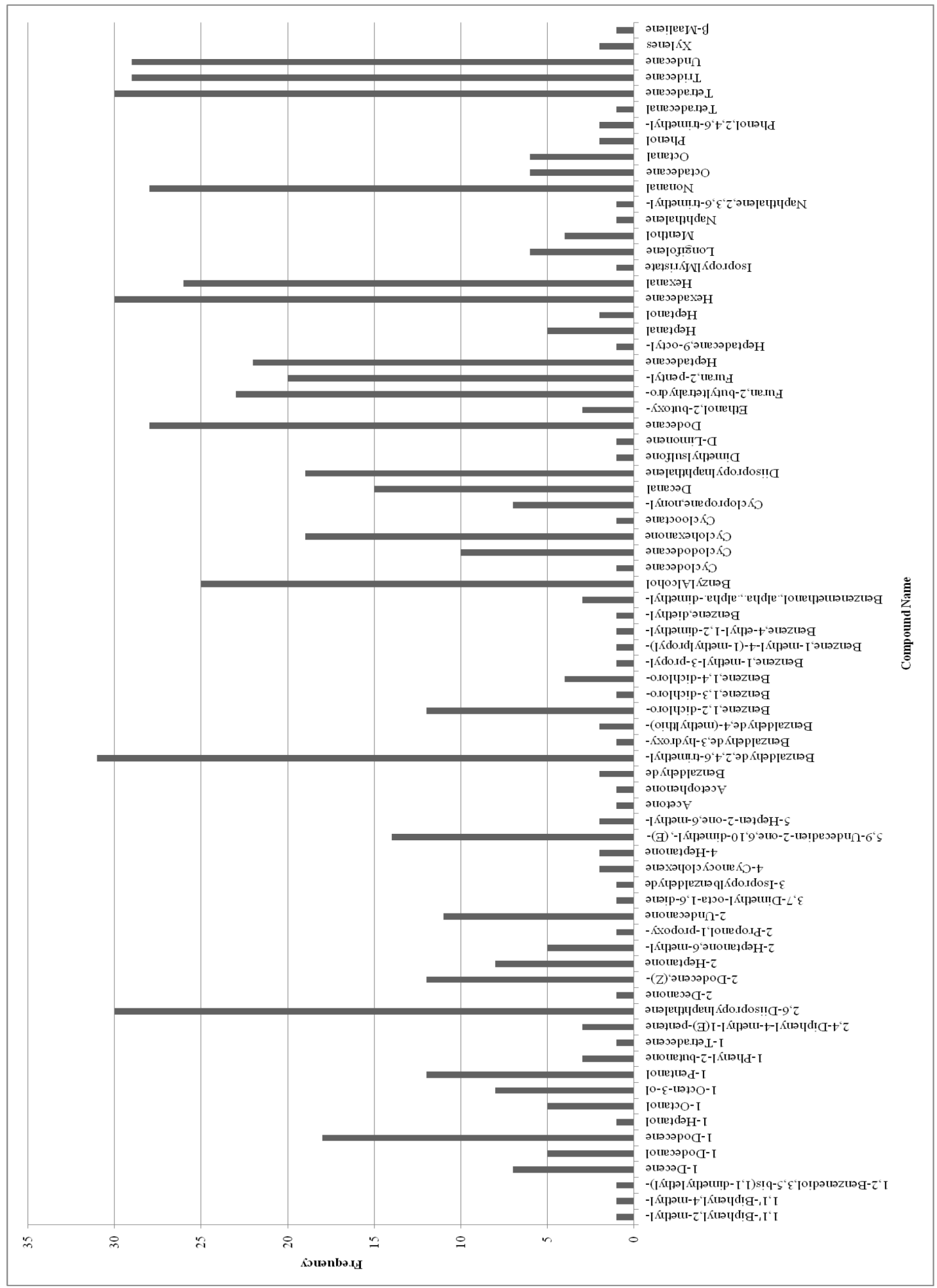


For urine, 87 volatile compounds were extracted across the samples collected from thirtyone individuals. Urinary VOCs were comprised of the widest range in functional groups compared to the other specimens. The functionality distribution of the extracted volatile compounds was as follows: acids (2.3\%), alcohols (24.1\%), aldehydes (11.5\%), aliphatics (13.8\%), alkylphenols (AP, 3.4\%), aromatics (10.3\%), esters (3.4\%), ketones (18.4\%), nitrogen compounds (4.6\%), $O$-heterocyclic compounds (3.4\%), and sulfur compounds $(5.7 \%)$ as seen in Figure 81. Where aromatics, aliphatics, and aldehydes comprised about $75 \%$ of the entire functional group distribution other specimens, those major hydrocarbon functional groups together only contributed to approximately one-third of the total range of functional groups found in urine. Over $20 \%$ of the urinary VOCs detected were alcohols, which was much higher than the percentage of alcohols present in other specimens. Additionally, sulfur compounds, nitrogen compounds, and O-heterocyclic compounds were present in urine. These functional groups are not completely absent in other specimens, however, they are present at extremely low frequencies or they may be present only at below-detection limit levels. The frequency of occurrence and histogram of the VOCs found in urine odor from thirty-one healthy individuals are shown in Table 41 and Figure 82. 4-Heptanone was the only compound with a frequency of occurrence higher than $80.0 \%(96.8 \%)$.

The volatile compounds ranging in the eleven functional groups are found regularly in human urine and their presences are mostly accounted for by nutrients, intermediates, and environmental contaminants. Many compounds including furans, terpines, pyrroles, carvones, and menthols originate from food and food additives. Some compounds such as 
2-ethyl-1-hexanol, p-benzoquinone, and 2,6-(1,1-dimethylethyl)-4-ethyl-phenol were omitted from the urine specimen compound database because they were also present in all water-blank samples that were stored in the same specimen collection container that the actual urine samples were collected and stored in.

Ketones are known to be prominent components of urinary volatiles and they are most of time decarboxylation products of their corresponding oxo-acids that are produced in urine. 4-Heptanone and 2-heptanone have been suggested to be exogenous compounds and their specific origins are still unknown. These ketones are widely observed in normal human urine and have been attributed to the in vivo metabolism of plasticizers in humans ${ }^{70}$. 2-Heptanone along with 2-pentanone are also known to be a naturally occurring VOCs in milk ${ }^{125}$.

Dichlorobenzenes which were observed in most healthy subjects' urine samples are unmetabolized VOCs that result from exposure. These compounds have been reported to be biomarkers of low level exposure to these chlorinated aromatic VOCs in indoor environments $^{80}$. Lastly, alkylphenols (AP), 4-nonylphenol (or simply nonylphenol, NP) and 4-(1,1,3,3-tetramethylbutyl)-phenol (or 4-tert-octylphenol, OP), are prominent constituents of urinary volatiles. These alkylphenols are used as surfactants commonly used in pesticides as "inert" ingredients and have been shown to be environmental contaminants in sewage wastewater, river water, sea water, and as a results in fish tissue $^{77,78,126}$. Alkylphenols are detected in normal human urine due to exposure from daily activities, air, and meals. 
Figure 81. Frequency distribution of functional groups of extracted volatile organic compounds for urine (Note: $\mathrm{AP}=$ alkylphenols)

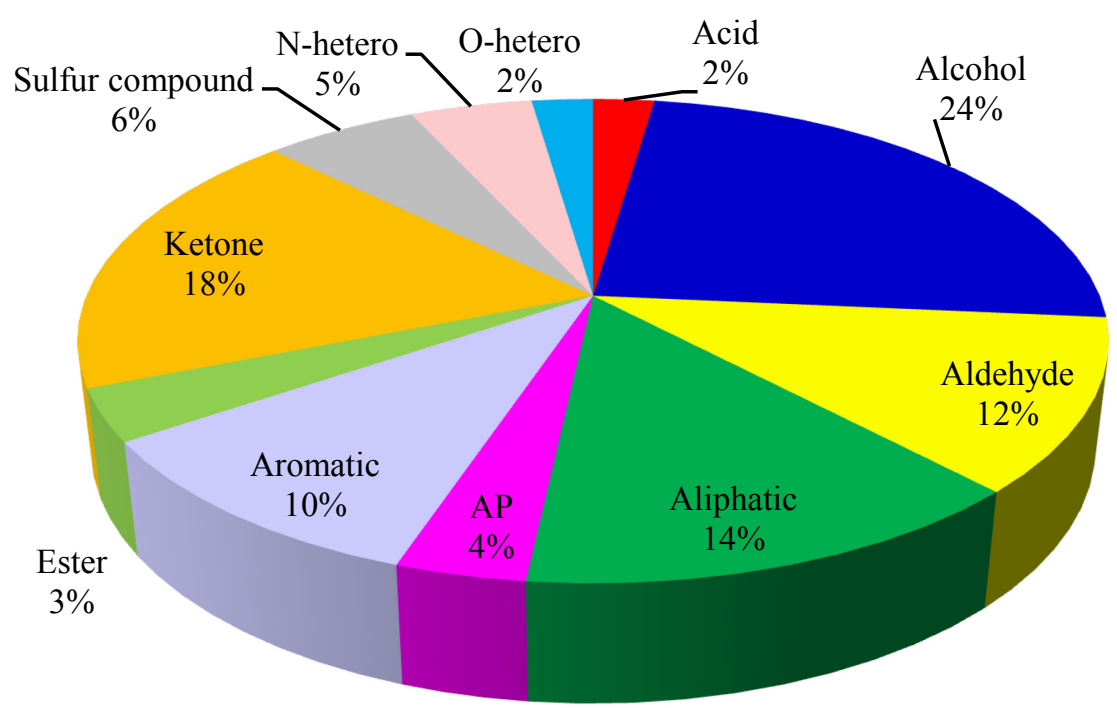

Table 41. Identified VOCs in urine odor of thirty-one healthy individuals ranked by frequency of occurrence (Note: * denotes compound was verified by reference standard)

\begin{tabular}{|l|ccc|c|}
\hline \multicolumn{1}{|c|}{ Compound Name } & \multicolumn{3}{c|}{ Frequency } & Occurrence \\
& Female & Male & Total & $(\%)$ \\
\hline 4-Heptanone* & 16 & 14 & 30 & 96.8 \\
\hline 4-Nonylphenol & 11 & 11 & 22 & 71.0 \\
\hline Carvone & 11 & 10 & 21 & 67.7 \\
\hline Pyrrole & 9 & 7 & 16 & 51.6 \\
\hline Benzene,1,4-dichloro- & 5 & 10 & 15 & 48.4 \\
\hline Phenol,4-(1,1,3,3-tetramethylbutyl)-* & 7 & 8 & 15 & 48.4 \\
\hline Nonanal* & 5 & 8 & 13 & 41.9 \\
\hline Menthol* & 5 & 7 & 12 & 38.7 \\
\hline Benzene,1-methyl-4-(1-methylethenyl)- & 5 & 5 & 10 & 32.3 \\
\hline.+ -.-4-Acetyl-1-methylcyclohexene & 6 & 4 & 10 & 32.3 \\
\hline 3-Cyclohexen-1-one2-isopropyl-5-methyl- & 7 & 2 & 9 & 29.0 \\
\hline 4-Terpineol & 5 & 3 & 8 & 25.5 \\
\hline Benzene,1,2-dichloro-* & 3 & 4 & 7 & 22.6 \\
\hline$\gamma$-Terpinene & 3 & 4 & 7 & 22.6 \\
\hline Benzaldehyde,3,5-dimethyl- & 4 & 3 & 7 & 22.6 \\
\hline
\end{tabular}




\begin{tabular}{|c|c|c|c|c|}
\hline 2-Isopropylbenzaldehyde & 6 & 1 & 7 & 22.6 \\
\hline Benzophenone* & 3 & 4 & 7 & 22.6 \\
\hline Cedrol & 3 & 3 & 6 & 19.4 \\
\hline Phenol,nonyl- & 2 & 4 & 6 & 19.4 \\
\hline$N, N$-Diethylcarbanilide & 2 & 4 & 6 & 19.4 \\
\hline$\alpha$-Terpinene & 1 & 4 & 5 & 16.1 \\
\hline Benzene,1-methyl-4-(1-methylethyl)- & 4 & 1 & 5 & 16.1 \\
\hline Phenol,4-methyl-(p-Cresol)* & 3 & 2 & 5 & 16.1 \\
\hline p-Menthan-3-one & 3 & 2 & 5 & 16.1 \\
\hline Decanal* & 1 & 4 & 5 & 16.1 \\
\hline 5,9-Undecadien-2-one,6,10-dimethyl-, (E)-* & 4 & 1 & 5 & 16.1 \\
\hline Benzaldehyde* & 2 & 2 & 4 & 12.9 \\
\hline p-Menth-1-en-3-one & 1 & 3 & 4 & 12.9 \\
\hline Dodecanoicacid* & 1 & 3 & 4 & 12.9 \\
\hline 2-Pentanone & 2 & 1 & 3 & 9.7 \\
\hline Cyclopropane, isothiocyanato- & 3 & 0 & 3 & 9.7 \\
\hline AllylIsothiocyanate & 2 & 1 & 3 & 9.7 \\
\hline 2-Heptanone* & 3 & 0 & 3 & 9.7 \\
\hline Dimethyltrisulfide* & 3 & 0 & 3 & 9.7 \\
\hline 1,6-Octadien-3-ol,3,7-dimethyl-* & 1 & 2 & 3 & 9.7 \\
\hline Benzeneacetaldehyde, $\alpha$-methyl- & 2 & 1 & 3 & 9.7 \\
\hline Ethanone,1-(3-methoxyphenyl)- & 2 & 1 & 3 & 9.7 \\
\hline$\beta$-Damascenone & 2 & 1 & 3 & 9.7 \\
\hline Dibutylphthalate & 1 & 2 & 3 & 9.7 \\
\hline Benzene,1,3-dichloro- & 2 & 0 & 2 & 6.5 \\
\hline Benzene,1-methyl-3-(1-methylethyl)- & 1 & 1 & 2 & 6.5 \\
\hline$\beta$-Thujene & 1 & 1 & 2 & 6.5 \\
\hline Eucalyptol & 0 & 2 & 2 & 6.5 \\
\hline 1,3,8-p-Menthatriene & 2 & 0 & 2 & 6.5 \\
\hline (E)-p-2-Menthen-1-ol & 1 & 1 & 2 & 6.5 \\
\hline 2-Nonenal,(E)-* & 1 & 1 & 2 & 6.5 \\
\hline Benzenemethanol,.alpha.,.alpha.,4-trimethyl- & 1 & 1 & 2 & 6.5 \\
\hline Benzene,(3-methyl-2-butenyl)- & 1 & 1 & 2 & 6.5 \\
\hline Ethanone,1-(4-methylphenyl)- & 1 & 1 & 2 & 6.5 \\
\hline Dihydrocarvone & 1 & 1 & 2 & 6.5 \\
\hline 2-Propenal,3-phenyl- & 1 & 1 & 2 & 6.5 \\
\hline Eugenol & 1 & 1 & 2 & 6.5 \\
\hline 1-Dodecanol & 2 & 0 & 2 & 6.5 \\
\hline Dibutanoylmorphine & 2 & 0 & 2 & 6.5 \\
\hline Dimethylsulfone & 0 & 1 & 1 & 3.2 \\
\hline 3-Heptanone,6-methyl- & 0 & 1 & 1 & 3.2 \\
\hline 2,3-Dehydro-1,8-cineole & 0 & 1 & 1 & 3.2 \\
\hline 2,3-Octanedione & 1 & 0 & 1 & 3.2 \\
\hline Benzene,1-methyl-2-(1-methylethyl)- & 0 & 1 & 1 & 3.2 \\
\hline
\end{tabular}




\begin{tabular}{|l|lll|l|}
\hline$\beta$-Phellandrene & 0 & 1 & 1 & 3.2 \\
\hline Benzeneacetaldehyde & 1 & 0 & 1 & 3.2 \\
\hline Cyclopentane,(methylthio)- & 0 & 1 & 1 & 3.2 \\
\hline$\beta$-Terpineol & 1 & 0 & 1 & 3.2 \\
\hline 1-Octanol* & 1 & 0 & 1 & 3.2 \\
\hline trans-p-Mentha-2,8-dienol & 1 & 0 & 1 & 3.2 \\
\hline BenzoicAcid & 0 & 1 & 1 & 3.2 \\
\hline Phenol,2,5-dichloro- & 0 & 1 & 1 & 3.2 \\
\hline (3E,5Z)-1,3,5-Undecatriene & 0 & 1 & 1 & 3.2 \\
\hline Pulegone & 1 & 0 & 1 & 3.2 \\
\hline Cyclodecane & 1 & 0 & 1 & 3.2 \\
\hline Phenol,4-ethyl-2-methoxy- & 1 & 0 & 1 & 3.2 \\
\hline 1-Undecanol & 0 & 1 & 1 & 3.2 \\
\hline Thymol & 0 & 1 & 1 & 3.2 \\
\hline p-Mentha-1,8-dien-7-ol & 1 & 0 & 1 & 3.2 \\
\hline 2-Methoxy-4-vinylphenol & 1 & 0 & 1 & 3.2 \\
\hline 3-Allyl-6-methoxyphenol & 1 & 0 & 1 & 3.2 \\
\hline Butanoicacid,butylester & 0 & 1 & 1 & 3.2 \\
\hline$\alpha-C e d r e n e$ & 0 & 1 & 1 & 3.2 \\
\hline 4'-(2-Methylpropyl)acetophenone & 1 & 0 & 1 & 3.2 \\
\hline$\alpha-C e d r e n e o x i d e$ & 0 & 1 & 1 & 3.2 \\
\hline DiethylPhthalate & 1 & 0 & 1 & 3.2 \\
\hline Hexadecane* & 0 & 1 & 1 & 3.2 \\
\hline Benzene,1-pentenyl- & 0 & 1 & 1 & 3.2 \\
\hline Diphenylamine* & 0 & 1 & 1 & 3.2 \\
\hline Heptadecane* & 0 & 1 & 1 & 3.2 \\
\hline Phenol,2-methyl-5-(1-methylethyl) & 0 & 1 & 1 & 3.2 \\
\hline 3,5-di-tert-Butyl-4-hydroxybenzaldehyde & & 1 & 3.2 \\
\hline
\end{tabular}


Figure 82. Histogram of VOCs in urine odor across thirty-one healthy individuals

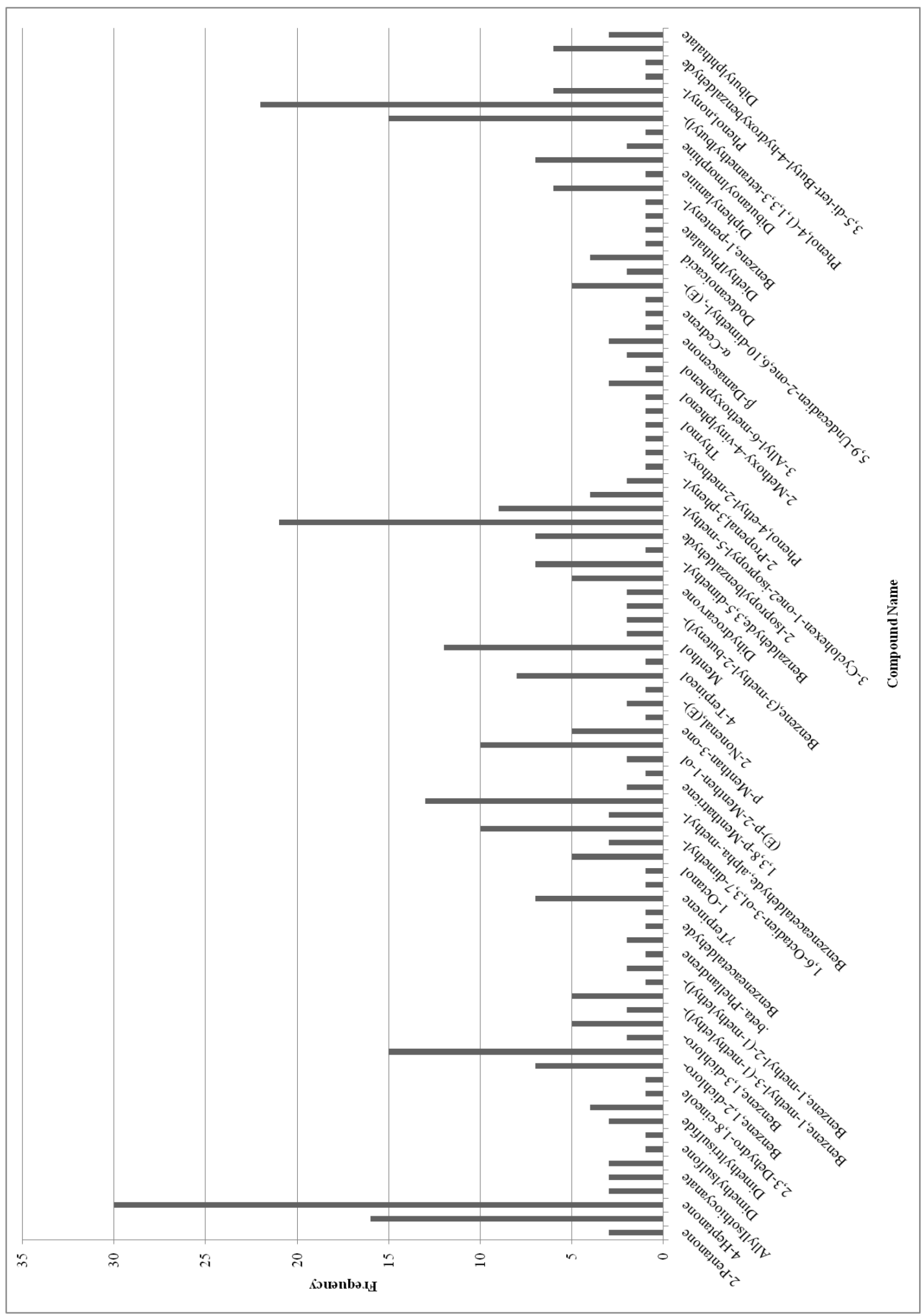




\subsection{Inter-Specimen Matching}

The similarities and differences of the VOC profiles of each collected specimens were investigated to determine whether inter-specimen samples could be used to match an individual (i.e. could subject A's blood samples be used to match with subject A's buccal swabs?). Figure 83 is a percent stacked column color chart of the relative peak area ratios of the VOCs extracted from the five specimens from subject Male 16. The color chart shows a visual representation of the similarities among the multiple samples from the same specimen (i.e., hand odor samples 1, 2, and 3) as well as the differences among the samples from varying specimens (i.e., buccal swab samples and blood samples). A semiquantitative method of analysis to represent these data is through Spearman rank correlation comparison. Table 42 demonstrates the correlation coefficients for the peak areas of the VOCs extracted from the five biological specimens of subject Male 16. The correlation coefficient values clearly indicate a high correlation among samples from the same specimen ( $>0.9)$; samples from different specimens show low correlations, with the Spearman rank correlation coefficient values ranging from 0.142 to -0.495 . Similar results were obtained from the other twenty-nine subjects sampled. Groupings according to specimens were seen through PCA analysis, similar to that demonstrated previously in Figure 60. 
Figure 83. Relative peak area ratios of VOCs extracted from M16 specimens

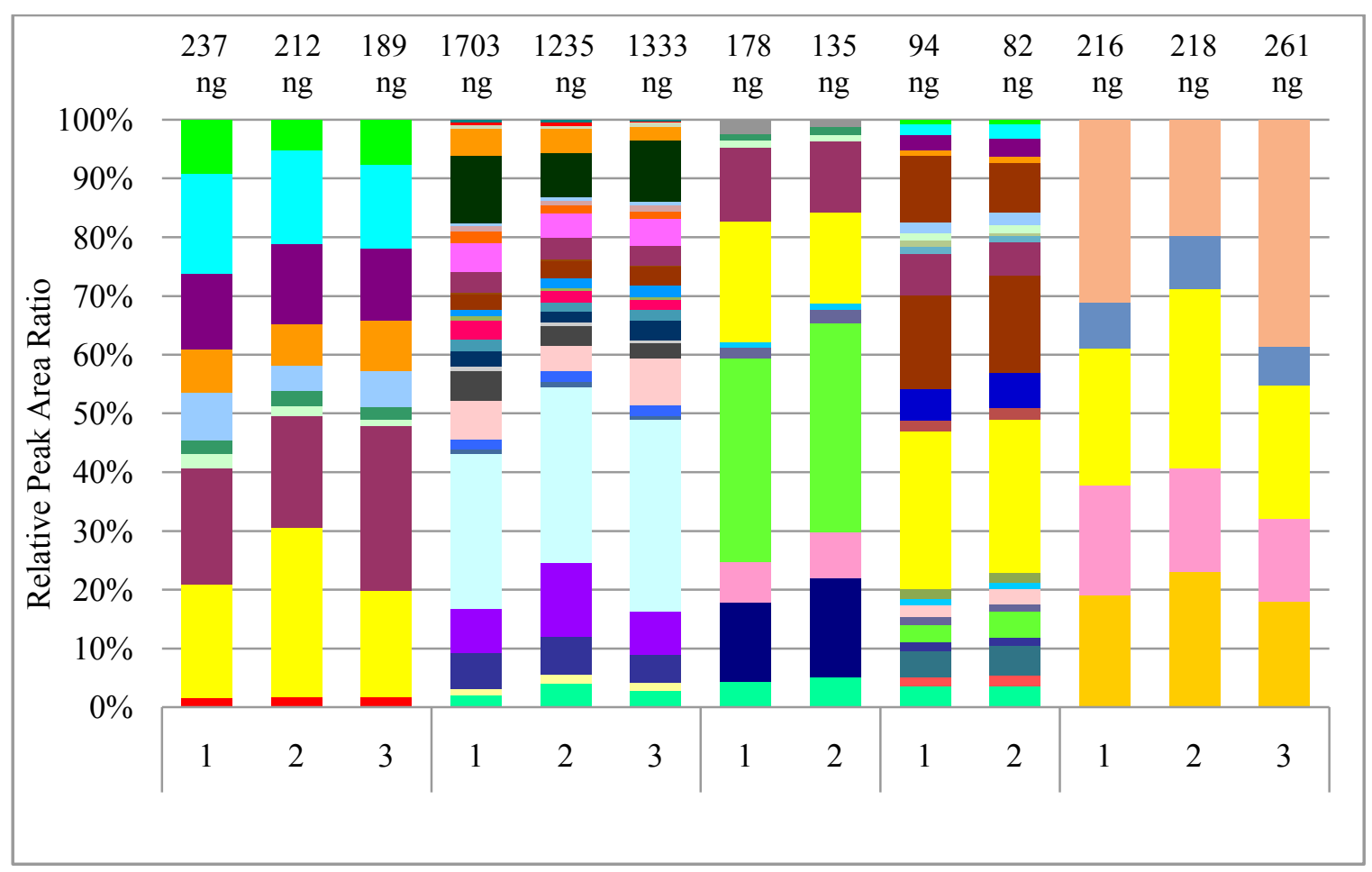

Table 42. Spearman rank correlation coefficients for the peak areas of the VOCs extracted from biological specimens from Male 16

\begin{tabular}{|l|c|c|c|c|c|c|c|c|c|c|c|c|c|}
\hline & HD1 & HD2 & HD3 & CH1 & CH2 & CH3 & BR1 & BR2 & BL1 & BL2 & UR1 & UR2 & UR3 \\
\hline HD1 & 1.000 & 0.915 & 0.927 & -0.353 & -0.348 & -0.358 & -0.140 & -0.170 & 0.116 & 0.142 & -0.495 & -0.439 & -0.495 \\
\hline HD2 & 0.915 & 1.000 & 0.988 & -0.334 & -0.327 & -0.352 & -0.147 & -0.167 & 0.092 & 0.117 & -0.470 & -0.409 & -0.470 \\
\hline HD3 & 0.927 & 0.988 & 1.000 & -0.313 & -0.306 & -0.329 & -0.155 & -0.170 & 0.087 & 0.111 & -0.495 & -0.439 & -0.495 \\
\hline CH1 & -0.353 & -0.334 & -0.313 & 1.000 & 0.960 & 0.947 & -0.426 & -0.426 & -0.380 & -0.392 & -0.412 & -0.412 & -0.412 \\
\hline CH2 & -0.348 & -0.327 & -0.306 & 0.960 & 1.000 & 0.945 & -0.394 & -0.394 & -0.352 & -0.366 & -0.412 & -0.412 & -0.412 \\
\hline CH3 & -0.358 & -0.352 & -0.329 & 0.947 & 0.945 & 1.000 & -0.403 & -0.403 & -0.356 & -0.373 & -0.372 & -0.372 & -0.372 \\
\hline BR1 & -0.140 & -0.147 & -0.155 & -0.426 & -0.394 & -0.403 & 1.000 & 0.958 & 0.093 & 0.089 & -0.047 & 0.009 & -0.047 \\
\hline BR2 & -0.170 & -0.167 & -0.170 & -0.426 & -0.394 & -0.403 & 0.958 & 1.000 & 0.015 & 0.005 & -0.068 & -0.016 & -0.068 \\
\hline BL1 & 0.116 & 0.092 & 0.087 & -0.380 & -0.352 & -0.356 & 0.093 & 0.015 & 1.000 & 0.976 & -0.248 & -0.207 & -0.248 \\
\hline BL2 & 0.142 & 0.117 & 0.111 & -0.392 & -0.366 & -0.373 & 0.089 & 0.005 & 0.976 & 1.000 & -0.234 & -0.194 & -0.234 \\
\hline UR1 & -0.495 & -0.470 & -0.495 & -0.412 & -0.412 & -0.372 & -0.047 & -0.068 & -0.248 & -0.234 & 1.000 & 0.929 & 1.000 \\
\hline UR2 & -0.439 & -0.409 & -0.439 & -0.412 & -0.412 & -0.372 & 0.009 & -0.016 & -0.207 & -0.194 & 0.929 & 1.000 & 0.929 \\
\hline UR3 & -0.495 & -0.470 & -0.495 & -0.412 & -0.412 & -0.372 & -0.047 & -0.068 & -0.248 & -0.234 & 1.000 & 0.929 & 1.000 \\
\hline
\end{tabular}


Results obtained through Spearman rank analysis of the relative peak areas of the common VOCs extracted across the five biological specimens from the same individual indicate that different specimens from the same individual are too different to be used for matching purposes. For instance, the blood sample and the buccal swab sample of subject A cannot be compared to one another in attempt to match the samples to be originating from subject A. Variations in the volatile organic compounds emanating from different biological specimens is most likely the result of distinct glandular secretions and their interactions with resident bacteria relative to each specimen. Even when comparing VOCs emanating from the skin, it has been previously reported that the site of emanation (skin from the back vs. skin from the forearm) influences the abundance of the same VOCs collected ${ }^{14}$. A number of exocrine glands are distributed throughout the body that contributes to the VOCs being excreted. Merocrine glands are found throughout the skin and include the eccrine (sweat) glands as well as salivary glands. Apocrine glands are present in the axillae and the pubic areas and are thought to function as scent glands. Holcrine glands include the sebaceous glands and are found mostly on the upper part of the body (such as the cheek mucosa, as described in the literature review section on oral fluids). Secretions from these various glands and their interactions with the different bacterial flora residing in surfaces of the body (skin vs. mouth/cheek mucosa), are the likely explanation for the differences in the VOC profiles of the different biological specimens. 


\subsection{Intra-Specimen Matching}

It has been demonstrated that volatile organic compound signatures of individuals can be differentiated through SPME-GC/MS. Previous research has successfully demonstrated the differentiation ability of human scent samples from hand and armpit of individuals using SPME-GC/MS ${ }^{13}$. The goal of the intra-specimen matching study was to investigate whether the same holds true for other biological specimens, namely buccal swabs, breath, blood, and urine.

Figure 84 through Figure 88 shows the three-dimensional scatter-plot of principal component analysis on correlations from ten randomly selected subjects ( 5 females and 5 males) from the healthy population sampled. The PCA plot demonstrates that samples from the same subject group together closely. Some samples from different individuals, however, clustered closely with one another making it difficult to differentiate between these individuals using PCA analysis. An example of samples from different individuals clustering together can be seen in Figure 85 for buccal swab samples between subjects Male 11 (represented by blue X) and Male 16 (represented by blue open square). These samples will most likely be misidentified. 
Figure 84. Three-dimensional PCA scatter plot of PCs 1, 2, and 3 of hand odor from 10 randomly selected healthy subjects

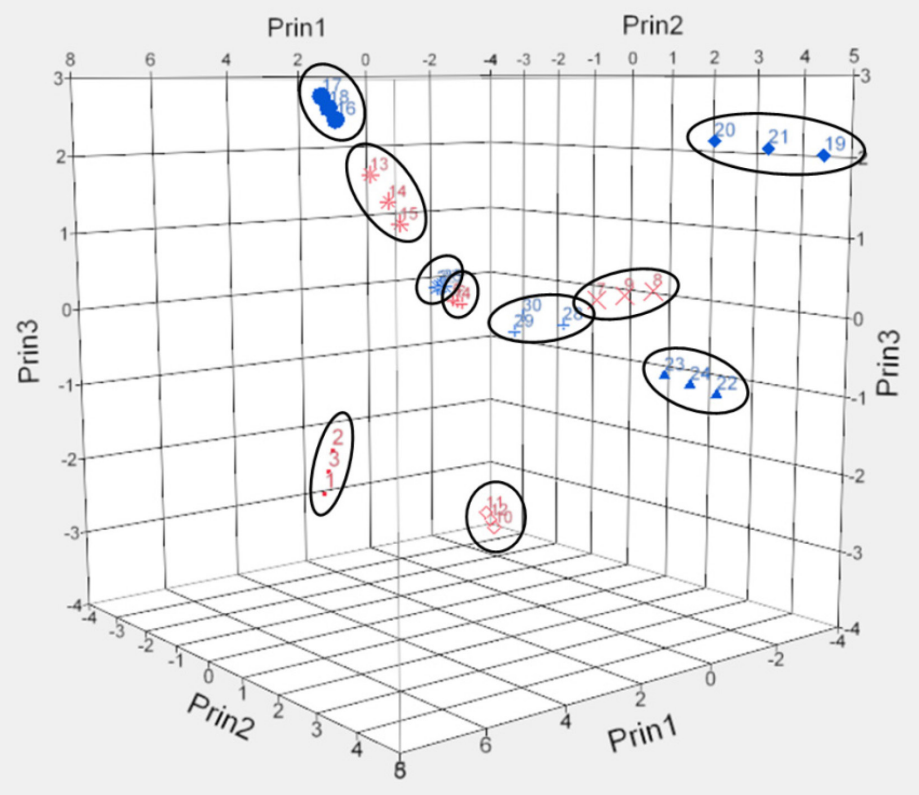

Figure 85. Three-dimensional PCA scatter plot of PCs 1, 2, and 3 of buccal swabs from 10 randomly selected healthy subjects

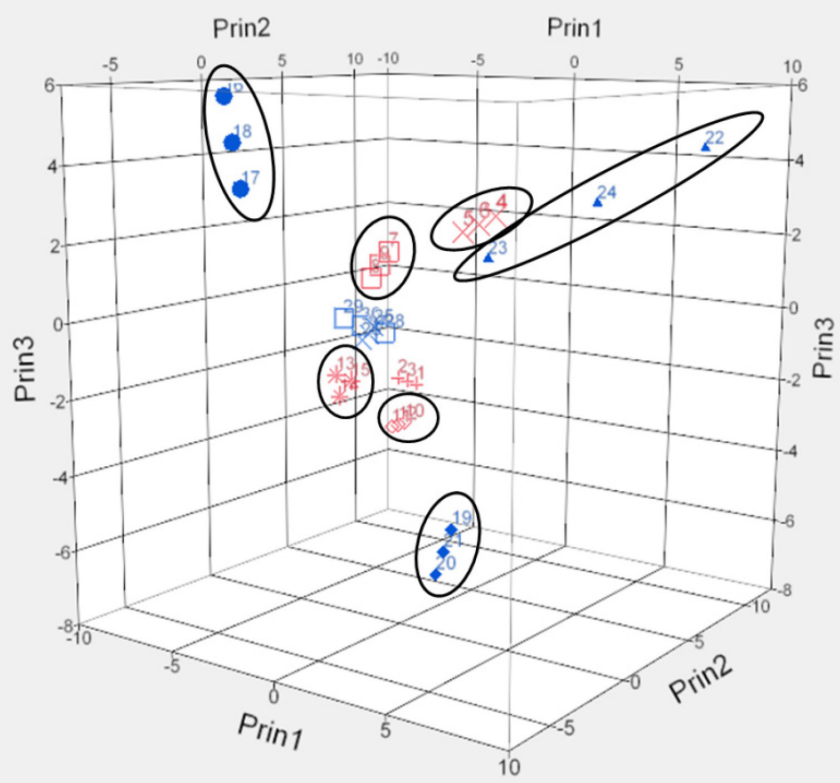


Figure 86. Three-dimensional PCA scatter plot of PCs 1, 2, and 3 of breath from 10 randomly selected healthy subjects

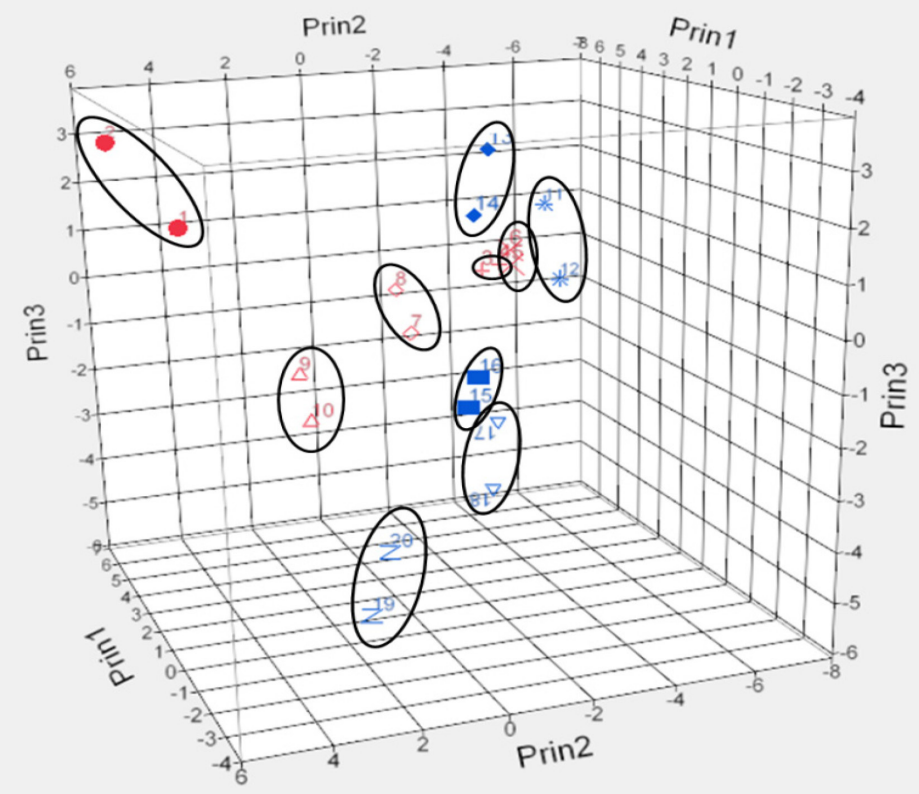

Figure 87. Three-dimensional PCA scatter plot of PCs 1, 2, and 3 of blood from 10 randomly selected healthy subjects

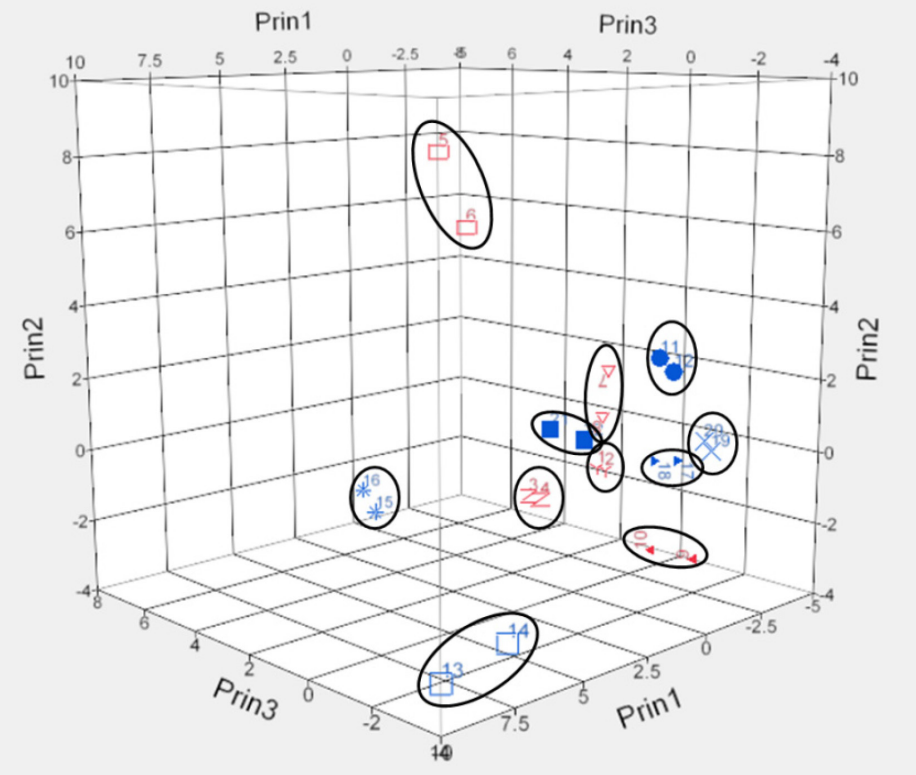


Figure 88. Three-dimensional PCA scatter plot of PCs 1, 2, and 3 of urine from 10 randomly selected healthy subjects

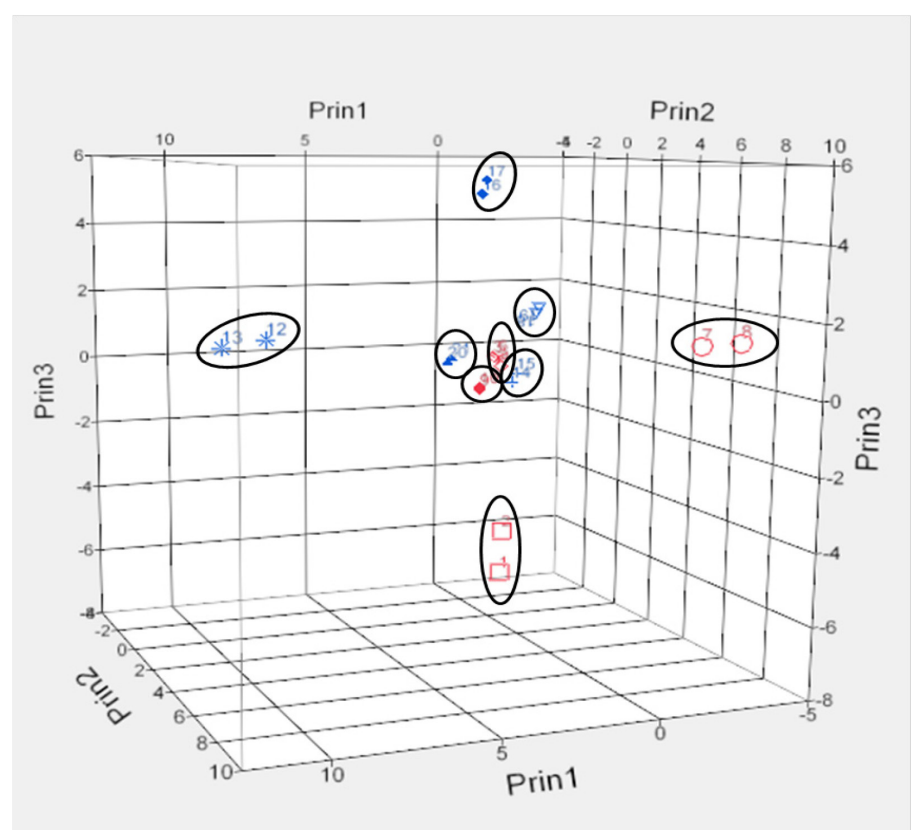

Spearman rank correlation coefficient comparisons were conducted with the samples obtained from the five biological specimens of the thirty-one healthy subjects. Occurrence of Type I and Type II errors were investigated under different correlation thresholds. Type I error is the process of incorrectly rejecting the null hypothesis in favor of the alternative, and is often referred to as a "false positive". In the present study, a Type I error is when two samples are implied to be from the same individual, when in reality they are from different individuals. This would result in a mismatch of samples (i.e. blood sample from subject A be positively identified as blood sample from subject B). Type II error is the opposite of Type I error and is the false acceptance of the null hypothesis. Often referred to as a "false negative", a Type II error implies that the two 
samples from the same individual are not a match to one another. Blood samples 1 and 2 from subject A would be identified as a non-match in this case.

\subsection{Type I Error}

The comparison of triplicate samples (for hand odor, buccal swab, and urine) collected from thirty-one subjects generates 4005 possible pairs. The comparison of duplicate samples (for breath and blood) collected from thirty-one subjects generates 1770 possible pairings. Examples where Type I errors were observed are shown in Table 43. At a correlation threshold of 0.8 , breath samples of Female 9 and Male 5 could not be distinguished. If the correlation threshold were lowered to 0.7, breath samples of Female 9, Male 5, Female 4, Female 12, and Male 7 were indistinguishable and may result in mismatched samples.

Table 44 is a summary of the number of Type I error occurrences for the comparison of the biological specimens scent samples from the thirty-one subjects. When considering a correlation threshold of 0.9 the individuals were distinguished in $99.98 \%$ of the cases for hand odor (or a mismatch percentage of $0.02 \%$ ), and $100 \%$ of the cases for buccal swabs, breath, blood, and urine. At a correlation threshold of 0.8 , these percentages were $99.93 \%$ $(0.07 \%$ mismatch) of the hand odor cases, $99.99 \%$ of the buccal swab and breath cases ( $0.01 \%$ mismatch), and $100 \%$ of the blood and urine cases (no mismatch). These values are promising in supporting the hypothesis the emanations of VOCs are different among individuals and can be used as distinguishing profiles among individuals. With the current sample size these percentages of distinguish-ability of individuals are extremely high. With increasing number of subjects and sample size, the likelihood of the sample 
pool to include individuals having similar odor profiles will increase resulting in the distinguish-ability percentage to decrease with larger sample size. In order to avoid Type I errors (false positives) as much as possible, it is recommended for the match/no-match cut-off threshold to be kept at least at 0.8 , and wherever possible, a cut-off threshold of 0.9 would be best.

Table 43. Example of Type I error occurrences

\begin{tabular}{|l|l|l|l|}
\hline \multicolumn{4}{|c|}{ F9 B reath } \\
\hline F9,1 & 1.000 & F9,2 & 1.000 \\
\hline F9,2 & 0.959 & F9,1 & 0.959 \\
\hline M5,2 & 0.870 & M5,2 & 0.839 \\
\hline M5,1 & 0.864 & M5,1 & 0.839 \\
\hline F4,2 & 0.764 & F4,2 & 0.785 \\
\hline F12,1 & 0.732 & F4,1 & 0.744 \\
\hline F4,1 & 0.722 & F12,1 & 0.743 \\
\hline M7,2 & 0.714 & M7,2 & 0.730 \\
\hline F12,2 & 0.708 & F12,2 & 0.725 \\
\hline M11,2 & 0.687 & M7,1 & 0.709 \\
\hline M7,1 & 0.681 & M11,2 & 0.688 \\
\hline M11,1 & 0.671 & M11,1 & 0.684 \\
\hline M4,1 & 0.663 & F10,1 & 0.665 \\
\hline F10,1 & 0.654 & F10,2 & 0.660 \\
\hline F10,2 & 0.650 & M4,1 & 0.654 \\
\hline M10,1 & 0.648 & M10,1 & 0.648 \\
\hline F8,1 & 0.646 & M6,2 & 0.646 \\
\hline M6,1 & 0.646 & M6,1 & 0.644 \\
\hline M6,2 & 0.644 & F8,1 & 0.625 \\
\hline M9,2 & 0.637 & M12,2 & 0.617 \\
\hline M12,2 & 0.619 & M9,2 & 0.614 \\
\hline M12,1 & 0.602 & M12,1 & 0.599 \\
\hline M10,2 & 0.598 & M10,2 & 0.598 \\
\hline
\end{tabular}

\begin{tabular}{|l|l|l|l|}
\hline \multicolumn{4}{|c|}{ M9 Blood } \\
\hline M9,1 & 1.000 & M9,2 & 1.000 \\
\hline M9,2 & 0.947 & M9,1 & 0.947 \\
\hline M6,1 & 0.732 & M6,1 & 0.718 \\
\hline F4,2 & 0.662 & F4,2 & 0.682 \\
\hline F8,2 & 0.612 & F4,1 & 0.641 \\
\hline F4,1 & 0.609 & F11,1 & 0.547 \\
\hline F11,1 & 0.578 & F9,2 & 0.541 \\
\hline F9,1 & 0.574 & F8,2 & 0.538 \\
\hline F11,2 & 0.572 & F11,2 & 0.531 \\
\hline F9,2 & 0.563 & F9,1 & 0.530 \\
\hline M6,2 & 0.550 & M6,2 & 0.518 \\
\hline F8,1 & 0.520 & M8,2 & 0.469 \\
\hline M16,2 & 0.504 & M16,2 & 0.466 \\
\hline M16,1 & 0.503 & M16,1 & 0.464 \\
\hline M5,1 & 0.496 & M5,1 & 0.456 \\
\hline F7,1 & 0.494 & M5,2 & 0.442 \\
\hline M8,2 & 0.481 & F8,1 & 0.439 \\
\hline M5,2 & 0.480 & F7,1 & 0.438 \\
\hline F6,1 & 0.478 & F6,1 & 0.426 \\
\hline M1,1 & 0.464 & M7,1 & 0.421 \\
\hline M1,2 & 0.459 & F15,1 & 0.416 \\
\hline M8,1 & 0.451 & M1,1 & 0.413 \\
\hline M7,1 & 0.445 & M8,1 & 0.413 \\
\hline
\end{tabular}


Table 44. Type I error occurrences for three correlation thresholds across biological specimens

\begin{tabular}{|l|c|c|c|}
\hline & \multicolumn{3}{|c|}{ Threshold } \\
\hline & $\mathbf{0 . 9}$ & $\mathbf{0 . 8}$ & $\mathbf{0 . 7}$ \\
\hline Hand Odor & 5 & 32 & 73 \\
\hline Buccal Swab & 0 & 1 & 8 \\
\hline Breath & 0 & 1 & 25 \\
\hline Blood & 0 & 0 & 3 \\
\hline Urine & 0 & 0 & 0 \\
\hline
\end{tabular}

\subsection{Type II Error}

Table 45 shows the Spearman rank correlation coefficients of buccal swab samples 1 and 2 from Female 5, breath samples 1 and 2 from Male 16, and blood samples 1 and 2 from Male 7. In each of these cases the duplicate samples from the same individual were identified as a non-match at all three thresholds of $0.7,0.8$, and 0.9 . Table 46 summarizes the Type II error occurrences among the thirty-one subjects compared in each of the biological specimens. As is expected the number of Type II errors increases as the cut-off threshold is raised. If the match/no-match threshold is set too high (i.e. at 0.9), the samples from the same individuals that have high correlation coefficients (as they should have) would be identified as a non-match, resulting in a false-negative. Breath and urine had the lowest number of Type II errors. Blood had the highest number of Type II errors; however, this could be due to sampling protocol. For example, there was an obvious volume difference between the duplicate blood samples collected onto the two FTA cards obtained from Male 7. 
Table 45. Example of Type II error occurrences

\begin{tabular}{|l|l|l|l|}
\hline \multicolumn{3}{|c|}{ F5 Buccal Swab } \\
\hline F5,1 & 1.000 & F5,2 & 1.000 \\
\hline F5,2 & 0.692 & F5,1 & 0.692 \\
\hline F6,2 & 0.673 & F6,2 & 0.565 \\
\hline F6,1 & 0.607 & F6,1 & 0.428 \\
\hline M2,2 & 0.425 & F17,3 & 0.353 \\
\hline M2,1 & 0.396 & F17,2 & 0.352 \\
\hline F17,3 & 0.311 & F17,1 & 0.342 \\
\hline F17,2 & 0.310 & M2,1 & 0.330 \\
\hline M7,1 & 0.309 & F14,2 & 0.326 \\
\hline F17,1 & 0.299 & M7,1 & 0.325 \\
\hline F14,2 & 0.291 & F14,1 & 0.291 \\
\hline F7,1 & 0.284 & M7,2 & 0.291 \\
\hline M7,2 & 0.280 & M2,2 & 0.264 \\
\hline F14,1 & 0.255 & $F 7,1$ & 0.231 \\
\hline F7,2 & 0.237 & F16,2 & 0.212 \\
\hline F16,2 & 0.222 & F16,3 & 0.206 \\
\hline F16,3 & 0.213 & F16,1 & 0.185 \\
\hline F16,1 & 0.176 & F7,2 & 0.159 \\
\hline F10,2 & 0.125 & F8,2 & 0.129 \\
\hline F10,1 & 0.097 & F10,2 & 0.081 \\
\hline F8,2 & 0.092 & F8,1 & 0.064 \\
\hline M6,1 & 0.029 & F10,1 & 0.056 \\
\hline M6,2 & 0.026 & M1,2 & 0.052 \\
\hline F8,1 & 0.023 & M1,1 & 0.051 \\
\hline
\end{tabular}

\begin{tabular}{|l|l|l|l|}
\hline \multicolumn{3}{|c|}{ M15 B reath } \\
\hline M15,1 & 1.000 & M15,2 & 1.000 \\
\hline M15,2 & 0.600 & M15,1 & 0.600 \\
\hline F5,2 & 0.442 & M9,2 & 0.596 \\
\hline F5,1 & 0.442 & F2,2 & 0.534 \\
\hline M9,2 & 0.440 & F2,1 & 0.527 \\
\hline F2,1 & 0.395 & F5,2 & 0.487 \\
\hline F2,2 & 0.388 & F5,1 & 0.487 \\
\hline M9,1 & 0.387 & M9,1 & 0.460 \\
\hline M16,1 & 0.290 & M16,1 & 0.396 \\
\hline M16,2 & 0.286 & F10,2 & 0.382 \\
\hline M4,1 & 0.260 & M4,1 & 0.380 \\
\hline F14,1 & 0.234 & F10,1 & 0.365 \\
\hline F14,2 & 0.207 & M16,2 & 0.364 \\
\hline F17,1 & 0.205 & F14,2 & 0.328 \\
\hline F4,2 & 0.197 & F14,1 & 0.322 \\
\hline F15,2 & 0.189 & F17,1 & 0.298 \\
\hline M4,2 & 0.184 & M5,2 & 0.298 \\
\hline M14,2 & 0.179 & F4,2 & 0.292 \\
\hline F10,1 & 0.179 & M5,1 & 0.286 \\
\hline F15,1 & 0.174 & F4,1 & 0.276 \\
\hline F4,1 & 0.166 & M12,2 & 0.270 \\
\hline F10,2 & 0.159 & F15,1 & 0.257 \\
\hline M12,2 & 0.157 & F15,2 & 0.249 \\
\hline M14,1 & 0.153 & F17,2 & 0.245 \\
\hline & & & \\
\hline
\end{tabular}

\begin{tabular}{|c|c|c|c|}
\hline \multicolumn{4}{|c|}{ M7 } \\
\hline $\mathrm{M} 7,1$ & 1.000 & $\mathrm{M} 7,2$ & 1.000 \\
\hline $\mathrm{M} 7,2$ & 0.620 & M7,1 & 0.620 \\
\hline $\mathrm{M} 2,1$ & 0.518 & $\mathrm{~F} 8,2$ & 0.526 \\
\hline $\mathrm{F} 7,1$ & 0.516 & M5,2 & 0.472 \\
\hline $\mathrm{F} 11,1$ & 0.502 & M5,1 & 0.468 \\
\hline $\mathrm{F} 11,2$ & 0.487 & $\mathrm{~F} 11,1$ & 0.462 \\
\hline $\mathrm{F} 8,2$ & 0.486 & F11,2 & 0.444 \\
\hline F7,2 & 0.477 & $\mathrm{M} 2,1$ & 0.427 \\
\hline M5,1 & 0.459 & $\mathrm{~F} 8,1$ & 0.426 \\
\hline M10,1 & 0.454 & F7,1 & 0.387 \\
\hline M10,2 & 0.449 & M1,1 & 0.380 \\
\hline M9,1 & 0.445 & F13,1 & 0.380 \\
\hline $\mathrm{M} 2,2$ & 0.437 & M10,2 & 0.377 \\
\hline M5,2 & 0.436 & $\mathrm{M} 10,1$ & 0.376 \\
\hline M6,1 & 0.422 & F6,1 & 0.370 \\
\hline M9,2 & 0.421 & $\mathrm{M} 1,2$ & 0.369 \\
\hline M13,1 & 0.408 & M2,2 & 0.341 \\
\hline $\mathrm{F} 9,1$ & 0.407 & F7,2 & 0.340 \\
\hline M16,1 & 0.402 & F13,2 & 0.326 \\
\hline M16,2 & 0.400 & M6,1 & 0.324 \\
\hline $\mathrm{F} 8,1$ & 0.395 & M16,2 & 0.307 \\
\hline M12,1 & 0.385 & M9,1 & 0.304 \\
\hline M13,2 & 0.383 & M12,1 & 0.304 \\
\hline $\mathrm{F9}, 2$ & 0.377 & M16,1 & 0.304 \\
\hline
\end{tabular}

Table 46. Type II error occurrences for three correlation thresholds across biological specimens

\begin{tabular}{|l|c|c|c|}
\hline & \multicolumn{3}{|c|}{ Threshold } \\
\hline & $\mathbf{0 . 9}$ & $\mathbf{0 . 8}$ & $\mathbf{0 . 7}$ \\
\hline Hand Odor & 15 & 9 & 7 \\
\hline Buccal Swab & 9 & 4 & 3 \\
\hline Breath & 11 & 4 & 1 \\
\hline Blood & 12 & 6 & 4 \\
\hline Urine & 8 & 3 & 1 \\
\hline
\end{tabular}


Table 47. Type I and Type II errors for five biological specimens amongst thirty-one healthy individuals

\begin{tabular}{|l|c|c|c|c|c|c|c|c|c|c|c|c|c|c|c|}
\hline & \multicolumn{3}{|c|}{ Hand Odor } & \multicolumn{3}{|c|}{ Buccal Swab } & \multicolumn{3}{c|}{ Breath } & \multicolumn{3}{c|}{ Blood } & \multicolumn{3}{c|}{ Urine } \\
\hline Threshold & 0.9 & 0.8 & 0.7 & 0.9 & 0.8 & 0.7 & 0.9 & 0.8 & 0.7 & 0.9 & 0.8 & 0.7 & 0.9 & 0.8 & 0.7 \\
\hline \hline Type I & 3 & 13 & 34 & 0 & 1 & 3 & 0 & 1 & 19 & 0 & 0 & 3 & 0 & 0 & 0 \\
\hline Type II & 15 & 9 & 7 & 9 & 4 & 3 & 11 & 4 & 1 & 12 & 6 & 4 & 8 & 3 & 1 \\
\hline Total Error & 18 & 22 & 41 & 9 & 5 & 6 & 11 & 5 & 20 & 12 & 6 & 7 & 8 & 3 & 1 \\
\hline \% Mismatch & 0.89 & 1.09 & 2.03 & 0.45 & 0.25 & 0.30 & 0.58 & 0.26 & 1.06 & 0.63 & 0.32 & 0.37 & 0.36 & 0.14 & 0.05 \\
\hline
\end{tabular}

Prior to the current study, it was not known if one could differentiate individuals by odor components other than odor emanating from skin (mainly hand, arm, and armpits) on gauze. For the population study of thirty-one healthy individuals, the headspace of scent samples collected on various biological specimens (hand odor, buccal swabs, breath, blood, and urine) can be distinguished chromatographically based on a combination of the relative peak area ratios of the common compounds present in these samples. Through the combined methods of chromatogram comparison, Spearman rank correlation comparison, and principal component analysis, it is possible to distinguish the VOC profiles of individuals for each of the specimens with high confidence. However, VOC profiles of different biological specimens from the same individual are too different to be used for matching purposes.

\subsubsection{Evaluation of Odor Profiles of Individuals with Type 2 Diabetes}

Odor profiles of nineteen individuals with diagnosed type 2 diabetes were evaluated using SPME-GC/MS. Compounds were identified by spectral library (NIST) and/or by standard reference comparison. Figure 89 demonstrates the frequency distribution of the different functional groups of VOCs that were extracted from hand odor, buccal swab, 
breath, blood, and urine across the 19 subjects. For hand odor, aldehyde and aliphatic VOCs (28\% each) comprised slightly over half of the total volatile compounds extracted, followed by ketones (16\%), carboxylic acids (16\%), and esters (12\%). No carboxylic acid VOCs were extracted from hand odor. Decanal and nonanal were extracted from all 19 diabetic subjects (Table 48). Other high-frequency occurring (>70\%) compounds for hand odor for type 2 diabetics were heptadecane, hexadecane, and tetradecane, each having an $89.5 \%$ occurrence among the 19 subjects sampled.

For buccal swab odor, ester VOCs had the highest frequency of occurrence $(24 \%)$, followed by aromatic (18\%), aliphatic (16\%), ketones (16\%), carboxylic acids $(9 \%)$, alcohols (8\%), and ether (1\%) compounds. There were eight high-frequency compounds across 19 diabetic individuals as seen in Table 49, of which hexanoic acid and decanal were present in all 19 diabetic individuals.

Aromatic compounds comprised about half of the VOCs extracted from breath odor at $45 \%$. The remaining frequency distribution of functional groups for breath odor was as follows: aliphatics (17\%), alcohols (10\%), ketones (10\%), aldehydes (8\%), esters $(8 \%)$, and other functional group compounds (2\%). Only two compounds (nonanal and decanal) were high-frequency compounds for breath, although xylenes were present in $78.9 \%$ of the 19 diabetic individuals (Table 50). Typical VOCs in exhaled breath are under $1 \mathrm{ppb}$ concentration range. It has been reported that disease states do not typically result in higher VOC concentrations, although there are certain disease states such as untreated diabetes or severe renal impairment that do result in significant differences in the VOC concentrations in breath ${ }^{49}$. For the present study, acetone was only detected in the breath 
samples of three of the diabetic patients sampled, and the amount of acetone extracted was not particularly higher than that of healthy individuals. The minimal difference in the acetone presence and the amount extracted can be likely explained by the fact that the diabetic subjects sampled in the present study were all fairly well-controlled (treated with diet and/or medication). Additionally, detection and measurement of acetone under the current instrumental condition are not always consistent due to acetone's nature of volatility and activity. Pre-concentration and/or on-fiber derivatization of acetone may need to be considered for future studies in order to effectively extract and accurately measure acetone in breath samples.

For blood, the frequency distribution of functional groups of the VOCs extracted was as follows: aromatics (40\%), aliphatics (26\%), alcohols (13\%), ketones $(10 \%)$, other functional groups $(4 \%)$, and esters $(2 \%)$. Other functional groups included ethers and nitrogen-containing compounds. There were seven high-frequency compounds, of which nonanal and hexadecane were present in all 19 diabetic individuals (Table 51).

Finally for urine, the frequency distribution of functional groups of the extracted VOCs was as follows: alcohols (22\%), aromatics $(20 \%)$, ketones $(17 \%)$, aldehydes $(11 \%)$, nitrogen-containing compounds (11\%), sulfur-containing compounds (6\%), aliphatics (5\%), carboxylic acids (4\%), and esters (4\%). No compound was present in high frequency; 4-heptanone was present at highest frequency among the 19 diabetic individuals sampled at $73.7 \%$ (Table 52). 
Figure 89. Frequency distribution of functional groups of extracted VOCs in hand odor, buccal swab, breath, blood, and urine across twenty individuals diagnosed with type 2 diabetes
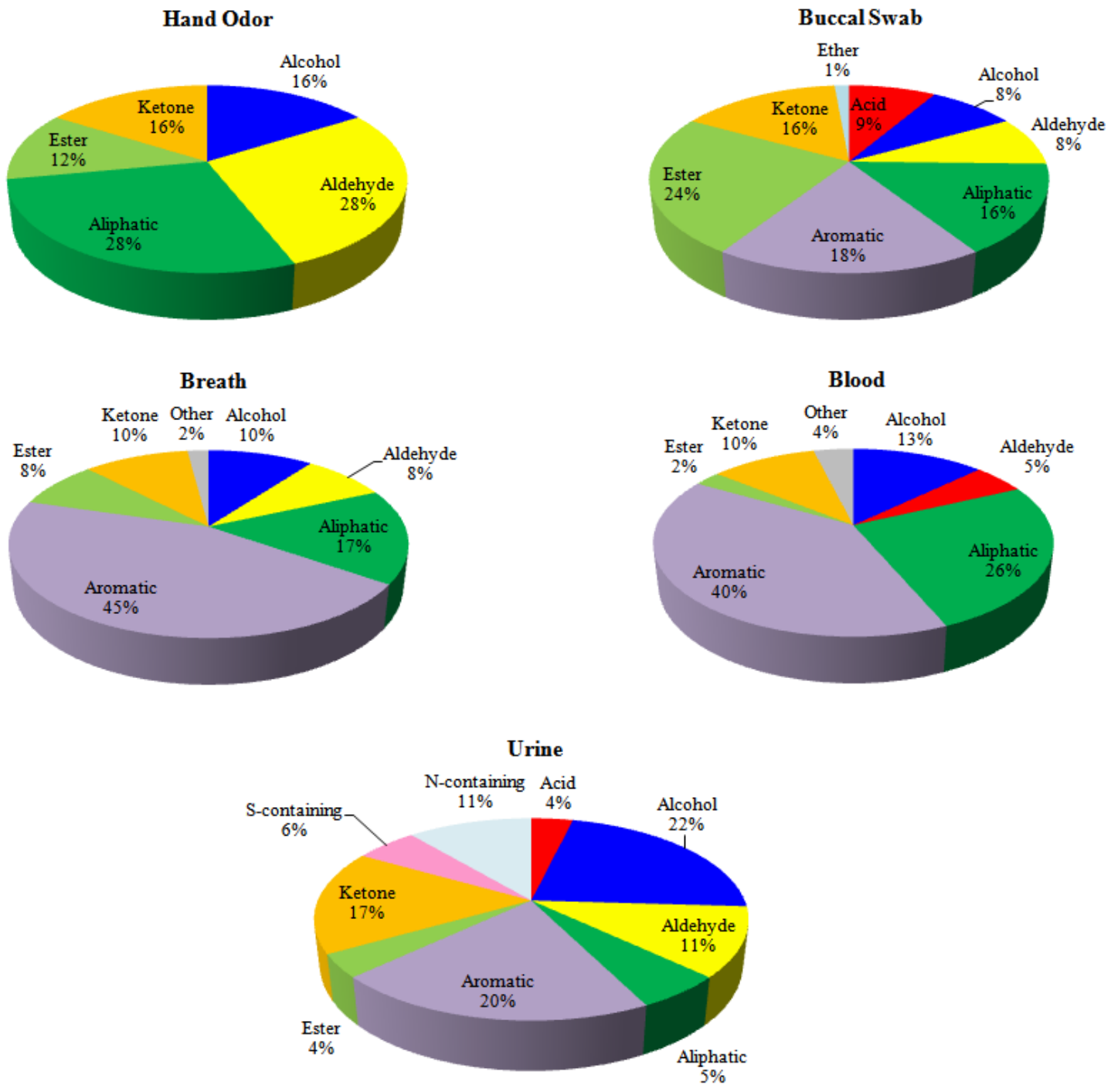
Table 48. Identified VOCs in hand odor of nineteen individuals with diagnosed type 2 diabetes ranked by frequency of occurrence (Note: *denotes compound was verified by reference standard)

\begin{tabular}{|l|ccc|c|}
\hline \multicolumn{1}{|c|}{ Compound Name } & \multicolumn{3}{|c|}{ Frequency } & Occurrence \\
& Female & Male & Total & $(\%)$ \\
\hline Decanal* & 8 & 11 & 19 & $100 \%$ \\
\hline Nonanal* $^{*}$ & 8 & 11 & 19 & $100 \%$ \\
\hline Heptadecane* & 7 & 10 & 17 & $89.5 \%$ \\
\hline Hexadecane* & 7 & 10 & 17 & $89.5 \%$ \\
\hline Tetradecane* & 7 & 10 & 17 & $89.5 \%$ \\
\hline Octadecane & 4 & 7 & 11 & $57.9 \%$ \\
\hline Undecane* & 5 & 6 & 11 & $57.9 \%$ \\
\hline Tridecane* & 3 & 6 & 9 & $47.4 \%$ \\
\hline 5,9-Undecadien-2-one,6,10-dimethyl-, (E)-* & 3 & 6 & 9 & $47.4 \%$ \\
\hline Undecanal* & 2 & 5 & 7 & $36.8 \%$ \\
\hline Dodecane* & 2 & 3 & 5 & $26.3 \%$ \\
\hline 2-Nonenal,(E)-* & 2 & 2 & 4 & $21.1 \%$ \\
\hline Dodecanoicacid,methylester & 0 & 4 & 4 & $21.1 \%$ \\
\hline Acetophenone* & 2 & 1 & 3 & $15.8 \%$ \\
\hline 1-Hexanol,2-ethyl-* & 1 & 1 & 2 & $10.5 \%$ \\
\hline Benzyl Alcohol* & 1 & 1 & 2 & $10.5 \%$ \\
\hline Dodecanal* & 1 & 1 & 2 & $10.5 \%$ \\
\hline 1,6-Octadien-3-ol,3,7-dimethyl-* & 0 & 1 & 1 & $5.3 \%$ \\
\hline Cedrol & 0 & 1 & 1 & $5.3 \%$ \\
\hline Benzaldehyde* & 1 & 0 & 1 & $5.3 \%$ \\
\hline Lilial & 1 & 0 & 1 & $5.3 \%$ \\
\hline Aceticacid,butylester & 1 & 0 & 1 & $5.3 \%$ \\
\hline Octanoicacid,methylester* & 0 & 1 & 1 & $5.3 \%$ \\
\hline 2-Pentanone & 1 & 0 & 1 & $5.3 \%$ \\
\hline 5-Hepten-2-one,6-methyl-* & 0 & 1 & 1 & $5.3 \%$ \\
\hline
\end{tabular}


Table 49. Identified VOCs in buccal swabs of nineteen individuals with diagnosed type 2 diabetes ranked by frequency of occurrence (Note: *denotes compound was verified by reference standard)

\begin{tabular}{|l|ccc|c|}
\hline \multicolumn{1}{|c|}{ Compound Name } & \multicolumn{3}{|c|}{ Frequency } & Occurrence \\
& Female & Male & Total & (\%) \\
\hline Hexanoicacid* & 8 & 11 & 19 & $100 \%$ \\
\hline Decana** & 8 & 11 & 19 & $100 \%$ \\
\hline Nonanoicacid & 6 & 11 & 17 & $89.5 \%$ \\
\hline 5,9-Undecadien-2-one,6,10-dimethyl-, (E)-* & 7 & 10 & 17 & $89.5 \%$ \\
\hline Nonanoicacid,ethylester* & 6 & 10 & 16 & $84.2 \%$ \\
\hline Furan,2-pentyl-* & 6 & 9 & 15 & $78.9 \%$ \\
\hline Dodecanoicacid,ethylester & 6 & 8 & 14 & $73.7 \%$ \\
\hline 6-Dodecanone & 5 & 9 & 14 & $73.7 \%$ \\
\hline 1-Tetradecene* & 4 & 9 & 13 & $68.4 \%$ \\
\hline Tetradecanoicacid,ethylester & 3 & 8 & 11 & $57.9 \%$ \\
\hline 1-Decene* & 6 & 5 & 11 & $57.9 \%$ \\
\hline 1,1'-Biphenyl,2,2'-diethyl- & 4 & 7 & 11 & $57.9 \%$ \\
\hline 2,4-Nonadienal,(E,E)-* & 3 & 7 & 10 & $52.6 \%$ \\
\hline Hexadecanoicacid,ethylester & 3 & 7 & 10 & $52.6 \%$ \\
\hline Tetradecane* & 4 & 6 & 10 & $52.6 \%$ \\
\hline 6-Methyl-3,5-heptadiene-2-one & 3 & 7 & 10 & $52.6 \%$ \\
\hline Heptanoicacid* & 2 & 6 & 8 & $42.1 \%$ \\
\hline Benzaldehyde* & 3 & 5 & 8 & $42.1 \%$ \\
\hline Octanoicacid,ethylester* & 3 & 4 & 7 & $36.8 \%$ \\
\hline 2-Nonenal,(E)-* & 2 & 4 & 6 & $31.6 \%$ \\
\hline 1-Dodecene* & 2 & 4 & 6 & $31.6 \%$ \\
\hline 1,1'-Biphenyl,4-methyl- & 2 & 4 & 6 & $31.6 \%$ \\
\hline 1-Hexanol* & 2 & 4 & 6 & $31.6 \%$ \\
\hline Cyclododecane & 3 & 2 & 5 & $26.3 \%$ \\
\hline 2(3H)-Furanone,dihydro-5-pentyl- & 2 & 3 & 5 & $26.3 \%$ \\
\hline Dodecanoicacid* & 3 & 2 & 5 & $26.3 \%$ \\
\hline 2(3H)-Furanone,5-ethyldihydro- & 3 & 2 & 5 & $26.3 \%$ \\
\hline 2-Tetradecene,(E)- & 3 & 1 & 4 & $21.1 \%$ \\
\hline 2-Octenoicacid & 1 & 3 & 4 & $21.1 \%$ \\
\hline Naphthalene,2-methyl- & 1 & 3 & 4 & $21.1 \%$ \\
\hline Benzothiazole & 0 & 4 & 4 & $21.1 \%$ \\
\hline Benzene,1-methyl-2-[(4-methylphenyl)methyl]- & 1 & 3 & 4 & $21.1 \%$ \\
\hline Phenol* & 1 & 3 & 4 & $21.1 \%$ \\
\hline Hexanoicacid,ethylester & 1 & 2 & 3 & $15.8 \%$ \\
\hline Heptanoicacid,ethylester* & 1 & 2 & 3 & $15.8 \%$ \\
\hline Caryophyllene* & 2 & 1 & 3 & $15.8 \%$ \\
\hline DiethylPhthalate & 1 & 2 & 3 & $15.8 \%$ \\
\hline & & & & \\
\hline
\end{tabular}




\begin{tabular}{|c|c|c|c|c|}
\hline Pentanoicacid* & 0 & 3 & 3 & $15.8 \%$ \\
\hline Naphthalene,2,7-dimethyl- & 1 & 2 & 3 & $15.8 \%$ \\
\hline Decanoicacid,ethylester* & 1 & 2 & 3 & $15.8 \%$ \\
\hline Acetophenone* & 1 & 2 & 3 & $15.8 \%$ \\
\hline Pentadecanoicacid,ethylester & 1 & 1 & 2 & $10.5 \%$ \\
\hline IsopropylMyristate & 1 & 0 & 1 & $10.5 \%$ \\
\hline Hexanoicacid,pentylester & 0 & 2 & 2 & $10.5 \%$ \\
\hline Hexadecanoicacid,methylester & 1 & 1 & 2 & $10.5 \%$ \\
\hline Dibutanoylmorphine & 1 & 1 & 2 & $10.5 \%$ \\
\hline Tridecane & 1 & 1 & 2 & $10.5 \%$ \\
\hline Naphthalene,1-methyl-* & 0 & 2 & 2 & $10.5 \%$ \\
\hline ButylatedHydroxytoluene & 1 & 1 & 2 & $10.5 \%$ \\
\hline 2(3H)-Furanone, dihydro-5-propyl- & 1 & 1 & 2 & $10.5 \%$ \\
\hline IsopropylPalmitate & 1 & 0 & 1 & $5.3 \%$ \\
\hline Hexanoicacid,propylester & 0 & 1 & 1 & $5.3 \%$ \\
\hline E-11-Hexadecenoicacid,ethylester & 1 & 0 & 1 & $5.3 \%$ \\
\hline Naphthalene,2,6-dimethyl- & 0 & 1 & 1 & $5.3 \%$ \\
\hline Benzene,1-methyl-4-nitro- & 1 & 0 & 1 & $5.3 \%$ \\
\hline Octadecane & 1 & 0 & 1 & $5.3 \%$ \\
\hline Benzene,1,2,4,5-tetramethyl- & 0 & 1 & 1 & $5.3 \%$ \\
\hline p-Menthan-3-one & 0 & 1 & 1 & $5.3 \%$ \\
\hline Hexadecane* & 0 & 1 & 1 & $5.3 \%$ \\
\hline Benzene,1,2,3,5-tetramethyl- & 1 & 0 & 1 & $5.3 \%$ \\
\hline 3,5,9-Undecatrien-2-one,6,10-dimethyl-, (E,Z)- & 0 & 1 & 1 & $5.3 \%$ \\
\hline Cyclohexadecane & 1 & 0 & 1 & $5.3 \%$ \\
\hline 1,1'-Biphenyl,3-methyl- & 0 & 1 & 1 & $5.3 \%$ \\
\hline Carvone & 0 & 1 & 1 & $5.3 \%$ \\
\hline p-Benzoquinone & 0 & 1 & 1 & $5.3 \%$ \\
\hline 1-Octanol* & 1 & 0 & 1 & $5.3 \%$ \\
\hline Hexanal* & 0 & 1 & 1 & $5.3 \%$ \\
\hline Menthol* & 1 & 0 & 1 & $5.3 \%$ \\
\hline Furfural* & 0 & 1 & 1 & $5.3 \%$ \\
\hline Linalool Oxide & 0 & 1 & 1 & $5.3 \%$ \\
\hline
\end{tabular}


Table 50. Identified VOCs in breath odor of nineteen individuals with diagnosed type 2 diabetes ranked by frequency of occurrence (Note: *denotes compound was verified by reference standard)

\begin{tabular}{|c|c|c|c|c|}
\hline \multirow{2}{*}{ Compound Name } & \multicolumn{3}{|c|}{ Frequency } & \multirow{2}{*}{$\begin{array}{c}\text { Occurrence } \\
(\%)\end{array}$} \\
\hline & Female & Male & Total & \\
\hline Nonanal* & 8 & 11 & 19 & $100 \%$ \\
\hline Decanal* & 7 & 11 & 18 & $94.7 \%$ \\
\hline Xylenes* & 5 & 10 & 15 & $78.9 \%$ \\
\hline Tridecane* & 5 & 8 & 13 & $68.4 \%$ \\
\hline Tetradecane* & 5 & 8 & 13 & $68.4 \%$ \\
\hline Dodecane* & 5 & 8 & 13 & $68.4 \%$ \\
\hline 1-Hexanol,2-ethyl-* & 5 & 6 & 11 & $57.9 \%$ \\
\hline Undecane* & 3 & 7 & 10 & $52.6 \%$ \\
\hline Benzene,1,2-dichloro-* & 4 & 6 & 10 & $52.6 \%$ \\
\hline Benzophenone* & 5 & 5 & 10 & $52.6 \%$ \\
\hline Toluene & 4 & 2 & 6 & $31.6 \%$ \\
\hline Styrene* & 2 & 4 & 6 & $31.6 \%$ \\
\hline ButylatedHydroxytoluene & 3 & 3 & 6 & $31.6 \%$ \\
\hline Benzene,1,3,5-trimethyl- & 1 & 5 & 6 & $31.6 \%$ \\
\hline p-Benzoquinone & 2 & 4 & 6 & $31.6 \%$ \\
\hline Benzene,4-ethyl-1,2-dimethyl- & 2 & 3 & 5 & $26.3 \%$ \\
\hline Benzene,2-ethyl-1,4-dimethyl- & 2 & 3 & 5 & $26.3 \%$ \\
\hline Benzene,1-methyl-3-propyl- & 0 & 5 & 5 & $26.3 \%$ \\
\hline Undecanal* $^{*}$ & 1 & 3 & 4 & $21.1 \%$ \\
\hline Benzene,1-ethyl-2,3-dimethyl- & 1 & 3 & 4 & $21.1 \%$ \\
\hline 4-Cyanocyclohexene & 2 & 2 & 4 & $21.1 \%$ \\
\hline Phenol* & 1 & 2 & 3 & $15.8 \%$ \\
\hline 1,3,5,7-Cyclooctatetraene & 2 & 1 & 3 & $15.8 \%$ \\
\hline Menthol* & 2 & 1 & 3 & $15.8 \%$ \\
\hline Naphthalene* & 1 & 2 & 3 & $15.8 \%$ \\
\hline Limonene* & 0 & 2 & 2 & $10.5 \%$ \\
\hline 2-Dodecene,(Z)- & 1 & 1 & 2 & $10.5 \%$ \\
\hline Diisopropylnaphthalene & 0 & 2 & 2 & $10.5 \%$ \\
\hline Benzene,2-ethyl-1,3-dimethyl- & 1 & 1 & 2 & $10.5 \%$ \\
\hline Benzene,1-ethyl-2-methyl- & 1 & 1 & 2 & $10.5 \%$ \\
\hline Benzene,1-ethyl-2,4-dimethyl- & 1 & 1 & 2 & $10.5 \%$ \\
\hline Benzene,1,2,3-trimethyl- & 1 & 1 & 2 & $10.5 \%$ \\
\hline 2,6-Diisopropylnaphthalene* & 0 & 2 & 2 & $10.5 \%$ \\
\hline Hexanedioicacid,bis(1-methylethyl) ester & 0 & 2 & 2 & $10.5 \%$ \\
\hline Acetone & 1 & 1 & 2 & $10.5 \%$ \\
\hline 2-Propanol,1-propoxy- & 1 & 0 & 1 & $5.3 \%$ \\
\hline Dodecanal* & 0 & 1 & 1 & $5.3 \%$ \\
\hline
\end{tabular}




\begin{tabular}{|l|lll|l|}
\hline 5-Dodecene,(E)- & 0 & 1 & 1 & $5.3 \%$ \\
\hline Naphthalene,2-methyl- & 0 & 1 & 1 & $5.3 \%$ \\
\hline Benzene,1-methyl-4-(1-methylpropyl)- & 0 & 1 & 1 & $5.3 \%$ \\
\hline Benzene,1,4-dichloro- & 0 & 1 & 1 & $5.3 \%$ \\
\hline Benzene,1,3-dichloro- & 0 & 1 & 1 & $5.3 \%$ \\
\hline Benzene,1,2,3,4-tetramethyl- & 0 & 1 & 1 & $5.3 \%$ \\
\hline Isobornylacetate & 1 & 0 & 1 & $5.3 \%$ \\
\hline DiethylPhthalate & 0 & 1 & 1 & $5.3 \%$ \\
\hline Dibutylphthalate & 0 & 1 & 1 & $5.3 \%$ \\
\hline 5,9-Undecadien-2-one,6,10-dimethyl-, (E)-* & 0 & 1 & 1 & $5.3 \%$ \\
\hline 3-Heptanone* & 0 & 1 & 1 & $5.3 \%$ \\
\hline
\end{tabular}


Table 51. Identified VOCs in blood odor of nineteen individuals with diagnosed type 2 diabetes ranked by frequency of occurrence (Note: *denotes compound was verified by reference standard)

\begin{tabular}{|c|c|c|c|c|}
\hline \multirow{2}{*}{ Compound Name } & \multicolumn{3}{|c|}{ Frequency } & \multirow{2}{*}{$\begin{array}{c}\text { Occurrence } \\
(\%)\end{array}$} \\
\hline & Female & Male & Total & \\
\hline Nonanal* & 8 & 11 & 19 & $100 \%$ \\
\hline Hexadecane* & 8 & 11 & 19 & $100 \%$ \\
\hline Tetradecane* & 7 & 9 & 16 & $84.2 \%$ \\
\hline Undecane* & 6 & 10 & 16 & $84.2 \%$ \\
\hline 2,6-Diisopropylnaphthalene* & 6 & 9 & 15 & $78.9 \%$ \\
\hline Tridecane* & 6 & 8 & 14 & $73.7 \%$ \\
\hline Benzophenone* & 4 & 10 & 14 & $73.7 \%$ \\
\hline Dodecane* & 6 & 7 & 13 & $68.4 \%$ \\
\hline Heptadecane* & 4 & 8 & 12 & $63.2 \%$ \\
\hline Benzaldehyde,2,4,6-trimethyl- & 4 & 8 & 12 & $63.2 \%$ \\
\hline Hexanal & 4 & 7 & 11 & $57.9 \%$ \\
\hline Decanal* & 7 & 2 & 9 & $47.4 \%$ \\
\hline Furan,2-butyltetrahydro- & 4 & 5 & 9 & $47.4 \%$ \\
\hline 1-Octen-3-ol* & 4 & 5 & 9 & $47.4 \%$ \\
\hline 1-Decene* & 4 & 4 & 8 & $42.1 \%$ \\
\hline Longifolene & 2 & 6 & 8 & $42.1 \%$ \\
\hline Benzene,1,3,5-trimethyl- & 2 & 5 & 7 & $36.8 \%$ \\
\hline Diisopropylnaphthalene & 3 & 4 & 7 & $36.8 \%$ \\
\hline Naphthalene* & 3 & 3 & 6 & $35.8 \%$ \\
\hline Benzyl Alcohol* & 3 & 3 & 6 & $31.6 \%$ \\
\hline 1-Dodecene* & 3 & 3 & 6 & $31.6 \%$ \\
\hline 2-Dodecene, $(\mathrm{Z})$ - & 4 & 2 & 6 & $31.6 \%$ \\
\hline Octadecane & 3 & 3 & 6 & $31.6 \%$ \\
\hline Furan,2-pentyl-* & 4 & 2 & 6 & $31.6 \%$ \\
\hline Benzene,1,2-dichloro-* & 2 & 3 & 5 & $26.3 \%$ \\
\hline Benzene,1-ethyl-2,4-dimethyl- & 1 & 4 & 5 & $26.3 \%$ \\
\hline Naphthalene,2-methyl- & 2 & 3 & 5 & $26.3 \%$ \\
\hline Menthol* & 3 & 1 & 4 & $21.1 \%$ \\
\hline 1-Dodecanol* & 1 & 3 & 4 & $21.1 \%$ \\
\hline Heptanal* & 2 & 2 & 4 & $21.1 \%$ \\
\hline 5,9-Undecadien-2-one, 6,10-dimethyl-, (E)-* & 1 & 3 & 4 & $21.1 \%$ \\
\hline 2-Propanol,1-propoxy- & 0 & 3 & 3 & $15.8 \%$ \\
\hline Benzene,1,2,3,5-tetramethyl- & 1 & 2 & 3 & $15.8 \%$ \\
\hline 2-Undecanone & 2 & 1 & 3 & $15.8 \%$ \\
\hline 3-Heptanone* & 1 & 2 & 3 & $15.8 \%$ \\
\hline Cyclohexanone* & 1 & 2 & 3 & $15.8 \%$ \\
\hline 4-Cyanocyclohexene & 2 & 1 & 3 & $15.8 \%$ \\
\hline
\end{tabular}




\begin{tabular}{|c|c|c|c|c|}
\hline 1-Octanol* & 2 & 0 & 2 & $10.5 \%$ \\
\hline 1-Pentanol & 0 & 2 & 2 & $10.5 \%$ \\
\hline 3-Dodecene,(Z)- & 0 & 2 & 2 & $10.5 \%$ \\
\hline Cyclododecane & 2 & 0 & 2 & $10.5 \%$ \\
\hline Benzene,1,2,3-trimethyl- & 1 & 1 & 2 & $10.5 \%$ \\
\hline Benzene,1,2,4,5-tetramethyl-* & 0 & 2 & 2 & $10.5 \%$ \\
\hline Benzene,1-ethyl-3,5-dimethyl- & 2 & 0 & 2 & $10.5 \%$ \\
\hline Benzene,2-ethyl-1,4-dimethyl- & 0 & 2 & 2 & $10.5 \%$ \\
\hline Naphthalene,1-methyl-* & 1 & 1 & 2 & $10.5 \%$ \\
\hline Naphthalene,2,7-dimethyl- & 1 & 1 & 2 & $10.5 \%$ \\
\hline p-Xylene* & 1 & 1 & 2 & $10.5 \%$ \\
\hline 2,5-Octanedione & 2 & 0 & 2 & $10.5 \%$ \\
\hline 2-Heptanone* & 1 & 1 & 2 & $10.5 \%$ \\
\hline 1-Heptanol* & 0 & 1 & 1 & $5.3 \%$ \\
\hline 2-Propanol,1-butoxy- & 1 & 0 & 1 & $5.3 \%$ \\
\hline Ethanol,2-butoxy- & 1 & 0 & 1 & $5.3 \%$ \\
\hline 1-Hexadecene & 1 & 0 & 1 & $5.3 \%$ \\
\hline 3,7-Dimethyl-octa-1,6-diene & 1 & 0 & 1 & $5.3 \%$ \\
\hline Cyclopropane,nonyl- & 0 & 1 & 1 & $5.3 \%$ \\
\hline Cyclotetradecane & 1 & 0 & 1 & $5.3 \%$ \\
\hline Decane & 0 & 1 & 1 & $5.3 \%$ \\
\hline Dodecane,2,6,10-trimethyl- & 1 & 0 & 1 & $5.3 \%$ \\
\hline Z-8-Hexadecene & 0 & 1 & 1 & $5.3 \%$ \\
\hline 1,1'-Biphenyl,2-methyl- & 0 & 1 & 1 & $5.3 \%$ \\
\hline 1,1'-Biphenyl,3-methyl- & 0 & 1 & 1 & $5.3 \%$ \\
\hline Benzene,1,2,3,4-tetramethyl- & 0 & 1 & 1 & $5.3 \%$ \\
\hline Benzene,1,3-dimethyl-5-(1-methylethyl)- & 0 & 1 & 1 & $5.3 \%$ \\
\hline Benzene,1,4-dichloro- & 0 & 1 & 1 & $5.3 \%$ \\
\hline Benzene,1-ethyl-2,3-dimethyl- & 1 & 0 & 1 & $5.3 \%$ \\
\hline Benzene,1-ethyl-2-methyl- & 0 & 1 & 1 & $5.3 \%$ \\
\hline Benzene,1-ethyl-3-methyl- & 0 & 1 & 1 & $5.3 \%$ \\
\hline Benzene,1-methyl-4-(1-methylethyl)- & 0 & 1 & 1 & $5.3 \%$ \\
\hline Benzene,1-methyl-4-(1-methylpropyl)- & 0 & 1 & 1 & $5.3 \%$ \\
\hline Benzene,2,4-dimethyl-1-(1-methylethyl)- & 1 & 0 & 1 & $5.3 \%$ \\
\hline Benzene,4-ethyl-1,2-dimethyl- & 0 & 1 & 1 & $5.3 \%$ \\
\hline Benzene,ethyl-1,2,4-trimethyl- & 0 & 1 & 1 & $5.3 \%$ \\
\hline Naphthalene,1,7-dimethyl- & 1 & 0 & 1 & $5.3 \%$ \\
\hline Naphthalene,2,3,6-trimethyl- & 1 & 0 & 1 & $5.3 \%$ \\
\hline 4-tert-Butylcyclohexylacetate & 0 & 1 & 1 & $5.3 \%$ \\
\hline Hexanedioicacid,bis(1-methylethyl) ester & 0 & 1 & 1 & $5.3 \%$ \\
\hline 2-Heptanone,6-methyl- & 1 & 0 & 1 & $5.3 \%$ \\
\hline
\end{tabular}


Table 52. Identified VOCs in urine odor of nineteen individuals with diagnosed type 2 diabetes ranked by frequency of occurrence (Note: *denotes compound was verified by reference standard)

\begin{tabular}{|l|ccc|c|}
\hline \multicolumn{1}{|c|}{ Compound Name } & \multicolumn{3}{|c|}{ Frequency } & Occurrence \\
& Female & Male & Total & (\%) \\
\hline 4-Heptanone* & 5 & 9 & 14 & $73.7 \%$ \\
\hline Cedrol & 4 & 7 & 11 & $57.9 \%$ \\
\hline Nonanal* $^{*}$ & 2 & 9 & 11 & $57.9 \%$ \\
\hline Menthol* & 4 & 4 & 8 & $42.1 \%$ \\
\hline Carvones & 3 & 4 & 7 & $36.8 \%$ \\
\hline ButylatedHydroxytoluene* & 2 & 3 & 5 & $26.3 \%$ \\
\hline Butanoicacid,butylester & 3 & 2 & 5 & $26.3 \%$ \\
\hline 4-Terpineol & 3 & 2 & 5 & $26.3 \%$ \\
\hline Benzaldehyde* & 3 & 1 & 4 & $21.1 \%$ \\
\hline Decanal* & 1 & 3 & 4 & $21.1 \%$ \\
\hline Benzene,1-methyl-4-(1-methylethenyl)- & 0 & 4 & 4 & $21.1 \%$ \\
\hline 2-Pentanone & 2 & 2 & 4 & $21.1 \%$ \\
\hline 2-Isopropylbenzaldehyde & 1 & 2 & 3 & $15.8 \%$ \\
\hline 1,4-Cyclohexadiene,1-methyl-4-(1-methylethyl)- & 2 & 1 & 3 & $15.8 \%$ \\
\hline Benzene,1-methyl-4-(1-methylethyl)- & 1 & 2 & 3 & $15.8 \%$ \\
\hline 3-Heptanone* & 1 & 2 & 3 & $15.8 \%$ \\
\hline ValproicAcid & 1 & 1 & 2 & $10.5 \%$ \\
\hline 2-Methoxy-4-vinylphenol & 2 & 0 & 2 & $10.5 \%$ \\
\hline Phenol,4-methyl- (p-Cresol) & 2 & 0 & 2 & $10.5 \%$ \\
\hline Benzaldehyde,3,5-dimethyl- & 0 & 2 & 2 & $10.5 \%$ \\
\hline 1,3-Cyclohexadiene,1-methyl-4-(1-methylethyl)- & 2 & 0 & 2 & $10.5 \%$ \\
\hline p-Menth-1-en-3-one & 1 & 1 & 2 & $10.5 \%$ \\
\hline Cyclopropane,isothiocyanato- & 0 & 2 & 2 & $10.5 \%$ \\
\hline N,N-Diethylcarbanilide & 2 & 0 & 2 & $10.5 \%$ \\
\hline Pyrrole & 2 & 0 & 2 & $10.5 \%$ \\
\hline Nonanoicacid & 1 & 0 & 1 & $5.3 \%$ \\
\hline$\alpha$-Terpineol & 0 & 1 & 1 & $5.3 \%$ \\
\hline BenzylAlcohol* & 0 & 1 & 1 & $5.3 \%$ \\
\hline E-2-Tetradecen-1-ol & 1 & 0 & 1 & $5.3 \%$ \\
\hline Eucalyptol & 0 & 1 & 1 & $5.3 \%$ \\
\hline Eugenol & 1 & 0 & 1 & $5.3 \%$ \\
\hline Phenol,2-methoxy- & 0 & 1 & 1 & $5.3 \%$ \\
\hline 3,5-di-tert-Butyl-4-hydroxybenzaldehyde & 0 & 1 & 1 & $5.3 \%$ \\
\hline$\alpha$-Cubebene & 0 & 1 & 1 & $5.3 \%$ \\
\hline Benzene,-(1-formylethyl)- & 1 & 0 & 1 & $5.3 \%$ \\
\hline Benzene,(2-isothiocyanatoethyl)- & 1 & 0 & 1 & $5.3 \%$ \\
\hline Benzene,1-(1,5-dimethyl-4-hexenyl)-4-methyl- & 1 & 0 & 1 & $5.3 \%$ \\
\hline & & & & \\
\hline
\end{tabular}




\begin{tabular}{|l|lll|l|}
\hline Benzene,1,2-dichloro-* & 1 & 0 & 1 & $5.3 \%$ \\
\hline Benzene,1,3-dichloro- & 0 & 1 & 1 & $5.3 \%$ \\
\hline Benzene,1-methyl-3-(1-methylethyl)- & 1 & 0 & 1 & $5.3 \%$ \\
\hline Naphthalene,2,3-dimethyl- & 1 & 0 & 1 & $5.3 \%$ \\
\hline Naphthalene,2-methyl- & 1 & 0 & 1 & $5.3 \%$ \\
\hline Isobornylacetate & 0 & 1 & 1 & $5.3 \%$ \\
\hline 3-Nonen-2-one & 1 & 0 & 1 & $5.3 \%$ \\
\hline 4-Hepten-3-one,4-methyl- & 1 & 0 & 1 & $5.3 \%$ \\
\hline Acetone & 0 & 1 & 1 & $5.3 \%$ \\
\hline Benzophenone* & 1 & 0 & 1 & $5.3 \%$ \\
\hline 1-Butene,4-isothiocyanato- & 0 & 1 & 1 & $5.3 \%$ \\
\hline Dibutanoylmorphine & 0 & 1 & 1 & $5.3 \%$ \\
\hline
\end{tabular}

\subsubsection{Evaluation of Odor Profiles of Individuals with Major Depressive}

\section{Disorder}

Odor profiles of twenty individuals with diagnosed Major Depressive Disorder (MDD) were evaluated using SPME-GC/MS. Compounds were identified by spectral library (NIST) and/or by standard reference comparisons. Figure 90 demonstrates the frequency distribution of the different functional groups of VOCs that were extracted from hand odor, buccal swab, breath, blood, and urine across the 20 subjects. Aliphatics (alkanes, alkenes, cycloalkanes) and aldehydes constructed over half (64\%) of the hand odor VOCs. Nonanal and decanal were present in all subjects, followed by tetradecane, hexadecane, heptadecane, and octadecane being present in more than $80 \%$ of the subjects sampled (Table 53).

For buccal swab, esters constructed slightly less than a third of the extracted VOCs (27\%), followed by aromatics (17\%) and aliphatics (14\%). Hexanoic acid and decanal were present in $100 \%$ of sampled subjects. Other VOCs present in high frequency 
$(<70 \%)$ were 2-pentylfuran, (E)-6,10-dimethyl-5-9-undecadien-2-one, nonanoic acid, nonanoic acid ethyl ester, (E,E)-2,4-nonadienal, dodecanoicacid ethyl ester, and 2,2'diethyl-1,1'-biphenyl (Table 54).

Aromatic VOCs comprised approximately half of the breath odor of the MDD subject group at $49 \%$. The remaining was comprised of aliphatics (17\%), ketones $(9 \%)$, carboxylic acids (8\%), esters (7\%), aldehydes (6\%), and alcohols (4\%). The two compounds that were present in all subjects were both aldehydes, namely nonanal and decanal (Table 55). Other breath VOCs with high frequency of occurrence were xylenes (p-xylene and m-xylene), 1,2-dichlorobezene, and 2-ethyl-1-hexanol, and benzophenone.

For blood, two-thirds of the VOCs extracted from the MDD subjects' blood samples were aromatics (35\%) and aromatics (26\%). The remainder of the functional groups present in the headspace of blood odor VOCs were aldehydes (17\%), alcohols (10\%), ketones $(9 \%)$, and ethers (3\%). Hexadecane and 2,6-diisopropylnaphthalene were present in all MDD subjects. Other compounds with high frequency of occurrence were undecane, nonanal, dodecane, 2,4,6-trimethylbenzaldehyde, tridecane, tetradecane, hexane, diisopropylnaphthalene, and heptadecane (Table 56).

Lastly for urine of MDD subjects, ketones had the highest frequency (23\%) in the extracted VOCs of urine odor, followed by aldehydes (17\%), aromatics $(16 \%)$ and alcohols (16\%). Nitrogen-containing VOCs were also present in urine odor (9\%), which were not found in the other specimens from the MDD group. 4-Heptanone and cedrol were the only two compounds that were present in over $70 \%$ of the depressed subjects sampled (Table 57). 
Figure 90. Frequency distribution of functional groups of extracted VOCs in hand odor, buccal swab, breath, blood, and urine across twenty individuals diagnosed with Major Depressive Disorder
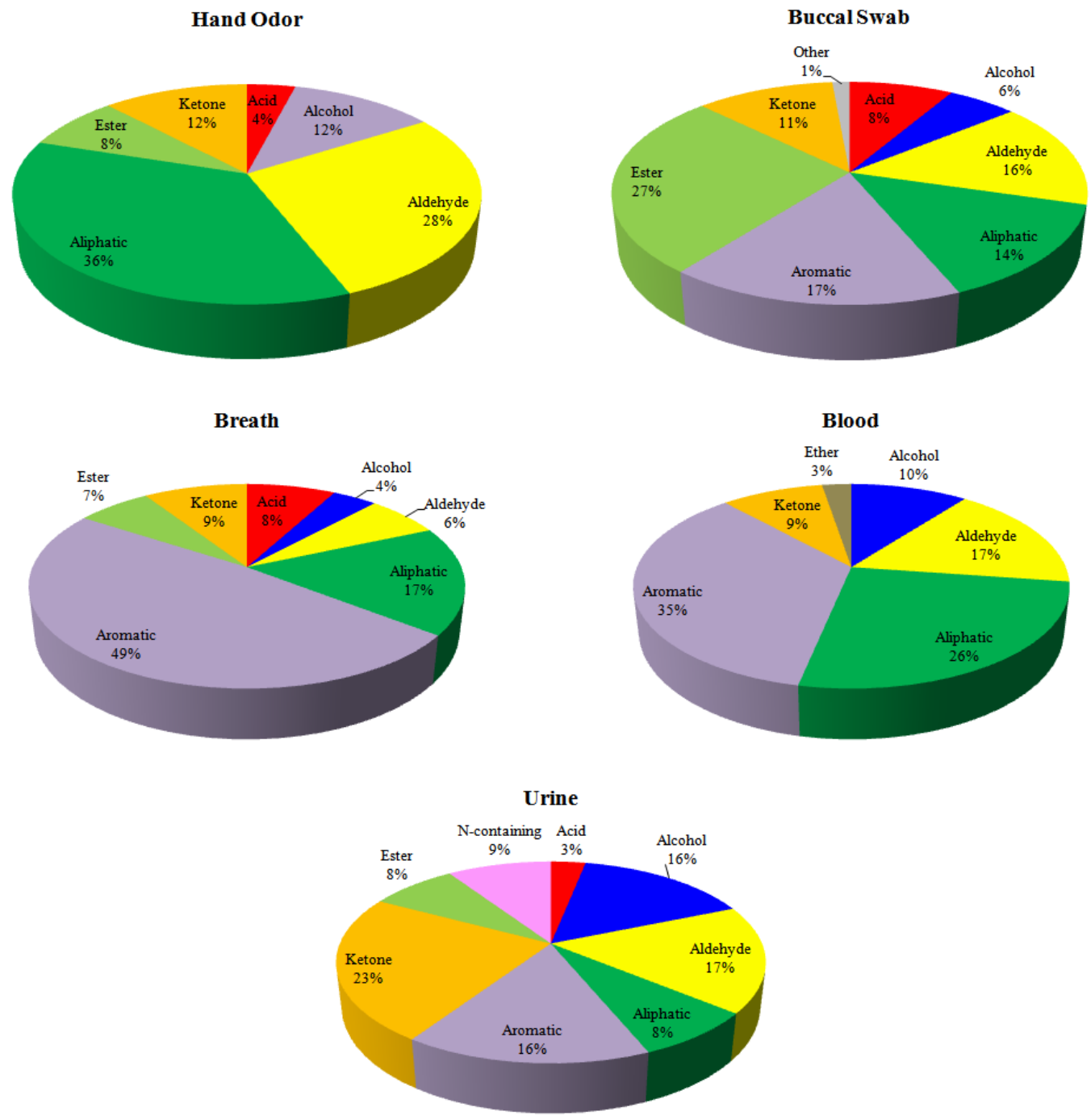
Table 53. Identified VOCs in hand odor of twenty individuals with diagnosed Major Depressive Disorder ranked by frequency of occurrence (Note: * denotes compound was verified by reference standard)

\begin{tabular}{|l|ccc|c|}
\hline \multicolumn{1}{|c|}{ Compound Name } & \multicolumn{3}{|c|}{ Frequency } & Occurrence \\
& Female & Male & Total & $(\%)$ \\
\hline Nonanal* & 10 & 10 & 20 & 100 \\
\hline Decanal* & 10 & 10 & 20 & 100 \\
\hline Tetradecane* & 10 & 9 & 19 & 95.0 \\
\hline Hexadecane* & 9 & 9 & 18 & 90.0 \\
\hline Heptadecan** & 9 & 9 & 18 & 90.0 \\
\hline Octadecane & 8 & 9 & 17 & 85.0 \\
\hline Undecane* & 6 & 6 & 12 & 60.0 \\
\hline Tridecane* & 6 & 6 & 12 & 60.0 \\
\hline Undecanal* & 6 & 6 & 12 & 60.0 \\
\hline 5,9-Undecadien-2-one-6,10-dimethyl-(E)-* & 6 & 6 & 12 & 60.0 \\
\hline Dodecane* & 2 & 5 & 7 & 35.0 \\
\hline Benzyl Alcohol* & 3 & 2 & 5 & 25.0 \\
\hline Dodecanal* & 2 & 2 & 4 & 20.0 \\
\hline 1-Hexanol,2-ethyl- & 3 & 0 & 3 & 15.0 \\
\hline 1,6-Octadien-3-ol,3,7-dimethyl-* & 2 & 1 & 3 & 15.0 \\
\hline Dodecanoicacid,methylester & 0 & 3 & 3 & 15.0 \\
\hline Benzaldehyde* & 2 & 0 & 2 & 10.0 \\
\hline Acetophenone* & 1 & 1 & 2 & 10.0 \\
\hline Heptanal & 1 & 0 & 1 & 5.0 \\
\hline 5-Hepten-2-one,6-methyl-* & 0 & 1 & 1 & 5.0 \\
\hline Hexanoicacid* & 1 & 0 & 1 & 5.0 \\
\hline Octanoicacid,methylester* & 1 & 0 & 1 & 5.0 \\
\hline 2-Nonenal, (E)-* & 0 & 1 & 1 & 5.0 \\
\hline 1-Pentadecene* & 0 & 1 & 1 & 5.0 \\
\hline Pentadecane* & 1 & 0 & 1 & 5.0 \\
\hline
\end{tabular}


Table 54. Identified VOCs in buccal swab of twenty individuals with diagnosed Major Depressive Disorder ranked by frequency of occurrence (Note: *denotes compound was verified by reference standard)

\begin{tabular}{|c|c|c|c|c|}
\hline \multirow[t]{2}{*}{ Compound Name } & \multicolumn{3}{|c|}{ Frequency } & \multirow{2}{*}{$\begin{array}{c}\text { Occurrence } \\
\text { (\%) }\end{array}$} \\
\hline & Female & Male & Total & \\
\hline Hexanoicacid* & 10 & 10 & 20 & 100 \\
\hline Decanal* & 10 & 10 & 20 & 100 \\
\hline Furan,2-pentyl-* & 9 & 10 & 19 & 95.0 \\
\hline 5,9-Undecadien-2-one,6,10-dimethyl,(E)-* & 10 & 9 & 19 & 95.0 \\
\hline Nonanoicacid & 8 & 10 & 18 & 90.0 \\
\hline Nonanoicacid,ethylester* & 9 & 9 & 18 & 90.0 \\
\hline 2,4-Nonadienal, (E,E)-v & 6 & 10 & 16 & 80.0 \\
\hline Dodecanoicacid,ethylester & 6 & 10 & 16 & 80.0 \\
\hline 1,1'-Biphenyl,2,2'-diethyl- & 5 & 10 & 15 & 75.0 \\
\hline 2-Nonenal, (E)-* & 5 & 8 & 13 & 65.0 \\
\hline Hexadecanoicacid,ethylester & 5 & 8 & 13 & 65.0 \\
\hline 1-Tetradecene* & 7 & 5 & 12 & 60.0 \\
\hline Tetradecanoicacid,ethylester & 4 & 8 & 12 & 60.0 \\
\hline 6-Dodecanone & 7 & 4 & 11 & 55.0 \\
\hline 6-Methyl-3,5-heptadiene-2-one & 3 & 7 & 10 & 50.0 \\
\hline 1-Dodecene* & 3 & 6 & 9 & 45.0 \\
\hline Octanoicacid,ethylester* & 6 & 3 & 9 & 45.0 \\
\hline Heptanoicacid* & 2 & 6 & 8 & 40.0 \\
\hline 1-Decene* & 4 & 4 & 8 & 40.0 \\
\hline 1,1'-Biphenyl, 4-methyl- & 2 & 6 & 8 & 40.0 \\
\hline Pentanoicacid* & 1 & 6 & 7 & 35.0 \\
\hline Tetradecane* & 1 & 6 & 7 & 35.0 \\
\hline Hexanoicacid,ethylester & 2 & 4 & 6 & 30.0 \\
\hline Heptanoicacid,ethylester* & 3 & 3 & 6 & 30.0 \\
\hline Hexanoicacid,pentylester & 4 & 2 & 6 & 30.0 \\
\hline Naphthalene,1-methyl-* & 3 & 3 & 6 & 30.0 \\
\hline 2-Tetradecene, (E)- & 1 & 5 & 6 & 30.0 \\
\hline Hexanal* & 3 & 2 & 5 & 25.0 \\
\hline Benzaldehyde* & 2 & 3 & 5 & 25.0 \\
\hline Decanoicacid,ethylester* & 2 & 3 & 5 & 25.0 \\
\hline Dodecanoicacid* & 0 & 5 & 5 & 25.0 \\
\hline Pentadecanoicacid,ethylester & 2 & 3 & 5 & 25.0 \\
\hline E-11-Hexadecenoicacid,ethylester & 1 & 4 & 5 & 25.0 \\
\hline Phenol* & 1 & 3 & 4 & 20.0 \\
\hline
\end{tabular}




\begin{tabular}{|c|c|c|c|c|}
\hline Acetophenone* & 2 & 2 & 4 & 20.0 \\
\hline Naphthalene,2-methyl- & 2 & 2 & 4 & 20.0 \\
\hline Naphthalene,2,7-dimethyl- & 2 & 2 & 4 & 20.0 \\
\hline Hexadecane* & 3 & 1 & 4 & 20.0 \\
\hline Furfural* & 0 & 3 & 3 & 15.0 \\
\hline 1-Hexanol* & 0 & 3 & 3 & 15.0 \\
\hline 2-Octenal, (E)-* & 3 & 0 & 3 & 15.0 \\
\hline 3-Nonen-2-one & 2 & 1 & 3 & 15.0 \\
\hline 2-Octenoicacid & 1 & 2 & 3 & 15.0 \\
\hline Benzene, 1,2,4,5-tetramethyl- & 1 & 2 & 3 & 15.0 \\
\hline p-Benzoquinone & 2 & 1 & 3 & 15.0 \\
\hline Galaxolide & 3 & 0 & 3 & 15.0 \\
\hline Homomenthylsalicylate & 3 & 0 & 3 & 15.0 \\
\hline Benzothiazole & 1 & 1 & 2 & 10.0 \\
\hline 2(3H)-Furanone, 5-ethyldihydro- & 2 & 0 & 2 & 10.0 \\
\hline 2(3H)-Furanone, dihydro-5-pentyl- & 1 & 1 & 2 & 10.0 \\
\hline Vanillin & 2 & 0 & 2 & 10.0 \\
\hline DiethylPhthalate & 2 & 0 & 2 & 10.0 \\
\hline Benzene,1-methyl-2-[(4-methylphenyl)methyl]- & 1 & 1 & 2 & 10.0 \\
\hline Dibutanoylmorphine & 0 & 2 & 2 & 10.0 \\
\hline IsopropylPalmitate & 2 & 0 & 2 & 10.0 \\
\hline Linalool Oxide & 1 & 0 & 1 & 5.0 \\
\hline 1-Octanol* & 1 & 0 & 1 & 5.0 \\
\hline Benzene,1-ethyl-2,3-dimethyl- & 0 & 1 & 1 & 5.0 \\
\hline Benzoicacid,ethylester & 0 & 1 & 1 & 5.0 \\
\hline 2-Decenal, (E)- & 1 & 0 & 1 & 5.0 \\
\hline Naphthalene,2,6-dimethyl- & 1 & 0 & 1 & 5.0 \\
\hline Dodecanal* & 1 & 0 & 1 & 5.0 \\
\hline Octadecanal & 1 & 0 & 1 & 5.0 \\
\hline 3,7-Dimethyl-octa-1,6-diene & 1 & 0 & 1 & 5.0 \\
\hline Caryophyllene* & 1 & 0 & 1 & 5.0 \\
\hline$\beta$-Cadinene & 1 & 0 & 1 & 5.0 \\
\hline ButylatedHydroxytoluene & 1 & 0 & 1 & 5.0 \\
\hline Octadecane & 1 & 0 & 1 & 5.0 \\
\hline 1-Hexadecene & 1 & 0 & 1 & 5.0 \\
\hline Hexadecanoicacid,methylester & 1 & 0 & 1 & 5.0 \\
\hline 11-Octadecenoicacid,methylester & 1 & 0 & 1 & 5.0 \\
\hline
\end{tabular}


Table 55. Identified VOCs in breath of twenty individuals with diagnosed Major Depressive Disorder ranked by frequency of occurrence (Note: *denotes compound was verified by reference standard)

\begin{tabular}{|c|c|c|c|c|}
\hline \multirow{2}{*}{ Compound Name } & \multicolumn{3}{|c|}{ Frequency } & \multirow{2}{*}{$\begin{array}{c}\text { Occurrence } \\
(\%)\end{array}$} \\
\hline & Female & Male & Total & \\
\hline Nonanal* & 10 & 10 & 20 & 100 \\
\hline Decanal* & 10 & 10 & 20 & 100 \\
\hline Xylenes* & 7 & 9 & 16 & 80 \\
\hline Benzene,1,2-dichloro-* & 6 & 10 & 16 & 80 \\
\hline 1-Hexanol,2-ethyl-* & 7 & 8 & 15 & 75 \\
\hline Benzophenone* & 6 & 8 & 14 & 70 \\
\hline Dodecane* & 5 & 8 & 13 & 65 \\
\hline Tetradecane* & 5 & 8 & 13 & 65 \\
\hline Styrene* & 5 & 7 & 12 & 60 \\
\hline Phenol* & 5 & 7 & 12 & 60 \\
\hline Undecane* & 4 & 8 & 12 & 60 \\
\hline Tridecane* & 4 & 8 & 12 & 60 \\
\hline ButylatedHydroxytoluene* & 6 & 6 & 12 & 60 \\
\hline Toluene & 3 & 4 & 7 & 35 \\
\hline Benzene,1,3,5-trimethyl- & 2 & 5 & 7 & 35 \\
\hline Benzene,1-methyl-3-propyl- & 1 & 6 & 7 & 35 \\
\hline Benzene,1,2,3-trimethyl- & 1 & 4 & 5 & 25 \\
\hline Benzene,4-ethyl-1,2-dimethyl- & 2 & 3 & 5 & 25 \\
\hline Benzene,1-ethyl-2,4-dimethyl- & 1 & 4 & 5 & 25 \\
\hline Undecanal* & 1 & 4 & 5 & 25 \\
\hline p-Benzoquinone & 0 & 5 & 5 & 25 \\
\hline Benzene, 1-ethyl-2-methyl- & 1 & 3 & 4 & 20 \\
\hline Nonanoicacid & 2 & 2 & 4 & 20 \\
\hline Benzene, 1,2,4,5-tetramethyl-* & 2 & 2 & 4 & 20 \\
\hline Naphthalene,2-methyl- & 1 & 3 & 4 & 20 \\
\hline 5,9-Undecadien-2-one, 6,10-dimethyl-(E)-* & 1 & 3 & 4 & 20 \\
\hline Naphthalene* & 1 & 3 & 4 & 20 \\
\hline Limonene* & 1 & 2 & 3 & 15 \\
\hline Benzene,1,2,3,4-tetramethyl- & 0 & 3 & 3 & 15 \\
\hline Dodecanal* & 0 & 3 & 3 & 15 \\
\hline 1,1'-Biphenyl,4-methyl- & 2 & 1 & 3 & 15 \\
\hline 5-Hepten-2-one,6-methyl-* & 1 & 1 & 2 & 10 \\
\hline Benzene,diethyl-* & 0 & 2 & 2 & 10 \\
\hline
\end{tabular}




\begin{tabular}{|c|c|c|c|c|}
\hline Acetophenone* & 1 & 1 & 2 & 10 \\
\hline Benzene,1-methyl-2-(1-methylethyl)- & 0 & 2 & 2 & 10 \\
\hline Benzene,1-ethyl-2,3-dimethyl- & 1 & 1 & 2 & 10 \\
\hline Benzene,1,2,3,5-tetramethyl- & 0 & 2 & 2 & 10 \\
\hline Triacetin & 0 & 2 & 2 & 10 \\
\hline DiethylPhthalate & 1 & 1 & 2 & 10 \\
\hline Acetone & 1 & 0 & 1 & 5 \\
\hline Aceticacid & 0 & 1 & 1 & 5 \\
\hline Hexanal* & 0 & 1 & 1 & 5 \\
\hline Tetrachloroethylene & 1 & 0 & 1 & 5 \\
\hline 2-Propanol,1-propoxy- & 0 & 1 & 1 & 5 \\
\hline 3-Heptanone* & 0 & 1 & 1 & 5 \\
\hline Benzene,(1-methylethyl)- & 0 & 1 & 1 & 5 \\
\hline Benzene,propyl-* & 0 & 1 & 1 & 5 \\
\hline Benzene,1-ethyl-3-methyl- & 0 & 1 & 1 & 5 \\
\hline Benzene,1,2,4-trimethyl- & 0 & 1 & 1 & 5 \\
\hline Benzene,1,4-dichloro- & 1 & 0 & 1 & 5 \\
\hline Benzene,2-ethyl-1,4-dimethyl- & 0 & 1 & 1 & 5 \\
\hline Decane,2-methyl- & 1 & 0 & 1 & 5 \\
\hline Benzene, 1-methyl-4-propyl- & 0 & 1 & 1 & 5 \\
\hline Benzene,2-ethyl-1,3-dimethyl- & 0 & 1 & 1 & 5 \\
\hline Benzene,1-methyl-4-(1-methylethyl)- & 0 & 1 & 1 & 5 \\
\hline 1-Dodecene* & 0 & 1 & 1 & 5 \\
\hline Benzene,1-methyl-4-(1-methylpropyl)- & 0 & 1 & 1 & 5 \\
\hline Undecane,2,6-dimethyl- & 1 & 0 & 1 & 5 \\
\hline 2-Dodecene, $(Z)$ - & 0 & 1 & 1 & 5 \\
\hline 3-Dodecene, $(Z)$ - & 0 & 1 & 1 & 5 \\
\hline Benzene,pentamethyl- & 0 & 1 & 1 & 5 \\
\hline Indole & 1 & 0 & 1 & 5 \\
\hline n-Decanoicacid* & 0 & 1 & 1 & 5 \\
\hline Hexanedioicacid,bis(1-methylethyl) ester & 0 & 1 & 1 & 5 \\
\hline Dodecanoicacid* & 0 & 1 & 1 & 5 \\
\hline Hexadecane* & 0 & 1 & 1 & 5 \\
\hline Methyldihydrojasmonate & 1 & 0 & 1 & 5 \\
\hline Diisopropylnaphthalene & 0 & 1 & 1 & 5 \\
\hline 2,6-Diisopropylnaphthalene* & 0 & 1 & 1 & 5 \\
\hline Tetradecanoicacid & 0 & 1 & 1 & 5 \\
\hline Galaxolide & 1 & 0 & 1 & 5 \\
\hline Homomenthylsalicylate & 1 & 0 & 1 & 5 \\
\hline n-Hexadecanoicacid* & 0 & 1 & 1 & 5 \\
\hline
\end{tabular}


Table 56. Identified VOCs in blood odor of twenty individuals with diagnosed Major Depressive Disorder ranked by frequency of occurrence (Note: *denotes compound was verified by reference standard)

\begin{tabular}{|c|c|c|c|c|}
\hline \multirow[t]{2}{*}{ Compound Name } & \multicolumn{3}{|c|}{ Frequency } & \multirow{2}{*}{$\begin{array}{c}\text { Occurrence } \\
(\%)\end{array}$} \\
\hline & Female & Male & Total & \\
\hline Hexadecane* & 10 & 10 & 20 & 100 \\
\hline 2,6-Diisopropylnaphthalene* & 10 & 10 & 20 & 100 \\
\hline Undecane* & 9 & 10 & 19 & 95.0 \\
\hline Nonanal* & 9 & 10 & 19 & 95.0 \\
\hline Dodecane* & 9 & 10 & 19 & 95.0 \\
\hline Benzenaldehyde,2,4,6-trimethyl- & 9 & 10 & 19 & 95.0 \\
\hline Tridecane* & 9 & 10 & 19 & 95.0 \\
\hline Tetradecane* & 9 & 9 & 18 & 90.0 \\
\hline Hexanal & 9 & 7 & 16 & 80.0 \\
\hline Diisopropylnaphthalene & 7 & 7 & 14 & 70.0 \\
\hline Heptadecane* & 5 & 9 & 14 & 70.0 \\
\hline Benzyl Alcohol* & 6 & 5 & 11 & 55.0 \\
\hline Longifolene & 2 & 7 & 9 & 45.0 \\
\hline Benzene,4-ethyl-1,2-dimethyl- & 1 & 7 & 8 & 40.0 \\
\hline Benzene,1,2,4,5-tetramethyl- & 2 & 6 & 8 & 40.0 \\
\hline 5,9-Undecadien-2-one,6,10-dimethyl,(E)- & 2 & 6 & 8 & 40.0 \\
\hline Octadecane & 2 & 6 & 8 & 40.0 \\
\hline Cyclohexanone* & 4 & 3 & 7 & 35.0 \\
\hline Furan,2-pentyl-* & 4 & 3 & 7 & 35.0 \\
\hline Benzene,1,3,5-trimethyl- & 2 & 5 & 7 & 35.0 \\
\hline Benzene,1,2,3,5-tetramethyl- & 3 & 4 & 7 & 35.0 \\
\hline 1-Decene* & 3 & 4 & 7 & 35.0 \\
\hline 2-Dodecene,(Z)- & 2 & 5 & 7 & 35.0 \\
\hline Decanal* & 3 & 4 & 7 & 35.0 \\
\hline 2-Undecanone & 3 & 4 & 7 & 35.0 \\
\hline Furan,2-butyltetrahydro- & 3 & 4 & 7 & 35.0 \\
\hline Naphthalene,2-methyl- & 2 & 4 & 6 & 30.0 \\
\hline 2-Heptanone* & 1 & 4 & 5 & 25.0 \\
\hline Heptanal* & 3 & 2 & 5 & 25.0 \\
\hline 1-Octen-3-ol* & 1 & 4 & 5 & 25.0 \\
\hline 1-Dodecene* & 3 & 2 & 5 & 25.0 \\
\hline 1-Pentanol & 1 & 3 & 4 & 20.0 \\
\hline p-Xylene* & 1 & 3 & 4 & 20.0 \\
\hline Benzene,1-ethyl-2,4-dimethyl- & 0 & 4 & 4 & 20.0 \\
\hline Naphthalene,1-methyl-* & 2 & 2 & 4 & 20.0 \\
\hline
\end{tabular}




\begin{tabular}{|c|c|c|c|c|}
\hline Benzene,1-ethyl-2-methyl- & 1 & 2 & 3 & 15 \\
\hline Benzene,1,2,3-trimethyl- & 1 & 2 & 3 & 15 \\
\hline Benzene,1,2-dichloro-* & 2 & 1 & 3 & 15 \\
\hline 4-Cyanocyclohexene & 1 & 2 & 3 & 15 \\
\hline Benzene,2-ethyl-1,4-dimethyl- & 3 & 0 & 3 & 15 \\
\hline Benzene,1,2,3,4-tetramethyl- & 2 & 1 & 3 & 15 \\
\hline Decane,1-chloro- & 1 & 2 & 3 & 15 \\
\hline 1-Dodecanol* & 0 & 3 & 3 & 15 \\
\hline Naphthalene* & 0 & 3 & 3 & 15 \\
\hline 2-Propanol,1-propoxy- & 0 & 2 & 2 & 10 \\
\hline Octanal & 0 & 2 & 2 & 10 \\
\hline Benzenemethanol, $\alpha, \alpha$-dimethyl- & 2 & 0 & 2 & 10 \\
\hline Benzaldehyde,4-(methylthio)- & 2 & 0 & 2 & 10 \\
\hline 2,4-Diphenyl-4-methyl-1(E)-pentene & 1 & 1 & 2 & 10 \\
\hline Tetrachloroethylene & 1 & 0 & 1 & 5 \\
\hline 3-Heptanone* & 0 & 1 & 1 & 5 \\
\hline Nonane* & 1 & 0 & 1 & 5 \\
\hline Ethanol,2-butoxy- & 1 & 0 & 1 & 5 \\
\hline Benzaldehyde* & 1 & 0 & 1 & 5 \\
\hline Benzene,1-ethyl-3-methyl- & 0 & 1 & 1 & 5 \\
\hline 2,5-Octanedione & 1 & 0 & 1 & 5 \\
\hline Phenol* & 1 & 0 & 1 & 5 \\
\hline Decane & 0 & 1 & 1 & 5 \\
\hline Benzene,1,4-dichloro- & 1 & 0 & 1 & 5 \\
\hline Benzaldehyde,2-hydroxy- & 1 & 0 & 1 & 5 \\
\hline Benzene,1-methyl-3-propyl- & 0 & 1 & 1 & 5 \\
\hline 3-Isopropylbenzaldehyde & 1 & 0 & 1 & 5 \\
\hline 3-Dodecene,(Z)- & 0 & 1 & 1 & 5 \\
\hline 1-Cyclohexene-1-carboxaldehyde,2,6,6-trimethyl- & 1 & 0 & 1 & 5 \\
\hline Benzaldehyde,ethyl- & 1 & 0 & 1 & 5 \\
\hline 1-Phenyl-2-butanone & 1 & 0 & 1 & 5 \\
\hline Naphthalene,1,6-dimethyl- & 1 & 0 & 1 & 5 \\
\hline Naphthalene,2,7-dimethyl- & 0 & 1 & 1 & 5 \\
\hline Naphthalene,2,6-dimethyl- & 1 & 0 & 1 & 5 \\
\hline Naphthalene,2,3-dimethyl- & 1 & 0 & 1 & 5 \\
\hline Cyclododecane & 1 & 0 & 1 & 5 \\
\hline 1,1'-Biphenyl,4-methyl- & 0 & 1 & 1 & 5 \\
\hline 1,1'-Biphenyl,2-methyl- & 0 & 1 & 1 & 5 \\
\hline Z-8-Hexadecene & 0 & 1 & 1 & 5 \\
\hline
\end{tabular}


Table 57. Identified VOCs in urine odor of twenty individuals with diagnosed Major Depressive Disorder ranked by frequency of occurrence (Note: *denotes compound was verified by reference standard)

\begin{tabular}{|l|ccc|c|}
\hline \multicolumn{1}{|c|}{ Compound Name } & \multicolumn{3}{|c|}{ Frequency } & Occurrence \\
& Female & Male & Total & $(\%)$ \\
\hline 4-Heptanone* & 10 & 9 & 19 & 95.0 \\
\hline Cedrol & 6 & 8 & 14 & 70.0 \\
\hline Nonanal* & 5 & 7 & 12 & 60.0 \\
\hline Carvones & 6 & 5 & 11 & 55.0 \\
\hline 2-Pentanone & 2 & 3 & 5 & 25.0 \\
\hline Phenol,4-methyl- (p-Cresol)* & 3 & 2 & 5 & 25.0 \\
\hline Benzene,1-methyl-4-(1-methylethenyl)- & 3 & 2 & 5 & 25.0 \\
\hline 2-Isopropylbenzaldehyde & 1 & 4 & 5 & 25.0 \\
\hline Pyrrole & 2 & 2 & 4 & 20.0 \\
\hline Menthol* & 5 & 5 & 10 & 20.0 \\
\hline Decanal* & 2 & 2 & 4 & 20.0 \\
\hline Benzaldehyde,3,5-dimethyl- & 2 & 2 & 4 & 20.0 \\
\hline 4-Nonylphenol & 3 & 1 & 4 & 20.0 \\
\hline Benzene,1,4-dichloro- & 2 & 1 & 3 & 15.0 \\
\hline ++-.-4-Acetyl-1-methylcyclohexene & 1 & 2 & 3 & 15.0 \\
\hline p-Menthan-3-one & 3 & 0 & 3 & 15.0 \\
\hline 4-Terpineol & 1 & 2 & 3 & 15.0 \\
\hline ButylatedHydroxytoluene* & 0 & 3 & 3 & 15.0 \\
\hline 3-Heptanone* & 0 & 2 & 2 & 10.0 \\
\hline Benzaldehyde* & 0 & 2 & 2 & 10.0 \\
\hline Benzene,1,2-dichloro-* & 2 & 0 & 2 & 10.0 \\
\hline Benzene,1-methyl-3-(1-methylethyl)- & 1 & 1 & 2 & 10.0 \\
\hline Benzeneacetaldehyde,.alpha.-methyl- & 2 & 0 & 2 & 10.0 \\
\hline ValproicAcid & 0 & 2 & 2 & 10.0 \\
\hline$\alpha$-Terpineol & 2 & 0 & 2 & 10.0 \\
\hline p-Chloroaniline & 0 & 2 & 2 & 10.0 \\
\hline 3-Cyclohexen-1-one,2-isopropyl-5-methyl- & 1 & 1 & 2 & 10.0 \\
\hline p-Menth-1-en-3-one & 1 & 1 & 2 & 10.0 \\
\hline Dodecanoicacid* & 1 & 1 & 2 & 10.0 \\
\hline Benzophenone* & 2 & 0 & 2 & 10.0 \\
\hline Phenol,4-(1,1,3,3-tetramethylbutyl)-* & 1 & 1 & 2 & 10.0 \\
\hline Toluene & 0 & 1 & 1 & 5.0 \\
\hline$\alpha$-Terpinene & 1 & 0 & 1 & 5.0 \\
\hline Benzene,1,3-dichloro- & 1 & 0 & 1 & 5.0 \\
\hline Benzene,4-ethyl-1,2-dimethyl- & 0 & 1 & 1 & 5.0 \\
\hline & & & & \\
\hline
\end{tabular}




\begin{tabular}{|c|c|c|c|c|}
\hline Benzene,1-methyl-4-(1-methylethyl)- & 0 & 1 & 1 & 5.0 \\
\hline Benzeneacetaldehyde & 1 & 0 & 1 & 5.0 \\
\hline 3-Octen-2-one & 0 & 1 & 1 & 5.0 \\
\hline y-Terpinene & 1 & 0 & 1 & 5.0 \\
\hline Benzene,-(1-formylethyl)- & 0 & 1 & 1 & 5.0 \\
\hline 1,6-Octadien-3-ol,3,7-dimethyl-* & 1 & 0 & 1 & 5.0 \\
\hline Benzaldehyde,3-chloro- & 1 & 0 & 1 & 5.0 \\
\hline Benzaldehyde,4-chloro- & 0 & 1 & 1 & 5.0 \\
\hline Ethanone,1-(2-hydroxyphenyl)- & 1 & 0 & 1 & 5.0 \\
\hline Benzenamine,4-chloro-2-(trifluoromethyl)- & 1 & 0 & 1 & 5.0 \\
\hline Phenol,2,5-dichloro- & 1 & 0 & 1 & 5.0 \\
\hline Benzenamine,2,6-dimethyl- & 0 & 1 & 1 & 5.0 \\
\hline Limonene & 0 & 1 & 1 & 5.0 \\
\hline Dihydrocarvone & 1 & 0 & 1 & 5.0 \\
\hline 2,4-Cycloheptadien-1-one,2,6,6-trimethyl- & 0 & 1 & 1 & 5.0 \\
\hline Isobornylacetate & 0 & 1 & 1 & 5.0 \\
\hline 2-Propenal,3-phenyl- & 1 & 0 & 1 & 5.0 \\
\hline 2-Methoxy-4-vinylphenol & 0 & 1 & 1 & 5.0 \\
\hline Ethanone,1-(4-chlorophenyl)- & 1 & 0 & 1 & 5.0 \\
\hline Benzoicacid,2-amino-,methylester & 1 & 0 & 1 & 5.0 \\
\hline Damascenone & 0 & 1 & 1 & 5.0 \\
\hline Butanoicacid,butylester & 1 & 0 & 1 & 5.0 \\
\hline 5,9-Undecadien-2-one,6,10-dimethyl,(E)-* & 1 & 0 & 1 & 5.0 \\
\hline Dibutanoylmorphine & 0 & 1 & 1 & 5.0 \\
\hline Cyclotetradecane & 0 & 1 & 1 & 5.0 \\
\hline IsopropylPalmitate & 1 & 0 & 1 & 5.0 \\
\hline
\end{tabular}




\subsubsection{Comparison of the Volatile Organic Compounds Present Above Collected}

Odor Samples from Five Biological Specimens across Populations

As investigated in sections 3.6.4.1.2.1 and 3.6.4.1.2.2, Type I and Type II errors were calculated for each of the five biological specimens for the type 2 diabetic and MDD subject groups. Summary of the total number of errors and the percentage of mismatching of individuals are shown in Table 58 through

Table 60 for healthy, type 2 diabetic and MDD subject groups, respectively. For all three subject groups, the number of total errors was highest for hand odor. However, this can be attributed to the fact that for hand odor, the volatile compounds categorized as "human scent compounds" have previously been determined and therefore the human compound database for hand odor holds a much smaller number of VOCs compared to the other specimens that were newly investigated in the present study. All extracted volatile compounds were included in the data analysis for the remaining four biological specimens investigated in the present study, resulting in a greater array of compounds and higher variations in the relative peak area ratios of compounds present in the headspace of each individual's specimen odor profiles, thereby lowering the Type I and Type II error occurrences.

The low percentages in the mismatch occurrences further support the hypothesis that VOCs emanations are different among individuals for the five biological specimens and can be used as distinguishing profiles among individuals. As previously mentioned in section 3.6.4.1.2.1 the match/no-match cut-off threshold is recommended to be kept at least at 0.8 correlation, and wherever possible, a cut-off threshold of 0.9 should be 
utilized. Preliminary comparison of the three subject groups at 0.9 correlation threshold as shown in Table 61 demonstrates that having a medical condition did not greatly affect in the mismatch occurrences for any of the biological specimens. Percentages of mismatch occurrences were almost identical across the three study groups for hand odor and buccal swabs. For breath and blood, misidentification occurrences were slightly higher in the diabetic and depressed study groups than in the healthy control group. For urine, diabetics had the lowest percentage of misidentification occurrence, while the depressed study group had the highest percentage of misidentification occurrence, although all are still under $1 \%$. There were no particular patterns in the increase or decrease in the percentage of misidentification of individuals relative to their physiological or psychological health statuses.

Thus far the present study has demonstrated that odor profiles from human biological specimens other than hand odor can differentiate individuals with high certainty. Specimen odor profiles from individuals with the same diagnosed medical condition were also found to achieve good differentiation which is important for forensic consideration. Because certain diseases are known to be associated with certain odors, it is expected that the odor profiles of individuals who are diagnosed with the same disease or disorder to have similar VOC profiles with one another than individuals who do not have the same disease or disorder. From the medical perspective this could be important as it could lead to identifying key volatile biomarkers for certain medical conditions. From the legal/forensic perspective, the ability to identify individuals with a given medical condition is potentially significant because the possibility of mismatching two individuals 
with the same medical condition becomes unlikely. This leads to less chance of false identification of suspects, for example by canine detection, on the basis of the two individuals having the same medical condition. With the sample size of the present study, having a diagnosed medical/psychological condition did not affect the misidentification rate of individuals. If an argument was made where suspect mismatching occurred because of the individual's health statuses, results from this preliminary study can be used as a counterargument on the basis that a high distinguish-ability of specimen VOC profiles was still achieved regardless of the subjects' health statuses.

Further studies with larger sample sizes for the three subject groups are needed to obtain a more definitive indication on whether physiological or psychological health statuses have an effect on the misidentification occurrences of individuals. Future studies should include individuals with extreme conditions from the diabetic and depressed subject groups. For the diabetic population, it would be desirable to collect samples from uncontrolled (by medication or diet) diabetic patients and perhaps even diabetic patients recently suffered from diabetic ketoacidosis. Similarly for the depressed population, sample collection from patients who are in severe depressed state at the time of sampling is desirable. The present study was not able to include such subjects as access to diabetic inpatients admitted as a result of uncontrolled diabetes was prohibited, and the severely depressed patients were unresponsive and unable to give consent for sample collection during the course of the study. 
Table 58. Type I and Type II errors for five biological specimens amongst healthy individuals

\begin{tabular}{|l|cc|cc|cc|cc|cc|}
\hline & \multicolumn{2}{|c|}{ Hand Odor } & \multicolumn{2}{|c|}{ Buccal Swab } & \multicolumn{2}{c|}{ Breath } & \multicolumn{2}{c|}{ Blood } & \multicolumn{2}{c|}{ Urine } \\
\hline Threshold & 0.9 & 0.8 & 0.9 & 0.8 & 0.9 & 0.8 & 0.9 & 0.8 & 0.9 & 0.8 \\
\hline \hline Type I & 3 & 13 & 0 & 1 & 0 & 1 & 0 & 0 & 0 & 0 \\
Type II & 15 & 9 & 9 & 4 & 11 & 4 & 12 & 6 & 8 & 3 \\
Total Error & 18 & 22 & 9 & 5 & 11 & 5 & 12 & 6 & 8 & 3 \\
\hline \% Mismatch & 0.89 & 1.09 & 0.45 & 0.25 & 0.58 & 0.26 & 0.63 & 0.32 & 0.36 & 0.14 \\
\hline
\end{tabular}

Table 59. Type I and Type II errors for five biological specimens amongst type 2 diabetic patients

\begin{tabular}{|l|cc|cc|cc|cc|cc|}
\hline & \multicolumn{2}{|c|}{ Hand Odor } & \multicolumn{2}{|c|}{ Buccal Swab } & \multicolumn{2}{c|}{ Breath } & \multicolumn{2}{c|}{ Blood } & \multicolumn{2}{c|}{ Urine } \\
\hline Threshold & 0.9 & 0.8 & 0.9 & 0.8 & 0.9 & 0.8 & 0.9 & 0.8 & 0.9 & 0.8 \\
\hline \hline Type I & 7 & 39 & 0 & 0 & 0 & 0 & 0 & 0 & 0 & 0 \\
Type II & 7 & 4 & 7 & 2 & 8 & 3 & 7 & 4 & 2 & 0 \\
Total Error & 14 & 43 & 7 & 2 & 8 & 3 & 7 & 4 & 2 & 0 \\
\hline \% Mismatch & 0.88 & 2.69 & 0.44 & 0.13 & 1.14 & 0.43 & 1.00 & 0.57 & 0.13 & 0 \\
\hline
\end{tabular}

Table 60. Type I and Type II errors for five biological specimens amongst individuals diagnosed with Major Depressive Disorder

\begin{tabular}{|l|cc|cc|cc|cc|cc|}
\hline & \multicolumn{2}{|c|}{ Hand Odor } & \multicolumn{2}{|c|}{ Buccal Swab } & \multicolumn{2}{c|}{ Breath } & \multicolumn{2}{c|}{ Blood } & \multicolumn{2}{c|}{ Urine } \\
\hline Threshold & 0.9 & 0.8 & 0.9 & 0.8 & 0.9 & 0.8 & 0.9 & 0.8 & 0.9 & 0.8 \\
\hline \hline Type I & 4 & 28 & 0 & 0 & 0 & 1 & 0 & 0 & 0 & 0 \\
Type II & 10 & 9 & 5 & 3 & 7 & 4 & 15 & 8 & 11 & 8 \\
Total Error & 14 & 37 & 5 & 3 & 7 & 5 & 15 & 8 & 11 & 8 \\
\hline \% Mismatch & 0.79 & 2.09 & 0.28 & 0.17 & 0.90 & 0.64 & 1.92 & 1.03 & 0.62 & 0.45 \\
\hline
\end{tabular}


Table 61. Comparison of mismatch occurrences for the five biological specimens across populations at 0.9 correlation cutoff threshold

\begin{tabular}{|l|c|c|c|c|c|}
\hline & Hand Odor & Buccal Swab & Breath & Blood & Urine \\
\hline Healthy & $0.89 \%$ & $0.45 \%$ & $0.58 \%$ & $0.63 \%$ & $0.36 \%$ \\
\hline Type 2 Diabetes & $0.88 \%$ & $0.44 \%$ & $1.14 \%$ & $1.00 \%$ & $0.13 \%$ \\
\hline MDD & $0.79 \%$ & $0.28 \%$ & $0.90 \%$ & $1.92 \%$ & $0.62 \%$ \\
\hline
\end{tabular}

\subsection{Chi-Square Significance Test}

Table 62 to Table 66 give the frequencies of the VOCs extracted from individuals within their population subgroups (healthy control, type 2 diabetics (T2DB), and clinically depressed (MDD)). From the entire list of compounds detected from each specimen, those compounds that appeared at least once in each subgroup were considered for further data analysis. 
Table 62. Frequency of occurrence of hand odor VOCs in total population and within subgroups (Notes: ${ }^{a} n=70,{ }^{b} n=31,{ }^{c} n=19,{ }^{d} n=20$ )

\begin{tabular}{|c|c|c|c|c|}
\hline \multirow{2}{*}{ Compound Name } & \multirow{2}{*}{$\begin{array}{c}\text { Occurrence in } \\
\text { Total } \\
\text { Population }^{\text {a }}\end{array}$} & \multicolumn{3}{|c|}{ Occurrence within Group } \\
\hline & & Healthy $^{b}$ & $\mathrm{~T} 2 \mathrm{DB}^{\mathrm{c}}$ & $\mathrm{MDD}^{\mathrm{d}}$ \\
\hline Decanal & $100 \%$ & 31 & 19 & 20 \\
\hline Nonanal & $100 \%$ & 31 & 19 & 20 \\
\hline Tetradecane & $96 \%$ & 31 & 17 & 19 \\
\hline Hexadecane & $93 \%$ & 30 & 17 & 18 \\
\hline Heptadecane & $90 \%$ & 28 & 17 & 18 \\
\hline Undecanal & $67 \%$ & 30 & 7 & 10 \\
\hline Octadecane & $66 \%$ & 18 & 11 & 17 \\
\hline 5,9-Undecadien-2-one,6,10-dimethyl-, (E)- & $63 \%$ & 23 & 9 & 12 \\
\hline Tridecane & $61 \%$ & 22 & 9 & 12 \\
\hline Undecane & $54 \%$ & 15 & 11 & 12 \\
\hline Dodecane & $37 \%$ & 14 & 5 & 7 \\
\hline BenzylAlcohol & $29 \%$ & 13 & 2 & 5 \\
\hline Dodecanal & $23 \%$ & 10 & 2 & 4 \\
\hline 1,6-Octadien-3-ol,3,7-dimethyl- & $16 \%$ & 7 & 1 & 3 \\
\hline 1-Hexanol,2-ethyl- & $16 \%$ & 6 & 2 & 3 \\
\hline Dodecanoicacid,methylester & $14 \%$ & 3 & 4 & 3 \\
\hline 2-Nonenal,(E)- & $11 \%$ & 3 & 4 & 1 \\
\hline Acetophenone & $11 \%$ & 3 & 3 & 2 \\
\hline Benzaldehyde & $10 \%$ & 4 & 1 & 2 \\
\hline 2-Decanone & $9 \%$ & 6 & 0 & 0 \\
\hline Nonanoicacid,methylester & $6 \%$ & 4 & 0 & 0 \\
\hline Octanoicacid,methylester & $6 \%$ & 2 & 1 & 1 \\
\hline Pentadecane & $4 \%$ & 2 & 0 & 1 \\
\hline 1-Pentadecene & $3 \%$ & 1 & 0 & 1 \\
\hline 5-Hepten-2-one,6-methyl- & $3 \%$ & 0 & 1 & 1 \\
\hline Dodecanoicacid & $3 \%$ & 2 & 0 & 0 \\
\hline Eicosane & $3 \%$ & 2 & 0 & 0 \\
\hline Furfural & $3 \%$ & 2 & 0 & 0 \\
\hline Heptanal & $3 \%$ & 1 & 0 & 1 \\
\hline Hexanoicacid & $3 \%$ & 1 & 0 & 1 \\
\hline Phenol & $3 \%$ & 2 & 0 & 0 \\
\hline 2-Pentanone & $1 \%$ & 0 & 1 & 0 \\
\hline Aceticacid,butylester & $1 \%$ & 0 & 1 & 0 \\
\hline Cedrol & $1 \%$ & 0 & 1 & 0 \\
\hline Docosane & $1 \%$ & 1 & 0 & 0 \\
\hline Heneicosane & $1 \%$ & 1 & 0 & 0 \\
\hline Lilial & $1 \%$ & 0 & 1 & 0 \\
\hline Octanal & $1 \%$ & 1 & 0 & 0 \\
\hline Propanedioicacid,dimethylester & $1 \%$ & 1 & 0 & 0 \\
\hline Tetradecanal & $1 \%$ & 1 & 0 & 0 \\
\hline
\end{tabular}


Table 63. Frequency of occurrence of buccal swab odor VOCs in total population and within subgroups (Notes: ${ }^{a} n=70,{ }^{b} n=31,{ }^{c} n=19,{ }^{d} n=20$ )

\begin{tabular}{|c|c|c|c|c|}
\hline \multirow{2}{*}{ Compound Name } & \multirow{2}{*}{$\begin{array}{l}\text { Occurrence } \\
\text { in Total } \\
\text { Population }\end{array}$} & \multicolumn{3}{|c|}{ Occurrence within Group } \\
\hline & & Healthy $^{\mathrm{b}}$ & $\mathrm{T} 2 \mathrm{DB}^{\mathrm{c}}$ & $\operatorname{MDD}^{d}$ \\
\hline Decanal & $100 \%$ & 31 & 19 & 20 \\
\hline Hexanoicacid & $100 \%$ & 31 & 19 & 20 \\
\hline Furan,2-pentyl- & $91 \%$ & 30 & 15 & 19 \\
\hline 5,9-Undecadien-2-one,6,10-dimethyl-, (E)- & $90 \%$ & 28 & 16 & 19 \\
\hline Nonanoicacid,ethylester & $87 \%$ & 27 & 16 & 18 \\
\hline Nonanoicacid & $84 \%$ & 24 & 17 & 18 \\
\hline 2,4-Nonadienal,(E,E)- & $77 \%$ & 28 & 10 & 16 \\
\hline Dodecanoicacid,ethylester & $77 \%$ & 24 & 14 & 16 \\
\hline 6-Dodecanone & $70 \%$ & 24 & 14 & 11 \\
\hline 2-Nonenal,(E)- & $63 \%$ & 25 & 6 & 13 \\
\hline Hexadecanoicacid,ethylester & $61 \%$ & 20 & 10 & 13 \\
\hline Tetradecanoicacid,ethylester & $61 \%$ & 20 & 11 & 12 \\
\hline 1-Tetradecene & $60 \%$ & 17 & 13 & 12 \\
\hline 1,1'-Biphenyl,2,2'-diethyl- & $56 \%$ & 13 & 11 & 15 \\
\hline Octanoicacid,ethylester & $50 \%$ & 19 & 7 & 9 \\
\hline 6-Methyl-3,5-heptadiene-2-one & $43 \%$ & 10 & 10 & 10 \\
\hline Hexanoicacid,ethylester & $41 \%$ & 20 & 3 & 6 \\
\hline 1-Dodecene & $40 \%$ & 13 & 6 & 9 \\
\hline 1-Decene & $39 \%$ & 8 & 11 & 8 \\
\hline Tetradecane & $39 \%$ & 10 & 10 & 7 \\
\hline Benzaldehyde & $36 \%$ & 12 & 8 & 5 \\
\hline Hexanal & $34 \%$ & 18 & 1 & 5 \\
\hline Naphthalene,1-methyl- & $31 \%$ & 14 & 2 & 6 \\
\hline Hexanoicacid,pentylester & $30 \%$ & 13 & 2 & 6 \\
\hline Naphthalene,2-methyl- & $29 \%$ & 12 & 4 & 4 \\
\hline 2-Tetradecene,(E)- & $24 \%$ & 7 & 4 & 6 \\
\hline Dodecanoicacid & $24 \%$ & 7 & 5 & 5 \\
\hline Heptanoicacid & $24 \%$ & 1 & 8 & 8 \\
\hline Heptanoicacid,ethylester & $24 \%$ & 8 & 3 & 6 \\
\hline 1,1'-Biphenyl,4-methyl- & $23 \%$ & 2 & 6 & 8 \\
\hline 1-Hexanol & $23 \%$ & 7 & 6 & 3 \\
\hline 2-Octenoicacid & $23 \%$ & 9 & 4 & 3 \\
\hline Dibutanoylmorphine & $21 \%$ & 11 & 2 & 2 \\
\hline Pentanoicacid & $21 \%$ & 5 & 3 & 7 \\
\hline
\end{tabular}




\begin{tabular}{|c|c|c|c|c|}
\hline Decanoicacid,ethylester & $20 \%$ & 6 & 3 & 5 \\
\hline DiethylPhthalate & $20 \%$ & 9 & 3 & 2 \\
\hline Linalool Oxide & $20 \%$ & 11 & 1 & 2 \\
\hline Pentadecanoicacid,ethylester & $20 \%$ & 7 & 2 & 5 \\
\hline 3-Nonen-2-one & $19 \%$ & 10 & 0 & 3 \\
\hline Cyclododecane & $19 \%$ & 8 & 5 & 0 \\
\hline Naphthalene,2,7-dimethyl- & $19 \%$ & 6 & 3 & 4 \\
\hline Furfural & $17 \%$ & 8 & 1 & 3 \\
\hline 1-Octanol & $16 \%$ & 9 & 1 & 1 \\
\hline Hexadecane & $16 \%$ & 6 & 1 & 4 \\
\hline 2(3H)-Furanone,5-ethyldihydro- & $14 \%$ & 3 & 5 & 2 \\
\hline 2(3H)-Furanone, dihydro-5-pentyl- & $14 \%$ & 3 & 5 & 2 \\
\hline 2-Octenal,(E)- & $14 \%$ & 7 & 0 & 3 \\
\hline Acetophenone & $14 \%$ & 3 & 3 & 4 \\
\hline Phenol & $14 \%$ & 2 & 4 & 4 \\
\hline Benzene,1-methyl-2-[(4-methylphenyl)methyl]- & $13 \%$ & 3 & 4 & 2 \\
\hline Benzothiazole & $13 \%$ & 3 & 4 & 2 \\
\hline Caryophyllene & $13 \%$ & 5 & 3 & 1 \\
\hline $\begin{array}{l}\text { 2,5-Cyclohexadiene-1,4-dione,2,6-bis(1,1- } \\
\text { dimethylethyl)- }\end{array}$ & $11 \%$ & 4 & 1 & 3 \\
\hline E-11-Hexadecenoicacid,ethylester & $11 \%$ & 2 & 1 & 5 \\
\hline IsopropylMyristate & $10 \%$ & 5 & 2 & 0 \\
\hline Vanillin & $10 \%$ & 5 & 0 & 2 \\
\hline 5,9-Undecadien-2-one,6,10-dimethyl-, (Z)- & $9 \%$ & 5 & 1 & 0 \\
\hline Benzoicacid,ethylester & $9 \%$ & 5 & 0 & 1 \\
\hline Galaxolide & $7 \%$ & 2 & 0 & 3 \\
\hline IsopropylPalmitate & $7 \%$ & 2 & 1 & 2 \\
\hline 1-Pentadecene & $6 \%$ & 4 & 0 & 0 \\
\hline 3,5,9-Undecatrien-2-one,6,10-dimethyl-, (E,Z)- & $6 \%$ & 3 & 1 & 0 \\
\hline 3,7-Dimethyl-octa-1,6-diene & $6 \%$ & 3 & 0 & 1 \\
\hline 5,9-Undecadien-2-one,6,10-dimethyl- & $6 \%$ & 4 & 0 & 0 \\
\hline Benzene, 1,2,4,5-tetramethyl- & $6 \%$ & 0 & 1 & 3 \\
\hline Benzophenone & $6 \%$ & 4 & 0 & 0 \\
\hline ButylatedHydroxytoluene & $6 \%$ & 1 & 2 & 1 \\
\hline Cyclopropane,nonyl- & $6 \%$ & 4 & 0 & 0 \\
\hline Hexadecanoicacid,methylester & $6 \%$ & 1 & 2 & 1 \\
\hline Octadecanal & $6 \%$ & 3 & 0 & 1 \\
\hline Octadecane & $6 \%$ & 2 & 1 & 1 \\
\hline 1-Hexadecene & $4 \%$ & 2 & 0 & 1 \\
\hline 2(3H)-Furanone,dihydro-5-propyl- & $4 \%$ & 1 & 2 & 0 \\
\hline
\end{tabular}




\begin{tabular}{|c|c|c|c|c|}
\hline Benzene, 1,1'-methylenebis[4-methyl- & $4 \%$ & 3 & 0 & 0 \\
\hline$\beta$-Bourbonene & $4 \%$ & 3 & 0 & 0 \\
\hline Calamenene & $4 \%$ & 3 & 0 & 0 \\
\hline Heptadecane & $4 \%$ & 3 & 0 & 0 \\
\hline Hexanoicacid,anhydride & $4 \%$ & 3 & 0 & 0 \\
\hline Homomenthylsalicylate & $4 \%$ & 0 & 0 & 3 \\
\hline OctanoicAcid & $4 \%$ & 3 & 0 & 0 \\
\hline Tributylphosphate & $4 \%$ & 1 & 1 & 1 \\
\hline 1,1'-Biphenyl,3-methyl- & $3 \%$ & 1 & 1 & 0 \\
\hline $\begin{array}{l}\text { 1,6,10-Dodecatriene,7,11-dimethyl-3-methylene-, } \\
(\mathrm{Z}) \text { - }\end{array}$ & $3 \%$ & 2 & 0 & 0 \\
\hline 1-Heptadecene & $3 \%$ & 2 & 0 & 0 \\
\hline 1-Pentanol & $3 \%$ & 2 & 0 & 0 \\
\hline Cyclodecane & $3 \%$ & 2 & 0 & 0 \\
\hline Cyclotetradecane & $3 \%$ & 2 & 0 & 0 \\
\hline Dodecanal & $3 \%$ & 1 & 0 & 1 \\
\hline Ethyl9-hexadecenoate & $3 \%$ & 2 & 0 & 0 \\
\hline Naphthalene,2,6-dimethyl- & $3 \%$ & 0 & 1 & 1 \\
\hline Tridecane & $3 \%$ & 0 & 2 & 0 \\
\hline$\gamma$-Cadinene & $3 \%$ & 1 & 0 & 1 \\
\hline 1,1'-Biphenyl,2-ethyl- & $1 \%$ & 1 & 0 & 0 \\
\hline 11-Octadecenoicacid,methylester & $1 \%$ & 0 & 0 & 1 \\
\hline 2,4-Decadienal,(E,E)- & $1 \%$ & 1 & 0 & 0 \\
\hline 2-Decenal,(E)- & $1 \%$ & 0 & 0 & 1 \\
\hline 2-Dodecenal,(E)- & $1 \%$ & 1 & 0 & 0 \\
\hline 2-Heptenoicacid & $1 \%$ & 1 & 0 & 0 \\
\hline 3-Eicosene,(E)- & $1 \%$ & 1 & 0 & 0 \\
\hline 3-Heptadecene,(Z)- & $1 \%$ & 1 & 0 & 0 \\
\hline 4,8-Dimethyl-nona-3,8-dien-2-one & $1 \%$ & 1 & 0 & 0 \\
\hline 7-Hexadecene,(Z)- & $1 \%$ & 1 & 0 & 0 \\
\hline 9-Octadecenoicacid,(E)- & $1 \%$ & 1 & 0 & 0 \\
\hline Aceticacid & $1 \%$ & 1 & 0 & 0 \\
\hline Benzaldehyde,4-(1-methylethyl)- & $1 \%$ & 1 & 0 & 0 \\
\hline Benzene, 1-methyl-2-[(3-methylphenyl)methyl]- & $1 \%$ & 1 & 0 & 0 \\
\hline Benzene, 1,2,3,5-tetramethyl- & $1 \%$ & 0 & 1 & 0 \\
\hline Benzene,1-ethyl-2,3-dimethyl- & $1 \%$ & 0 & 0 & 1 \\
\hline Benzene,1-methyl-4-nitro- & $1 \%$ & 0 & 1 & 0 \\
\hline Carvone & $1 \%$ & 0 & 1 & 0 \\
\hline Cyclododecanol,1-ethenyl- & $1 \%$ & 1 & 0 & 0 \\
\hline Cyclohexadecane & $1 \%$ & 0 & 1 & 0 \\
\hline
\end{tabular}




\begin{tabular}{|l|c|c|c|c|}
\hline trans-p-Menthan-3-one & $1 \%$ & 0 & 1 & 0 \\
\hline$\Delta$-Cadinene & $1 \%$ & 1 & 0 & 0 \\
\hline Docosane & $1 \%$ & 1 & 0 & 0 \\
\hline Dodecanoicacid,methylester & $1 \%$ & 1 & 0 & 0 \\
\hline Eicosane & $1 \%$ & 1 & 0 & 0 \\
\hline Ethyltridecanoate & $1 \%$ & 1 & 0 & 0 \\
\hline Glycocyanidine & $1 \%$ & 1 & 0 & 0 \\
\hline Heneicosane & $1 \%$ & 1 & 0 & 0 \\
\hline Hexanoicacid,propylester & $1 \%$ & 0 & 1 & 0 \\
\hline Menthol & $1 \%$ & 0 & 1 & 0 \\
\hline Nonadecanoicacid,ethylester & $1 \%$ & 1 & 0 & 0 \\
\hline Pentadecane,7-methyl- & $1 \%$ & 1 & 0 & 0 \\
\hline Tetradecanoicacid,2-methyl-,methyl ester & $1 \%$ & 1 & 0 & 0 \\
\hline
\end{tabular}


Table 64. Frequency of occurrence of breath odor VOCs in total population and within subgroups (Notes: ${ }^{a} n=70,{ }^{b} n=31,{ }^{c} n=19,{ }^{d} n=20$ )

\begin{tabular}{|l|c|c|c|c|}
\hline \multicolumn{1}{|c|}{ Compound Name } & \multicolumn{3}{|c|}{ Occurrence in } & \multicolumn{3}{|c|}{ Occurrence within Group $^{\text {Total Population }}{ }^{\mathrm{a}}$} & Healthy $^{\mathrm{b}}$ & $\mathrm{T}^{\mathrm{N}} \mathrm{DB}^{\mathrm{c}}$ & MDD $^{\mathrm{d}}$ \\
\hline Decanal & $99 \%$ & 31 & 18 & 20 \\
\hline Nonanal & $99 \%$ & 30 & 19 & 20 \\
\hline Xylenes & $76 \%$ & 22 & 15 & 16 \\
\hline Benzophenone & $76 \%$ & 29 & 10 & 14 \\
\hline 1-Hexanol,2-ethyl- & $73 \%$ & 25 & 11 & 15 \\
\hline Benzene,1,2-dichloro- & $73 \%$ & 25 & 10 & 16 \\
\hline Styrene & $66 \%$ & 29 & 6 & 11 \\
\hline ButylatedHydroxytoluene & $60 \%$ & 24 & 6 & 12 \\
\hline Dodecane & $56 \%$ & 13 & 13 & 13 \\
\hline Tetradecane & $53 \%$ & 11 & 13 & 13 \\
\hline Tridecane & $50 \%$ & 10 & 13 & 12 \\
\hline Undecane & $50 \%$ & 13 & 10 & 12 \\
\hline Phenol & $47 \%$ & 18 & 3 & 12 \\
\hline Toluene & $30 \%$ & 8 & 6 & 7 \\
\hline Undecanal & $26 \%$ & 9 & 4 & 5 \\
\hline Benzene,1,3,5-trimethyl- & $24 \%$ & 4 & 6 & 7 \\
\hline Dibutylphthalate & $24 \%$ & 16 & 1 & 0 \\
\hline 2,6-Diisopropylnaphthalene & $23 \%$ & 13 & 2 & 1 \\
\hline 5,9-Undecadien-2-one,6,10-dimethyl-, (E)- & $20 \%$ & 9 & 1 & 4 \\
\hline p-Benzoquinone & $17 \%$ & 1 & 6 & 5 \\
\hline Acetophenone & $17 \%$ & 10 & 0 & 2 \\
\hline Benzene,1-methyl-3-propyl- & $17 \%$ & 0 & 5 & 7 \\
\hline Benzene,4-ethyl-1,2-dimethyl- & $17 \%$ & 2 & 5 & 5 \\
\hline Dodecanal & $17 \%$ & 8 & 1 & 3 \\
\hline 1-Dodecene & $16 \%$ & 10 & 0 & 1 \\
\hline Longifolene & $14 \%$ & 10 & 0 & 0 \\
\hline Naphthalene & $14 \%$ & 5 & 3 & 2 \\
\hline Benzene,1,2,3-trimethyl- & $13 \%$ & 2 & 2 & 5 \\
\hline Nonanoicacid & $13 \%$ & 5 & 0 & 4 \\
\hline Benzene,1-ethyl-2,4-dimethyl- & $11 \%$ & 1 & 2 & 5 \\
\hline Benzene,1-ethyl-2-methyl- & $10 \%$ & 1 & 2 & 4 \\
\hline Diisopropylnaphthalene & $10 \%$ & 4 & 2 & 1 \\
\hline Indole & $10 \%$ & 6 & 0 & 1 \\
\hline Phenol,2-(1,1-dimethylethyl)- & $10 \%$ & 7 & 0 & 0 \\
\hline Limonene & $10 \%$ & 2 & 2 & 3 \\
\hline
\end{tabular}




\begin{tabular}{|c|c|c|c|c|}
\hline Benzene,1-ethyl-2,3-dimethyl- & $9 \%$ & 0 & 4 & 2 \\
\hline Benzene,2-ethyl-1,4-dimethyl- & $9 \%$ & 0 & 5 & 1 \\
\hline Naphthalene,2-methyl- & $9 \%$ & 1 & 1 & 4 \\
\hline 1,1'-Biphenyl,4-methyl- & $7 \%$ & 2 & 0 & 3 \\
\hline 1,3,5,7-Cyclooctatetraene & $7 \%$ & 1 & 3 & 1 \\
\hline 4-Cyanocyclohexene & $7 \%$ & 1 & 4 & 0 \\
\hline Benzene,1,4-dichloro- & $7 \%$ & 3 & 1 & 1 \\
\hline 5-Hepten-2-one,6-methyl- & $6 \%$ & 2 & 0 & 2 \\
\hline Benzene,1,2,3,4-tetramethyl- & $6 \%$ & 0 & 1 & 3 \\
\hline Benzene, 1,2,4,5-tetramethyl- & $6 \%$ & 0 & 0 & 4 \\
\hline DiethylPhthalate & $6 \%$ & 1 & 1 & 2 \\
\hline Hexadecane & $6 \%$ & 3 & 0 & 1 \\
\hline Menthol & $6 \%$ & 1 & 3 & 0 \\
\hline 2-Dodecene, $(\mathrm{Z})$ - & $4 \%$ & 0 & 2 & 1 \\
\hline 2-Propanol,1-propoxy- & $4 \%$ & 1 & 1 & 1 \\
\hline Acetone & $4 \%$ & 0 & 2 & 1 \\
\hline Benzene,1-methyl-4-(1-methylpropyl)- & $4 \%$ & 1 & 1 & 1 \\
\hline Benzene,2-ethyl-1,3-dimethyl- & $4 \%$ & 0 & 2 & 1 \\
\hline Benzene, diethyl- & $4 \%$ & 1 & 0 & 2 \\
\hline Cyclododecane & $4 \%$ & 3 & 0 & 0 \\
\hline Hexanedioicacid,bis(1-methylethyl) ester & $4 \%$ & 0 & 2 & 1 \\
\hline Longicyclene & $4 \%$ & 3 & 0 & 0 \\
\hline n-Decanoicacid & $4 \%$ & 2 & 0 & 1 \\
\hline 3-Heptanone & $3 \%$ & 0 & 1 & 1 \\
\hline Benzene,1,2,3,5-tetramethyl- & $3 \%$ & 0 & 0 & 2 \\
\hline Benzene,1-methyl-2-(1-methylethyl)- & $3 \%$ & 0 & 0 & 2 \\
\hline Benzene,propyl- & $3 \%$ & 1 & 0 & 1 \\
\hline$\beta$-Maaliene & $3 \%$ & 2 & 0 & 0 \\
\hline Galaxolide & $3 \%$ & 1 & 0 & 1 \\
\hline Heptadecane & $3 \%$ & 2 & 0 & 0 \\
\hline Hexanal & $3 \%$ & 1 & 0 & 1 \\
\hline Homomenthylsalicylate & $3 \%$ & 1 & 0 & 1 \\
\hline Triacetin & $3 \%$ & 0 & 0 & 2 \\
\hline Undecane,2,6-dimethyl- & $3 \%$ & 1 & 0 & 1 \\
\hline 1S- $\alpha$-Pinene & $1 \%$ & 1 & 0 & 0 \\
\hline 3-Dodecene,(Z)- & $1 \%$ & 0 & 0 & 1 \\
\hline 3-Octanol,3,7-dimethyl-,(.+/-.)- & $1 \%$ & 1 & 0 & 0 \\
\hline 5-Dodecene,(E)- & $1 \%$ & 0 & 1 & 0 \\
\hline Aceticacid & $1 \%$ & 0 & 0 & 1 \\
\hline Benzaldehyde & $1 \%$ & 1 & 0 & 0 \\
\hline
\end{tabular}




\begin{tabular}{|c|c|c|c|c|}
\hline Benzaldehyde,4-methoxy- & $1 \%$ & 1 & 0 & 0 \\
\hline Benzene,(1-methylethyl)- & $1 \%$ & 0 & 0 & 1 \\
\hline Benzene,1,2,4-trimethyl- & $1 \%$ & 0 & 0 & 1 \\
\hline Benzene,1,3-dichloro- & $1 \%$ & 0 & 1 & 0 \\
\hline Benzene,1,3-diethyl-5-methyl- & $1 \%$ & 1 & 0 & 0 \\
\hline Benzene,1-ethyl-3,5-dimethyl- & $1 \%$ & 1 & 0 & 0 \\
\hline Benzene,1-ethyl-3-methyl- & $1 \%$ & 0 & 0 & 1 \\
\hline Benzene,1-methyl-3-(1-methylethyl)- & $1 \%$ & 1 & 0 & 0 \\
\hline Benzene,1-methyl-4-(1-methylethyl)- & $1 \%$ & 0 & 0 & 1 \\
\hline Benzene,1-methyl-4-propyl- & $1 \%$ & 0 & 0 & 1 \\
\hline Benzene,3-cyclohexen-1-yl- & $1 \%$ & 1 & 0 & 0 \\
\hline Benzene,pentamethyl- & $1 \%$ & 0 & 0 & 1 \\
\hline$\beta$-Gurjunene & $1 \%$ & 1 & 0 & 0 \\
\hline Caryophyllene & $1 \%$ & 1 & 0 & 0 \\
\hline Cyclohexane,methyl- & $1 \%$ & 1 & 0 & 0 \\
\hline Decane,2-methyl- & $1 \%$ & 0 & 0 & 1 \\
\hline Decane,3-methyl- & $1 \%$ & 1 & 0 & 0 \\
\hline Dodecanoicacid & $1 \%$ & 0 & 0 & 1 \\
\hline Dodecanoicacid,methylester & $1 \%$ & 1 & 0 & 0 \\
\hline Eicosane & $1 \%$ & 1 & 0 & 0 \\
\hline Ethanol,2-phenoxy- & $1 \%$ & 1 & 0 & 0 \\
\hline Indolizine & $1 \%$ & 1 & 0 & 0 \\
\hline Isobornylacetate & $1 \%$ & 0 & 1 & 0 \\
\hline IsopropylPalmitate & $1 \%$ & 1 & 0 & 0 \\
\hline Methyldihydrojasmonate & $1 \%$ & 0 & 0 & 1 \\
\hline MethylSalicylate & $1 \%$ & 1 & 0 & 0 \\
\hline n-Hexadecanoicacid & $1 \%$ & 0 & 0 & 1 \\
\hline Nonadecane & $1 \%$ & 1 & 0 & 0 \\
\hline Nonane,3-methyl- & $1 \%$ & 1 & 0 & 0 \\
\hline Octanal & $1 \%$ & 1 & 0 & 0 \\
\hline Tetrachloroethylene & $1 \%$ & 0 & 0 & 1 \\
\hline Tetradecanal & $1 \%$ & 1 & 0 & 0 \\
\hline Tetradecanoicacid & $1 \%$ & 0 & 0 & 1 \\
\hline Undecane,3,6-dimethyl- & $1 \%$ & 1 & 0 & 0 \\
\hline
\end{tabular}


Table 65. Frequency of occurrence of breath odor VOCs in total population and within subgroups (Notes: ${ }^{a} n=70,{ }^{b} n=31,{ }^{c} n=19,{ }^{d} n=20$ )

\begin{tabular}{|c|c|c|c|c|}
\hline \multirow{2}{*}{ Compound Name } & \multirow{2}{*}{$\begin{array}{l}\text { Occurrence in } \\
\text { Total Population }\end{array}$} & \multicolumn{3}{|c|}{ Occurrence within Group } \\
\hline & & Healthy $^{\mathrm{b}}$ & $\mathrm{T}_{2} \mathrm{DB}^{\mathrm{c}}$ & $M D^{d}$ \\
\hline Hexadecane & $99 \%$ & 30 & 19 & 20 \\
\hline Nonanal & $94 \%$ & 28 & 19 & 19 \\
\hline 2,6-Diisopropylnaphthalene & $93 \%$ & 30 & 15 & 20 \\
\hline Tetradecane & $91 \%$ & 30 & 16 & 18 \\
\hline Undecane & $91 \%$ & 29 & 16 & 19 \\
\hline Benzaldehyde,2,4,6-trimethyl- & $89 \%$ & 31 & 12 & 19 \\
\hline Tridecane & $89 \%$ & 29 & 14 & 19 \\
\hline Dodecane & $86 \%$ & 28 & 13 & 19 \\
\hline Hexanal & $76 \%$ & 26 & 11 & 16 \\
\hline Heptadecane & $69 \%$ & 22 & 12 & 14 \\
\hline BenzylAlcohol & $60 \%$ & 25 & 6 & 11 \\
\hline Diisopropylnaphthalene & $57 \%$ & 19 & 7 & 14 \\
\hline Furan,2-butyltetrahydro- & $56 \%$ & 23 & 9 & 7 \\
\hline Furan,2-pentyl- & $47 \%$ & 20 & 6 & 7 \\
\hline Decanal & $44 \%$ & 15 & 9 & 7 \\
\hline 1-Dodecene & $41 \%$ & 18 & 6 & 5 \\
\hline Cyclohexanone & $41 \%$ & 19 & 3 & 7 \\
\hline 5,9-Undecadien-2-one,6,10-dimethyl-, (E)- & $37 \%$ & 14 & 4 & 8 \\
\hline 2-Dodecene,(Z)- & $36 \%$ & 12 & 6 & 7 \\
\hline Longifolene & $33 \%$ & 6 & 8 & 9 \\
\hline 1-Decene & $31 \%$ & 7 & 8 & 7 \\
\hline 1-Octen-3-ol & $31 \%$ & 8 & 9 & 5 \\
\hline 2-Undecanone & $30 \%$ & 11 & 3 & 7 \\
\hline Benzene,1,2-dichloro- & $29 \%$ & 12 & 5 & 3 \\
\hline Octadecane & $29 \%$ & 6 & 6 & 8 \\
\hline 1-Pentanol & $26 \%$ & 12 & 2 & 4 \\
\hline 2-Heptanone & $21 \%$ & 8 & 2 & 5 \\
\hline Benzene,1,3,5-trimethyl- & $20 \%$ & 0 & 7 & 7 \\
\hline Heptanal & $20 \%$ & 5 & 4 & 5 \\
\hline Cyclododecane & $19 \%$ & 10 & 2 & 1 \\
\hline 1-Dodecanol & $17 \%$ & 5 & 4 & 3 \\
\hline Naphthalene,2-methyl- & $16 \%$ & 0 & 5 & 6 \\
\hline Benzene,1,2,3,5-tetramethyl- & $14 \%$ & 0 & 3 & 7 \\
\hline Benzene, 1,2,4,5-tetramethyl- & $14 \%$ & 0 & 2 & 8 \\
\hline Benzene,4-ethyl-1,2-dimethyl- & $14 \%$ & 1 & 1 & 8 \\
\hline Naphthalene & $14 \%$ & 1 & 6 & 3 \\
\hline
\end{tabular}




\begin{tabular}{|c|c|c|c|c|}
\hline Benzene,1-ethyl-2,4-dimethyl- & $13 \%$ & 0 & 5 & 4 \\
\hline Xylenes & $13 \%$ & 3 & 2 & 4 \\
\hline 4-Cyanocyclohexene & $11 \%$ & 2 & 3 & 3 \\
\hline Cyclopropane,nonyl- & $11 \%$ & 7 & 1 & 0 \\
\hline Menthol & $11 \%$ & 4 & 4 & 0 \\
\hline Octanal & $11 \%$ & 6 & 0 & 2 \\
\hline 1-Octanol & $10 \%$ & 5 & 2 & 0 \\
\hline 2-Heptanone,6-methyl- & $9 \%$ & 5 & 1 & 0 \\
\hline 2-Propanol,1-propoxy- & $9 \%$ & 1 & 3 & 2 \\
\hline Benzene,1,4-dichloro- & $9 \%$ & 4 & 1 & 1 \\
\hline Naphthalene,1-methyl- & $9 \%$ & 0 & 2 & 4 \\
\hline 2,4-Diphenyl-4-methyl-1(E)-pentene & $7 \%$ & 3 & 0 & 2 \\
\hline Benzenemethanol,.alpha.,.alpha.-dimethyl- & $7 \%$ & 3 & 0 & 2 \\
\hline Ethanol,2-butoxy- & $7 \%$ & 3 & 1 & 1 \\
\hline 1-Phenyl-2-butanone & $6 \%$ & 3 & 0 & 1 \\
\hline 3-Heptanone & $6 \%$ & 0 & 3 & 1 \\
\hline Benzaldehyde,4-(methylthio)- & $6 \%$ & 2 & 0 & 2 \\
\hline Benzene,1,2,3,4-tetramethyl- & $6 \%$ & 0 & 1 & 3 \\
\hline Benzene,1,2,3-trimethyl- & $6 \%$ & 0 & 1 & 3 \\
\hline Benzene,1-ethyl-2-methyl- & $6 \%$ & 0 & 1 & 3 \\
\hline Benzene,2-ethyl-1,4-dimethyl- & $6 \%$ & 0 & 1 & 3 \\
\hline Heptanol & $6 \%$ & 3 & 1 & 0 \\
\hline 1,1'-Biphenyl,2-methyl- & $4 \%$ & 1 & 1 & 1 \\
\hline 2,5-Octanedione & $4 \%$ & 0 & 2 & 1 \\
\hline 3-Dodecene, $(Z)$ - & $4 \%$ & 0 & 2 & 1 \\
\hline Benzaldehyde & $4 \%$ & 2 & 0 & 1 \\
\hline Dodecane,1-chloro- & $4 \%$ & 0 & 0 & 3 \\
\hline Naphthalene,2,7-dimethyl- & $4 \%$ & 0 & 2 & 1 \\
\hline Phenol & $4 \%$ & 2 & 0 & 1 \\
\hline 1,1'-Biphenyl,4-methyl- & $3 \%$ & 1 & 0 & 1 \\
\hline 3,7-Dimethyl-octa-1,6-diene & $3 \%$ & 1 & 1 & 0 \\
\hline 3-Isopropylbenzaldehyde & $3 \%$ & 1 & 0 & 1 \\
\hline 4-Heptanone & $3 \%$ & 2 & 0 & 0 \\
\hline 5-Hepten-2-one,6-methyl- & $3 \%$ & 2 & 0 & 0 \\
\hline Benzene,1,4-diethyl-2-methyl- & $3 \%$ & 0 & 1 & 1 \\
\hline Benzene,1-ethyl-3,5-dimethyl- & $3 \%$ & 0 & 2 & 0 \\
\hline Benzene,1-ethyl-3-methyl- & $3 \%$ & 0 & 1 & 1 \\
\hline Benzene,1-methyl-3-propyl- & $3 \%$ & 1 & 0 & 1 \\
\hline Benzene,1-methyl-4-(1-methylpropyl)- & $3 \%$ & 1 & 1 & 0 \\
\hline Decane & $3 \%$ & 0 & 1 & 1 \\
\hline Naphthalene,2,3,6-trimethyl- & $3 \%$ & 1 & 1 & 0 \\
\hline Phenol,2,4,6-trimethyl- & $3 \%$ & 2 & 0 & 0 \\
\hline
\end{tabular}




\begin{tabular}{|c|c|c|c|c|}
\hline Phenol,4-methoxy-2-nitro- & $3 \%$ & 2 & 0 & 0 \\
\hline Z-8-Hexadecene & $3 \%$ & 0 & 1 & 1 \\
\hline 1,1'-Biphenyl,3-methyl- & $1 \%$ & 0 & 1 & 0 \\
\hline 1,2-Benzenediol,3,5-bis(1,1-dimethylethyl)- & $1 \%$ & 1 & 0 & 0 \\
\hline$\beta$-Cyclocitral & $1 \%$ & 0 & 0 & 1 \\
\hline 1-Hexadecene & $1 \%$ & 0 & 1 & 0 \\
\hline 1-Tetradecene & $1 \%$ & 1 & 0 & 0 \\
\hline 2-Decanone & $1 \%$ & 1 & 0 & 0 \\
\hline 2-Propanol,1-butoxy- & $1 \%$ & 0 & 1 & 0 \\
\hline 4-tert-Butylcyclohexylacetate & $1 \%$ & 0 & 1 & 0 \\
\hline Acetone & $1 \%$ & 1 & 0 & 0 \\
\hline Acetophenone & $1 \%$ & 1 & 0 & 0 \\
\hline Benzaldehyde,2-hydroxy- & $1 \%$ & 0 & 0 & 1 \\
\hline Benzaldehyde,3-hydroxy- & $1 \%$ & 1 & 0 & 0 \\
\hline Benzaldehyde,ethyl- & $1 \%$ & 0 & 0 & 1 \\
\hline Benzene,1,3-dichloro- & $1 \%$ & 1 & 0 & 0 \\
\hline Benzene,1,3-dimethyl-5-(1-methylethyl)- & $1 \%$ & 0 & 1 & 0 \\
\hline Benzene,1-ethyl-2,3-dimethyl- & $1 \%$ & 0 & 1 & 0 \\
\hline Benzene,1-methyl-4-(1-methylethyl)- & $1 \%$ & 0 & 1 & 0 \\
\hline Benzene,2,4-dimethyl-1-(1-methylethyl)- & $1 \%$ & 0 & 1 & 0 \\
\hline Benzene, diethyl- & $1 \%$ & 1 & 0 & 0 \\
\hline Benzene,ethyl-1,2,4-trimethyl- & $1 \%$ & 0 & 1 & 0 \\
\hline Cyclodecane & $1 \%$ & 1 & 0 & 0 \\
\hline Cyclooctane & $1 \%$ & 1 & 0 & 0 \\
\hline Cyclooctane,1,2-dimethyl- & $1 \%$ & 1 & 0 & 0 \\
\hline Cyclotetradecane & $1 \%$ & 0 & 1 & 0 \\
\hline Dimethylsulfone & $1 \%$ & 1 & 0 & 0 \\
\hline Limonene & $1 \%$ & 1 & 0 & 0 \\
\hline Dodecane,2,6,10-trimethyl- & $1 \%$ & 0 & 1 & 0 \\
\hline Heptadecane,9-octyl- & $1 \%$ & 1 & 0 & 0 \\
\hline Hexanedioicacid,bis(1-methylethyl) ester & $1 \%$ & 0 & 1 & 0 \\
\hline IsopropylMyristate & $1 \%$ & 1 & 0 & 0 \\
\hline Naphthalene,1,6-dimethyl- & $1 \%$ & 0 & 0 & 1 \\
\hline Naphthalene,1,7-dimethyl- & $1 \%$ & 0 & 1 & 0 \\
\hline Naphthalene,2,3-dimethyl- & $1 \%$ & 0 & 0 & 1 \\
\hline Naphthalene,2,6-dimethyl- & $1 \%$ & 0 & 0 & 1 \\
\hline Nonane & $1 \%$ & 0 & 0 & 1 \\
\hline Tetrachloroethylene & $1 \%$ & 0 & 0 & 1 \\
\hline Tetradecanal & $1 \%$ & 1 & 0 & 0 \\
\hline
\end{tabular}


Table 66. Frequency of occurrence of breath odor VOCs in total population and within subgroups (Notes: ${ }^{a} n=70,{ }^{b} n=31,{ }^{c} n=19,{ }^{d} n=20$ )

\begin{tabular}{|c|c|c|c|c|}
\hline \multirow{2}{*}{ Compound Name } & \multirow{2}{*}{$\begin{array}{l}\text { Occurrence in } \\
\text { Total Population }\end{array}$} & \multicolumn{3}{|c|}{ Occurrence within Group } \\
\hline & & Healthy ${ }^{b}$ & $\mathrm{~T}_{2} \mathrm{DB}^{\mathrm{c}}$ & $\mathrm{MDD}^{\mathrm{c}}$ \\
\hline 4-Heptanone & $90 \%$ & 30 & 14 & 19 \\
\hline Carvone & $56 \%$ & 21 & 7 & 11 \\
\hline Nonanal & $51 \%$ & 13 & 11 & 12 \\
\hline Cedrol & $44 \%$ & 6 & 11 & 14 \\
\hline Menthol & $43 \%$ & 12 & 8 & 10 \\
\hline 4-Nonylphenol & $37 \%$ & 22 & 0 & 4 \\
\hline Pyrrole & $31 \%$ & 16 & 2 & 4 \\
\hline Benzene,1-methyl-4-(1-methylethenyl)- & $27 \%$ & 10 & 4 & 5 \\
\hline Benzene,1,4-dichloro- & $26 \%$ & 15 & 0 & 3 \\
\hline Phenol,4-(1,1,3,3-tetramethylbutyl)- & $24 \%$ & 15 & 0 & 2 \\
\hline 4-Terpineol & $23 \%$ & 8 & 5 & 3 \\
\hline 2-Isopropylbenzaldehyde & $21 \%$ & 7 & 3 & 5 \\
\hline.+ - -.-4-Acetyl-1-methylcyclohexene & $19 \%$ & 10 & 0 & 3 \\
\hline Benzaldehyde,3,5-dimethyl- & $19 \%$ & 7 & 2 & 4 \\
\hline Decanal & $19 \%$ & 5 & 4 & 4 \\
\hline 2-Pentanone & $17 \%$ & 3 & 4 & 5 \\
\hline p-Cresol & $17 \%$ & 5 & 2 & 5 \\
\hline 3-Cyclohexen-1-one2-isopropyl-5-methyl- & $16 \%$ & 9 & 0 & 2 \\
\hline $\mathrm{y}$-Terpinene & $16 \%$ & 7 & 3 & 1 \\
\hline Benzaldehyde & $14 \%$ & 4 & 4 & 2 \\
\hline Benzene,1,2-dichloro- & $14 \%$ & 7 & 1 & 2 \\
\hline Benzophenone & $14 \%$ & 7 & 1 & 2 \\
\hline Benzene,1-methyl-4-(1-methylethyl)- & $13 \%$ & 5 & 3 & 1 \\
\hline p-Menthan-3-one & $13 \%$ & 6 & 0 & 3 \\
\hline ButylatedHydroxytoluene & $11 \%$ & 0 & 5 & 3 \\
\hline N,N-Diethylcarbanilide & $11 \%$ & 6 & 2 & 0 \\
\hline p-Menth-1-en-3-one & $11 \%$ & 4 & 2 & 2 \\
\hline$\alpha$-Terpinene & $11 \%$ & 5 & 2 & 1 \\
\hline Butanoicacid,butylester & $10 \%$ & 1 & 5 & 1 \\
\hline 5,9-Undecadien-2-one,6,10-dimethyl-, (E)- & $9 \%$ & 5 & 0 & 1 \\
\hline Dodecanoicacid & $9 \%$ & 4 & 0 & 2 \\
\hline Phenol,nonyl- & $9 \%$ & 6 & 0 & 0 \\
\hline 3-Heptanone & $7 \%$ & 0 & 3 & 2 \\
\hline Benzene,1-methyl-3-(1-methylethyl)- & $7 \%$ & 2 & 1 & 2 \\
\hline Benzeneacetaldehyde,.alpha.-methyl- & $7 \%$ & 3 & 0 & 2 \\
\hline Cyclopropane,isothiocyanato- & $7 \%$ & 3 & 2 & 0 \\
\hline 1,6-Octadien-3-ol,3,7-dimethyl- & $6 \%$ & 3 & 0 & 1 \\
\hline 2-Methoxy-4-vinylphenol & $6 \%$ & 1 & 2 & 1 \\
\hline
\end{tabular}




\begin{tabular}{|c|c|c|c|c|}
\hline Benzene,1,3-dichloro- & $6 \%$ & 2 & 1 & 1 \\
\hline Dibutanoylmorphine & $6 \%$ & 2 & 1 & 1 \\
\hline ValproicAcid & $6 \%$ & 0 & 2 & 2 \\
\hline$\beta$-Damascenone & $6 \%$ & 3 & 0 & 1 \\
\hline 2-Heptanone & $4 \%$ & 3 & 0 & 0 \\
\hline 2-Propenal,3-phenyl- & $4 \%$ & 2 & 0 & 1 \\
\hline Allylisothiocyanate & $4 \%$ & 3 & 0 & 0 \\
\hline Dibutylphthalate & $4 \%$ & 3 & 0 & 0 \\
\hline Dihydrocarvone & $4 \%$ & 2 & 0 & 1 \\
\hline Dimethyltrisulfide & $4 \%$ & 3 & 0 & 0 \\
\hline Ethanone,1-(3-methoxyphenyl)- & $4 \%$ & 3 & 0 & 0 \\
\hline Eucalyptol & $4 \%$ & 2 & 1 & 0 \\
\hline Eugenol & $4 \%$ & 2 & 1 & 0 \\
\hline$\alpha$-Terpineol & $4 \%$ & 0 & 1 & 2 \\
\hline (E)-p-2-Menthen-1-ol & $3 \%$ & 2 & 0 & 0 \\
\hline 1,3,8-p-Menthatriene & $3 \%$ & 2 & 0 & 0 \\
\hline 1-Dodecanol & $3 \%$ & 2 & 0 & 0 \\
\hline 2-Nonenal,(E)- & $3 \%$ & 2 & 0 & 0 \\
\hline 3,5-di-tert-Butyl-4-hydroxybenzaldehyde & $3 \%$ & 1 & 1 & 0 \\
\hline 3-Ethylcyclopentanone & $3 \%$ & 2 & 0 & 0 \\
\hline Benzene,-(1-formylethyl)- & $3 \%$ & 0 & 1 & 1 \\
\hline Benzene,(3-methyl-2-butenyl)- & $3 \%$ & 2 & 0 & 0 \\
\hline Benzeneacetaldehyde & $3 \%$ & 1 & 0 & 1 \\
\hline Benzenemethanol, $\alpha, \alpha, 4$-trimethyl- & $3 \%$ & 2 & 0 & 0 \\
\hline Ethanone,1-(2-hydroxyphenyl)- & $3 \%$ & 0 & 1 & 1 \\
\hline Ethanone,1-(4-methylphenyl)- & $3 \%$ & 2 & 0 & 0 \\
\hline Isobornylacetate & $3 \%$ & 0 & 1 & 1 \\
\hline p-Chloroaniline & $3 \%$ & 0 & 0 & 2 \\
\hline Phenol,2,5-dichloro- & $3 \%$ & 1 & 0 & 1 \\
\hline$\beta$-Thujene & $3 \%$ & 2 & 0 & 0 \\
\hline (3E,5Z)-1,3,5-Undecatriene & $1 \%$ & 1 & 0 & 0 \\
\hline 1-Butene,4-isothiocyanato- & $1 \%$ & 0 & 1 & 0 \\
\hline p-Mentha-1,8-dien-7-ol & $1 \%$ & 1 & 0 & 0 \\
\hline 1-Octanol & $1 \%$ & 1 & 0 & 0 \\
\hline 1-Undecanol & $1 \%$ & 1 & 0 & 0 \\
\hline 2,3-Dehydro-1,8-cineole & $1 \%$ & 1 & 0 & 0 \\
\hline 2,3-Octanedione & $1 \%$ & 1 & 0 & 0 \\
\hline 2,4-Cycloheptadien-1-one,2,6,6-trimethyl- & $1 \%$ & 0 & 0 & 1 \\
\hline 3-Allyl-6-methoxyphenol & $1 \%$ & 1 & 0 & 0 \\
\hline 3-Heptanone,6-methyl- & $1 \%$ & 1 & 0 & 0 \\
\hline 3-Nonen-2-one & $1 \%$ & 0 & 1 & 0 \\
\hline 3-Octen-2-one & $1 \%$ & 0 & 0 & 1 \\
\hline 4'-(2-Methylpropyl)acetophenone & $1 \%$ & 1 & 0 & 0 \\
\hline 4-Hepten-3-one,4-methyl- & $1 \%$ & 0 & 1 & 0 \\
\hline
\end{tabular}




\begin{tabular}{|c|c|c|c|c|}
\hline Acetone & $1 \%$ & 0 & 1 & 0 \\
\hline Benzaldehyde,3-chloro- & $1 \%$ & 0 & 0 & 1 \\
\hline Benzaldehyde,4-chloro- & $1 \%$ & 0 & 0 & 1 \\
\hline Benzenamine,2,6-dimethyl- & $1 \%$ & 0 & 0 & 1 \\
\hline Benzenamine,4-chloro-2-(trifluoromethyl)- & $1 \%$ & 0 & 0 & 1 \\
\hline Benzene,(2-isothiocyanatoethyl)- & $1 \%$ & 0 & 1 & 0 \\
\hline $\begin{array}{l}\text { Benzene,1-(1,5-dimethyl-4-hexenyl)-4- } \\
\text { methyl- }\end{array}$ & $1 \%$ & 0 & 1 & 0 \\
\hline Benzene,1-methyl-2-(1-methylethyl)- & $1 \%$ & 1 & 0 & 0 \\
\hline Benzene,1-pentenyl- & $1 \%$ & 1 & 0 & 0 \\
\hline Benzene,4-ethyl-1,2-dimethyl- & $1 \%$ & 0 & 0 & 1 \\
\hline BenzoicAcid & $1 \%$ & 1 & 0 & 0 \\
\hline Benzoicacid,2-amino-,methylester & $1 \%$ & 0 & 0 & 1 \\
\hline BenzylAlcohol & $1 \%$ & 0 & 1 & 0 \\
\hline cis- $\beta$-Terpineol & $1 \%$ & 1 & 0 & 0 \\
\hline Cyclodecane & $1 \%$ & 1 & 0 & 0 \\
\hline Cyclopentane,(methylthio)- & $1 \%$ & 1 & 0 & 0 \\
\hline Cyclotetradecane & $1 \%$ & 0 & 0 & 1 \\
\hline DiethylPhthalate & $1 \%$ & 1 & 0 & 0 \\
\hline Dimethylsulfone & $1 \%$ & 1 & 0 & 0 \\
\hline Diphenylamine & $1 \%$ & 1 & 0 & 0 \\
\hline E-2-Tetradecen-1-ol & $1 \%$ & 0 & 1 & 0 \\
\hline Ethanone,1-(4-chlorophenyl)- & $1 \%$ & 0 & 0 & 1 \\
\hline Heptadecane & $1 \%$ & 1 & 0 & 0 \\
\hline Hexadecane & $1 \%$ & 1 & 0 & 0 \\
\hline IsopropylPalmitate & $1 \%$ & 0 & 0 & 1 \\
\hline Naphthalene,2,3-dimethyl- & $1 \%$ & 0 & 1 & 0 \\
\hline Naphthalene,2-methyl- & $1 \%$ & 0 & 1 & 0 \\
\hline Nonanoicacid & $1 \%$ & 0 & 1 & 0 \\
\hline Phenol,2-methoxy- & $1 \%$ & 0 & 1 & 0 \\
\hline Phenol,2-methyl-5-(1-methylethyl) & $1 \%$ & 1 & 0 & 0 \\
\hline Phenol,4-ethyl-2-methoxy- & $1 \%$ & 1 & 0 & 0 \\
\hline Pulegone & $1 \%$ & 1 & 0 & 0 \\
\hline Thymol & $1 \%$ & 1 & 0 & 0 \\
\hline Toluene & $1 \%$ & 0 & 0 & 1 \\
\hline trans-p-Mentha-2,8-dienol & $1 \%$ & 1 & 0 & 0 \\
\hline$\alpha$-Cedrene & $1 \%$ & 1 & 0 & 0 \\
\hline$\alpha$-Cedreneoxide & $1 \%$ & 1 & 0 & 0 \\
\hline$\alpha$-Cubebene & $1 \%$ & 0 & 1 & 0 \\
\hline$\beta$-Phellandrene & $1 \%$ & 1 & 0 & 0 \\
\hline
\end{tabular}


The chi-square significance test was performed on the frequency of occurrences of each of the volatile compounds extracted from the headspace above the collected biological specimen samples across the three population subgroups of interest. The chi-square test was used to test whether the observed frequencies of occurrence (proportions of the compound occurrence within the subgroup) differ significantly from those which would be expected on the null hypothesis. The null hypothesis $\left(\mathrm{H}_{0}\right)$ in this case is that there is no significant difference in the proportion of the volatile compound subjected to the chisquare test between the compared population subgroups. The alternate hypothesis $\left(\mathrm{H}_{\mathrm{a}}\right)$ is that the population subgroups do not have the same proportions of the volatile compounds subjected to the chi-square test (statistically significantly different). The chisquare equation for two-way tables is as follows:

$$
\chi^{2}=\sum \quad \frac{(\text { Observed-Expected })^{2}}{\text { Expected }} \quad \text { Equation } 6
$$

Table 67 to Table 71 list the compounds that were found to be different $(p<0.05)$ between the healthy, diabetic, and depressed subgroups on the basis of results of chi-square tests. At a $95 \%$ confidence interval and two degrees of freedom, compounds with a chi-square value of greater than 5.99 was assumed to be significant in the proportion of that particular compound being present in a given specimen across the three subgroups. For the five biological specimens studied, the number of compounds that were assumed to be significantly different between three subgroups was as follows: fifteen for hand odor, fourty-seven for buccal swabs, twenty-nine for both breath and blood, and twenty-seven for urine. It should be noted that the frequency differences in majority of the compounds 
listed in Table 62 through Table 66 are not statistically significant and therefore will not be used for future studies.

Performing the chi-square test to the data obtained through the present study is a way of data mining in order to reduce the data volume for further future statistical analyses. The greater the dataset size, the greater the probability of encountering errors, where a greater number of compounds will be found to be significantly different by chance. Therefore, by reducing the dataset size, the chances of error can thereby be reduced also. The reduced dataset obtained from the chi-square test will be utilized in the future statistical analyses using principal component analysis and discriminant analysis, in an attempt to identify any potential biomarker that would eventually help in identifying the difference in the VOC profiles of the samples collected from the different population subgroups. 
Table 67. Occurrences of 15 VOCs with significant difference between population subgroups for hand odor through the chi-square test $(p=0.05, \mathrm{dF}=2)$

\begin{tabular}{|c|c|c|c|c|c|c|c|}
\hline \multirow{2}{*}{ Compound Name } & \multicolumn{3}{|c|}{ Observed } & \multicolumn{3}{|c|}{ Expected } & \multirow{2}{*}{$\chi^{2}$ value } \\
\hline & Healthy & T2DB & MDD & He althy & T2DB & MDD & \\
\hline Undecanal & 30 & 7 & 10 & 31 & 19 & 20 & 12.61 \\
\hline Octadecane & 18 & 11 & 17 & 31 & 19 & 20 & 9.27 \\
\hline 5,9-Undecadien-2-one,6,10-dimethyl-, (E)- & 23 & 9 & 12 & 31 & 19 & 20 & 10.53 \\
\hline Tridecane & 22 & 9 & 12 & 31 & 19 & 20 & 11.08 \\
\hline Undecane & 15 & 11 & 12 & 31 & 19 & 20 & 14.83 \\
\hline Dodecane & 14 & 5 & 7 & 31 & 19 & 20 & 28.09 \\
\hline BenzylAlcohol & 13 & 2 & 5 & 31 & 19 & 20 & 36.91 \\
\hline Dodecanal & 10 & 2 & 4 & 31 & 19 & 20 & 42.24 \\
\hline 1,6-Octadien-3-ol,3,7-dimethyl- & 7 & 1 & 3 & 31 & 19 & 20 & 50.08 \\
\hline 1-Hexanol,2-ethyl- & 6 & 2 & 3 & 31 & 19 & 20 & 49.82 \\
\hline Dodecanoicacid,methylester & 3 & 4 & 3 & 31 & 19 & 20 & 51.58 \\
\hline 2-Nonenal,(E)- & 3 & 4 & 1 & 31 & 19 & 20 & 55.18 \\
\hline Acetophenone & 3 & 3 & 2 & 31 & 19 & 20 & 54.96 \\
\hline Benzaldehyde & 4 & 1 & 2 & 31 & 19 & 20 & 56.77 \\
\hline Octanoicacid,methylester & 2 & 1 & 1 & 31 & 19 & 20 & 62.23 \\
\hline
\end{tabular}


Table 68. Occurrences of 47 VOCs with significant difference between population subgroups for buccal swab through the chi-square test $(p=0.05, \mathrm{dF}=2)$

\begin{tabular}{|c|c|c|c|c|c|c|c|}
\hline \multirow{2}{*}{ Compound Name } & \multicolumn{3}{|c|}{ Observed } & \multicolumn{3}{|c|}{ Expected } & \multirow{2}{*}{$\chi^{2}$ value } \\
\hline & Healthy & T2DB & MDD & Healthy & T2DB & MDD & \\
\hline 6-Dodecanone & 24 & 14 & 11 & 30 & 19 & 20 & 6.57 \\
\hline 2-Nonenal,(E)- & 25 & 6 & 13 & 30 & 19 & 20 & 12.18 \\
\hline Hexadecanoicacid,ethylester & 20 & 10 & 13 & 30 & 19 & 20 & 10.05 \\
\hline Tetradecanoicacid,ethylester & 20 & 11 & 12 & 30 & 19 & 20 & 9.90 \\
\hline 1-Tetradecene & 17 & 13 & 12 & 30 & 19 & 20 & 10.73 \\
\hline 1,1'-Biphenyl,2,2'-diethyl- & 13 & 11 & 15 & 30 & 19 & 20 & 14.25 \\
\hline Octanoicacid,ethylester & 19 & 7 & 9 & 30 & 19 & 20 & 17.66 \\
\hline 6-Methyl-3,5-heptadiene-2-one & 10 & 10 & 10 & 30 & 19 & 20 & 22.60 \\
\hline Hexanoicacid,ethylester & 20 & 3 & 6 & 30 & 19 & 20 & 26.61 \\
\hline 1-Dodecene & 13 & 6 & 9 & 30 & 19 & 20 & 24.58 \\
\hline 1-Decene & 8 & 11 & 8 & 30 & 19 & 20 & 26.70 \\
\hline Tetradecane & 10 & 10 & 7 & 30 & 19 & 20 & 26.05 \\
\hline Benzaldehyde & 12 & 8 & 5 & 30 & 19 & 20 & 28.42 \\
\hline Hexanal & 18 & 1 & 5 & 30 & 19 & 20 & 33.10 \\
\hline Naphthalene,1-methyl- & 14 & 2 & 6 & 30 & 19 & 20 & 33.54 \\
\hline Hexanoicacid,pentylester & 13 & 2 & 6 & 30 & 19 & 20 & 34.64 \\
\hline Naphthalene,2-methyl- & 12 & 4 & 4 & 30 & 19 & 20 & 35.44 \\
\hline 2-Tetradecene,(E)- & 7 & 4 & 6 & 30 & 19 & 20 & 39.28 \\
\hline Dodecanoicacid & 7 & 5 & 5 & 30 & 19 & 20 & 39.20 \\
\hline Heptanoicacid & 1 & 8 & 8 & 30 & 19 & 20 & 41.60 \\
\hline Heptanoicacid,ethylester & 8 & 3 & 6 & 30 & 19 & 20 & 39.41 \\
\hline 1,1'-Biphenyl,4-methyl- & 2 & 6 & 8 & 30 & 19 & 20 & 42.23 \\
\hline 1-Hexanol & 7 & 6 & 3 & 30 & 19 & 20 & 40.98 \\
\hline 2-Octenoicacid & 9 & 4 & 3 & 30 & 19 & 20 & 40.99 \\
\hline Dibutanoylmorphine & 11 & 2 & 2 & 30 & 19 & 20 & 43.44 \\
\hline Pentanoicacid & 5 & 3 & 7 & 30 & 19 & 20 & 42.76 \\
\hline Decanoicacid,ethylester & 6 & 3 & 5 & 30 & 19 & 20 & 43.92 \\
\hline DiethylPhthalate & 9 & 3 & 2 & 30 & 19 & 20 & 44.37 \\
\hline Linalool Oxide & 11 & 1 & 2 & 30 & 19 & 20 & 45.29 \\
\hline Pentadecanoicacid,ethylester & 7 & 2 & 5 & 30 & 19 & 20 & 44.09 \\
\hline Naphthalene,2,7-dimethyl- & 6 & 3 & 4 & 30 & 19 & 20 & 45.47 \\
\hline Furfural & 8 & 1 & 3 & 30 & 19 & 20 & 47.64 \\
\hline 1-Octanol & 9 & 1 & 1 & 30 & 19 & 20 & 49.80 \\
\hline Hexadecane & 6 & 1 & 4 & 30 & 19 & 20 & 49.05 \\
\hline 2(3H)-Furanone,5-ethyldihydro- & 3 & 5 & 2 & 30 & 19 & 20 & 50.82 \\
\hline 2(3H)-Furanone, dihydro-5-pentyl- & 3 & 5 & 2 & 30 & 19 & 20 & 50.82 \\
\hline Acetophenone & 3 & 3 & 4 & 30 & 19 & 20 & 50.57 \\
\hline Phenol & 2 & 4 & 4 & 30 & 19 & 20 & 50.78 \\
\hline Benzene,1-methyl-2-[(4-methylphenyl)methyl]- & 3 & 4 & 2 & 30 & 19 & 20 & 52.34 \\
\hline Benzothiazole & 3 & 4 & 2 & 30 & 19 & 20 & 52.34 \\
\hline Caryophyllene & 5 & 3 & 1 & 30 & 19 & 20 & 52.36 \\
\hline 2,5-Cyclohexadiene-1,4-dione,2,6-bis(1,1-dimethylethyl)- & 4 & 1 & 3 & 30 & 19 & 20 & 54.04 \\
\hline E-11-Hexadecenoicacid,ethylester & 2 & 1 & 5 & 30 & 19 & 20 & 54.44 \\
\hline IsopropylPalmitate & 2 & 1 & 2 & 30 & 19 & 20 & 59.39 \\
\hline ButylatedHydroxytoluene & 1 & 2 & 1 & 30 & 19 & 20 & 61.29 \\
\hline Hexadecanoicacid,methylester & 1 & 2 & 1 & 30 & 19 & 20 & 61.29 \\
\hline Tributylphosphate & 1 & 1 & 1 & 30 & 19 & 20 & 63.14 \\
\hline
\end{tabular}


Table 69. Occurrences of 29 VOCs with significant difference between population subgroups for breath through the chi-square test $(p=0.05, \mathrm{dF}=2)$

\begin{tabular}{|l|c|c|c|c|c|c|c|}
\hline \multicolumn{1}{|c|}{ Compound Name } & \multicolumn{3}{|c|}{ Observed } & \multicolumn{3}{c|}{ Expected } & \multirow{2}{*}{ Healthy } \\
\cline { 2 - 8 } & T2DB & MDD & Healthy & T2DB & MDD & $\chi^{2}$ value \\
\hline Benzophenone & 29 & 10 & 14 & 31 & 19 & 20 & 6.19 \\
\hline Benzene,1,2-dichloro- & 25 & 10 & 16 & 31 & 19 & 20 & 6.22 \\
\hline Styrene & 29 & 6 & 11 & 31 & 19 & 20 & 13.07 \\
\hline ButylatedHydroxytoluene & 24 & 6 & 12 & 31 & 19 & 20 & 13.68 \\
\hline Dodecane & 13 & 13 & 13 & 31 & 19 & 20 & 14.80 \\
\hline Tetradecane & 11 & 13 & 13 & 31 & 19 & 20 & 17.25 \\
\hline Tridecane & 10 & 13 & 12 & 31 & 19 & 20 & 19.32 \\
\hline Undecane & 13 & 10 & 12 & 31 & 19 & 20 & 17.91 \\
\hline Phenol & 18 & 3 & 12 & 31 & 19 & 20 & 22.13 \\
\hline Toluene & 8 & 6 & 7 & 31 & 19 & 20 & 34.41 \\
\hline Undecanal & 9 & 4 & 5 & 31 & 19 & 20 & 38.71 \\
\hline Benzene,1,3,5-trimethyl- & 4 & 6 & 7 & 31 & 19 & 20 & 40.86 \\
\hline 2,6-Diisopropylnaphthalene & 13 & 2 & 1 & 31 & 19 & 20 & 43.71 \\
\hline 5,9-Undecadien-2-one,6,10-dimethyl-, (E)- & 9 & 1 & 4 & 31 & 19 & 20 & 45.47 \\
\hline p-Benzoquinone & 1 & 6 & 5 & 31 & 19 & 20 & 49.18 \\
\hline Benzene,4-ethyl-1,2-dimethyl- & 2 & 5 & 5 & 31 & 19 & 20 & 48.69 \\
\hline Dodecanal & 8 & 1 & 3 & 31 & 19 & 20 & 48.57 \\
\hline Naphthalene & 5 & 3 & 2 & 31 & 19 & 20 & 51.48 \\
\hline Benzene,1,2,3-trimethyl- & 2 & 2 & 5 & 31 & 19 & 20 & 53.59 \\
\hline Benzene,1-ethyl-2,4-dimethyl- & 1 & 2 & 5 & 31 & 19 & 20 & 55.49 \\
\hline Benzene,1-ethyl-2-methyl- & 1 & 2 & 4 & 31 & 19 & 20 & 57.04 \\
\hline Diisopropylnaphthalene & 4 & 2 & 1 & 31 & 19 & 20 & 56.78 \\
\hline Limonene & 1 & 2 & 3 & 31 & 19 & 20 & 58.69 \\
\hline Naphthalene,2-methyl- & 1 & 1 & 4 & 31 & 19 & 20 & 58.88 \\
\hline 1,3,5,7-Cyclooctatetraene & 1 & 3 & 1 & 31 & 19 & 20 & 60.56 \\
\hline Benzene,1,4-dichloro- & 3 & 1 & 1 & 31 & 19 & 20 & 60.39 \\
\hline DiethylPhthalate & 1 & 1 & 2 & 31 & 19 & 20 & 62.28 \\
\hline 2-Propanol,1-propoxy- & 1 & 1 & 1 & 31 & 19 & 20 & 64.13 \\
\hline Benzene,1-methyl-4-(1-methylpropyl)- & 1 & 1 & 1 & 31 & 19 & 20 & 64.13 \\
\hline
\end{tabular}


Table 70. Occurrences of 29 VOCs with significant difference between population subgroups for blood through the chi-square test $(p=0.05, \mathrm{dF}=2)$

\begin{tabular}{|c|c|c|c|c|c|c|c|}
\hline \multirow{2}{*}{ Compound Name } & \multicolumn{3}{|c|}{ Observed } & \multicolumn{3}{|c|}{ Expected } & \multirow{2}{*}{$\chi^{2}$ value } \\
\hline & Healthy & T2DB & MDD & Healthy & T2DB & MDD & \\
\hline Heptadecane & 22 & 12 & 14 & 31 & 19 & 20 & 6.99 \\
\hline BenzylAlcohol & 25 & 6 & 11 & 31 & 19 & 20 & 14.11 \\
\hline Diisopropylnaphthalene & 19 & 7 & 14 & 31 & 19 & 20 & 14.02 \\
\hline Furan,2-butyltetrahydro- & 23 & 9 & 7 & 31 & 19 & 20 & 15.78 \\
\hline Furan,2-pentyl- & 20 & 6 & 7 & 31 & 19 & 20 & 21.25 \\
\hline Decanal & 15 & 9 & 7 & 31 & 19 & 20 & 21.97 \\
\hline 1-Dodecene & 18 & 6 & 5 & 31 & 19 & 20 & 25.60 \\
\hline Cyclohexanone & 19 & 3 & 7 & 31 & 19 & 20 & 26.57 \\
\hline 5,9-Undecadien-2-one,6,10-dimethyl-, (E)- & 14 & 4 & 8 & 31 & 19 & 20 & 28.36 \\
\hline 2-Dodecene,(Z)- & 12 & 6 & 7 & 31 & 19 & 20 & 28.99 \\
\hline Longifolene & 6 & 8 & 9 & 31 & 19 & 20 & 32.58 \\
\hline 1-Decene & 7 & 8 & 7 & 31 & 19 & 20 & 33.40 \\
\hline 1-Octen-3-ol & 8 & 9 & 5 & 31 & 19 & 20 & 33.58 \\
\hline 2-Undecanone & 11 & 3 & 7 & 31 & 19 & 20 & 34.83 \\
\hline Benzene,1,2-dichloro- & 12 & 5 & 3 & 31 & 19 & 20 & 36.41 \\
\hline Octadecane & 6 & 6 & 8 & 31 & 19 & 20 & 36.26 \\
\hline 1-Pentanol & 12 & 2 & 4 & 31 & 19 & 20 & 39.66 \\
\hline 2-Heptanone & 8 & 2 & 5 & 31 & 19 & 20 & 43.53 \\
\hline Heptanal & 5 & 4 & 5 & 31 & 19 & 20 & 44.90 \\
\hline Cyclododecane & 10 & 2 & 1 & 31 & 19 & 20 & 47.49 \\
\hline 1-Dodecanol & 5 & 4 & 3 & 31 & 19 & 20 & 48.10 \\
\hline Benzene,4-ethyl-1,2-dimethyl- & 1 & 1 & 8 & 31 & 19 & 20 & 53.28 \\
\hline Naphthalene & 1 & 6 & 3 & 31 & 19 & 20 & 52.38 \\
\hline Xylenes & 3 & 2 & 4 & 31 & 19 & 20 & 53.30 \\
\hline 4-Cyanocyclohexene & 2 & 3 & 3 & 31 & 19 & 20 & 55.05 \\
\hline 2-Propanol,1-propoxy- & 1 & 3 & 2 & 31 & 19 & 20 & 58.71 \\
\hline Benzene,1,4-dichloro- & 4 & 1 & 1 & 31 & 19 & 20 & 58.62 \\
\hline Ethanol,2-butoxy- & 3 & 1 & 1 & 31 & 19 & 20 & 60.39 \\
\hline 1,1'-Biphenyl,2-methyl- & 1 & 1 & 1 & 31 & 19 & 20 & 64.13 \\
\hline
\end{tabular}


Table 71. Occurrences of 27 VOCs with significant difference between population subgroups for urine through the chi-square test $(p=0.05, \mathrm{dF}=2)$

\begin{tabular}{|l|c|c|c|c|c|c|c|}
\hline \multicolumn{1}{|c|}{ Compound Name } & \multicolumn{3}{|c|}{ Observed } & \multicolumn{3}{c|}{ Expected } & $\chi^{2}$ value \\
\cline { 2 - 9 } & Healthy & T2DB & MDD & Healthy & T2DB & MDD & (11 \\
\hline Carvone & 21 & 7 & 11 & 31 & 19 & 20 & 14.85 \\
Nonanal & 13 & 11 & 12 & 31 & 19 & 20 & 17.02 \\
\hline Cedrol & 6 & 11 & 14 & 31 & 19 & 20 & 25.33 \\
\hline Menthol & 12 & 8 & 10 & 31 & 19 & 20 & 23.01 \\
\hline Pyrrole & 16 & 2 & 4 & 31 & 19 & 20 & 35.27 \\
\hline Benzene,1-methyl-4-(1-methylethenyl)- & 10 & 4 & 5 & 31 & 19 & 20 & 37.32 \\
\hline 4-Terpineol & 8 & 5 & 3 & 31 & 19 & 20 & 41.83 \\
\hline 2-Isopropylbenzaldehyde & 7 & 3 & 5 & 31 & 19 & 20 & 43.30 \\
\hline Benzaldehyde,3,5-dimethyl- & 7 & 2 & 4 & 31 & 19 & 20 & 46.59 \\
\hline Decanal & 5 & 4 & 4 & 31 & 19 & 20 & 46.45 \\
\hline 2-Pentanone & 3 & 4 & 5 & 31 & 19 & 20 & 48.38 \\
\hline -Cresol & 5 & 2 & 5 & 31 & 19 & 20 & 48.27 \\
\hline $\boldsymbol{\gamma}$-Terpinene & 7 & 3 & 1 & 31 & 19 & 20 & 50.10 \\
\hline Benzaldehyde & 4 & 4 & 2 & 31 & 19 & 20 & 51.56 \\
\hline Benzene,1,2-dichloro- & 7 & 1 & 2 & 31 & 19 & 20 & 51.83 \\
\hline Benzophenone & 7 & 1 & 2 & 31 & 19 & 20 & 51.83 \\
\hline Benzene,1-methyl-4-(1-methylethyl)- & 5 & 3 & 1 & 31 & 19 & 20 & 53.33 \\
\hline$\alpha$-Terpinene & 5 & 2 & 1 & 31 & 19 & 20 & 55.07 \\
\hline p-Menth-1-en-3-one & 4 & 2 & 2 & 31 & 19 & 20 & 54.93 \\
\hline Butanoicacid,butylester & 1 & 5 & 1 & 31 & 19 & 20 & 57.40 \\
\hline Benzene,1-methyl-3-(1-methylethyl)- & 2 & 1 & 2 & 31 & 19 & 20 & 60.38 \\
\hline 2-Methoxy-4-vinylphenol & 1 & 2 & 1 & 31 & 19 & 20 & 62.29 \\
\hline Benzene,1,3-dichloro- & 2 & 1 & 1 & 31 & 19 & 20 & 62.23 \\
\hline Dibutanoylmorphine & 2 & 1 & 1 & 31 & 19 & 20 & 62.23 \\
\hline
\end{tabular}




\subsection{Human Biological Specimen Compound Database}

Four hundred fifty three (453) compounds were extracted across all samples of hand odor, buccal swab, breath, blood, and urine from the 70 subjects ( 31 healthy, 19 diabetic, 20 depressed) taken during this study. Of these 453 compounds, 111 have been omitted from the biological specimen database for one or more of the following reasons: compound identity was questionable or completely unidentifiable, compound was a known background compound of environment and/or collection material, compound was a known laboratory solvent. The remaining 342 compounds extracted and identified from the headspace of five biological specimen samples from all 70 subjects of this study are presented in Table 72. Compounds were identified by the standard mass spectrum in the NIST library. Where standard reference compounds were available, the compounds were identified by using both the standard mass spectrum in the NIST library and the corresponding reference standard retention time and spectrum. As noted in Table 72, many of the identified compounds can be attributed to exogenous sources, as the majority of the identified compounds are used in the flavor and fragrance industries. Common names for some of the compounds are given in parentheses and italicized. Many of the presented compounds have been previously reported to be volatile emanation compounds of human $\operatorname{skin}^{5,14,28,29,123}$, saliva ${ }^{127}$, breath ${ }^{44,48,97,128}$, volatile constituents of human blood $^{18,62,66,67}$, and urine $\mathrm{e}^{70-72}$. Compounds denoted by "en" are possibly endogenous compounds, while compounds denoted by "ex" are possibly of exogenous origin. Compounds denoted by "food" are known to be present in food and beverages (vegetables, fruits, dairy, meat, alcohol, beverages, etc.). The compounds that are denoted with both "en" and "ex" are compounds used in flavor and/or fragrance industry, but can 
also be found occurring naturally in the body. However, whether those compounds are actually occurring naturally in the body or seem to be occurring naturally due to food consumption or exposure to the exogenous-origin compounds is unclear. 
Table 72 Biological specimen compound database (Notes:*Identity verified by standard comparison; en=possibly endogenous; ex=probably exogenous; food=food origin)

\begin{tabular}{|c|c|c|c|c|c|c|c|c|c|}
\hline Compound Name & HD & & $\begin{array}{c}\text { ecim } \\
\text { BR }\end{array}$ & & UR & CAS\# & Natural Occurrence & Use & Class \\
\hline$(3 E, 5 Z)-1,3,5$-Undecatriene & & & & & $\mathrm{x}$ & $51447-08-6$ & & & \\
\hline.+ +/.-.4-Acetyl-1-methylcyclohexene & & & & & $\mathrm{x}$ & $6090-09-1$ & natural & & \\
\hline 1,1'-Biphenyl,2,2'-diethyl- & & $\mathrm{x}$ & & & & & & & \\
\hline 1,1'-Biphenyl,2-ethyl- & & $\mathrm{x}$ & & & & $1812-51-7$ & & & \\
\hline 1,1'-Biphenyl,2-methyl- & $\mathrm{x}$ & & & $\mathrm{x}$ & & $643-58-3$ & & & \\
\hline 1,1'-Biphenyl,3-methyl- & & $\mathrm{x}$ & & $\mathrm{x}$ & & $643-93-6$ & & & \\
\hline 1,1'-Biphenyl,4-methyl- & $\mathrm{x}$ & $\mathrm{x}$ & $\mathrm{x}$ & $\mathrm{x}$ & & $644-08-6$ & not found in nature & flavor & food \\
\hline 1,2-Benzenediol,3,5-bis(1,1-dimethylethyl)- & $\mathrm{x}$ & & & $\mathrm{x}$ & & $1020-31-1$ & & & \\
\hline 1,3,5,7-Cycl ooctatetraene (Annulene) & & & $\mathrm{x}$ & & & $629-20-9$ & fungi & & \\
\hline 1,3,8-p-Menthatriene & & & & & $\mathrm{x}$ & $21195-59-5$ & & food flavor, chinese medicine & food/ex \\
\hline 1,3-Cycl ohexadiene, 1-methyl-4-(1-methylethyl)- ( $\alpha$-Terpinene) & & & & & $x$ & $99-86-5$ & plant & flavor/fragranœ & food \\
\hline 1,4-Cycl ohexadiene, 1-methyl-4-(1-methylethyl)- ( $\gamma$-Terpinene) & & & & & $\mathrm{x}$ & $99-85-4$ & plant oil & flavor/fragranœ & food/ex \\
\hline 1,6,10-Dodecatriene, 7,11-dimethyl-3-methylene, (Z)- ((Z)- $\beta$-Farnesene) & & $\mathrm{x}$ & & & & $28973-97-9$ & plant oil & & \\
\hline 1,6-Octadien-3-ol,3,7-dimethyl- (Linalool; Linalool oxide) & $\mathrm{x}$ & $\mathrm{x}$ & & & $\mathrm{x}$ & $78-70-6$ & plant & flavor/fragranœ & en/food/ex \\
\hline 11-Od adeœnoicad d,methyl ester & & $\mathrm{x}$ & & & & $52380-33-3$ & & & \\
\hline 1-Butene,4-isothiocyanato- & & & & & $\mathrm{x}$ & $3386-97-8$ & natural, food & flavor & food \\
\hline 1-Cycl ohexene-1-carboxaldehyde,2,6,6-trimethyl- (B -Cyclocitral) & & & & $\mathrm{x}$ & & $432-25-7$ & natural, food & flavor/fragrance & $\mathrm{ex}$ \\
\hline 1-Cyclohexene-1-methanol,4-(1-methylethenyl)- ( $p$-Mentha-1,8-dien-7-ol) & & & & & $\mathrm{x}$ & $536-59-4$ & plant oil & flavor/fragranœ & ex \\
\hline 1-Deøene $e^{*}$ & $\mathrm{x}$ & $\mathrm{x}$ & & $\mathrm{x}$ & & $872-05-9$ & & & \\
\hline 1-Dodecanol* & $x$ & & & $\mathrm{x}$ & $\mathrm{x}$ & $112-53-8$ & plant oil & flavor/fragranœ; pharmaœuticals & food/ex \\
\hline 1-Dodecene ${ }^{*}$ & $\mathrm{x}$ & $\mathrm{x}$ & $x$ & $\mathrm{x}$ & & $112-41-4$ & plant & flavor; solvent; viscosity controlling agent & ex \\
\hline 1-Heptadeœne & & $\mathrm{x}$ & & & & $6765-39-5$ & & & \\
\hline 1-Hexadecene & & $\mathrm{x}$ & & $\mathrm{x}$ & & $629-73-2$ & & & \\
\hline 1-Hexanol* & & $x$ & & & & $111-27-3$ & plant oil, alcohol & & \\
\hline 1-Hexanol,2-ethyl-* & $x$ & & $x$ & $\mathrm{x}$ & $x$ & 104-76-7 & plant & & ex \\
\hline 1-Octanol ${ }^{*}$ & $\mathrm{x}$ & $\mathrm{x}$ & & $\mathrm{x}$ & $\mathrm{x}$ & $111-87-5$ & plant oil & flavor/fragranœ & ex \\
\hline 1-Octen-3-ol* & $\mathrm{x}$ & & & $\mathrm{x}$ & & $3391-86-4$ & plant oil, food & & en/ex \\
\hline 1-Pentadeœne* & $\mathrm{x}$ & $\mathrm{x}$ & & & & $13360-61-7$ & & & \\
\hline 1-Pentanol & $\mathrm{x}$ & $\mathrm{x}$ & & $\mathrm{x}$ & & $71-41-0$ & plant oil, food & & \\
\hline 1-Phenyl-2-butanone (Benzyl ethyl ketone) & $\mathrm{x}$ & & & $\mathrm{x}$ & & $1007-32-5$ & & & \\
\hline 1S- $\alpha$-Pinene* & & & $\mathrm{x}$ & & & $7785-26-4$ & turpentine & flavor/fragranœ & \\
\hline 1-Tetradeone* ${ }^{*}$ & $x$ & $\mathrm{x}$ & & $\mathrm{x}$ & & $1120-36-1$ & & & \\
\hline 1-Undecanol & & & & & $\mathrm{x}$ & $112-42-5$ & plant oil & & \\
\hline 2(3H)-Furanone,5-ethyldihydro- ( $\gamma$-Caprolactone) & & $\mathrm{x}$ & & & & 695-06-7 & natural, insect pheromone & flavor/fragranœ & ex \\
\hline 2(3H)-Furanone, dihydro-5-pentyl- ( $\gamma$-Amylbutyrolactone) & & $\mathrm{x}$ & & & & 104-61-0 & natural, food & flavor/fragranœ & $\mathrm{ex}$ \\
\hline 2(3H)-Furanone, dihydro-5-propyl- ( $\gamma$-Heptanolactone) & & $x$ & & & & $105-21-5$ & natural, food & flavor/fragranœ & ex \\
\hline 2,3-Dehydro-1,8-cineole & & & & & $x$ & $66113-06-2$ & & flavor & \\
\hline 2,3-Octanedione & & & & & $\mathrm{x}$ & $585-25-1$ & fermentation byproduct & flavor & food \\
\hline 2,4-Cycloheptadien-1-one,2,6,6-trimethyl- (Eucarvone) & & & & & $\mathrm{x}$ & $503-93-5$ & natural; pheromone & & food \\
\hline
\end{tabular}




\begin{tabular}{|c|c|c|c|c|c|c|c|c|c|}
\hline 2,4-Nonadienal & & $\mathrm{x}$ & & & & $6750-03-4$ & coffee & & \\
\hline 2,4-Nonadienal,(E,E)-* & & $\mathrm{x}$ & & & & $5910-87-2$ & food & & \\
\hline 2,5-Cycl ohexadiene-1,4-dione,2,6-bis(1,1-dimethylethyl)- (p-Benzoquinone) & $\mathrm{x}$ & $\mathrm{x}$ & $\mathrm{x}$ & $\mathrm{x}$ & $x$ & $719-22-2$ & & pharmaceutical, herbidide, œ smetics & $\mathrm{ex}$ \\
\hline 2,5 -Octanedione & & & & $\mathrm{x}$ & & $3214-41-3$ & & & food \\
\hline 2,6-Diisopropylnaphthalene ${ }^{*}$ & $\mathrm{x}$ & & $\mathrm{x}$ & $\mathrm{x}$ & & $24157-81-1$ & & & \\
\hline 2-Cycl ohexen-1-ol,1-methyl-4-(1-methylethyl)-, trans- ((E)-p-2-Menthen-1-ol) & & & & & $x$ & $29803-81-4$ & & & \\
\hline 2-Cycl ohexen-1-one,2-methyl-5-(1-methylethenyl)- (DL-Carvone) & & & & & $x$ & $99-49-0$ & plant oil & flavor/fragranœ & food/ex \\
\hline 2-Cycl ohexen-1-one,2-methyl-5-(1-methylethenyl)-, (S)- ((+)-Carvone) & & $\mathrm{x}$ & & & $\mathrm{x}$ & $2244-16-8$ & plant oil & flavor/fragranœ & food/ex \\
\hline 2-Cycl ohexen-1-one,3-methyl-6-(1-methylethyl)- (p-Menth-1-en-3-one) & & & & & $\mathrm{x}$ & $89-81-6$ & plant oil & flavor/fragranœ & food/ex \\
\hline 2-Decanone* & $\mathrm{x}$ & & & $\mathrm{x}$ & & $693-54-9$ & plant oil & & \\
\hline 2-Deœnal,(E)- & & $\mathrm{x}$ & & & & $3913-81-3$ & plant oil, food & flavor/fragranœ & food/ex \\
\hline 2-Dodecenal,(E)- & & $x$ & & & & $20407-84-5$ & plant oil, food & flavor/fragranœ & food/ex \\
\hline 2-Dodecene,(Z)- & $\mathrm{x}$ & & $\mathrm{x}$ & $\mathrm{x}$ & & 7206-26-0 & & & \\
\hline 2-Heptanone* & $x$ & & & $\mathrm{x}$ & $x$ & $110-43-0$ & plant oil, alcohol, chese & flavor/fragranœ & food/ex \\
\hline 2-Heptanone,6-methyl- & $\mathrm{x}$ & & & $\mathrm{x}$ & & $928-68-7$ & found in nature & flavor/fragranœ & food/ex \\
\hline 2-Heptenoicacid & & $\mathrm{x}$ & & & & 18999-28-5 & & & \\
\hline 2-Isopropylbenzaldehyde & & & & & $x$ & $6502-22-3$ & & & \\
\hline 2-Methoxy-4-vinylphenol & & & & & $x$ & $7786-61-0$ & natural, food & flavor/fragranœ & ex \\
\hline 2-Nonenal,(E)-* & $\mathrm{x}$ & $x$ & & & $x$ & $18829-56-6$ & food & flavor/fragranœ & food/ex \\
\hline 2 -Octenal, $(\mathrm{E}))^{*}$ & & $\bar{x}$ & & & & $2548-87-0$ & food & flavor/fragranœ & food/ex \\
\hline 2-Octenoicacid & & $\mathrm{x}$ & & & & $1470-50-4$ & & & \\
\hline 2-Pentanone ${ }^{*}$ & $\mathrm{x}$ & & & & $x$ & $107-87-9$ & food & flavor/fragranœ & food/ex \\
\hline 2-Propanol,1-butoxy- & & & & $\mathrm{x}$ & & $5131-66-8$ & & fragranœ & ex \\
\hline 2-Propanol,1-propoxy- & $\mathrm{x}$ & & $\mathrm{x}$ & $\mathrm{x}$ & & 1569-01-3 & & fragranœ & $\mathrm{ex}$ \\
\hline 2-Propenal,3-phenyl- (Cinnamaldehyde) & & & & & $\mathrm{x}$ & $104-55-2$ & plant & flavor/fragranœ & $\mathrm{ex}$ \\
\hline 2-Tetradeœene,(E)- & & $\mathrm{x}$ & & & & $35953-53-8$ & & & \\
\hline 2-Undecanone (Methyl nonyl ketone) & $\mathrm{x}$ & & & $x$ & & $112-12-9$ & plant & flavor/fragranœ; insed/animal repellent & food/ex \\
\hline 3,5,9-Undecatrien-2-one,6,10-dimethyl-, (E,Z)- ((E,Z)-Pseudoionone) & & $\mathrm{x}$ & & & & & plant & flavor & ex \\
\hline 3,5-di-tert-Butyl-4-hydroxybenzaldehyde & & & & & $x$ & $1620-98-0$ & & & \\
\hline 3,7-Dimethyl-octa-1,6-diene (Dyhydromyrcene) & $\mathrm{x}$ & $\mathrm{x}$ & & $\mathrm{x}$ & & $2436-90-0$ & plant & fragranœ & food/ex \\
\hline 3-Allyl-6-methoxyphenol & & & & & $\mathrm{x}$ & $501-19-9$ & & & \\
\hline 3-Cycl ohexen-1-ol,4-methyl-1-(1-methylethyl)- (4-Terpineol) & & & & & $\mathrm{x}$ & $562-74-3$ & plant oil & flavor/fragranœ & food/ex \\
\hline 3-Cyclohexen-1-ol,4-methyl-1-(1-methylethyl)-, (R)- ((-)-4-Terpineol) & & & & & $\mathrm{x}$ & $20126-76-5$ & & & \\
\hline 3-Cyclohexen-1-one2-isopropyl-5-methyl- & & & & & $x$ & & & food additive & food \\
\hline 3-Cycl ohexene-1-methanol, $\alpha, \alpha, 4$-trimethyl-, (S)- ( $\alpha$-Terpineol) & & & & & $x$ & $98-55-5$ & plant oil/fruits & flavor/fragranœ & en/food/ex \\
\hline 3-Dodecene,(Z)- & & & $\mathrm{x}$ & $\mathrm{x}$ & & $7239-23-8$ & & & \\
\hline 3-Eicosene,(E)- & & $\mathrm{x}$ & & & & $74685-33-9$ & & & \\
\hline 3-Ethylcycl opentanone & & & & & $\mathrm{x}$ & $10264-55-8$ & urine & & \\
\hline 3-Heptadeœne,(Z)- & & $\mathrm{x}$ & & & & & & & \\
\hline 3-Heptanone ${ }^{*}$ & & & $\mathrm{x}$ & $\mathrm{x}$ & $x$ & $106-35-4$ & natural & flavor/fragranœ & \\
\hline 3-Heptanone,6-methyl- & & & & & $x$ & $624-42-0$ & pheromone, urine & & \\
\hline 3-Isopropylbenzaldehyde & $\mathrm{x}$ & & & $\mathrm{x}$ & & $34246-57-6$ & & & \\
\hline 3-Nonen-2-one & & $x$ & & & $\mathrm{x}$ & $14309-57-0$ & natural & flavor/fragranœ & \\
\hline 3-Octanol,3,7-dimethyl-,(.+/-.)- & & & $\mathrm{x}$ & & & $57706-88-4$ & synthetic & flavor/fragranœ & $\mathrm{ex}$ \\
\hline
\end{tabular}


3-Octen-2-one

4'-(2-Methyl propyl)acetophenone

4,8-Dimethyl-nona3,8-dien-2-one (Citrinone)

4-Cyanocycl ohexene

4-Heptanone $(G B L)^{*}$

4-Nonylphenol

4-tert-Butylcyclohexylacetate

5,9-Undecadien-2-one,6,10-dimethyl- (Geranyl acetone) ${ }^{*}$

5,9-Undecadien-2-one,6, 10-dimethyl-, (E)- (trans-Geranyl acetone)

5,9-Undecadien-2-one,6,10-dimethyl-, (Z)- (Neryl acetone)*

5-Dodecene, (E)-

5-Hepten-2-one,6-methyl- (Sulcaatone) *

6-Dodecanone

6-Methyl-3,5-heptadiene-2-one

7-Hexadeoene,(Z)-

9-Octadecenoicacid,(E)-

Aceticacid

Aœeticadid,butyl ester

Acetone

Acetophenone* $^{*}$

Allyll sothiocyanate

Azulene

Benzaldehyde ${ }^{*}$

Benzaldehyde,2,4,5-trimethyl- (Duraldehyde)

Benzaldehyde,2,4,6-trimethyl- (Mesitaldehyde)

Benzaldehyde,2-hydroxy- (Salicylaldehyde)

Benzaldehyde,3,5-dimethyl-

Benzaldehyde,3-chloro-

\begin{tabular}{|l|}
\hline Benzaldehyde,3-hydroxy- \\
\hline Benzaddehyde-(1-mehyle
\end{tabular}

Benzaldehyde,4-(1-methylethyl)- ( $p$-Cumic aldehyde)

Benzaldehyde,4-(methylthio)-

Benzaldehyde,4-chloro-

Benzaldehyde,4-methoxy-

Benzaldehyde, ethyl-

Benzenamine,2,6-dimethyl-

Benzenamine,4-chloro-2-(trifluoromethyl)-

Benzene,(1-formylethyl)- (Cumene aldehyde)

Benzene,(1-methyl ethyl)- (Cumene)

Benzene,(2-isothiocyanatoethyl)-

Benzene,(3-methyl-2-butenyl)-

Benzene, 1-(1,5-dimethyl-4-hexenyl)-4-methyl-

Benzene, 1,1'-methylenebis[4-methyl- ( $\alpha$-Curcumene)

Benzene, 1,2,3,4-tetramethyl- (Prehnitene)

Benzene,1,2,3,5-tetramethyl- (/sodurene)

\begin{tabular}{|c|c|c|c|c|c|c|}
\hline & & & & $x$ & $1669-44-9$ & natural \\
\hline & & & & $x$ & $38861-78-8$ & \\
\hline & $\mathrm{x}$ & & & & $817-88-9$ & not found in nature \\
\hline $\mathrm{x}$ & & $\mathrm{x}$ & $\mathrm{x}$ & & $100-45-8$ & \\
\hline \multirow[t]{4}{*}{$x$} & & & $\mathrm{x}$ & $\mathrm{x}$ & $123-19-3$ & food \\
\hline & & & & $\mathrm{x}$ & $104-40-5$ & synthetic, sewage \\
\hline & & & $\mathrm{x}$ & & $32210-23-4$ & synthetic \\
\hline & $\mathrm{x}$ & & & $\mathrm{x}$ & $689-67-8$ & plant oil \\
\hline \multirow[t]{3}{*}{$x$} & $x$ & $\mathrm{x}$ & $x$ & $\mathrm{x}$ & $3796-70-1$ & plant \\
\hline & $x$ & & & & $3879-26-3$ & coffe \\
\hline & & $\mathrm{x}$ & & & $7206-16-8$ & \\
\hline \multirow[t]{6}{*}{$\mathrm{x}$} & & $\mathrm{x}$ & $\mathrm{x}$ & & $110-93-0$ & natural, pheromone \\
\hline & $\mathrm{x}$ & & & & $6064-27-3$ & \\
\hline & $\mathrm{x}$ & & & & 1604-28-0 & plant oil, plant \\
\hline & $\mathrm{x}$ & & & & $35507-09-6$ & \\
\hline & $x$ & & & & $112-79-8$ & \\
\hline & $\mathrm{x}$ & $\mathrm{x}$ & & & $64-19-7$ & plant \\
\hline$x$ & & & & & $123-86-4$ & food \\
\hline$x$ & & $\mathrm{x}$ & $\mathrm{x}$ & $\mathrm{x}$ & 67-64-1 & plant oil, food \\
\hline \multirow[t]{3}{*}{$x$} & $\mathrm{x}$ & $x$ & $x$ & & $98-86-2$ & plant oil, food \\
\hline & & & & $\mathrm{x}$ & 57-06-7 & plant oil \\
\hline & & $\mathrm{x}$ & $\mathrm{x}$ & & $275-51-4$ & \\
\hline$x$ & $\mathrm{x}$ & $x$ & $\bar{x}$ & $x$ & $100-52-7$ & plant oil, food \\
\hline$x$ & & & $\mathrm{x}$ & & $5779-72-6$ & \\
\hline \multirow[t]{4}{*}{$x$} & & & $\mathrm{x}$ & & $487-68-3$ & \\
\hline & & & $\mathrm{x}$ & & $90-02-8$ & plant oil \\
\hline & & & & $\mathrm{x}$ & $5779-95-3$ & \\
\hline & & & & $\mathrm{x}$ & $587-04-2$ & \\
\hline \multirow[t]{2}{*}{$x$} & & & $\mathrm{x}$ & & $100-83-4$ & \\
\hline & $\mathrm{x}$ & & & & $122-03-2$ & plant oil \\
\hline \multirow[t]{14}{*}{$\mathrm{x}$} & & & $\mathrm{x}$ & & $3446-89-7$ & \\
\hline & & & & $\mathrm{x}$ & $104-88-1$ & \\
\hline & & $\mathrm{x}$ & & & $123-11-5$ & plant oil, plant \\
\hline & & & $\mathrm{x}$ & & 53951-50-1 & \\
\hline & & & & $\mathrm{x}$ & $87-62-7$ & \\
\hline & & & & $\mathrm{x}$ & $445-03-4$ & \\
\hline & & & & $x$ & $93-53-8$ & not found in nature \\
\hline & & $\mathrm{x}$ & & & $98-82-8$ & crude oil; refined fuels \\
\hline & & & & $\mathrm{x}$ & $2257-09-2$ & food \\
\hline & & & & $\mathrm{x}$ & $4489-84-3$ & \\
\hline & & & & $\mathrm{x}$ & 644-30-4 & plant oil \\
\hline & $\mathrm{x}$ & & & & $4957-14-6$ & \\
\hline & & $x$ & $\mathrm{x}$ & & $488-23-3$ & \\
\hline & $x$ & $\mathrm{x}$ & $\mathrm{x}$ & & $527-53-7$ & \\
\hline
\end{tabular}

\begin{tabular}{|c|c|}
\hline d garette ingredient; flavor/fragranœ & $\mathrm{ex}$ \\
\hline Ibuprofen related & food \\
\hline \multicolumn{2}{|l|}{ flavor } \\
\hline & en/ex \\
\hline \multirow{2}{*}{ flavor/fragranœ } & food/ex \\
\hline & ex \\
\hline fragranœ & ex \\
\hline flavor/fragranœ & food/ex \\
\hline fragranœ & food/ex \\
\hline & food \\
\hline & \\
\hline \multirow[t]{2}{*}{ flavor/fragranœ } & en/food/ex \\
\hline & \\
\hline flavor & en/food/ex \\
\hline flavor/fragranœ (œ smetic solvent) & food/ex \\
\hline flavor, extraction solvent & en/food/ex \\
\hline \multirow[t]{2}{*}{ flavor/fragranœ } & food/ex \\
\hline & food \\
\hline flavor/fragranœ & en/food/ex \\
\hline \multirow{3}{*}{ flavor/fragranœ } & ex \\
\hline & \\
\hline & \\
\hline $\begin{array}{l}\text { flavor/fragranœe } \\
\text { intermediate (pharmaœuticals, pestioi des) }\end{array}$ & ex \\
\hline intermediate (pharmacuticals, pesticides) & $\mathrm{ex}$ \\
\hline flavor/fragranœ & food/ex \\
\hline synthesis of lidocane & ex \\
\hline dye & $\mathrm{ex}$ \\
\hline $\begin{array}{l}\text { flavor/fragranœe } \\
\text { varnish, cement, primer, auto product }\end{array}$ & $\mathrm{ex}$ \\
\hline $\begin{array}{l}\text { varnish, cement, primer, auto product } \\
\text { flavor }\end{array}$ & ex \\
\hline flavor & food \\
\hline \multicolumn{2}{|l|}{ flavor/fragranœ } \\
\hline & \\
\hline & \\
\hline
\end{tabular}


Benzene, 1,2,3-trimethyl-

\begin{tabular}{|l}
\hline Benzene, 1,2,4,5-tetramethyl- (Durene) \\
\end{tabular}

Benzene, 1,2,4-trimethyl- ( $\psi$-Cumene)

Benzene, 1,2-dichloro-*

Benzene, 1,3,5-trimethyl- (Mesitylene)

Benzene, 1,3-dichloro-

Benzene, 1,3-diethyl-5-methyl-

Benzene, 1,3-dimethyl- ( $m$-Xylene) $)^{*}$

Benzene,1,3-dimethyl-5-(1-methylethyl)-

Benzene, 1,4-dichloro-

Benzene,1-ethyl-2,3-dimethyl-

Benzene, 1-ethyl-2,4-dimethyl-

Benzene, 1-ethyl-2-methyl-

Benzene, 1-ethyl-3,5-dimethyl-

Benzene, 1-ethyl-3-methyl-

Benzene, 1-methyl-2-(1-methylethyl)-

Benzene, 1-methyl-2-[(3-methylphenyl)methyl]

Benzene, 1-methyl-2-[(4-methylphenyl)methyl]-

Benzene, 1-methyl-3-(1-methylethyl)- ( $\beta$-Cymene)

Benzene, 1-methyl-3-propyl-

\begin{tabular}{l|l}
$\sim$ & Benzene,1-methyl-4-(1-methylethenyl)- (a,p-Dimethylstyrene) \\
\cline { 2 - 2 } &
\end{tabular}

Benzene, 1-methyl-4-(1-methylethyl)- ( $p$-Cymene)

Benzene, 1-methyl-4-(1-methylpropyl)-

\begin{tabular}{|l}
\hline Benzene, 1-methyl-4-nitro- \\
\hline Benzene1-meny-4-propy- \\
\hline
\end{tabular}

Benzene, 1-methyl-4-propyl-

Benzene, 1-pentenyl-

Benzene,2,4-dimethyl-1-(1-methylethyl)-

Benzene,2-ethyl-1,3-dimethyl-

Benzene,2-ethyl-1,4-dimethyl-

Benzene,3-cycl ohexen-1-yl-

Benzene,4-ethyl-1,2-dimethyl-

Benzene, diethyl -

Benzene,pentamethyl-

Benzene,propyl-

Benzeneacetaldehyde

Benzeneacetaldehyde, $\mathrm{\alpha}$-methy - (Cumene aldehyde)

Benzeneethanol,2-methyl-

Benzenemethanol, $\alpha, \alpha, 4$-trimethyl- ( $p$-Cymenol)

Benzenemethanol, $\mathrm{\alpha}, \mathrm{\alpha}$-dimethyl- ( $\alpha$-Cumyl alcohol)

BenzoicAcid

Benzoicacid,2-amino-,methylester

Benzophenone*

Benzothiazole

BenzylAlcohol $^{*}$

\begin{tabular}{|c|c|c|c|c|c|c|}
\hline & & $\mathrm{x}$ & $\mathrm{x}$ & & $526-73-8$ & \\
\hline & $x$ & $\mathrm{x}$ & $x$ & & $95-93-2$ & natural \\
\hline & & $\mathrm{x}$ & & & $95-63-6$ & \\
\hline $\mathrm{x}$ & & $x$ & $\mathrm{x}$ & $\mathrm{x}$ & $95-50-1$ & \\
\hline & & $x$ & $x$ & & $108-67-8$ & \\
\hline $\mathrm{x}$ & & $\mathrm{x}$ & $\mathrm{x}$ & $\mathrm{x}$ & 541-73-1 & \\
\hline & & $\mathrm{x}$ & & & $2050-24-0$ & \\
\hline$x$ & & $x$ & $\mathrm{x}$ & & $108-38-3$ & \\
\hline & & & $\mathrm{x}$ & & $4706-90-5$ & \\
\hline $\mathrm{x}$ & & $x$ & $x$ & $\mathrm{x}$ & $106-46-7$ & \\
\hline & $\mathrm{x}$ & $\mathrm{x}$ & $\mathrm{x}$ & & 933-98-2 & \\
\hline & & $\mathrm{x}$ & $\mathrm{x}$ & & 874-41-9 & \\
\hline & & $\mathrm{x}$ & $\mathrm{x}$ & & $611-14-3$ & \\
\hline & & $x$ & $x$ & & 934-74-7 & \\
\hline & & $x$ & $x$ & & $620-14-4$ & \\
\hline & & $x$ & & $\mathrm{x}$ & $527-84-4$ & \\
\hline & $\mathrm{x}$ & & & & $21895-13-6$ & \\
\hline & $x$ & & & & 21895-17-0 & \\
\hline & & $\mathrm{x}$ & & $\mathrm{x}$ & $535-77-3$ & species pheromone \\
\hline $\mathrm{x}$ & & $x$ & $\mathrm{x}$ & & 1074-43-7 & \\
\hline & & & & $\mathrm{x}$ & 1195-32-0 & plant oil \\
\hline & & $\mathrm{x}$ & $\mathrm{x}$ & $x$ & $99-87-6$ & plant oil \\
\hline $\mathrm{x}$ & & $\mathrm{x}$ & $\mathrm{x}$ & & $1595-16-0$ & \\
\hline & $\mathrm{x}$ & & & & $99-99-0$ & \\
\hline & & $\mathrm{x}$ & & & 1074-55-1 & \\
\hline & & & & $\mathrm{x}$ & $826-18-6$ & \\
\hline & & & $\mathrm{x}$ & & $4706-89-2$ & \\
\hline & & $x$ & & & $2870-04-4$ & \\
\hline & & $\mathrm{x}$ & $\mathrm{x}$ & & $1758-88-9$ & \\
\hline & & $\mathrm{x}$ & & & $4994-16-5$ & \\
\hline $\mathrm{x}$ & & $\mathrm{x}$ & $\mathrm{x}$ & $\mathrm{x}$ & $934-80-5$ & \\
\hline $\mathrm{x}$ & & $\mathrm{x}$ & $\mathrm{x}$ & & $25340-17-4$ & not found in nature \\
\hline & & $x$ & & & $700-12-6$ & \\
\hline & & $x$ & & & $103-65-1$ & \\
\hline & & & & $\mathrm{x}$ & 122-78-1 & plant oil \\
\hline & & & & $\mathrm{x}$ & 93-53-8 & not found in nature \\
\hline & & & & & 19819-98-8 & not found in nature \\
\hline & & & & $\mathrm{x}$ & $1197-01-9$ & plant oil \\
\hline$x$ & & & $x$ & & $617-94-7$ & cocoa \\
\hline & & & & $\mathrm{x}$ & $65-85-0$ & plant \\
\hline & & & & $\mathrm{x}$ & 134-20-3 & plant oil \\
\hline $\mathrm{x}$ & $\mathrm{x}$ & $\mathrm{x}$ & $\mathrm{x}$ & $\mathrm{x}$ & 119-61-9 & plant \\
\hline & $x$ & & & & $95-16-9$ & plant oil \\
\hline$x$ & & & $\mathrm{x}$ & $\mathrm{x}$ & $100-51-6$ & plants, plant oil \\
\hline
\end{tabular}

\begin{tabular}{|c|c|}
\hline & $\mathrm{ex}$ \\
\hline \multicolumn{2}{|l|}{ fragranœ } \\
\hline fragranœ & ex \\
\hline & \\
\hline & \\
\hline & \\
\hline & \\
\hline & \\
\hline & \\
\hline & \\
\hline & \\
\hline & \\
\hline flavor/fragranœ & food/ex \\
\hline flavor/fragranœe & ex \\
\hline & \\
\hline & \\
\hline & \\
\hline & \\
\hline & \\
\hline fragranœ & ex \\
\hline & \\
\hline flavor/fragranœ & $\mathrm{ex}$ \\
\hline flavor/fragranœ & ex \\
\hline fragranœ & ex \\
\hline flavor/fragranœ & food/ex \\
\hline flavor/fragranœ & ex \\
\hline food preservative; antimicrobial & food \\
\hline flavor/fragrance, bird repellant for plants & $\mathrm{ex}$ \\
\hline photo-initiator, UV blocker & food/ex \\
\hline flavor/pharmaceuticals & ex \\
\hline flavor/fragranœ, preservative, solvent & food/ex \\
\hline
\end{tabular}


Bicycl o[3.1.0] hex-2-ene,4-methyl-1-(1-methylethyl)- ( $\beta$-Thujene) Butanoicacid,butylester

ButylatedHydroxyt oluene ${ }^{*}$

Calamenene

Caryophyllene*

Cedrol

cis- $\beta$-Terpineol

Cycl odecane

Cycl ododecane

Cyd ohexadecane

Cyd ohexane, methyl-

Cycl ohexane, pentyl-

Cycl ohexanol,5-methyl-2-(1-methylethyl)-, (1 $\alpha, 2 \beta, 5 \alpha)-(D L-M e n t h o l)^{*}$

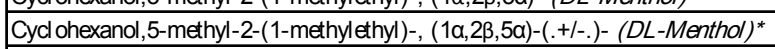

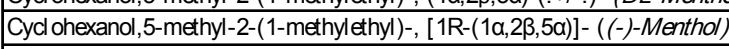

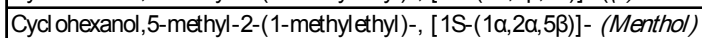

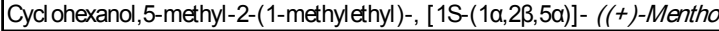

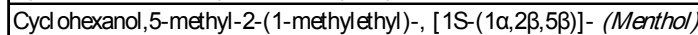

Cyclohexanone $^{*}$

Cyclohexanone,2-methyl-5-(1-methylethenyl)- (Dihydrocarvone)

Cycl ohexanone,5-methyl-2-(1-methylethyl)- (p-Menthan-3-one)

Cycl ohexanone,5-methyl-2-(1-methylethyl)-, cis- (cis-p-Menthan-3-one

Cycl ohexanone,5-methyl-2-(1-methylethyl)-, trans- (trans-p-Menthan-3-one)

Cyclohexene,1-methyl-4-(1-methylethenyl)-, (.+/-.)- (DL-Limonene)*

Cycl ooctane

Cycl opentane,(methylthio)-

Cycl opropane, isothiocyanato-

Cycl opropane, nonyl-

Cyd otetradecane

Decanal $^{*}$

Decane

Decane,2-methy -

Decane,3-methyl -

Decanoicad d,ethyl ester

Dibutanoylmorphine

Dibutylphthalate

DiethylPhthalate

Diisopropylnaphthalene

Dimethylsulfone

Dimethyltrisulfide*

Diphenylamine*

Docosane*

Dodecanal $^{*}$

\begin{tabular}{|c|c|c|c|c|c|c|}
\hline & & & & $\mathrm{x}$ & 28634-89-1 & plant oil \\
\hline & & & & $\mathrm{x}$ & $109-21-7$ & fruits \\
\hline & $\mathrm{x}$ & $\mathrm{x}$ & & $x$ & $128-37-0$ & \\
\hline & $x$ & & & & $483-77-2$ & plant oil \\
\hline & $x$ & $\mathrm{x}$ & & & $87-44-5$ & plant oil \\
\hline $\mathrm{x}$ & & & & $\mathrm{x}$ & $77-53-2$ & plant oil \\
\hline & & & & $\mathrm{x}$ & $7299-40-3$ & \\
\hline $\mathrm{x}$ & $\mathrm{x}$ & & $\mathrm{x}$ & $\mathrm{x}$ & 293-96-9 & \\
\hline $\mathrm{x}$ & $\mathrm{x}$ & $\mathrm{x}$ & $\mathrm{x}$ & & $294-62-2$ & not found in nature \\
\hline & $\mathrm{x}$ & & & & $295-65-8$ & \\
\hline & & $\mathrm{x}$ & & & $108-87-2$ & \\
\hline & & $\mathrm{x}$ & & & $4292-92-6$ & \\
\hline $\mathrm{x}$ & $\mathrm{x}$ & $\mathrm{x}$ & $\mathrm{x}$ & $\mathrm{x}$ & $89-78-1$ & not found in nature \\
\hline $\mathrm{x}$ & & $\mathrm{x}$ & $\mathrm{x}$ & $\mathrm{x}$ & $15356-70-4$ & not found in nature \\
\hline$x$ & & $x$ & $x$ & $x$ & $2216-51-5$ & plant oil \\
\hline$x$ & & $x$ & $x$ & & $2216-52-6$ & not found in nature \\
\hline & & & & $\mathrm{x}$ & & not found in nature \\
\hline & & & & $\mathrm{x}$ & 23283-97-8 & not found in nature \\
\hline $\mathrm{x}$ & & & $\mathrm{x}$ & & 108-94-1 & plant oil \\
\hline & & & & $\mathrm{x}$ & $7764-50-3$ & plant oil \\
\hline & & & & $\mathrm{x}$ & $5948-04-9$ & plant oil \\
\hline & & & & $\mathrm{x}$ & 10458-14-7 & plant oil \\
\hline & & & & $x$ & $491-07-6$ & plant oil \\
\hline & $\mathrm{x}$ & & & $\mathrm{x}$ & $89-80-5$ & plant oil \\
\hline $\mathrm{x}$ & & $\mathrm{x}$ & $\mathrm{x}$ & & $138-86-3$ & plant oil/fruits \\
\hline $\mathrm{x}$ & & & $\mathrm{x}$ & & $292-64-8$ & \\
\hline & & & & $\mathrm{x}$ & $7133-36-0$ & \\
\hline & & & & $\mathrm{x}$ & $56601-42-4$ & \\
\hline $\mathrm{x}$ & $\mathrm{x}$ & & $\mathrm{x}$ & & 74663-85-7 & \\
\hline & $\mathrm{x}$ & & $\mathrm{x}$ & $\mathrm{x}$ & $295-17-0$ & \\
\hline $\mathrm{x}$ & $\mathrm{x}$ & $\mathrm{x}$ & $x$ & $\mathrm{x}$ & $112-31-2$ & plant oil, fruits \\
\hline & & & $x$ & & 124-18-5 & natural \\
\hline & & $\mathrm{x}$ & & & $6975-98-0$ & plant spedies \\
\hline & & $x$ & & & 13151-34-3 & \\
\hline & $\mathrm{x}$ & & & & $110-38-3$ & plant oil \\
\hline & $\mathrm{x}$ & & & $\mathrm{x}$ & 66641-03-0 & synthetic diester of morphine \\
\hline & & $\mathrm{x}$ & & $\mathrm{x}$ & $84-74-2$ & plant oil \\
\hline & $\mathrm{x}$ & $\mathrm{x}$ & & $x$ & $84-66-2$ & not found in nature \\
\hline $\mathrm{x}$ & & $x$ & $\mathrm{x}$ & & $38640-62-9$ & \\
\hline$x$ & & & $x$ & $\mathrm{x}$ & $67-71-0$ & plant \\
\hline & & & & $\mathrm{x}$ & $3658-80-8$ & food \\
\hline & & & & $x$ & $122-39-4$ & natural, species pheromone \\
\hline & $\mathrm{x}$ & & & & $629-97-0$ & plant oil \\
\hline$x$ & $\mathrm{x}$ & $\mathrm{x}$ & & & $112-54-9$ & plant oil \\
\hline
\end{tabular}

\begin{tabular}{|c|c|}
\hline & food \\
\hline flavor/fragranœ & food/ex \\
\hline \multirow{2}{*}{ antioxi dant additive, preservative } & food/ex \\
\hline & food \\
\hline flavor/fragranœ & food \\
\hline \multirow[t]{2}{*}{ flavor/fragranœ } & en/ex \\
\hline & \\
\hline intermediate, volatile binding media & ex \\
\hline \multirow[t]{2}{*}{ organic solvent, jet fuel } & ex \\
\hline & \\
\hline flavor/fragranœ & food/ex \\
\hline flavor/fragranœ & food/ex \\
\hline flavor/fragranœ & food/ex \\
\hline flavor/fragranœ & food/ex \\
\hline flavor/fragranœ & food/ex \\
\hline flavor/fragranœ & food/ex \\
\hline precursor to nylon & ex \\
\hline flavor/fragranœ & food/ex \\
\hline fragranœ & $\mathrm{ex}$ \\
\hline fragranœ & ex \\
\hline fragranœ & ex \\
\hline \multirow[t]{4}{*}{ flavor/fragranœ } & food/ex \\
\hline & \\
\hline & \\
\hline & \\
\hline flavor/fragranœ & en/food/ex \\
\hline solvent/diluent; fragrance & food/ex \\
\hline \multirow[b]{2}{*}{ flavor/fragranœ } & \\
\hline & $\mathrm{ex}$ \\
\hline solvent/diluent for flavor/fragrance agents & $\mathrm{ex}$ \\
\hline solvent (cosmetics); plasticizer & ex \\
\hline food; solvent & en/food/ex \\
\hline flavor/fragranœ & food \\
\hline \multicolumn{2}{|l|}{ scald-inhibitant for apples } \\
\hline fragranœe & ex \\
\hline flavor/fragranœ & ex \\
\hline
\end{tabular}

flavor/fragranœ

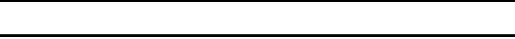

(2)

ter $^{*}$

\begin{tabular}{|l|l}
\hline & \\
\hline & \\
& \\
\hline & \\
\hline & \\
\hline & \\
\hline &
\end{tabular}

$112-54-9$

plant oil 


\begin{tabular}{|c|c|c|c|c|c|c|c|c|c|}
\hline Dodecane* & $\mathrm{x}$ & & $\mathrm{x}$ & $\mathrm{x}$ & & $112-40-3$ & plant oil, food & solvent/diluent; fragrance & ex \\
\hline \begin{tabular}{|l|} 
Dodecane,1-chloro- \\
\end{tabular} & & & & $x$ & & $112-52-7$ & & & \\
\hline Dodecane,2,6,10-trimethyl- & & & & $\mathrm{x}$ & & $3891-98-3$ & & & \\
\hline Dodecanoicacid ${ }^{*}$ & & $\mathrm{x}$ & $\mathrm{x}$ & & $\mathrm{x}$ & $143-07-7$ & plant oil, human milk & & en \\
\hline Dodecanoicacid,ethyl ester & & $\mathrm{x}$ & & & & $106-33-2$ & plant oil, alcohol & flavor/fragranœe & ex \\
\hline Dodecanoicad d,methyl ester ${ }^{*}$ & $\mathrm{x}$ & $\mathrm{x}$ & $\mathrm{x}$ & & & $111-82-0$ & natural; food & flavor/fragranœ & $\mathrm{ex}$ \\
\hline Eicosane ${ }^{*}$ & & $\mathrm{x}$ & $\mathrm{x}$ & & & $112-95-8$ & plant oil & fragranœ & $\mathrm{ex}$ \\
\hline \begin{tabular}{|l|} 
Ethanol,2-butoxy- \\
\end{tabular} & $\mathrm{x}$ & & & $\mathrm{x}$ & & $111-76-2$ & cheese & sanitizing agent, solvent (cosmetics) & ex \\
\hline Ethanol,2-phenoxy- & & & $\mathrm{x}$ & & & $122-99-6$ & plant & preservative (flavor/fragranœ) & food/ex \\
\hline Ethanone,1-(2-hydroxyphenyl)- & & & & & $\mathrm{x}$ & $118-93-4$ & food & flavor/fragranœ & food \\
\hline Ethanone,1-(3-methoxyphenyl)- & & & & & $\mathrm{x}$ & $586-37-8$ & plant oil & flavor/fragranœ & food/ex \\
\hline Ethanone,1-(4-chlorophenyl)- & & & & & $\mathrm{x}$ & $99-91-2$ & & & \\
\hline Ethanone,1-(4-methylphenyl)- & & & & & $\mathrm{x}$ & $122-00-9$ & plant oil, food & flavor/fragranœ & food/ex \\
\hline \begin{tabular}{|l|} 
Ethyl9-hexadecenoate \\
\end{tabular} & & $\mathrm{x}$ & & & & $54546-22-4$ & species pheromone & & \\
\hline Ethyltridecanoate & & $\mathrm{x}$ & & & & $28267-29-0$ & & & \\
\hline Eucalyptol & & & & & $\mathrm{x}$ & $470-82-6$ & plant oil & flavor/fragranœ & ex \\
\hline \begin{tabular}{|l|} 
Eugenol \\
\end{tabular} & & & & & $\mathrm{x}$ & $97-53-0$ & plant oil & flavor/fragranœ & \\
\hline Furan,2-butyltetrahydro- & $\mathrm{x}$ & & & $\mathrm{x}$ & & $1004-29-1$ & not found in nature & flavor & \\
\hline Furan,2-pentyl-** & $\mathrm{x}$ & $\mathrm{x}$ & & $x$ & & $3777-69-3$ & natural; food & $\mid$ flavor/fragranœe & ex \\
\hline \begin{tabular}{|l|} 
Furfural $^{*}$ \\
\end{tabular} & & $\mathrm{x}$ & & & & $98-01-1$ & plant oil & flavor, solvent (cosmetics) & $\mathrm{ex}$ \\
\hline Galaxolide & & $\mathrm{x}$ & $\mathrm{x}$ & & & $1222-05-5$ & not found in nature & fragranœ & ex \\
\hline Heneiøsane* & & $\mathrm{x}$ & & & & $629-94-7$ & plant oil & fragranœ & $\mathrm{ex}$ \\
\hline Heptadecane ${ }^{*}$ & $\mathrm{x}$ & $\mathrm{x}$ & $x$ & $\mathrm{x}$ & $\mathrm{x}$ & $629-78-7$ & plant oil & fragranœ & food/ex \\
\hline Heptadecane,9-oct y- & $\mathrm{x}$ & & & $\mathrm{x}$ & & $7225-64-1$ & plant oil & & food \\
\hline${\text { Heptana }{ }^{*}}$ & $\mathrm{x}$ & & & $\mathrm{x}$ & & $111-71-7$ & fruits, food & flavor/fragranœ & food/ex \\
\hline Heptanoicacid $^{*}$ & & $\mathrm{x}$ & & & & $111-14-8$ & fruits, alcohol & flavor/fragranœ, d garette additive & food/ex \\
\hline Heptanoicaci d,ethyl ester* & & $\mathrm{x}$ & & & & $106-30-9$ & food, alcohol & flavor/fragrance & food/ex \\
\hline Heptanol $^{*}$ & $\mathrm{x}$ & & & $x$ & & $111-70-6$ & food, alcohol & flavor/fragranœe & food/ex \\
\hline Hexadecane* & $\mathrm{x}$ & $\mathrm{x}$ & $\mathrm{x}$ & $\mathrm{x}$ & $\mathrm{x}$ & $544-76-3$ & plant oil & fragranœ & food/ex \\
\hline Hexadecanoicadid,ethyl ester (Ethyl palmitate) & & $\mathrm{x}$ & & & & $628-97-7$ & plant oil, rice, vanilla & flavor/fragranœ & ex \\
\hline Hexadecanoicadid,methyl ester (Methyl palmitate) & & $\mathrm{x}$ & & & & $112-39-0$ & plant oil & flavor/fragranœ & $\mathrm{ex}$ \\
\hline Hexana $^{*}$ & $\mathrm{x}$ & $\mathrm{x}$ & $\mathrm{x}$ & $\mathrm{x}$ & & $66-25-1$ & plant oil, food & flavor/fragranœ & food/ex \\
\hline Hexanedioicad d,bis(1-methyl ethyl) ester (Diisopropyl adipate) & & & $\mathrm{x}$ & $\mathrm{x}$ & & $230-072-0$ & not found in nature & solvent for flavor/fragrance agents & $\mathrm{ex}$ \\
\hline Hexanoicad d ${ }^{*}$ & $\mathrm{x}$ & $\mathrm{x}$ & & & & $142-62-1$ & plant oil, food & flavor/fragranœ & food/ex \\
\hline Hexanoicacid, anhydride & & $\mathrm{x}$ & & & & $2051-49-2$ & & & \\
\hline Hexanoicad d,ethyl ester & & $\mathrm{x}$ & & & & $123-66-0$ & fruits, food & flavor/fragranœ & food/ex \\
\hline Hexanoicad d, pentyl ester & & $\mathrm{x}$ & & & & $540-07-8$ & fruits, cheese & flavor/fragranœ & food/ex \\
\hline Hexanoicacid,propylester & & $\mathrm{x}$ & & & & $626-77-7$ & food, alcohol & flavor/fragranœ & food \\
\hline Hexatriacontane & & & & $\mathrm{x}$ & & $630-06-8$ & & & $\mathrm{ex}$ \\
\hline Homomenthylsalicyl ate & & $\mathrm{x}$ & $\mathrm{x}$ & & & $52253-93-7$ & not found in nature & UV adsorbing agent (sunscreen) & ex \\
\hline Indole & & & $\mathrm{x}$ & & & $120-72-9$ & plant oil, food & flavor/fragranœ & $\mathrm{ex}$ \\
\hline Indolizine & & & $\mathrm{x}$ & & & $274-40-8$ & & & \\
\hline Isobornylacetate & & & $\mathrm{x}$ & & $\mathrm{x}$ & $125-12-2$ & plant oil & flavor/fragranœ & food/ex \\
\hline IsopropylMyristate & $\mathrm{x}$ & $\mathrm{x}$ & & $\mathrm{x}$ & & $110-27-0$ & plant oil & solvent/diluent for flavor/fragrance agents & food/ex \\
\hline
\end{tabular}




\begin{tabular}{|c|c|c|c|c|c|c|c|c|c|}
\hline IsopropylPalmitate & & $\mathrm{x}$ & $\mathrm{x}$ & & $\mathrm{x}$ & 142-91-6 & not found in nature & solvent/diluent for flavor/fragrance agents & ex \\
\hline Lilial & $\mathrm{x}$ & & & & & $80-54-6$ & not found in nature & fragranœ & ex \\
\hline Longicycl ene & & & $\mathrm{x}$ & & & $1137-12-8$ & plant oil & & $\mathrm{ex}$ \\
\hline Longifolene & $\mathrm{x}$ & & $\mathrm{x}$ & $\mathrm{x}$ & & $475-20-7$ & plant oil & flavor/fragranœ & $\mathrm{ex}$ \\
\hline Methyldihydrojasmonate & & & $\mathrm{x}$ & & & $24851-98-7$ & plant & flavor/fragranœ & ex \\
\hline Methyl Salicy ate & & & $\mathrm{x}$ & & $\mathrm{x}$ & $119-36-8$ & plant oil, fruits & flavor/fragranœ, aspirin & food/ex \\
\hline $\mathrm{N}, \mathrm{N}$-Diethylcarbanilide (Ethyl centralite) & & & & & $\mathrm{x}$ & $85-98-3$ & & celluloid plasticizer, explosives stabilizer & $\mathrm{ex}$ \\
\hline Naphthalene ${ }^{*}$ & $\mathrm{x}$ & & $\mathrm{x}$ & $\mathrm{x}$ & & $91-20-3$ & natural & & \\
\hline Naphthalene,1,6-dimethyl- & & & & $\mathrm{x}$ & & $575-43-9$ & & & \\
\hline Naphthalene, 1,7-dimethyl- & & & & $\mathrm{x}$ & & $575-37-1$ & & & \\
\hline Naphthalene,1-methyl-** & & $\mathrm{x}$ & & $\mathrm{x}$ & & $90-12-0$ & food & flavor & food \\
\hline Naphthalene,2,3,6-trimethyl- & $\mathrm{x}$ & & & $\mathrm{x}$ & & $829-26-5$ & & & \\
\hline Naphthalene,2,3-dimethyl- & & & & $\mathrm{x}$ & $\mathrm{x}$ & $581-40-8$ & & & \\
\hline Naphthalene,2,6-dimethyl- & & $\mathrm{x}$ & & $\mathrm{x}$ & & $581-42-0$ & & & \\
\hline Naphthalene,2,7-dimethyl- & & $\mathrm{x}$ & & $\mathrm{x}$ & & $582-16-1$ & & & \\
\hline Naphthalene,2-methyl- & & $\mathrm{x}$ & $\mathrm{x}$ & $\mathrm{x}$ & $\mathrm{x}$ & $91-57-6$ & plant/fruits & flavor/fragranœ & ex \\
\hline$n$-Decanoicacid ${ }^{*}$ & & & $\mathrm{x}$ & & & $334-48-5$ & plant oil, food, mamalian milk & flavor/fragrance, food additive, phamaceuticals & food/ex \\
\hline n-Hexadecanoicadi d* & & & $\mathrm{x}$ & & & $57-10-3$ & plant oil, food & thicke ner, emulsifier,stabilizer, pharmaœuticals & food/ex \\
\hline Nonadecane & & & $\mathrm{x}$ & & & $629-92-5$ & plant & fragranœ & food/ex \\
\hline Nonadecanoicacid,ethyl ester & & $\mathrm{x}$ & & & & 18281-04-4 & alcohol & & food \\
\hline Nonana $\left.\right|^{*}$ & $\mathrm{x}$ & & $\mathrm{x}$ & $\mathrm{x}$ & $\mathrm{x}$ & $124-19-6$ & plant oil, food & flavor/fragranœ & en/food/ex \\
\hline Nonane $^{*}$ & & & & $\mathrm{x}$ & & $111-84-2$ & plant oil & fragranœ & $\mathrm{ex}$ \\
\hline Nonane,3-methyl- & & & $\mathrm{x}$ & & & $5911-04-6$ & & & \\
\hline Nonanoicacid & & $\mathrm{x}$ & $\mathrm{x}$ & & $\mathrm{x}$ & $112-05-0$ & plant oil, fruits & flavor, cosmetic (solvent, perfumery) & food/ex \\
\hline Nonanoicacid,ethylester* & & $\mathrm{x}$ & & & & $123-29-5$ & fruits, alcohol & flavor/fragranœ & ex \\
\hline Odt adecanal & & $\mathrm{x}$ & & & & $638-66-4$ & plant oil, insect pheromone & $\infty$ smetic & food \\
\hline Od adecane & $\mathrm{x}$ & $\mathrm{x}$ & & $\mathrm{x}$ & & $593-45-3$ & plant oil & fragranœ & en/food/ex \\
\hline Od anal & $x$ & & $\mathrm{x}$ & $\mathrm{x}$ & & $124-13-0$ & plant oil, food & flavor/fragranœ, antimiar obial & food/ex \\
\hline OctanoicAcid* & & $\mathrm{x}$ & & & & 124-07-2 & plant oil, fruits, mamalian milk & flavor/fragranœ & food/ex \\
\hline Od anoicad d,ethyl ester* & & $\mathrm{x}$ & & & & 106-32-1 & fruits, alcohol & flavor/fragranœ & food/ex \\
\hline Oct anoicad d,methyl ester & $\mathrm{x}$ & & & & & $111-11-5$ & plant/fruits, species pheromone & flavor/fragranœ & food/ex \\
\hline p-Chloroaniline & & & & & $\mathrm{x}$ & $106-47-8$ & & precursor to antiseptic & ex \\
\hline Pentadecane ${ }^{*}$ & $\mathrm{x}$ & & & & & $629-62-9$ & plant oil, food & fragranœ & food/ex \\
\hline Pentadecane,7-methyl- & & $\mathrm{x}$ & & & & $6165-40-8$ & & & \\
\hline Pentadecanoicaci d,ethyl ester & & $\mathrm{x}$ & & & & $41114-00-5$ & not found in nature & flavor & ex \\
\hline Pentanoicacid (Valeric acid) ${ }^{*}$ & & $\mathrm{x}$ & & & & $109-52-4$ & fruits, food & flavor/fragranœ & food/ex \\
\hline Phenol $^{*}$ & $\mathrm{x}$ & $\mathrm{x}$ & $\mathrm{x}$ & $\mathrm{x}$ & & $108-95-2$ & plant oil & plastics production, pharmaceuticals & food \\
\hline Phenol,2-(1,1-dimethylethyl)- & & & $\mathrm{x}$ & & & $88-18-6$ & & fragranœ & $\mathrm{ex}$ \\
\hline Phenol,2,4,6-trimethyl- (Mesitol) & $\mathrm{x}$ & & & $\mathrm{x}$ & & $527-60-6$ & not found in nature & flavor/fragranœ & ex \\
\hline Phenol,2,5-dichloro- & & & & & $\mathrm{x}$ & $583-78-8$ & p-dichlorobenzene metabolite & & \\
\hline Phenol,2,6-bis(1,1-dimethylethyl)-4-ethyl- & & & & & $\mathrm{x}$ & $4130-42-1$ & & & \\
\hline Phenol,2-methoxy- (o-guaiacol) & & & & & $\mathrm{x}$ & $90-05-1$ & food & flavor/fragranœ & food/ex \\
\hline Phenol,2-methyl-5-(1-methylethyl) $(p-C y m e n-2-o l)$ & & & & & $\mathrm{x}$ & $499-75-2$ & plant oil & flavor/fragranœe & \\
\hline Phenol,4-(1,1,3,3-tetramethylbutyl)-* & & & & & $\mathrm{x}$ & $140-66-9$ & not found in nature & adjuvant for pesticide & $\mathrm{ex}$ \\
\hline
\end{tabular}




\begin{tabular}{|c|c|c|c|c|c|c|c|c|c|}
\hline \begin{tabular}{|l|} 
Phenol,4-ethyl-2-methoxy- \\
\end{tabular} & & & & & $\mathrm{x}$ & $2785-89-9$ & alcohol, produced by yeast & flavor/fragranœ & $\mathrm{ex}$ \\
\hline Phenol,4-methyl- (p-Cresol) ${ }^{*}$ & & & & & $x$ & $106-44-5$ & plant oil, food & flavor/fragranœ (œstmetics) & en/food \\
\hline Phenol,nonyl- & & & & & $x$ & $25154-52-3$ & & & \\
\hline Propanoicacid,2-methyl-,butylester & & & & & $\mathrm{x}$ & $97-87-0$ & plant oil & flavor/fragranœ & ex \\
\hline Pulegone & & & & & $\mathrm{x}$ & $15932-80-6$ & plant oil & flavor/fragranœe & food/ex \\
\hline p-Xyl ene (Benzene, 1,4-dimethyl-)* & $x$ & & $\mathrm{x}$ & $x$ & & $106-42-3$ & & & \\
\hline Pyrrole & & & & & $\mathrm{x}$ & $109-97-7$ & plant & flavor & food \\
\hline Styr ene ${ }^{*}$ & & & $\mathrm{x}$ & & & $100-42-5$ & plant, food & cosmetics, plastics & food/ex \\
\hline Tetrachloroethylene & & & $\mathrm{x}$ & $x$ & & $127-18-4$ & environmental/soil contaminant & solvent; dry cleaning & $\mathrm{ex}$ \\
\hline Tetradecanal (Myristaldehyde) & $\mathrm{x}$ & & $\mathrm{x}$ & $\mathrm{x}$ & & $124-25-4$ & plant oil, food & flavor/fragranœ & food/ex \\
\hline Tetradecane $^{*}$ & $\mathrm{x}$ & $\mathrm{x}$ & $\mathrm{x}$ & $\mathrm{x}$ & & 629-59-4 & plant, food & flavor/fragranœ & en/food/ex \\
\hline Tetradecanoicadid (Myristic acid) & & & $\mathrm{x}$ & & & $544-63-8$ & plant oil, food & food additive; fragrance & ex \\
\hline Tetradecanoicacid,2-methyl-,methyl ester & & $\mathrm{x}$ & & & & 55554-09-1 & & & \\
\hline Tetradecanoicad d,ethyl ester & & $\mathrm{x}$ & & & & $124-06-1$ & food & flavor/fragranœ & food/ex \\
\hline Thymol (p-Cymen-3-ol) & & & & & $\mathrm{x}$ & $89-83-8$ & plant oil & flavor/fragranœ; antiseptic , d garette additive & ex \\
\hline Toluene & & & $\mathrm{x}$ & & $\mathrm{x}$ & $108-88-3$ & plant, plant oil & solvent; antioxidant & food/ex \\
\hline trans-p-Mentha2,8-dienol & & & & & $\mathrm{x}$ & & & & \\
\hline Triactin & & & $\mathrm{x}$ & & & $102-76-1$ & not found in nature & solvent (flavor); food additive; cigarette additive & ex \\
\hline Tridecane ${ }^{*}$ & $\mathrm{x}$ & $\mathrm{x}$ & $\mathrm{x}$ & $\mathrm{x}$ & & $629-50-5$ & plant oil & fragranœ & food/ex \\
\hline Undecanal $^{\star}$ & $x$ & & $\mathrm{x}$ & & & $112-44-7$ & plant oil, food & flavor/fragranœ & en/food/ex \\
\hline Undecane $^{*}$ & $x$ & & $\mathrm{x}$ & $\mathrm{x}$ & & $1120-21-4$ & plant oil & fragranœ & food \\
\hline Undecane,2,6-dimethyl- & & & $\mathrm{x}$ & & & 17301-23-4 & & & \\
\hline ValproicAcid & & & & & $x$ & $99-66-1$ & synthetic & pharmaceutical (anticonvulsant) & $\mathrm{ex}$ \\
\hline Vanillin & & $\mathrm{x}$ & & & & $148-53-8$ & plant & flavor/fragranœ & \\
\hline Z-8-Hexadeœene & & & & $\mathrm{x}$ & & & plant & & \\
\hline$\alpha$-Cedrene & & & & & $x$ & $469-61-4$ & plant oil & flavor/fragranœ & \\
\hline$\alpha$-Cedreneoxide & & & & & $x$ & $29597-36-2$ & not found in nature & fragranœ & ex \\
\hline a-Cubebene & & & & & $\mathrm{x}$ & 17699-14-8 & plant oil & & food/ex \\
\hline$\beta$-Bourbonene & & $\mathrm{x}$ & & & & $5208-59-3$ & plant oil, species pheromone & flavor/fragranœ & ex \\
\hline$\beta$-Cadinene & & $\mathrm{x}$ & & & & $523-47-7$ & plant oil, plant species & & food \\
\hline$\beta$-Damasœenone & & & & & $x$ & $23696-85-7$ & plant & fragranœ & ex \\
\hline$\beta$-Damasœenone & & & & & $\mathrm{x}$ & $23726-93-4$ & plant/fruits & flavor & $\mathrm{ex}$ \\
\hline$\beta$-Gurjunene & & & $x$ & & & 17334-55-3 & plant oil & flavor/fragranœ & \\
\hline$\beta$-Maaliene & & & $\mathrm{x}$ & & & 489-29-2 & plant oil & flavor/fragranœ & \\
\hline$\beta$-Phellandrene & & & & & $\mathrm{x}$ & $555-10-2$ & plant oil & fragranœe & $\mathrm{ex}$ \\
\hline y-Cadinene & & $\mathrm{x}$ & & & & $39029-41-9$ & fruits & & food \\
\hline$\Delta$-Cadinene & & $\mathrm{x}$ & & & & $483-76-1$ & plant oil & & food \\
\hline
\end{tabular}




\section{CONCLUSIONS}

The SPME-GC/MS method developed and optimized has been demonstrated to be capable of sampling, identifying and differentiating the VOCs present in various biological specimens of forensic and medical importance. While previous studies have generally looked at individual or pairs of biological specimens for diagnostic purposes, the current method allows for the direct comparison between the major samples consisting of hand odor, buccal swabs, breath, blood, blood, and urine taken from the same individuals.

The pre-treatment method developed allowed the removal of the targeted VOCs from the sampling kits prior to sampling, extraction and analysis. Optimized SPME-GC/MS conditions yielded excellent detection limits for the VOCs from blood, breath, buccal cells, and urine with average limits of detection of $8.3 \mathrm{ng}$. The VOCs from breath were detected with the lowest limit of detection while urine samples showing the highest limit of detection which was four times higher than breath for breath samples. The data obtained using this optimized method yielded promising results as for each of the specimens investigated, the VOC profiles of different subjects are distinct and show reproducible ratios of characteristic VOCs present. Visual, spearman rank correlation, and PCA comparisons of the most abundant and frequent VOCs (from replicate samples of the same individuals) demonstrates that each specimen has characteristic VOCs and that within specimens there is correlation of VOCs for replicates from individuals and differentiation (lack of correlation) among individuals. The present method, for the first time, allows for a large scale study to be completed simultaneously comparing these five biological specimens from a larger population of individuals over an extended period of 
time in order to evaluate the utility of these biological specimens for profiling (individualization) and diagnostic (disease state) purposes.

Prior to this study, no work had been done to investigate the potential for differentiating people in terms of odor components other than skin emanations (mainly hand, arm, and armpits) on gauze. Through the combined methods of chromatogram comparison, Spearman rank correlation comparison, and principal component analysis, it is possible to distinguish the VOC profiles of individuals for each of the specimens with high confidence. The data obtained from this study also revealed that VOC profiles of different biological specimens from the same individual are too different to be used for the purpose of individual profiling.

Comparison of VOC profiles of healthy individuals, patients with Type 2 diabetes, and patients with MDD revealed that it was possible to distinguish individuals even when they are diagnosed with the same physiological or psychological condition. Chi-square test on the preliminary comparison of VOCs present in odor profiles across the three populations revealed a group of volatile compounds that could be used for potential biomarkers to differentiate between the healthy, diabetic, and clinically depressed study groups.

Finally, a human biological specimen compound database has been created compiling the volatile compounds present in the emanations of human hand odor, oral fluids (buccal cells and saliva), breath, blood, and urine. Compounds were classified as possibly endogenous, exogenous, originating from food and beverage sources, or a combination of any of the three. Majority of the volatile compounds are of plant origin and are widely 
used in the flavor and/or fragrance industries. Because the VOCs emanated from the human body may be the results of exogenous VOC inhalation and/or absorption or the production of endogenous VOCs by the body's metabolic processes, it is unwise to attempt to derive/determine the genetic basis of human odor compounds at the present time.

Results from the present study all further support the individual odor hypothesis in that each individual possesses a scent profile that is distinguishable from others. However, because many of the extracted VOCs are known to be exogenous and/or from food or food additives while the metabolic origins of the other extracted volatile compounds remain unclear, individual human odor should be considered as an "extended phenotype" as initially mentioned in the introduction of this research study. Extended phenotype is most suitable to describe human odor, as variations in the volatile compounds present in the human odor may be due to either genetic or environmental factors or a combination of both. 


\section{LIST OF REFERENCES}

1. Welsh JS; Barton D; Ahuja H A case of breast cancer detected by a pet dog. Community Oncology 2005, 2 (4), 324-326.

2. Williams H; Pembroke A Sniffer dogs in the melanoma clinic? The Lancet 1989, 333 (8640), 734.

3. Saferstein, R. Forensic Science Handbook; 2nd ed. ed.; Prentice Hall: Upper Saddle River, 2002; Vol. I.

4. Daubert v. Merrell Dow Pharmaceuticals, Inc. 1993. 509 U.S. 579.

5. Curran AM; Ramirez CF; Schoon AA; Furton KG The frequency of occurrence and discriminatory power of compounds found in human scent across a population determined by SPME-GC/MS. Journal of Chromatography B 2007, 846, 86-97.

6. U.S. v. Joshua Alan Wade. U.S. v. Wade. [583]. 2009.

7. The People, Plaintiff and Respondent vs. Jeffrey Dewayne Mitchell et al. 110 Cal. App. 4th 772. 2003. Court of Appeal of California, Second Appellate District, Division One.

8. The People, Plaintiff and Respondent vs. Ryan Willis. 115 Cal. App. 4th 379. 2004. Court of Appeal of California, Second Appellate District, Division Two.

9. People of the State of California vs. Benigno Salcido. 115 Cal. App. 4th 379. 2005. Los Angeles Superior Court.

10. Schoon GAA; De Bruin JC The ability of dogs to recognize and cross-match human odours. Forensic Science International 1994, 69, 111-118.

11. Settle RH; Sommerville BA; McCormick J; Broom DM Human scent matching using specially trained dogs. Animal Behaviour 1994, 48, 1443-1448.

12. Hudson DT; Curran AM; Furton KG The stability of collected human scent under various environmental conditions. Journal of Forensic Sciences 2009, 54 (6), 12701277.

13. Curran AM; Prada PA; Furton KG The differentiation of the volatile organic signatures of individuals through SPME-GC/MS of characteristic human scent compounds. Journal of Forensic Sciences 2010, 55 (1), 50-57. 
14. Gallagher M; Wysocki CJ; Leyden JJ; Spielman AI; Sun X; Preti G Analysis of volatile organic compounds from human skin. British Journal of Dermatology 2008, 159, 780-791.

15. Pauling L; Robinson AB; Teranishi R; Cary P Quantitative analysis of urine vapor and breath by gas-liquid partition chromatography. Proc. Nat. Acad. Sci. 1971, 68 (10), 2374-2376.

16. Andreoli R; Manini P; Bergamaschi E; Brustolin A; Mutti A Solid-phase microextraction and gas chromatography-mass spectrometry for determination of monoaromatic hydrocarbons in blood and urine: application to people exposed to air pollutants. Chromatographia 1999, 50 (3/4), 167-172.

17. O'Hara ME; Clutton-Brock TH; Green S; O'Hehir S; Mayhew CA Mass spectrometric investigations to obtain the first direct comparisons of endogenous breath and blood volatile organic compound concentrations in healthy volunteers. International Journal of Mass Spectrometry 2009, 281 (1-2), 92-96.

18. Statheropoulous M.; Sianos E; Agapiou A; Pappa A; Tzamtzis N; Giotaki H; Papageorgiou C; Kolostoumbis D Preliminary investigation of using volatile organic compounds from human expired air, blood and urine for locating entrapped people in earthquakes. Journal of Chromatography B 2005, 822, 112-117.

19. Pawliszyn J Solid phase microextraction. Theory and practice.; John Wiley \& Sons, Ltd: New York, NY, 1997.

20. Pawliszyn J Theory of solid-phase microextraction. Journal of Chromatographic Science 2000, 38, 270-278.

21. Solid Phase Microextration: A Practical Guide; Marcel Dekker, Inc.: New York, NY, 1999.

22. Niessen WMA Principles and instrumentation of gas chromatography-mass spectrometry. In Current Practice of Gas Chromatography-Mass Spectrometry, Niessen WMA, Ed.; Marcel Dekker, Inc.: New York, NY, 2001; pp 1-29.

23. Miller JM Chromatography: Concepts and Contrasts; John Wiley \& Sons, Inc.: New York, 1988.

24. Robards K; Haddad PR; Jackson PE Principles and Practice of Modern Chromatographic Methods; Elsevier Ltd: San Diego, 2004.

25. De Hoffmann E; Stroobant V Mass spectrometry: principles and applications; 3rd ed.; John Wiley \& Sons Lotd: 2007. 
26. Doyle C Secret cloud that surround us. Family Health 1970, 32-35.

27. Romanes GJ Experiments on the sense of smell in dogs. Nature 1887, 36, 273-274.

28. Penn DJ; Oberzaucher E; Grammer K; Fischer G; Soini HA; Wiesler D; Novotny MV; Dixon SJ; Xu Y; Brereton RG Individual and gender fingerprints in human body odour. Journal of the Royal Society Interface 2007, 4, 331-340.

29. Zhang ZM; Cai JJ; Ruan GH; Li GK The study of fingerprint characteristics of the emanations from human arm skin using the original sampling system by SPMEGC/MS. Journal of Chromatography B 2005, 822, 244-252.

30. Curran AM; Rabin SI; Furton KG Analysis of the uniqueness and persistence of human scent. Forensic Science Communications 2005, 7 (2).

31. Schoon GAA The effect the ageing of crime scene objects on the results of scent identification line-ups using trained dogs. Forensic Science International 2005, 147, 43-47.

32. Chiappin S; Antonelli G; Gatti R; De Palo EF Saliva specimen: a new laboratory tool for diagnostic and basic investigation. Clinica Chimica Acta 2007, 383, 30-40.

33. de Almeida PDV; Gregio AMT; Machado MAN; de Lima AAS; Azevedo LR Saliva composition and functions: a comprehensive review. The Journal of Contemporary Dental Practice 2008, 9 (3), 072-080.

34. Kaufman E; Lamster IB The diagnostic applications of saliva - a review. Critical Reviews in Oral Biology \& Medicine 2002, 13 (2), 197-212.

35. Schenkels LCPM; Veerman ECI; Amerongen AVN Biochemical composition of human saliva in relation to other mucosal fluids. Critical Reviews in Oral Biology \& Medicine 1995, 6 (2), 161-175.

36. Causes of Dry Mouth. morefocus goup, inc. 2010.

37. Kidwell DA; Holland JC; Athanaselis S Testing for drugs of abuse in saliva and sweat. Journal of Chromatography B 1998, 713, 111-135.

38. Dodds MWJ; Johnson DA; Yeh CK Health benefits of saliva: a review. Journal of Dentistry 2005, 33, 223-233.

39. Tonzetich J; Richter VJ Evaluation of volatile odoriferous components of saliva. Archives of Oral Biology 1964, 9, 39-45. 
40. Kostelc JG; Preti G; Zelson PR; Stoller NH Salivary volatiles as indicators of periodontitis. Journal of Periodontal Research 1980, 15, 185-192.

41. Lochner A; Weisner S; Zlatkis A Gas chromatographic-mass spectrometric analysis of volatile constituents in saliva. Journal of Chromatography B 1986, 378, 267-282.

42. Kostelc JG; Zelson PR; Preti G; Tonzetich J Quantitative differences in volatiles from healthy mouths and mouths with periodontitis. Clinical Chemistry 1981, 27 (6), 842-845.

43. Fucci N; de Giovanni D; Chiarotti M Simultaneous detection of some drugs of abuse in saliva samples by SPME technique. Forensic Science International 2003, $134,40-45$.

44. Statheropoulous M.; Agapiou A; Georgiadou A Analysis of expired air of fasting male monks at Mount Athos. Journal of Chromatography B 2006, 832, 274-279.

45. Miekisch W.; Schubert JK; Noeldge-Schomburg GFE. Diagnostic potential of breath analysis - focus on volatile organic compounds. Clinica Chimica Acta 2004, $347,25-39$.

46. Vander A; Sherman J; Luciano D Human physiology: the mechanisms of body function; 7th ed. ed.; McGrawHill: Boston, 1998.

47. Phillips M; Greenberg J; Cataneo RN Effect of age on profile of alkanes in normal human breath. Free Radical Research 1999, 33, 57-63.

48. Phillips M; Herrera J; Krishnan S; Zain M; Greenberg J; Cataneo RN Variation in volatile organic compounds in the breath of normal humans. Journal of Chromatography B 1999, 729, 75-88.

49. Buszewski B; Kesy M; Ligor M; Amann A Human exhaled air analytics: biomarkers of diseases. Biomedical Chromatography 2007, 21, 553-566.

50. Cailleux A; Allain P Is pentane a normal constituent of human breath? Free Radical Research Communications 1993, 18 (6), 323-327.

51. Deng C; Zhang J; Yu X; Zhang W; Zhang X Determination of acetone in human breath by gas chromatography-mass spectrometry and solid-phase microextraction with on-fiber derivatization. Journal of Chromatography B 2004, 810, 269-275.

52. Francesco FD; Fuoco R; Trivella MG; Ceccarini A Breath analysis: treands in techniques and clinical applications. Microchemical Journal 2005, 75, 405-410. 
53. Phillips M; Greenberg J; Awad J Metabolic and environmetal origins of volatile organic compounds in breath. Journal of Clinical Pathology 1994, 47, 1052-1053.

54. Phillips M; Cataneo RN; Cummin ARC; Gagliardi AJ; Gleeson K; Greenberg J; Maxfield RA; Rom Wn Detection of lung cancer with volatile markers in the breath. Chest 2003, 123, 2115-2123.

55. Phillips M; Cataneo RN; Cheema T; Greenberg J Increased breath biomarkers of oxidative stress in diabetes mellitus. Clinica Chimica Acta 2004, 344, 189-194.

56. Phillips M; Cataneo RN; Condos R; Erickson GA; Greenberg J; La Bombardi V; Munawar MI; Tietje O Volatile biomarkers of pulmonary tuberculosis in the breath. Tuberculosis 2007, 87 (1), 44-52.

57. Bajtarevic A; Ager C; Pienz M; Klieber M; Schwarz K; Ligor M; Ligor T; Filipiak W; Denz H; Fiegl M; Hilbe W; Weiss W; Lukas P; jamnig H; hackl M; Haidenberger A; Buszewski B; Miekisch W; Schubert J; Amann A Noninvasive detection of lung cancer by analysis of exhaled breath. BMC Cancer 2009, 9 (348).

58. Thibodeau GA; Patton KT Structure \& Function of the Body; 10th ed. ed.; MosbyYear Book, Inc.: St.Louis, 1997.

59. Lote, C. Principles of renal physiology; 4th ed. ed.; Kluwer Academic Publishers: Dordrecht, The Netherlands, 2000.

60. Cohen BJ; Wood DL The Human Body in Health and Disease; 9th ed. ed.; Lippincott Williams \& Wilkns: Philadephia, 2000.

61. Hajimiragha H; Ewers U; Brockhaus A; Boettger A Levels of benzene and other volatile aromatic compounds in the blood of non-smokers and smokers. International Archives of Occupational and Environmental Health 1989, 61, 513518.

62. Ashley DL; Bonin MA; Cardinali FL; McCraw JM; Wooten JV Measurement of volatile organic compounds in human blood. Environmental Health Perspectives 1996, 104, 871-877.

63. Blount BC; KObelski RJ; McElprang DO; Ashley DL; Chambers DM; Cardinali FL Quantification of 31 volatile organic compounds in whole blood using solid-phase microextraction and gas chromatography-mass spectrometry. Journal of Chromatography B 2006, 832, 292-301.

64. Schimming E; LEvsen K; Kohme C; Schurmann W Biomonitoring of benzene and toluene in human blood by headspace-solid-phase microextraction. Fresenius Journal of Analytical Chemistry 1999, 363, 88-91. 
65. Miekisch W; Schubert JK; Vagts DA; Geiger K Analysis of volatile disease markers in blood. Clinical Chemistry 2001, 47 (6), 1053-1060.

66. Deng C; Li N; Zhang X Development of headspace solid-phase microextraction with on-fiber derivatization for determination of hexanal and heptanal in human blood. Journal of Chromatography B 2004, 813, 47-52.

67. Deng C; Zhang X; Li N Investigation of volatile biomarkers in lung cancer blood using solid-phase microextraction and capillary gas chromatography-mass spectrometry. Journal of Chromatography B 2004, 808, 269-277.

68. Xue R; Dong L; Zhang S; Deng C; Liu T; Wang J; Shen X Investigation of volatile biomarkers in liver cancer blood using solid-phase microextraction and gas chromatography/mass spectrometry. Rapid Communications in Mass Spectrometry 2008, 22, 1181-1186.

69. Hladky SB; Rink TJ Body Fluid and Kidney Physiology; Edward Arnold: London, 1986.

70. Mills GA; Walker V Headspace solid-phase microextraction profiling of volatile compounds in urine: application to metabolic investigation. Journal of Chromatography B 2001, 753, 259-268.

71. Wahl HG; Hoffmann A; Luft D; Liebich HM Analysis of volatile organic compounds in human urine by headspace gas chromatography-mass spectrometry with a multipurpose sampler. Journal of Chromatography A 1999, 847, 117-125.

72. Zlatkis A; Liebich HM Profile of volatile metabolites in human urine. Clinical Chemistry 1971, 17 (7), 592-594.

73. Zlatkis A; Brazell RW; Poole CF The role of organic volatile profiles in clinical diagnosis. Clinical Chemistry 1981, 27 (6), 789-797.

74. Brown SD; Rhodes DJ; Pritchard BJ A validated SPME-GC-MS method for simultaneous quantification of club drugs in human urine. Forensic Science International 2007, 171, 142-150.

75. Kumazawa T; Lee XP; Tsai MC; Seno H; Ishii A; Sato K Simple extraction of tricyclic antidepressants in human urine by headspace solid-phase microextraction (SPME). Japanese Journal of Forensic Toxicology 1995, 13 (1), 25-30.

76. Yashiki M; Kojima T; Miyazaki T; Nagasawa N; Iwasaki Y; Hara K Detection of amphetamines in urine using head space-solid phase microextraction and chemical ionization selected ion monitoring. Forensic Science International 1995, 76 (3), 169-177. 
77. Calafat AM; Kuklenyik Z; Reidy JA; Caudill SP; Ekon J; Needham LL Urinary concentrations of bisphenol A and 4-nonylphenol in a human reference population. Environmental Health Perspectives 2005, 113 (4), 391-395.

78. Inoue K; Kawaguchi M; Okada F; Takai N; Yoshimura Y; Horie M; Izumi S; Makino T; Nakazawa H Measurement of 4-nonylphenol and 4-tert-octylphenol in human urine by column-switching liquid chromatography-mass spectrometry. Analytica Chimica Acta 2003, 486, 41-50.

79. Poli D; Manini P; Andreoli R; Franchini I; Mutti A Determination of dichloromethane, trichloroethylene and perchloroethylene in urine samples by headspace solid phase microextraction gas chromatography-mass spectrometry. Journal of Chromatography B 2005, 820, 95-102.

80. Wang BL; Takigawa T; Takeuchi A; Yamasaki Y; Kataoka H; Wang DG; Ogino K Unmetabolized VOCs in urine as biomarkers of low level exposure in indoor environments. Journal of Occupational Health 2007, 49, 104-110.

81. Stahlhut RW; van Wijngaarden E; Dye TD; Cook S; Swan SH Concentrations of urinary phthalate metabolites are associated with increased waist circumference and insulin resistance in adult U.S. males. Environmental Health Perspectives 2007, $115(6), 876-882$.

82. Liebich HM; Al-Babbili O Gas chromatographic-mass spectrometric study of volatile organic metabolites in urines of patients with diabetes mellitus. Journal of Chromatography A 1975, 112, 539-550.

83. Burke DG; Halpern B; Malegan D; McCairns E; Danks D; Schlesinger P; Wilken B Profiles of urinary volatiles from metabolic disorders characterized by unusual odors. Clinical Chemistry 1983, 29 (10), 1834-1838.

84. Curran AM The Analytical Determination of the Uniqueness and Persistence of the Volatile Components of Human Scent Using Optimized Collection Methods. Florida International University, Miami, FL, 2005.

85. Miller JN; Miller JC Statistics and Chemometrics for Analytical Chemistry; 5th ed. ed.; Pearson Education Limited: Harlow, England, 2005.

86. Liddel K Smell as a diagnostic marker. Postgraduate Medical Journal 1976, 52, 136-138.

87. Phillips M; Sabas M; Greenberg J Increased pentane and carbon disulfide in the breath of patients with schizophrenia. Journal of Clinical Pathology 1993, 46 (861), 864. 
88. Phillips M; Erickson GA; Sabas M; Smith JP; Greenberg J Volatile organic compounds in the breath of patients with schizophrenia. Journal of Clinical Pathology 1994, 48, 466-469.

89. Phillips M; Gleeson K; Hughes MB; Greenberg J; Cataneo RN; Baker L; McVay WP Volatile organic compounds in breath as markers of lung cancer: a crosssectional study. Lancet 1999, 353, 1930-1933.

90. Mazzone PJ Analysis of volatile organic compounds in the exhaled breath for the diagnosis of lung cancer. Journal of Thoracic Oncology 2008, 3 (7), 774-780.

91. Olopade CO; Zakkar M; Swedler Wi; Rubinstein I Exhaled pentane levels in acute asthma. Chest 1997, 111, 862-865.

92. Montuschi P; Corradi M; Ciabattoni G; Nightingale J; Kharitonov SA; Barnes PJ Increased 8-isoprostane, a marker of oxidative stress, in exhaled condensate of asthma patients. American Journal of Respiratory and Critical Care Medicine 1999, 160, 216-220.

93. Paredi P; Kharitonov SA; Barnes PJ Elevation of exhaled ethane concentration in asthma. American Journal of Respiratory and Critical Care Medicine 2000, 162, $1450-1454$.

94. Lebovits HE Diabetic ketoacidosis. Lancet 1995, 945, 767-772.

95. Probert CSJ; Ahmed I; Khalid T; Johnson E; Smith S; Ratcliffe N Volatile organic compounds as diagnostic biomarkers in gastrointestinal and liver diseases. Journal of Gastrointestinal and Liver Diseases 2009, 18 (3), 337-343.

96. Phillips M; Cataneo RN; Greenberg J; Munawar MI; Samtani S Pilot study of a breath test for volatile organic compounds associated with oral malodor: evidence for the role of oxidated stress. Oral Diseases 2005, 11 (1), 32-34.

97. Poli D; Carbognani P; Corradi M; Goldoni M; Acampa O; Balbi B; Bianchi L; Rusca M; Mutti A Exhaled volatile organic compounds in patients with non-small cell lung cancer: cross sectional and nested short-term follow-up study. Respiratory Research 2005, 6 (71).

98. Barker M; Hengst M; Schmid J; Buers H-J; Mittermaier B; Klempt D; Koppmann R Volatile organic compounds in the exhaled breath of young patiends with cystic fibrosis. European Respiratory Journal 2006, 27 (5), 929-936.

99. Podebrad F; Heil M; Reichert S; Mosandl A; Sewell AC; Bohles H 4,5-Dimeethyl3-hydroxy-2[5H]-furanone (sotolone) - the odour of maple syrup urine disease. Journal of Inherited Metabolic Disease 1999, 22, 107-114. 
100. Mitchell SC; Smith RL Trimethylaminuria: the fish malodor syndrome. Drug Metabolism and Disposition 2001, 29 (4), 517-521.

101. Church J; Williams H Another sniffer dog for the clinic? The Lancet 2001, 358 (9285), 930.

102. Smith K; Sines JO Demonstration of a peculiar odor in the sweat of schizophrenic patients. AMA Archives of General Psychiatry 1960, 2, 184-188.

103. Voet D; Voet JG; Pratt CW Fundamentals of Biochemistry; Wiley: 2002.

104. National Institute of Health . National Diabetes Information Clearinghouse (NDIC). 2008. NIH Publication. 3-10-2010.

105. Mahler RJ; Adler ML Type 2 diabetes mellitus: update on diagnosis, pathophysiology, and treatment. The Journal of Clinical Endocrinology \& Metabolism 1999, 84 (4), 1165-1171.

106. CDC . National Diabetes Fact Sheet, 2007. 2007. 3-9-2010.

107. World Health Organization Definition, Diagnosis and Classification of Diabetes Mellitus and its Complications; World Health Organization: 99.

108. Moller DE New drug targets for type 2 diabetes and the metabolic syndrome. Nature 2001, 414, 821-827.

109. National Institute of Mental Health . The Numbers Count: Mental Disorders in America. 2009. 3-11-2010.

110. World Health Organization . Depression. 2010. 3-11-2010.

111. American Psychiatric Association Diagnostic and statistical manual of mental disorders: DSM-IV-TR; 4th ed., text revision ed.; American Psychiatric Association: Washington, DC, 2000.

112. Butcher JN; Mineka S; Hooley JM Abnormal Psychology; 12th ed. ed.; Pearson Education, Inc.: Boston, 2004.

113. Beidel DC; Bulik CM; Stanley MA Abnormal psychology; Prentice Hall: Upper Saddle River, NJ, 2010.

114. Rovner BWR; German PS; Brant LJ; Clark R; Burton L; Folstein MF Depression and mortality. Journal of the American Medical Association 1991, 265 (8), 993996. 
115. Penninx BW; Geerlings SW; Deeg DJH; Van Eijk JTM; Van Tilburg W Minor and major depression and the risk of death in older persons. Archives of General Psychiatry 1999, 56 (10), 889-895.

116. American Psychiatric Association Practice Guidelines for the Treatment of Patients with Major Depressive Disorder; 2nd ed. ed.; American Psychiatric PUblishing, Inc.: 2000.

117. Anderson RJ; Fredland KE; Clouse RE; Lustman PJ The prevalence of comorbid depression in adults with diabetes. Diabetes Care 2001, 24 (6), 1069-1078.

118. Gavard JA; Lustman PJ; Clouse RE Prevalence of depression in adults with diabetes. An epidemiological evaluation. Diabetes Care 1993, 16, 1167-1178.

119. Eatin WW; Armenian H; Gallo J; Pratt L; Ford DE Depression and risk for onset of type II diabetes. A prospective population-based study. Diabetes Care 1996, 19, 1097-1102.

120. Peyrot M; Rubin RR Persistence of depressive symptoms in diabetic adults. Diabetes Care 1999, 22 (448), 452.

121. Kawakami N; Shimizu H; Takatsuka N; Ishibashi H Depressive symptoms and occurrence of type 2 diabetes among Japanese men. Diabetes Care 1999, 22 (7), 1071-1076.

122. Talbot F; Nouwen A A review of the relationship between depression and diabetes in adults. Diabetes Care 2000, 23 (10), 1556-1562.

123. Bernier UR; Kline DL; Barnard DR; Schreck CE; Yost RA Analysis of human skin emanations by gas chromatography/mass spectrometry. 2. Identification of volatile compounds that are candidate attractants for the yellow fever mosquito (Aedes aegypti). Analytical Chemistry 200, 72 (4), 747-756.

124. Kojima S; Nakagawa M; Suzuki R; Horio M; Tanaka Y Alkylnaphthalenes. I. Absorption, tissue distribution and excretion of 2,6-diisopropylnaphthalene in rats. Chemical \& Pharmaceutical Bulletin 1978, 26 (10), 3007-3009.

125. Solano-Lopez CE; Ji TH; Alvarez VB Volatile compounds and chemical changes in ultrapasteurized milk packaged in polyethylene terephthalate containers. Journal of Food Science 2005, 70 (6), C407-C412.

126. Cox C Nonyl phenol and related chemicals. Journal of Pesticide Reform 1996, 16 (1), 15-20. 
127. Soini HA; Klouckova I; Wiesler D; Oberzaucher E; Grammer K; Dixon SJ; Xu Y; Brereton RG; Penn DJ; Novotny MV Analysis of volatile organic compounds in human saliva by a static sorptive extraction method and gas chromatography-mass spectrometry. Journal of Chemical Ecology 2010, 36, 1035-1042.

128. Statheropoulous M.; Mikedi K; Agapiou A; Georgiadou A.; Karma S Discriminant analysis of volatile organic compounds data related to a new location method of entrapped people in collapsed buildings of an earthquake. Analytica Chimica Acta 2006, 566, 207-216. 


\section{APPENDICES}

Appendix A

A1 Calibration curves for selected VOCs found in hand odor

Figure 91. Calibration curve for furfural

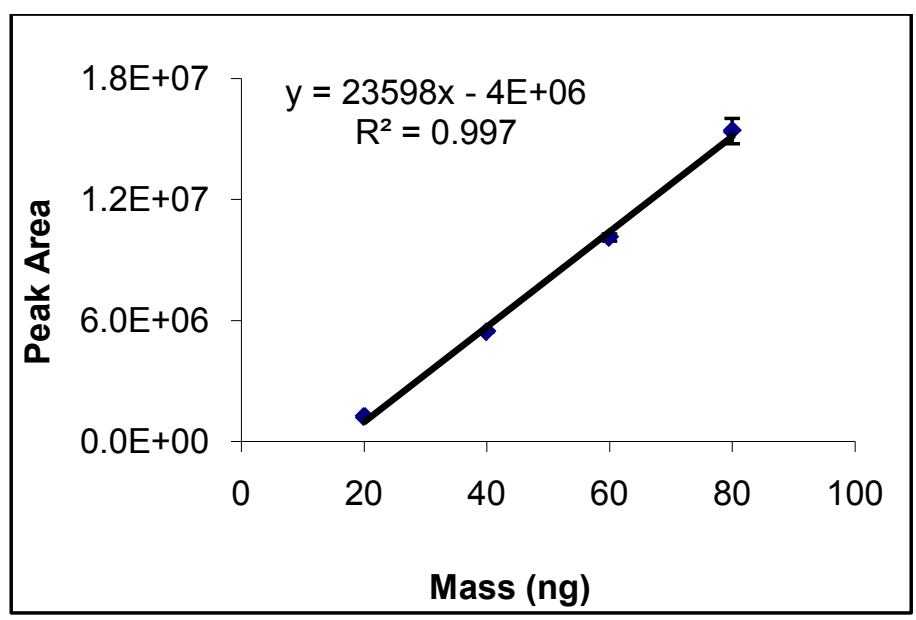

Figure 92. Calibration curve for 2-furanmethanol

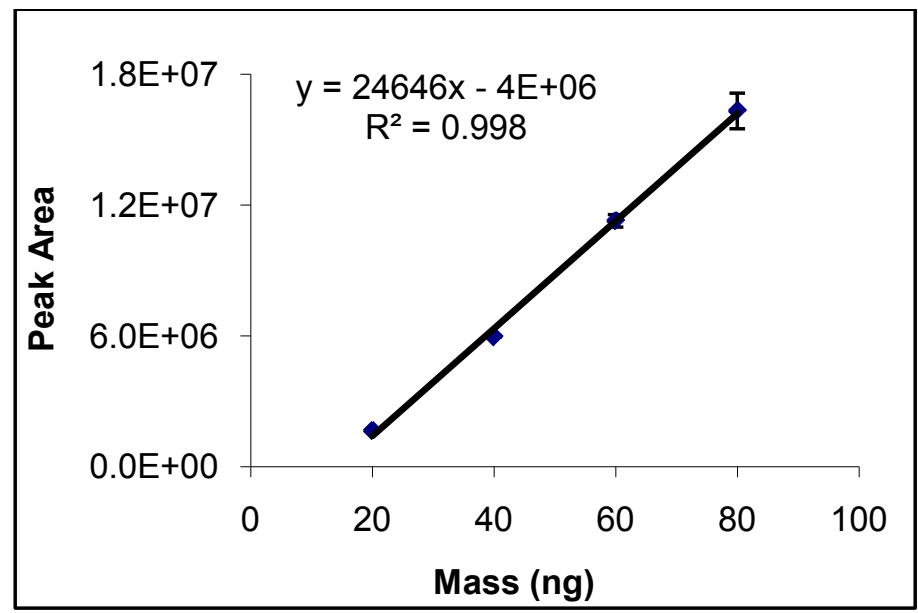


Figure 93. Calibration curve for dimethyl malonate

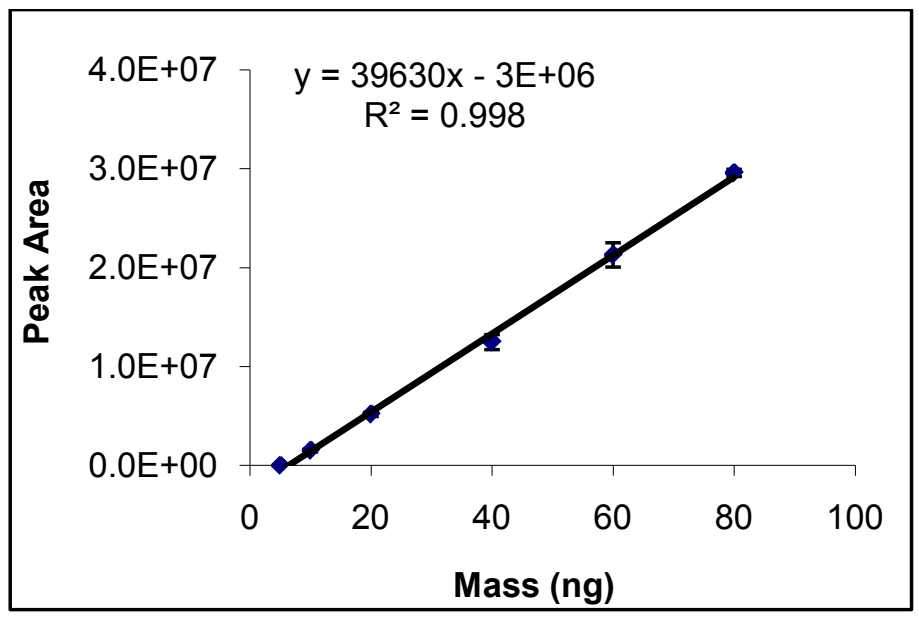

Figure 94. Calibration curve for undecane

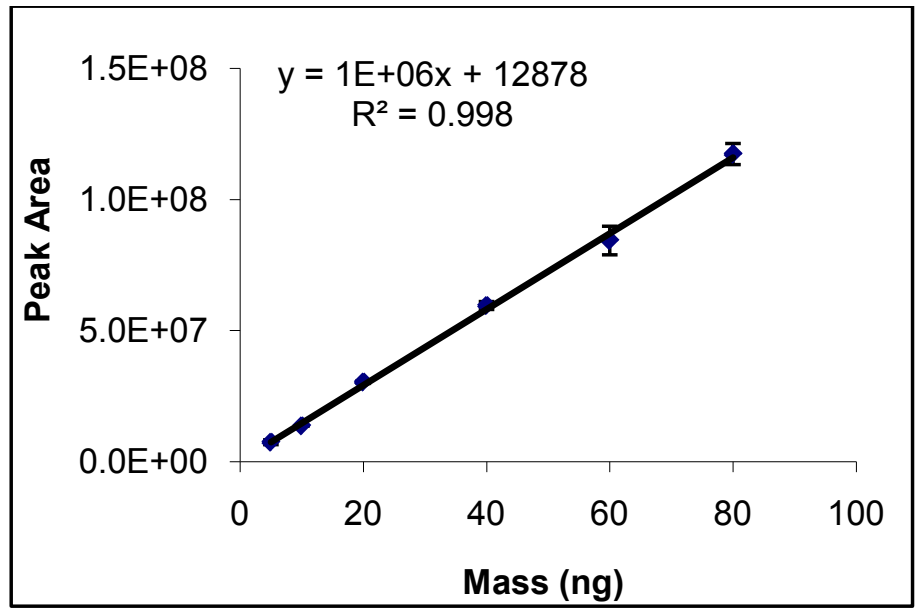

Figure 95. Calibration curve for (E)-6,10-Dimethyl-5,9-undecadien-2-one

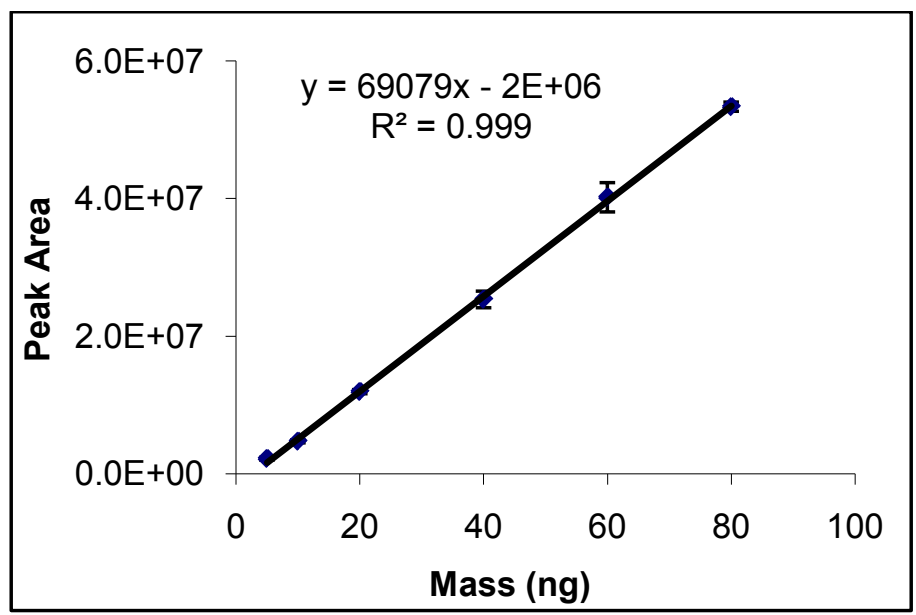


Figure 96. Calibration curve for dodecanoic acid

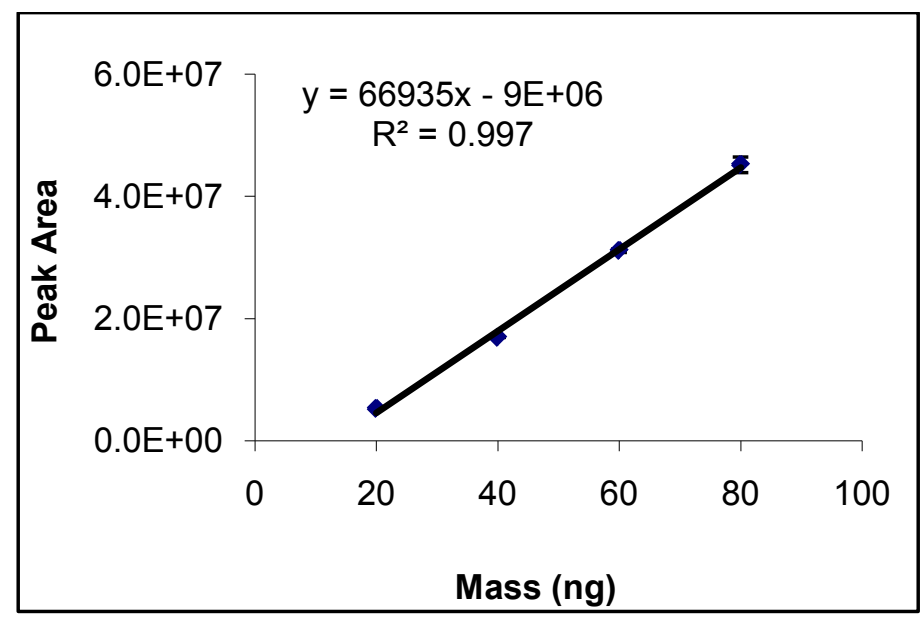

A2 Calibration curves for selected VOCs found in buccal swab

Figure 97. Calibration curve for hexanal

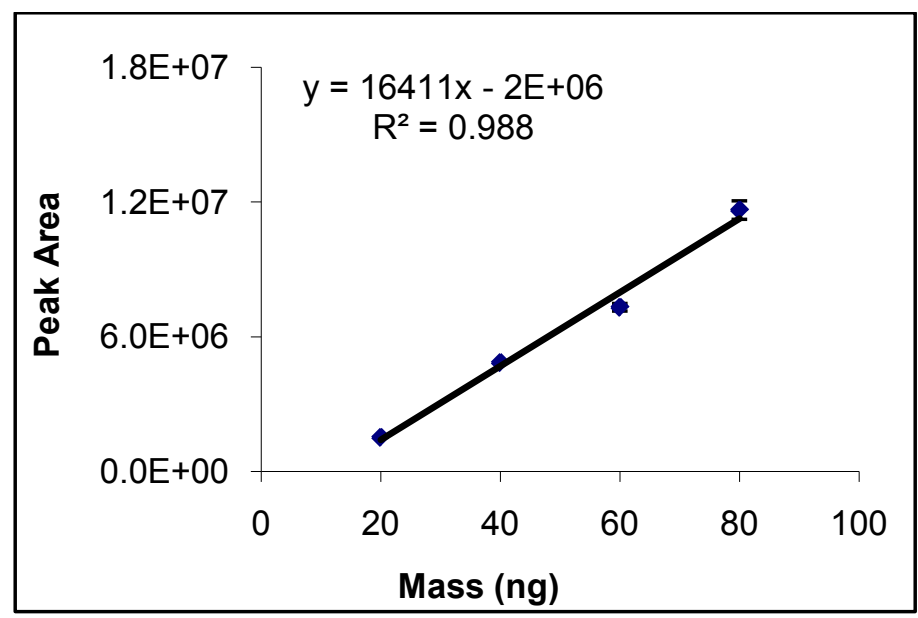


Figure 98. Calibration curve for hexanoic acid

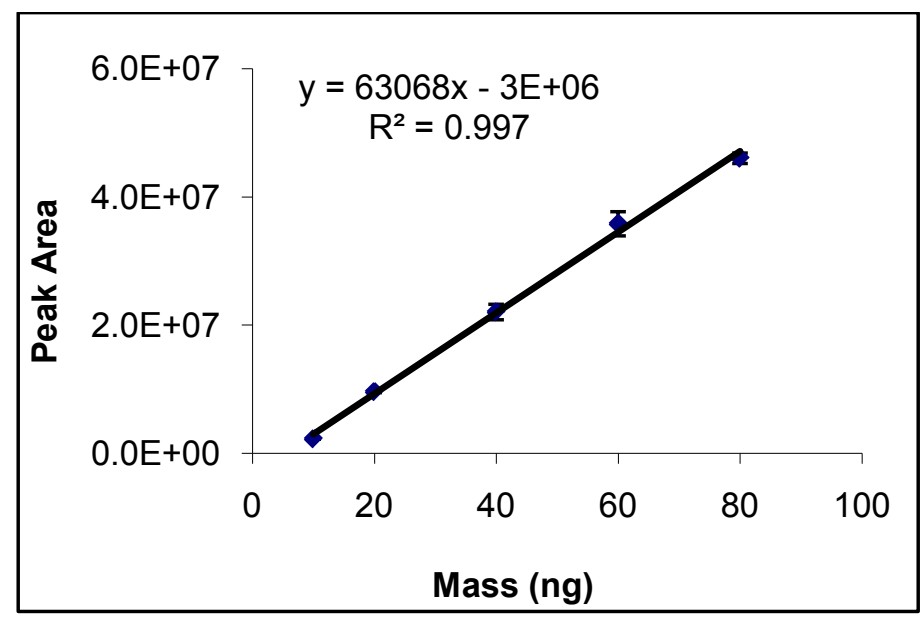

Figure 99. Calibration curve for acetophenone

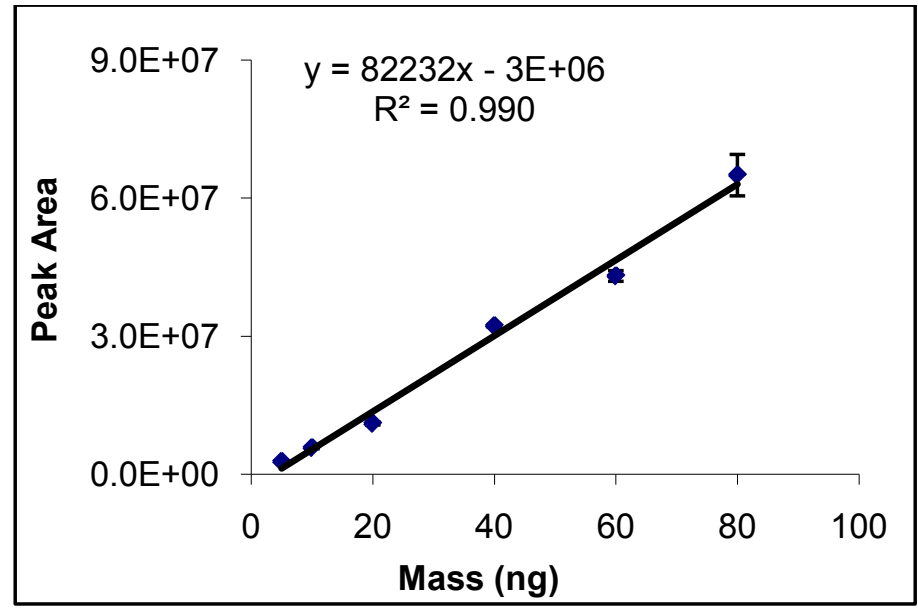

Figure 100. Calibration curve for 1-octanol

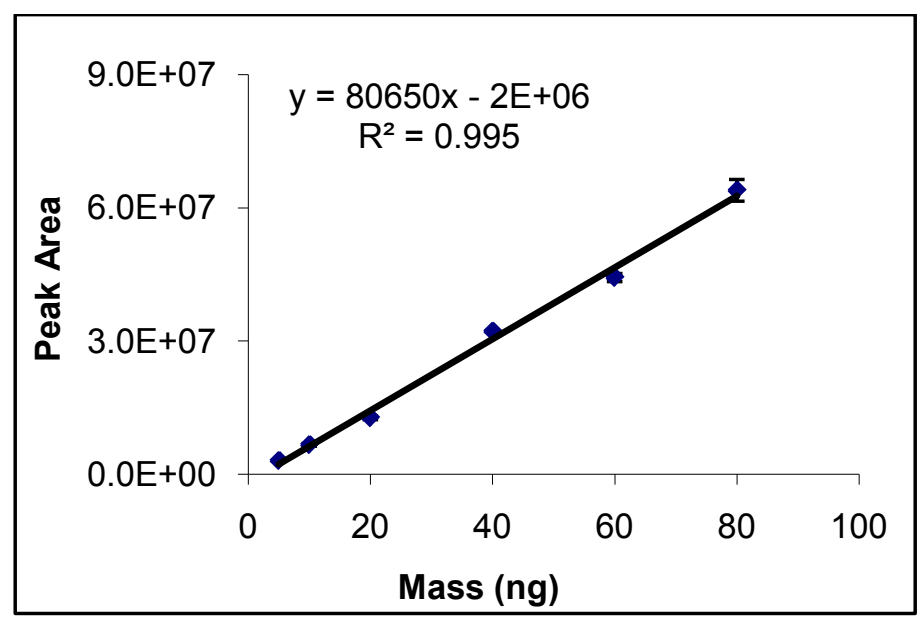


Figure 101. Calibration curve for octanoic acid, ethyl ester

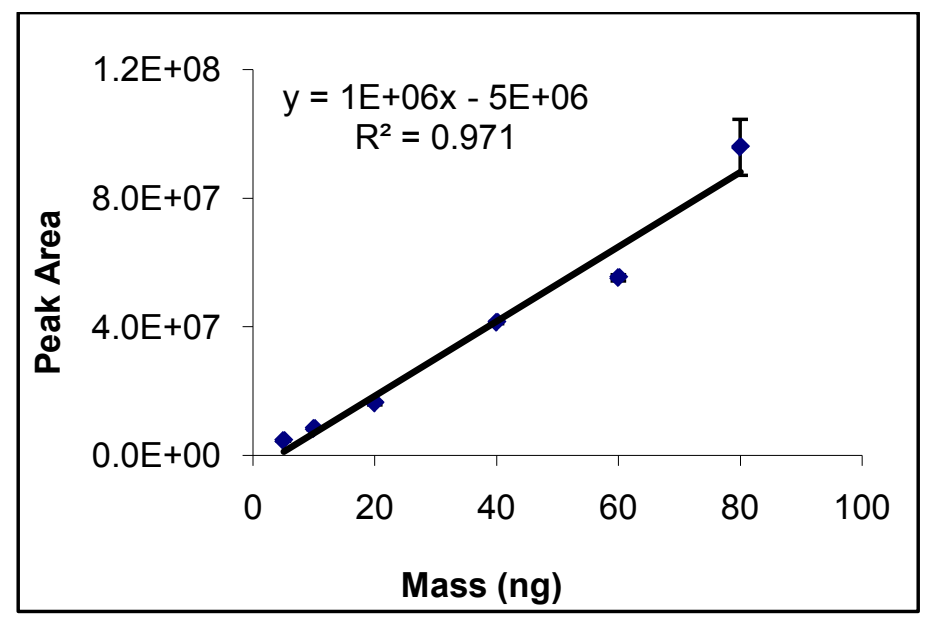

Figure 102. Calibration curve for 1-methylnaphthalene

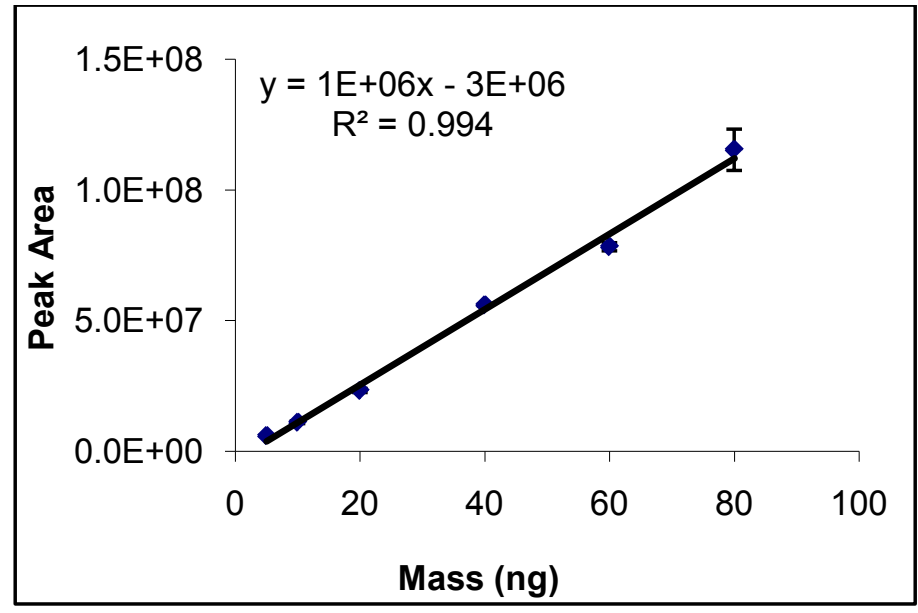

Figure 103. Calibration curve for tetradecane

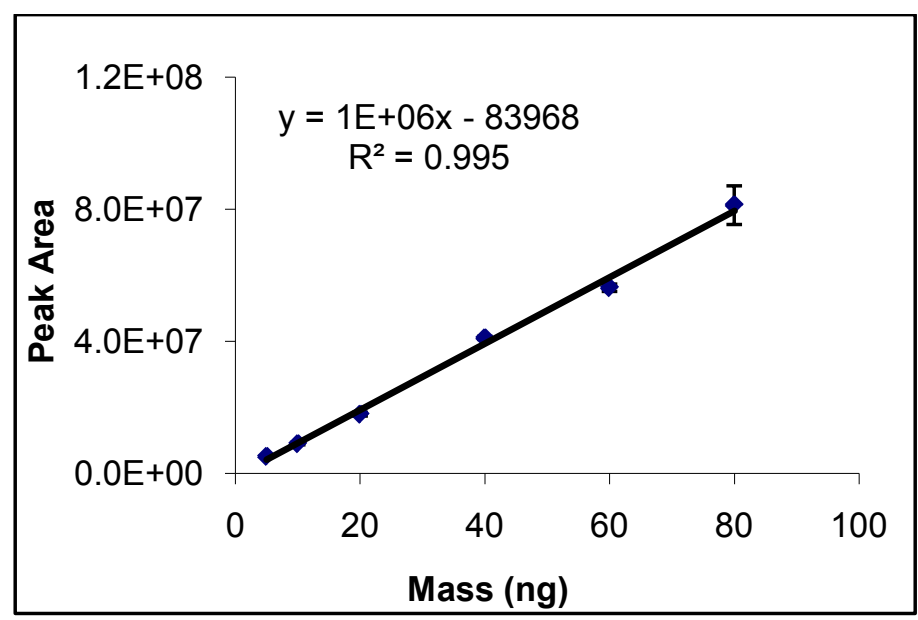


A3 Calibration curves for selected VOCs found in breath

Figure 104. Calibration curve for 3-heptanone

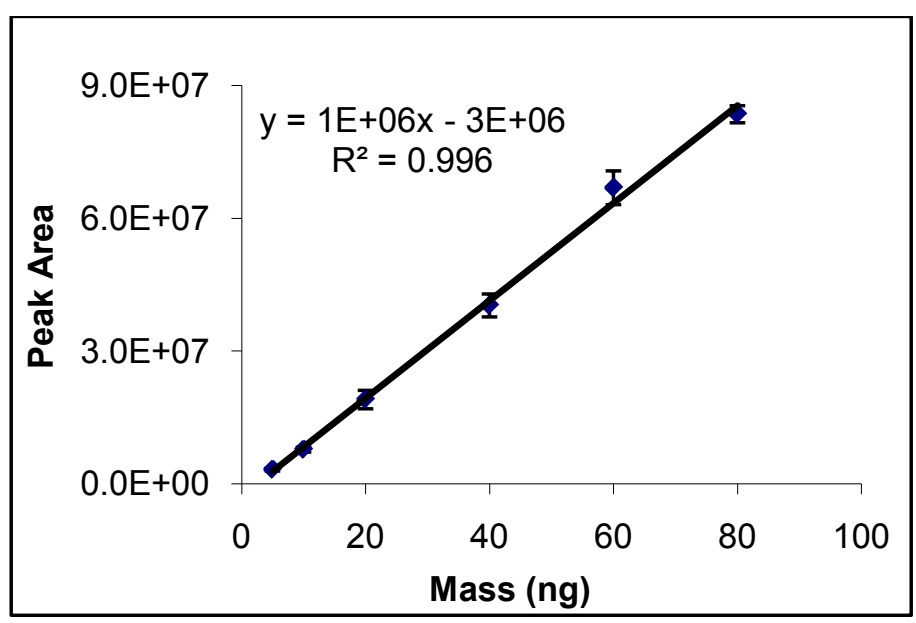

Figure 105. Calibration curve for styrene

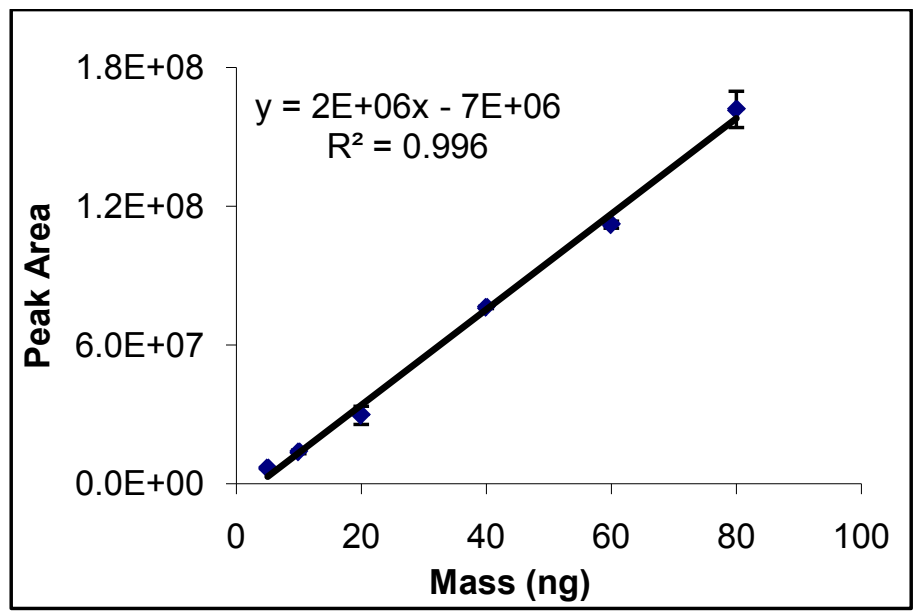


Figure 106. Calibration curve for phenol

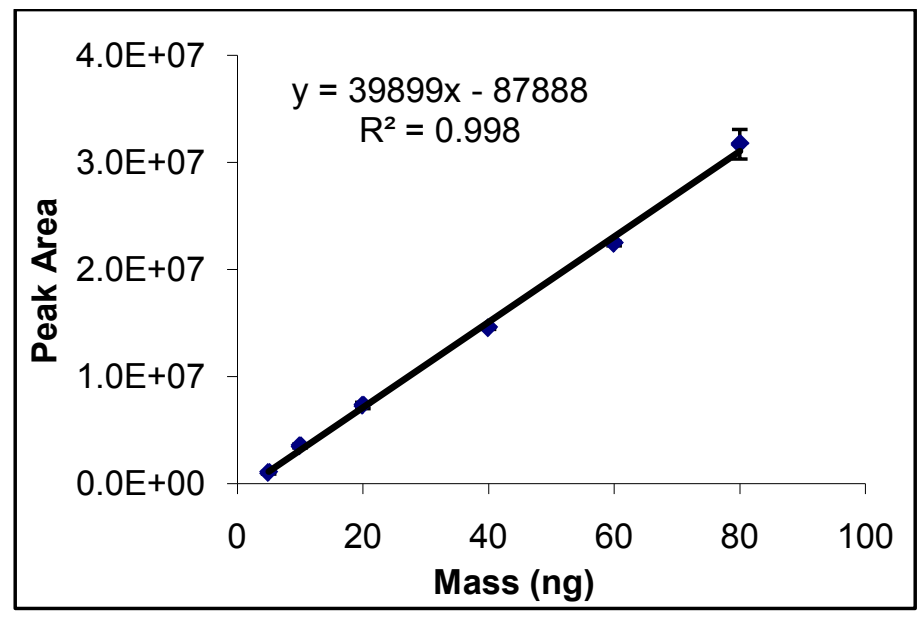

Figure 107. Calibration curve for nonanal

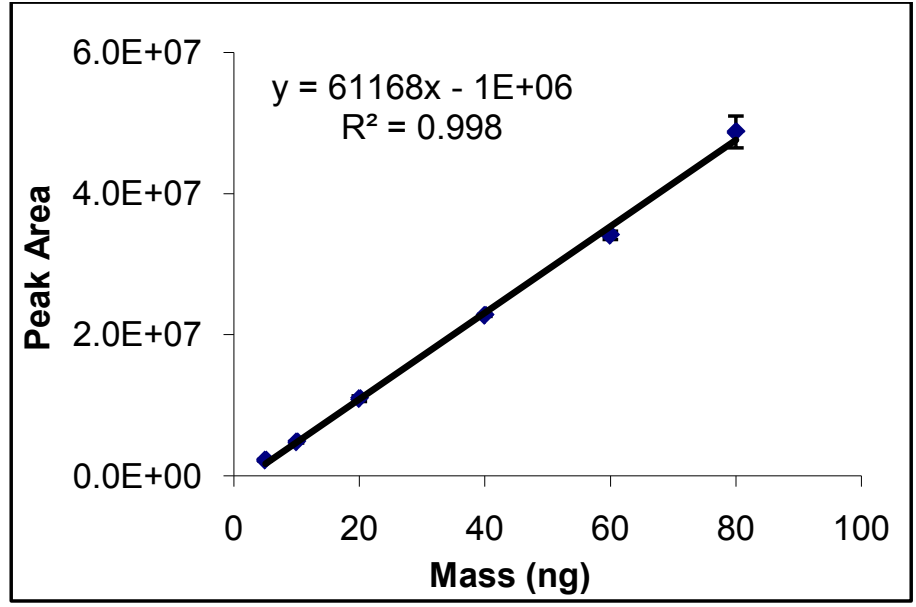

Figure 108. Calibration curve for 1-methylnaphthalene

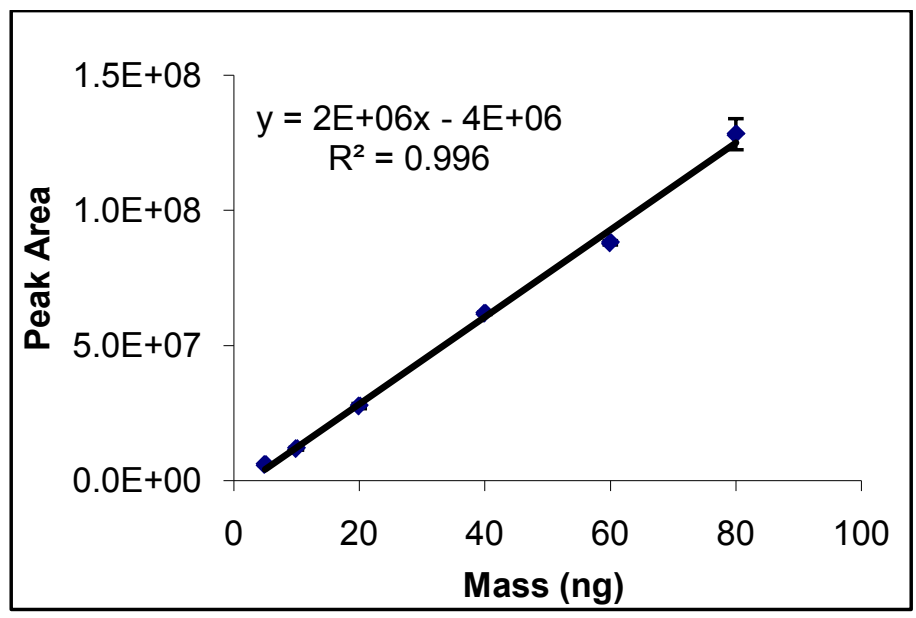


Figure 109. Calibration curve for caryophyllene

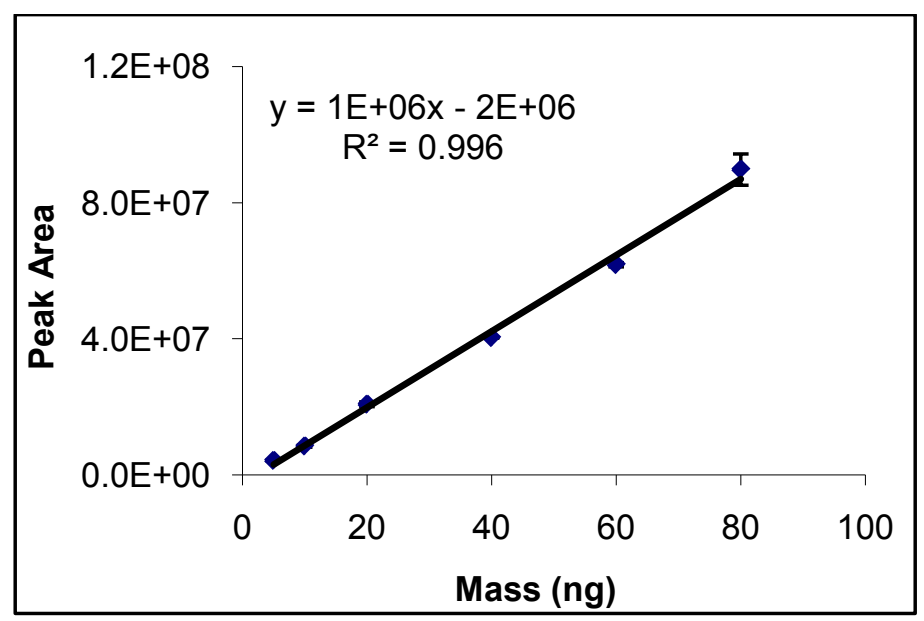

A4 Calibration curves for selected VOCs found in blood

Figure 110. Calibration curve for cyclohexanone

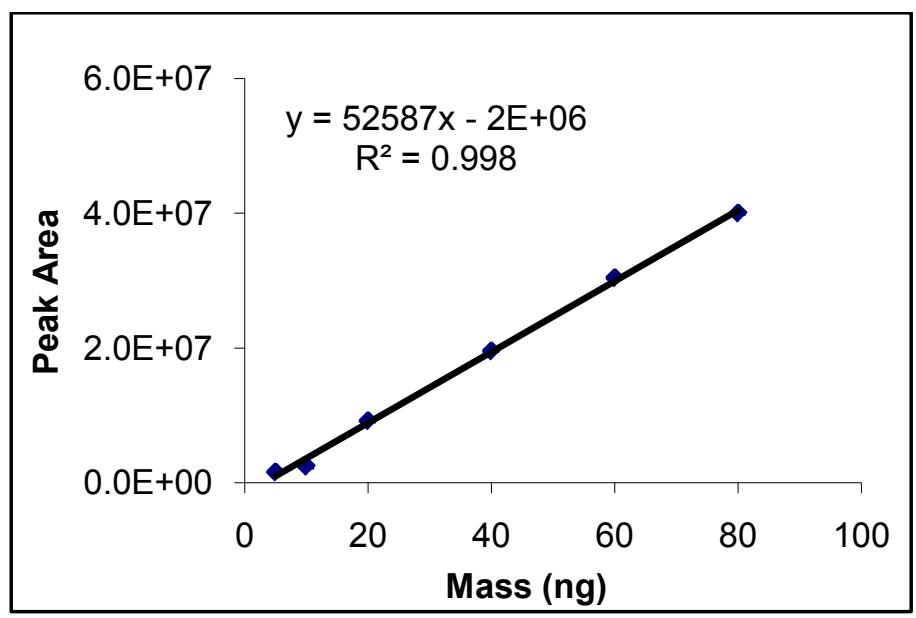


Figure 111. Calibration curve for heptanal

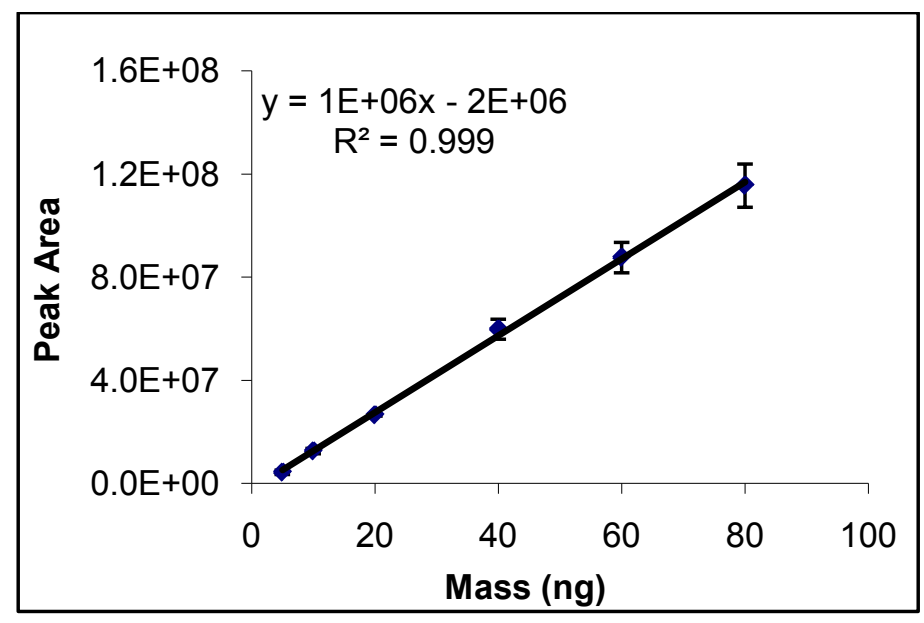

Figure 112. Calibration curve for 1-octen-3-ol

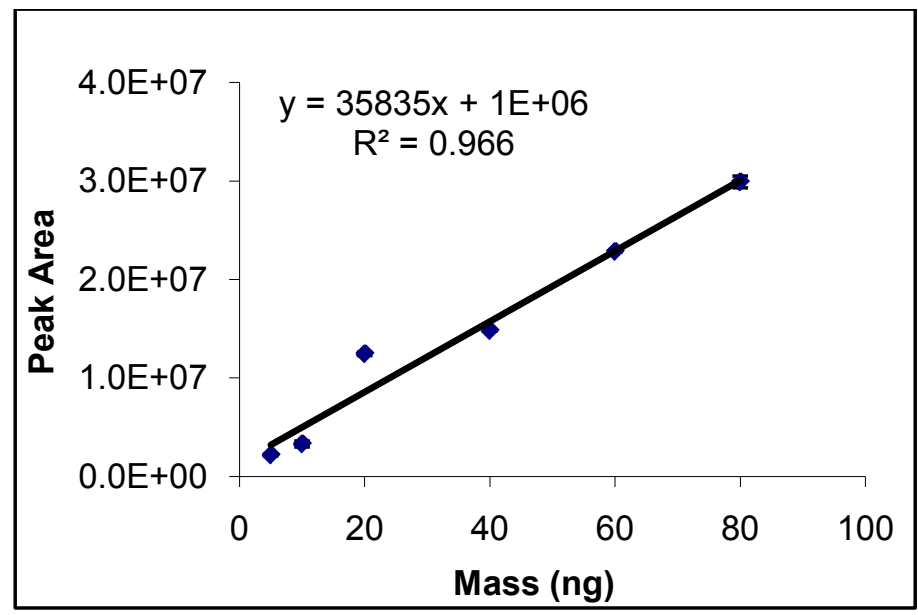

Figure 113. Calibration curve for 2-pentylfuran

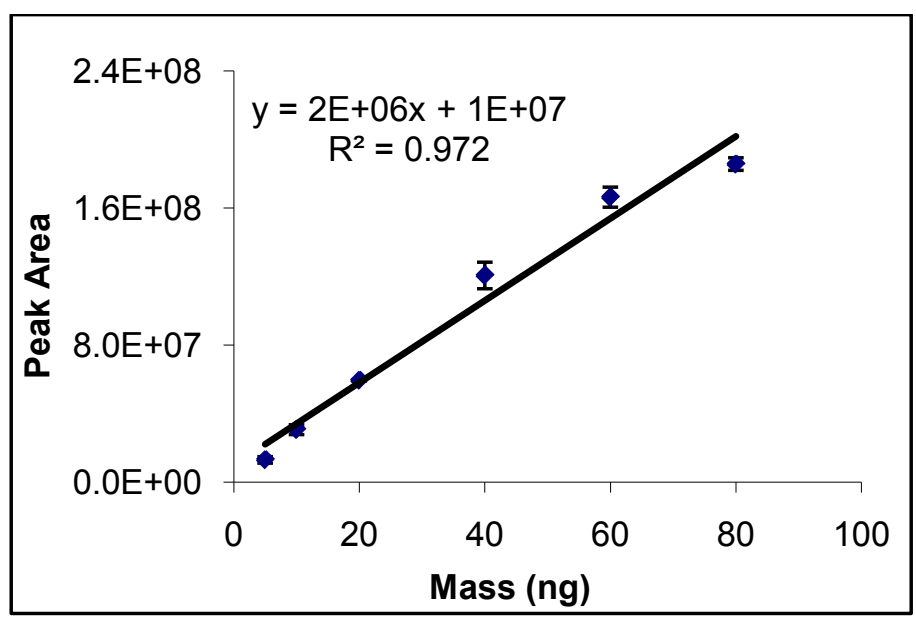


Figure 114. Calibration curve for 1-tetradecene

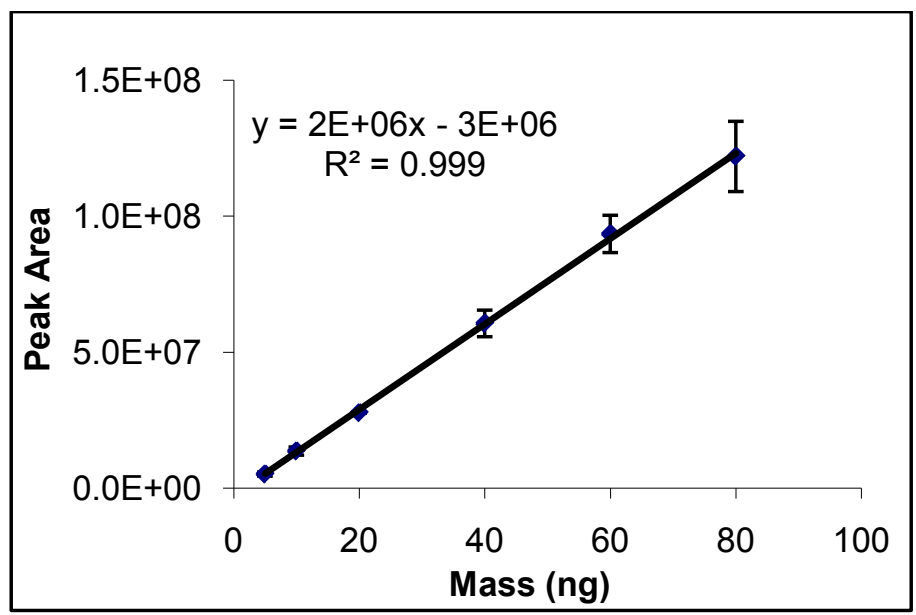

Figure 115. Calibration curve for 2,6-diisopropylnaphthalene

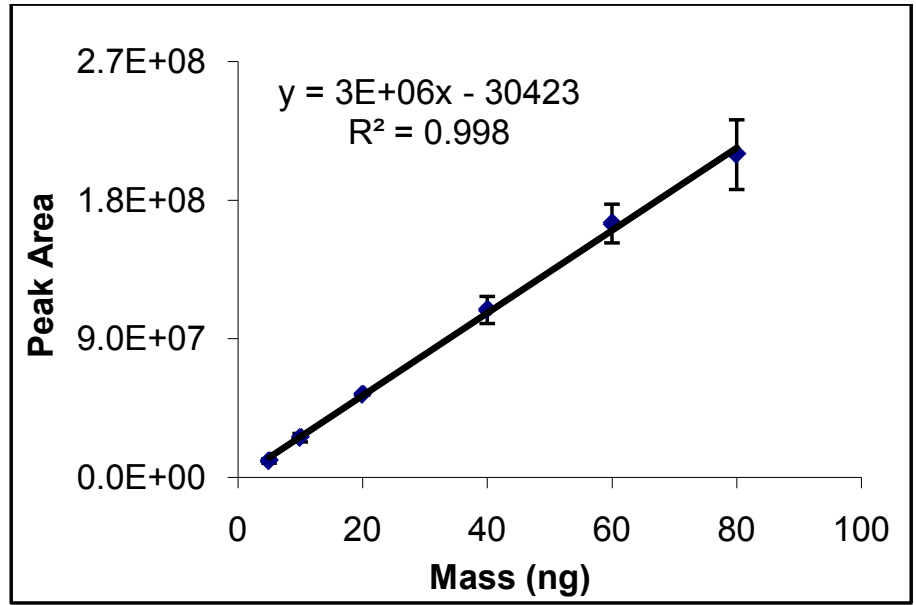


A5 Calibration curves for selected VOCs found in urine

Figure 116. Calibration curve for 4-heptanone

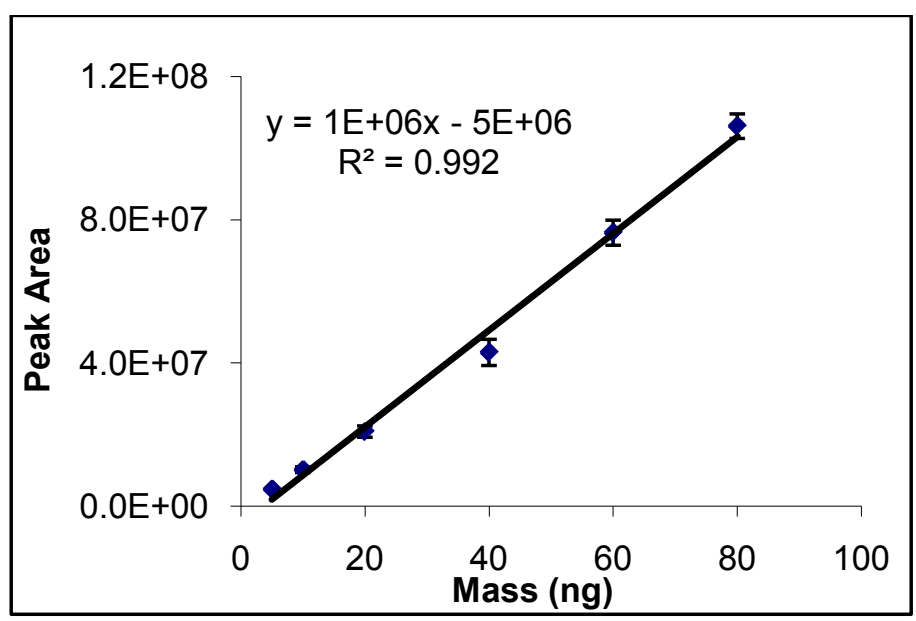

Figure 117. Calibration curve for dimethyl trisulfide

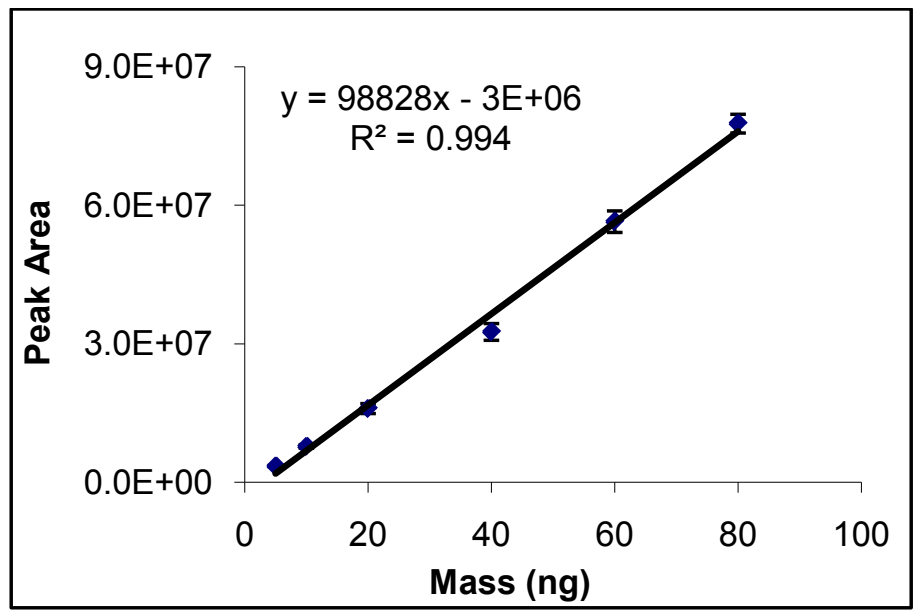


Figure 118. Calibration curve for 1,2-dichlorobenzene

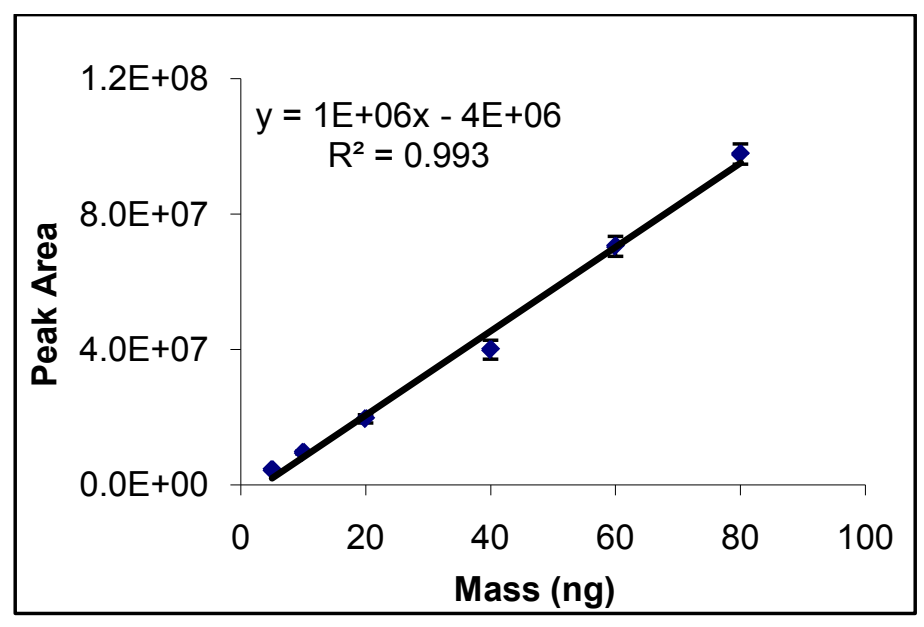

Figure 119. Calibration curve for linalool

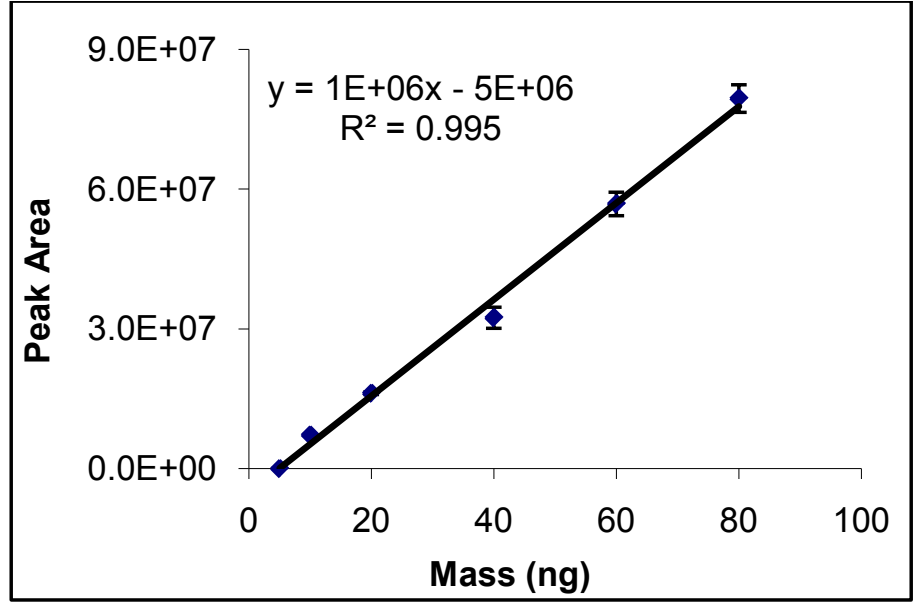

Figure 120. Calibration curve for (E)-2-nonenal

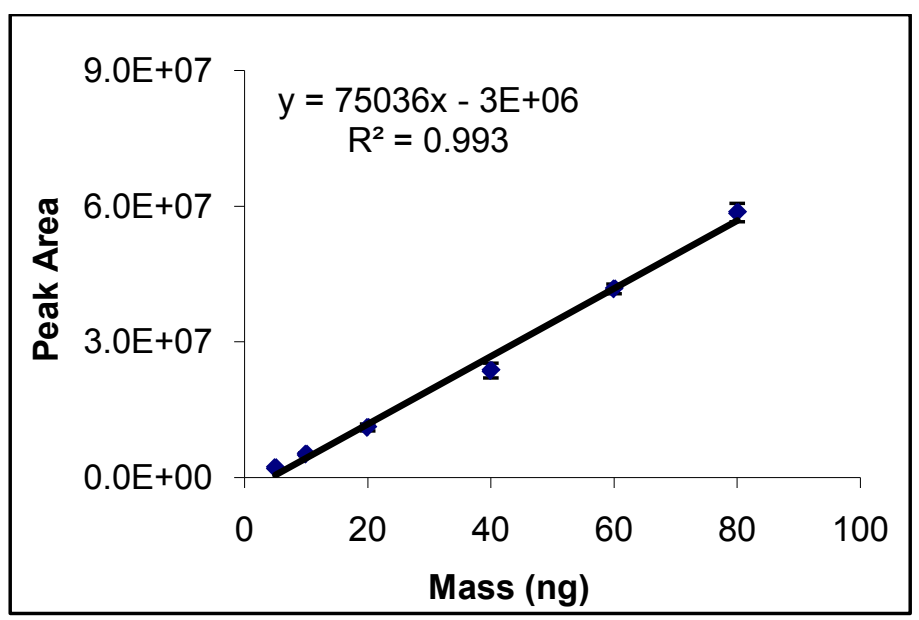


Figure 121. Calibration curve for diphenylamine

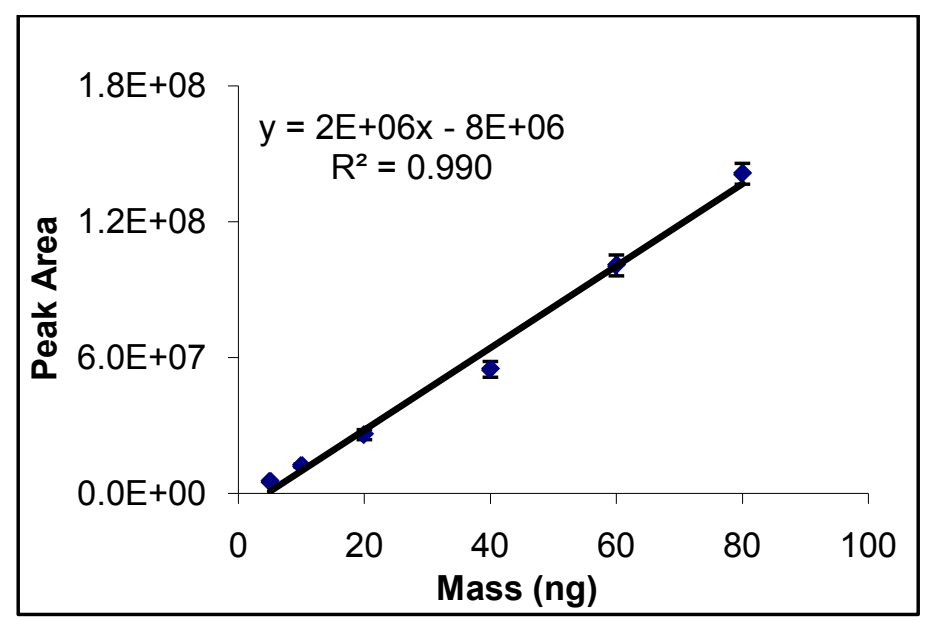




\section{Appendix B}

\section{B1 Table 73. Target ion and qualifying ions for frequently occurring hand odor}

\section{VOCs used for identification and quantitation}

\begin{tabular}{|c|c|c|c|c|c|c|}
\hline RT (min) & Compound Name & Target & Q1 & Q2 & Q3 & MW (g/mol) \\
\hline & 2-Pentanone & 43 & 86 & 41 & 58 & 86 \\
\hline 6.25 & Furfural & 96 & 96 & 95 & 39 & 96 \\
\hline 6.93 & 2-Furanmethanol & 98 & 81 & 53 & 41 & 98 \\
\hline 8.10 & Heptanal & 70 & 44 & 55 & 81 & 114 \\
\hline 8.85 & Propanedioicacid,dimethylester & 101 & 59 & 74 & 42 & 132 \\
\hline 9.44 & Benzaldehyde & 106 & 77 & 51 & 105 & 106 \\
\hline 9.98 & Phenol & 94 & 66 & 65 & 39 & 94 \\
\hline 10.06 & 6-Methyl-5-hepten-2-one & 43 & 108 & 69 & 55 & 126 \\
\hline 10.25 & Hexanoicacid & 60 & 73 & 41 & 87 & 116 \\
\hline 10.36 & Octanal & 41 & 43 & 57 & 84 & 128 \\
\hline 10.97 & BenzylAlcohol & 79 & 108 & 107 & 77 & 108 \\
\hline 11.56 & Acetophenone & 105 & 77 & 120 & 51 & 120 \\
\hline 12.14 & Undecane & 57 & 43 & 71 & 85 & 156 \\
\hline 12.16 & 3,7-dimethyl-1,6-Octadien-3-ol & 71 & 41 & 55 & 93 & 154 \\
\hline 12.22 & Nonanal & 57 & 41 & 56 & 98 & 142 \\
\hline 12.56 & Octanoicacid,methylester & 74 & 87 & 127 & 55 & 158 \\
\hline 13.14 & (E)-2-Nonenal & 41 & 55 & 70 & 43 & 140 \\
\hline 13.31 & Nonanol & 56 & 55 & 43 & 70 & 144 \\
\hline 13.65 & 2-Decanone & 58 & 43 & 71 & 59 & 156 \\
\hline 13.75 & Dodecane & 57 & 43 & 71 & 41 & 170 \\
\hline 13.85 & Decanal & 41 & 57 & 43 & 55 & 156 \\
\hline 15.21 & Tridecane & 57 & 43 & 71 & 85 & 184 \\
\hline 15.33 & Undecanal & 57 & 43 & 71 & 85 & 170 \\
\hline 16.57 & Tetradecane & 57 & 43 & 71 & 85 & 198 \\
\hline 16.70 & Dodecanal & 57 & 41 & 43 & 55 & 184 \\
\hline 17.04 & (Z)-6,10-dimethyl-5,9-Undecadien-2-one & 43 & 69 & 41 & 151 & 194 \\
\hline 17.27 & (E)-6,10-dimethyl-5,9-Undecadien-2-one & 43 & 69 & 41 & 151 & 194 \\
\hline 17.74 & 1-Pentadecene & 55 & 41 & 83 & 97 & 210 \\
\hline 17.83 & Pentadecane & 57 & 43 & 71 & 85 & 212 \\
\hline 18.65 & Dodecanoic acid & 73 & 60 & 43 & 129 & 200 \\
\hline 19.02 & Hexadecane & 57 & 71 & 43 & 85 & 226 \\
\hline 20.16 & Heptadecane & 57 & 43 & 71 & 85 & 240 \\
\hline 23.23 & Eicosane & 57 & 71 & 43 & 85 & 282 \\
\hline 24.17 & Heneicosane & 57 & 71 & 43 & 85 & 296 \\
\hline 25.06 & Docosane & 57 & 71 & 43 & 281 & 310 \\
\hline
\end{tabular}




\section{B2 Table 74. Target ion and qualifying ions for frequently occurring buccal}

\section{swab VOCs for identification and quantitation}

\begin{tabular}{|c|c|c|c|c|c|c|}
\hline RT (min) & Compound Name & Target & Q1 & $\mathbf{Q 2}$ & $\mathbf{Q 3}$ & MW (g/mol) \\
\hline & Aceticacid & 43 & 45 & 60 & 42 & 60 \\
\hline & Nonanoicacid & 60 & 73 & 57 & 115 & 158 \\
\hline & 1-Pentanol & 42 & 55 & 70 & 41 & 88 \\
\hline & Ethanol & 31 & 29 & 45 & 46 & 46 \\
\hline 5.24 & Hexanal & 44 & 56 & 41 & 57 & 100 \\
\hline 6.25 & Furfural & 96 & 96 & 95 & 39 & 96 \\
\hline 7.29 & 1-Hexanol & 56 & 55 & 43 & 41 & 102 \\
\hline 8.46 & Pentanoicacid & 60 & 73 & 41 & 45 & \\
\hline 9.43 & Benzaldehyde & 106 & 105 & 77 & 51 & 106 \\
\hline 9.95 & Phenol & 94 & 66 & 65 & 95 & 94 \\
\hline 10.12 & 2-Pentylfuran & 81 & 82 & 138 & 53 & 138 \\
\hline 10.13 & 1-Decene & 56 & 55 & 41 & 70 & 140 \\
\hline 10.4 & Hexanoicacid & 60 & 73 & 41 & 87 & 116 \\
\hline 11.41 & (E)-2-Octenal & 55 & 57 & 70 & 83 & 126 \\
\hline 11.57 & Acetophenone & 105 & 77 & 120 & 51 & 120 \\
\hline 11.66 & 1-Octanol & 56 & 55 & 41 & 69 & 130 \\
\hline 12.07 & Heptanoicacid & 60 & 73 & 43 & 41 & 130 \\
\hline 12.13 & Heptanoicacid,ethylester & 88 & 43 & 113 & 60 & 158 \\
\hline 13.14 & (E)-2-Nonenal & 41 & 55 & 70 & 43 & 140 \\
\hline 13.41 & Menthol & 71 & 81 & 95 & 41 & 156 \\
\hline 13.55 & OctanoicAcid & 60 & 73 & 55 & 144 & 144 \\
\hline 13.63 & 1-Dodecene & 55 & 41 & 69 & 56 & 168 \\
\hline 13.72 & Octanoicacid,ethylester & 88 & 101 & 127 & 57 & 172 \\
\hline 13.86 & Decanal & 41 & 57 & 43 & 55 & 156 \\
\hline 13.99 & (E,E)-2,4-Nonadienal & 81 & 41 & 67 & 138 & 138 \\
\hline 15.18 & Nonanoicacid,ethylester & 88 & 101 & 141 & 41 & 186 \\
\hline 15.23 & Tridecane & 57 & 43 & 71 & 85 & 184 \\
\hline 15.47 & 1-Methylnaphthalene & 142 & 141 & 115 & 143 & 142 \\
\hline 16.48 & 1-Tetradecene & 55 & 41 & 83 & 69 & 196 \\
\hline 16.52 & Decanoicacid,ethylester & 88 & 101 & 43 & 155 & 200 \\
\hline 16.58 & Tetradecane & 57 & 43 & 71 & 85 & 198 \\
\hline 16.72 & Dodecanal & 57 & 41 & 43 & 55 & 184 \\
\hline 16.98 & Caryophyllene & 93 & 133 & 91 & 79 & 204 \\
\hline 17.04 & (Z)-6,10-dimethyl-5,9-Undecadien-2-one & 43 & 69 & 41 & 151 & 194 \\
\hline 17.28 & (E)-6,10-dimethyl-5,9-Undecadien-2-one & 43 & 69 & 41 & 151 & 194 \\
\hline 17.75 & 1-Pentadecene & 55 & 43 & 83 & 69 & 210 \\
\hline 18.14 & Dodecanoicacid,methylester & 74 & 87 & 43 & 55 & 214 \\
\hline 18.65 & Dodecanoicacid & 73 & 60 & 200 & 43 & 200 \\
\hline 19.04 & Hexadecane & 57 & 71 & 43 & 85 & 226 \\
\hline 19.49 & Benzophenone & 105 & 182 & 77 & 51 & 182 \\
\hline 20.17 & Heptadecane & 57 & 71 & 43 & 85 & 240 \\
\hline 23.25 & Eicosane & 57 & 71 & 43 & 85 & 282 \\
\hline 24.18 & Heneicosane & 57 & 71 & 43 & 85 & 296 \\
\hline 25.08 & Docosane & 57 & 71 & 43 & 85 & 310 \\
\hline
\end{tabular}




\section{B3 Table 75. Target ion and qualifying ions for frequently occurring breath}

\section{VOCs for identification and quantitation}

\begin{tabular}{|c|c|c|c|c|c|c|}
\hline RT (min) & Compound Name & Target & Q1 & Q2 & Q3 & MW (g/mol) \\
\hline & Acetone & 43 & 58 & 42 & & 58 \\
\hline & Carbon dioxide & 44 & 28 & 16 & & 44 \\
\hline & Carbon disulfide & 76 & 78 & 44 & 32 & 76 \\
\hline & Heptane & 43 & 100 & 71 & 57 & 100 \\
\hline & Isoprene & 67 & 68 & 53 & 39 & 68 \\
\hline & Pentane & 43 & 72 & 57 & 42 & 72 \\
\hline & Toluene & 91 & 92 & 65 & 63 & 92 \\
\hline 4.16 & Octane & 43 & 85 & 41 & 57 & 114 \\
\hline 4.28 & Hexanal & 44 & 56 & 41 & 57 & 100 \\
\hline 5.28 & Ethylbenzene & 91 & 106 & 51 & & 106 \\
\hline 5.42 & p-Xylene & 91 & 106 & 105 & 77 & 106 \\
\hline 5.75 & 3-Heptanone & 57 & 85 & 114 & 72 & 114 \\
\hline 5.79 & Styrene & 104 & 103 & 78 & 51 & 104 \\
\hline 6.52 & $\alpha$-Pinene & 93 & 91 & 92 & 77 & 136 \\
\hline 6.89 & Propylbenzene & 91 & 120 & 92 & & 120 \\
\hline 7.01 & Benzaldehyde & 106 & 105 & 77 & 51 & 106 \\
\hline 7.37 & Phenol & 94 & 66 & 65 & 39 & 94 \\
\hline 7.48 & 6-Methyl-5-hepten-2-one & 43 & 41 & 108 & 55 & 126 \\
\hline 8.16 & D-Limonene & 68 & 93 & 67 & 79 & 136 \\
\hline 8.26 & 1,2-Dichlorobenzene & 147 & 111 & 112 & 75 & 146 \\
\hline 8.80 & Acetophenone & 105 & 77 & 120 & 51 & 120 \\
\hline 9.29 & Undecane & 57 & 43 & 71 & 41 & 156 \\
\hline 9.37 & Nonanal & 57 & 41 & 56 & 55 & 142 \\
\hline 9.59 & 1,2,4,5-Tetramethylbenzene & 119 & 134 & 91 & 133 & 134 \\
\hline 10.48 & Menthol & 71 & 81 & 95 & 41 & 156 \\
\hline 10.65 & Naphthalene & 128 & 127 & 129 & 102 & 128 \\
\hline 10.70 & 1-Dodecene & 55 & 41 & 83 & 97 & 168 \\
\hline 10.82 & Dodecane & 57 & 43 & 71 & 170 & 170 \\
\hline 10.91 & Decanal & 41 & 57 & 43 & 55 & 156 \\
\hline 12.25 & Tridecane & 57 & 43 & 71 & 85 & 184 \\
\hline 12.36 & Undecanal & 43 & 57 & 41 & 55 & 170 \\
\hline 12.50 & 1-Methylnaphthalene & 142 & 141 & 115 & 143 & 142 \\
\hline 13.26 & n-Decanoicacid & 73 & 60 & 129 & 41 & 172 \\
\hline 13.58 & Tetradecane & 57 & 43 & 71 & 85 & 198 \\
\hline 13.72 & Dodecanal & 41 & 55 & 43 & 57 & 184 \\
\hline 13.99 & Caryophyllene & 93 & 133 & 91 & 41 & 204 \\
\hline 14.04 & (Z)-6,10-dimethyl-5,9-Undecadien-2-one & 43 & 69 & 41 & 151 & 194 \\
\hline 14.28 & (E)-6,10-dimethyl-5,9-Undecadien-2-one & 43 & 69 & 41 & 151 & 194 \\
\hline 15.08 & ButylatedHydroxytoluene & 205 & 220 & 206 & 57 & 220 \\
\hline 15.65 & Dodecanoicacid & 73 & 60 & 41 & 43 & 200 \\
\hline 16.04 & Hexadecane & 57 & 71 & 43 & 85 & 226 \\
\hline 16.50 & Benzophenone & 105 & 182 & 77 & 51 & 182 \\
\hline 17.17 & Heptadecane & 57 & 71 & 43 & 85 & 240 \\
\hline 17.59 & 2,6-Diisopropylnaphthalene & 197 & 212 & 155 & 198 & 212 \\
\hline 19.74 & n-Hexadecanoicacid & 73 & 60 & 43 & 41 & 256 \\
\hline 20.03 & Eicosane & 57 & 71 & 43 & 85 & 282 \\
\hline
\end{tabular}




\section{B4 Table 76. Target ion and qualifying ions for frequently occurring blood}

\section{VOCs for identification and quantitation}

\begin{tabular}{|c|c|c|c|c|c|c|}
\hline RT (min) & Compound Name & Target & Q1 & Q2 & Q3 & MW (g/mol) \\
\hline & Acetone & 43 & 58 & 42 & & 58 \\
\hline & Octanal & 43 & 44 & 56 & 84 & 128 \\
\hline & Hexanal & 44 & 41 & 56 & 57 & 100 \\
\hline & 1-Pentanol & 42 & 55 & 70 & 41 & 88 \\
\hline 4.80 & 1-Hexanol & 56 & 55 & 43 & 41 & 102 \\
\hline 4.82 & Benzene,1,3-dimethyl- [m-xylene] & 91 & 106 & 105 & 77 & 106 \\
\hline 4.83 & p-Xylene & 91 & 106 & 105 & 77 & 106 \\
\hline 4.88 & 4-Heptanone & 71 & 43 & 114 & 41 & 114 \\
\hline 5.09 & 3-Heptanone & 57 & 85 & 72 & 41 & 114 \\
\hline 5.16 & 2-Heptanone & 43 & 58 & 71 & 59 & 114 \\
\hline 5.23 & Cyclohexanone & 55 & 42 & 98 & 69 & 98 \\
\hline 5.24 & Nonane & 43 & 57 & 71 & 41 & 128 \\
\hline 5.30 & Heptanal & 70 & 44 & 41 & 55 & 114 \\
\hline 6.23 & Benzaldehyde & 106 & 105 & 77 & 51 & 106 \\
\hline 6.35 & 1-Heptanol & 70 & 56 & 55 & 43 & 116 \\
\hline 6.51 & 1-Octen-3-ol & 57 & 43 & 72 & 41 & 128 \\
\hline 6.55 & Phenol & 94 & 66 & 65 & 55 & 94 \\
\hline 6.66 & 6-Methyl-hepten-2-one & 43 & 42 & 126 & 69 & 126 \\
\hline 6.67 & 1-Decene & 56 & 55 & 41 & 70 & 140 \\
\hline 6.69 & 2-Pentylfuran & 81 & 82 & 138 & 53 & 138 \\
\hline 7.29 & D-Limonene & 68 & 93 & 67 & 79 & 136 \\
\hline 7.39 & BenzylAlcohol & 79 & 77 & 108 & 107 & 108 \\
\hline 7.39 & 1,2-Dichlorobenzene & 146 & 148 & 111 & 113 & 146 \\
\hline 7.89 & Acetophenone & 105 & 77 & 120 & 51 & 120 \\
\hline 7.99 & 1-Octanol & 55 & 41 & 69 & 70 & 130 \\
\hline 8.36 & Undecane & 57 & 43 & 71 & 41 & 156 \\
\hline 8.43 & Nonanal & 57 & 41 & 56 & 55 & 142 \\
\hline 8.65 & $1,2,4,5$-Tetramethylbenzene & 119 & 134 & 91 & & 134 \\
\hline 9.52 & Menthol & 71 & 81 & 95 & 41 & 156 \\
\hline 9.69 & Naphthalene & 128 & 127 & 129 & 102 & 128 \\
\hline 9.73 & 1-Dodecene & 55 & 43 & 41 & 69 & 168 \\
\hline 9.76 & 2-Decanone & 58 & 43 & 71 & 59 & 156 \\
\hline 9.85 & Dodecane & 57 & 43 & 71 & 41 & 170 \\
\hline 9.94 & Decanal & 57 & 43 & 41 & 55 & 156 \\
\hline 11.26 & Tridecane & 57 & 43 & 71 & 85 & 184 \\
\hline 11.51 & 1-Methylnaphthalene & 142 & 141 & 115 & 143 & 142 \\
\hline 12.49 & 1-Tetradecene & 41 & 55 & 83 & 69 & 196 \\
\hline 12.59 & Tetradecane & 57 & 43 & 71 & 85 & 198 \\
\hline 12.72 & Dodecanal & 57 & 41 & 43 & 67 & 184 \\
\hline 13.06 & (Z)-6,10-dimethyl-5,9-Undecadien-2-one & 43 & 69 & 41 & 151 & 194 \\
\hline 13.29 & (E)-6,10-dimethyl-5,9-Undecadien-2-one & 43 & 69 & 41 & 151 & 194 \\
\hline 13.53 & 1-Dodecanol & 55 & 69 & 83 & 168 & 186 \\
\hline 15.04 & Hexadecane & 57 & 71 & 43 & 85 & 226 \\
\hline 16.17 & Heptadecane & 57 & 71 & 43 & 85 & 240 \\
\hline 16.58 & 2,6-Diisopropylnaphthalene & 197 & 212 & 155 & 198 & 212 \\
\hline
\end{tabular}


B5 Table 77. Target ion and qualifying ions for frequently occurring urine

VOCs for identification and quantitation

\begin{tabular}{|c|c|c|c|c|c|c|}
\hline RT (min) & Compound Name & Target & Q1 & Q2 & Q3 & MW (g/mol) \\
\hline & Toluene & 91 & 92 & 65 & & 92 \\
\hline & 2-Pentanone & 43 & 86 & 41 & 58 & 86 \\
\hline & Pyrrole & 67 & 39 & 41 & 28 & 67 \\
\hline & 4-Nonylphenol & 107 & 220 & 108 & & 220 \\
\hline 7.08 & \begin{tabular}{|l|} 
4-Heptanone \\
\end{tabular} & 71 & 43 & 114 & 41 & 114 \\
\hline 7.38 & 3-Heptanone & 57 & 85 & 72 & 41 & 114 \\
\hline 7.46 & 2-Heptanone & 43 & 58 & 71 & 59 & 114 \\
\hline 8.68 & Benzaldehyde & 106 & 105 & 77 & 51 & 106 \\
\hline 8.80 & Dimethyltrisulfide & 126 & 79 & 45 & 64 & 126 \\
\hline 9.15 & 1-Decene & 41 & 55 & 56 & 70 & 140 \\
\hline 9.77 & 1,2-Dichlorobenzene & 146 & 148 & 111 & 75 & 146 \\
\hline 10.24 & 1-Octanol & 56 & 55 & 41 & 69 & 130 \\
\hline 10.30 & 4-Methylphenol & 107 & 108 & 77 & 79 & 108 \\
\hline 10.62 & 3,7-dimethyl-,6-Octadien-3-ol & 93 & 71 & 41 & 43 & 154 \\
\hline 10.65 & Nonanal & 57 & 41 & 56 & 98 & 142 \\
\hline 11.29 & (E)-2-Nonenal & 41 & 55 & 70 & 43 & 140 \\
\hline 11.47 & Menthol & 71 & 81 & 95 & 41 & 156 \\
\hline 11.71 & MethylSalicylate & 120 & 98 & 152 & 121 & 152 \\
\hline 11.77 & Decanal & 57 & 43 & 41 & 55 & 156 \\
\hline 12.67 & 5-(2-propenyl)-1,3-Benzodioxole & 162 & 131 & 104 & 103 & 162 \\
\hline 13.94 & (E)-6,10-dimethyl-5,9-Undecadien-2-one & 43 & 69 & 41 & 151 & 194 \\
\hline 14.10 & (Z)-6,10-dimethyl-5,9-Undecadien-2-one & 43 & 69 & 41 & 151 & 194 \\
\hline 14.64 & ButylatedHydroxytoluene & 205 & 220 & 206 & 57 & 220 \\
\hline 14.99 & Dodecanoicacid & 73 & 60 & 200 & 43 & 200 \\
\hline 15.26 & Hexadecane & 57 & 71 & 43 & 85 & 226 \\
\hline 15.39 & 4-(1,1,3,3-Tetramethylbutyl)-phenol & 135 & 107 & & & 206 \\
\hline 15.55 & Diphenylamine & 169 & 168 & 167 & 170 & 169 \\
\hline 15.63 & Benzophenone & 105 & 182 & 77 & 51 & 182 \\
\hline 16.02 & Heptadecane & 57 & 43 & 71 & 85 & 240 \\
\hline
\end{tabular}


VITA

\section{MAIKO KUSANO}

$\begin{array}{ll}\text { April 29, } 1981 & \text { Born, Tokyo, Japan } \\ \text { 1999-2003 } & \text { B.A. Chemistry: Concentration in Biochemistry (Cum Laude) } \\ & \text { Washington University in St. Louis } \\ & \text { St. Louis, MO, USA } \\ & \text { Toxicology Laboratory Intern } \\ & \text { St. Louis County Medical Examiner's Office } \\ & \text { Berkeley, MO, USA } \\ & \text { Teaching Assistant } \\ & \text { Florida International University } \\ & \text { Miami, FL, USA } \\ & \text { Graduate Assistant } \\ & \text { Florida International University } \\ & \text { Miami, FL, USA } \\ & 3^{\text {rd }} \text { Place Graduate Student Association's Scholarly Forum } \\ & \text { Florida International University } \\ & \text { Miami, FL, USA }\end{array}$

\section{PUBLICATIONS AND PRESENTATIONS:}

Kusano M, Mendez E, Furton KG. Development of a SPME-GC/MS method capable of differentiating VOC profiles from human blood, breath, buccal cells and urine, Journal of Chromatography B, in submission.

Furton KG, Kusano M, Macias MS. Gas Chromatography: Forensic Applications (L3), in Encyclopedia of Separation Science, First On-line Update, Elsevier Ltd., 2007. 
Kusano M and Furton KG. A 6-month study of the characteristic human scent VOCs present in various biological specimens. Presented at the $61^{\text {st }}$ Pittsburgh Conference on Analytical Chemistry and Applied Spectroscopy, Orlando, FL, March 2010.

Kusano M and Furton KG. HS-SPME/GC-MS analysis of various biological specimens using VOCs. Presented at the $61^{\text {st }}$ Annual Meeting, American Academy of Forensic Sciences, Denver, CO, February 2009.

Kusano M and Furton KG. Pilot study of the potential for using different biological specimens as human scent sources. Presented at the $60^{\text {th }}$ Annual Meeting, American Academy of Forensic Sciences, Washington DC, February 2008.

Kusano M and Furton KG. Comparison of the volatile biomarkers from biological specimens for profiling potential. Presented at the $59^{\text {th }}$ Annual Meeting, American Academy of Forensic Sciences, San Antonio, TX, February 2007.

Kusano $\mathrm{M}$ and McCord BR. The development of the microfluidic approaches to the detection of GHB. Presented at the $58^{\text {th }}$ Annual Meeting, American Academy of Forensic Sciences, Seattle, WA, February, 2006 and Forensic Science Symposium, Fort Laudert Lauderdale Forensic Conference, South Florida Section of American Chemical Society, Fort Lauderdale, FL, 2006. 